\title{
Development of Extrospective Systems for Mobile Robotic Vehicles.
}

\author{
A thesis \\ submitted in partial fulfilment \\ of the requirements for the Degree \\ of \\ Master of Engineering in \\ Electronic and Computer Systems Engineering \\ at \\ Victoria University of Wellington \\ By \\ Johnny Robert Keogh McClymont

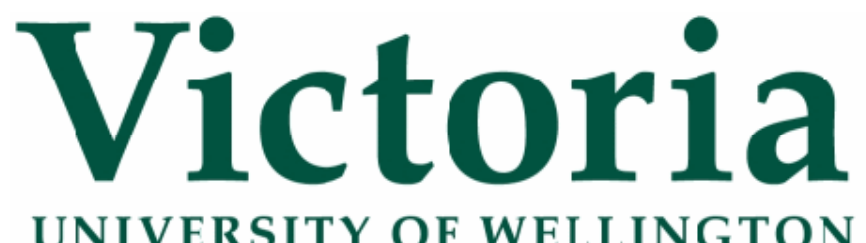 \\ Te Whare Wānanga \\ o te Ūpoko o te Ika a Māui

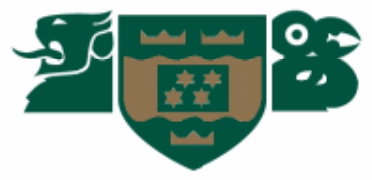

2010 


\section{Abstract}

Extrospection is the process of receiving knowledge of the outside world through the senses. On robotic platforms this is primarily focussed on determining distances to objects of interest and is achieved through the use of ranging sensors. Any hardware implemented on mobile robotic platforms, including sensors, must ideally be small in size and weight, have good power efficiency, be self-contained and interface easily with the existing platform hardware. The development of stable, expandable and interchangeable mobile robot based sensing systems is crucial to the establishment of platforms on which complex robotic research can be conducted and evaluated in real world situations.

This thesis details the design and development of two extrospective systems for incorporation in the Victoria University of Wellington's fleet of mobile robotic platforms. The first system is a generic intelligent sensor network. Fundamental to this system has been the development of network architecture and protocols that provide a stable scheme for connecting a large number of sensors to a mobile robotic platform with little or no dependence on the existing hardware configuration of the platform. A prototype sensor network comprising fourteen infrared position sensitive detectors providing a short to medium distance ranging system $(0.2-3 \mathrm{~m})$ with a $360^{\circ}$ field of view has been successfully developed and tested.

The second system is a redesign of an existing prototype full-field image ranger system. The redesign has yielded a smaller, mobile version of the prototype system capable of ranging medium to long distances $(0-15 \mathrm{~m})$ with a $22.2^{\circ} \times 16.5^{\circ}$ field-of-view. This ranger system can now be incorporated onto mobile robotic platforms for further research into the capabilities of full-field image ranging as a form of extrospection on a mobile platform. 


\section{Acknowledgements}

I wish to express my sincere gratitude and thanks to the following people, who have provided invaluable support and guidance to me, as well as patience, during the course of this project.

To Professor Dale Carnegie, my project supervisor, I am thankful for his patience and expertise in guiding me through the process of completing this project. His assistance, especially in the challenging final stages of this project, has been invaluable in meeting project deadlines and ensuring the successful completion of this project. Finally thank you for providing a sounding board through the stressful stages of the project.

A big thank you to Adrian Jongenelen, the $\mathrm{PhD}$ student who designed the prototype ranger system, for all the time spent explaining the intricacies of the system and his research that has been invaluable in the system's redesign and in the writing of this thesis and several conference papers.

Thank you to Ben Drayton, the PhD student who will employ the mobile ranger system in his studies, for assistance in designing the Image Capture board and the effort and talent provided in providing VHDL code in debugging the new system hardware and for porting code segments from the prototype system to the mobile system.

My gratitude to the School of Chemical and Physical Sciences and the School of Engineering and Computer Science for allowing me to work part time while this project was conducted.

Many thanks to Alan Rennie, Manu Pouajen-Blakiston and Nick Grinter, the talented and friendly staff of the mechanical workshop. Their knowledge, ability and professionalism with the material aspects of the sensor network chassis development are greatly appreciated.

Thanks to my fellow work colleagues, Jason Edwards and Tim Exley, whose enthusiasm and willingness to take on the extra workload has allowed me to work part time and concentrate on this project.

To my parents and friends Bob and Colleen McClymont, words cannot express the appreciation and indebtedness I have for them. Thanks to their endless support, both 
emotionally and financially in providing me with the privileged opportunity to earn an education. Special thanks to Colleen for the hours of proof-reading this thesis.

Finally, I owe enormous gratitude to Duc Nguyen, my partner, for his continual support and incredible patience during the undertaking of this project. Thanks to his willingness to sacrifice two years of his life waiting for the completion of this thesis before I could move countries to be with him. 


\section{Table of Contents}

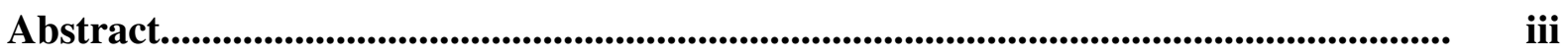

Acknowledgements..

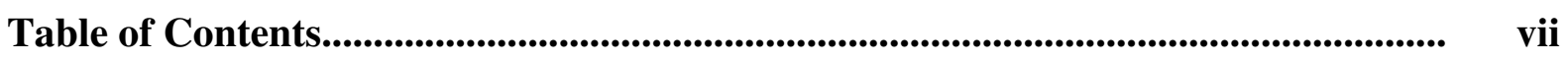

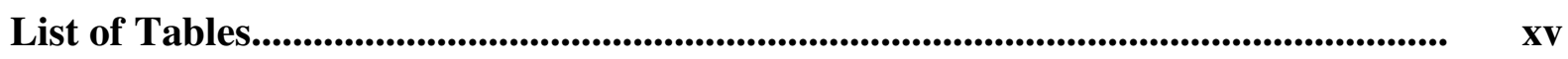

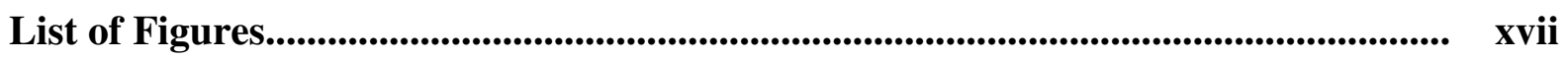

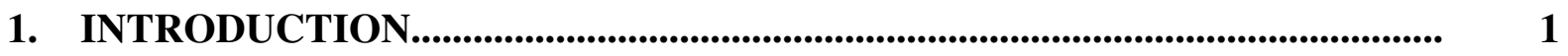

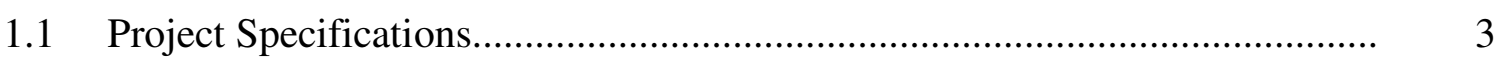

1.2 Project Management............................................................................. 5

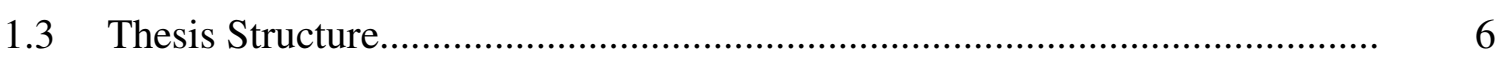

2. BACKGROUND THEORY ....................................................................................... 9

2.1 Techniques of Extrospection for Range Finding.........................................

2.1.1 Triangulation................................................................................... 9

2.1.1.1 Position Sensitive Detection.......................................................... 11

2.2.1.2 Laser Line Scanning............................................................... 12

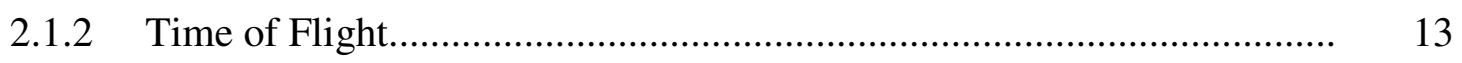

2.1.2.1 Acoustic Waves......................................................................... 15

2.1.2.2 Electromagnetic Waves............................................................. 18

2.2 Sensor Technology on the VUW Robotic Fleet............................................. 22

2.2.1 Sharp Position Sensitive Detectors......................................................... 22

2.2.2 SensComp Ultrasonic Ranging Sensors................................................ 24

2.2.3 Laser Line Scanning System............................................................... 25

2.2.4 Heterodyne Full-Field Image Ranger System....................................... 25

2.2.5 Sensor Technology Summary............................................................ 28 
2.3 Network Characteristics........................................................................ 28

2.3.1 Network Topology............................................................................ 29

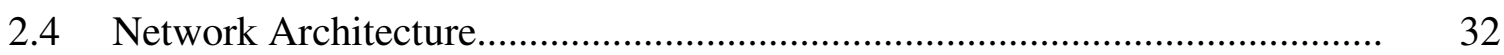

2.4.1 Open Systems interconnection (OSI) Model....................................... 32

3 HARDWARE METHODOLOGY - SENSOR NETWORK INTERFACE....... 35

3.1 Fundamentals of a Sensor Network........................................................... 35

3.1.1 Selection of Network Topology....................................................... 36

3.1.2 Communication Technology........................................................... 38

3.1.2.1 Serial Peripheral Interface ............................................................ 39

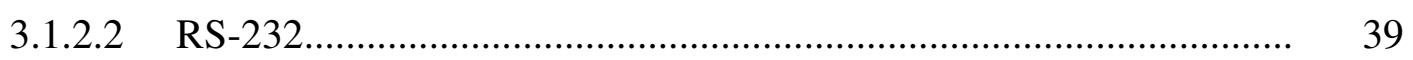

3.1.2.3 Ethernet............................................................................... 39

3.1.2.4 Universal Serial Bus................................................................ 40

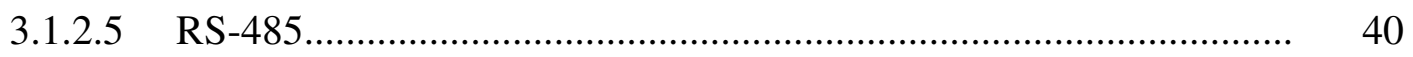

3.1.3 Network Bus.............................................................................. 42

3.2 Sensor Network Interface Module................................................................ 43

3.2.1 Ethernet Interface......................................................................... 45

3.2.2 Universal Serial Bus Interface............................................................. 46

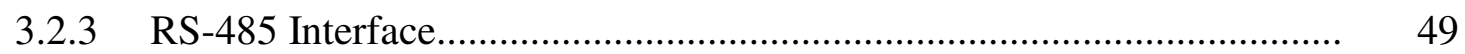

3.2.4 Power Supply.............................................................................. 51

3.2.5 Assembled Module....................................................................... 52

4 HARDWARE METHODOLOGY - SENSOR NETWORK NODES................. 53

4.1 Infrared Ranging Node............................................................................ 53

4.1.1 Sharp IR PSD Ranging Modules...................................................... 53

4.1.2 RS-485 Interface......................................................................... 56

4.1.3 Sharp IR PSD Interface ............................................................. 58

4.1.4 Assembled Module........................................................................ 60

4.2 Ultrasonic Range Node........................................................................ 61

4.2.1 SensComp Ultrasonic Ranging Modules............................................... 61

4.2.2 RS-485 Interface......................................................................... 65

4.2.3 Ultrasonic Sensor Interface............................................................. 65 


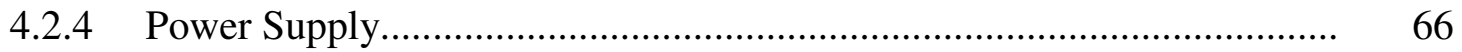

4.2.5 Assembled Module.................................................................................. 67

4.3 Prototype Sensor Network....................................................................... $\quad 68$

4.3.1 Assembled Sensor Network Chassis.......................................................... 71

5 FIRMWARE METHODOLOGY - SENSOR NETWORK............................... 73

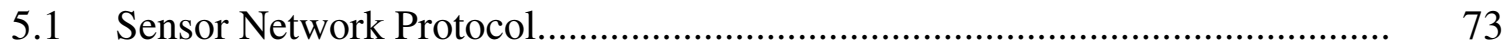

5.1.1 Sensor Model....................................................................................... $\quad 73$

5.1.2 Host Layer Protocol...............................................................................

5.1.2.1 Sensor Tasks.............................................................................. 76

5.1.2.2 HLP Packet Format................................................................. 77

5.1.3 Media Layer Protocol........................................................................ 79

5.1.3.1 MLP Packets............................................................................... 81

5.1.3.2 Physical Addresses................................................................. 83

5.1.3.3 MLP Packet Types..................................................................... 83

5.2 Network Layer Stack........................................................................... 86

5.2.1 Physical Layer.............................................................................. 88

5.2.2 Data Link Layer......................................................................... 90

5.2.3 Network Layer................................................................................ 93

5.2.4 Sensor Network Layer..................................................................... 95

5.3 Control Computer / Network Command Interface ...................................... 98

5.4 IR PSD Sensor Control....................................................................... 102

5.5 Ultrasonic Sensor Control............................................................................ 104

6 SOFTWARE METHODOLOGY - SENSOR NETWORK................................. 107

6.1 Development Environment................................................................. 107

6.2 C\# Sensor Network Libraries................................................................. 107

6.2.1 Packet Communication....................................................................... 108

6.2.1.1 Packet Class.............................................................................. 109

6.2.1.2 PacketCom Class............................................................................ 111

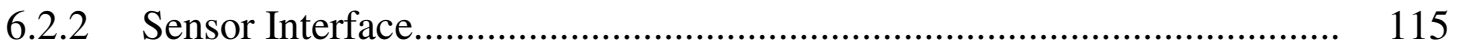

6.2.2.1 Sensor Node Class............................................................................ 116

6.2.2.2 Sensor Interface Class................................................................ 120 
6.3 Command Packet Interface ..................................................................... 128

6.4 Test Sensor Network Graphical Application................................................. 129

7 HARDWARE METHODOLOGY - FULL-FIELD IMAGE RANGER............ 131

7.1 Initial Prototype Design.................................................................... 131

7.1.1 FPGA Development Board.............................................................. 132

7.1.2 Illumination Board............................................................................. 133

7.1.3 PMD Daughter Board...................................................................... 134

7.1.4 PMD Main Board......................................................................... 135

7.1.5 Ethernet / VGA Board........................................................................ 135

7.2 Mobile Platform Design............................................................................ 136

7.2.1 Circuit Board Dimensions................................................................. 138

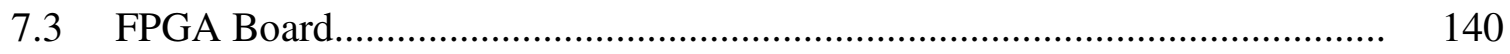

7.3.1 FPGA Selection.............................................................................. 140

7.3.2 External Memory Selection................................................................... 141

7.3.2.1 Selected Memory Devices............................................................. 145

7.3.3 FPGA Configuration Scheme................................................................ 146

7.3.4 General Purpose Interface................................................................... 149

7.3.5 Power Supply........................................................................... 150

7.3.5.1 Unregulated Power Bus............................................................. 150

7.3.5.2 FPGA Power Supply............................................................. 151

8 HARDWARE METHODOLOGY - IMAGE RANGER PERIPHERALS........ 155

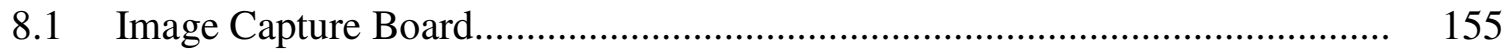

8.1.1 Imaging Sensor............................................................................. 156

8.1.2 Analogue to Digital Conversion.............................................................. 158

8.1.3 Low Voltage Differential Signals........................................................ 158

8.1.4 Power Supply............................................................................. 159

8.2 Laser Illumination Board.......................................................................... 161

8.2.1 Laser Diode Current......................................................................... 162

8.2.2 Power Supply.............................................................................. 163

8.3 External Interface Board....................................................................... 165 
8.3.1 Video Graphics Array (VGA) Interface................................................. 166

8.3.2 Ethernet Interface......................................................................... 167

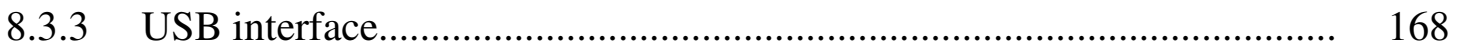

8.3.4 Power Supply............................................................................ 168

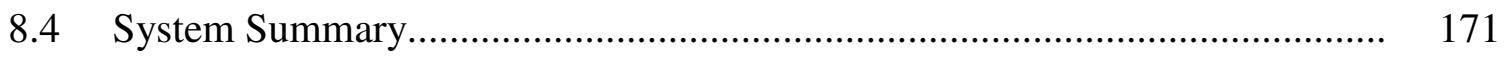

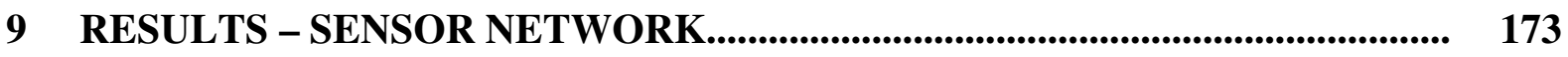

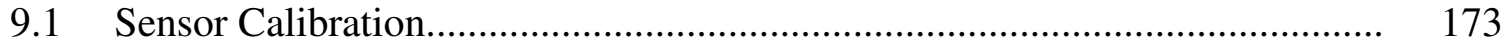

9.1.1 IR Ranging Modules.......................................................................... 173

9.1.2 Ultrasonic Ranging Modules................................................................. 184

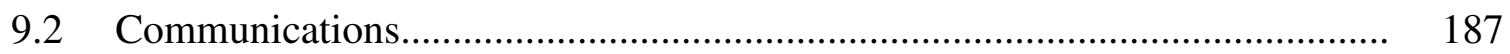

9.2.1 Network Frame........................................................................... 188

9.3 Sensor Control................................................................................... 189

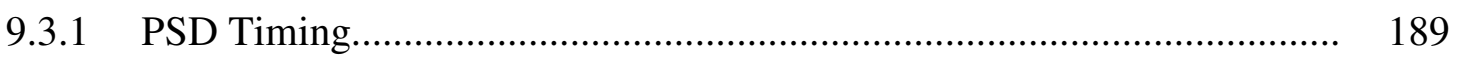

9.3.2 Ultrasonic Timing............................................................................ 191

9.3.3 Measurement Window....................................................................... 191

9.4 Prototype Sensor Network ....................................................................... 192

9.4.1 Sensor Accuracy................................................................................ 192

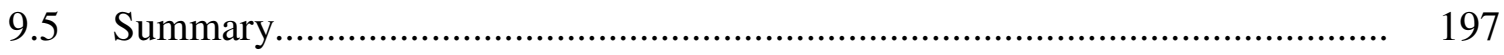

10 RESULTS - FULL-FIELD IMAGE RANGER_.................................................. 199

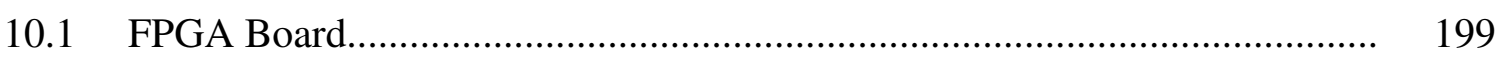

10.1.1 FPGA Power Supply..................................................................... 199

10.1.2 FPGA Configuration and Programming.............................................. 201

10.1.3 FPGA External Memory..................................................................... 204

10.1.4 FPGA Generic I/O Interface......................................................... 206

10.2 External Interface Board................................................................... 208

10.2.1 Power Supply.................................................................................. 208

10.2.2 Generic I/O Interface...................................................................... 208

$10.3 \quad$ Image Capture Board............................................................................. 209

10.3.1 Power Supply.................................................................................. 209

10.3.2 Generic I/O Interface......................................................................... 209

10.3.3 Signal Modulation........................................................................... 209 
10.3.4 Pin Driver Power Consumption........................................................ 210

$10.4 \quad$ Laser Illumination Board...................................................................... 212

10.4.1 Power Supply............................................................................ 212

10.4.2 Laser Switching.......................................................................... 212

10.4.3 Protection Circuit................................................................................ 214

10.4.4 DAC Two Wire Interface................................................................ 215

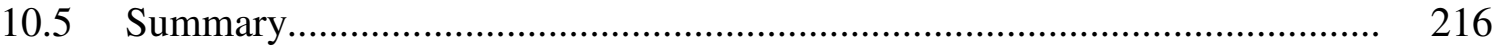

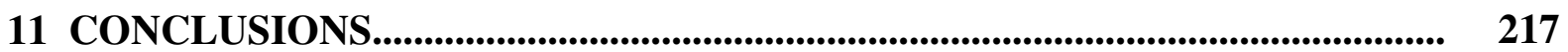

$11.1 \quad$ Project Review................................................................................ 217

11.1.1 Sensor Network........................................................................ 217

11.1.2 Full-Field Image Ranger............................................................... 219

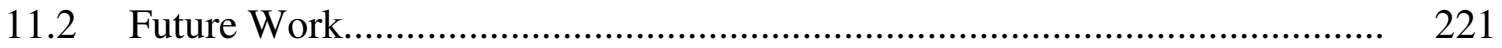

11.2.1 Cyclone III FPGA Code......................................................................... 221

11.2.2 FPGA Configuration.......................................................................... 222

11.2.3 DDR2 SDRAM....................................................................... 222

11.2.4 Thermal Management....................................................................... 222

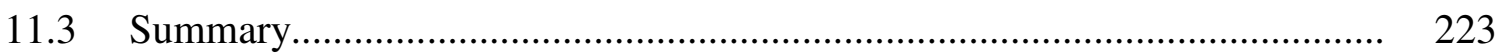

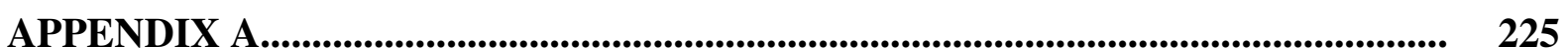

A.1 Sensor Network Interface MCU Schematic................................................... 225

A.2 Sensor Network Interface USB Schematic................................................... 226

A.3 Infrared Ranging Node Schematic........................................................... 227

A.4 Ultrasonic Ranging Node Schematic............................................................. 228

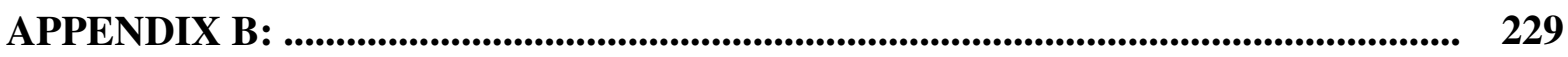

B.1 FPGA Board - Power 1 Schematic.......................................................... 229

B.2 FPGA Board - Power 2 Schematic........................................................... 230

B.3 FPGA Board - FPGA Power Bank Schematic.............................................. 231

B.4 FPGA Board - FPGA Configuration Schematic........................................... 232

B.5 FPGA Board - FPGA Bank 1 \& 2 Schematic.............................................. 233

B.6 FPGA Board - FPGA Bank 3 \& 4 Schematic.............................................. 234 
B.7 FPGA Board - FPGA Bank 5 \& 6 Schematic......................................... 235

B.8 FPGA Board - FPGA Bank 7 \& 8 Schematic........................................... 236

B.9 FPGA Board - Accumulator SDRAM Schematic....................................... 237

B.10 FPGA Board - Output Buffer SDRAM Schematic................................... 238

B.11 FPGA Board - NIOS SDRAM Schematic.............................................. 239

B.12 FPGA Board - Ethernet Buffer SDRAM Schematic.................................... 240

B.13 FPGA Board - HSM-A Generic Interface Schematic................................. 241

B.14 FPGA Board - HSM-B Generic Interface Schematic................................. 242

B.15 FPGA Board - Power Decoupling Schematic........................................... 243

B.16 External Interface - Power Schematic..................................................... 244

B.17 External Interface - Ethernet Schematic.............................................. 245

B.18 External Interface - USB Schematic..................................................... 246

B.19 External Interface - VGA Schematic................................................... 247

B.20 External Interface - Generic Interface Schematic.................................... 248

B.21 Image Capture - LVDS Schematic......................................................... 249

B.22 Image Capture - Daughter Interface Schematic........................................ 250

B.23 Image Capture - Generic Interface Schematic......................................... 251

B.24 Laser Illumination - Power Schematic................................................. 252

B.25 Laser Illumination - Control Interface Schematic..................................... 253

B.26 Laser Illumination - Laser Bank 1 Schematic......................................... 254

B.27 Laser Illumination - Laser Bank 2 Schematic............................................ 255

APPENDIX C: CD CONTENTS............................................................................................. 257

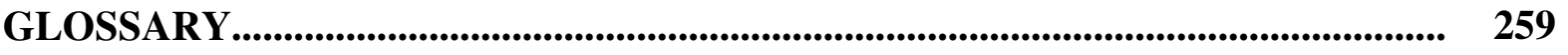

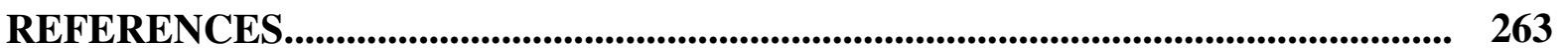




\section{List of Tables}

Table 4.1 SensComp Electronic modules Control and Output Lines........................... 63

Table 5.1 Host Layer Protocol Packet Descriptions.................................................... 78

Table 5.2 MLP Packet Types................................................................................ 84

Table 5.3 Sensor Network Layer Functions................................................................. 97

Table 5.4 Command Interface Packet Types................................................................ 101

Table 5.5 Command Interface Error Codes................................................................... 102

Table 6.1 Public Fields of the Packet Class................................................................... 109

Table 6.2 Configuration File Data Fields............................................................... 121

Table 7.1 Cyclone III FPGA Information................................................................ 141

Table 7.2 External Memory Devices of Mobile Platform System.............................. 146

Table 7.3 FPGA Board Voltage Rails..................................................................... 151

Table 8.1 Image Capture Board Voltage Rails........................................................ 159

Table 8.2 Laser Illumination Board Voltage Rails...................................................... 163

Table 8.3 External Interface Board Voltage Rails.................................................. 169 


\section{List of Figures}

Figure 1.1: (Left) CamCube 3-D Ranging Camera from PMDTechnologies, (Right)

Ranging Camera from Mesa Imaging...

Figure 1.2: $\quad$ Range Image Captured by prototype Image Ranger System...................... 3

Figure 2.1: Method of Triangulation......................................................................... 10

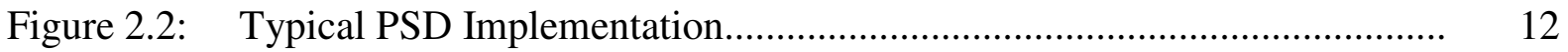

Figure 2.3: $\quad$ Principle of Laser Line Scanning.............................................................. 13

Figure 2.4: $\quad$ Time of Flight Principle............................................................................ 14

Figure 2.5: Principle of Ultrasonic Range Finding.................................................... 15

Figure 2.6: (Left) LRB20000 Military Laser Range Finder, (Right) Leica HDS-3000 LIDAR Scanner.......................................................................... 18

Figure 2.7: Indirect Time of Flight Ranging Principle................................................. 19

Figure 2.8: Demonstration of pixel intensity as a function of phase delay................... 20

Figure 2.9: LEFT: Tank, Itchy \& Scratchy, RIGHT: ROV, USAR ........................... 22

Figure 2.10: Sharp GP2Y3A002K0F and GP2Y3A003K0F PSD Sensors.................... 23

Figure 2.11: PSD Orientation for ranging moving objects............................................. 23

Figure 2.12: SensComp 6500 Ranging Board and 600 Series Ultrasonic Transducer.... 24

Figure 2.13: Beam pattern of 600 Series transducer................................................... 25

Figure 2.14: Modulation Frequency vs. phase and distance standard deviation............. 26

Figure 2.15: Ring Topology................................................................................... 29

Figure 2.16: Mesh Topology................................................................................ 30

Figure 2.17: Star Topology................................................................................ 30

Figure 2.18: Tree Topology................................................................................. 31

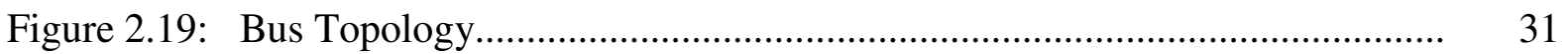

Figure 2.20: Layers of OSI Model........................................................................ 33

Figure 3.1: Control Computer to Network interface................................................. 37

Figure 3.2: $\quad$ Star / Bus Hybrid Network Topology..................................................... 38

Figure 3.3: A Half Duplex RS-485 Bussed Network................................................. 41

Figure 3.4: Daisy Chain format of Network Bus.................................................... 42

Figure 3.5: Mass Terminal Assembly Connectors.................................................. 43

Figure 3.6: Block Diagram of Sensor Network Interface Master Module................... 44

Figure 3.7: Ethernet Interface System Diagram....................................................... 45 
Figure 3.8: Interface between Ethernet Controller and Ethernet Network................... 46

Figure 3.9: USB System Diagram......................................................................... 47

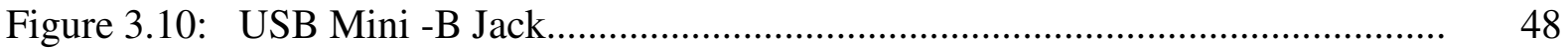

Figure 3.11: USB bus to transceiver interface..............................................................

Figure 3.12: RS-485 Network Interface Version 1...................................................... 49

Figure 3.13: RS-485 Network Interface Version 2 ................................................. 50

Figure 3.14: HDD Power Socket.............................................................................. 51

Figure 3.15: Sensor Network Interface module layout................................................ 52

Figure 4.1: $\quad$ Sharp wide angle IR PSD block diagram............................................... 54

Figure 4.2: Sharp wide angle IR PSD timing diagram............................................. 55

Figure 4.3: Block Diagram of Infrared Ranging Node.............................................. 56

Figure 4.4: Infrared Ranging Module RS-485 Interface.......................................... 57

Figure 4.5: Sharp IR PSD Interface System Diagram............................................. 58

Figure 4.6: Sharp GP2Y3A003K0F Output Voltage vs. Distance............................... 59

Figure 4.7: Sharp GP2Y3A002K0F Output Voltage vs. Distance................................. 60

Figure 4.8: $\quad$ Assembled Infrared Ranging Module (GP2Y3A003K0F)........................ 60

Figure 4.9: Waveform Examples of a Single Echo Mode cycle without blanking

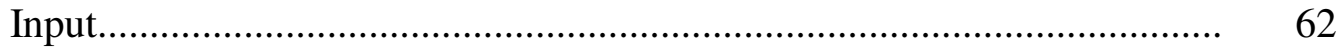

Figure 4.10: Waveform Examples of a Multiple Echo Mode cycle with blanking input 63

Figure 4.11: Block Diagram of Ultrasonic Ranging Node.............................................. 64

Figure 4.12: $\quad$ Ultrasonic Sensor - MCU Interface.......................................................... 65

Figure 4.13: Assembled Ultrasonic Ranging module................................................... 67

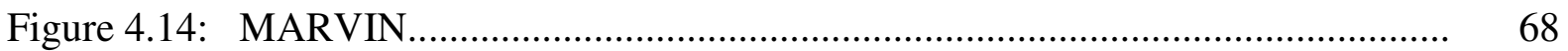

Figure 4.15: Mounting Bracket for Infrared Ranging Modules.................................... 70

Figure 4.16: $\quad$ Mounting Bracket with Sharp GP2Y3A003K0F sensor attached.............. 70

Figure 4.17: Detection Zone of Test Sensor Network................................................. 71

Figure 4.18: Assembled Sensor Network Chassis....................................................... 71

Figure 4.19: Assembled Test Sensor Network Unit...................................................... 72

Figure 4.20: Test Sensor Network mounted on MARVIN.......................................... 72

Figure 5.1: Black Box Sensor Model........................................................................ 74

Figure 5.2: $\quad$ Stepped Measurement Example................................................................. 77

Figure 5.3: $\quad$ Example of a Network Frame.............................................................. 80

Figure 5.4: Transfer of HLP Packet between Network Nodes.................................... 81

Figure 5.5: $\quad$ MLP Packet Structure.......................................................................... 82 
Figure 5.6: Firmware Network Stack Model.......................................................... 86

Figure 5.7: $\quad$ Physical Layer State Transition Diagram................................................... 89

Figure 5.8: $\quad$ Data Link Layer State Transition Diagram................................................. 91

Figure 5.9: Structure definition for a Sensor Node...................................................... 93

Figure 5.10: Master Node's Network Stack Model...................................................... 95

Figure 5.11: Structure definition for master node's sensor table.................................. 95

Figure 5.12: Structure definition for master node's sensor node.................................. 96

Figure 5.13: Packet Structure for Command Interface................................................ 99

Figure 5.14: State Transition Diagram for IR PSD Finite State Machine...................... 103

Figure 5.15: State Transition Diagram for Ultrasonic Sensor FSM.............................. 105

Figure 6.1: $\quad$ Structure of Sensor Interface Library................................................... 108

Figure 6.2: Constructor Function for Packet Object................................................. 110

Figure 6.3: Function to add data to packet data payload........................................... 110

Figure 6.4: Function to read data from a packet data payload..................................... 111

Figure 6.5: Constructor for PacketComm Object........................................................ 111

Figure 6.6: Transmit Packet Code Segment................................................................ 112

Figure 6.7: $\quad$ Transmit Packet Data Overview............................................................... 113

Figure 6.8: $\quad$ Receive Packet Code Segment.................................................................. 114

Figure 6.9: Orientation of Sensor measurement in 3-D Environment.......................... 117

Figure 6.10: Constructor for Sensor Node Object........................................................ 119

Figure 6.11: Distance Calculation Code Segment....................................................... 119

Figure 6.12: Function to set calibration values from configuration file......................... 120

Figure 6.13: Example Configuration File for a Sensor Network.................................. 122

Figure 6.14: SensorInterface Constructor Process....................................................... 123

Figure 6.15: Code Example: ResetNetwork Function................................................... 124

Figure 6.16: Code Example: GetNetworkDefinition Function...................................... 125

Figure 6.17: Code Example: SetNodeMeasurement Function...................................... 126

Figure 6.18: Command Packet Interface Application.................................................... 128

Figure 6.19: Sensor Network Interface Application.................................................... 130

Figure 7.1: $\quad$ System Layout of Prototype Range Imaging System................................ 132

Figure 7.2: $\quad$ The Prototype Full-Field Image Ranger System...................................... 136

Figure 7.3: $\quad$ System Architecture of Full-field Imaging System................................... 137

Figure 7.4: Minimum Size Limitations for Laser Illumination Printed Circuit Board.. 139 
Figure 7.5: Printed Circuit Board Template for Mobile Platform Full-field Imaging System.

Figure 7.6: External Memory Banks of Mobile Platform System.

Figure 7.7: Passive Serial Configuration

Figure 7.8: Active Serial Configuration.

Figure 7.9: Active Parallel Configuration.

Figure 7.10: Fast Passive Parallel Configuration.

Figure 7.11: JTAG Configuration.

Figure 7.12: Voltage Supply Power Up Sequence for FPGA Board.

Figure 7.13: FPGA PCB layout - Top Side

Figure 7.14: FPGA PCB layout - Bottom Side

Figure 7.15: Mobile Platform Imaging Ranger - FPGA Block Diagram.

Figure 8.1: Image Capture Board Block Diagram.

Figure 8.2: The PhotonICs PMD 19K-2 Sensor Array.

Figure 8.3: High Current Pin Driver Rise and Fall Times Vs Supply Voltage.

Figure 8.4: Image Contrast vs. Modulation Signal Voltage for PMD 19K2 Sensor.....

Figure 8.5: Image Capture PCB layout - Top Side

Figure 8.6: Image Capture PCB layout - Bottom Side.

Figure 8.7: Laser Illumination Board Block Diagram.

Figure 8.8: $\quad$ Laser Diode Current Control.

Figure 8.9: Protection Circuit for Laser Diode Current.

Figure 8.10: Laser Illumination PCB layout - Top Side....

Figure 8.11: Laser Illumination PCB layout - Bottom Side.

Figure 8.12: External Interface Board Block Diagram.

Figure 8.13: Raster Scan of 640x480 pixel image.

Figure 8.14: Horizontal and Vertical Sync signals in VGA Display........

Figure 8.15: External Interface PCB layout - Top Side.

Figure 8.16: External Interface PCB layout - Bottom Side.

Figure 9.1: Code Segment of Sensor Calibration Application.

Figure 9.2: IR Module (GP2Y3A003K0F) Calibration Data.....

Figure 9.3: Average ADC Values vs Distance for Five channels of GP2Y3A003K0F Sensor.

Figure 9.4: Variation in ADC Readings for Lights On and Off Compared with Standard Deviation. 
Figure 9.5: Variation in ADC Readings between different GP2Y3A003K0F Modules.

Figure 9.6: Variation in ADC Readings for a GP2Y3A003K0F Module Under Sunlight and Artificial Illumination.

Figure 9.7: IR Module (GP2Y3A002K0F) Calibration Data.....

Figure 9.8: Average ADC Values vs Distance for Five channels of GP2Y3A002K0F Sensor.

Figure 9.9: Variation in ADC Readings for Lights On and Off Compared with Standard Deviation.

Figure 9.10: Variation in ADC Readings between different GP2Y3A002K0F Modules.

Figure 9.11: Error Analysis of GP2Y3A003K0F IR Module.

Figure 9.12: Error Analysis of GP2Y3A002K0F IR Module.

Figure 9.13: Calculated Distance vs Actual Distance for Calibrated GP2Y3A003K0F IR Module.

Figure 9.14: Calculated Distance vs Actual Distance for Calibrated GP2Y3A002K0F IR Module.

Figure 9.15: SensComp Ultrasonic Module Calibration Data.

Figure 9.16: Theoretical and Measured Tick Count Vs Distance for Ultrasonic Sensor

Figure 9.17: Error Analysis of SensComp Ultrasonic Module.

Figure 9.18: Sensor Network Frame...

Figure 9.19: Example of a Cancelled Network Frame.

Figure 9.20: IR PSD Timing Oscilloscope Trace.

Figure 9.21: Example of Step Measurements on the Ultrasonic Sensor Node.

Figure 9.22: Prototype Sensor network Testing Enclosure.

Figure 9.23: Screen Capture of Sensor Network Data for Test Enclosure.

Figure 9.24: Radar Map for Sensor Network Data in Test Enclosure.

Figure 9.25: Test Enclosure Setup for Object Detection

Figure 9.26: Radar Graph for Test Enclosure with Plastic Object at $497 \mathrm{~mm}$......

Figure 9.27: Radar Graph for Test Enclosure with Cardboard Object at $500 \mathrm{~mm}$.

Figure 9.28: Radar Graph for Test Enclosure with Aluminium Object at $498 \mathrm{~mm}$

Figure 10.1: FPGA Board Power Supply Design Error.

Figure 10.2: I/O Bank Allocation in Current FPGA Design.

Figure 10.3: Suggested I/O Bank Allocation to Allow Active Parallel Configuration...

Figure 10.4: Block Diagram of Simple Counter Program for Testing FPGA Configuration. 
Figure 10.6: SignalTap II Captured Data for Output Buffer Memory Bank Test.......... 205

Figure 10.7: Generic I/O Interface Design Error on Image Capture Board................... 207

Figure 10.8: $10 \mathrm{MHz}$ and $40 \mathrm{MHz}$ Modulation Signals Received at High Current Pin Driver Chip.

Figure 10.9: Temperature of High Current Pin Driver With and Without Active Cooling vs Time for $40 \mathrm{MHZ}$ Modulation Signal...

Figure 10.10: Temperature of High Current Pin Driver With and Without Active Cooling vs Time for $10 \mathrm{MHZ}$ Modulation Signal.....

Figure 10.11: $10 \mathrm{MHz}$ and $40 \mathrm{MHz}$ Modulation Signals received at Laser Switch.....

Figure 10.12: Silicon Detector signal for $10 \mathrm{MHz}$ modulation signal.

Figure 10.13: Silicon Detector signals for $20 \mathrm{MHz}$ and $40 \mathrm{MHz}$ Modulation Signals....

Figure 10.14: Laser Switch Enable Signal Response for DC Drive Signal.....

Figure 11.1: Size Comparison between the Mobile and Prototype Full-Field Image Ranger Systems.....

Figure 11.2: Completed Intelligent Sensor Network for Mobile Robotic Platforms.......

Figure 11.3: Completed Full-Field Image Ranger System for Mobile Robotic Platforms. 


\section{Chapter 1 Introduction}

The field of robotics progresses at an ever increasing rate, producing remarkably complex systems. But regardless of how complex the system, it can be modelled, like any electronic device, as a black box where the system produces outputs based on its current internal state and environmental inputs. The ability of a robotic system to operate successfully in an environment is based on the information it can sense from the environment. This is known as extrospection. Consequently improvements in the area of extrospection are a crucial field of robotic research.

The key areas of extrospection that limit a robotic system's ability to operate in its environment are sensor resolution, field of view, accuracy and sampling rate. A significant amount of the research into extrospection focuses on overcoming these limiting factors.

Victoria University has a fleet of mobile robotic vehicles that use a range of electronic sensors to provide both extrospective and introspective information that allow the vehicles to monitor themselves and their environment. The main variables that these robotic vehicles monitor are:

1.) Distance to external obstacles.

2.) Remaining battery charge.

3.) Distance the robotic vehicle has travelled.

4.) Orientation, with respect to gravity.

The integration of sensors into robotic platforms in the Victoria fleet has been performed over several iterations by various student projects. This has led to fragmented implementations of sensor integration that utilise various communication protocols, power supplies, a mixture of manual and computer controlled functionality and a dependence on expensive data acquisition cards (DAQ). This non-uniform approach has inhibited the ability to transfer sensors from one platform to another without some level of (normally time consuming) customisation. Development of a uniform approach to sensor integration across the robotic fleet is desired to provide stable, expandable and interchangeable sensor hardware. 
Commercially available extrospective systems such as the Time-of Flight cameras shown in Figure 1.1 are the end product of a number of iteration cycles of the development process and are frequently designed to be small, self contained systems capable of being interfaced to a variety of hardware. Ideally the extrospective systems utilised at Victoria University of Wellington should adopt a similar model which would assist the employment of such systems onto mobile robotic platforms. Key design specifications that should be considered when developing systems to be mounted onto mobile robotic platforms include:

- A small footprint.

- Low weight.

- Good power efficiency.

- Able to interface to computer systems found on a mobile platform.

- Flexibility for system expansion and alternative configurations depending on the platform's primary task.
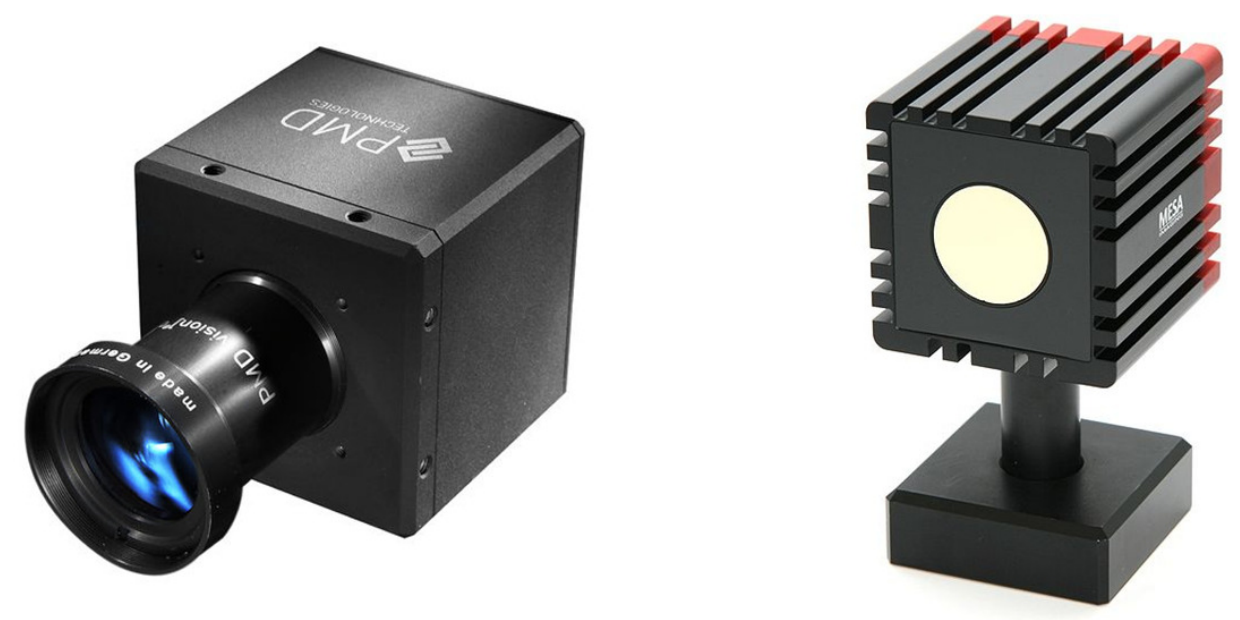

Figure 1.1 (Left) CamCube 3-D Ranging Camera from PMDTechnologies, (Right) Ranging Camera from Mesa Imaging [1,2].

Several VUW research projects have focussed [3, 5, 20] on developing improved extrospective systems, primarily for measuring distance to external objects. Unfortunately these development systems have been designed for use in a laboratory and are not easily mountable to the mobile robotic vehicles in Victoria University's fleet. One such example is a full-field image ranger system. This is an active system that can determine the range to any object in a standard camera lens' field of view. An example of range data captured by the system is shown in Figure 1.2 [3]. This prototype system can perform 19200 simultaneous 
range measurements at sampling frequencies of up to $24 \mathrm{~Hz}$ to centimetre precision and can achieve millimetre precision at sampling frequencies of the order of $0.1 \mathrm{~Hz}$. It has a configurable maximum unambiguous range between $1.5-14.99 \mathrm{~m}$. The field of view of the system is limited only by the optical hardware and it currently employs a $16 \mathrm{~mm}$ focal length lens that provides a field of view (horizontal $\times$ vertical) of $22.2^{\circ} \times 16.5^{\circ}$. This system provides significant advantages over currently adopted single beam range finding sensors but has several limitations. This prototype range finder has dimensions $400 \times 200 \times 250 \mathrm{~mm}$ and requires two separate power supplies; a $10 \mathrm{~V}, 1.5 \mathrm{~A}$ supply for the illumination circuit and a $16 \mathrm{~V}, 1.5 \mathrm{~A}$ supply for the image capture and processing circuit. Such size and power requirements are unrealistic for implementation on a mobile robotic platform.
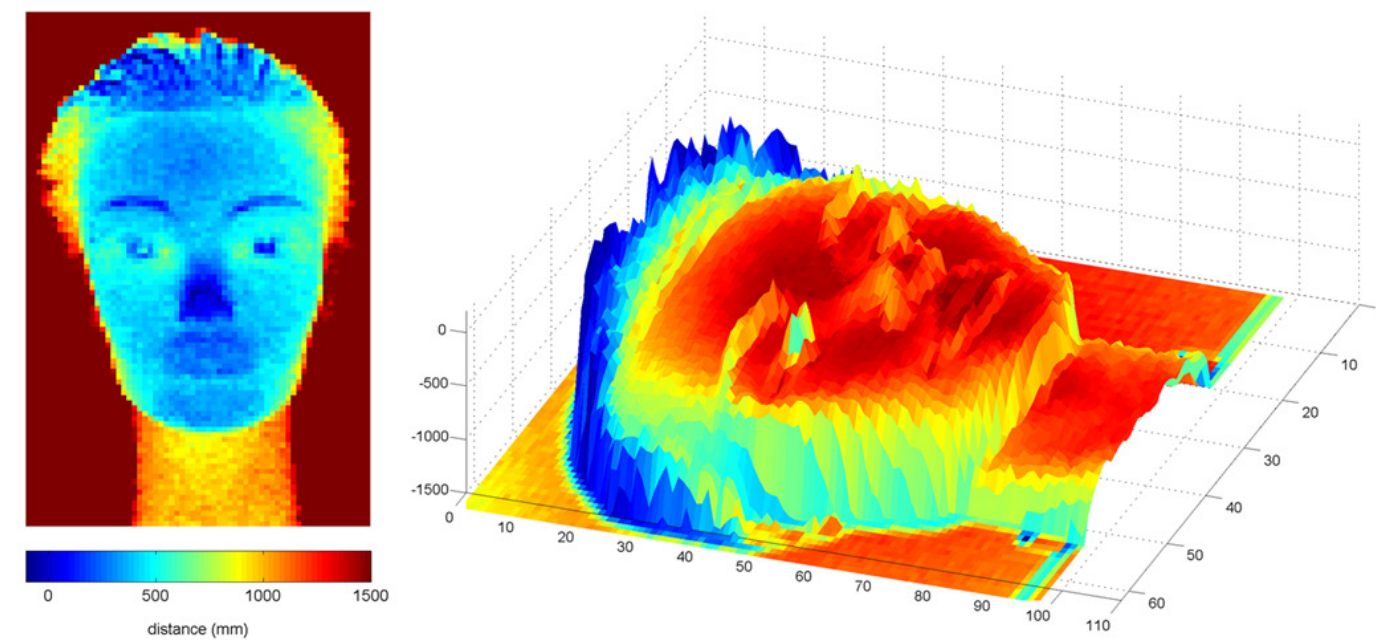

Figure 1.2 Range Image Captured by prototype Image Ranger System [3]

\subsection{Project Specifications}

The primary objective of this project is the development of two extrospective systems capable of being mounted onto mobile robotic platforms.

The first system will be a generic intelligent sensor network utilising sensors currently installed on the robots in the VUW fleet. Fundamental to this system is the development of network architecture and protocols to provide a stable scheme for connecting a large number of sensors to a mobile robotic platform with little or no dependence on the existing hardware configuration of the platform. Ideally this network architecture will be adopted across Victoria's robotic fleet. 
The second system is a redesign of the existing prototype full-field image ranger system. The redesign must yield a smaller, mobile version of the prototype system and should incorporate the flexibility to facilitate upgrades to the image sensor and optical hardware if required in the future.

Both extrospective systems encompass mechanical, electronic and software elements. Performance specifications for these systems include:

- Physical: Both systems must be capable of being mounted onto a mobile robotic platform. As such they should have a compact footprint and be self-contained modules. The sensor network should take up as little space as possible and be mechanically designed to reduce the effects of sensor dead zones. The ranger setup should be flexible enough to accommodate changes to its optical configuration.

- Environment: In the first instance, operation in an internally lit indoor office environment is required. Such an environment is defined as being a confined area with smooth, flat indoor surfaces with objects of interest at short to medium distances $(0-10 \mathrm{~m})$. These extrospective systems should be capable of ranging objects within such an environment and provide sufficient resolution to allow a mobile robotic platform to manoeuvre safely.

- Communication: Both systems should interface to a robotic platform's control computer. They should adopt a communication scheme that does not require hardware modifications to the computer to facilitate portability between robotic platforms. Typically, mobile robotic platforms tend to have hardware that produces moderate levels of noise. Such hardware includes motors and switching power supplies. The communication technology of the sensor network should be capable of data rates of $1 \mathrm{Mbit} / \mathrm{s}$ even in the presence of such noise. The ranger should provide a video output to allow a user to visually examine range images in real time.

- Power Supply: Mobile robotic platforms tend to have a single bank of power cells from which their hardware is powered, typically for VUW robots this is provided by a pair of sealed lead acid batteries. Both systems must be able to be powered from existing power sources on the robotic platform fleet. They must meet all power requirements locally eliminating the need for modifications or additions to a robotic 
platform's power supply therefore facilitating portability between robotic platforms. Importance should be placed on operating efficiency to maximise the operating time from a platform's power source.

- Future Work: The design and implementation of these systems must consider upgrades and additions that may occur during future development. The sensor network should be flexible to allow the addition of new sensor technology. It would be ideal that the image processing and storage capacity of the ranger should be sufficient to support upgrades to higher resolution image sensors at least up to one megapixel.

\subsection{Project Management}

The project objectives require the following steps to be completed.

- Research and evaluation of existing extrospective systems on Victoria University's fleet of mobile robots.

- Research and select appropriate network architecture and topology.

- Design and develop sensor network hardware.

- Design and develop communication and task allocation protocols for network.

- Develop the firmware for control of sensors.

- Develop a software interface for network control and sensor feedback.

- Calibrate the sensors.

- Test and evaluate a prototype sensor network.

- Research and evaluation of the prototype full-field image ranger system.

- Development of a design specification for the mobile ranger system.

- Develop ranger system hardware.

- Test, debug and evaluate ranger hardware. 


\subsection{Thesis Structure}

The thesis is presented as follows:

Chapter 2 presents several examples of existing extrospective techniques for determining distances. It reviews a selection of current sensor technology utilised on Victoria University's robotic fleet and technologies utilised by other researchers. It presents a background into network topology and the Open System Interconnectivity model widely used in modern networks.

Chapter 3 covers the network topology and communication technology adopted for the sensor network and the development of the Sensor Network Interface Module hardware to control the network and communicate with an external computer.

Chapter 4 covers the development of two sensor nodes for the network: a mid-range ultrasonic sensor based node and a short to mid-range infrared Position Sensitive Detector based node.

Chapter 5 details the embedded software algorithms written to control network communication and sensor data capture. It outlines the two main communication protocols developed to co-ordinate tasks, and the transfer of information on the network.

Chapter 6 details the PC based development of a software library to interface to the sensor network. It presents two applications, built on top of the software library, to test the network and communication with an external computer.

Chapter 7 details the prototype image ranger system and covers the development of a FPGA supervisory board for the new mobile image ranger system.

Chapter 8 covers the development of the peripheral hardware for the mobile image ranger system.

Chapter 9 presents the results of sensor calibration, characterisation of the sensors under varying conditions and trials of the test sensor network,

Chapter 10 presents the results of hardware testing undertaken on the full-field image ranger and initial porting of FPGA code to the Cyclone III FPGA. 
Chapter 11 concludes the project with recommendations for future work and a summary of achievements. 


\section{Chapter 2 Background Theory}

This chapter describes varying techniques of extrospection employed by robotic systems to measure distance ranges to environmental objects. It discusses types of electronic sensors that exploit these extrospection techniques and presents several examples of sensors that have been used on robotic platforms at Victoria University. Finally a discussion on different types of network topology and architecture is presented to provide background into the design of the sensor network.

\subsection{Techniques of Extrospection for Range Finding}

Extrospection is defined as the act of observation consisting of receiving knowledge of the outside world through the senses. The ability of a robotic system to operate successfully in an environment is primarily determined by its ability to sense its environment. One of the fundamental variables of an environment that a mobile robotic system must monitor is distance to external objects. Distance information allows a robotic system to map its external environment, detect and avoid obstacles, and to potentially manipulate objects in its environment.

The majority of distance determination schemes exploited on robotic platforms $[4,5]$ involve active extrospection. Active extrospection involves actively probing a scene with a signal and capturing any signal reflected back from objects in that scene. These reflected signals can be used to calculate distances to objects in the scene. The majority of active extrospective sensors on robotic systems exploit either the principle of triangulation or time of flight.

\subsubsection{Triangulation}

Triangulation is a method of distance determination that exploits basic geometry to calculate the distance to an object. Triangulation determines the location of a point by measuring the angles to it from two known fixed points, rather than directly measuring the distance to the point. Figure 2.1 presents the basic geometry of triangulation. The two known fixed points (Points A and B) and the 3rd unknown point (Point C) form a triangle with the distance between the two fixed points forming the baseline of the triangle. Trigonometric formulae 
can be used to determine the distance $\mathrm{D}$, to point $\mathrm{C}$, from the baseline formed by points $\mathrm{A}$ and $\mathrm{B}$ and the angles of points $\mathrm{A}$ and $\mathrm{B}$ to point $\mathrm{C}\left(\Theta_{1}\right.$ and $\left.\Theta_{2}\right)[6]$.

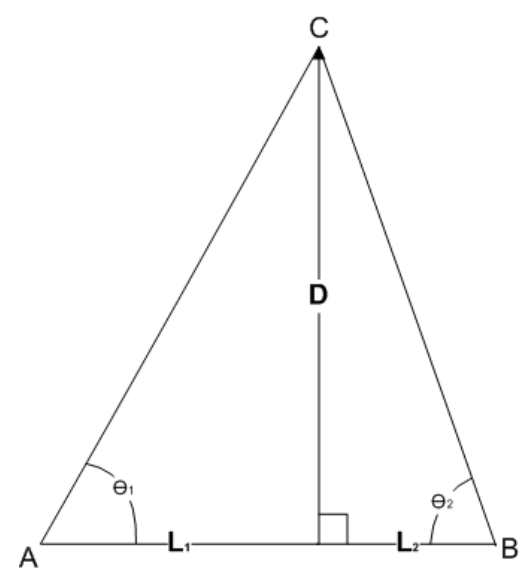

Figure 2.1 Method of Triangulation

Both lengths $\mathrm{L}_{1}$ and $\mathrm{L}_{2}$, which together form the triangle's baseline, can be defined by the following trigonometric equations:

$$
L_{1}=\frac{D}{\tan \theta_{1}} \text { and } L_{2}=\frac{D}{\tan \theta_{2}}
$$

In triangulation the angles $\Theta_{1}$ and $\Theta_{2}$ and the lengths $L_{1}$ and $L_{2}$ are known. These equations can be combined to form the following relationship relating the angles $\Theta_{1}$ and $\Theta_{2}$ and the lengths $\mathrm{L}_{1}$ and $\mathrm{L}_{2}$ to the unknown distance $\mathrm{D}$ by:

$$
L_{1}+\mathrm{L}_{2}=\frac{D}{\tan \theta_{1}}+\frac{D}{\tan \theta_{2}}
$$

Equation 2.2

This equation can be rearranged to define the distance $\mathrm{D}$ in terms of the known lengths and angles to:

$$
D=\frac{L_{1}+L_{2}}{\left(\frac{1}{\tan \theta_{1}}+\frac{1}{\tan \theta_{2}}\right)}
$$

From this formula it is straightforward to determine the distance D as well as the distance from point $\mathrm{A}$ or point $\mathrm{B}$ to point $\mathrm{C}$ [6]. 
In practice triangulation using an electronic sensor is accomplished through wave reflection. A signal pulse is generated at Point A and received at point B after it has reflected back from an object at Point C. Typically light (including infrared and microwave) is used as a signal source due to its ability to be collimated and focused into a beam [4]. Acoustic waves can be used but suffer from the effects of multiple reflections and are more difficult to focus than light waves $[8,9]$.

\subsubsection{Position Sensitive Detection}

A position sensitive detector (PSD) is a non-contact detector that uses triangulation to calculate the distance from the sensor to an object.

In practice, electronic PSD sensors, such as the sensors discussed in Section 2.2.1 Sharp Position Sensitive Detectors, do not mathematically calculate the position of a point using equation 2.3 but rather exploit the geometrical property of triangulation [17, 18]. For a PSD sensor, point A of the triangle is usually a light emitting source and point $\mathrm{B}$ is a light sensitive detector. Typically a PSD does not use a fixed baseline but a fixed angular position of the light emitter to triangulate an object's position. If the angular position of the light emitter is fixed, then the position on the light sensor at which a reflected beam returns, varies with the distance of the object that reflected the light. PSDs use a geometrically linear photosensitive sensor to detect the position of the reflected beam.

Figure 2.2 illustrates this principle. A light beam reflected from object 1, the closer object, strikes the photo sensor at a geometrically different position than a reflected beam from object 2. The photo sensor outputs a different output signal depending on where the reflected beam geometrically strikes it. The maximum and minimum distance a PSD can measure is characterised by the angular position of the emitter, the length of the photo sensor and the separation between the emitter and the photo sensor. If object 1 was too close to the emitter, the reflected beam would return to a point between the emitter and sensor and thus not register on the sensor. If object 2 was too far away from the PSD, the reflected beam would return past the far end of the photo sensor. 


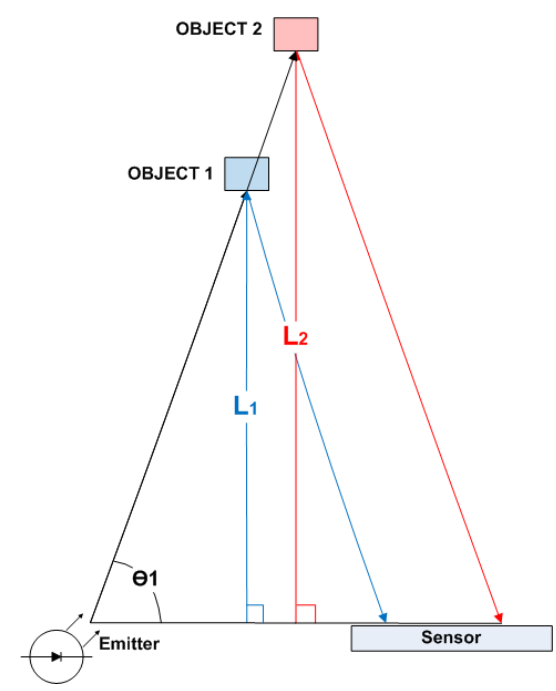

Figure 2.2 Typical PSD Implementation

Typically the output of a PSD's photo sensor is calibrated to objects within the PSD's range to determine a relationship between sensor output and distance.

Due to the geometric relationship of the PSD, this method of distance determination is not significantly dependent on the intensity of the reflected light beam. Therefore artefacts of the environment under measure that attenuate the light intensity, such as the reflectivity of the object, do not significantly affect the measurements $[17,18]$.

The light emitting source is typically an infrared laser or light emitting diode, which offers higher intensity and better reflectivity than standard visible light.

\subsubsection{Laser Line Scanning}

The basic position sensitive detector, described in the previous section, captures only one dimensional data of a scene; the depth to a single point. Laser Line scanning allows the triangulation technique to be expanded to capture two dimensional data of a scene by utilising a light source that produces a line rather than a dot. In this situation a two dimensional light sensor is required to capture the position of the reflected laser line. Typically a charge coupled device (CCD) camera is used as a 2D light sensor [20]. Laser line scanning operates in a similar manner to the basic PSD. The laser and camera are mounted a fixed distance apart and at a fixed angle. The laser emits a laser line onto a scene where it is reflected back by objects at varying distances. The reflected laser line strikes geometrically different locations on the camera sensor depending on the distance from the sensor to an object in the scene. The geometric variance in the location of the reflected laser line on the camera can be 
used to determine the distance to the object that reflected the laser line. Figure 2.3 illustrates this process.

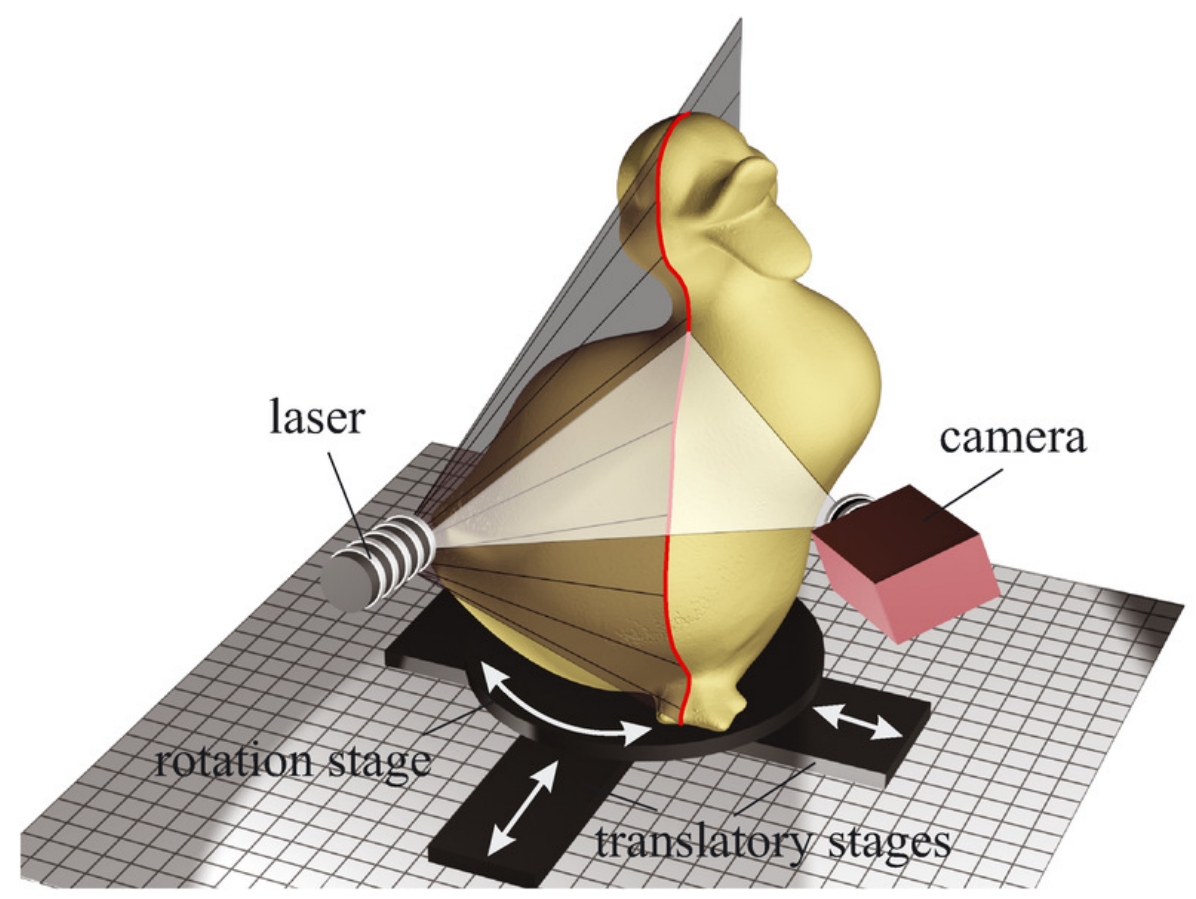

Figure 2.3 Principle of Laser Line Scanning [7]

The laser line triangulation technique can be modified to capture three dimensional data from a scene by sweeping, in discrete steps, the laser line. By capturing frames at each sweep step, a three dimensioned model of the scene can be constructed.

\subsubsection{Time of Flight}

An unknown distance can be determined by timing how long a particle, travelling at a fixed speed, takes to travel from the observer to the object. This method of distance determination is known as time of flight. Ideally a wave particle is used, as it is not subject to external forces of gravity and friction compared with a macroscopic particle.

If the speed of a wave is known then, using Newton's kinematic equations [6], the time it takes for the wave to travel from the wave source to a receiver can be used to determine the distance between the wave source and receiver.

$$
D=\frac{V}{t}
$$


There are three basic configurations for distance ranging using the time of flight principle. These are:

1. Mono-static, where the wave source and receiver are located in the same place.

2. Bi-static, where the wave source and receiver are separated.

3. Multi-static, where there can be multiple wave sources or receivers.

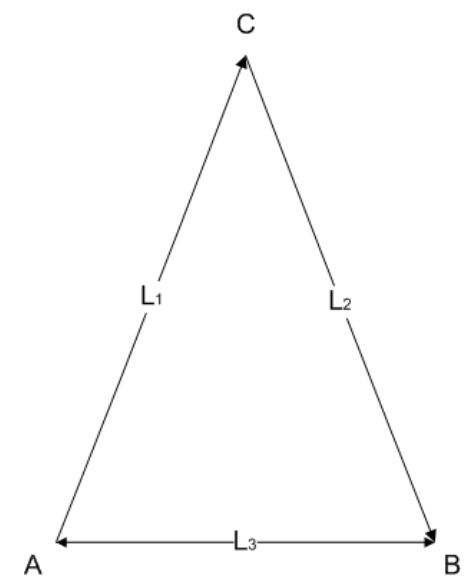

Figure 2.4 Time of Flight Principle

Figure 2.4 illustrates a bi-static configuration where point $\mathrm{A}$ is the wave source, point $\mathrm{B}$ is the wave receiver and point $\mathrm{C}$ is the unknown object. A wave that travels from point $\mathrm{A}$ to point $\mathrm{C}$ and back to point $\mathrm{B}$ has travelled a distance $\mathrm{L}_{1}+\mathrm{L}_{2}$. If the velocity of the wave is known, and the time taken for the wave to travel the path is measured, then the distance the wave has travelled is given by the following equation:

$$
L_{1}+L_{2}=\frac{v_{\text {wave }}}{t}
$$

Equation 2.5

It is important to note that the point at which the source wave strikes the unknown object acts as a point source for the reflected wave. Therefore a percentage of the reflected wave will reach the wave receiver irrespective of its separation from the wave source.

In the majority of distance measuring applications, a mono-static setup is used as it provides a simple geometry and, in robotics, the wave source and receiver are mounted on the same robotic entity.

Primarily acoustic waves have been used for the wave source in this technique, but through recent improvements in the speed of electronics and by using an indirect time of flight 
method, by encoding the time of flight into a phase shift, electromagnetic waves are now also being utilised [11 - 13].

\subsubsection{Acoustic Waves}

The speed of an acoustic wave can be accurately determined if the medium the wave is propagating through is known. Therefore knowing the time an acoustic wave takes to travel a distance, and the velocity of the wave, the distance can be calculated.

Measuring a distance to an object by means of an acoustic wave is a simple process. An acoustic emitter produces a short burst of sound. The sound travels through a medium, typically air, as a compression wave. The compression wave reflects from an object and returns to the emitter where it can be detected by a transducer. The time for the round trip can be determined and, knowing the speed of sound for the medium the compression wave propagates through, the distance to the object can be calculated [8].

Figure 2.5 illustrates the principle of range finding with acoustic waves, using a mono-static setup.

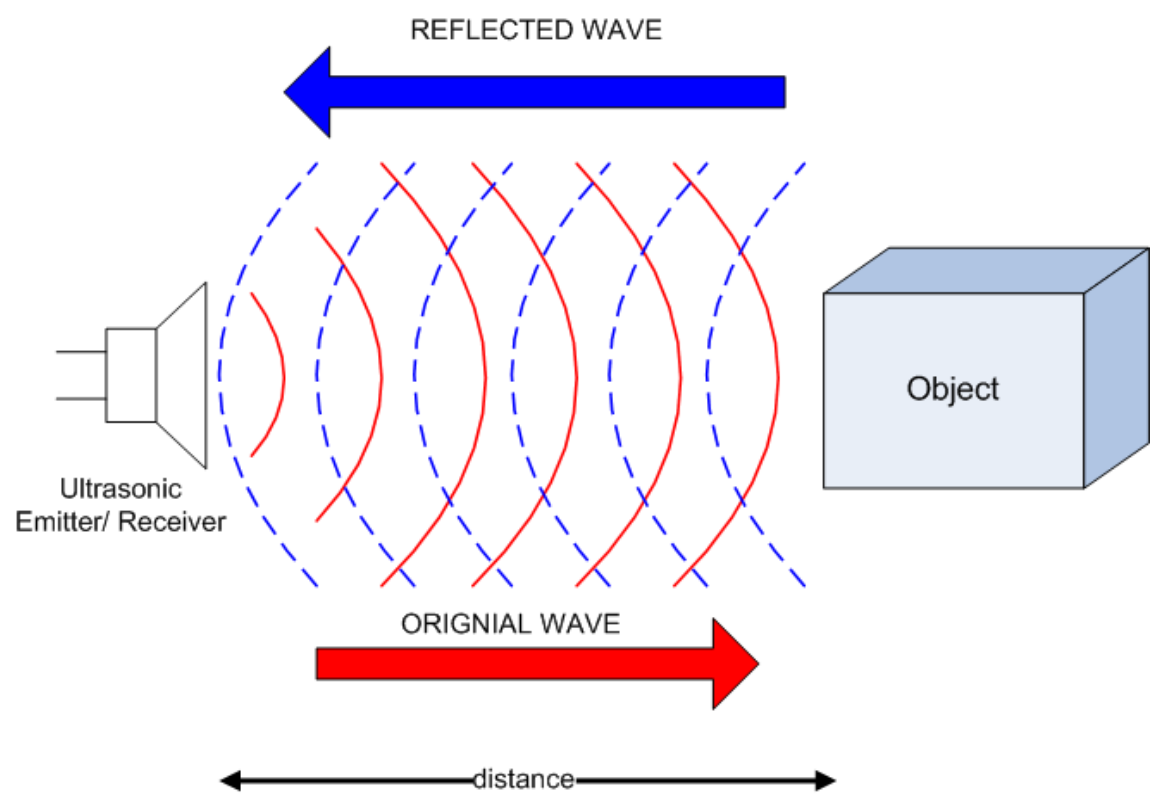

Figure 2.5 Principle of Ultrasonic Range Finding

Generally in robotics ultrasonic frequencies commonly spanning $20 \mathrm{kHz}$ to $2 \mathrm{MHz}$ are used as the wave source [4]. An electrostatic transducer is employed to generate a chirp of changing frequency. The same transducer can be used as the wave receiver to detect the 
incoming reflected wave. The choice of frequency depends on the application of the sensor. Sound absorption in air, beam directivity and resolution are all affected by frequency. Low ultrasonic frequencies attenuate less in air than high frequencies so are more suited for applications involving long range measurements. The speed of sound in air varies only slightly with humidity, about $0.35 \%$ at $20^{\circ} \mathrm{C}$, between $10 \%$ and $90 \%$ humidity [9], and is virtually independent of pressure. Changes in temperature cause the most significant effect on the speed of sound, typically $7 \%$ variation from $0^{\circ}$ to $40^{\circ} \mathrm{C}$. Therefore for accurate measurements the temperature of the medium must be known.

The transmitted power, of any wave emitted in three dimensions from a point source, decreases as $1 / d^{2}$ (where $d=$ distance). Most objects will scatter the wave signal randomly, decreasing the reflected signal power with the same factor $1 / d^{2}$. In addition, the medium in which the wave propagates through absorbs energy from the wave exponentially and attenuates the wave energy with distance. Eventually the medium will absorb all the wave energy. The relationship between signal power and distance, due to absorption, is defined by the following equation:

$$
P=P_{0} e^{-\alpha d}
$$

Equation 2.6

where $P_{0}$ is the signal power at the wave source and $\alpha$ is the absorption co-efficient of the medium the wave is propagating through [6]. The absorption co-efficient has a frequency dependence in that higher frequency waves will have a larger absorption co-efficient in air. Combining the three sources of energy loss, the reflected signal power varies with distance by:

$$
P=\frac{P_{0} e^{-\alpha d}}{d^{4}}
$$

Therefore a $60 \mathrm{kHz}$ signal, at $20^{\circ} \mathrm{C}$, will have echo signal strength approximately $60 \mathrm{~dB}$ weaker at $5 \mathrm{~m}$ than at $0.25 \mathrm{~m}[9]$.

This loss of signal power with distance can be compensated for by increasing the sensitivity of the wave receiver with time.

The strength of a reflected signal is dependent on the surface structure of the objects under measure. The phases of the reflected waves can interfere with each other leading to a large 
variation in reflected wave strength depending on the object's surface geometry. These interference effects are wavelength dependent: echoes at one frequency may be relatively weaker compared to echoes at other frequencies. To overcome this wavelength dependence and provide a higher reliability of receiving a reflected signal, many ultrasonic systems produce a wave pulse of varying frequencies, known as a chirp.

In mono-static configurations, the transmitter will resonate for a fixed period of time after the ultrasonic chirp has been emitted. While the transmitter is resonating it cannot accurately detect a reflected wave. Therefore a certain amount of time must pass to allow the transmitter resonance to decay below the threshold for detecting a reflected wave. This time produces a dead zone in the range the sensor can measure. Objects within the dead zone will not be detected as they will reflect waves that will be received by the transmitter while it is still resonating.

Several situations exist where multiple reflected echoes can be received by an ultrasonic transducer. These are:

1. A single transmitted ultrasonic chirp can reflect from multiple objects in a scene resulting in several reflected echoes arriving at the transmitter at varying times.

2. In a system with multiple ultrasonic transducers a single transducer can receive the reflected echo from the chirp it transmitted and a reflect echo from a chirp generated by another transducer.

3. A transmitted chirp from a transducer can reflect at varying angles from an irregular surface. In this situation a reflected wave can further reflect off another object and back to the transducer. In this case the doubly reflected wave has travelled multiple paths and the time of the flight does not accurately equate to the distance to either object. This is known as the multiple path problem.

Several methods can be exploited to manage these situations. In situation one the transducer can be disabled after receiving the first reflected pulse until any other echo has been received or dropped in intensity enough to not be detected by the transducer. In situation two positioning of the transducers and careful control of their operation can reduce the likelihood of transducers receiving reflected signals generated from other transducers. In situation three multipath waves tend to be received off-axis from the transducer. By reducing the admittance angle of the transducer the detection of these off axis reflections can be reduced. 


\subsubsection{Electromagnetic Waves}

Recent advances in the switching speed of electronics now allow electromagnetic waves to be used as a wave source for time of flight measurements. Current implementations of time of flight systems, utilising electromagnetic waves, are known as laser range finders and time of flight cameras.

Laser range finders, such as the commercial rangers pictured in Figure 2.6, determine the distance to an object by emitting a laser pulse in a narrow beam towards an object and measuring the time taken by the pulse to be reflected off the object and returned to the rangefinder. Due to the high speed of light, this technique is not suitable for high precision sub-millimetre measurements [10].
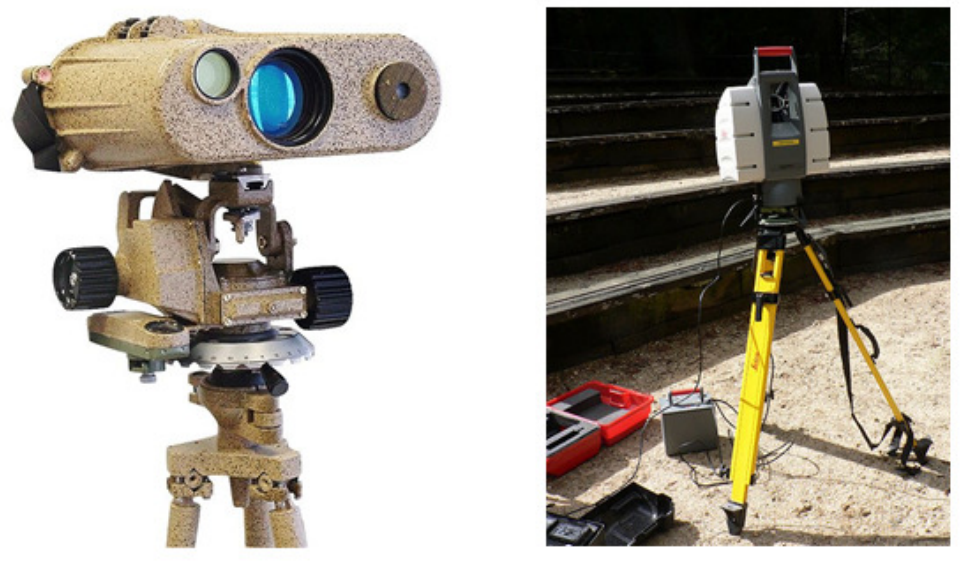

Figure 2.6 (Left) LRB20000 Military Laser Range Finder, (Right) Leica HDS-3000 LIDAR Scanner.

The precision of a range finder is determined by the rise and fall time of the laser pulse and the speed of the receiver. Top of the line laser rangefinders that exhibit very sharp laser pulses and fast detectors are capable of ranging objects within a few millimetres. Even though a laser has a narrow beam, it will eventually spread over a long distance due to the divergence of the laser beam and beam wander effects caused by atmospheric distortions, such as lens effects caused by water vapour in the air. These effects have to be taken into account to ensure accurate readings of distances to objects.

It is possible for laser rangefinders to achieve sub-millimetre precision through use of an in-direct time of flight method. This method does not measure the time light takes to travel to 
the scene and back but determines the range by measuring the phase shift between a modulated light source and its reflect pulse train. Figure 2.7 illustrates the basic set up for an indirect time of flight based distance ranger.

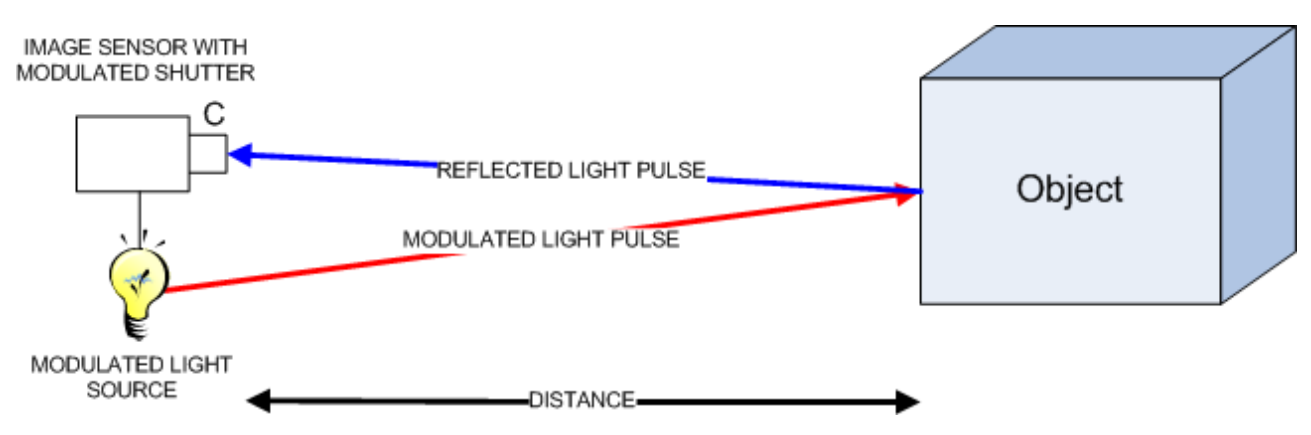

Figure 2.7 Indirect Time of Flight Ranging Principle

The Amplitude Modulated Continuous Wave (AMCW) indirect time of flight method [11] requires that a scene be actively illuminated by a light source modulated at a high frequency, typically between 10 and $100 \mathrm{MHz}$. The light is reflected from objects within the scene and received by a sensor with an electronic shutter that is modulated at the same frequency and in phase with the light source modulation frequency. This is known as homodyne modulation. Due to the time taken for the light to travel to and from objects in the scene, a phase delay is introduced between the reflected wave and the originally emitted light wave.

The sensor measures an average intensity value that is proportional to this phase difference. The phase difference is proportional to the distance from the sensor and an object within the scene.

Figure 2.8 illustrates an example of two waveforms reflected from two objects at differing distances within a scene. The reflected signals are mixed with the original modulation waveform of the emitted light, producing intensities on pixel ${ }_{1}$ and pixel $_{2}$. These intensities are averaged over time to give intensities $I_{1}$ and $I_{2}$ which are relative to the phase differences $\Theta_{1}$ and $\Theta_{2}$ of the reflected waveforms [11]. 


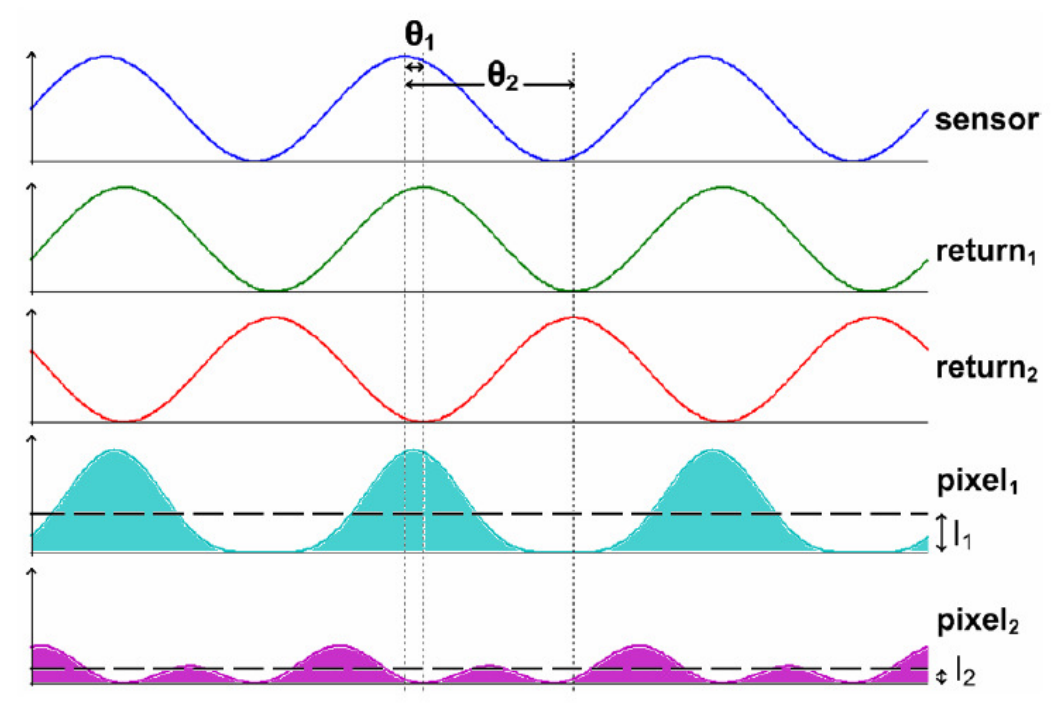

Figure 2.8 Demonstration of pixel intensity as a function of phase delay

An ambiguity exists in that it is impossible to discern if a pixel's averaged intensity is due to the phase shift of the reflected light or from artefacts of the scene under measure, such as varying object's reflectance or the reduction in light intensity $\left(1 / d^{2}\right)$ expected with distance. To resolve this ambiguity, $N$ frames can be taken while introducing a phase step in the modulated sensor's shutter signal, relative to the modulated light signal, incrementing by $2 \pi / N$ radians between each frame [12]. This allows the phase delay to be calculated using a Discrete Fourier Transform as:

$$
\theta=\tan ^{-1}\left(\frac{\sum I_{i} \cos \frac{2 \pi i}{N}}{\sum I_{i} \sin \frac{2 \pi i}{N}}\right)
$$

where $I_{i}$ is the pixel intensity for the $i^{\text {th }}$ frame. If $N=4$ then the equation can be simplified to:

$$
\theta=\tan ^{-1}\left(\frac{I_{0}-I_{2}}{I_{1}-I_{3}}\right)
$$

From the phase shift $\theta$, the distance can be calculated as:

$$
d=\frac{c \theta}{4 \pi f_{m}}
$$

where $c$ is the speed of light and $f_{m}$ is the modulation frequency of the light source and sensor shutter. 
The wavelength of the modulated light source determines the maximum unambiguous range an indirect time of flight ranging system can determine. Distances greater than this unambiguous range are effectively wrapped back into the range. Therefore a system with an unambiguous range of $10 \mathrm{~m}$ will determine an object at a range of $11 \mathrm{~m}$ to have a range of $1 \mathrm{~m}$. The wavelength of the light source is related to the modulation frequency by the following relationship:

$$
\lambda={ }^{c} / f_{m}
$$

Equation 2.11

The maximum unambiguous range that can be determined is equal to half the wavelength of the modulated light. For a modulation frequency of $10 \mathrm{MHz}$ an unambiguous range of $14.99 \mathrm{~m}$ is possible.

The measurement accuracy can be improved by implementing a heterodyne modulation setup. Heterodyne modulation is where the light source and image sensor are modulated at slightly different frequencies. The light source is modulated at a base frequency of $f_{m}$ and the image sensor is modulated at $f_{m}+f_{B}$. With $f_{B}$ being beat frequency that is an integer fraction of the sampling rate of the system, a continuous phase shift results rather than the discrete phase steps in the homodyne modulation method. The continuous phase shift in the heterodyne method minimizes the effects of harmonics compared to the homodyne method resulting in enhanced measurement accuracy [13].

Time of Flight cameras use the same indirect time of method but use a two dimensional array of pixels to image the intensity of light reflected from the scene and also encode distance from the camera to objects in the scene. This allows a three dimensional model of the scene to be reconstructed. This type of distance determination is known as full-field image range finding. 


\subsection{Sensor Technology on the VUW Robotic Fleet}

Victoria University of Wellington (VUW) has a fleet of mobile robotic platforms that currently use a range of sensors to provide extrospective capabilities [5, 14 - 16]. As mentioned above, these sensors are based on the principle of triangulation or time of flight to determine distances. VUW's robotic platforms most commonly operate in an enclosed environment, typically large rooms, and as such the majority of on-board sensors measure mid-range distances, $0.1-10 \mathrm{~m}$. Figure 2.9 presents some of the robotic platforms in the VUW fleet.
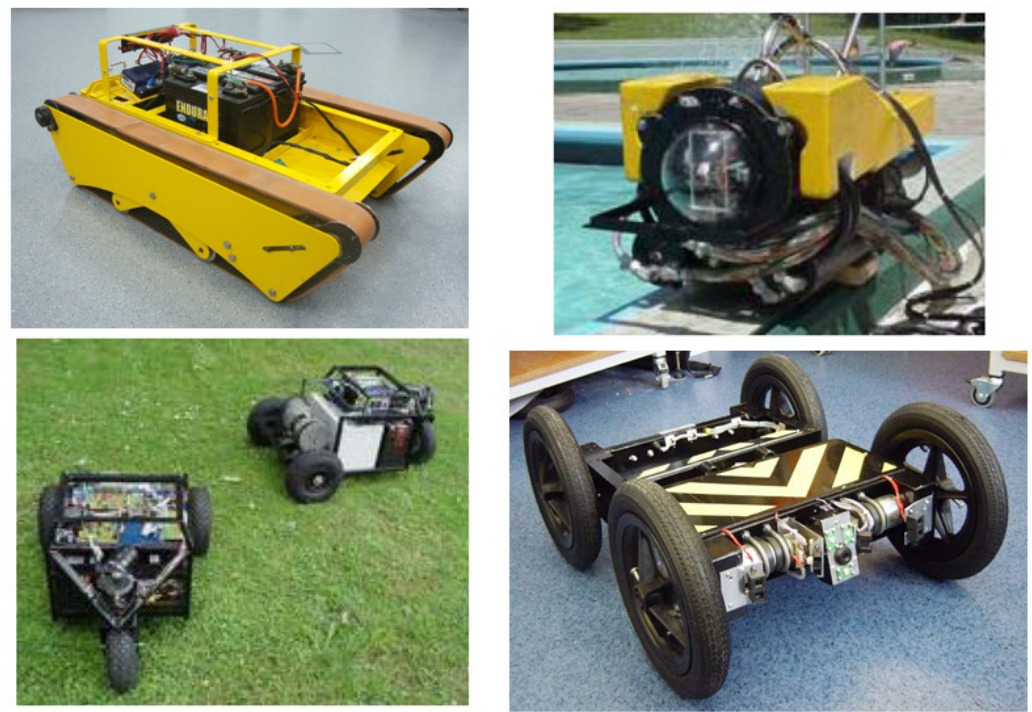

Figure 2.9 LEFT: Tank, Itchy \& Scratchy, RIGHT: ROV, USAR

\subsubsection{Sharp Position Sensitive Detectors}

Several of VUWs robotic vehicle use PSD sensors from Sharp for extrospection. The Sharp GP2Y3A003K0F and GP2Y3A002K0F position sensitive detectors, pictured in Figure 2.10, consist of an integrated PSD, five infrared $870 \mathrm{~nm}$ LEDs and a signal processing unit. They provide mid-range distance measurement capabilities, $0.2-1.5 \mathrm{~m}$ for the GP2Y3A002K0F and $0.4-3 \mathrm{~m}$ for the GP2Y3A003K0F. The 5 infrared LEDs are mounted at $5^{\circ}$ intervals to provide a $25^{\circ}$ field of view $[17,18]$.

These devices output an analogue voltage for each LED channel, which is proportional to the distance measured. They are not greatly affected by the varying reflectivity of objects, the environmental temperature, or the operating duration. 

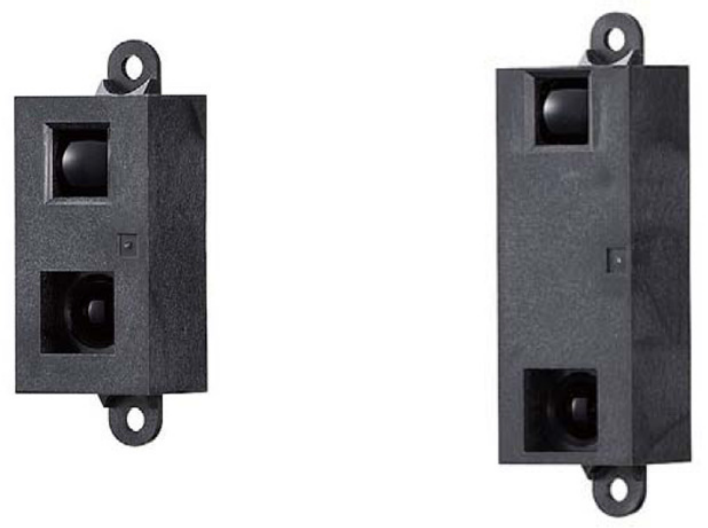

Figure 2.10 Sharp GP2Y3A002K0F and GP2Y3A003K0F PSD Sensors

These sensors provide an efficient means of mid-range distance determination but do suffer from several short comings. Because these sensors use infrared light to actively illuminate a scene, other sources of infrared light can affect the ability of the sensors to accurately measure distances. Such external sources of infrared light include direct sunlight, tungsten lamps or a radiant heat source.

Highly reflective surfaces such as mirrors, and significantly transparent surfaces such as glass, provide extreme cases of reflection that can cause the PSD to measure distances inaccurately.

The Sharp datasheet specifies that these sensors exhibit an asymmetrical characteristic in determining distances to objects moving in a scene. As illustrated in Figure 2.11, the distances to objects moving perpendicular to the sensor are more accurately determined than objects that move parallel with the sensor.
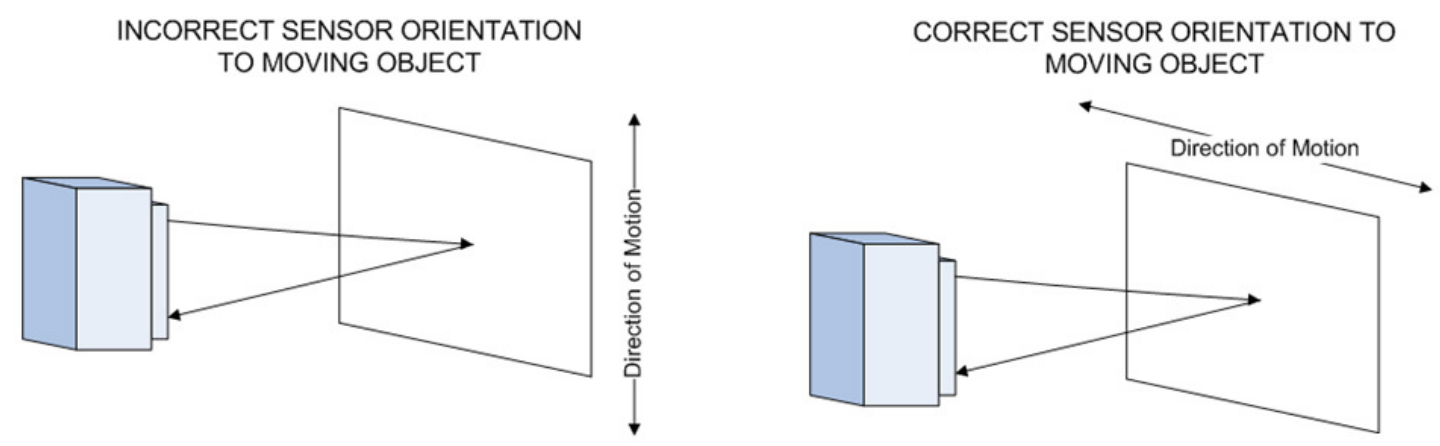

Figure 2.11 PSD Orientation for ranging moving objects 


\subsubsection{SensComp Ultrasonic Ranging Sensors}

The SensComp 6500 Ranging board and 600 series ultrasonic transducer, pictured in Figure 2.12, are used on a selection of Victoria University's robotic vehicles. These sensors operate in a mono-static arrangement and provide mid to long range distance measurements $(0.4 \mathrm{~m}$ to $15 \mathrm{~m})$.

The transducer consists of a thin plastic foil with a conductive gold coating on the front side. It is stretched over a metallic back plate such that the back plate and foil represent an electrical capacitor. When charged, an electrostatic force is exerted on the foil. If an AC voltage of a given frequency is applied, the foil will move at the same frequency and emit sound waves [19].

The major acoustical factors affecting the performance of the ultrasonic ranging system are related to the transducer performance, operating frequency and the desired maximum range.

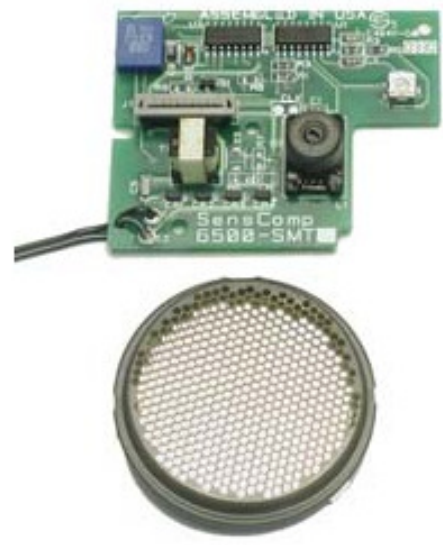

Figure 2.12 SensComp 6500 Ranging Board and 600 Series Ultrasonic Transducer

To maximise the sound output, the phase and distribution of the output across the transducer have to be well defined and uniform. The quality of these characteristics determines the lobe pattern of the transducer. The larger the diameter of the transducer, coupled with a high frequency creates a narrow beam for transmission and reception. From Figure 2.13 it can be seen that the transducer yields a $3 \mathrm{~dB}$ full angle beam width of approximately $15^{\circ}$ at $50 \mathrm{kHz}$. 


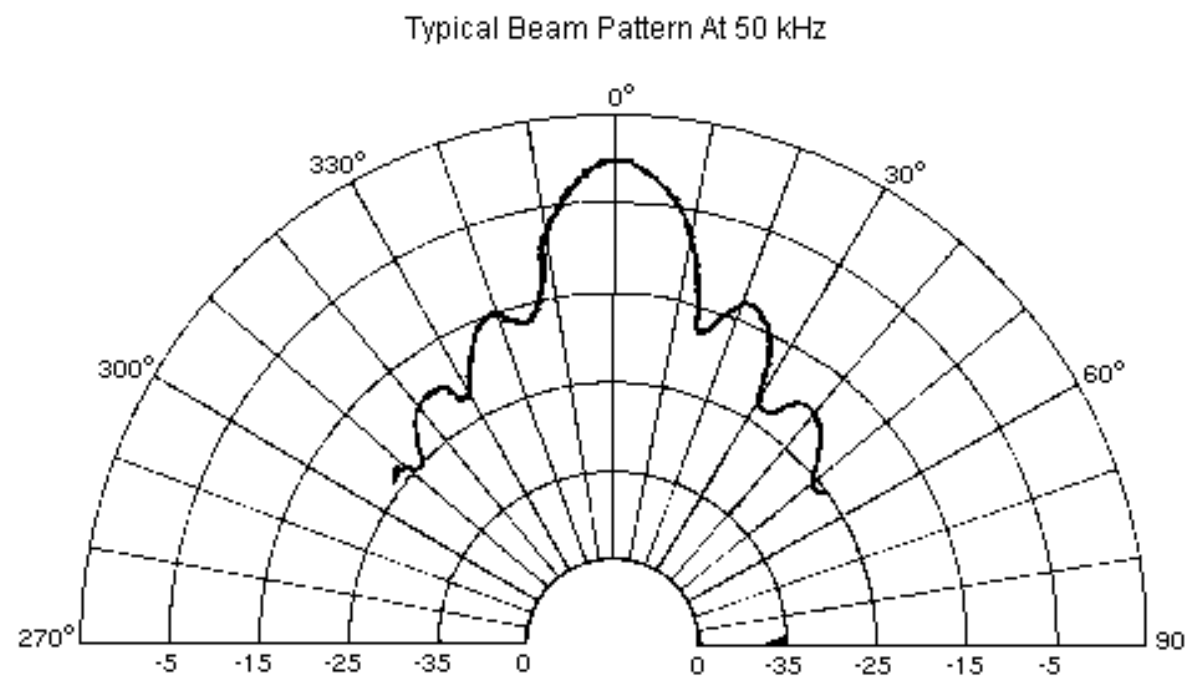

Note: 此 normalized to on-axis response.

Figure 2.13 Beam pattern of 600 Series transducer [19]

\subsubsection{Laser Line Scanning System}

Several iterations of a laser range finding systems have been developed and integrated onto robotic platforms in the VUW fleet [20]. The latest iteration makes use of laser optics to produce a laser line allowing 2-D distance data to be captured. The laser line range finder has been designed to have a mid-range measurement capability and can measure distances between 0.27 and $12.3 \mathrm{~m}$. The precision of this system is related to the resolution of the web camera and the angular set up of the laser source and camera. The current set-up of the system provides a precision of $0.1 \mathrm{~m}$. The upper limit of the measurement range is limited by the power of the laser source.

The system consists of a $5 \mathrm{~mW}$ green laser $(532 \mathrm{~nm})$, a cylindrical lens to generate the laser line, and a 2.0 mega-pixel DSE web camera to capture the reflected laser line. Mounted to the front of the web camera is a $532 \mathrm{~nm}$ band pass filter to exclude the ambient visible light from the web camera image.

\subsubsection{Full-Field Image Ranger System}

Victoria University has developed a prototype full-field image ranger system to investigate the capabilities of such systems. The latest prototype is a solid state system constructed from off-the-shelf components and has been set up in both homodyne and heterodyne configuration[13]. The system can operate at modulation frequencies between $10-100 \mathrm{MHz}$ 
allowing short to mid-range distance measurements. The maximum unambiguous range is determined by the modulation frequency. With a $10 \mathrm{MHz}$ modulation frequency the unambiguous range corresponds to $14.99 \mathrm{~m}$ and at $100 \mathrm{MHz}$ to $1.5 \mathrm{~m}$. The precision of the system is related to the standard deviation of the phase measurements [13].

Figure 2.14 illustrates the standard deviation of phase and distance measurements for the VUW full-field image ranger prototype. It can be seen that the standard deviation of the phase remains constant for modulation frequencies from $10 \mathrm{MHz}$ to $40 \mathrm{MHz}$. Above $40 \mathrm{MHz}$ the bandwidth limitation of the electronics causes the precision of the phase measurements to get progressively worse.

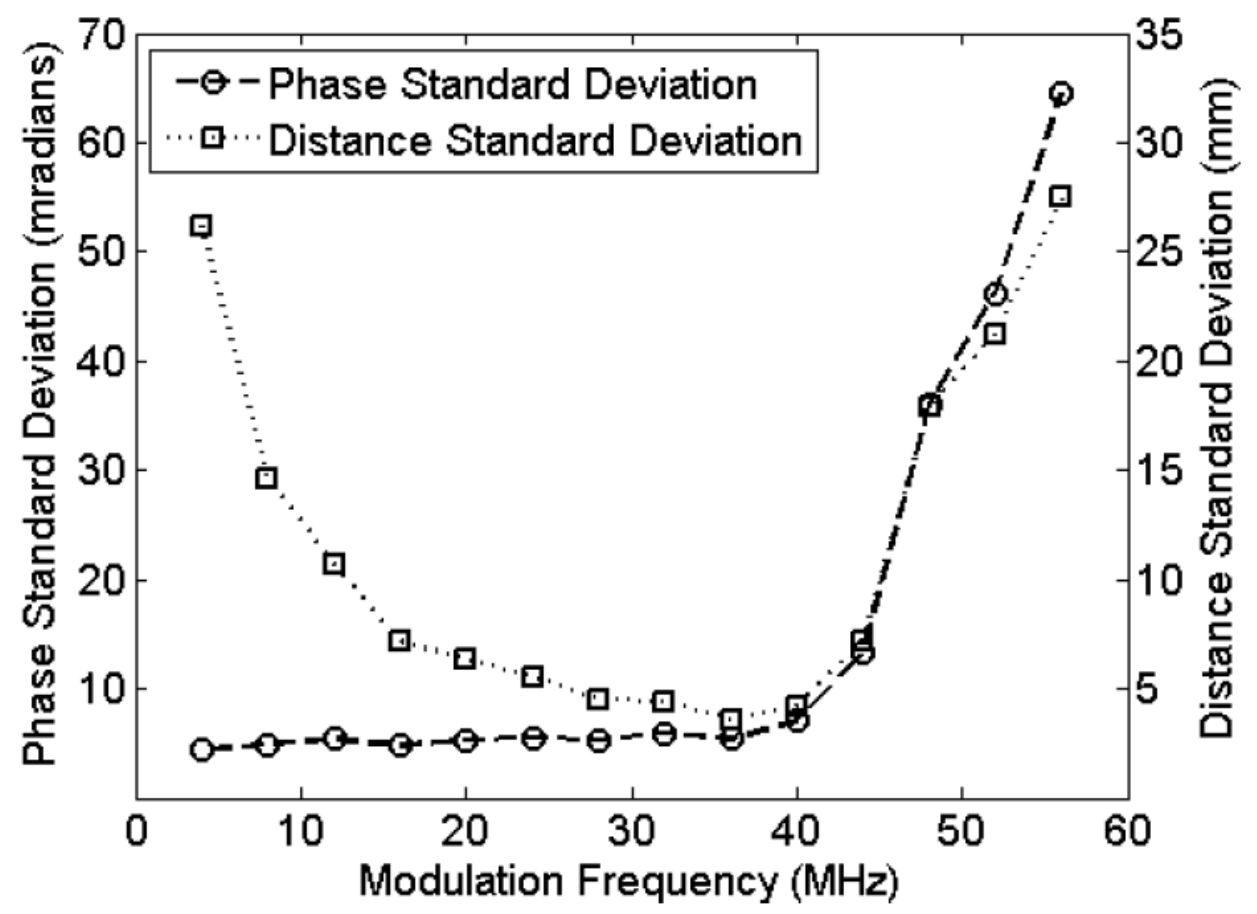

Figure 2.14 Modulation Frequency vs. phase and distance standard deviation [13]

Since the measured distance is inversely proportional to the modulation frequency, the precision of the system increases with frequency, over the frequency range where the phase standard deviation remains constant. This corresponds to a best case precision of approximately $3.5 \mathrm{~mm}$ at $36 \mathrm{MHz}$. At $10 \mathrm{MHz}$ the precision is approximately $12 \mathrm{~mm}$. Greater precision can be achieved through using longer exposure times to capture range images. 
The use of an FPGA development board provides the system with 12 easily configurable phase lock loops (PLL) enabling heterodyne modulation to be easily investigated [39, 40]. These PLLs allow the system to reconfigure the phase, frequency and duty cycle of modulation frequencies in real time, allowing the modulation signal to be changed between frames as well as during sensor integration time. The FPGA also allows the system the ability to be easily configured through software to take long exposures, high resolution depth pictures with millimetre precision, or alternately video frame rate depth images at the expense of precision.

The system uses two banks of laser diodes to actively illuminate the scene under measurement. Each bank consists of 8 laser diodes that can be modulated at frequencies up to $155 \mathrm{MHz}$. One bank consists of near infrared laser diodes $(808 \mathrm{~nm})$ and the other of red laser diodes $(658 \mathrm{~nm})$ [13]. The use of two different wavelength light sources allows the investigation of wavelength on the ranger's performance.

The system uses the PMDTechnology 19k 3D video image sensor to capture ranging data from the scene. The sensor features a $160 \times 120$ array of pixels grouped into four independently modulated blocks of $40 \times 120$ pixels [41]. Mounted above the sensor is a cage plate designed to hold a C-mount lens. Currently the system uses a Goyo Optical $16 \mathrm{~mm}$ focal length lens with manual focus and iris. This gives a field-of-view (horizontal $\times$ vertical) of $22.2^{\circ} \times 16.5^{\circ}$. The lens assembly has a LP645 filter from Midwest Optical Systems that passes $90 \%$ of the reflected light of the red and NIR laser diodes while excluding the shorter visible wavelength light that the image sensor would otherwise detect.

The system has an Ethernet port to allow raw and processed image frames to be stored on an external computer. Image frames can be stored temporarily onboard the system in the FPGA's internal RAM. The system can display image frames in real-time by connecting a standard $640 \times 480$ resolution VGA monitor via an onboard VGA connector.

The system has been successful in developing and characterising homodyne and heterodyne configurations but currently is too bulky $(120 \times 200 \times 120 \mathrm{~mm})$ and power hungry requiring two separate power supplies, a $10 \mathrm{~V} 1.5$ A supply for the illumination circuit and a $16 \mathrm{~V}$ 1.5 A supply for the FPGA board and imaging circuitry. This configuration is not suitable to be mounted on a mobile robotic vehicle. 


\subsubsection{Sensor Technology Summary}

Investigation of the existing implementations of sensor networks on the VUW robotic fleet and research on extrospective technologies have identified two key areas to improve the extrospective capabilities of VUW robotic platforms:

1. The incorporation of existing PSD, ultrasonic, infrared and laser sensors into a flexible and expandable network that is independent of the control hardware. The ability of a network to provide these advantages relies primarily on its architecture and protocols. An investigation into potential communication technologies, topological layouts of sensors on robotic platforms and software implementation of network protocols will be paramount to ensure a successful sensor network integration scheme onto the range of robotic platforms in the VUW fleet.

2. The implementation of a full-field range imaging system. Such a system offers faster acquisition times and accuracy compared with the sensor technologies currently employed on the VUW robotic fleet. This can be implemented by a redesign of an existing VUW prototype ranger system. This redesign must cater for the operating limitations that exist on a mobile robotic platform, specifically consideration must be given to the physical size, power consumption and interfacing to control hardware.

\subsection{Network Characteristics}

The development of a $360^{\circ}$ distance ranging system requires the organised control and layout of a collection of distance ranging sensors. These sensors collectively form a web of devices that must communicate their measurement data to a central control computer. It is therefore logical to view and organise them as a network of sensors. Any network implementation has a topology and architecture that define it. Careful selection of both the topology and architecture can result in a robust network capable of growth and change. This section discusses the fundamental types of network topology and the commonly used Open Systems Interconnectivity model exploited by the majority of computer networks as the network architecture. 


\subsubsection{Network Topology}

Networks can be physically implemented in a variety of ways. Fundamental to how a network is implemented, is how a communication link is established between a network node and the rest of the network. The nature of the tasks of the network can help define the most suitable communication link for each network node and therefore the topology of the network. There are several topologies a network could be based on. The five fundamental topologies are:

1. Ring - where each network node has a communication link to its two adjacent nodes and the network forms a closed loop, as illustrated in Figure 2.15. Network messages pass in one direction through each node connected to the ring. This topology allows only one network node to talk at a time, but saves costs as each network node requires only one receiver and one driver. The major disadvantage of a ring topology is that a fault in one network node will cause the entire network to fail.

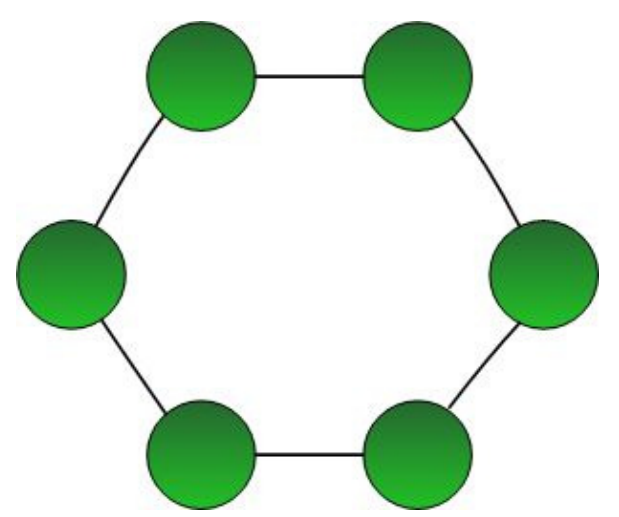

Figure 2.15 Ring Topology

2. Mesh - where each node has a direct point to point communication link with every other node in the network, as illustrated in Figure 2.16. This topology is inherently costly and complicated as every network node requires one pair of driver/receivers per communication link for every node in the network. This topology is predominantly used when there are only a small number of nodes to be interconnected. 


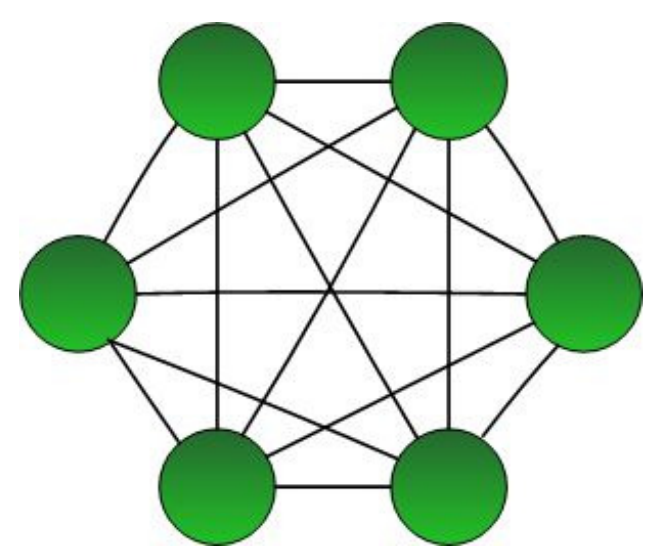

Figure 2.16 Mesh Topology

3. Star - Where every network node has a point to point communication link with a central network hub, as illustrated in Figure 2.17. All network messages must pass through the network hub. This topology allows easy implementation of a network but the number of network nodes allowed is limited by the amount of point-to-point communication links the central network hub can physically support. It should be noted that the existing ad-hoc sensor networks on the robotic fleet follow this topology where the central network hub is the control computer.

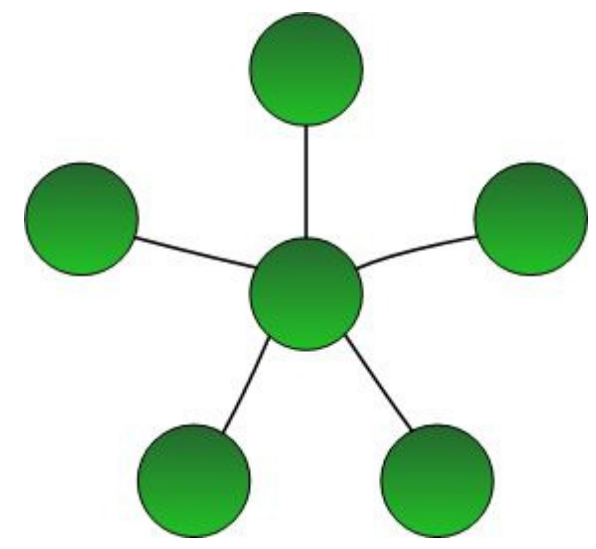

Figure 2.17 Star Topology

4. Tree - where a central 'root' network node has point-to-point communication links to a fixed number of network nodes at a secondary level, as illustrated in Figure 2.18. Every secondary level network node acts as a 'root' node for network nodes in a tertiary level, etc. This network topology is symmetrical in that each network node has a fixed number of point-to-point communication links to nodes in the lower level. Expansion of the network requires the addition of network levels. The tree topology offers advantages when the network itself is required to perform processing of the 
data that is transmitted between nodes on the network in a hierarchical manner. Nodes in a higher level perform more processing operations on behalf of nodes in the lower network levels.

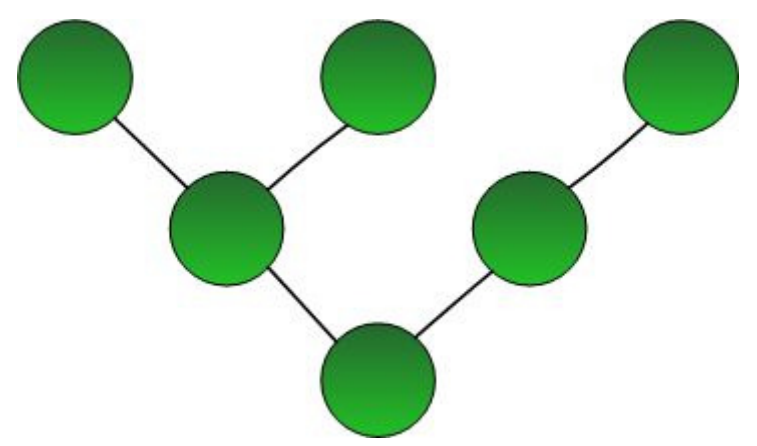

Figure 2.18 Tree Topology

5. Bus - where all network nodes share a single communication link to each other, as illustrated in Figure 2.19. In this topology a network message travels to all nodes connected on the link until it finds the intended recipient. This topology is inherently inexpensive as it requires only one network link, and each node only requires one driver/receiver pair. This topology typically follows a master/slave protocol, where one network node is designated the master and manages all network traffic, the remaining nodes are designated slaves and can only access the network when they receive permission from the master node.

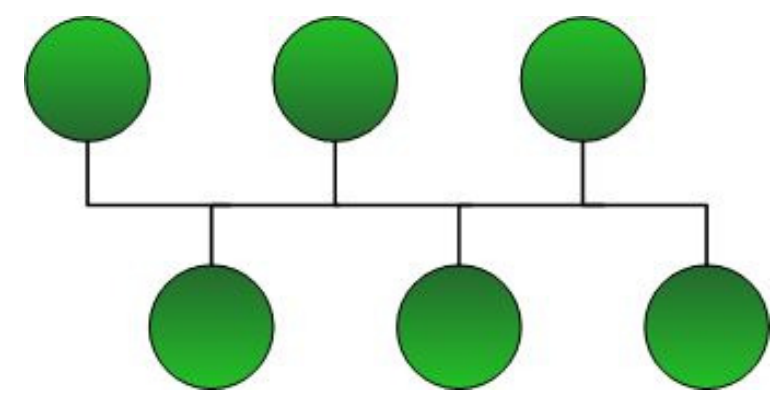

Figure 2.19 Bus Topology 


\subsection{Network Architecture}

Network architecture is the design of a communication network. It describes and specifies the network's physical components and their functional organisation and configuration and the services the network provides.

\subsubsection{Open Systems Interconnection (OSI) Model}

The open systems interconnection model is a way of sub-dividing communication systems, such as networks, into smaller parts called layers. A layer is a collection of conceptually similar functions that provide services to the layer above it and receives services from the layer below it.

For example, a layer that provides error free communication across a network provides the path needed by applications above it, while it calls the next lower layer to send and receive packets that make up the contents of the path.

The OSI model is based on 7 layers. Figure 2.20 illustrates the layers that constitute the OSI model. The Physical, Data Link and Network layers are known as the media layers as they provide services that correspond directly to the media that make up the network. The higher layers are known as the host layers and are layers that provide services specific to the host machine they run on.

The Physical layer defines the relationship between the device and a physical medium. It contains the electrical and physical specifications for the devices. This can include pin layout, voltage levels, cable specifications and repeaters [21, 22].

The Data Link layer provides the functional and procedural means to transfer data between network nodes and may provide the means to detect and possibly correct errors that occur in the physical layer. This layer configures bits from the physical layer into logical sequences called frames. Delivery of frames to devices requires unambiguous hardware addressing.

The Network layer provides the functional and procedural means of transferring variable length data sequences from a source to a destination. The network layer performs data 
fragmentation and reassembly and reports delivery errors. It uses a logical addressing scheme that allows hierarchical addressing.

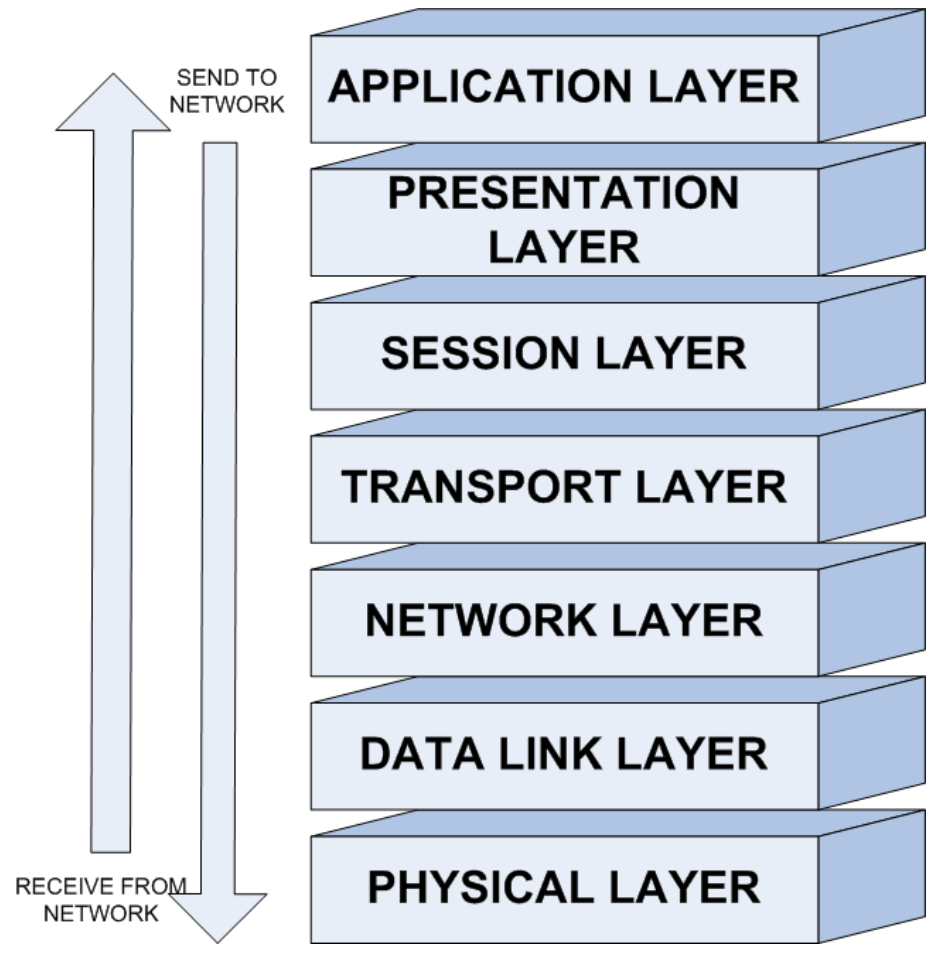

Figure 2.20 Layers of OSI Model

The Transport layer provides transparent data transfer between nodes, allowing end-to-end communication services for applications. It provides, through connection oriented data streams, in-order delivery of a bit or byte stream from one node to another.

The Session Layer provides the mechanism for opening, closing and managing a session between end-user's applications. It allows information of different streams, potentially originating from different sources, to be properly combined and synchronised. For example, in web conferencing it would synchronise the video and audio streams to avoid lip synch problems.

The Presentation layer is responsible for the delivery and formatting of information to the application layer for further processing or display. This layer relieves the application layer of concern regarding syntactical difference in data representation such as converting between ASCII and EBCDIC coded text files. Encryption is usually done at this layer. 
The Application layer provides the means for the user to access information on the network through an application. This layer is the main interface for the user to interact with the application and therefore the network.

By separating the network communications into logical smaller pieces, the OSI model simplifies how network protocols are designed. Network architectures that follow the OSI model guarantee that network devices, designed by different engineers, will be able to communicate. The OSI model also makes network designs more extensible as new protocols and other network services are generally easier to add to a layered architecture than to a monolithic one.

A network architecture based on the OSI model provides the following benefits:

- It helps a user understand the big picture of networking.

- It helps a user understand how the hardware and software elements function together.

- It makes trouble shooting easier by separating networks into manageable pieces. 


\section{Chapter 3 Hardware Methodology - Sensor Network Interface}

This chapter describes the hardware methodology adopted for development of an intelligent sensor network. The following sections outline the network topology and communication technology selected. The development of a sensor network interface board, for controlling the network and communicating with a robotic vehicle's control computer is presented.

\subsection{Fundamentals of a Sensor Network}

The collection of sensors onboard a robotic platform can be viewed as a network of nodes, where each node is a sensor, except for one node being the robotic platform's control computer. All sensors must be able to report measurements to the main control computer; therefore a communication link must exist between each sensor and the computer. These communication links form the structure of the sensor network, and define its topology. Due to the variety of sensors, and the previous incremental approach taken to add new sensors to a platform, the VUW fleet's existing sensor networks have evolved with differing topologies and implementations of control across the robotic fleet [14 - 16]. Without a clear and defined network topology and architecture, the addition of new sensors only complicates the interface between the control computer and the robotic platform's peripheral hardware.

A uniform approach to sensor implementation across the robotic fleet can provide several benefits. These include:

1. The ability to transplant sensor hardware from one robotic platform to the next with minimal or no customisation required.

2. The ability for a network to dynamically grow by allowing the addition of new sensors with no changes required to hardware or software.

3. The ability to add new sensor technology, which currently might not be available, to any robotic vehicle with minimal changes to existing sensors.

4. A stable and defined communication protocol between the control computer and the sensors.

5. With correct selection of the hardware it can provide a cheap and easy method to physically mount sensors to a robotic platform. 
6. A true digital communication interface connecting the control computer to the sensors. This would replace the existing mixed analogue and digital interface currently employed on the robotic fleet. Converting analogue signals immediately at the sensor, instead of running them through long wire traces back to an ADC board on the control computer, would reduce a sensor's susceptibility to noise.

A uniform approach can be achieved through the clear definition of a sensor network that can be adopted across the fleet. This would also allow a user to quickly and easily construct and configure a new sensor network without the need for an in-depth knowledge of how the network operates.

Key to the definition of a sensor network is both the topology and architecture adopted. The topology defines how each sensor is physically attached to the network, while the protocol of the network architecture defines how each sensor logically interacts with the network.

\subsubsection{Selection of Network Topology}

The main task of the sensor network is for sensor nodes to report measurements back to the control computer, allowing the robotic platform to process the data. It is unlikely that sensor nodes will need to communicate with each other over the network. The network task, of a sensor reporting readings back to the control computer, follows a Master/Slave relationship where the sensor nodes are slaves to the control computer (the master). Although the Master/Slave relationship has a basic hierarchical structure, it only requires a flat network structure as all network nodes, apart from the control computer, are slaves and treated the same.

The make, footprint and style of the control computer on a robotic platform varies across the fleet. Some platforms utilise a small form factor 'Shuttle' PC which is rapidly becoming obsolete. Others employ a laptop, and some use a single board computer. It is highly likely that a robotic platform's control computer will require upgrading in the future and as such could change in make and style. It would be ideal for the sensor network to adopt a communication link with the control computer that is likely to be supported in future computer upgrades. The use of third party Input / Output (IO) and Data Acquisition (DAQ) 
cards limits the network's ability to adapt to future computer upgrades as third party cards tend to be hardware specific; currently the DAQ cards used on the robotic platforms only work with a PC style computer offering a PCI slot. Adoption of a communication technology that is standard on computer systems would allow the sensor network to be connected to upgraded computer systems without the need for proprietary hardware. Current trends in computing hardware indicate two communication technologies that will remain standard on computers for the foreseeable future $[23,24]$. These technologies are USB and Ethernet. To take advantage of utilising one of these communication technologies the network master node should be a peripheral hardware device that can communicate with the control computer via these technologies. This would require the network to be intelligent so that it is able to adapt and control itself independent of the robotic platform's computer. Figure 3.1 illustrates how a sensor network interface board acts as the communication interface between the control computer and the sensors.

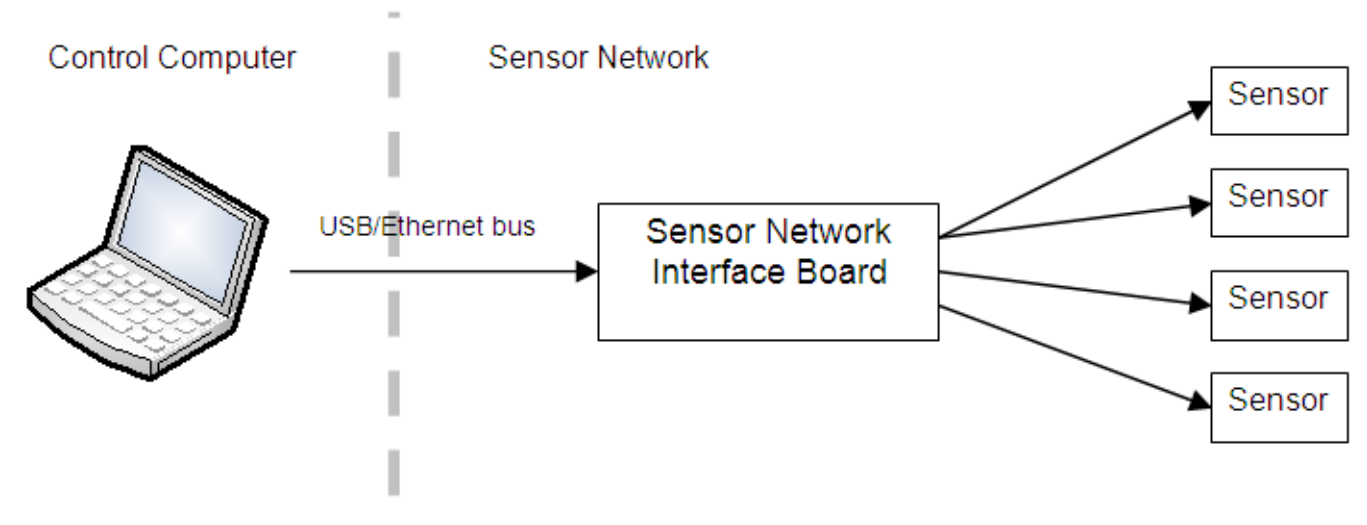

Figure 3.1 Control Computer to Network interface

The sensor network adopted is a hybrid scheme between the star and bus topologies. At the top level the network has a star topology where the network master node acts as the central hub for the network. But unlike a standard star topology, where the central network hub has direct point to point communication links with every sensor node, the central network hub has communication links to sub-networks that utilise a bus topology. The bus topology of the sub-networks allows the use of a single network cable to connect all the sensor nodes. Figure 3.2 illustrates the hybrid topology utilised on the sensor network. This is advantageous as it allows for a simple wiring loom on the robotic platforms as well as offering ease of expansion, as any additional sensor nodes can be added onto the wiring loom without the need to connect additional wires back to the central network hub. The star 
topology allows the central network hub to act as the sensor network interface to the control computer, where the central hub manages the sensor sub-networks and communicates to the control computer via Ethernet/USB.

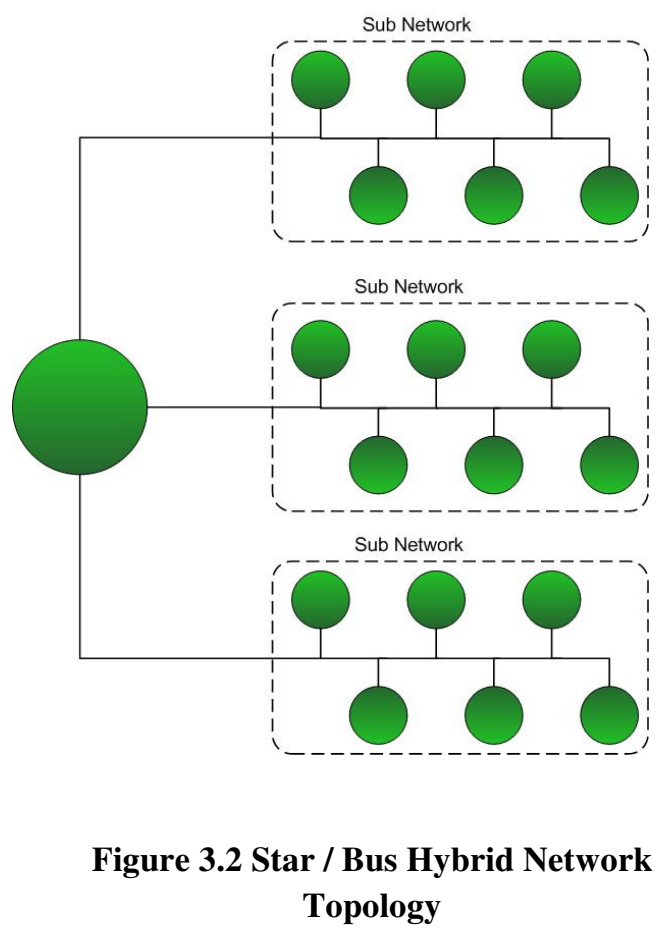

\subsubsection{Communication Technology}

Following the OSI model for network architecture, the Physical layer must define the electrical and mechanical connection between a network node and the network media. There are several communication technologies that have been developed to allow embedded controllers, such as microcontrollers, to communicate with peripheral devices. Each technology has its own advantages and disadvantages.

The main characteristics considered in deciding on the communication technology to adopt for the sub-networks are the following:

- The interface must allow a bus topology to be adopted.

- It should provide high noise immunity to high current noise sources, such as motors or switch mode power supplies.

- It should provide a simple wiring loom.

- It should provide reasonably fast data communication $(100 \mathrm{~kb} / \mathrm{s}-1 \mathrm{Mb} / \mathrm{s})$.

- It should support network lengths between 1 to 25 metres. 


\subsubsection{Serial Peripheral Interface}

Previous distributed control and sampling systems for sensors on the VUW fleet of robotic platforms have relied on a Serial Peripheral Interface (SPI) to communicate between an embedded controller and the sample/control unit. While this interface works well for communicating with peripheral devices located physically near the embedded controller, it lacks the ability to communicate over medium length distances reliably and the use of single ended data lines offers very limited noise immunity. The SPI technology also provides no address space. Addressing of a device is achieved through the use of chip select (CS) lines in hardware $[25,26]$. In a bussed network the network master would require a CS line for each connected network node, dramatically increasing the number of lines needed in the bus wiring loom. Because of these characteristics the SPI technology is more suited to a star topology where distances to network nodes can be guaranteed to be below a maximum length. SPI was not considered as an option for the communication technology due to these limitations.

\subsubsection{RS-232}

RS-232 provides an improvement over SPI with regard to the maximum length of a connection, upwards of $15 \mathrm{~m}$. The bipolar nature of the logic signals, typically $-3 \mathrm{~V}$ for a high and $+3 \mathrm{~V}$ for a low, also provides an improvement in noise immunity over SPI. RS-232 has single ended data lines which limit the maximum length of a connection and the speed of data transfer. RS232 was originally developed for data speeds of $20 \mathrm{~kb} / \mathrm{s}$ by reducing the length of a connection the data speed can be increased. At $15 \mathrm{~m}$ the maximum speed is given as $20 \mathrm{~kb} / \mathrm{s}$ while connection lengths of less than $0.5 \mathrm{~m}$ can reach speeds up to $115 \mathrm{~kb} / \mathrm{s}$ [27].

RS-232 was not selected as the communication technology for the network because its single ended data lines do not provide the speed nor the noise immunity comparable to other technologies investigated.

\subsubsection{Ethernet}

Using Ethernet as a communication technology has several advantages. If the network nodes are designed with a Transport Control / Internet Protocol (TCP/IP) stack they could potentially be connected to any Ethernet network allowing sensor networks to be established over existing network infrastructure [23]. A fully internet enabled node would also be able to be connected to the internet allowing any authorised computer to access the sensor network. 
However, implementing a fully enabled Ethernet connection would require each node to have a unique Media Access Controller (MAC) address that would have to be purchased. Also each node would have to conform rigidly to the Ethernet standard IEEE 802.3 which would require the use of switches or hubs on a network and cause the sensor network to develop a star topology. This would add extra hardware and complexity to the network node design for the benefit of advantages that do not greatly improve the performance of this type of sensor network; a small area network confined to a robotic platform. Due to these factors it was decided not to use Ethernet as the network communication technology.

\subsubsection{Universal Serial Bus}

The Universal Serial Bus has been developed to operate in a star topology. Additional network nodes can be added to the network through intermediary network hubs. The star topology complicates network wiring as it requires each network node to be connected to a central hub [24]. As mentioned in Section 3.1.1 Selection of Network Topology, the bus topology is preferred as it would require only one network cable to connect to a central network master. Additional nodes can be added to the network by connecting them to any network connected node. Due to the inherent star topology of the USB technology it was not selected as a communication technology for the sensor network.

\subsubsection{RS-485}

RS-485 was selected as the communication technology for the sensor network. It provides high data speeds for medium to long connection lengths, good noise immunity and lends itself well to a bus configuration.

RS-485 is a balanced differential signal based system. Digital data is transmitted by the driver inducing voltage differentials across a pair of signal lines, typically the magnitude of the differential voltage ranges between $2-6 \mathrm{~V}$. The differential signal removes the requirement that the driver and receiver share a common ground. This greatly improves noise immunity of the communication lines.

RS-485 offers high data transmission speeds (35 Mbit/s up to $10 \mathrm{~m}$ ) and its use of differential balanced lines over a twisted pair allows it to span relatively large distances (up to $1200 \mathrm{~m}$, with a transmission speed of $100 \mathrm{kBit} / \mathrm{s}$ ) [28]. RS-485 drivers are equipped with an enable line that allows the implementation of linear bus topologies using only one pair of twisted 
wires. This type of implementation allows half duplex transmissions, where communication is permitted in both directions but only one direction at a time. The recommended arrangement of network bus is usually a connected series of point-to-point (multi-dropped) nodes, where each node has input and output connectors and the network is expanded by connecting the output of one node to the input of another node, in a daisy chain style [29]. Ideally the two ends of the cable that forms the communication link will have a termination resistor connected across the twisted wire pair. The termination resistors help to reduce reflections of fast driver edges which can cause data corruption. The value of each termination resistor should be equal to the cable impedance (typically $120 \Omega$ for twisted pairs).

The RS-485 standard specifies an input impedance of $12 \mathrm{k} \Omega$ per receiver. This input impedance allows up to 32 nodes to connect to a single network bus. Higher input impedance receivers are available, increasing the maximum number of nodes to 256 .

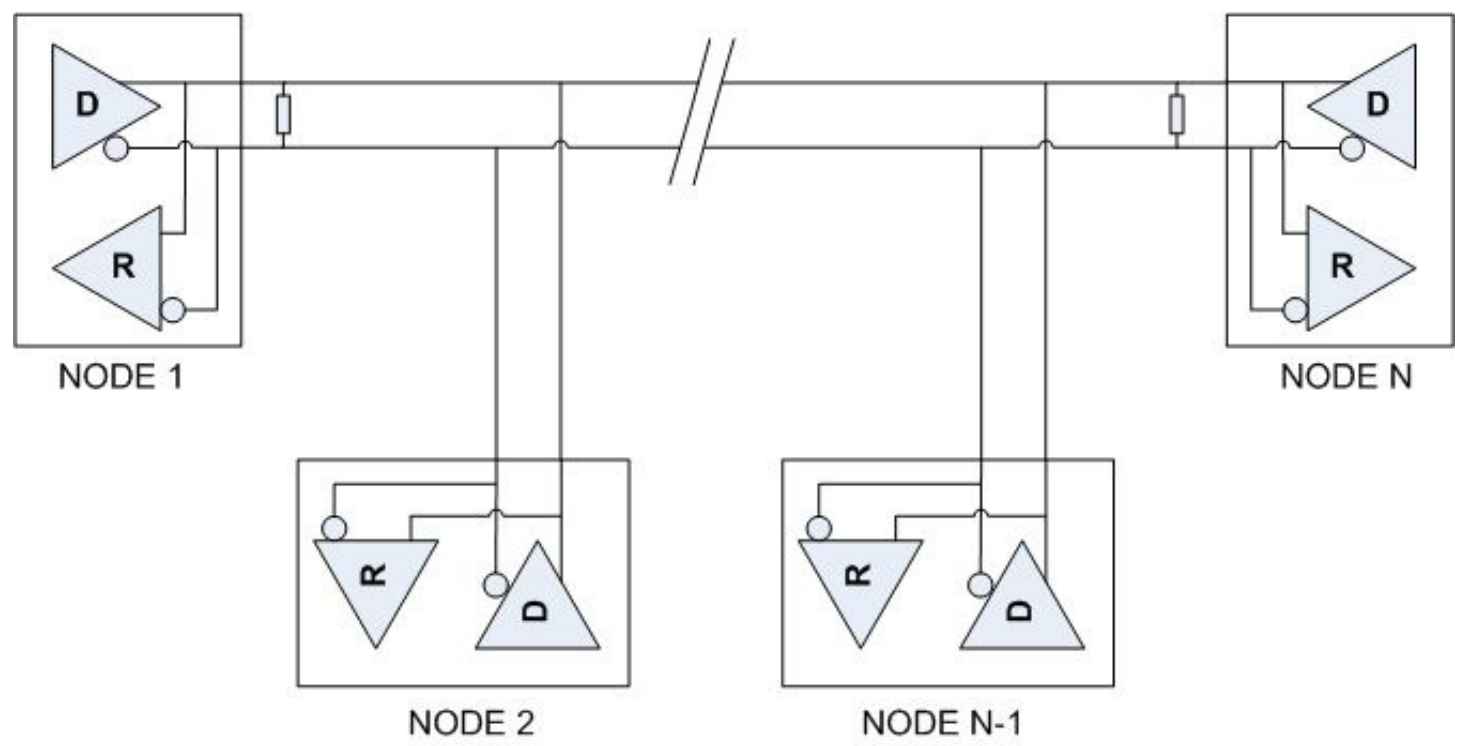

Figure 3.3 A Half Duplex RS-485 Bussed Network

Figure 3.3 illustrates the implementation of a half-duplex, multi-drop network using RS-485 drivers/receivers. The half duplex operation works by all drivers initially being in a high impedance tri-state and all receivers in an active state. When a node has data to send on the network, it enables its driver and commences communication. Since all other drivers are in their high impedance tri-state, the active driver has full use of the network bus. Once the 
node has finished transmitting the data it switches its driver back into the high impedance tristate.

\subsubsection{Network Bus}

The network bus consists of two twisted wire pairs. One pair carries the differential data signals on the network while the second pair supplies power to network nodes. Differential data transmission is used to reliably transmit data at high rates over long distances and through noisy environments. Twisted pair cables tend to cancel common-mode noise and also cause cancellation of the magnetic fields generated by currents flowing through each wire. Because the network is of a multi-drop configuration, the physical wiring of the network only requires separate network cables from each node, as illustrated in Figure 3.4.

Category 5 (CAT5) network cable is used as the network medium. CAT5 cable has a nominal characteristic impedance of $100 \Omega$ at $100 \mathrm{MHz}$, which provides a good impedance match to RS-485 driver/receivers which require differential line termination impedances of approximately $100-120 \Omega$.

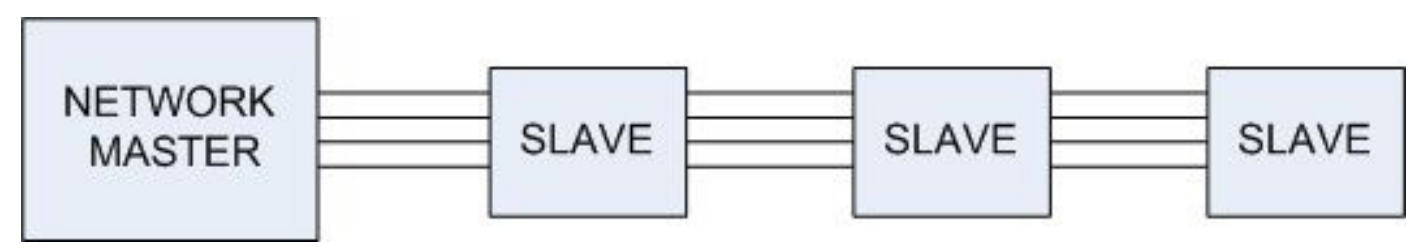

Figure 3.4 Daisy Chain format of Network Bus

The power rails on the network bus provide a regulated $+12 \mathrm{~V}$ supply to connected network nodes. The $+12 \mathrm{~V}$ supply is provided from the network master and is fused at $3 \mathrm{~A}$. Each network node has a $10 \mu \mathrm{F}$ capacitor to provide filtering of the power supply to reduce voltage variations due to extended network cable runs and intermittent high current draws from devices such as ultrasonic transducers.

Mass Terminal Assembly (MTA) connectors (pictured in Figure 3.5) have been used to connect network nodes to the network bus. These connectors are polarised to ensure that network cables are connected in the correct orientation and have been selected due to their relatively low price (tens of cents) and ease of assembly. 

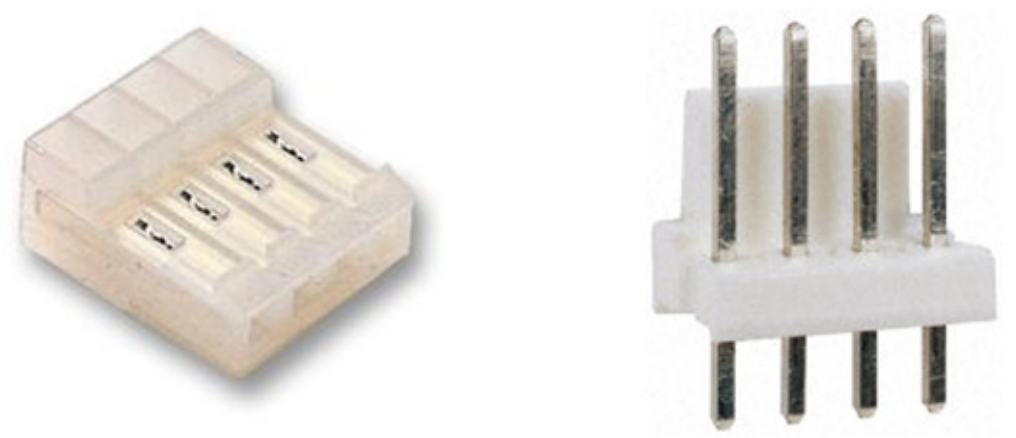

Figure 3.5 Mass Terminal Assembly Connectors

\subsection{Sensor Network Interface Module}

To allow the sensor network to operate independently of a robotic platform's control computer, a network interface module, operating as a master node, is required to control the network. This interface module communicates with the control computer to report back sensor data. The interface module is responsible for defining the sensor network at initialisation allowing a control computer to query the size and operational capabilities of the network. As mentioned in Section 3.1.1 Selection of Network Topology, the master node also provides a communication translator between the RS-485 communication technology of the network and either an Ethernet or USB communication interface of a control computer.

Figure 3.6 illustrates the main components of the Network Interface node. To control the network and manage communications between the control computer and the sensors, the interface node employs an ATMega128 micro-controller (MCU). The ATMega128 is an 8-bit AVR MCU with $128 \mathrm{kB}$ of flash memory and $4 \mathrm{kB}$ of SRAM [30]. The MCU has a JTAG interface allowing programming and in-circuit emulation. A single supply rail of $+5 \mathrm{~V}$ is required for MCU operation. 
Featured peripherals relevant to the network interface's requirements include:

- $2 \times$ Universal Synchronous/Asynchronous Receiver Transmitters (USART).

- Master/Slave Serial Peripheral Interface.

- $2 \times 16$-bit Timer/Counters.

- $2 \times 8$-bit Timer/Counters.

- JTAG interface.

- Watch Dog Timer.

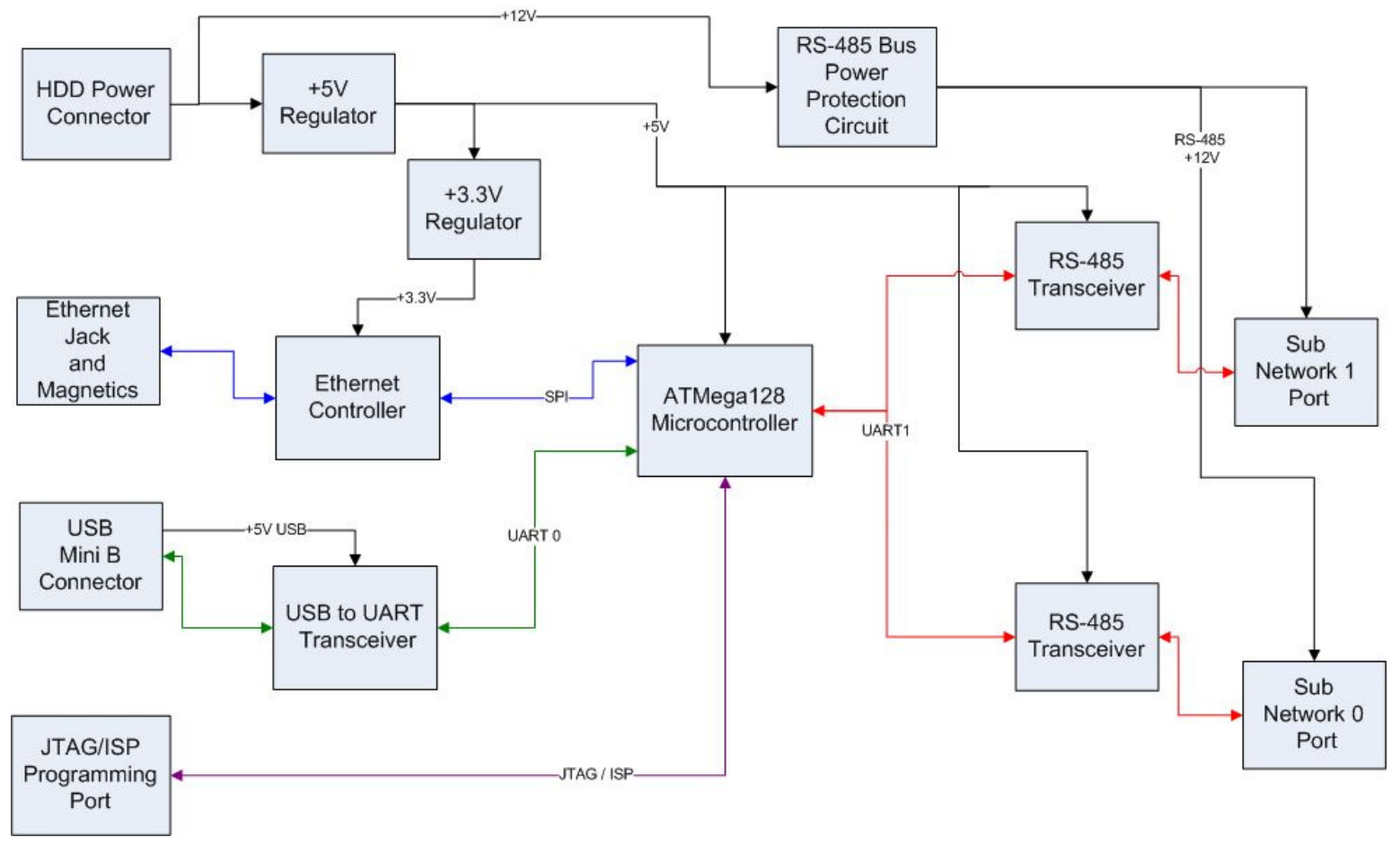

Figure 3.6 Block Diagram of Sensor Network Interface Master Module

A $16 \mathrm{MHz}$ crystal provides the base clock frequency for the MCU. Due to the AVR's RISC architecture, a $16 \mathrm{MHz}$ clock allows up to 16 million instructions per second (MIPS). The maximum data speed of the MCU's USARTs is limited by the system clock. At $16 \mathrm{MHz}$ the maximum data rate possible is $2 \mathrm{Mb} / \mathrm{s}$. 


\subsubsection{Ethernet Interface}

To enable Ethernet communication between the MCU and a control computer, an external physical layer (PHY) transceiver, media access controller layer (MAC) transceiver and appropriate line transformer are provided. A stand-alone Ethernet controller from Microchip (ENC28J60) provides both a MAC and PHY transceiver to meet the IEEE 802.3 specification [23] for Ethernet connections. The Ethernet controller is equipped with an industry standard serial peripheral interface. The controller chip is interfaced to the ATMega128 via the MCU SPI interface and a single interrupt pin. The SPI interface can work at speeds up to $10 \mathrm{Mbit} / \mathrm{s}$ [31]. The Ethernet controller requires a $+3.3 \mathrm{~V}$ supply which is provided from a $+3.3 \mathrm{~V}$ regulator (National Semiconductor LP3964-3.3). Figure 3.7 illustrates the main components of the Ethernet interface.

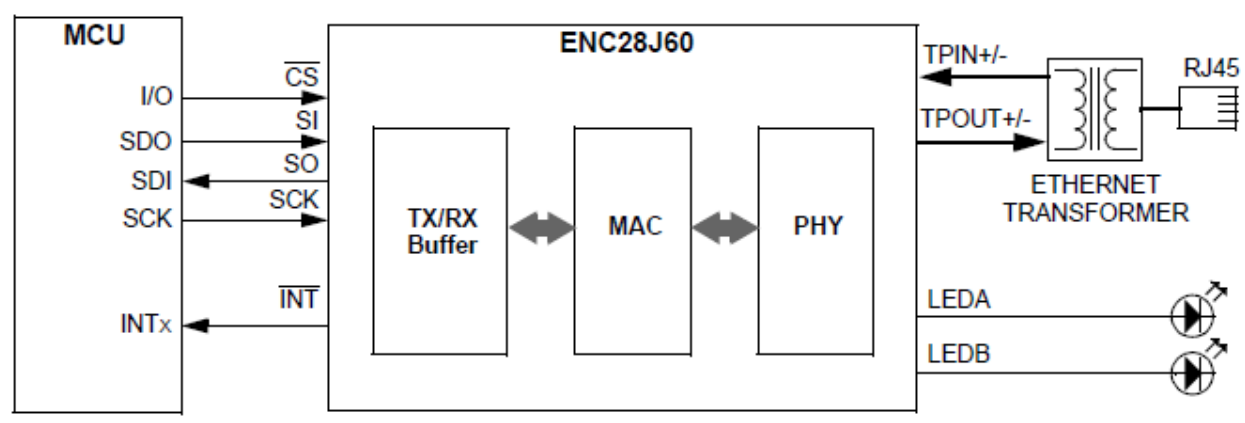

Figure 3.7 Ethernet Interface System Diagram

The SPI interface allows received data packets to be transferred from the Ethernet controller to the MCU, as well as allowing the MCU to configure the controller with important parameters, such as a MAC address, and to prioritise interrupts. An external $25 \mathrm{MHz}$ crystal provides the clock source for both the MAC and PHY transceivers. The SPI clock is derived from the MCU clock source.

Use of an RJ-45 jack with integrated Ethernet transformer and status light emitting diodes (LED) has reduced the component count of this circuit.

Microchip's recommended circuit layout for the ENC28J60 has been followed to ensure correct termination of the differential data signals to meet the IEEE 802.3 specification [23]. Figure 3.8 details the circuit implemented to interface the Ethernet controller to the standard 


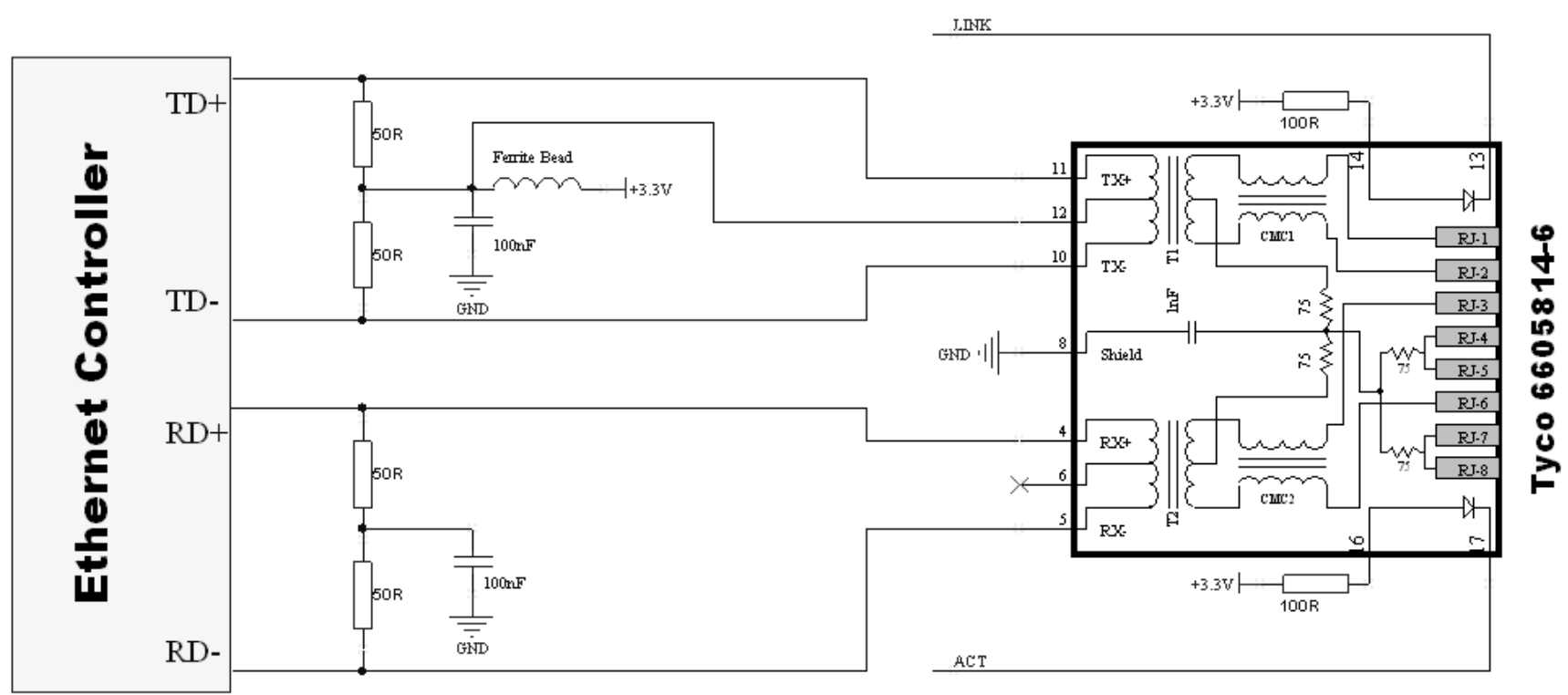

Figure 3.8 Interface between Ethernet Controller and Ethernet Network

Ethernet twisted pair network. The $50 \Omega$ resistors provide active sink and source termination of the differential transmit and receive lines from the Ethernet jack. These terminating resistors are placed as close as possible to the transmit and receive pins of the Ethernet controller to minimise signal reflections from the Ethernet data signals. The ENC28J60 features both hardware and software reset options. The hardware reset line has been connected to a MCU I/O line to allow the MCU to perform reset of the controller, after the MCU has itself initialised.

Level shifting, between the $+3.3 \mathrm{~V}$ logic level of the Ethernet controller and the $+5 \mathrm{~V}$ logic level of the MCU, is provided through the use of a quad buffer (74AHC125). The SPI interface pins on the Ethernet Controller are $+5 \mathrm{~V}$ tolerant. Level shifting is provided on the MISO data line and interrupt line to ensure clear communication between the Ethernet controller and the MCU.

\subsubsection{Universal Serial Bus Interface}

To provide a Universal Serial Bus interface between the control computer and the network interface module, a USB transceiver is utilised. A USB to USART converter from Future Technology Devices (FT232BM) is used to create a virtual Com port between the control computer and the interface module [32]. The FT232 has a USART interface that connects to 
one of the MCU's onboard USARTs (USART0). Figure 3.9 illustrates the main components of the USB interface.

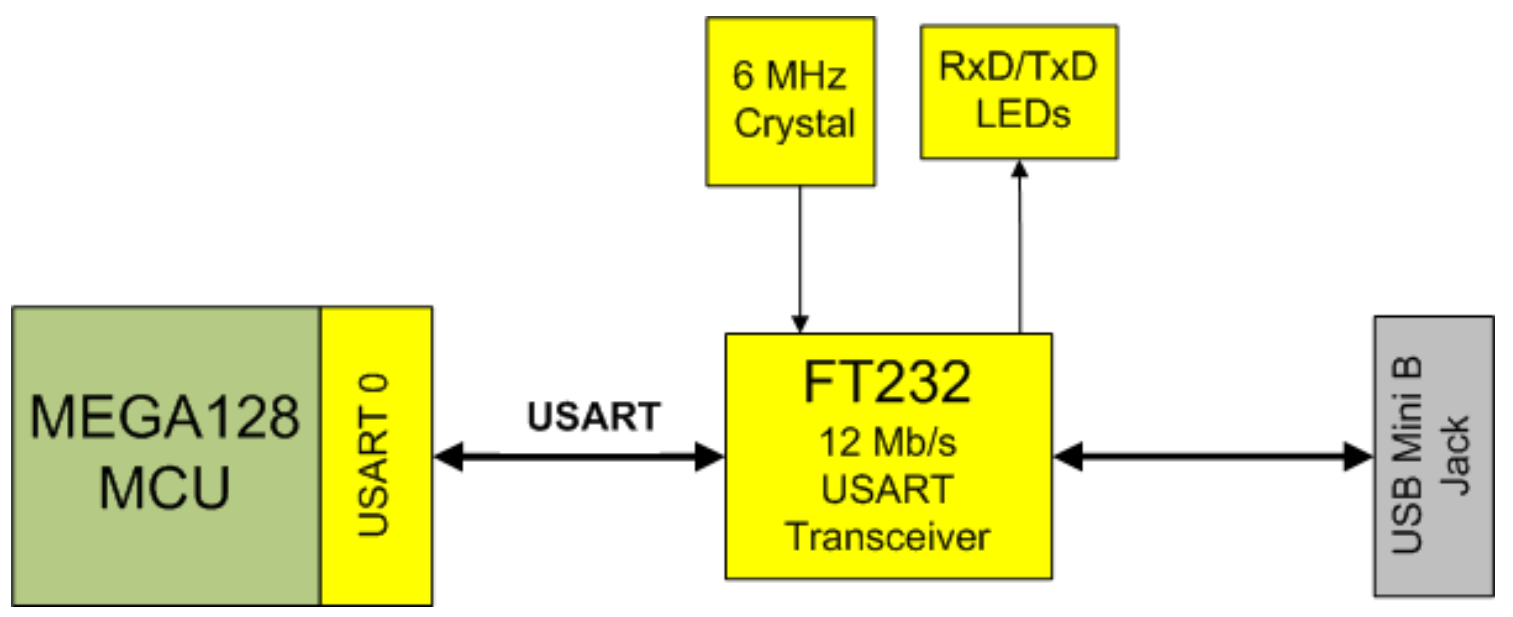

Figure 3.9 USB System Diagram

The USB transceiver of the FT232 operates from a $48 \mathrm{MHz}$ clock that is generated from an internal $\times 8$ multiplier connected to an external $6 \mathrm{MHz}$ crystal. The transceiver has a 128 byte transmit buffer and a 384 byte receive buffer to provide onboard buffering of data.

The FT233 transceiver is powered from the USB bus allowing it to be immediately detected when connected to the control computer. A USB power LED is included to indicate that the FT232 transceiver is powered from the USB bus. Having the transceiver bus powered allows the network interface module power to be cycled off and on without the control computer having to close and then reopen the Com port. This feature is advantageous when the control computer wishes to manage power on the robotic platform by controlling which peripheral hardware is powered. The transceiver can run at speeds of up to $3 \mathrm{Mb} / \mathrm{s}$. The USB specification [24] requires standard connectors for USB end devices, such as the FT232 USBUSART transceiver. Hence a Mini -B USB connector, pictured in Figure 3.10, is used. Transmit and Receive status LEDs are used to indicate operation of the USB interface. 


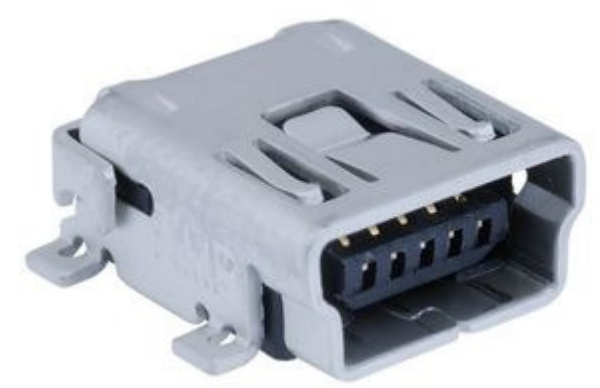

Figure 3.10 USB Mini -B Jack

The differential data lines of the USB connection require correct termination to ensure any connected device does not degrade the data signals. The Future Technology Devices recommended circuit layout for the USB transceiver in a bus powered configuration has been followed to ensure correct operation of the device. This configuration requires the use of $27 \Omega$ resistors in series with the USB differential data lines to ensure the USB transceiver impedance matches the line impedance of the USB connection. Figure 3.11 illustrates the interface between the USB bus and the transceiver.

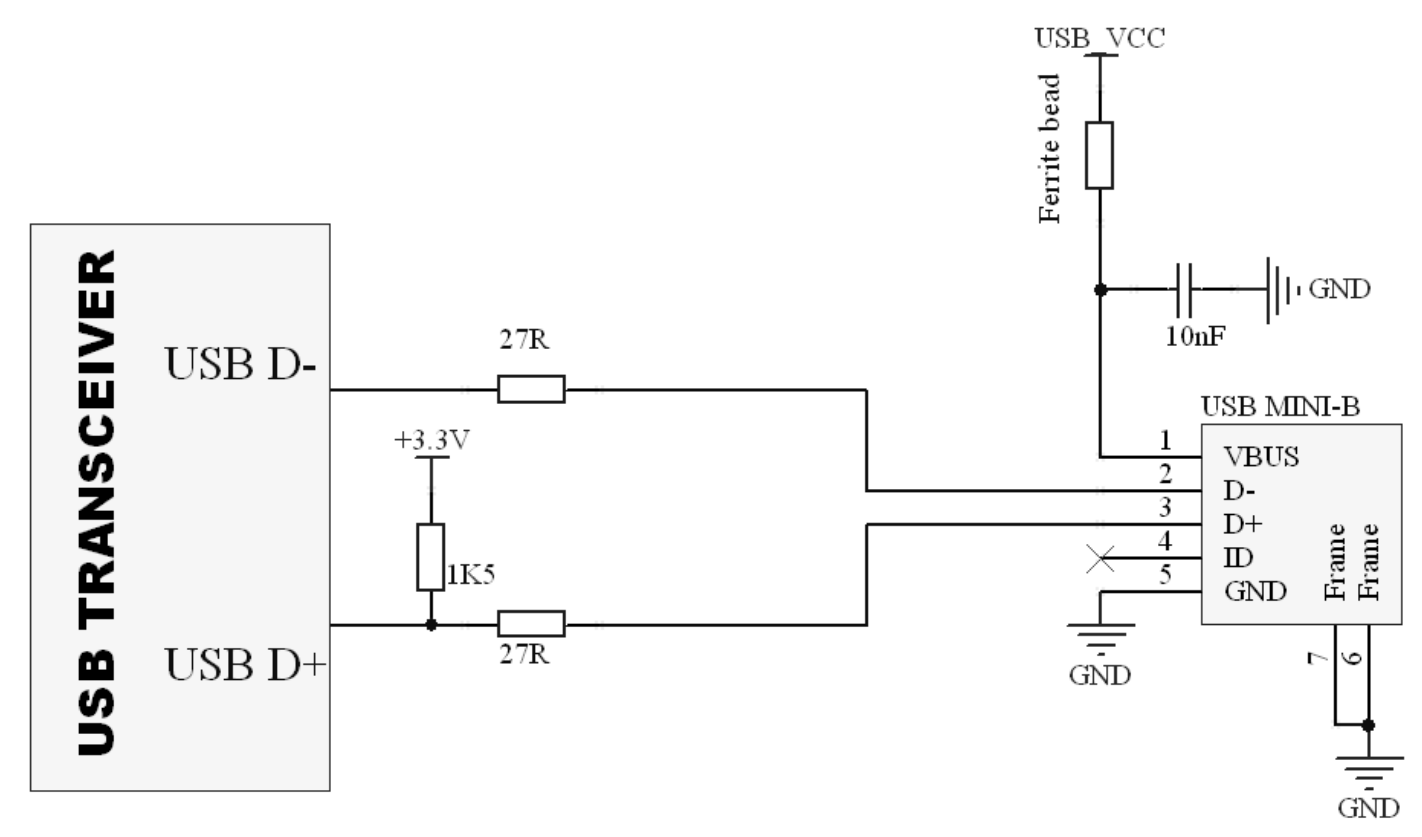

Figure 3.11 USB bus to transceiver interface

A $1.5 \mathrm{k} \Omega$ pull-up resistor is attached to the positive data line of the USB bus, as specified by the USB standard, to indicate to the control computer that the USB end device can operate at the full data speed. A ferrite bead is connected in series with the USB power to prevent noise 
from the device and associated circuitry (EMI) being radiated down the USB cable to the control computer.

\subsubsection{RS-485 Interface}

The sensor network interface module has two RS-485 interfaces allowing two sub-networks of sensors to be controlled. Two RS-485 transceivers (ADM1485, from Analog Devices) are used, one per sub-network, which connect to the MCU through one of the MCU's onboard USARTs (USART1). The ADM1485 is a differential line transceiver designed for high speed (up to $30 \mathrm{Mb} / \mathrm{s}$ ) bi-directional data communication on multipoint transmission lines [33]. The MCU controls which of the sub-network interfaces is accessed by enabling or disabling the RS-485 transceivers. With two sub-network interfaces the MCU can address up to 62 sensor nodes ( 31 per sub-network) without the need of a high impedance repeater. If a repeater is employed then the number of sensor nodes possible increases to 255 per sub-network. Logically the MCU treats both sub-networks as one large network by switching between the two interfaces.

To ensure that only one sub-network interface receiver is connected to the MCU's USART at one time a 'receiver enable' control signal is generated by the MCU. An inverted value of this signal is generated in hardware by a Schmitt Not gate. The inverted signal feeds the enable line of the receiver of sub-network interface 2 while the non-inverted signal feeds the enable line of the receiver of sub-network interface 1. This allows only one sub-network interface receiver to be active at one time. Figure 3.12 illustrates the initial implementation of the RS-485 network interface circuit.

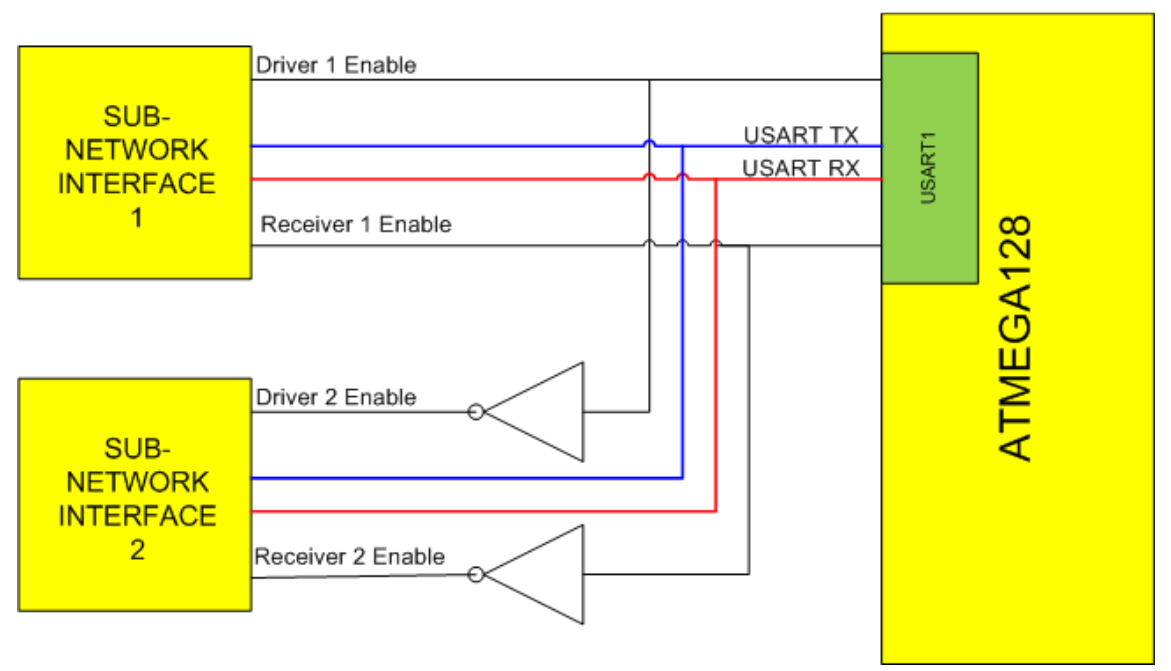

Figure 3.12 RS-485 Network Interface Version 1 
As the network utilises half duplex communication, it is important that the MCU can disable both sub-network interface drivers to allow other devices on the network to send data. The original circuit design utilised a single 'driver enable' signal generated by the MCU to enable the driver of sub-network interface 1, and an inverted 'driver enable' signal generated with a Schmitt Not gate to enable the driver of sub-network 2. It was realised that this configuration prevented the MCU from writing global data to the entire network as one of the two subnetwork interface drivers was always disabled, preventing network devices on that subnetwork from receiving the global data.

The design was altered to allow the MCU to generate two separate 'driver enable' signals allowing the MCU to enable both sub-network interface drivers simultaneously. Figure 3.13 illustrates the improved RS-485 network interface circuit.

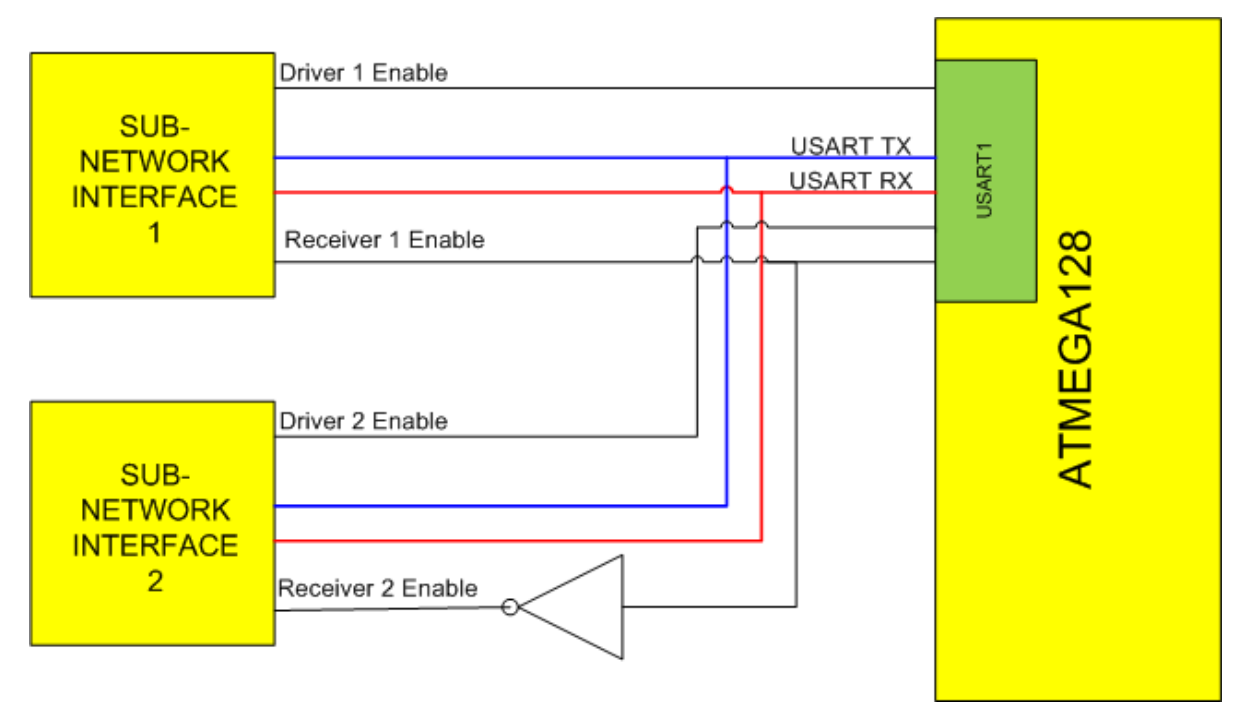

Figure 3.13 RS-485 Network Interface Version 2

A characteristic of the half duplex bus is that any data transmitted by the driver is automatically echoed back to the transmitting node on its receiver. This feature can be exploited to provide error checking and to sense bus collisions with another network device. This error checking is discussed further in Section 5.2.1 Physical Layer.

LED indicators on the transmit and receive lines of the MCU USART provide a visual indication of network traffic. 


\subsubsection{Power Supply}

The network interface module has 3 voltage rails:

1. $+12 \mathrm{~V}$ for the RS-485 bus,

2. $+5 \mathrm{~V}$ for the MCU and RS-485 transceivers,

3. $+3.3 \mathrm{~V}$ for the Ethernet controller.

As mentioned above, the USB circuit is powered from a $+5 \mathrm{~V}$ supply derived from the USB bus. The interface module has a standard hard disk drive (HDD) power connector, as illustrated in Figure 3.14, allowing it to be powered by any standard computer switch mode power supply which provides $+5 \mathrm{~V}$ and $+12 \mathrm{~V}$. The $+12 \mathrm{~V}$ supply powers the RS -485 bus. The RS-485 $+12 \mathrm{~V}$ supply is fused at $3 \mathrm{~A}$ and has overvoltage protection limiting the maximum voltage to $15 \mathrm{~V}$.

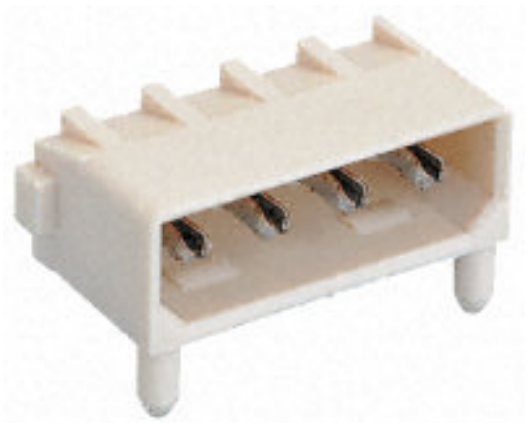

Figure 3.14 HDD Power Socket

The $+5 \mathrm{~V}$ supply is regulated from the $+12 \mathrm{~V}$ HDD power through a $+5 \mathrm{~V}$ regulator (LF50ABDT from STMicroelectronics). The regulator has a maximum input operating voltage of $+16 \mathrm{~V}$ and a maximum output current of $0.5 \mathrm{~A}$ [34].

The +3.3 V supply is regulated by a $+3.3 \mathrm{~V}$ regulator (LP3964-3.3 from National Semiconductor). The $+3.3 \mathrm{~V}$ regulator is cascaded off the output of the $+5 \mathrm{~V}$ regulator to provide a stable input voltage. The $+3.3 \mathrm{~V}$ regulator has a maximum output current of $1.1 \mathrm{~A}$ and a maximum input voltage of $+7.5 \mathrm{~V}[35]$. 


\subsubsection{Assembled Module}

Each of the design sub-systems discussed in previous sections has been integrated into a single printed circuit board (PCB). Figure 3.15 illustrates the placement of the sub-systems and primary interface headers. Mounting of the PCB is via four M3 standoffs.

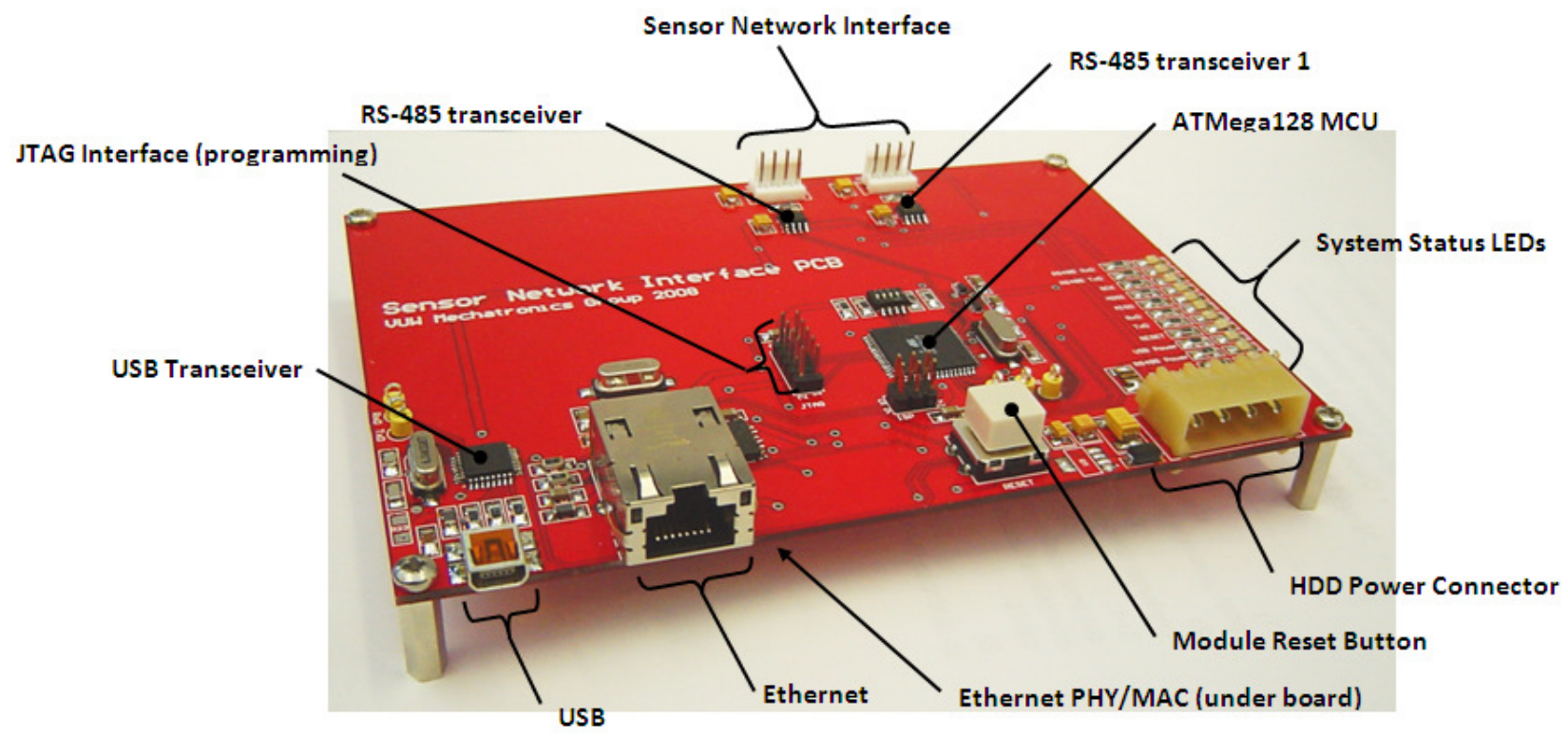

Figure 3.15 Sensor Network Interface module layout 


\section{Chapter 4 Hardware Methodology - Sensor Network Nodes}

This chapter describes the two hardware platforms that have been developed to provide different types of network nodes:

1. Infrared Ranging Node - a network device for controlling a Sharp PSD sensor.

2. Ultrasonic Ranging Node - a network device for controlling a SensComp ultrasonic ranging module.

The chapter also discusses a prototype sensor network constructed from the network nodes, including the mechanical mounting of this network onto a robotic vehicle.

\subsection{Infrared Ranging Node}

Infrared ranging modules have been extensively used on robotic platforms in VUW's fleet as they provide a low cost and effective solution for short and medium ranging $[5,11,12]$. The position sensitive detectors, first mentioned in Section 2.2.1 Sharp Position Sensitive Detectors, provide a multi-beam ranging module giving a ranging field of view of $25^{\circ}$. The design of an interface module between these PSD sensors and the sensor network has been designed to allow multiple numbers of these sensors to be connected into a sensor network.

\subsubsection{Sharp IR PSD Ranging Modules}

Each PSD sensor employs optical triangulation to determine range measurements. Due to the trigonometric nature of triangulation, these sensors produce a non-linear relationship between analogue output voltage and distance. This non-linearity is compensated for by empirically calibrating the analogue output to distance, covered in Section 8.1.4 Calibration of IR PSD Ranging Modules. Internal control circuitry provides multiplexing of the five infrared beams into a single analogue output signal.

Figure 4.1 illustrates the main functional blocks within a Sharp wide angle PSD module. Only one IR LED can be illuminated at a time to ensure that the IR light incident on the PSD sensor can be attributed to that LED. The $\mathrm{V}_{\text {in }}$ signal is used to control set-up and hold times for a sampled channel. $V_{\text {in }}$ also resets the signal processing circuit after each transition to the next LED. 


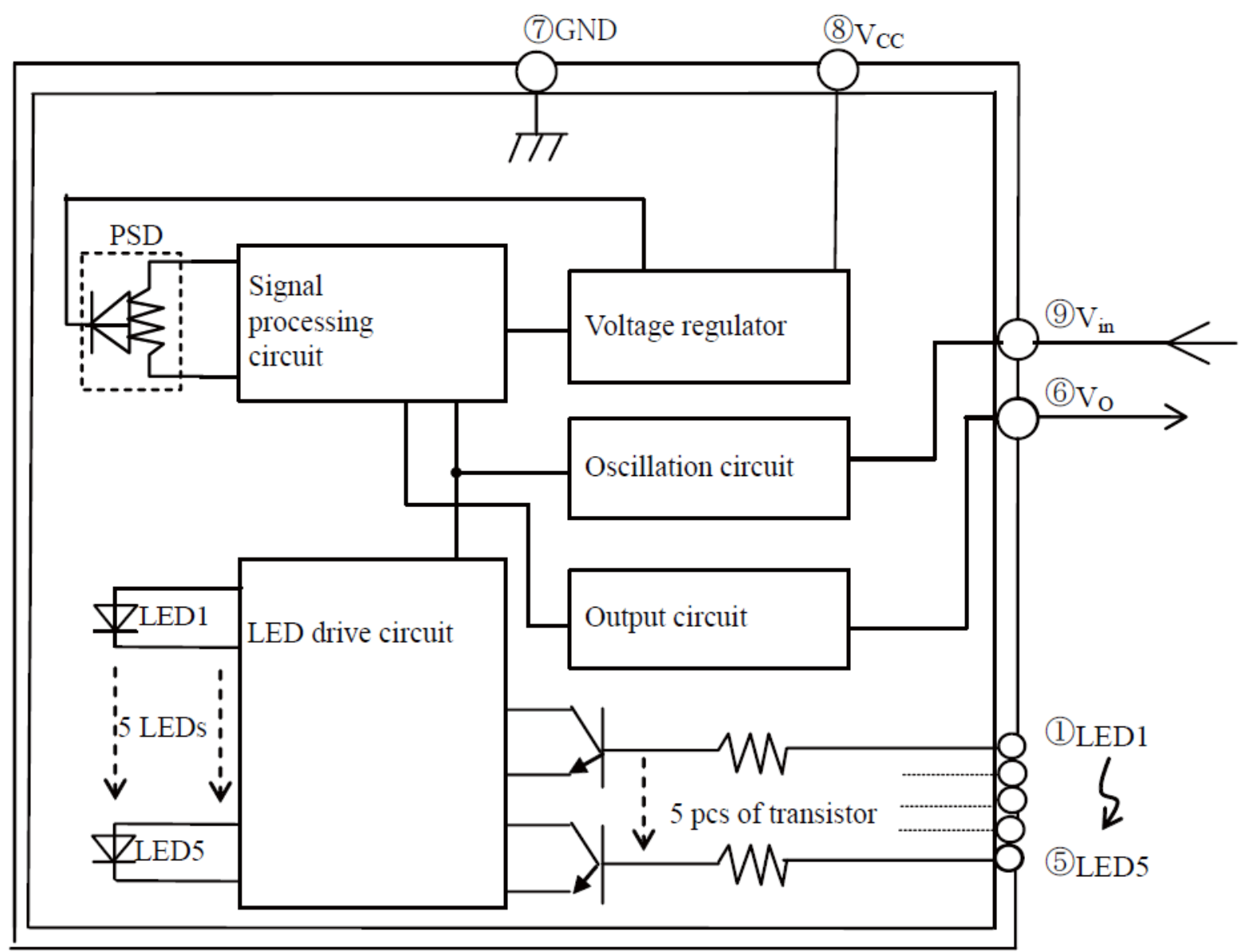

Figure 4.1 Sharp wide angle IR PSD block diagram [17]

Figure 4.2 provides details on the timing of the control signals to obtain valid distance readings for the first, second and subsequent IR beams. From the timing diagram it can be seen that a minimum sample time of $21.5 \mathrm{~ms}$ is required per LED channel. This limits the measurement cycle time across all five LED channels to $107.5 \mathrm{~ms}$, allowing a maximum sample rate of approximately $9 \mathrm{~Hz}$. The maximum sample rate for one LED channel is limited to approximately $45 \mathrm{~Hz}$. Multiple IR LEDs can be activated at the same time and it is possible to cycle through the LEDs in any order. Control of the IR module and sampling of the analogue output voltage is managed by a MCU, allowing increased flexibility over the operation of the IR module. Various IR LED activation timing and patterns can be uploaded to the MCU to allow it to operate the IR module in an organised fashion with other IR modules connected to the sensor network.

The MCU can also perform basic signal processing on the sampled analogue output signal, such as various forms of averaging, to improve the accuracy of the ranging. 


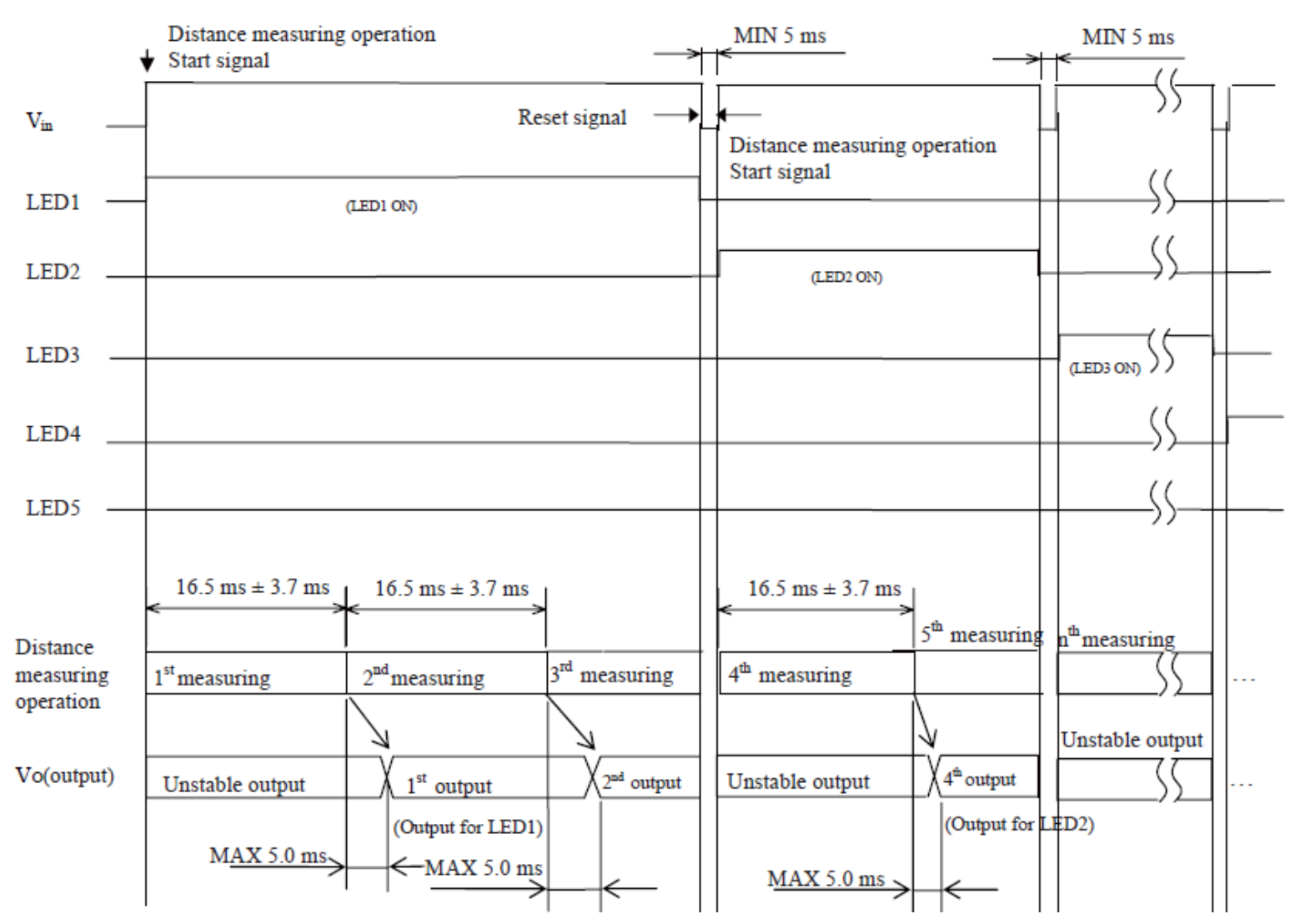

Figure 4.2 Sharp wide angle IR PSD timing diagram [17]

The infrared ranging node has been designed to interface both the Sharp GP2Y3A002K0F and GP2Y3A003K0F PSD sensors to the sensor network.

Figure 4.3 illustrates the main components of the infrared ranging node. An ATMega32 AVR 8-bit MCU controls all of the operations of the node, including IR module control, ADC sampling of ranging voltages, and communication interface to the sensor network. The ATMega32 has $32 \mathrm{kB}$ of flash memory, $1 \mathrm{kB}$ of EEPROM and $2 \mathrm{kB}$ of SRAM [36]. The MCU can be programmed through its JTAG interface or via Atmel's In-System Programming (ISP) interface. A single $+5 \mathrm{~V}$ supply rail is required for MCU operation.

Featured peripherals relevant to the infrared ranging node's requirements include:

- $1 \times$ Universal Synchronous/Asynchronous Receiver Transmitter (USART).

- $1 \times 16$-bit Timer/Counter.

- $2 \times 8$-bit Timer/Counters.

- $8 \times$ 10-bit Single Ended ADCs.

- 32 programmable I/O lines. 
- JTAG interface.

- Watch Dog Timer.

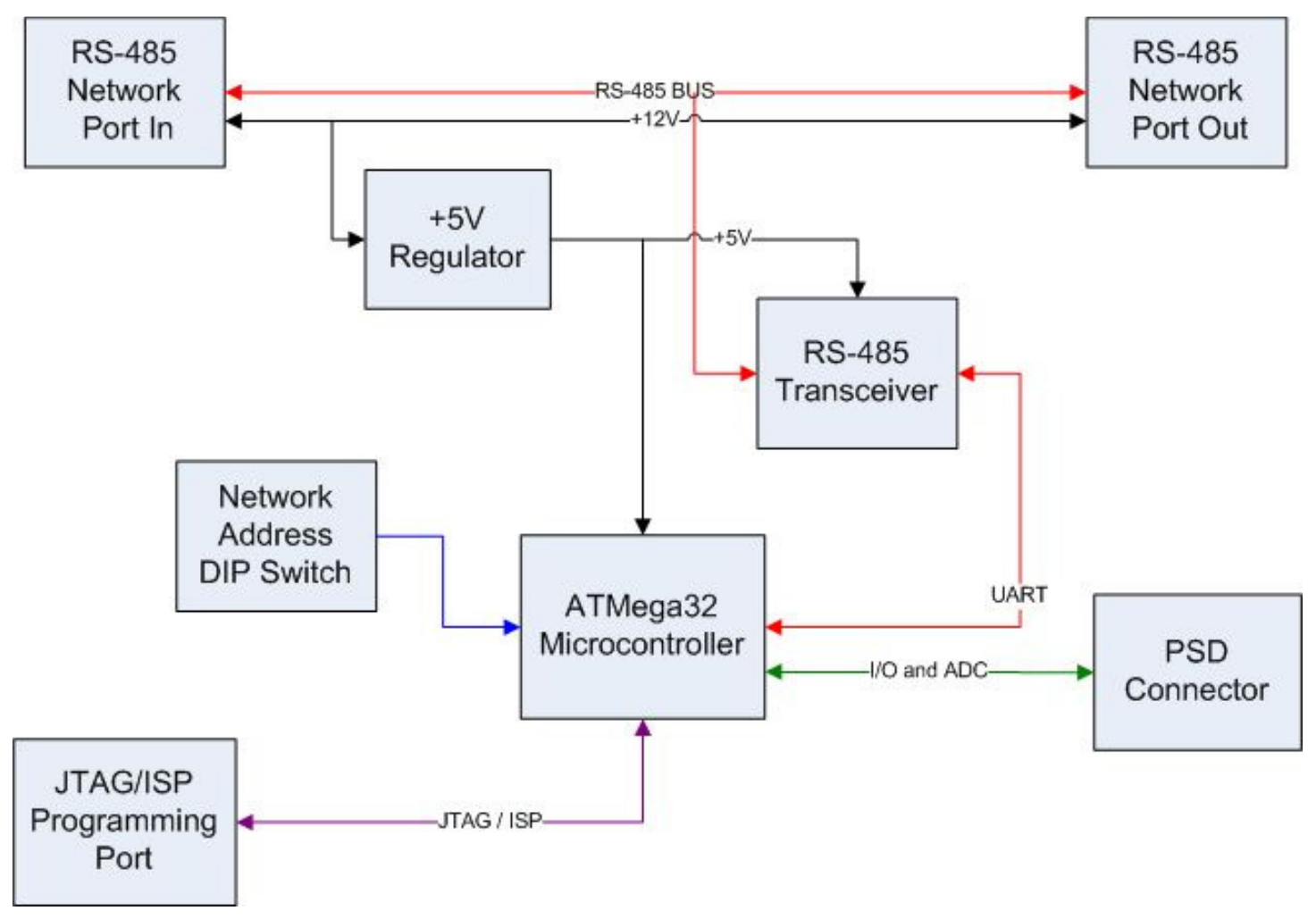

Figure 4.3 Block Diagram of Infrared Ranging Node

A five-way DIP switch allows a user to specify the network address of the node (0-31). The MCU derives its system clock from an external $16 \mathrm{MHz}$ crystal. This clock frequency matches the clock frequency of the Sensor Network Interface Module mentioned in Section 3.2 Sensor Network Interface Module allowing the infrared ranging node to communicate at an identical maximum data rate of $2 \mathrm{Mb} / \mathrm{s}$.

\subsubsection{RS-485 Interface}

The RS-485 interface on the infrared ranging node has both an input and output connector (four-way MTA) which are wired one-to-one. This allows further sensor nodes to be daisy chained onto the RS-485 bus, with the final network node having a $120 \Omega$ termination resistor on its output connector to reduce reflections. 


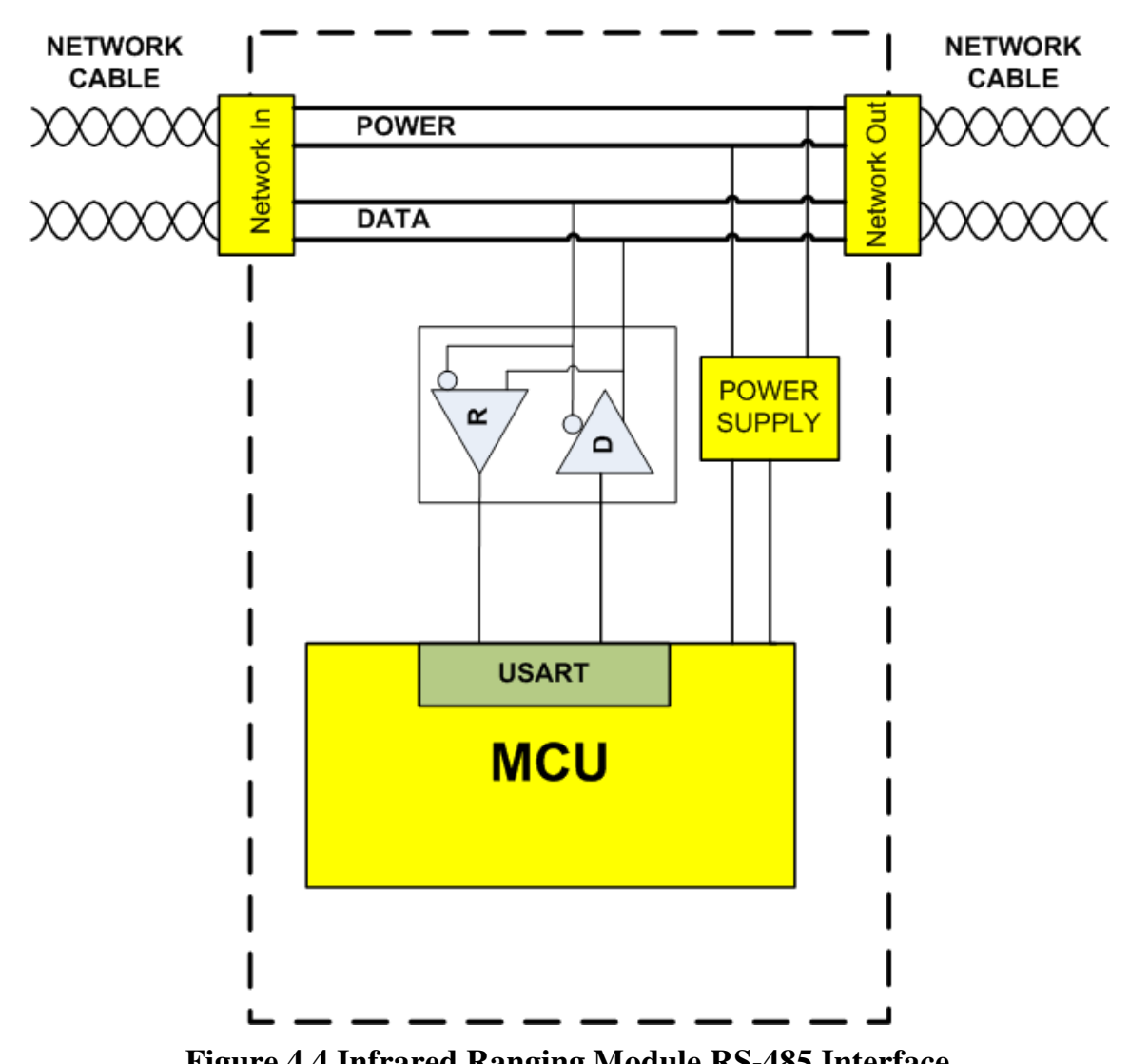

Figure 4.4 Infrared Ranging Module RS-485 Interface

Figure 4.4 illustrates the network bus connections passing through the infrared ranging node. A half duplex RS-485 transceiver (ADM1485) is tapped off the differential data pair of the RS-485 bus and the power supply circuit of the node derives its input power from the network bus power lines.

By permanently enabling the receiver of the RS-485 transceiver, the network node can receive and monitor all network traffic. The enabling and disabling of the driver is controlled by the MCU. When the driver is disabled, its output is placed in a high impedance state preventing it from loading the network data lines, thereby allowing other network nodes to transmit data across the network. By default the MCU disables the driver and only enables it when acknowledging network data addressed to the MCU.

The data input and output lines of the ADM1485 are connected to the Receive Data (RxD) and Transmit Data (TxD) lines of the MCU USART.

A +5 V $150 \mathrm{~mA}$ regulator (MIC5205-5.0BM5, from Micrel [37]) is employed to provide a stable $+5 \mathrm{~V}$ supply for the sensor node from the $+12 \mathrm{~V}$ voltage lines on the Network bus. 


\subsubsection{Sharp IR PSD Interface}

The control interface of both the Sharp GP2Y3A002K0F and GP2Y3A003K0F sensors are identical. The identical interface simplifies the interface on the infrared ranging node allowing it to accommodate either sensor with no hardware modifications required. As seen in Figure 4.5, the sensor's $V_{\text {in }}$ and LED $1-5$ control lines are connected to I/O lines on the MCU allowing the MCU to activate all of the 5 IR ranging beams in any order.

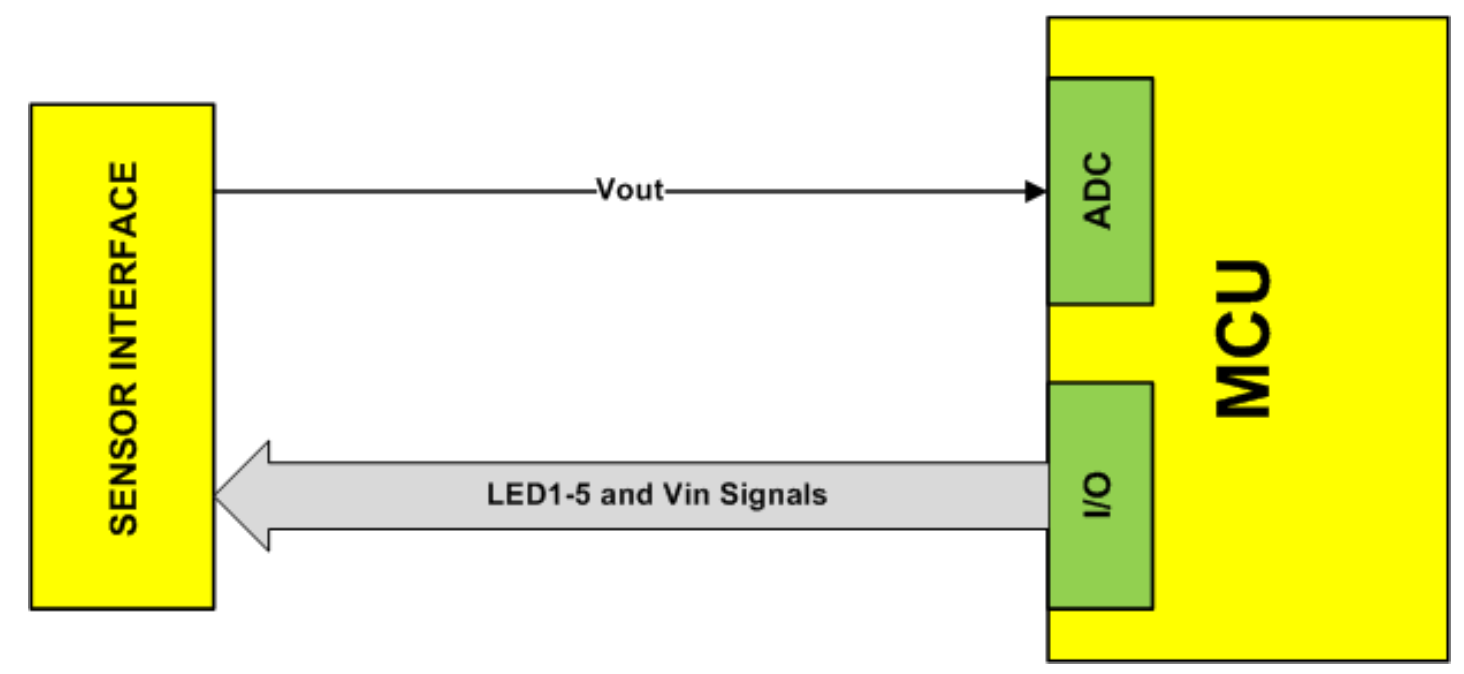

Figure 4.5 Sharp IR PSD Interface System Diagram

The sensor's analogue output signal, $\mathrm{V}_{\text {out }}$, is connected to the MCU's onboard 10-bit successive approximation ADC. A low equivalent series resistance (ESR) $10 \mu \mathrm{F}$ tantalum by-pass capacitor is connected between $\mathrm{V}_{\mathrm{cc}}$ and GND to help stabilize the power supply, as recommended by the Sharp datasheet. The $\mathrm{V}_{\mathrm{cc}}$ line of the sensor is connected to the same filtered power supply $\left(\mathrm{AV}_{\mathrm{cc}}\right)$ that powers the MCU's $\mathrm{ADC}$.

The MCU's onboard ADC is powered by a filtered $+5 \mathrm{~V}$ supply but is referenced from an internal $+2.56 \mathrm{~V}$ reference voltage of the MCU. Figure 4.6 illustrates the relationship between the output analogue voltage and distance for a GP2Y3A003K0F medium range sensor. The analogue output voltage reaches its maximum value at approximately $0.4 \mathrm{~m}$. The maximum value can vary from sensor to sensor due to slight differences in the PSD sensor placement during manufacturing. The typical maximum value is specified by the Sharp datasheet $[17,18]$ as $+2.3 \mathrm{~V} \pm 0.3 \mathrm{~V}$. This means a worst case output voltage of $+2.6 \mathrm{~V}$ which would saturate the ADC by $+0.04 \mathrm{~V}$. The use of the $+2.56 \mathrm{~V}$ reference may increases the dead zone range in a small number of IR PSD modules but is an acceptable trade off against the increased ADC resolution compared with the alternative of a $+5 \mathrm{~V}$ reference. 


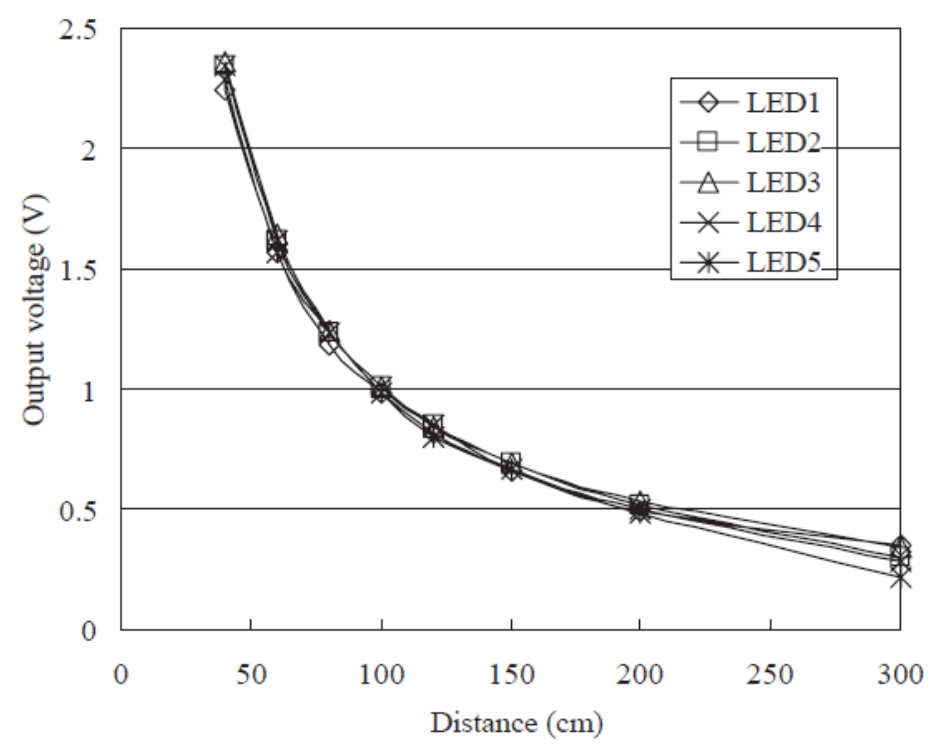

Figure 4.6 Sharp GP2Y3A003K0F Output Voltage vs. Distance [17]

Given the typical values of the output voltage specified by the data sheet, the useful output spans between $2.3 \mathrm{~V}$ and $0.25 \mathrm{~V}$. Using the MCU's internal $2.56 \mathrm{~V}$ reference as the ADC reference, one least significant bit (LSB) equates to $2.5 \mathrm{mV}$. Given the voltage value for one LSB, the specified analogue output voltage range spans 820 (100 - 920) LSB.

As the relationship between output voltage and distance is non-linear, a linear correlation between the precision of the ADC measurement and the precision of distance measured cannot be obtained. But from Figure 4.6 the slowest rate of change of the voltage curve occurs between the distances $200 \mathrm{~cm}$ and $300 \mathrm{~cm}$. Over this distance the output voltage changes by approximately $0.25 \mathrm{~V}$. Given the precision of the ADC is $2.5 \mathrm{mV}$, the approximate worst case precision of a distance measured would be $1 \%$ or, for the GP2Y3A003K0F sensor, $0.01 \mathrm{~m}$. 


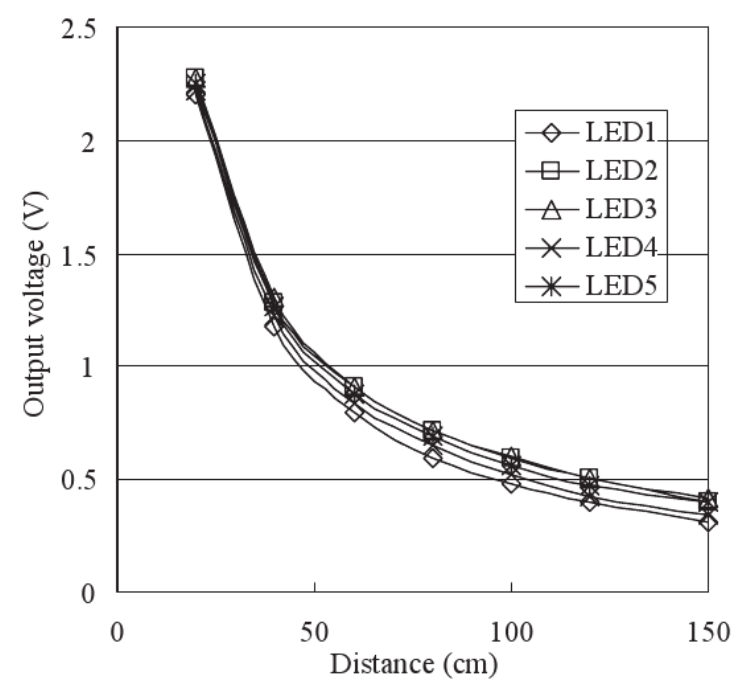

Figure 4.7 Sharp GP2Y3A002K0F Output Voltage vs. Distance [18]

Figure 4.7 illustrates the relationship between the output analogue voltage and distance for a GP2Y3A002K0F short range sensor. The slowest rate of change in output voltage occurs from $100 \mathrm{~cm}$ to $150 \mathrm{~cm}$. Given a worst case precision of $1 \%$ for an ADC measurement over this range, the precision of a distance measurement is approximately $0.006 \mathrm{~m}$.

\subsubsection{Assembled Modulle}

Each of the design sub-systems discussed in previous sections have been integrated into a single PCB. Figure 4.8 illustrates the placement of the sub-systems and primary interface headers. The shape and size of the PCB has been designed to mount directly on the rear of either the GP2Y3A002K0F or GP2Y3A003K0F sensors.

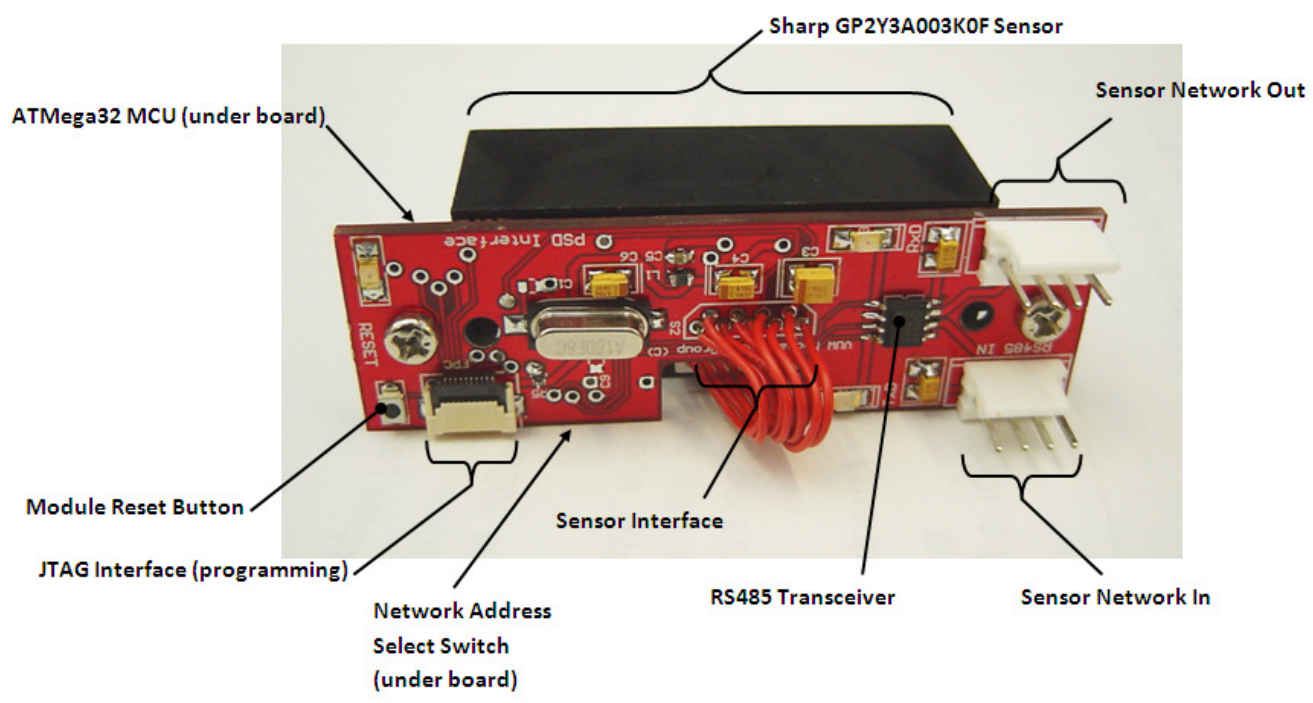

Figure 4.8 Assembled Infrared Ranging Module (GP2Y3A003K0F) 


\subsection{Ultrasonic Ranging Node}

Ultrasonic ranging modules have been selectively implemented on a small number of robotic platforms in VUW's fleet. These modules provide a medium-to-long distance ranging capability that can augment the short-to-medium distance ranging capability provided by the infrared ranging sensors discussed in the previous section. The SensComp ultrasonic ranging module, first mentioned in Section 2.2.2 SensComp Ultrasonic Ranging Sensors, provides a low cost, uni-directional ranging option.

\subsubsection{SensComp Ultrasonic Ranging Modules}

The SensComp ultrasonic sensor utilises the Polaroid 6500 Series Ranging Module, used in auto focus systems on Polaroid cameras. A transducer is used to transmit a short ultrasonic pulse, the echo of which is received by the same transducer. The elapsed time between the transmitted and received pulse is proportional to the distance that the pulse has travelled. Since the speed of sound is known, then the distance can be calculated from the time of travel.

The SensComp sensor comprises an electrostatic transducer to transmit the ultrasonic pulse and receive its echo, and the electronics module, which contains all the circuitry to generate the transmit signal, drive the transducer, receive the echo and process the information received by the transducer. The sensor works over a distance range of 0.26 to $10.7 \mathrm{~m}$ [19].

The ultrasonic sensor can operate in a single echo mode and a multiple echo mode. Single echo mode is primarily used for situations where there is only one target in a scene. When there are several targets in a scene, the multiple echo mode allows the sensor to detect the multiple echoes. This requires the reception circuit to be reset after receiving each echo signal by pulsing the Blanking (BLNK) control signal on the SensComp module.

Because the transducer is used as both the loudspeaker of the ultrasonic chirp as well as the microphone to detect the reflected chirp, it is necessary to inhibit the echo reception circuitry until all the ringing of the transmitted chirp has been eliminated from the transducer to prevent it being registered as a reflected echo. The time the reception circuit is inhibited defines the minimum distance the sensor can measure. The standard inhibit time is set to $2.38 \mathrm{~ms}$ corresponding to a minimum measurement range of $0.41 \mathrm{~m} \mathrm{[19].} \mathrm{It} \mathrm{is} \mathrm{possible} \mathrm{to}$ measure distances less than $0.41 \mathrm{~m}$ by activating the reception circuit. This is done by 
asserting the Blank Inhibit (BINH) control signal of the SensComp module before the internal blanking period of $2.38 \mathrm{~ms}$ finishes. This allows accurate readings providing the transducer is damped enough to prevent ringing from the transmitted chirp registering as an echo.

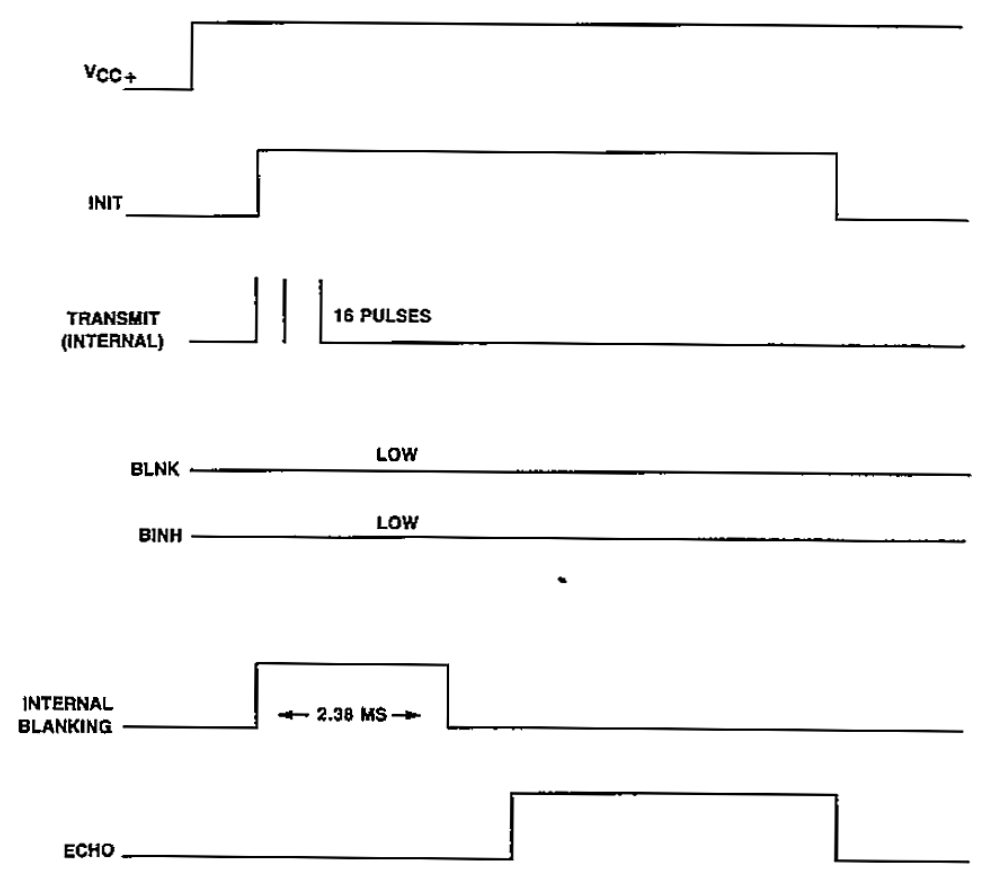

Figure 4.9 Waveform Examples of a Single Echo Mode cycle without blanking input

Figure 4.9 illustrates the control waveforms for single echo mode. The BINH signal is held low to allow the internal $2.38 \mathrm{~ms}$ blanking signal to be activated to provide enough time for the ringing of the transmitted echo to dissipate on the transducer. The BLANK signal is held low to ensure the internal echo detection circuit is not reset, preventing the sensor from detecting further echoes. 


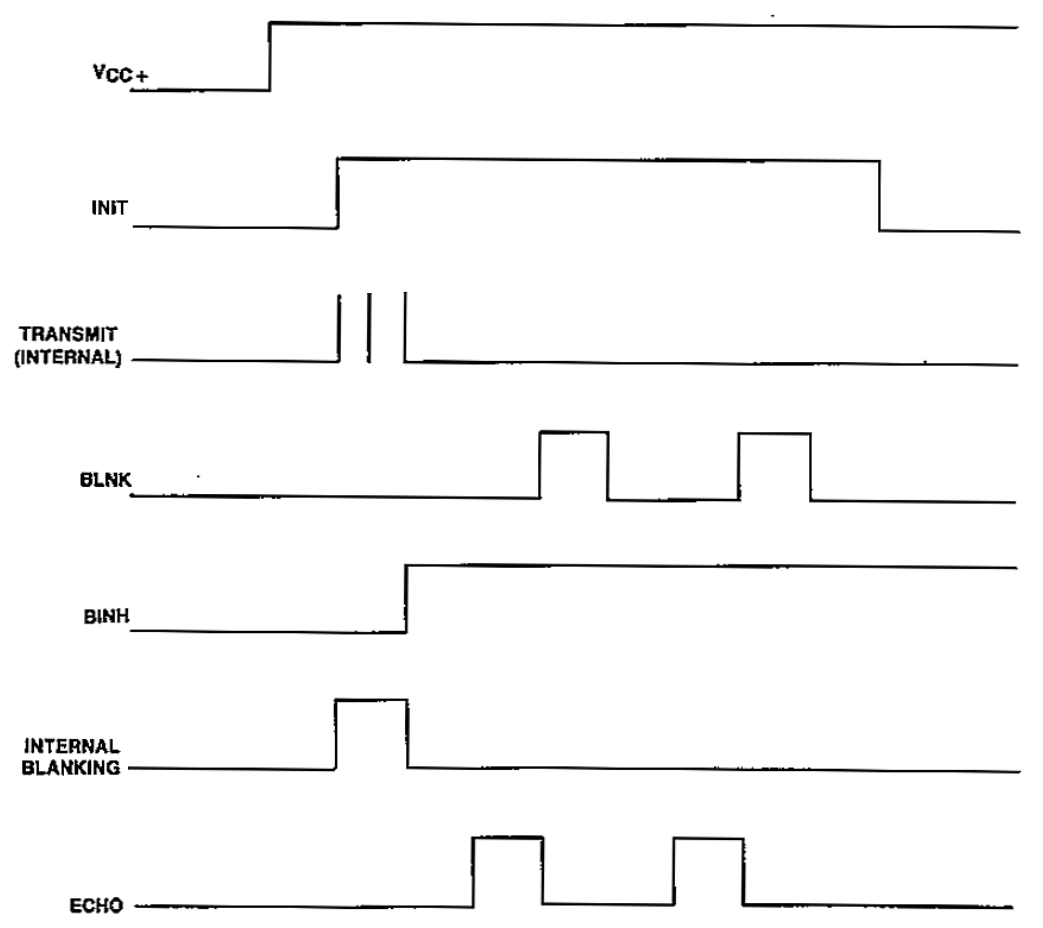

Figure 4.10 Waveform Examples of a Multiple Echo Mode cycle with blanking input

Figure 4.10 illustrates the control signal waveforms required to achieve multiple echo mode on the SensComp ranging module. In this case the BINH signal is held high to allow the transducer to detect echo signals from objects closer than $0.41 \mathrm{~m}$ away. The BLANK signal is repeatedly asserted high after each received echo, to reset the echo detection circuit for subsequent echoes.

The electronics module has several control and output lines. These lines are listed in table 4.1:

Table 4.1 SensComp Electronic modules Control and Output Lines

\begin{tabular}{|c|c|c|}
\hline Control Line & Input/Output & Function \\
\hline Init & Input & Signal to transmit a ultrasonic chirp \\
\hline Blnk & Input & $\begin{array}{c}\text { Signal to reset echo detect for multiple echo } \\
\text { measurements }\end{array}$ \\
\hline Binh & Input & $\begin{array}{c}\text { Signal used for close range measurements, } \\
\text { less than } 0.41 \mathrm{~m}\end{array}$ \\
\hline Echo & Output & $\begin{array}{c}\text { Output signal to indicate an echo has been } \\
\text { received }\end{array}$ \\
\hline Osc & Output & $\begin{array}{c}\text { Clock output that can be used for external } \\
\text { timing functions. }\end{array}$ \\
\hline
\end{tabular}


Figure 4.11 illustrates the main components of the ultrasonic ranging node. The same ATMega32 AVR 8-bit MCU [36] used in the infrared ranging node is utilised in the ultrasonic ranging node. The MCU controls all of the operations of the node, including control of the SensComp electronics module, timing of the ultrasonic pulse flight, and communication interface to the sensor network. The MCU can be programmed through its JTAG interface or via Atmel's In-System Programming (ISP) interface. A single +5 V supply rail is required for MCU operation.

Featured peripherals relevant to the ultrasonic ranging node's requirements include:

- $1 \times$ Universal Synchronous/Asynchronous Receiver Transmitter (USART).

- $1 \times$ 16-bit Timer/Counter.

- $2 \times 8$-bit Timer/Counters.

- 32 programmable I/O lines.

- 3 external interrupts.

- JTAG interface.

- Watch Dog Timer.

As on the infrared ranging module, a five-way DIP switch allows a user to specify the network address of the node (0-31). An external $16 \mathrm{MHz}$ crystal is the source of the MCU's system clock.

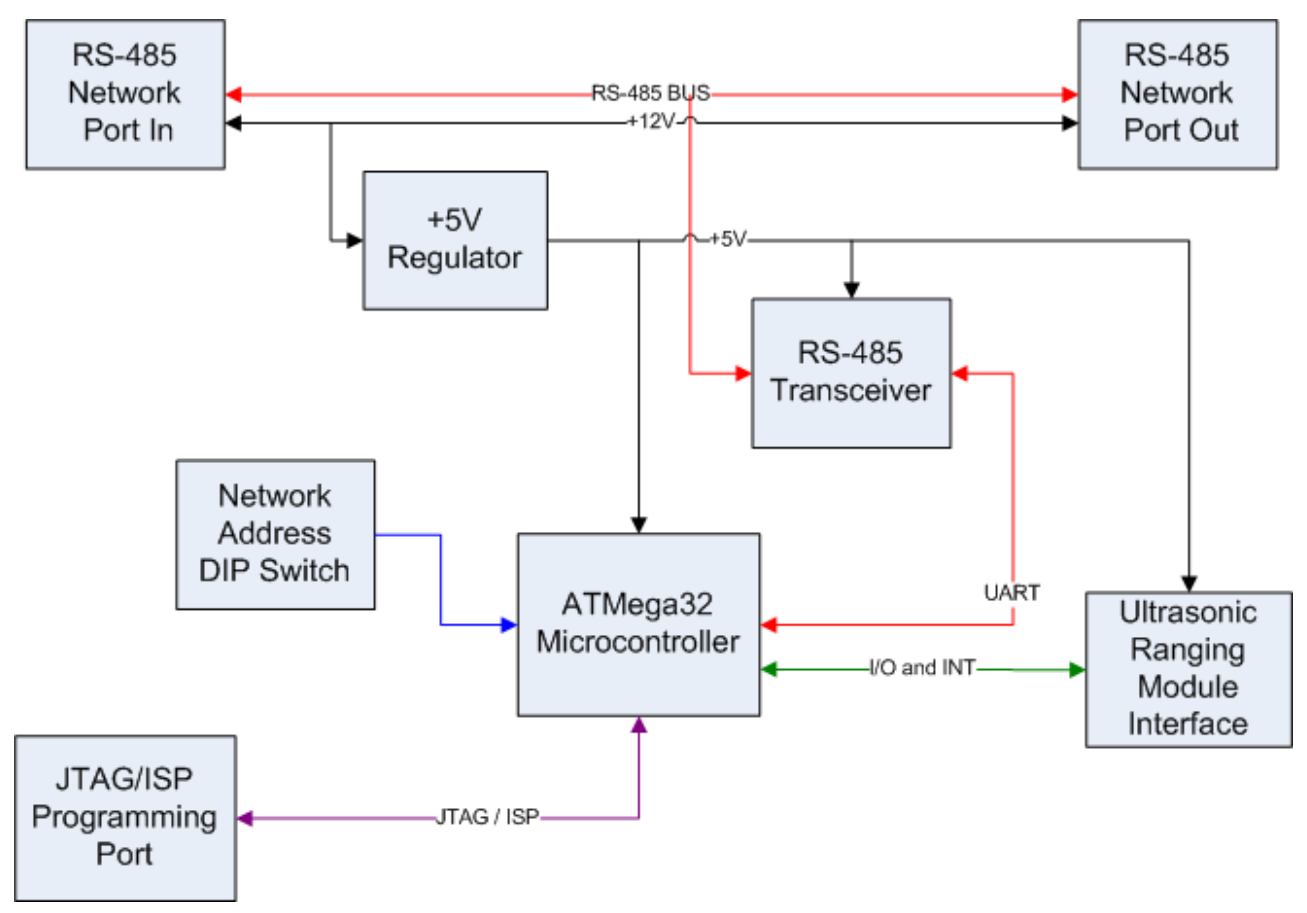

Figure 4.11 Block Diagram of Ultrasonic Ranging Node 
The $16 \mathrm{MHz}$ clock frequency matches the clock frequency of the Sensor Network Interface Module, mentioned in Section 3.2 Sensor Network Interface Module, and the infrared ranging module, mentioned in Section 4.1.1 Sharp IR PSD Ranging Module, allowing the ultrasonic ranging node to communicate at an identical maximum data rate of $2 \mathrm{Mb} / \mathrm{s}$.

\subsubsection{RS-485 Interface}

The RS-485 interface is a replica of the infrared ranging node's RS-485 interface, first mentioned in Section 4.1.2 RS-485 Interface. Refer to that section for information on the interface.

\subsubsection{Ultrasonic Sensor Interface}

The SensComp electronic module's control signals are connected to I/O lines on the MCU (Figure 4.12). The MCU can start an ultrasonic transmission pulse burst, reset the reception circuitry to allow receipt of multiple echoes and inhibit the internal blanking signal to allow the transducer to detect echo signals from objects closer than $0.4 \mathrm{~m}$. The echo output signal from the SensComp module is connected to an external interrupt line of the MCU.

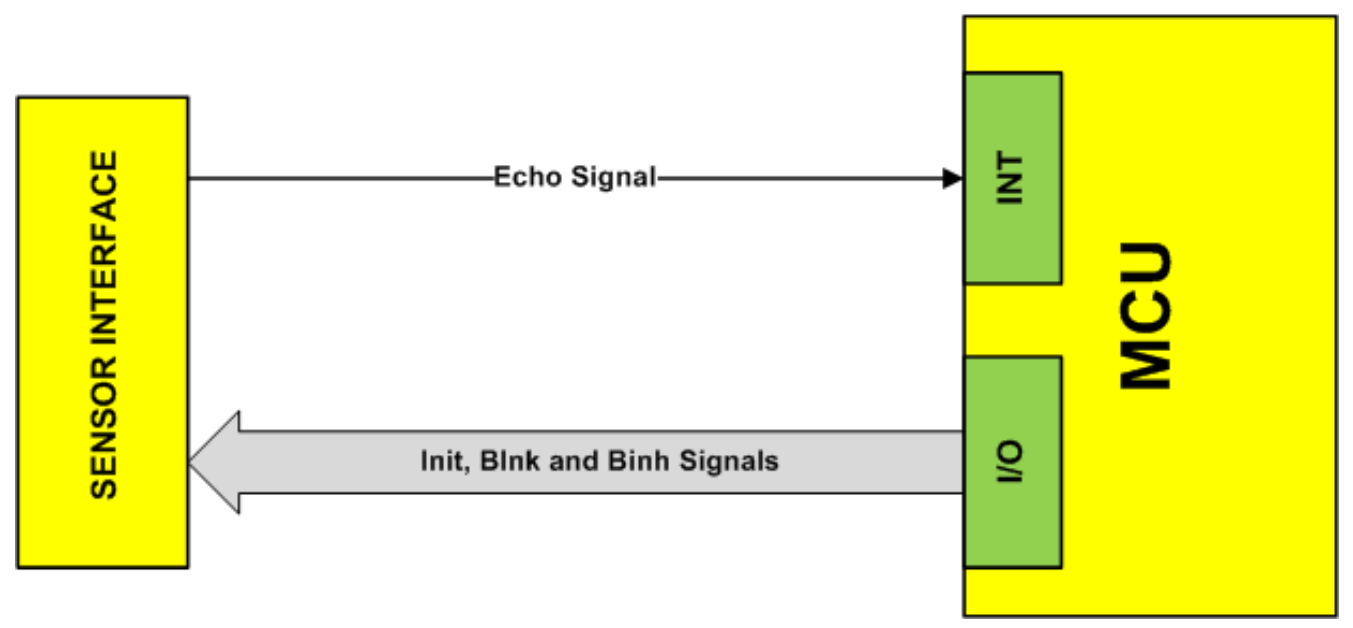

Figure 4.12 Ultrasonic Sensor - MCU Interface

The MCU measures the time of flight of the transmitted pulse by using an internal 16 bit counter to measure the time between setting the Init signal high and receiving an echo signal on its external interrupt. LED indicators on the Init and Echo signals allow a user to visually monitor the activity of the ultrasonic ranging node. 


\subsubsection{Power Supply}

The ultrasonic ranging node derives its power from the network bus. It utilises a $+5 \mathrm{~V}$, $500 \mathrm{~mA}$ voltage regulator, LF50ABDT from STMicroelectronics [34], to supply a $+5 \mathrm{~V}$ rail to the MCU and peripheral electronics. The $+5 \mathrm{~V}$ rail also provides the power for the SensComp electronics module and transducer. The $+5 \mathrm{~V}$ rail must be able to handle transient currents of 2 A due to the high current draw of the SensComp electronic module. The transient current draw is required only for the period of time the ultrasonic transducer is transmitting pulses. The SensComp module transmits sixteen pulses at $49.9 \mathrm{kHz}$. This limits the transient current draw period to approximately $400 \mu \mathrm{s}$. Selection of an appropriate bypass capacitor provides the necessary instantaneous current supply during the pulse transmission period.

The amount of charge delivered to the SensComp module during this period is defined by:

$$
Q=I t
$$

Equation (4.1)

where $Q$ equals the amount of charge, in coulombs, delivered, $I$ equals the transient current, and $t$ equals the period of time of the transient current draw. In this case the charge delivered to the module is $800 \mu \mathrm{C}$. The minimum time before the SensComp module can commence another transmission burst is set by the module's internal blanking time of $2.38 \mathrm{~ms}$. This allows a bypass capacitor a period of $1.98 \mathrm{~ms}$ (internal blanking time minus transmission burst time) to recover the amount of charge delivered to the transducer. From Equation 3.1 it can be calculated that the current required to recover the amount of charge in this time period is approximately $400 \mathrm{~mA}$, which is comfortably within the ability of the ultrasonic ranging module's +5 V $500 \mathrm{~mA}$ regulator.

The voltage drop across the bypass capacitor during the transient current period is determined by:

$$
V=\frac{Q}{C}
$$

Equation (4.2)

where $V$ is the voltage drop across the capacitor, $C$ is the capacitance and $Q$ is the amount of charge lost. The selection of a $22 \mathrm{mF}$ capacitor limits the voltage drop across the capacitor and on the $+5 \mathrm{~V}$ rail to be no greater than $37 \mathrm{mV}$. 


\subsubsection{Assembled Modulle}

Each of the design sub-systems discussed in previous sections have been integrated into a single PCB. Figure 4.13 illustrates the placement of the sub-systems and primary interface headers. The shape and size of the PCB has been designed to allow the SensComp electronics module to be soldered and mounted directly onto the ultrasonic ranging node PCB. This provides a compact integrated unit that can be easily mounted onto a robotic platform and connected to the sensor network.

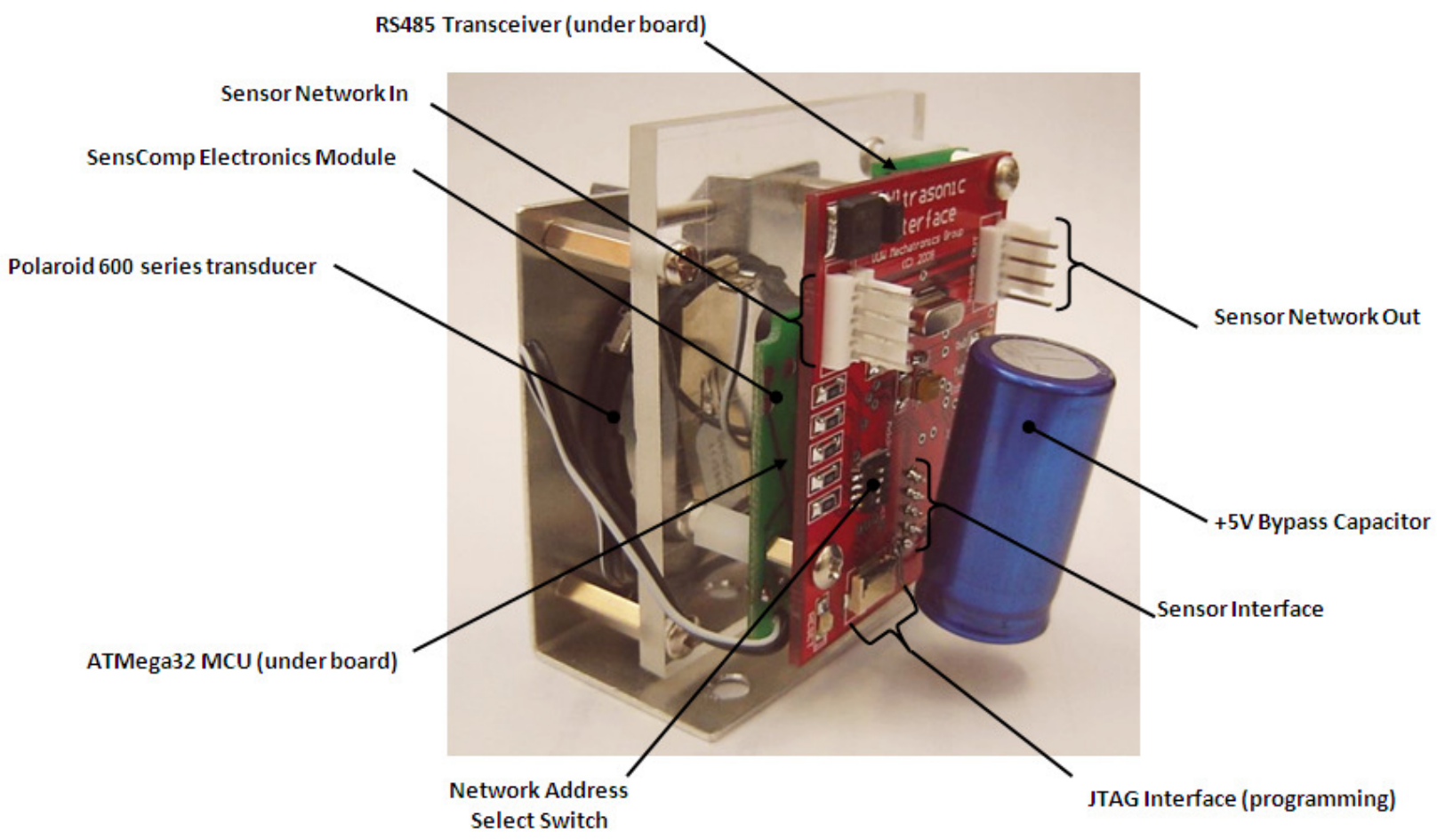

Figure 4.13 Assembled Ultrasonic Ranging module 


\subsection{Prototype Sensor Network}

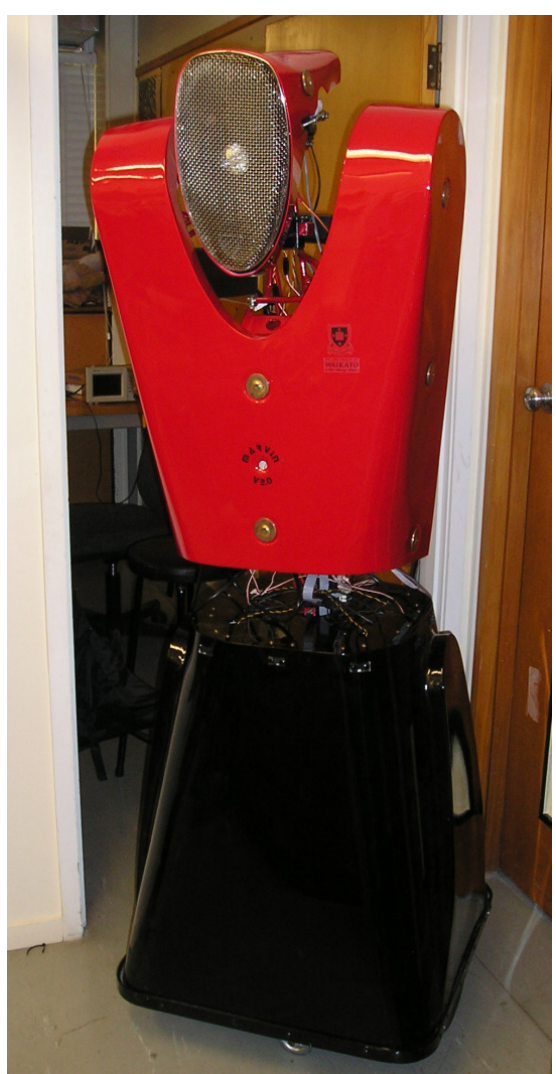

Figure 4.14 MARVIN

A prototype sensor network has been designed to be mounted on MARVIN, one of the robotic platforms in the VUW's fleet (pictured in Figure 4.14). MARVIN (backronymed to Mobile Autonomous Robotic Vehicle for Indoor Navigation) is a custom-built mobile robot intended for security and public relations applications. It is intended that MARVIN be capable of autonomous patrolling of indoor office block environments.

MARVIN originally was provided with a range of sensors that were primarily used for obstacle detection and localisation [15]. As the initial design brief defined MARVIN's operating environment as an office block, the sensors for detecting obstacles are required to measure short to medium distances $(0.2-10 \mathrm{~m})$.

MARVIN's frame stands at 1.77 metres tall. The hardware can be divided into two main sections: a base section that contains the battery supply, control computer and the majority of the peripheral hardware that controls MARVIN's transducers, and an upper torso section that is modelled on a humanoid upper body to provide aesthetic appeal and help facilitate human-machine interaction. This torso section comprises several transducers that can alter the torso's shape and orientation to imitate human body positions and emotions.

The earlier sensors employed on MARVIN for distance ranging were Sharp GP2Y0A02YK Position Sensitive detectors. These sensors are single beam sensors that have a detection range of $0.2 \mathrm{~m}$ to $1.5 \mathrm{~m}$. The sensor outputs an analogue voltage that is proportional to the distance measured.

MARVIN's current sensor implementation is based on a star topology. The main bank of distance sensors consist of ten GP2Y0A02YK PSDs mounted at the top of MARVIN's base section. Each PSD sensor analogue output is connected to an ADC card mounted in the control computer. This current implementation is less than ideal for the following reasons: 
1. For the 10 PSD sensors there are 30 connections to the control computer in a star topology (3 connections per sensor, VCC, GND, O/P). This results in a complicated wiring loom and requires each sensor to be disconnected each time MARVIN's base section requires servicing.

2. The analogue outputs are connected to the ADC card in the control computer by relatively long lengths of wire $(400 \mathrm{~mm})$ and as such are prone to noise pick-up.

3. The PSD sensors are mounted parallel to the ground. The PSD datasheet $[17,18]$ suggests mounting the sensors $90^{\circ}$ to the direction of motion for accurate distance readings, as mentioned in Section 2.2.1 Sharp Position Sensitive Detectors. This recommended orientation requires the sensors to be mounted perpendicular to the ground.

4. The ten PSD sensors do not provide a $360^{\circ}$ scan of the environment that MARVIN operates in. Dead zones range from $20^{\circ}-40^{\circ}$ based on the current mounting positions of the sensors.

The prototype sensor network has been designed to provide a $360^{\circ}$ field of view utilising a mix of four Sharp short range GP2Y3A002K0F and ten medium range GP2Y3A003K0F sensors. The sensors will be mounted at the top of MARVIN's base section, approximately 1 metre above the ground.

A mechanical chassis has been designed to mount the test sensor network to MARVIN. As each infrared ranging module has a wide angle IR PSD sensor with a field of view of $25^{\circ}$, a total of fourteen infrared ranging modules are needed to provide a $360^{\circ}$ field of view. The mechanical chassis houses these fourteen ranging modules spaced evenly at $25.7^{\circ}$ intervals around its base, allowing the sensors to probe, in a horizontal plane, MARVIN's environment. As mentioned in Section 2.2.1, the Sharp IR PSD sensors have been mounted vertically to ensure that accurate distance measurements are captured for objects moving parallel to the sensor network.

The mechanical mounting chassis consists of an aluminium base plate, an aluminium top plate, and fourteen aluminium brackets to mount the infrared ranging modules. Both the chassis base plate and top plate are $0.2 \mathrm{~m}$ in diameter. To ensure accurate ranging measurements are acquired, each mounting bracket is attached to the top plate and base plate by 2 screws respectively. To mount the network chassis to MARVIN's mid-section both the top and base plates have a rectangular $40 \mathrm{~mm} \times 60 \mathrm{~mm}$ hole to allow the assembled test 
sensor chassis to be attached to MARVIN's main support beam connecting his base unit to his torso unit.

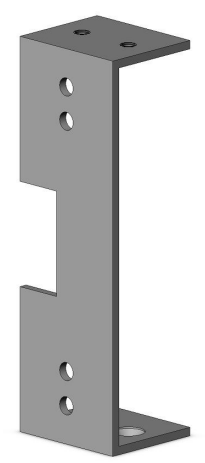

Figure 4.15 Mounting Bracket for Infrared Ranging Modules

Figure 4.15 illustrates the mounting bracket designed to hold either the GP2Y3A002K0F short distance IR sensor or the GP2Y3A003K0F medium distance IR sensor. The infrared ranging node PCB mounts inside the bracket, while the Sharp IR sensor mounts on the outside of the bracket. Figure 4.16 illustrates a mounting bracket with a Sharp GP2Y3A003K0F sensor mounted on the front.

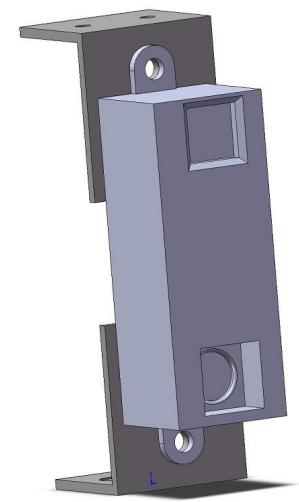

Figure 4.16 Mounting Bracket with Sharp GP2Y3A003K0F sensor attached.

When the infrared ranging modules (GP2Y3A003K0F and GP2Y3A002K0F) are mounted to the base plate the sensor network has a detection field $6.2 \mathrm{~m}$ in diameter with a $1 \mathrm{~m}$ diameter dead zone in the centre. This dead zone is due to the fixed diameter of the base plate $(0.2 \mathrm{~m})$ and the dead zone of the Sharp sensors, $0.4 \mathrm{~m}$ for GP2Y3A003K0F and $0.2 \mathrm{~m}$ for GP2Y3A002K0F. As MARVIN is primarily operating in an office block environment, the four short range IR modules (GP2Y3A002K0F) have been mounted in lateral locations of the network chassis to reduce the dead zone from $1 \mathrm{~m}$ to $0.6 \mathrm{~m}$ to provide ranging capability to allow MARVIN to pass through office doorways that have a typical clearance of $0.8 \mathrm{~m}$. 
Figure 4.17 illustrates the active detection zone of the sensor network including the $1 \mathrm{~m}$ diameter dead zone.

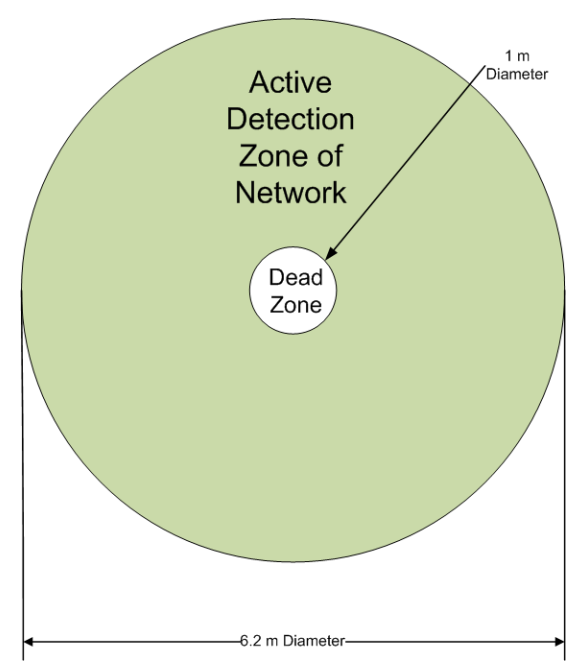

Figure 4.17 Detection Zone of Test Sensor Network

Figure 4.18 illustrates the assembled mechanical mounting chassis.

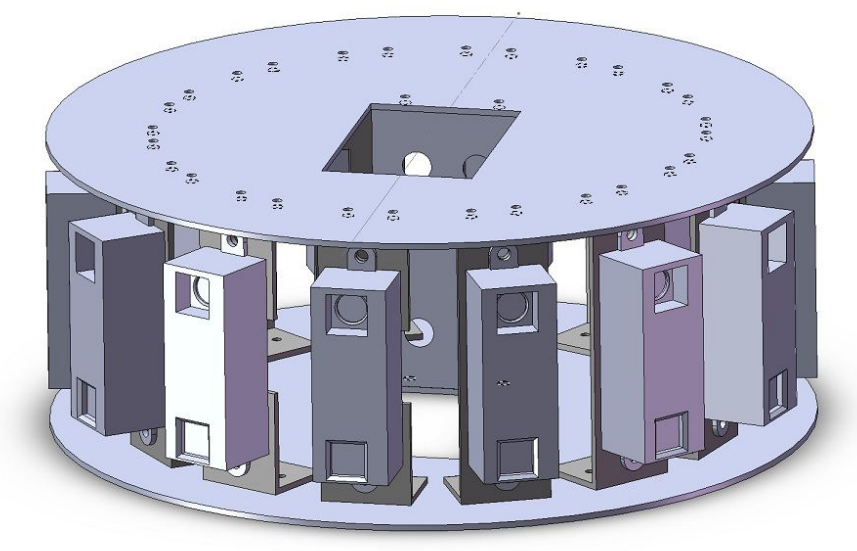

Figure 4.18 Assembled Sensor Network Chassis

\subsubsection{Assembled Sensor Network Chassis}

A mechanical mounting chassis has been manufactured out of aluminium. It has been anodised black to aesthetically match the existing style of MARVIN's appearance. The assembled prototype sensor network chassis is shown in Figure 4.19. Figure 4.20 shows the assembled prototype sensor network mounted onto MARVIN. 


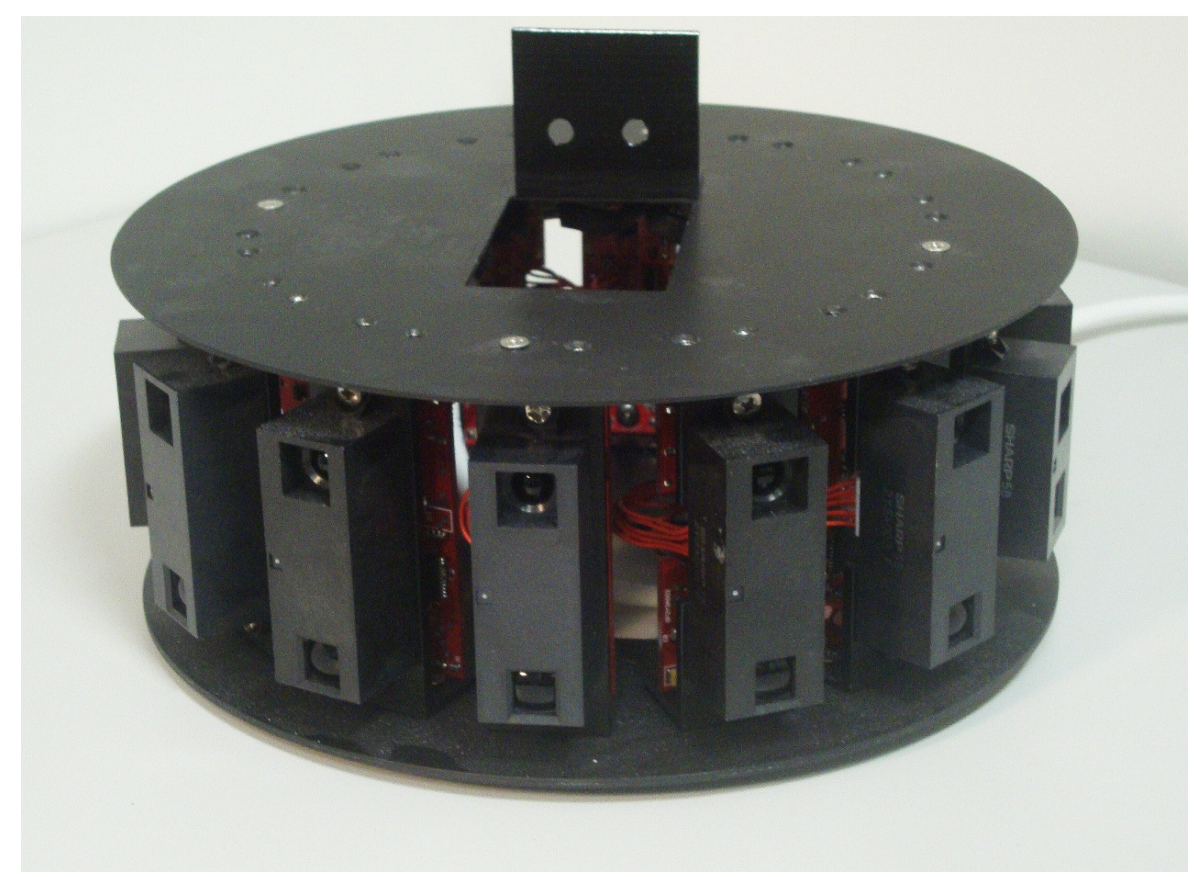

Figure 4.19 Assembled Test Sensor Network Unit

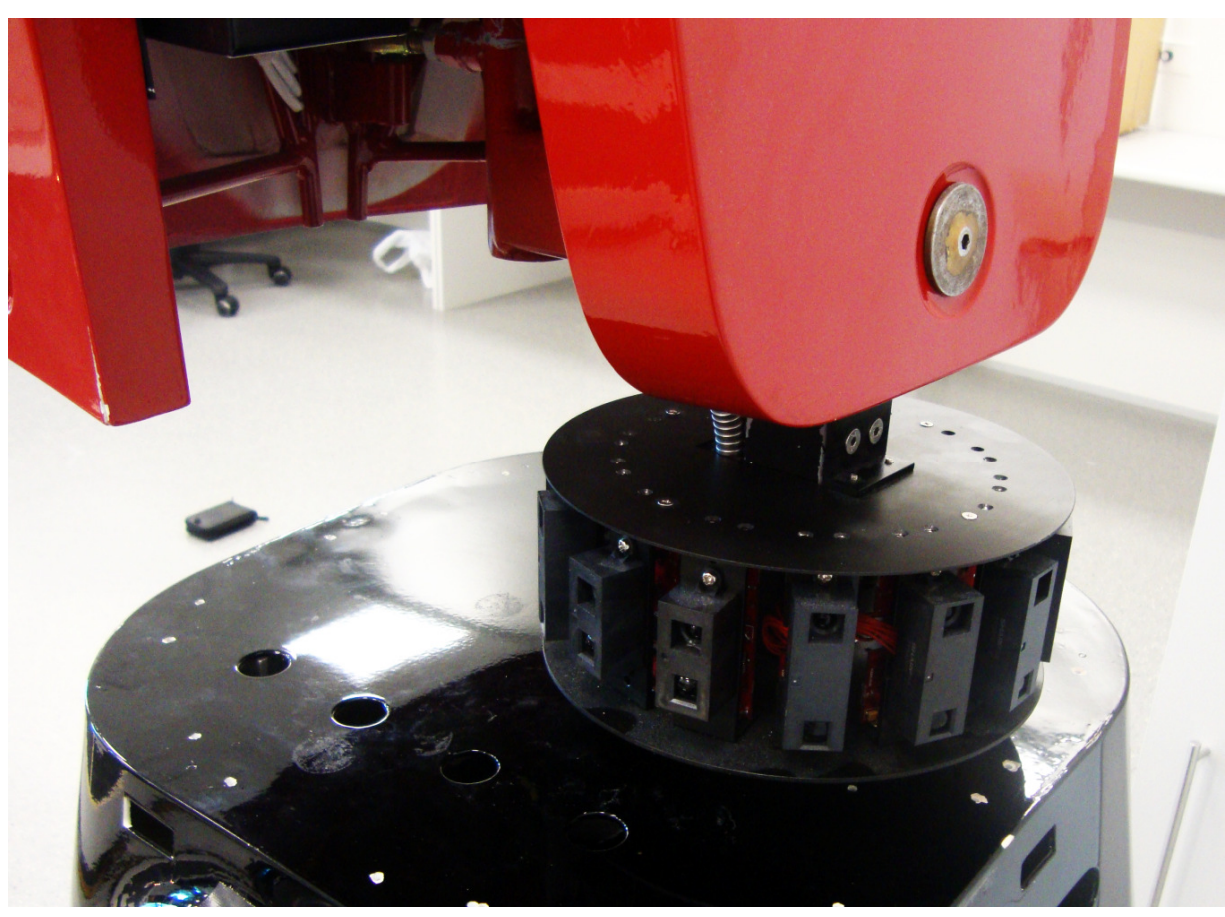

Figure 4.20 Test Sensor Network mounted on MARVIN 


\section{Chapter 5 Firmware Methodology - Sensor Network}

This chapter presents the firmware that provides the operational functionality of the sensor network. It discusses the sensor network protocol adopted to allow the network to operate, the network layer stack and firmware controlling network communication shared across all hardware platforms on the network and the communication protocol that allows a control computer to interface to the network. Finally the firmware that controls the tasks unique to each hardware platform is discussed.

\subsection{Sensor Network Protocol}

As mentioned in Section 3.1Fundamentals of a Sensor Network, the three hardware platforms designed for the sensor network all incorporate a MCU to implement control of the hardware. Although the three hardware platforms perform different tasks they also share similar functionality, primarily sending and receiving data on the network. The task of network communication can be separated into two key areas, these being:

1. The media layer communication protocol - which allows network devices to send and receive data across the network. This protocol is concerned with $\underline{\text { how }}$ to move data on the network and in the OSI model is handled by the media layers.

2. The host layer communication protocol - which allows the operation of sensors as a co-ordinated group. This protocol is concerned with what data is moved on the network and in the OSI model is handled by the host layers.

Definition of the communication protocol for the host layers provides the sensor network with its capabilities.

\subsubsection{Sensor Model}

Ideally any sensor can be modelled as a black box that reports a mathematical value. This mathematical value represents a measured quantity of an environmental variable. It is possible that a sensor is capable of measuring more than one environmental variable but even in this case it can be reduced to a model with the constraint that only one value is reported back for each environmental quantity measured. Figure 5.1 illustrates the black box model for a sensor. 


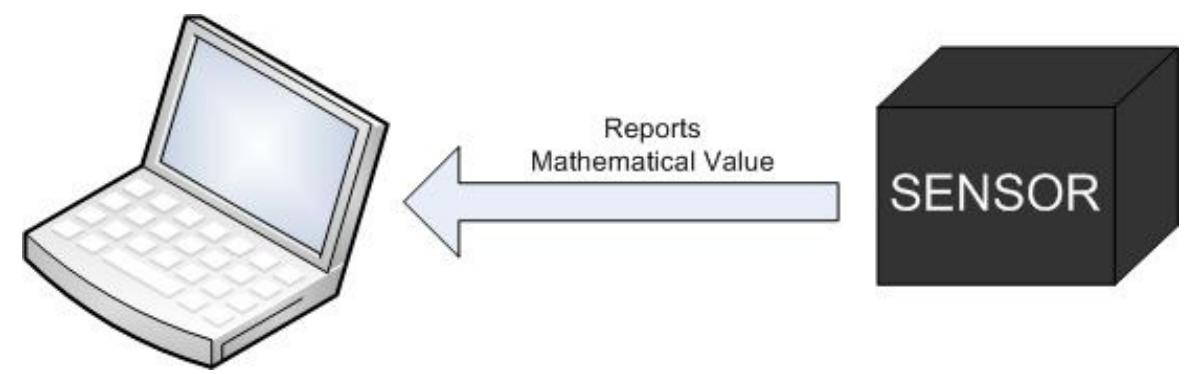

Figure 5.1 Black Box Sensor Model

Using this black box model it is possible to define a network communication protocol that will allow any sensor to be added to the network regardless of its functionality or limitations.

\subsubsection{Host Layer Protocol}

The host layer protocol (HLP) provides the structure for how sensors interact logically on the network. For the HLP to function all sensor nodes have to be intelligent. This means the network nodes must be able to control the sensor itself and convert the sensor data to a form that is compatible with the HLP. This intelligence removes the burden on the control computer of having to control and monitor the sensors. Key to the functionality of the HLP is that the protocol views every sensor node as identical and as such does not require any functional knowledge of the type of sensor attached to the node to allow the node to operate. If every node is viewed as being identical to every other node, then the HLP must define the basic requirements of a sensor that need to be known to allow the network to function. The HLP must provide enough information to allow the control computer to reconstruct data, received from a sensor node, back into a sensible value that represents the environmental variable measured. For example, the control computer must be able to determine the range of a distance sensor to be able to process the dimensionless sensor data back into a distance measurement.

As described previously, a sensor can be modelled as a black box reporting a mathematical value which represents a measurement quantity of an environmental variable. This defines a fundamental requirement of a sensor to be that it must report a mathematical value. Furthermore a sensor can be viewed as an array of sensors if it is able to measure more than one environmental variable. This modifies the initial fundamental requirement of a sensor to be that it must report back an array, with a minimum size of one, of mathematical values where each value represents one measurement of an environmental variable. 
It is important to note that a sensor belongs to a network of sensors and as such does not act completely independently of the other sensors in the network. The most obvious case of this is where operation of one sensor may affect the measurements of another sensor. An example of this would be cross talk between infrared position sensitive detectors. For successful operation of a sensor network it must be possible for the network master to assign measurement tasks to a sensor, allowing it to operate in a co-ordinated fashion with other sensors in the network.

For network tasks to be assigned by the network master to sensor nodes, each node must have a unique network address.

The type of environmental variable a sensor measures must be known by the control computer so that it can understand what a sensor's reported mathematical value actually represents. The most commonly measured environmental variable by the robotic fleet is distance, but other variables can include gravity, light intensity, magnetic intensity, battery charge etc. The HLP must be able to report the environmental variable the sensor measures.

Finally all sensors require calibration to ensure accurate measurements of environmental variables. This calibration defines how sensor data should be processed to determine the magnitude of the actual environmental variable measured.

Given these basic parameters of a sensor, the HLP must handle the following data types:

1. Sensor Data - the mathematical values the sensor has measured. These values are dimensionless as they represent a fraction of the maximum value that can be measured by the sensor.

2. Number of Sensor Channels - the number of unique environmental variables the sensor can measure. This value determines how many mathematical values can be reported back by the sensor.

3. Assigned Task - the task that the sensor is designated to undertake. This task allows the network to operate in a co-ordinated fashion by allowing the network master to assign tasks to specific sensor nodes allowing network-wide co-ordination of measurements.

4. Network Address - the unique identifier that allows the network master to assign tasks to a specific sensor node. 
5. Sensor Type - the type of sensor to indicate to the control computer the environmental variable the sensor measures.

6. Calibration Identification - the unique identifier that allows the control computer to process the dimensionless sensor data back into a measured magnitude of an environmental variable.

\subsubsection{Sensor Tasks}

Sensor tasks are the measurement jobs that are given to the sensor nodes by the network master. These tasks determine how and when a sensor should undertake a measurement. These measurement jobs can be defined as modes of operation of the sensor. These measurement modes are:

- STOP - in this mode the sensor is not active and will not take any further measurements.

- FREE - in this mode the sensor is free running and will actively take measurements as fast as it can across all its channels. The length of the measurement cycle is fixed by the speed of the sensor.

- FAST - in this mode the sensor will actively take measurements as fast as possible on one selected channel in the sensor. The network master must specify the active sensor channel to be sampled in this mode. This allows the update rate from a sensor to be increased at the expensive of the number of channels sampled.

- STEP- in this mode the sensor will take a measurement of one sensor channel then wait a fixed period of time before taking a measurement on the sensor's next channel. This mode allows the network master to control the entire sensor network's measurements in an organised fashion by time multiplexing measurements across the whole sensor network. This measurement mode is reliant on the sensor network having a universal network time that all sensor nodes are synchronised to.

This measurement mode is achieved by defining a fixed length network measurement window of $60,000 \mathrm{~ms}$ which is synchronised to the network time. Sensor nodes are issued a start time and a step time. The start time defines when in the measurement window a sensor will commence its first measurement, while the step time defines how long to wait after taking a measurement before starting a subsequent measurement. A sampling rate of $10 \mathrm{~Hz}$ per sensor requires sensors to have start and step times defined in milliseconds. A measurement window length of $60,000 \mathrm{~ms}$ has been adopted as it can be 
stored as a 16-bit number. Once the network measurement window ends the sensor will stop taking measurements until another measurement window begins. Figure 5.2 illustrates a Step measurement cycle for two sensors over a measurement window.

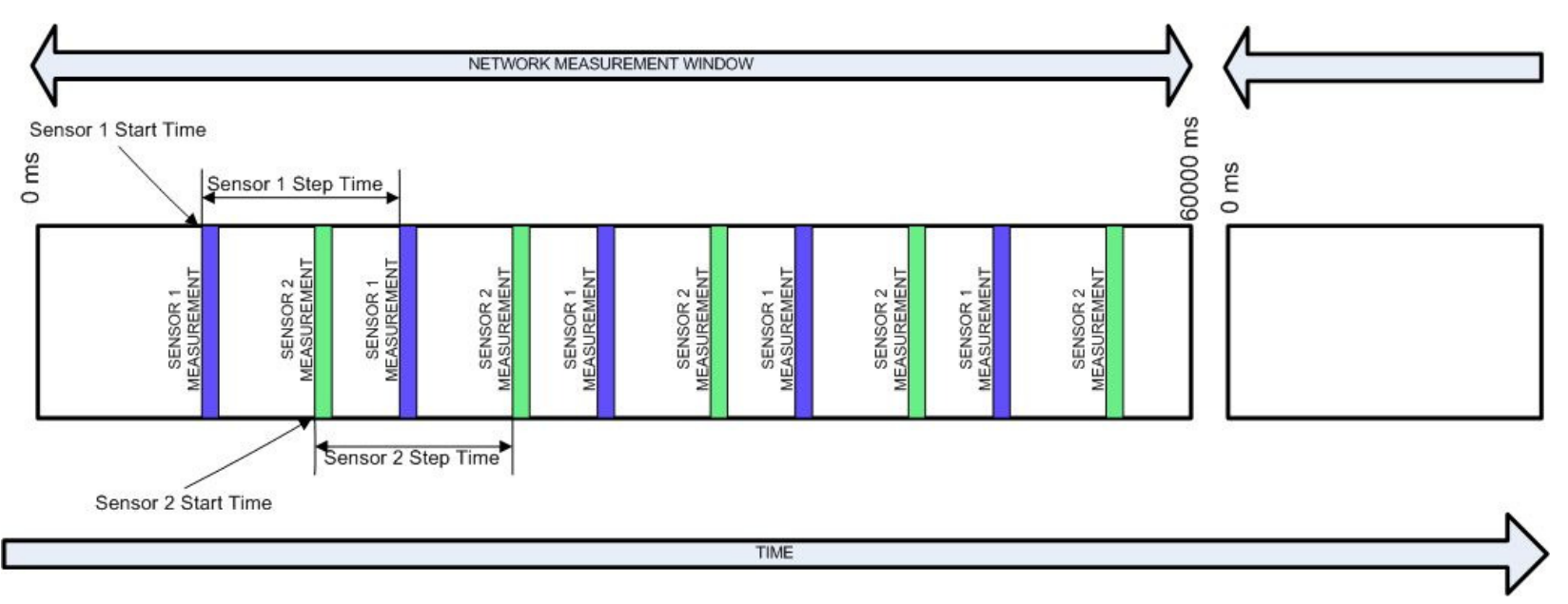

Figure 5.2 Stepped Measurement Example

Given these defined operating modes of a sensor, a measurement task should contain the following information:

- Measurement Mode - [STOP, FAST, FREE, STEP]

- Start Time - [0 - $60000 \mathrm{~ms}]$

- Step Time - $[0-60000 \mathrm{~ms}]$

- Active Channel - the channel to be sampled in FAST mode.

Note that some of these measurement variables are not required for certain measurement modes but still should be provided to allow measurement configurations to be set up prior to any measurement mode being selected.

The HLP must also provide a means of synchronising the universal time on all sensor nodes to the network time established in the network master.

\subsubsection{HLP Packet Format}

The HLP sends instructions and data to sensors on the network by issuing HLP packets. HLP packets allow the network master to configure the sensor network, issue sensor tasks and request measurement data. An HLP packet is comprised of a packet type identifier and any associated data payload required. Once an HLP packet has been configured it is passed to the media layers to be sent via the media layer protocol to the designated sensor. Table 5.1 describes the types of HLP packets and their function. 
Table 5.1 Host Layer Protocol Packet Descriptions

\begin{tabular}{|c|c|c|}
\hline Packet ID & Packet Type & Description \\
\hline $\mathbf{0 x 0 0}$ & Get Sensor Info & $\begin{array}{l}\text { This packet requests the sensor type, number of } \\
\text { channels and the calibration ID. }\end{array}$ \\
\hline 0x01 & Get Calibration ID & This packet requests the sensor's calibration ID. \\
\hline $0 x 02$ & Get Measurement Setup & $\begin{array}{l}\text { This packet requests the sensor's current sensor task } \\
\text { information. This includes the measurement mode, } \\
\text { start time, step time and current active channel. }\end{array}$ \\
\hline 0x03 & Get Measurement Mode & $\begin{array}{l}\text { This packet requests the sensor's current mode of } \\
\text { measurement, defined in its sensor task. }\end{array}$ \\
\hline 0x04 & Get Channel Data & $\begin{array}{l}\text { This packet requests the most recent sampled } \\
\text { measurement of a specified channel on the sensor. }\end{array}$ \\
\hline $0 \times 05$ & Get Every Channels data & $\begin{array}{l}\text { This packet requests the most recent sampled } \\
\text { measurements across all the sensor's channels. }\end{array}$ \\
\hline $0 x 06$ & Get Channel Reading & $\begin{array}{l}\text { This packet instructs the sensor to take a new } \\
\text { reading on a specified channel and return the result. }\end{array}$ \\
\hline 0x07 & $\begin{array}{l}\text { Get Every Channels } \\
\text { reading }\end{array}$ & $\begin{array}{l}\text { This packet instructs the sensor to take new readings } \\
\text { across all the sensor's channels and return the } \\
\text { results. }\end{array}$ \\
\hline 0x11 & Set Calibration ID & $\begin{array}{l}\text { This packet issues the sensor with a new calibration } \\
\text { ID. }\end{array}$ \\
\hline $0 \times 12$ & Set Measurement Setup & This packet issues the sensor with a new sensor task. \\
\hline $\mathbf{0 x 1 3}$ & Set Measurement Mode & $\begin{array}{l}\text { This packet issues the sensor with a new } \\
\text { measurement mode. }\end{array}$ \\
\hline $0 \times 7 F$ & Dummy & $\begin{array}{l}\text { This packet is a dummy packet. It is used to test } \\
\text { network frames. }\end{array}$ \\
\hline $0 \times 80$ & Sync Network Time & $\begin{array}{l}\text { This is a global packet and instructs all network } \\
\text { devices to reset their internal clock to match the } \\
\text { master node's network clock. }\end{array}$ \\
\hline $0 \times 81$ & $\begin{array}{c}\text { Set Global Measurement } \\
\text { Mode }\end{array}$ & $\begin{array}{l}\text { This is a global packet allowing the network master } \\
\text { node to globally change the measurement mode for } \\
\text { every sensor. }\end{array}$ \\
\hline
\end{tabular}

The HLP is a request-response protocol. Communication is initiated only by the network master through requesting data from a designated sensor. The sensor will respond with the requested information, by replying with an HLP packet. This request - response protocol follows the Master/Slave relationship between the network master and the sensors. 
An example of using HLP packets to initially configure a network after power-up is the following.

1. The master node will send Dummy packets to every possible network address to detect if a sensor is attached. Attached sensor nodes will reply to the dummy packet with an acknowledgement. From this the master node can construct a register of sensors available on the network.

2. The master node will send a Get Sensor Information packet, one at a time, to every registered sensor. Each sensor will reply with its sensor information.

3. The master will stop every sensor from taking measurements, while it configures the network, by sending a Set Global Measurement Mode packet with the measurement mode set to Stop.

4. The master node will send Set Measurement Setup packets to each sensor, one at a time, to specify the type of measurement the sensor will conduct.

5. The master node will send a global Sync Network Time packet to the network to ensure every sensor is synchronised to the master's network time to allow accurate measurement windows.

6. The master node will start measurements on the network by sending a Set Global Measurement Mode with the measurement mode set to Step.

7. The master node will repeatedly, at a fixed interval, send Get Every Channels data packets to every sensor, one at a time, to receive the sensor's latest measurements. These measurements will be stored on the master node and be available to the control computer when requested.

\subsubsection{Media Layer Protocol}

As the network is half duplex, a managed access system needs to be established to prevent multiple nodes from communicating at the same time. This is achieved by the media layer protocol (MLP). The MLP employs frames and packet formats to control the flow of data across the network. Data is encapsulated in a packet and sent across the network in a network frame.

Figure 5.3 illustrates the sequencing of a network frame. If a network node wishes to initiate data transfer across the network, it must check to see if the network is free and then open a network frame by sending a data packet to a destination node. As all connected network 
devices listen to network traffic when a network frame is opened, all nodes register that a frame is open, and only the destination node and initiator node have permission to write to the network. The network frame is closed when the destination node sends an acknowledgment packet to the initiator node. Once the network frame is closed the network is available for data transfer by another node.

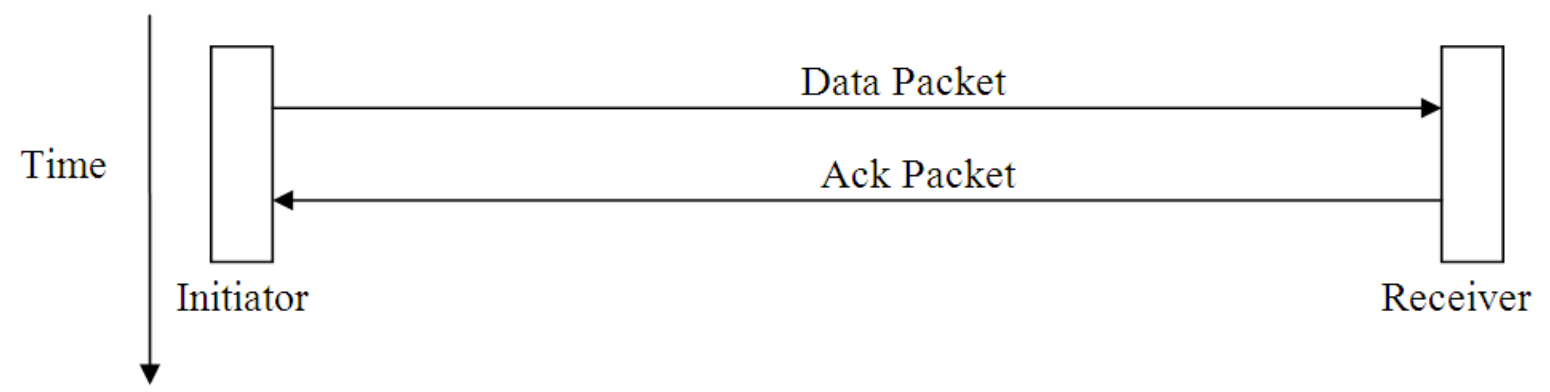

Figure 5.3 Example of a Network Frame

Network frames have a fixed life span of $5 \mathrm{~ms}$. If the initiator node does not receive an acknowledge packet $5 \mathrm{~ms}$ after opening the network frame it issues a Cancel Frame packet to close the frame. All network devices will receive the Cancel Frame packet, register the frame is closed and that the network is now available for new frames. The fixed life span of network frames prevents the network from being stuck in an opened frame state in the possibility that the destination node fails. 


\subsubsection{MLP Packets}

Data is transferred between network nodes in MLP packets. Figure 5.4 illustrates how the MLP transfers an HLP packet between network nodes.

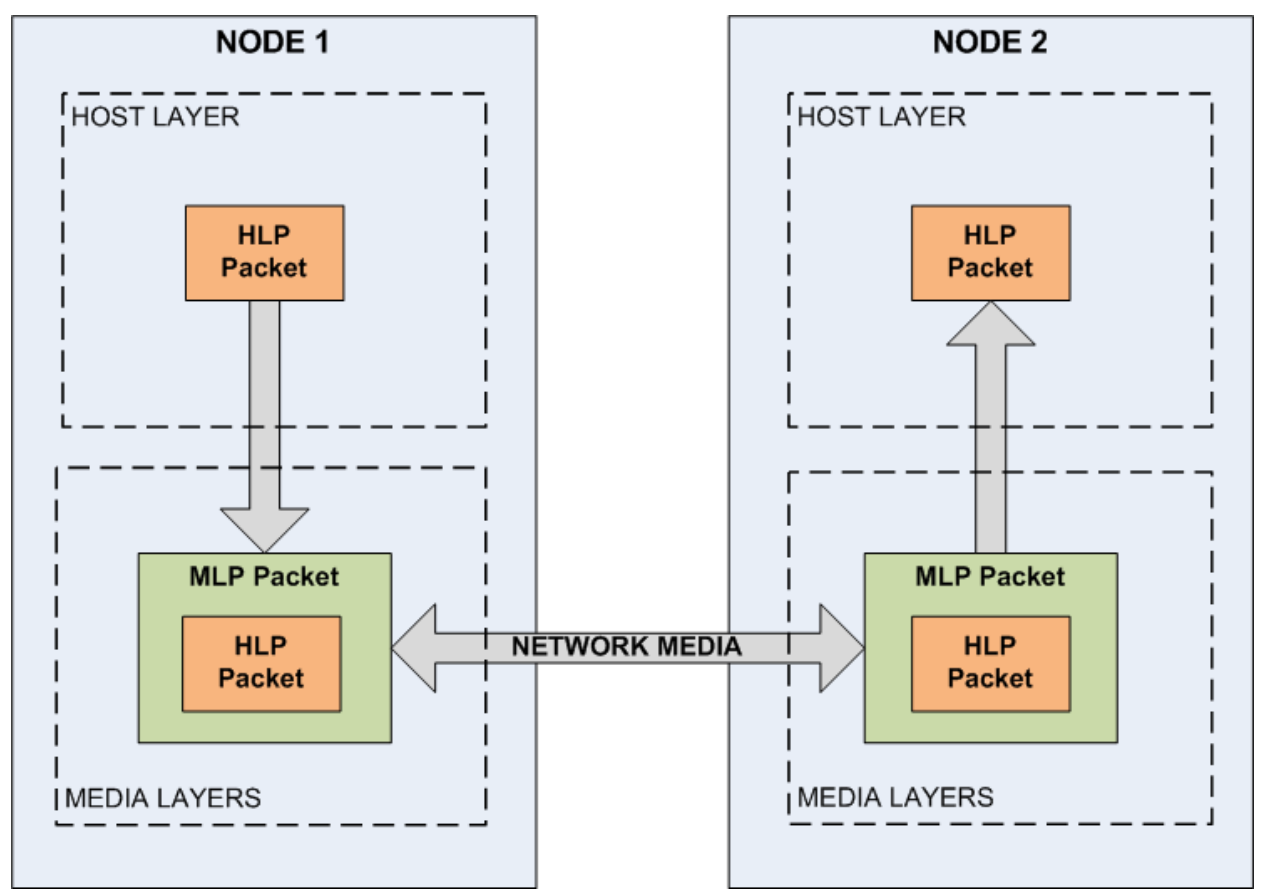

Figure 5.4 Transfer of HLP Packet between Network Nodes

An HLP packet is passed to the media layers with information on where it is destined for. The media layers process the HLP packet and embed it into an MLP packet whereupon it is sent to the destination node via a network frame. The destination node's media layers process the MLP packet and strip out the embedded HLP packet to pass to its host layers for further processing.

The MLP packet consists of two types of data: control information and a data payload. The control information provides data that the network needs to deliver the data payload to a network node. A MLP packet can vary in length from six to twenty one bytes, depending on the size of the data payload. Figure 5.5 present the structure of a MLP packet. The MLP packet is framed with a start and end byte to allow the Physical layer of the media layers to detect the beginning and end of a packet. The control information is contained within the packet header and trailer. 


\begin{tabular}{|c|c|c|}
\hline $\begin{array}{c}\text { Bit } \\
\text { Offset }\end{array}$ & \multicolumn{2}{|c|}{$\mathbf{0 - 3}-7$} \\
\hline 0 & \multicolumn{2}{|c|}{ Start Byte } \\
\hline 8 & \multicolumn{2}{|c|}{ Destination Address } \\
\hline 16 & \multicolumn{2}{|c|}{ Source Address } \\
\hline 24 & Packet ID & Data Payload Size \\
\hline 32 & \multicolumn{2}{|c|}{} \\
& \multicolumn{2}{|c|}{ Data Payload } \\
\hline $32-$ & \multicolumn{2}{|c}{} \\
\hline 152 & Packet Checksum \\
\hline $40-$ & \multicolumn{2}{|c|}{ Stop Byte } \\
\hline 160
\end{tabular}

Figure 5.5 MLP Packet Structure

The packet header consists of the following information:

- Destination Address - this field contains the physical address of the network node the packet is to be delivered to. The address is an 8 bit value allowing a maximum number of 256 network nodes to be addressed per sub-network. Network nodes on separate sub-networks can share the same physical address but are given different logical addresses by the network master. For example, a network node with the physical address of 0x01 will have a logical address of 0x0001 if it is connected to sub-network one and a logical address of 0x0101 if it is connected to sub-network two.

- Source Address - this field contains the physical address of the network node that has sent the packet.

- Packet ID - this 4 bit field identifies the type of MLP packet. The type of MLP packet assists the media layers with the sequencing of a network frame. For example a Data packet type indicates a network frame is open while a Cancel Frame packet type indicates that the frame is closed. MLP packet types are discussed further in section 5.1.3.2 MLP Packet Types. 
- Data Payload Size - this 4 bit field indicates the size of the data payload. Combined with the framing start byte and end byte, this field helps to indicate when a packet has been received. As the data payload byte is a 4 bit nibble it limits the size of a data payload to a maximum of 15 bytes.

The packet trailer consists of a checksum byte that is used for error detection. The checksum is calculated from a Modular Sum algorithm. Excluding the framing start and end bytes, the checksum is calculated by adding the MLP packet bytes together, discarding any overflow bits, and taking the two's complement of the result. When the destination node receives the MLP packet it can check the validity of the packet by adding together all the packet bytes, including the checksum value. The result will be zero if no errors occurred during transmission.

\subsubsection{Physical Addresses}

As mentioned previously, there are a maximum of 256 possible physical addresses on a subnetwork. The physical address $0 x 00$ is reserved for the network master node. As the master node is connected to every sub-network, the master's logical address is permanently defined as 0x00 so that it always matches its physical address thus allowing any node on a subnetwork to find it without having to compute a physical address from a logical address. This way only the master is required to keep track of how logical addresses map to physical addresses and sub-networks. When broadcasting a global packet on the network, the network master node will address the packet to itself.

\subsubsection{MLP Packet Types}

As mentioned in the section 5.1.3.1 MLP Packets, there are several types of MLP packets that assist in the sequencing of a network frame. The Data and Acknowledge packet types are used during normal operation to open and close a network frame. The remaining packet types are used to help configure the media layers and deal with error situations. It should be noted that packets can be classified into two forms, either NEW or ACK. NEW packets are packets that open a network frame while ACK packets are packets that close a network frame. Table 5.2 describes the types of MLP packets and their function. 
Table 5.2 MLP Packet Types

\begin{tabular}{|c|c|c|c|}
\hline $\begin{array}{c}\text { Packet } \\
\text { ID }\end{array}$ & Packet Type & New/Ack & Description \\
\hline 0x00 & Data & NEW & $\begin{array}{l}\text { Sent by the source node when transmitting } \\
\text { data to another network node. }\end{array}$ \\
\hline $0 \times 01$ & Acknowledge & ACK & $\begin{array}{l}\text { Sent by the destination node to the source } \\
\text { node in response to receiving a Data type } \\
\text { packet. }\end{array}$ \\
\hline 0x02 & Corrupt Data & ACK & $\begin{array}{l}\text { Sent by the destination node when the } \\
\text { checksum byte does not match the packet } \\
\text { checksum. Indicates an unsuccessful data } \\
\text { transmission. }\end{array}$ \\
\hline $0 \times 03$ & Deactivate & NEW & $\begin{array}{l}\text { Sent by the network master node to } \\
\text { deactivate a network node until the next } \\
\text { network initialisation. This is used primarily } \\
\text { if a physical address conflict occurs and } \\
\text { cannot be resolved. }\end{array}$ \\
\hline 0x04 & Reset & NEW & $\begin{array}{l}\text { This is a broadcast packet sent by the } \\
\text { network master node to reset all network } \\
\text { nodes. }\end{array}$ \\
\hline $0 \times 05$ & Cancel Frame & NEW & $\begin{array}{l}\text { Sent by the source node if no } \\
\text { acknowledgment is received to a transmitted } \\
\text { Data type packet after the network frame has } \\
\text { expired. This is a broadcast packet to } \\
\text { indicate to all network nodes that the current } \\
\text { network frame is now closed. }\end{array}$ \\
\hline $0 \times 06$ & Broadcast & NEW & $\begin{array}{l}\text { This is a broadcast packet sent by the } \\
\text { network master node to send data to all } \\
\text { network nodes. }\end{array}$ \\
\hline $\begin{array}{l}\text { 0x07 - } \\
\text { 0x0F }\end{array}$ & $\begin{array}{l}\text { Reserved for } \\
\text { future use }\end{array}$ & N/A & Reserved. \\
\hline
\end{tabular}


An example of using MLP packets to request a sensor reading is the following:

1. The network master passes a Get Sensor Data HLP packet, with the logical address of the sensor, to the media layers for transmission.

2. The media layers embed the HLP packet into a Data type MLP packet as the data payload.

3. The physical network address is computed from the logical address and inserted into the MLP packet header.

4. The remaining MLP packet header information is inserted and the checksum calculated.

5. The Data type MLP packet is transmitted on the network, opening a network frame between the master node and the destination node.

6. The destination node receives the Data type MLP packet. It checks the checksum and, provided no errors have occurred, strips the HLP packet from the data payload of the MLP packet and passes it to the Host layer.

7. The Host layer processes the Get Sensor Data HLP packet. The host layer gets the latest sensor data and creates a new Get Sensor Data HLP packet with the sensor data as the HLP packet's data payload.

8. The host layer passes the new Get Sensor Data HLP packet to the media layers with the physical address of the source node.

9. The media layers embed the HLP packet into an Acknowledge type MLP packet as the data payload.

10. The MLP packet header information is inserted and the checksum calculated.

11. The Acknowledge type MLP packet is transmitted on the network, closing the network frame between the master node and the destination node.

12. The master node receives the Acknowledge type MLP packet. It checks the checksum and, provided no errors have occurred, strips the HLP packet from the data payload of the MLP packet and passes it to the Host layer.

13. The Host layer processes the received Get Sensor Data HLP packet data payload to obtain the sensor readings. 


\subsection{Network Layer Stack}

The sensor network communication firmware has been written based upon an OSI model where the network system has been divided into smaller parts, called layers [22]. A layer is a collection of similar functions that provide services to the layer above it and receives services from the layer below it. Figure 5.6 presents the network stack model for the sensor network.
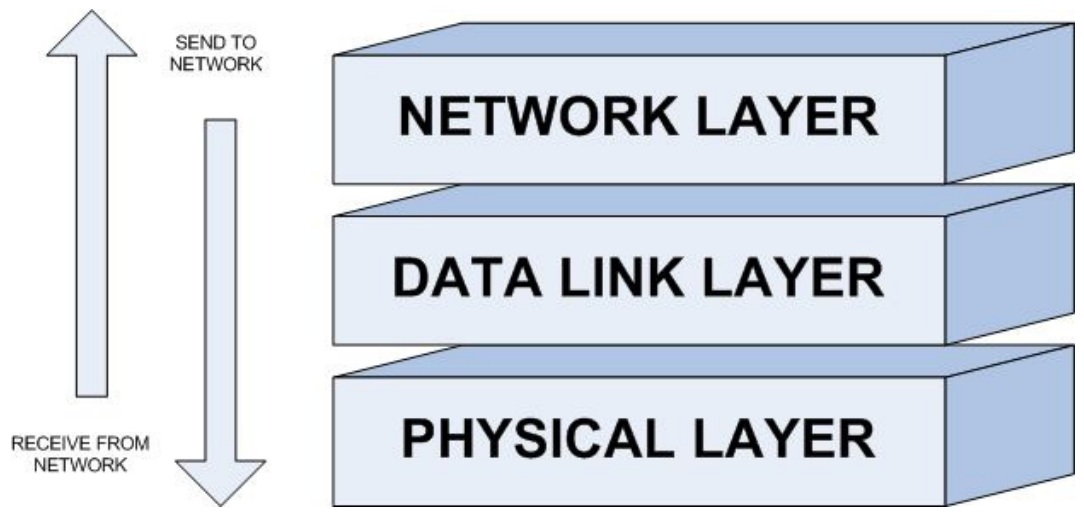

Figure 5.6 Firmware Network Stack Model

The division into layers allows additional functionality and services to be added to the network by the addition of another top-level host layer. Each layer guarantees a suite of stable services to the level above it. For example, a user can make changes to the Network layer by knowing only how to interface it with the Data Link layer to guarantee a functional network.

As the purpose of the network is dedicated to controlling and operating sensors the full seven layer OSI model is not necessary. The higher host layers can be collapsed down into the main MCU loop. This allows the Network layer stack model to be based on a three layer system. These layers are;

\section{Physical Layer}

This layer deals with the actual manipulation and control of the hardware. It handles the control signals for the MCU USART and the RS-485 driver chip. It also deals with sending MLP packets across the MCU USART and reconstructing MLP packets from bytes received from the USART. The Physical layer is one of the two layers that make up the Media layers. 


\section{Data Link Layer}

The Data Link layer provides the functionality to transfer data between network nodes. It is also responsible for detecting and possibly correcting errors that occur in the Physical layer. Any data received from the layer above is compiled into a MLP packet and passed to the Physical Layer for transmission. This layer is responsible for:

- Initiating a Network frame, by sending a MLP packet on the network.

- Receiving MLP packets.

- Checking the integrity of the data of all received MLP packets addressed to it.

- Ending a Network frame by sending an acknowledgment packet to the Network frame initiator.

- Deactivating itself if it receives a Deactivate packet.

- Reactivating itself if it receives a Reset packet.

The Data Link layer, along with the Physical layer, forms the Media Layers.

\section{Network Layer}

The Physical and Data Link layers together control how data is communicated on the sensor network. The Network layer is the Host layer. While the Data Link and Physical layers deal with how data is handled on the network, the Network layer deals with what data is sent across the network. It is at this layer that tasks and commands, specific to a sensor's function, are interpreted. The Sensor Network protocol is utilised at this layer to ensure the sensor network operates correctly. This layer is responsible for:

- Allowing the MCU's main application loop to write data to the network.

- Allowing the MCU's main application loop to read data from network.

- Collision detection on the network.

- Collision resolution.

- Storing/altering network node parameters such as network address. 


\subsubsection{Physical Layer}

The Physical layer (PHY) has two major roles to play: the transmission and reception of MLP packets over the USART and RS-485 transceiver. The PHY firmware is built on top of the basic USART functions provided for AVR MCUs. The PHY is implemented as a finite state machine (FSM). Data is received and transmitted by the USART in byte form. An interrupt routine is executed for every byte received by the USART. As the network is based on half duplex communication any transmitted byte will be echoed back to the USART's receiver. This means that every transmitted byte will also trigger the byte received interrupt service routine (ISR). The states of the PHY's FSM allow the USART byte received ISR to keep track of writing and reading MLP packets to and from the network.

The Data Link layer and the PHY share two MLP packet buffers to enable MLP packets to be passed from one layer to the other. One buffer (transmit buffer) is used by the Data Link layer to pass packets to the PHY and the other buffer (receive buffer) is used by the PHY to pass MLP packets to the Data Link layer. When the Data Link layer has an MLP packet to transmit, it stores it in the MLP packet transmit buffer and instructs the PHY to open a network frame. Once the PHY has opened a network frame it reads the MLP packet bytes from the transmit buffer and transmits them over the network.

When the PHY has received a MLP packet from the network it stores the packet contents in the shared MLP packet receive buffer and instructs the Data Link layer to process the packet. The Data Link layer can then read the MLP packet contents from the receive buffer and process them. Figure 5.7 presents the state transition diagram for the PHY FSM. 


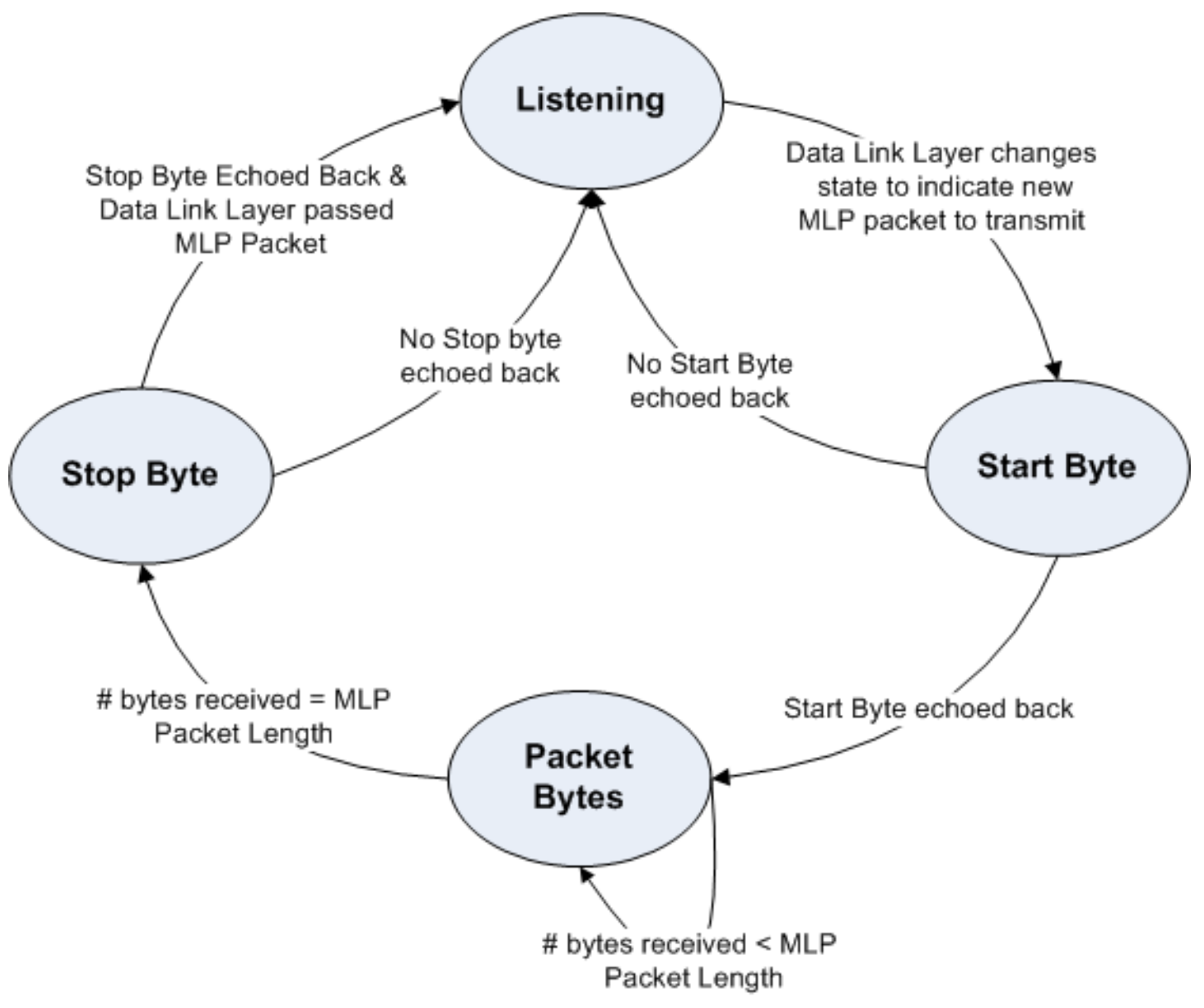

Figure 5.7 Physical Layer State Transition Diagram

The PHY evaluates whether a state transition should occur every time the byte-received ISR is called.

The default state of the PHY is the Listening state. In this state the PHY is listening for new network frames and MLP packets on the network. Note in this state the PHY disables the RS-485 transmitter. When the PHY detects a Start byte, indicating a new network frame has opened, it begins to store received bytes in a MLP packet buffer. Once a start byte has been received the PHY processes the incoming bytes that form the MLP packet header to read the MLP packet Data Payload Size field. The PHY then uses the Data Payload Size value, in conjunction with the Stop byte, to determine when a complete MLP packet has been received. Upon reception of a complete MLP packet the PHY layer passes the MLP packet to the Data Link layer for processing.

When the Data Link layer has an MLP packet ready for transmitting it forces the PHY layer to the Start Byte state and calls the PHY layer transmit function to send the start byte character. The PHY's transmit function activates the RS-485 transmitter, writes the byte to 
the network and then deactivates the RS-48 transmitter. Once the start byte is transmitted, it is echoed back on the half duplex network and triggers the PHY's byte-received ISR. When in the Start Byte state the PHY checks to see if the received byte matches the start byte character. If they do not match a problem has occurred with data transmission, most likely a network collision, and the PHY sets an error flag and returns to Listening state. The error flag indicates to the Data Link layer that the MLP packet could not be transmitted. If the received byte matches the start byte character, the PHY transmits the first byte in the MLP packet transmit buffer and changes to the Packet Bytes state to start receiving the transmitted packet bytes.

In the Packet Bytes state the PHY will check that the received byte matches the last transmitted byte to ensure no bus collisions have occurred. If there is a mismatch then the PHY sets an error flag to indicate to the Data Link layer that a transmission error has occurred. If the bytes match, the PHY transmits the next byte in the MLP packet transmit buffer. The PHY will also read the Data Payload Size field from the MLP packet header bytes and use this value to calculate the MLP packet size indicating how many bytes will be received in this MLP packet. Once the number of received bytes equals the calculated packet size, the PHY transmits the stop byte character and changes its state to the Stop Byte state.

In the Stop Byte state the PHY checks to see if the received byte equals the stop byte character. If they do not match, the PHY sets an error flag to indicate to the Data Link layer that a data transmission error has occurred and changes its state to the Listening state. If the received byte does match, the stop byte character, the PHY calls the Data Link layer to process the received packet and changes its state to the Listening state.

\subsubsection{Data Link Layer}

The Data Link layer (DLL) is responsible for the transmission and reception of data to and from another network node. For transmission this involves constructing MLP packets with the data embedded in the packet's data payload field and passing the MLP packet to the PHY layer for transmission. For reception this involves decoding the received MLP packet control information to check if the packet is intended for the node, checking the data integrity of the packet, and passing the data payload to the Host layer for processing.

The DLL is implemented as a finite state machine. The FSM states indicate how the DLL should interact with the network. State transitions occur whenever the DLL receives a MLP 
packet. The DLL uses the type of MLP packet to determine which state to be in. Figure 5.8 presents the state transition diagram for the DLL.

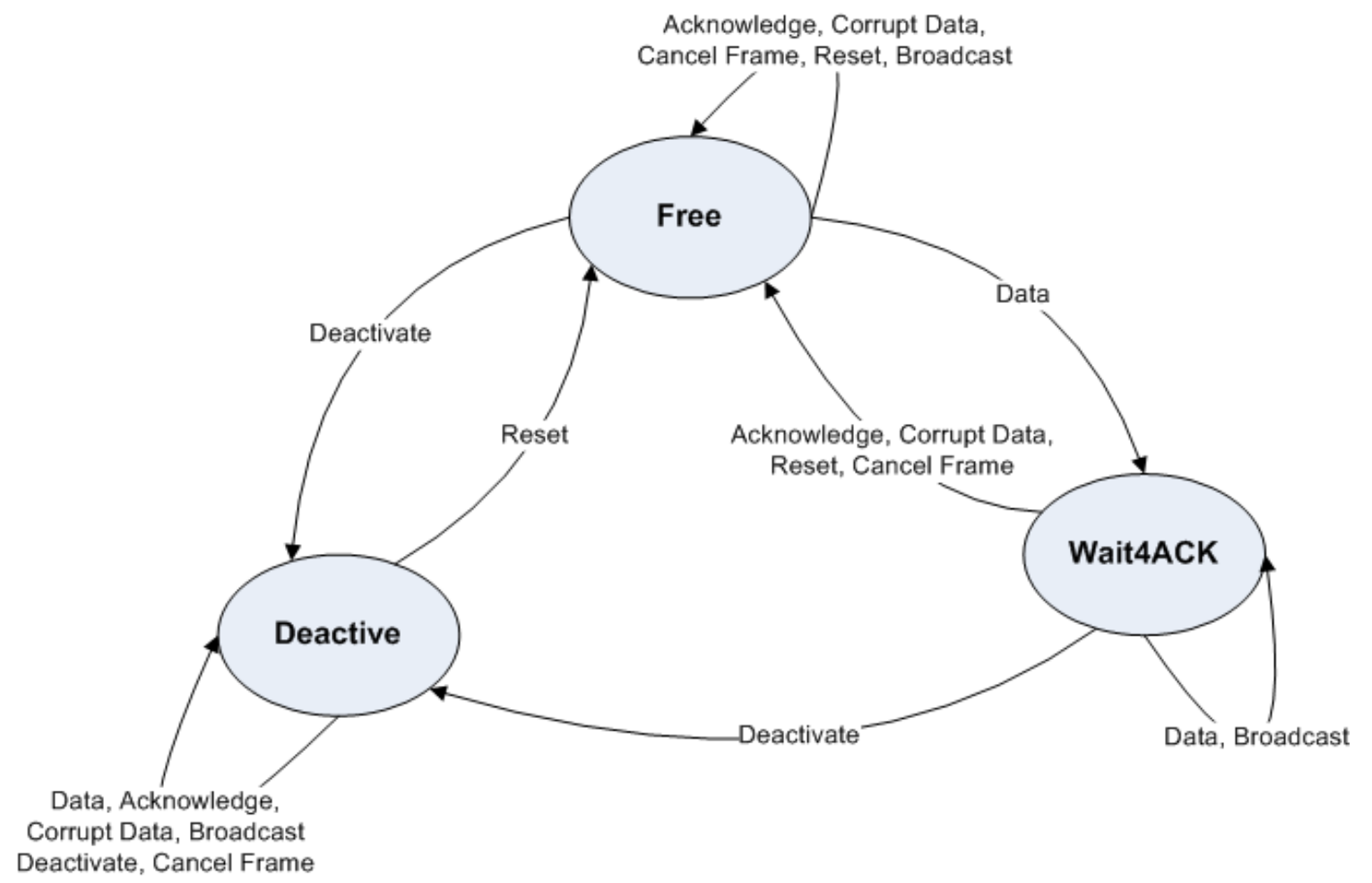

Figure 5.8 Data Link Layer State Transition Diagram

The default state of the DLL is the Free state. This state indicates that the network is free to transmit data (i.e. there are no open network frames).

The Wait4ACK state indicates there is a current network frame open and the DLL is waiting for an Acknowledge type MLP packet to close the network frame. In this state the network is not free for data transmission.

The Deactive state indicates that the network node has been deactivated from the network and should not access the network until it receives a Reset type MLP packet. This state is used to remove network nodes from the network if a physical address conflict cannot be resolved.

In the Free state the DLL will transition to the Wait4ACK upon receipt of a Data type MLP packet, indicating a Network frame is now open. The DLL will change to the Deactive state if it receives a Deactivate type packet addressed to it. All other MLP packet types will leave the DLL in the Free state.

In the Wait4ACK state the DLL will transition to the Free state if it receives either an Acknowledge, Corrupt data, Reset or Cancel Frame type MLP packet. All these packets 
indicate that any open network frames should be closed and that the network should be free for new data transmissions. The DLL will change to the Deactive state if it receives a Deactivate type packet addressed to it. The DLL will remain in the Wait4ACK state if it receives a Broadcast or Data type packet.

In the Deactive state the DLL will only transition to the Free state if it receives a Reset type MLP packet. All other packet types will leave the DLL in the Deactive state.

When the DLL receives a MLP packet from the PHY it checks the current DLL state. If the current state is Deactive then it will ignore the MLP packet. If the DLL state is either Free or Wait4ACK the DLL decodes the MLP packet control information. In the Free state the source address is extracted and stored as the frame initiator and the destination address is extracted and stored as the frame receiver. These variables are used by the Wait4ACK state to determine if a Network frame can be closed. If the MLP packet is a Data type packet and the destination address matches the DLL's physical address, the packet checksum is calculated and confirmed. If the checksum does not match the MLP packet checksum then the DLL will reply with a Corrupt Data type MLP packet to the source node. If the checksum matches, the DLL passes the MLP packet data payload to the Host layer.

If the MLP packet is a Broadcast type packet the DLL will calculate and confirm the checksum. If there is a mismatch the DLL will ignore the Broadcast packet. If the checksum is valid, the DLL passes the MLP packet data payload to the Host layer.

In the Wait4ACK state the DLL will register a network frame as closed only if it receives an Acknowledge or Corrupt Data type MLP packet addressed to the frame initiator from the frame receiver that both were defined when the network frame was opened, or if a Cancel Frame type MLP packet is received from the frame initiator that originally opened the Network frame. A Reset type MLP packet will cause all network nodes to reinitialise and start in the default Free state.

When the DLL initiates a data transmission on the PHY it begins a timer to monitor if the network frame lifespan has expired. If the frame does expire, the DLL will instruct the PHY to send a Cancel frame MLP packet to close the network frame.

The DLL transmit function also checks the DLL state to determine how to proceed. If the DLL state is Free then the DLL will proceed with instructing the PHY to transmit the packet. 
If the DLL is in the Wait4ACK state, the DLL will transmit Acknowledge and Corrupt Data MLP packets only if the packet is addressed to the frame initiator from the frame receiver. The DLL will transmit Cancel Frame MLP packets only if the source address matches the frame initiator. The DLL will always transmit Reset type MLP packets in this state.

In the Deactive state the DLL will not transmit any packets including resets.

The Reset type MLP packet resets the network interface by executing a soft reset on the MCU. This resets the network interface of the MCU as well as resetting the sensor control routines, causing the sensor node to operate in its default start up mode.

\subsubsection{Network Layer}

The Network layer is responsible for interpreting data from the network. It uses the HLP to decode data payloads from MLP packets received by the DLL. The HLP uses a C language Struct type to store the sensor node information required by the protocol. Figure 5.9 presents the structure definition of a sensor node.

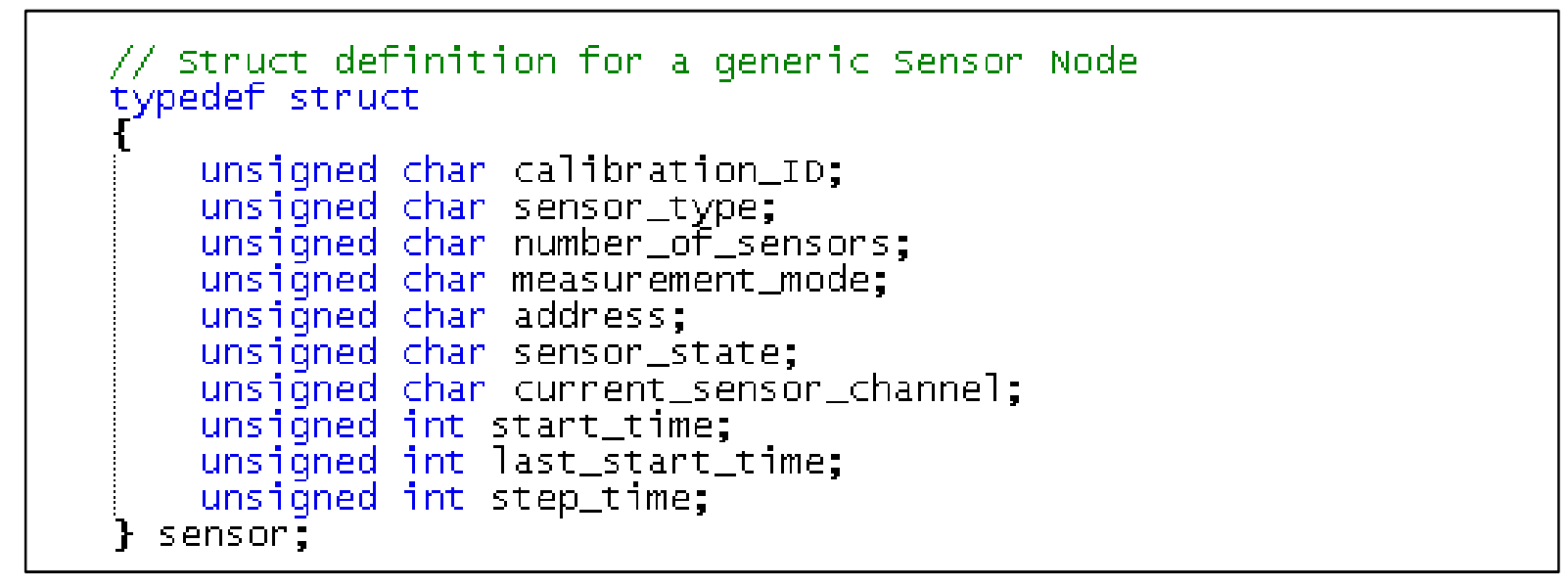

Figure 5.9 Structure definition for a Sensor Node

The HLP data types, mentioned in section 5.1.2 Host Layer Protocol, are stored in the sensor struct. Additional information stored in the sensor struct includes:

- $\quad$ sensor_state - all sensors are implemented as a finite state machine to control the flow of a measurement. The current state of the state machine is stored in this variable.

- last_start_time - this variable is used for Step measurement modes. It stores the last time, during a measurement window, that a measurement was taken and is used with 
the step_time variable to calculate when the next time, in the measurement window, a sensor should take a reading.

The sensor measurements are stored in an array. This array has as many elements as there are sensor channels.

Upon receipt of an HLP packet the network layer decodes the packet type, executes any functions required to fulfil the instruction from the HLP packet, interrogates the sensor struct for the HLP data types and responds with any required sensor data by passing a new HLP packet to the DLL for transmission to the master node.

On all network nodes, apart from the master node, the Network layer after initialisation is accessed only by the DLL. The main loop has no requirement to access the Network layer as the sensor node is a slave device and will access the network only when prompted by receiving an HLP packet from the network master. As the PHY is interrupt-driven this removes any requirement on the main loop to poll the Network layer and as such the main loop is dedicated to the control of the sensor interface. The Network layer firmware implemented on network nodes, apart from the master node, comprises two functions. These functions are:

1. Network_Init - called by the main loop on start-up to initialise the DLL and PHY.

2. Network_packetReceived - called by the DLL to instruct the Network layer to process an HLP packet that has been delivered to the sensor from the network master.

The network master's Network layer has a further function, Network_transmitPacket, which allows the master node to initiate data transfer on the network. This function decodes the logical network addresses into physical addresses on the two sub-networks. It also determines if the packet should be broadcast across the entire network or only on the sub-network that the addressed node resides on. A further host layer is implemented on the master node's network stack to control access to the network. Figure 5.10 illustrates the network stack model of the firmware on the master node. The Sensor Network layer is a host layer that provides services to allow the MCU's main loop to access the network layer. 


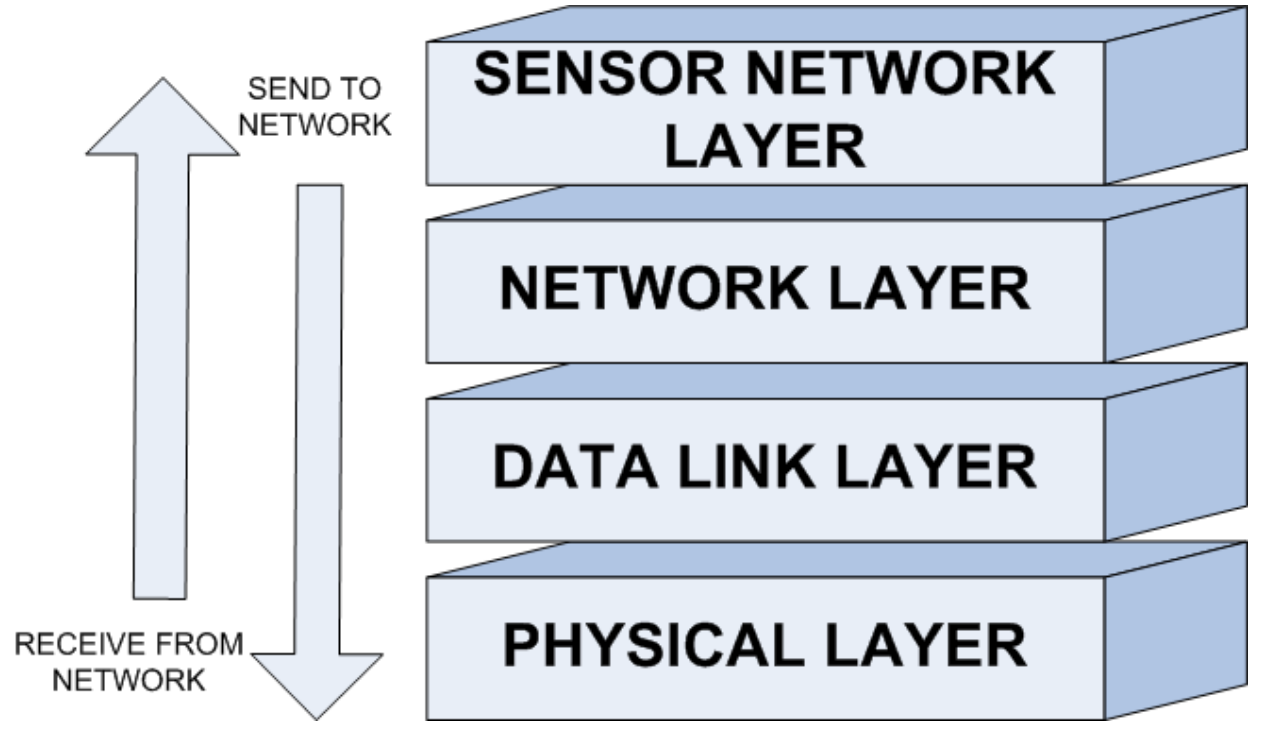

Figure 5.10 Master Node's Network Stack Model

It is the Sensor Network layer that defines the services available to the master node in managing the network.

\subsubsection{Sensor Network Layer}

The Sensor Network layer manages control of the sensor network. It maintains a table that defines the sensor network. Each element of the table provides information on a connected sensor node. The table also stores a local copy of sensor data that was last received from the entire network. This allows a control computer to access the locally stored sensor data to determine the state of the environment that the network resides in. The table is implemented on the MCU as a sensor_control structure. Figure 5.11 presents the definition of the sensor_control structure.

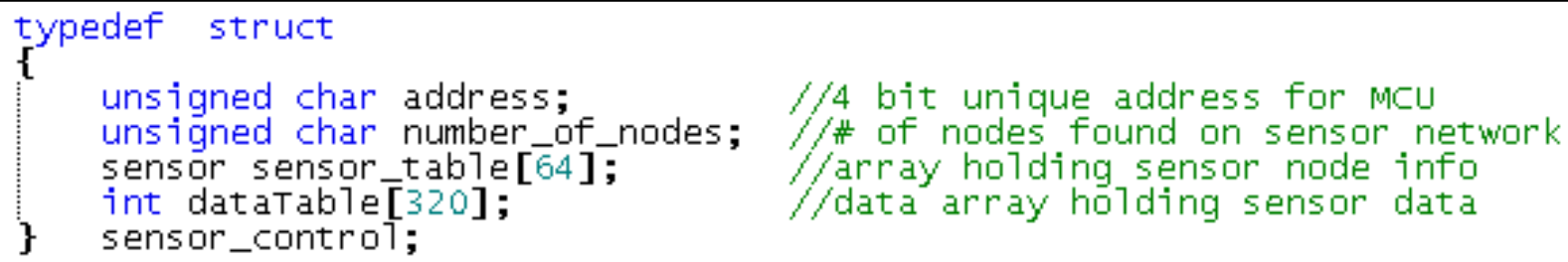

Figure 5.11 Structure definition for master node's sensor table 
The sensor_control structure has the following fields:

- Address - to identify the sensor network interface board from other peripheral hardware connected to a control computer. This address is read by the MCU from DIP switches on the sensor network interface board, allowing the user to set the address in hardware.

- Number of nodes - the field keeps a count of the number of sensor nodes detected on the network during initialisation.

- Sensor table - This is an array that holds sensor structures described further below. Each entry provides the key information about an attached sensor. The sensor table is indexed by the list of possible logical addresses on the network.

- Data Table - the data table is an array that holds all the measurements received from sensors on the network. The data table is a flat memory structure. An individual sensor's data is indexed in the data table by a data pointer stored in each sensor entry in the sensor table. This allows sensors to be allocated memory in the data table when the network is initialised, which provides the sensor network interface board with the capability to store sensor information for a variety of network configurations.

The sensor structure, used to store sensor information in the sensor table, is similar to the structure implement on the slave nodes. Figure 5.12 presents the definition of the sensor structure.

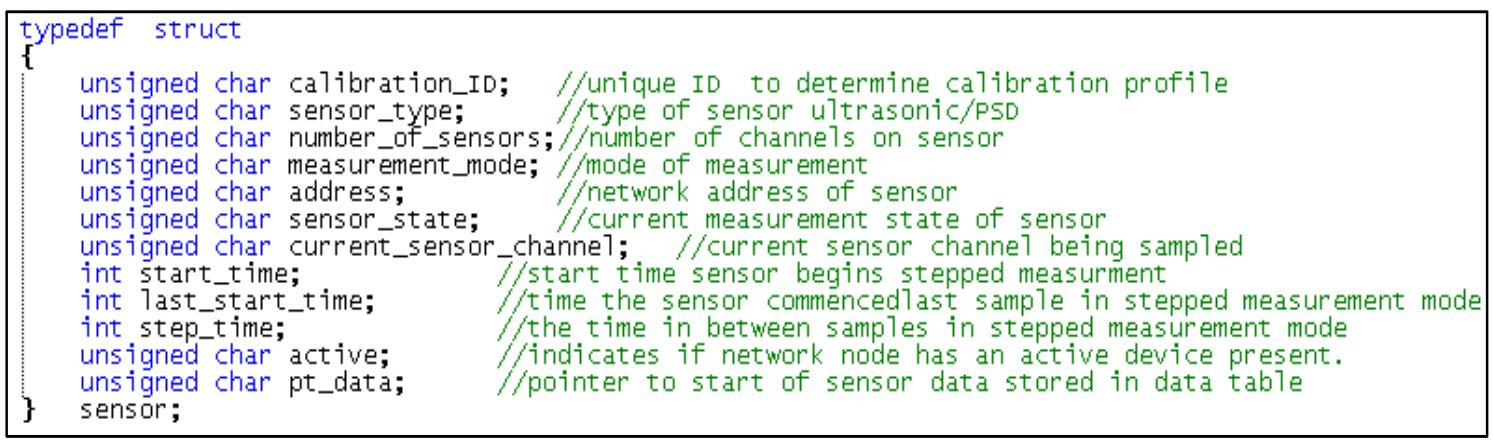

Figure 5.12 Structure definition for master node's sensor node

It has the additional fields, on top of the slave node sensor structure:

- Active - this is a flag to indicate that a sensor has been found at the logical network address that indexes this sensor structure in the sensor table. The address field of the sensor structure holds the physical network address for the sensor. 
This allows the master network node to map logical network addresses to physical addresses.

- $p t \_d a t a$ - this is a pointer to an element in the data table at which the sensor's data is stored. The data pointer and number of channels fields of the sensor structure define the block of memory allocated in the data table to this sensor.

The Sensor network layer functions provide services to the MCU and any connected control computer to configure the network and receive sensor network data. The functions that provide these services are described in Table 5.3.

Table 5.3 Sensor Network Layer Functions

\begin{tabular}{|c|c|c|c|}
\hline Function & Parameters & Returns & Description \\
\hline Sensor_Network_init & void & void & $\begin{array}{l}\text { This function initialises the PHY, } \\
\text { DLL and Network layers. It polls all } \\
\text { possible logical addresses on the } \\
\text { network and populates the Sensor } \\
\text { Table with information of found } \\
\text { sensors. }\end{array}$ \\
\hline $\begin{array}{c}\text { Sensor_Network_ } \\
\text { update }\end{array}$ & void & void & $\begin{array}{l}\text { This function polls every sensor on } \\
\text { the network for the last measurement } \\
\text { data. It populates the data table with } \\
\text { the measurement data it receives. }\end{array}$ \\
\hline Setup_Measurement & $\begin{array}{l}\text { Node } \\
\text { Address, } \\
\text { Mode, Start } \\
\text { Time, Step } \\
\text { Time, } \\
\text { Channel }\end{array}$ & $\begin{array}{l}\text { Success } \\
\text { Flag }\end{array}$ & $\begin{array}{l}\text { This function allows the MCU to } \\
\text { define a sensor task for a specified } \\
\text { sensor. It returns a success flag to } \\
\text { indicate if the sensor task was } \\
\text { received by the sensor node. }\end{array}$ \\
\hline Set_Calib_ID & $\begin{array}{l}\text { Node } \\
\text { Address, } \\
\text { Calib ID }\end{array}$ & $\begin{array}{l}\text { Success } \\
\text { Flag }\end{array}$ & $\begin{array}{l}\text { This function allows the MCU to } \\
\text { allocate a new calibration ID to a } \\
\text { specified sensor. The new calibration } \\
\text { ID is stored in volatile memory and } \\
\text { will be lost when power is removed } \\
\text { from the sensor node. It returns a } \\
\text { success flag to indicate if the } \\
\text { calibration ID was received by the } \\
\text { sensor node. }\end{array}$ \\
\hline Set_Mode & $\begin{array}{l}\text { Node } \\
\text { Address, } \\
\text { Mode }\end{array}$ & $\begin{array}{l}\text { Success } \\
\text { Flag }\end{array}$ & $\begin{array}{l}\text { This function allows the MCU to set } \\
\text { the measurement mode of a specified } \\
\text { sensor. It returns a success flag to } \\
\text { indicate if the measurement mode } \\
\text { was received by the sensor node. }\end{array}$ \\
\hline Set_Global_Mode & Mode & $\begin{array}{l}\text { Success } \\
\text { Flag }\end{array}$ & $\begin{array}{l}\text { This function allows the MCU to } \\
\text { globally set the measurement for all } \\
\text { sensors. It returns a success flag to }\end{array}$ \\
\hline
\end{tabular}




\begin{tabular}{|c|c|c|c|}
\hline & & & $\begin{array}{l}\text { indicate if the master received the } \\
\text { global network packet. }\end{array}$ \\
\hline Sync_Network_Time & void & $\begin{array}{l}\text { Success } \\
\text { Flag }\end{array}$ & $\begin{array}{l}\text { This function allows the MCU to } \\
\text { synchronise all sensors' clocks to the } \\
\text { network clock. It returns a success } \\
\text { flag to indicate if the master received } \\
\text { the global network packet. }\end{array}$ \\
\hline Get_Sensor_Info & $\begin{array}{l}\text { Node } \\
\text { Address }\end{array}$ & $\begin{array}{l}\text { Success } \\
\text { Flag }\end{array}$ & $\begin{array}{l}\text { This function retrieves the sensor } \\
\text { information from a specified sensor. } \\
\text { It returns a success flag to indicate if } \\
\text { the master received the sensor } \\
\text { information. }\end{array}$ \\
\hline $\begin{array}{c}\text { Get_Measurement } \\
\text { Setup }\end{array}$ & $\begin{array}{l}\text { Node } \\
\text { Address }\end{array}$ & $\begin{array}{l}\text { Success } \\
\text { Flag }\end{array}$ & $\begin{array}{l}\text { This function retrieves the sensor task } \\
\text { from a specified sensor. It returns a } \\
\text { success flag to indicate if the master } \\
\text { received the sensor task information. }\end{array}$ \\
\hline Get_All_Data & $\begin{array}{l}\text { Node } \\
\text { Address }\end{array}$ & $\begin{array}{l}\text { Success } \\
\text { Flag }\end{array}$ & $\begin{array}{l}\text { This function retrieves the last } \\
\text { measurement data from all the } \\
\text { channels of a specified sensor. It } \\
\text { returns a success flag to indicate if the } \\
\text { master received the sensor data. }\end{array}$ \\
\hline Get_Node_Reading & $\begin{array}{l}\text { Node } \\
\text { Address }\end{array}$ & $\begin{array}{l}\text { Success } \\
\text { Flag }\end{array}$ & $\begin{array}{l}\text { This function instructs a specified } \\
\text { sensor to take a sensor measurement } \\
\text { on all channels and to return the } \\
\text { result. It returns a success flag to } \\
\text { indicate if the master received the } \\
\text { sensor data. }\end{array}$ \\
\hline $\begin{array}{c}\text { Get_Node_Channel_ } \\
\text { Reading }\end{array}$ & $\begin{array}{l}\text { Node } \\
\text { Address, } \\
\text { Channel }\end{array}$ & $\begin{array}{l}\text { Success } \\
\text { Flag }\end{array}$ & $\begin{array}{l}\text { This function instructs a specified } \\
\text { sensor to take a sensor measurement } \\
\text { on a specified channel and return the } \\
\text { result. It returns a success flag to } \\
\text { indicate if the master received the } \\
\text { sensor data. }\end{array}$ \\
\hline
\end{tabular}

\subsection{Control Computer / Network Command Interface}

The interface between the control computer and the network master provides a robotic platform with the ability to configure the sensor network. A command interface has been written in firmware to allow the control computer to issue instructions to the master node on how to configure the network and the master. This command interface currently is supported only by the USB interface. The Ethernet interface can be enabled to support the command interface by the implementation of a TCP/IP stack on the MCU. Time restraints have prevented the Ethernet interface from being completed. 
The command interface is based on a request - response protocol. The network master will communicate on the USB interface only in response to a request from the control computer. The interface utilises a packet format to ensure correct operation of the interface and to guarantee data integrity. The command interface uses the ASCII control characters Data Link Escape (DLE) and End of Text (ETX) for packet framing. New frames begin with a single DLE character. Frames end with a single DLE character followed by an ETX character. To prevent DLE characters within the data payload or packet header bytes from being interpreted as a framing character, the command protocol uses byte stuffing. Byte stuffing involves adding extra control character bytes into the packet message to indicate that DLE characters within the packet body should not be interpreted as framing characters. When the packet is being transmitted, the packet data is scanned for DLE characters. If a DLE character is embedded in the packet, an extra DLE character is added directly after the first DLE. The byte stuffing means that only a single DLE character represents a framing character. Figure 5.13 presents the structure of the command interface packet.

\begin{tabular}{|c|c|}
\hline $\begin{array}{c}\text { Byte } \\
\text { Offset }\end{array}$ & Packet Field \\
\hline 0 & Data Link Escape \\
\hline 1 & Packet ID \\
\hline 2 & Error Code \\
\hline 3 & Packet Size \\
\hline 4 & \\
& Data Payload \\
\hline $4-$ & \\
132 & Packet Checksum \\
\hline $132-$ & Data Link Escape \\
\hline 133 & End of Text \\
\hline 134 &
\end{tabular}

Figure 5.13 Packet Structure for Command Interface 
The command interface packet has the following fields:

- Packet ID - this field identifies the type of command packet. The type of command packet indicates the command being requested by the control computer.

- Error Code - this field holds a code that indicates an error has occurred during transmission. A typical error can be invalid command parameters in a data payload, for example, requesting data from a specified sensor node that doesn't exist on the network.

- Packet Size - this field indicates the number of bytes in the command packet. As this field is 8 bits in size, the maximum allowed packet size is limited to 255 bytes. The packet header and trailer information consists of 4 bytes, meaning that the maximum data payload size is limited to 251 bytes.

- Data Payload - this field holds the data payload for the command. When issued by the control computer this payload contains command parameters and when issued by the network master it contains requested sensor data.

- Packet Checksum - this field contains a checksum byte to indicate whether data corruption has occurred during transmission. The check sum is calculated from a Modular Sum algorithm by adding the command packet bytes together, excluding the framing start and end characters and discarding any overflow bits, and taking the two's complement of the result.

The command interface has thirteen different command packet IDs providing the control computer with the ability to issue instructions to the sensor network. The command packet types provide all the functionality to allow the control computer to reconfigure the sensor network, request sensor data, and identify and configure the network master. Table 5.4 describes the command interface packet types. 
Table 5.4 Command Interface Packet Types

\begin{tabular}{|c|c|c|c|}
\hline $\begin{array}{c}\text { Packet } \\
\text { ID }\end{array}$ & Packet Type & Parameters & Description \\
\hline $0 \times 01$ & Dummy & - & $\begin{array}{l}\text { This packet has no data payload and is } \\
\text { primarily used to check correct communication } \\
\text { on the command interface. }\end{array}$ \\
\hline $0 \times 02$ & Reset & - & $\begin{array}{l}\text { This packet resets the Network Master and } \\
\text { Sensor Network. }\end{array}$ \\
\hline $0 \times 20$ & Get Address & - & $\begin{array}{l}\text { This packet requests the Network Master's } \\
\text { hardware address. It is used to indicate to the } \\
\text { control computer that the attached hardware is } \\
\text { a sensor network. }\end{array}$ \\
\hline $0 \times 21$ & $\begin{array}{l}\text { Get Network } \\
\text { Definition }\end{array}$ & - & $\begin{array}{l}\text { This packet requests the network definition } \\
\text { from the Network Master. The network } \\
\text { definition is comprised of the number of } \\
\text { detected nodes and, for each node, the node } \\
\text { address and its calibration ID. }\end{array}$ \\
\hline $0 \times 22$ & Get Node Data & Node & $\begin{array}{l}\text { This packet requests the sensor data for a } \\
\text { specified sensor. The data returned is the latest } \\
\text { data the Network Master has read from the } \\
\text { network. }\end{array}$ \\
\hline $0 \times 26$ & $\begin{array}{l}\text { Get Node } \\
\text { Reading }\end{array}$ & Node & $\begin{array}{l}\text { This packet requests that the Network Master } \\
\text { instruct a specified sensor to take a reading and } \\
\text { return the data back to the master and the } \\
\text { control computer. }\end{array}$ \\
\hline $0 \times 28$ & $\begin{array}{l}\text { Get Auto } \\
\text { Update }\end{array}$ & - & $\begin{array}{l}\text { This packet requests the state of the Network } \\
\text { Master's auto update flag. The auto update } \\
\text { flag indicates if the master is automatically } \\
\text { polling the sensor network, every } 50 \mathrm{~ms} \text {, for } \\
\text { new sensor data. }\end{array}$ \\
\hline $0 \times 43$ & $\begin{array}{c}\text { Set Node } \\
\text { Measurement }\end{array}$ & $\begin{array}{l}\text { Node, Mode, } \\
\text { Start Time, Stop } \\
\text { Time, Channel }\end{array}$ & $\begin{array}{l}\text { This packet instructs the Network Master to } \\
\text { issue new measure task data to a specified node } \\
\text { on the network. }\end{array}$ \\
\hline $0 \times 44$ & Set Node Mode & Node, Mode & $\begin{array}{l}\text { This packet instructs the Network Master to } \\
\text { change a specified sensor's measurement mode } \\
\text { to the specified mode. }\end{array}$ \\
\hline $0 \times 45$ & $\begin{array}{l}\text { Set Global } \\
\text { Mode }\end{array}$ & Mode & $\begin{array}{l}\text { This packet instructs the Network Master to } \\
\text { globally change the measurement mode of all } \\
\text { sensors on the network to the specified mode. }\end{array}$ \\
\hline $0 \times 46$ & $\begin{array}{l}\text { Set Auto } \\
\text { Update }\end{array}$ & $\begin{array}{c}\text { Auto Update } \\
\text { Flag }\end{array}$ & $\begin{array}{l}\text { This packet instructs the Network Master to } \\
\text { change its auto update flag to the specified } \\
\text { value. }\end{array}$ \\
\hline $0 \times 47$ & $\begin{array}{l}\text { Set Network } \\
\text { Time }\end{array}$ & & $\begin{array}{l}\text { This packet instructs the Network Master to } \\
\text { reset and sync the network time. }\end{array}$ \\
\hline
\end{tabular}


The error code field allows the command interface to indicate standard errors that may occur. These standard errors can then be rectified by either the control computer or network master. As more than one error can occur at a time, the error code byte represents errors by setting or clearing a bit. This allows a maximum of 8 error codes to be defined in a byte. Table 5.5 lists and describes the different error codes.

Table 5.5 Command Interface Error Codes

\begin{tabular}{|c|c|l|}
$\begin{array}{c}\text { Error } \\
\text { Code }\end{array}$ & \multicolumn{2}{|c|}{ Error Type } \\
\hline $\mathbf{0 x 0 1}$ & No Error & This bit is set if no error has occurred. \\
\hline $\mathbf{0 x 0 2}$ & $\begin{array}{c}\text { Packet ID } \\
\text { Invalid }\end{array}$ & $\begin{array}{l}\text { This bit indicates that the received packet ID does not match a } \\
\text { packet ID defined on the master or control computer. }\end{array}$ \\
\hline $\mathbf{0 x 0 4}$ & $\begin{array}{c}\text { Checksum } \\
\text { Error }\end{array}$ & $\begin{array}{l}\text { This bit indicates that the checksum byte did not match the } \\
\text { checksum value of the packet. Data corruption must have } \\
\text { occurred during transmission. }\end{array}$ \\
\hline $\mathbf{0 x 0 8}$ & $\begin{array}{c}\text { Parameter } \\
\text { Invalid }\end{array}$ & $\begin{array}{l}\text { This bit indicates that a parameter sent in the data payload is } \\
\text { invalid, for example, if the control computer requests sensor data } \\
\text { from a specified sensor that cannot be found on the network. }\end{array}$ \\
\hline $\mathbf{0 x 1 0}$ & Frame Error & $\begin{array}{l}\text { This bit indicates that a communication error occurred on the } \\
\text { sensor network. This code is set when the control computer } \\
\text { instructs the network master to send data to a sensor node but the } \\
\text { master could not achieve the data transfer. }\end{array}$ \\
\hline $\mathbf{0 x 2 0}$ & reserved & Reserved for future use. \\
\hline $\mathbf{0 x 4 0}$ & reserved & Reserved for future use. \\
\hline $\mathbf{0 x 8 0}$ & reserved & Reserved for future use. \\
\hline
\end{tabular}

\subsection{IR PSD Sensor Control}

As the network interface on all sensor nodes is interrupt driven, the main loop of the sensor node's MCU is required only to manage control of the sensor. As mentioned in Section 4.1.1 Sharp IR PSD Ranging Module, the IR PSD requires timing of its control signals to ensure accurate sensor readings are obtained. This timing constraint allows the action of taking a measurement to be separated into defined states which can be implemented as a finite state machine to ensure the sensor is controlled correctly. Figure 5.14 illustrates the state transition diagram for the finite state machine implemented to control the IR PSD sensor measurements. 


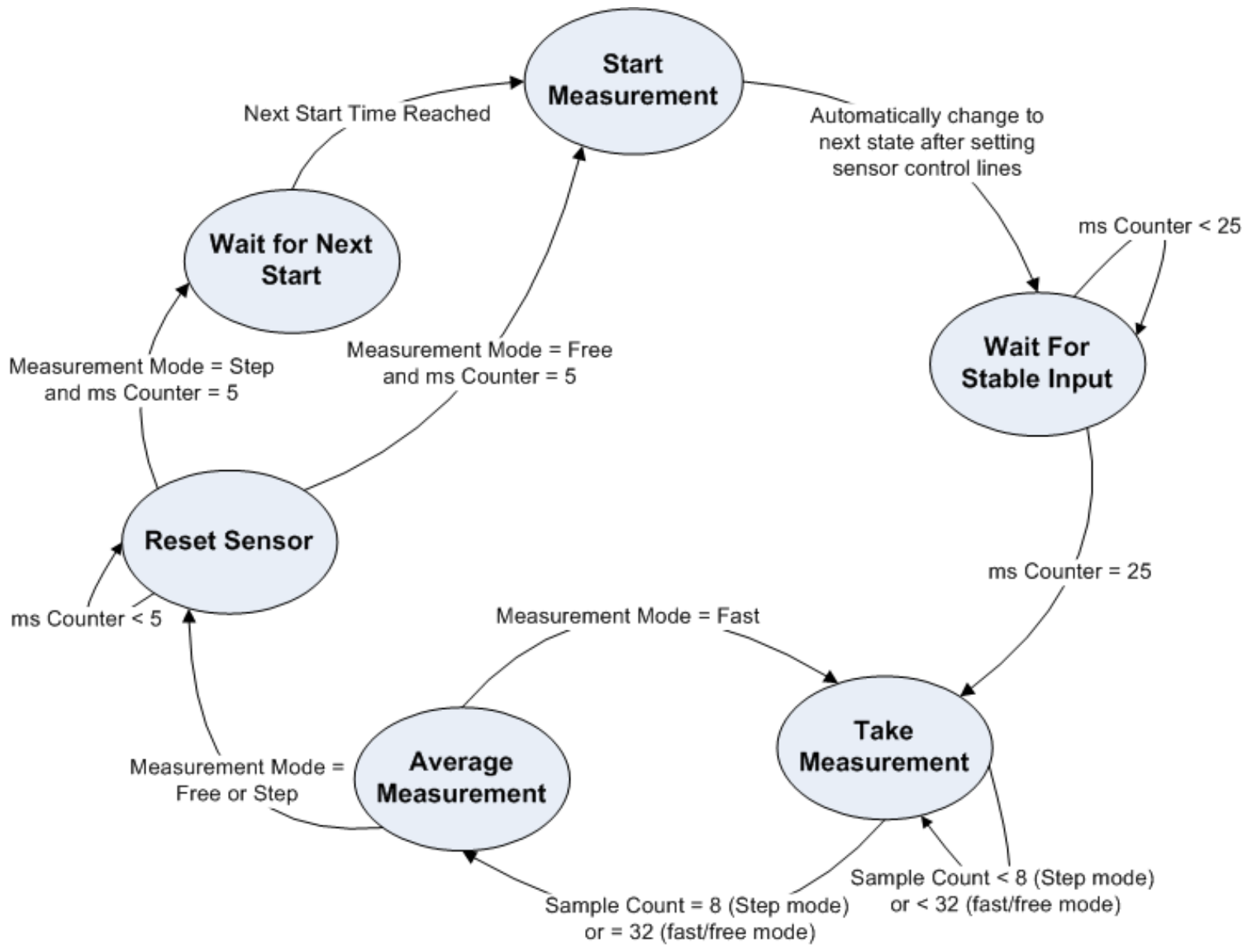

Figure 5.14 State Transition Diagram for IR PSD Finite State Machine

The IR PSD finite state machine has the following states:

- Start Measurement - in this state the MCU sets the control lines of the IR PSD to specify which LED to illuminate and to begin the PSD's signal processing circuit. The MCU uses a pointer to indicate which sensor channel / LED is currently being sampled. The MCU also starts a millisecond counter to monitor how long the FSM has waited before commencing ADC measurements. Once these control lines have been set, the FSM automatically moves to the Wait For Stable Measurement state.

- Wait For Stable Measurement - in this state the MCU waits until the millisecond counter has reached 25. At this point the voltage output of the IR PSD should be stable enough for ADC measurements to begin. The MCU will then instruct the ADC to start a measurement and move the FSM to the Take Measurement state.

- Take Measurement - in this state the MCU instructs the ADC to take samples and sums the ADC samples for averaging. In Free and Fast measurement modes the MCU takes 32 ADC samples. In the Step measurement mode the MCU takes 8 ADC samples for averaging, to ensure the FSM finishes a complete machine cycle before 
the next measurement begins. When the sample number has been reached the FSM moves to the Average Measurement state.

- Average Measurement - in this state the MCU averages the accumulated ADC samples. The ADC sample counts of 32 and 8 have been selected to provide easy division on the MCU. The Free and Fast measurement mode samples are averaged by right shifting the accumulated sample sum by 5 . The Step measurement mode samples are averaged by right shifting the accumulated sample sum by 3 . In Fast measurement mode only one channel of the IR PSD is sampled. Therefore the FSM changes to the Take Measurement state to collect a new set of ADC samples on the current sensor channel. In the other measurement modes the MCU increments the pointer that indicates which sensor channel is active, switches off the control lines to reset the IR PSD sensor circuitry, begins a millisecond counter, and changes the FSM state to Reset Sensor.

- Reset Sensor - in this state the FSM waits until the millisecond counter reaches $5 \mathrm{~ms}$ to ensure the IR PSD sensor has had enough time to reset its internal circuitry. After $5 \mathrm{~ms}$, if in the Free measurement mode, the FSM will change to the Start Measurement state to begin a measurement on the next sensor channel. If in the Step measurement mode, after $5 \mathrm{~ms}$ the FSM will change to the Wait For Next Start state.

- Wait For Next Start - the FSM will remain in this state until the MCU detects the next measurement start time in the measurement window. At this point the FSM will change to the Start Measurement state to begin a measurement on the next sensor channel.

The main loop of the MCU continually calls a function to evaluate the FSM. If the measurement mode is set to Stop, the FSM will be reset to the Start Measurement state and not be evaluated by the function.

\subsection{Ultrasonic Sensor Control}

Control of the ultrasonic sensor is implemented in a similar scheme as the IR PSD sensor. A finite state machine is used to control the process of the measurement. Similar to the IR PSD sensor node, the MCU's main loop is required only to control the ultrasonic sensor. The ultrasonic sensor FSM is simpler than the IR PSD sensor as it does not require setup time to begin a measurement, averaging has not been implemented on sensor readings and the sensor has only one sensor channel to sample. Averaging has not been implemented on the 
ultrasonic sensor due to the timing restrains of the measurement itself. Each ultrasonic measurement requires at worst $60 \mathrm{~ms}$ when ranging an object at $10 \mathrm{~m}$. To achieve a sample rate of $10 \mathrm{~Hz}$ would only allow one sample to be completed before an average would have to be calculated. Figure 5.15 illustrates the implemented FSM.

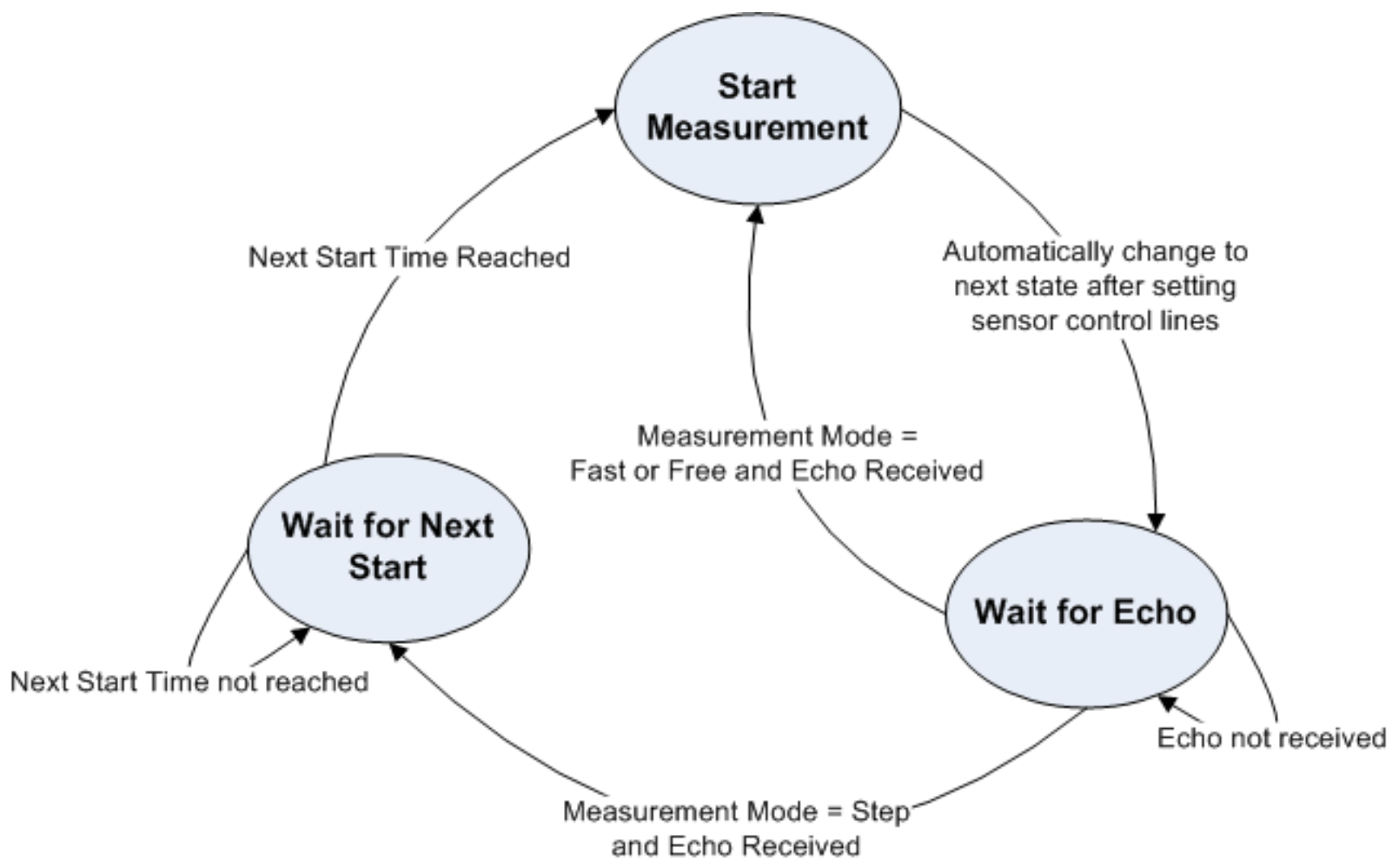

Figure 5.15 State Transition Diagram for Ultrasonic Sensor FSM

The ultrasonic sensor FSM has the following states:

- Start Measurement - in this state the MCU sets the control lines of the ultrasonic sensor to begin a new measurement. The MCU begins a timer to monitor the time between the start of a measurement and the receipt of the ultrasonic echo. The FSM automatically changes to the Wait For Echo state.

- Wait For Echo - the FSM remains in this state until an echo has been received. Once an echo has been received the MCU stops the timer and records the timer count. In the Fast and Free measurement mode, the FSM will change to the Start Measurement state to begin another ultrasonic measurement. If the MCU is in the Step measurement mode, the FSM will change to the Wait For Next Start state.

- Wait For Next Start - the FSM will remain in this state until the MCU detects the next measurement start time in the measurement window. At this point the FSM will change to the Start Measurement state to begin another ultrasonic measurement. 
Similar to the IR PSD sensor node, the main loop of the MCU continually calls a function that evaluates the FSM. In the Stop measurement mode the function does not evaluate the FSM and forces the FSM into the Start Measurement state. In any other measurement mode the FSM is evaluated and ultrasonic measurements are conducted.

The firmware described in the previous sections has been implemented and tested on the three hardware systems: the Sensor Network Interface board, the IR PSD Module and the Ultrasonic module. PC Software, discussed in Chapter 6 Methodology - Sensor Network PC Software, has been developed to test the operation of both the network communications and the sensor control firmware. 


\section{Chapter 6 Software Methodology - Sensor Network}

This chapter details the development of PC based software that interfaces a control computer to the sensor network. It presents the $\mathrm{C \#} \mathrm{libraries} \mathrm{written} \mathrm{to} \mathrm{access} \mathrm{the} \mathrm{sensor} \mathrm{network,} \mathrm{a}$ basic graphical command packet interface and an application to graphically illustrate the distance data from the test sensor network developed for MARVIN.

\subsection{Development Environment}

Currently the majority of robotic platforms in the VUW fleet have a control computer running variants of the Microsoft Windows operating system (OS). It has been anticipated that future control computers will continue to run the Windows operating system and as such the development of the software interface to the sensor network has been targeted to run on a Windows XP OS.

The software libraries and application programs that access the sensor network have been developed to run on the .NET framework. The .NET framework supports multiple programming languages and allows language interoperability [38]. This interoperability allows future users to design software applications in other languages that can access the sensor network software libraries.

Microsoft Visual Studio has been used as the integrated design environment (IDE) to develop software for the sensor network. The IDE allows libraries, console and graphical applications to be designed, programmed, debugged and finally published. All software libraries and applications have been written in the $\mathrm{C \#}$ programming language.

C\# is a general purpose, object-oriented programming language. It is suitable for writing applications for both hosted and embedded systems.

\subsection{C\# Sensor Network Libraries}

Several C\# libraries have been written to allow access to the sensor network. These libraries form the basic framework upon which graphical and console applications can be written to allow a control computer to manipulate the sensor network. 
The control computer accesses the sensor network interface board over a USB connection which appears on the Windows OS as a virtual serial port. The .Net frame work provides a SerialPort class that allows a C\# program to access serial ports available on the OS. The main purpose of the Sensor Interface library is to provide communication between the control computer and the sensor network over the virtual serial port. Therefore the Sensor Interface library is an extension of the SerialPort class with additional services to ensure the serial port communication meets the specifications of the command interface described in the previous chapter. The Sensor Interface library is built on top of other C\# classes that provide encapsulated functionality that allow robust operation and reuse of classes. Figure 6.1 presents the structure of the Sensor Interface library.

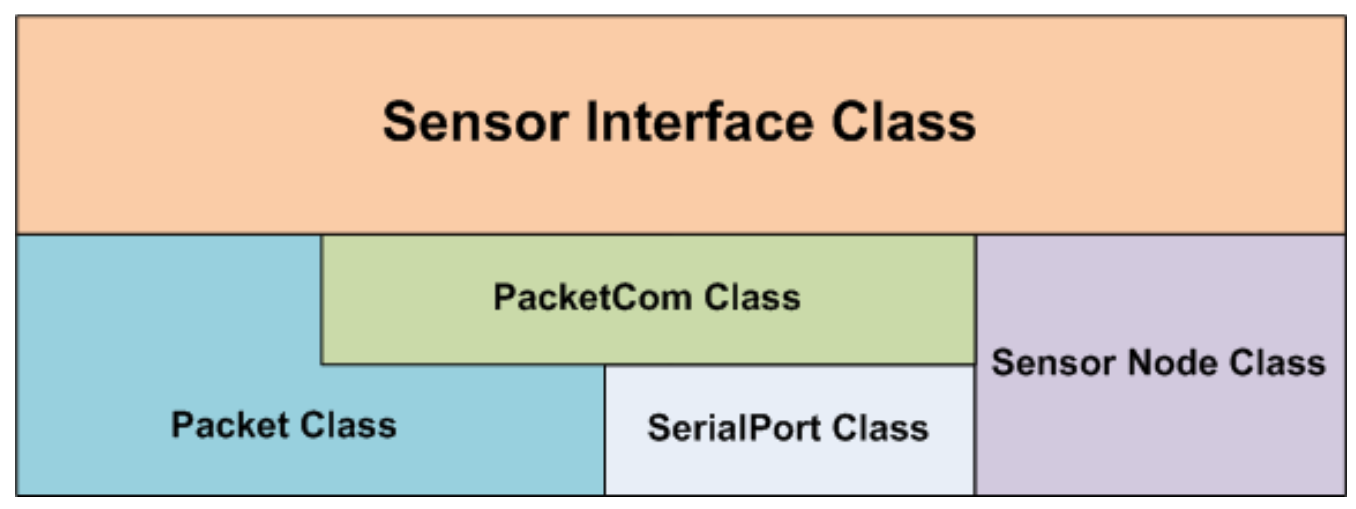

Figure 6.1 Structure of Sensor Interface Library

The Sensor Interface library has been written as a C\# class. The Packet, PacketCom and SerialPort classes provide all the functionality required to create, transmit and receive packets, which meet the command interface specification, over the virtual serial port. The Sensor Interface and Sensor Node classes provide the services to access and reconfigure the sensor network and compute calibrated sensor measurements.

\subsubsection{Packet Communication}

The SerialPort class provides only the basic functionality to open, configure, close a serial port and send and receive data bytes. The PacketCom and Packet classes have been developed to extend the capabilities of the SerialPort class so that together they can open, configure and close a serial port but also have the ability to create, send and receive packets that meet the packet format used in the command interface. 


\subsubsection{Packet Class}

The packet class provides the framework to create packets that meet the command interface packet format. It has internal private functions that automatically calculate the packet checksum whenever data is added, as well as checking the validity of any data written to packet fields. The software interface must be able to handle two types of packets: request and response. A request packet is one created by the software interface to be sent to the sensor network. A response packet is the packet that is received by the software interface from the sensor network. Although these packets have the same format they must be handled in different ways. For example, when creating a request packet, the software interface should not be able to manually set the checksum field. This field should be read-only as the packet class should automatically compute the checksum every time new data is added to the packet. Conversely when bytes are received from the sensor network interface board on the virtual serial port they must be reconstructed into a packet. This requires the checksum field to be writable for reconstructing response packets from the byte stream of the virtual serial port.

The packet class has several public fields accessible to the software interface. Table 6.1 presents these fields.

Table 6.1 Public Fields of the Packet Class

\begin{tabular}{|c|c|l|}
\hline $\begin{array}{c}\text { Packet Field } \\
\text { Packet ID }\end{array}$ & Data Type & \multicolumn{1}{c|}{ Description } \\
\hline $\begin{array}{c}\text { Packet Byte } \\
\text { Count }\end{array}$ & int & $\begin{array}{l}\text { This is a read/write field for the ID of the packet. } \\
\text { packets. It is automatically calculated by a private function } \\
\text { within the packet class. }\end{array}$ \\
\hline Checksum & int & $\begin{array}{l}\text { This is a read-only field for request packets and a read/write } \\
\text { field for response packets. For request packets a private } \\
\text { function within the packet class calculates the checksum } \\
\text { value. }\end{array}$ \\
\hline $\begin{array}{c}\text { Error Code } \\
\text { Request }\end{array}$ & int & $\begin{array}{l}\text { This is a read-only field for request packets and a read/write } \\
\text { field for response packets. For request packets the error code } \\
\text { is always set to 0x01 as the error code field is only utilised in } \\
\text { response packets. }\end{array}$ \\
\hline bool & $\begin{array}{l}\text { This is read/write field that allows the software interface to } \\
\text { specify if the packet is a request or response packet. This } \\
\text { field determines which of the other packet fields are read- } \\
\text { only. }\end{array}$ \\
\hline
\end{tabular}


The packet class also provides several public functions to allow the software interface to configure the packet. These functions are:

\section{Packet()}

This constructor (Figure 6.2) is called whenever a new packet is instantiated. It creates a default packet which the software interface can then reconfigure by calling the other public functions and writing to the packet fields. The default packet has a Dummy packet ID code with a zero size data payload. Figure 6.2 shows the structure.

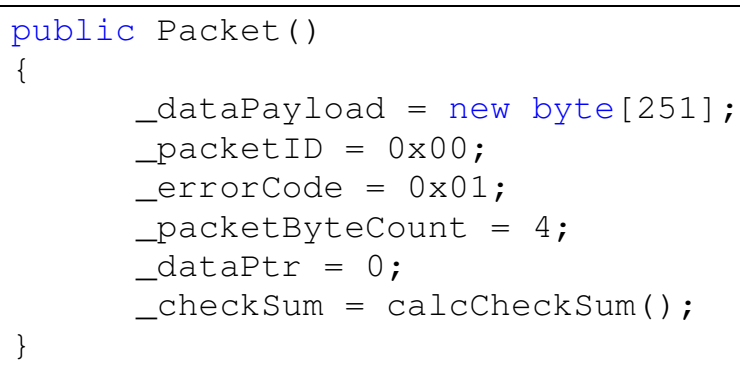

Figure 6.2 Constructor Function for Packet Object.

\section{void addData(byte data)}

This function (Figure 6.3) allows the software interface to add a data byte to the packet data payload. The internal data payload buffer can hold 251 data bytes. It calls private functions to recalculate the checksum and packet byte count field each time a data byte is added to the data payload.

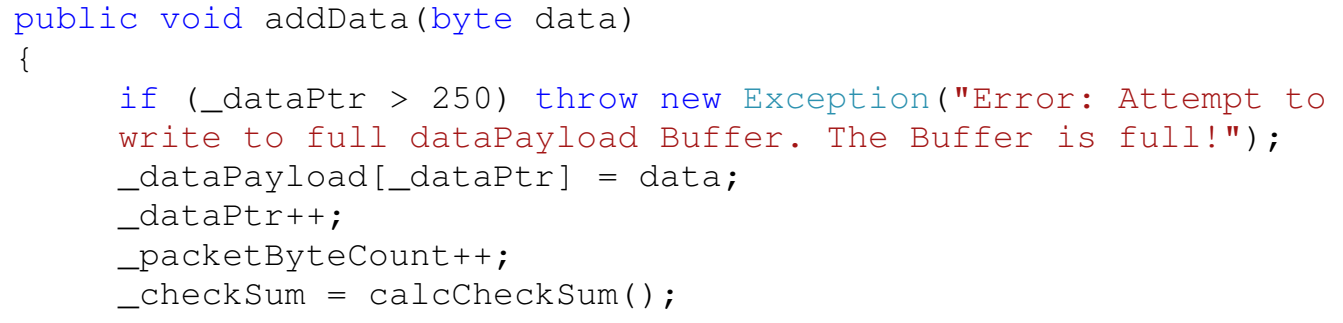

Figure 6.3 Function to add data to packet data payload.

\section{byte readData(int ptr)}

This function (Figure 6.4) allows the software interface to read a data byte, specified as a function parameter, from the data payload buffer. It has internal checking to ensure that the specified ptr value indexes a valid location in the data payload buffer. 


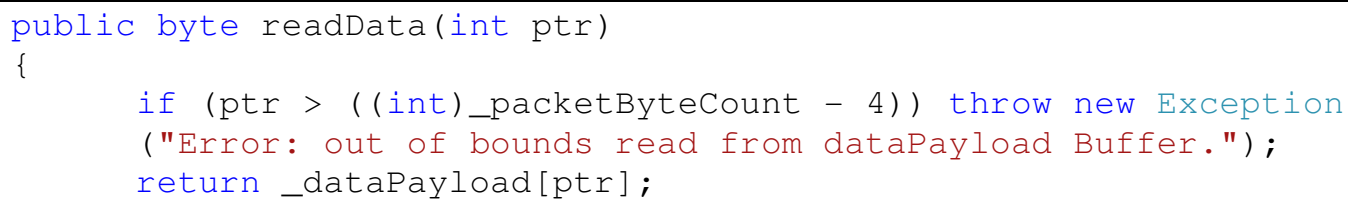

Figure 6.4 Function to read data from a packet data payload.

\subsubsection{PacketCom Class}

The PacketCom class provides the functionality to open, configure and close a serial port, as well as the ability to send and receive data packets and basic functionality to convert data packets fields into human readable characters. The PacketCom class utilises the Packet class so that it can handle packets across the serial port. The PacketCom class has two public fields that allow the software interface to configure a serial port. These fields are:

\section{string PortName}

This field allows the software interface to define which serial port will be used for communication between the control computer and the sensor network interface board. Any value of this field is checked against a list of the available serial ports on the control computer to ensure it is a valid port.

\section{int BaudRate}

This field allows the software interface to set the data transfer speed of the serial port.

The PacketCom class also has several public functions allowing packet transfer across the serial port. The main functions are:

\section{public PacketComm()}

This constructor (Figure 6.5) allows the software interface to create a new serial port object to access an available serial port on the control computer.

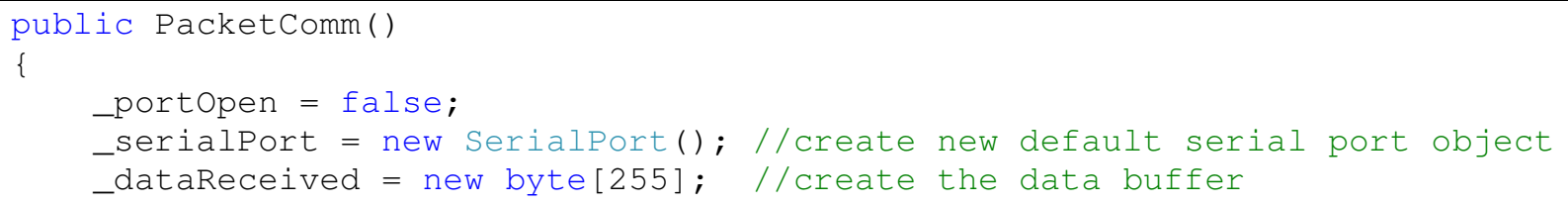

Figure 6.5 Constructor for PacketComm Object. 


\section{void OpenComPort()}

This function instructs the PacketCom class to open a serial port object specified by the PacketCom fields.

\section{void CloseComPort()}

This function instructs the PacketCom class to close a serial port object specified by the PacketCom fields.

\section{void WritePacket(Packet TXPacket, Packet RXPacket)}

This function is passed two packet objects: a request packet and a response packet. The request packet object holds the packet information that will be sent over the serial port as a byte stream. The function automatically checks for control character bytes in the byte stream and carries out byte stuffing, as mentioned in section 4.3 Control Computer/Network Command Interface, when control characters are found. Figure 6.6 shows the code segment that performs the transmission of the packet bytes and byte stuffing.

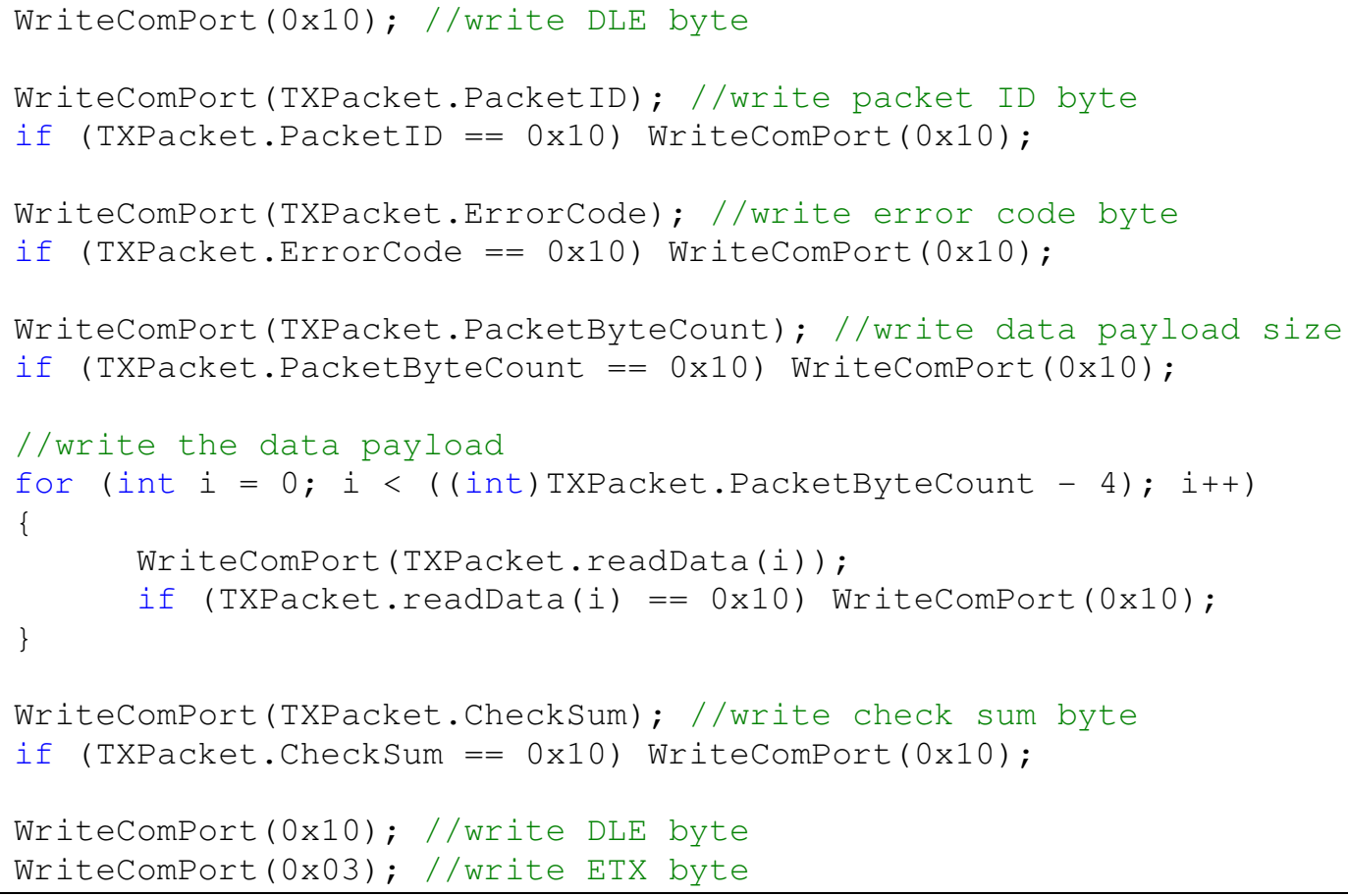

Figure 6.6 Transmit Packet Code Segment

Figure 6.7 illustrates the transmission of packet bytes and how byte stuffing is implemented to help distinguish control bytes from valid packet bytes. An odd 
number of consecutive control bytes indicates the bytes should be interpreted as control bytes. An even number of control bytes indicates the bytes should be interpreted as packet bytes.

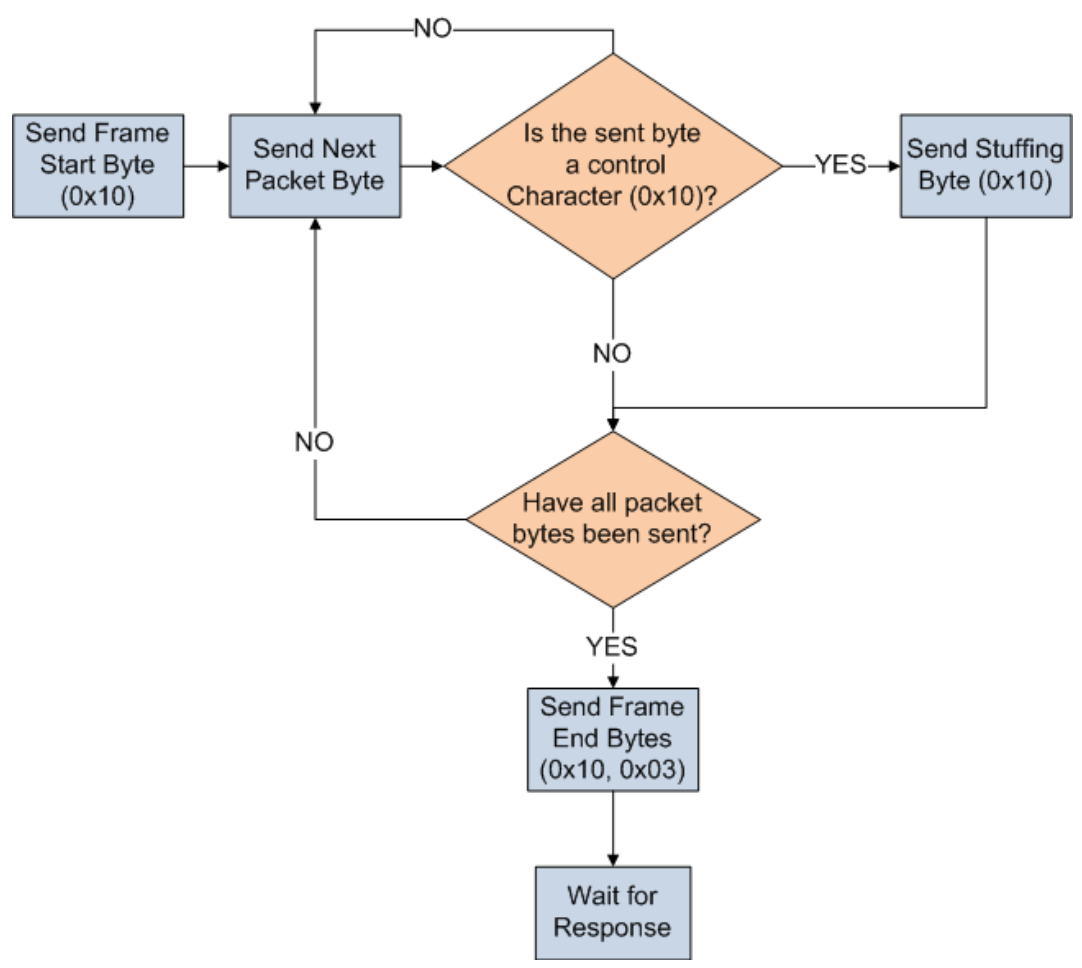

Figure 6.7 Transmit Packet Data Overview

The function remains in a While loop after packet transmission to wait for a response from the sensor network. The internal event-driven data receive functions of the serial port object receive the byte stream from the sensor network and parse the stream for a response packet. Once a response packet has been found in the byte stream the WritePacket function extracts the packet bytes from the byte stream and writes them to the response packet object. The function automatically parses the byte stream for stuffed bytes and removes them. Figure 6.8 shows the code segment that parses the serial byte stream for packet bytes and removal of stuffed bytes. 


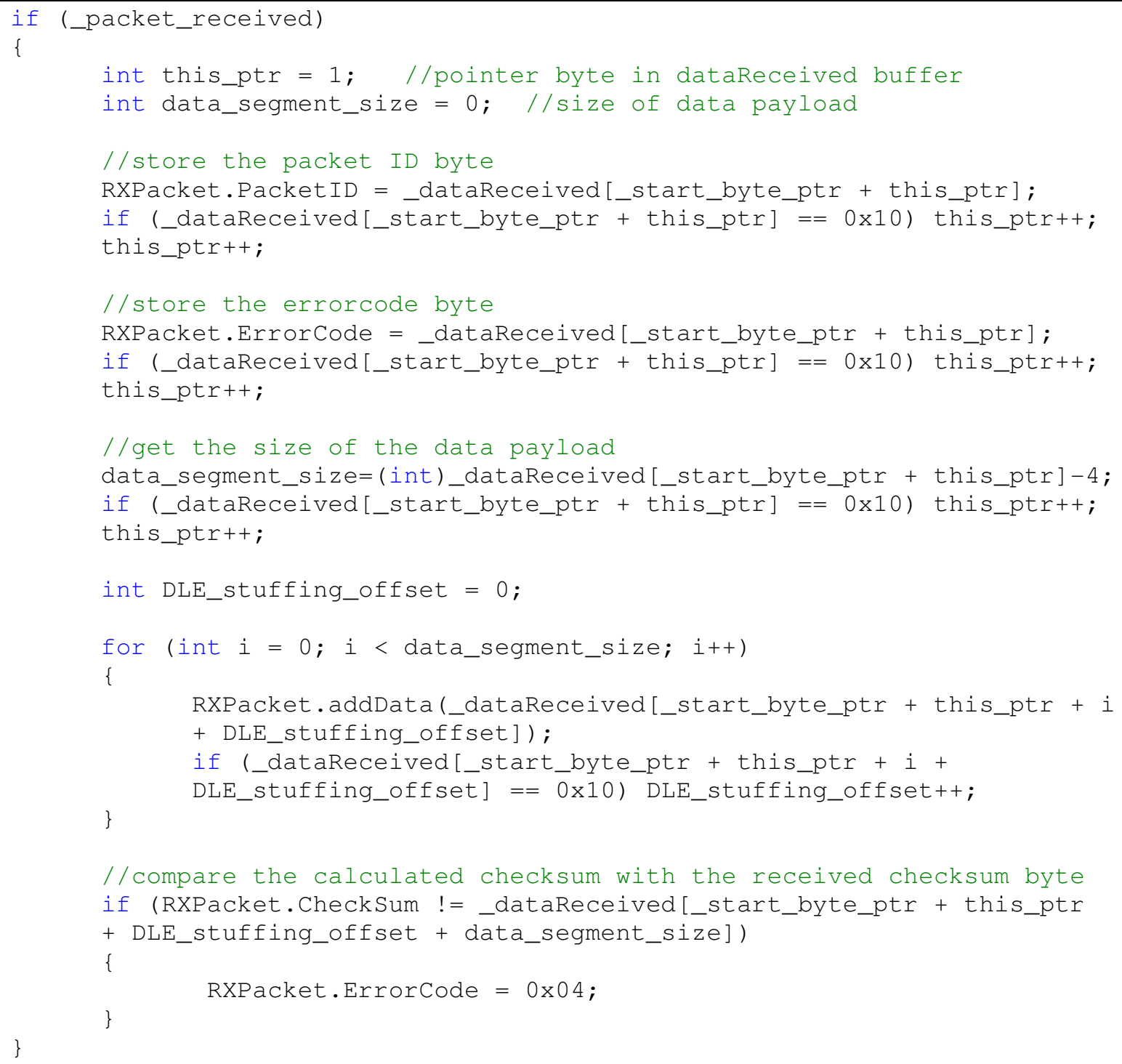

Figure 6.8 Receive Packet Code Segment

The PacketCom class has two formatting functions to convert packet bytes to and from human-readable characters to assist in debugging of the class. These functions are:

\section{int convertHex2Int(char HexValue)}

This function allows a user to convert an ASCII character, representing a hexidecimal character, into an integer value of the hexidecimal value. For example, the ASCII charcater ' $F$ ' will return the integer value 15. 


\section{string convertInt $2 \mathrm{Hex}$ (int number, int places)}

This function allows a user to convert an integer value into an ASCII character string that represents the integer in hexidecimal form. For example, the integer value 255 will return the ASCII charcater string 'FF'. The places parameter allows the user to specify how many hexi-decimal places should be returned.

An example of a user application using the PacketCom and Packet class for sending data over the serial port is the following:

1. The user application opens a serial port by calling the PacketCom() constructor with a valid serial port name and a baudrate supported by the Sensor Network interface board.

2. The user application then opens the serial port by calling the OpenComPort() function.

3. The user application creates a request packet object by calling the Packet() constructor and setting the Request packet field to true.

4. The user application creates a response packet object by calling the Packet() constructor and setting the request packet field to false.

5. The user application configures the request packet object with a packet ID, by writing to the Packet ID field, and Data Payload, by calling the addData() function.

6. The user application calls the WritePacket(Packet TXPacket, Packet RXPacket) function with the request and response packet objects as the function parameters. The request packet object is sent to the sensor network as a byte stream over the serial port. The response packet object is populated with data received from the Sensor Network interface board as a byte stream over the serial port.

7. The user application can now parse the response packet object's data payload for the information it requested by calling the getData() function.

\subsubsection{Sensor Interface}

The software interface to the Sensor Network is composed of two classes. A sensor node class that stores information on an individual sensor node, and a sensor interface class that provides functionality to access and configure the sensor network. 


\subsubsection{Sensor Node Class}

To maintain information on individual sensors in the network, the software interface uses sensor node objects. The sensor node class provides the definition of a sensor node object. A sensor node class represents an actual sensor connected to the network. The class contains fields that store the information that uniquely defines each sensor, as well as functionality to calculate distance values from sensor data.

It is important for each sensor node object to maintain the configuration information that has been sent to the actual sensors. This allows the control computer to provide accurate information on the sensor network sampling behaviour and model the external environment from sensor data. The sensor node fields that keep a copy of the configuration information on a sensor are the following:

\section{int network_address}

This field stores the logical network address for a sensor. It allows the control computer to instruct the network master to send information to a specific sensor.

\section{uint calibrationID}

This field stores the unique calibration ID for each sensor. This calibration ID is used by the control computer to look up calibration and orientation information on the sensor from a configuration file.

\section{uint number_of_channels}

This field stores the number of channels available on the sensor.

\section{uint measurement_mode}

This field stores the current measurement mode that the sensor has been configured with.

\section{uint start_time}

This field stores the start time in a measurement window when the sensor has been configured with a Step mode measurement task. 


\section{uint step_time}

This field stores the time between sensor measurements in a measurement window for Step mode measurements.

\section{uint active_channel}

This field stores the channel selected for Fast mode measurements.

A distance sensor allows the robotic platform to determine the distance to an object in a three dimensional environment. The orientation and position of the sensor must be known for the control computer to accurately model this environment. As illustrated in Figure 6.9, in order to accurately determine the location of the cardboard box not only does the distance from the sensor to the box need to be known but also the angle, inclination and mounting position of the sensor. In Figure 6.9 the sensor is mounted at the origin.

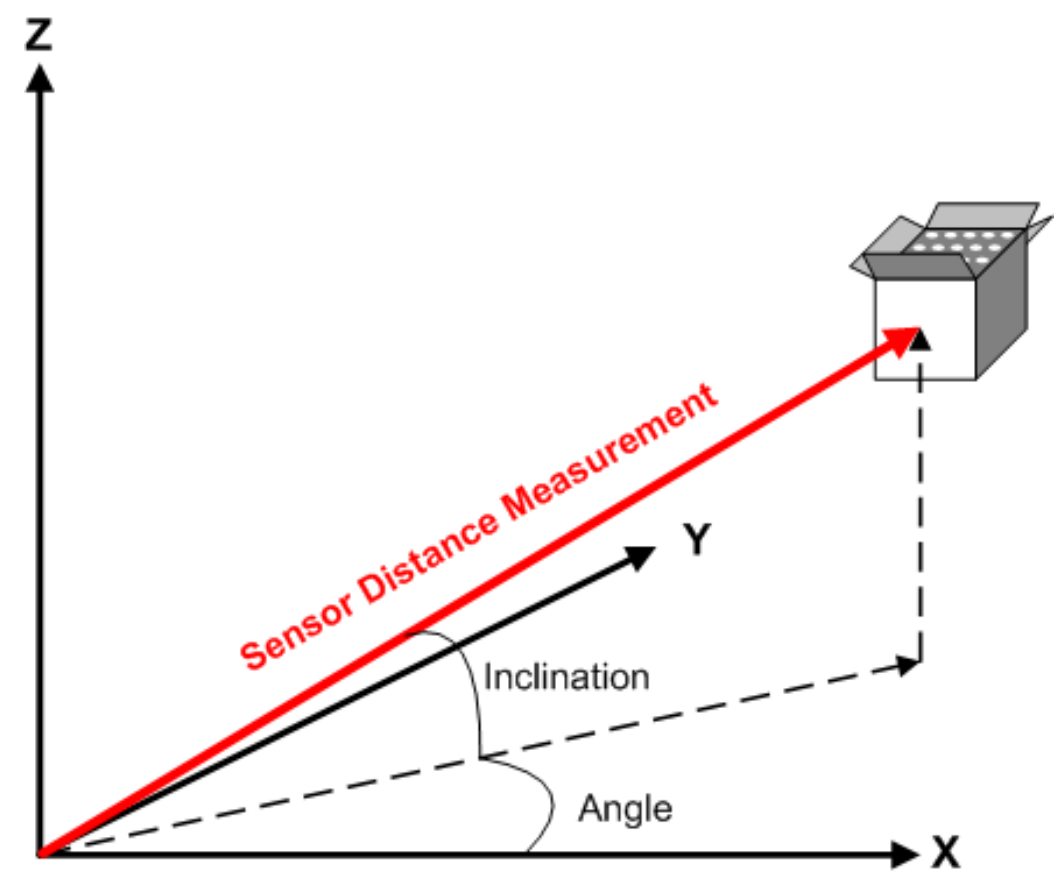

Figure 6.9 Orientation of Sensor measurement in 3-D Environment

When a sensor is fixed to a robotic platform, the mounting point, inclination and angle can be measured. Provided the mounted sensor's position remains static, these values will not change and therefore can be continually used to calculate location of obstacles from sensor distance measurements. In three dimensions, the mounting point can be defined by three Cartesian co-ordinates from a referenced origin, ideally the centre point of a robotic platform. The sensor node object stores this orientation information to allow the control computer to 
determine where, in a three dimensional space, the sensor has detected an object. These fields are:

\section{uint mountX}

The X co-ordinate of the sensor mounting point from a referenced origin.

\section{uint mountY}

The Y co-ordinate of the sensor mounting point from a referenced origin.

\section{uint mountZ}

The $\mathrm{Z}$ co-ordinate of the sensor mounting point from a referenced origin.

\section{double inclination}

The angle from the X-Y plane that the sensor is facing.

\section{double angle}

The angle from the $\mathrm{X}$ axis that the sensor is facing.

The sensor node object also contains functionality to calculate the magnitude of the distance measured from the dimensionless sensor data received from the network. This requires the sensor node object to store the calibration data for the sensor it represents. The calibration data varies from sensor to sensor so must be loaded into the sensor node object at instantiation. Currently distance measurements for the Sharp wide angle sensors and SensComp ultrasonic sensors are calculated from a power equation. The calibration data is stored as the co-efficient, exponential and offset of Equation 6.1.

$$
\text { Distance }=A x^{B}+C
$$

\section{Equation 6.1}

In equation $6.1 x$ represents the dimensionless sensor data, $A$ represents the co-efficient term, $B$ represents the exponential term and $C$ represents an offset. The $A, B$ and $C$ terms uniquely define the relationship between distance and sensor data for a specific sensor channel. 
The sensor node object functions are:

\section{sensor_node()}

This constructor (Figure 6.10) is called whenever a new sensor node object is created.

It populates the sensor node object's fields with default data.

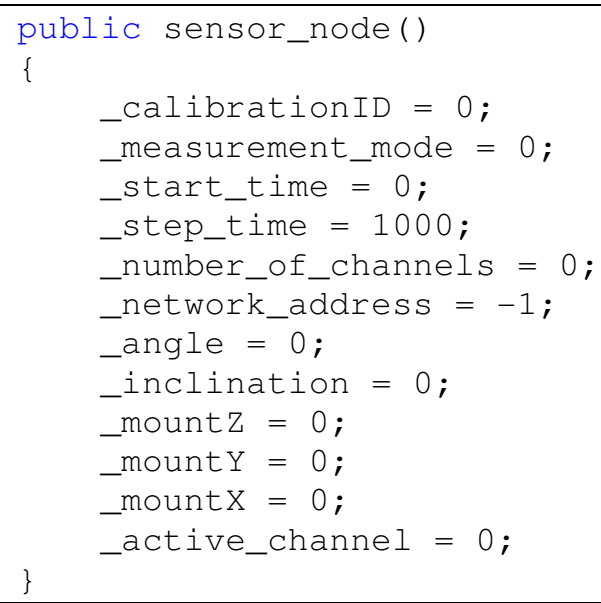

Figure 6.10 Constructor for Sensor Node Object.

\section{double getDistance(int channel, int adc_reading)}

This function, presented in Figure 6.11, takes an ADC reading and returns a double representing the distance the sensor has measured. As a sensor can have multiple channels and each channel will have a different calibration equation, the sensor channel must be specified to compute the distance.

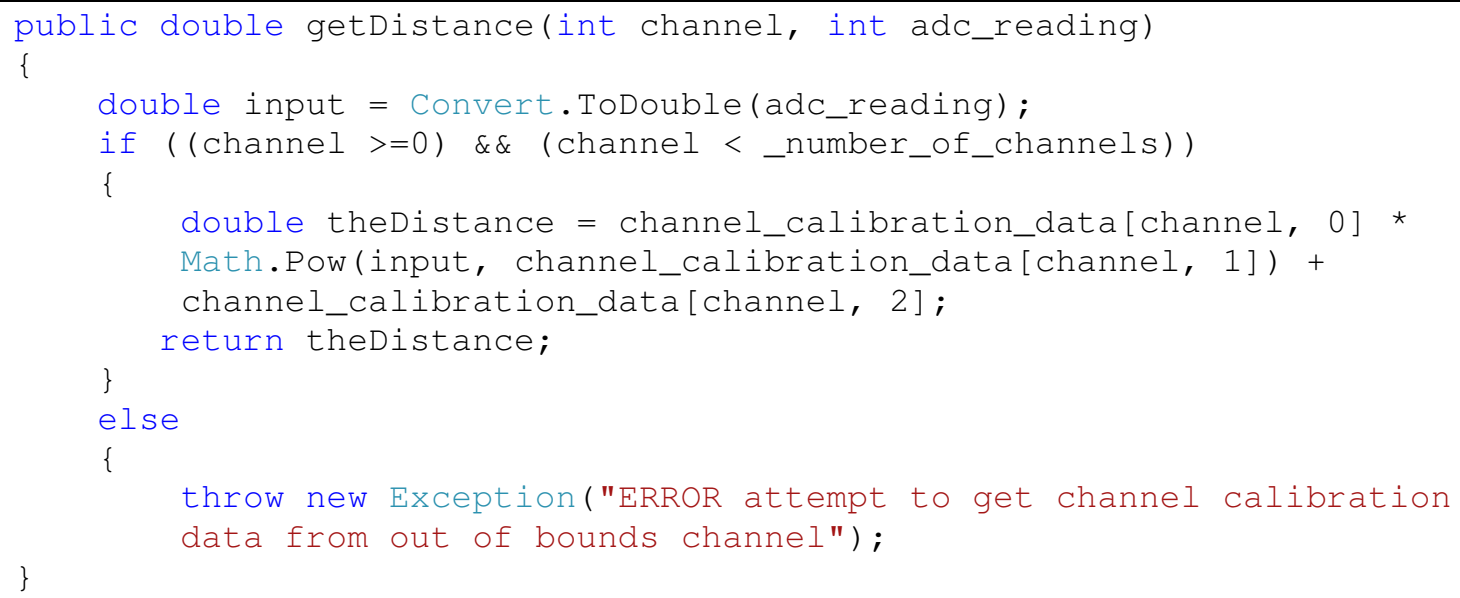

Figure 6.11 Distance Calculation Code Segment. 
void setChannelCalibrationValues(uint channel, double A, double B, double C)

This function (Figure 6.12) allows a sensor node object to be loaded with calibration data unique to the sensor. Calibration co-efficient, exponential and offset values must be loaded for each of the sensor's channels.

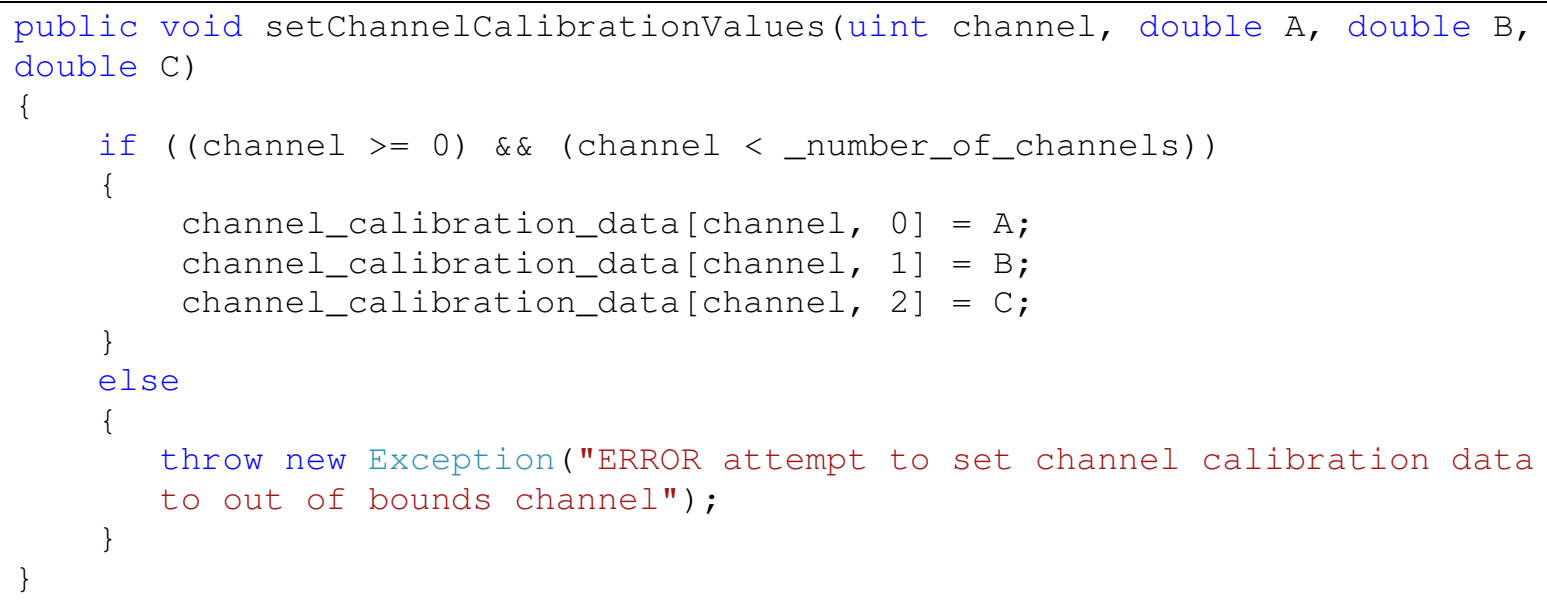

Figure 6.12 Function to set calibration values from configuration file.

\section{double C(uint channel)}

This function returns the calibration offset term. The sensor channel must be selected to specify which $\mathrm{C}$ term is requested.

\section{double B(uint channel)}

This function returns the calibration exponential term of the power function. The sensor channel must be selected to specify which B term is requested.

\section{double A(uint channel)}

This function returns the calibration co-efficient of the power function. The sensor channel must be selected to specify which A term is requested.

The data fields and functionality of a sensor node object allow a software interface to store and process information unique to a sensor attached to the sensor network.

\subsubsection{Sensor Interface Class}

The sensor interface class is the top layer of the sensor network interface between a software application running on the control computer and the sensor network. The sensor interface class provides all the services required to configure the sensor network, as well as maintain 
information on the sensors that make up the network. The sensor class utilises the Packet and PacketCom classes to communicate to the sensor network interface board, and utilises the sensor node class to maintain information on sensors attached to the network.

A sensor network can be constructed from any number of sensors, mounted in a variety of ways and orientations. The sensor interface must be flexible enough to allow different variations of networks to be managed by the control computer. A configuration file is used to store information on individual sensors. When the sensor interface asks for the network definition from the sensor network interface board, it uses the unique calibration ID of a sensor to search the configuration file for further information on the sensor. A user is required only to alter the configuration file, after making changes to the network, to ensure that the sensor interface works correctly.

Table 6.2 lists the data fields of the configuration file.

Table 6.2 Configuration File Data Fields

\begin{tabular}{|c|c|c|}
\hline Config Field & Data Type & Description \\
\hline sensor_port & string & $\begin{array}{l}\text { This field contains the name of the serial port that the sensor } \\
\text { network is connected to. }\end{array}$ \\
\hline baudrate & int & $\begin{array}{l}\text { This field contains the recommended baud rate for the serial } \\
\text { port that the sensor network is connected to. }\end{array}$ \\
\hline nodeTotal & int & $\begin{array}{l}\text { This field contains the total number of sensor entries in the } \\
\text { configuration file. }\end{array}$ \\
\hline nodes & string & $\begin{array}{l}\text { This field contains the information unique to the sensors on } \\
\text { the network. The field is comma delimited and every sensor } \\
\text { requires twelve comma delimited terms. The construction of } \\
\text { a sensor entry is the following: } \\
\text { [Calibration ID], [Angle], [MountX], [MountY], [MountZ], } \\
\text { [Inclination], [Measurement Mode], [Start Time], [Step } \\
\text { Time], [Number of Channels], [Active Channel], [Pointer to } \\
\text { Calibration Data] }\end{array}$ \\
\hline $\begin{array}{l}\text { Calibration } \\
\text { Data Pointer }\end{array}$ & string & $\begin{array}{l}\text { This field contains the calibration values for a sensor. The } \\
\text { [Pointer to Calibration Data] entry in the nodes field links a } \\
\text { sensor with calibration data. The string is comma delimited } \\
\text { where each term holds a calibration value. The number of } \\
\text { terms in the string is dependent on the number of channels in } \\
\text { a sensor. For a wide angle IR PSD sensor with five channels, } \\
\text { there will fifteen terms (three terms for each channel). The } \\
\text { construction of a calibration entry for a two channel sensor is } \\
\text { the following: } \\
\text { [Channel 0 A Term], [Channel 0 B Term], [Channel } 0 \text { C } \\
\text { Term] [Channel } 1 \mathrm{~A} \text { Term], [Channel } 1 \mathrm{~B} \text { Term], [Channel } 1 \\
\text { C Term]. }\end{array}$ \\
\hline
\end{tabular}


The sensor interface class contains an array of sensor node objects, where each sensor node represents a sensor attached to the network.

Figure 6.13 presents an example of a configuration file for a sensor network. An application parses the configuration file for key indexes and returns the associated value string. A user can alter the value string to specify the configuration of the network.

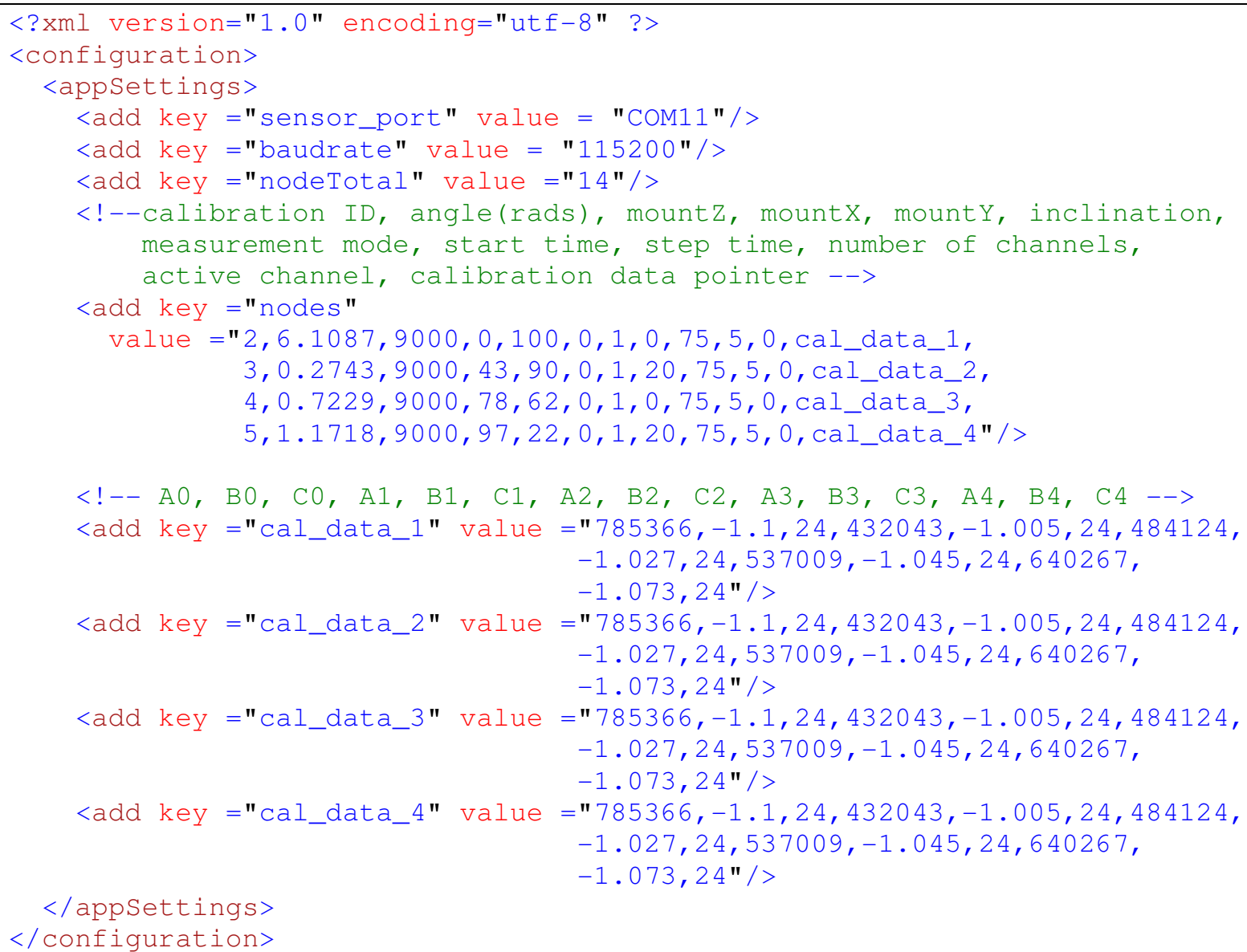

Figure 6.13 Example Configuration File for a Sensor Network

The sensor network class provides the following functions to access the network:

\section{SensorInterface()}

This function is a constructor for a Sensor Interface object. It opens a serial port via the PacketCom object, resets the network and requests the network definition from the Sensor Network interface board. The constructor searches the configuration file for sensor information that matches the sensors defined in the network definition. When a match is found the constructor extracts the sensor information from the configuration file and stores it in a sensor node object that is mapped to the sensor on the network. The configuration information contains a default measurement set up for 
the sensor that the constructor uses to instruct the network master to configure the sensor accordingly. Once every sensor has been configured the constructor resets the network time. Figure 6.14 illustrates the process of the constructor.

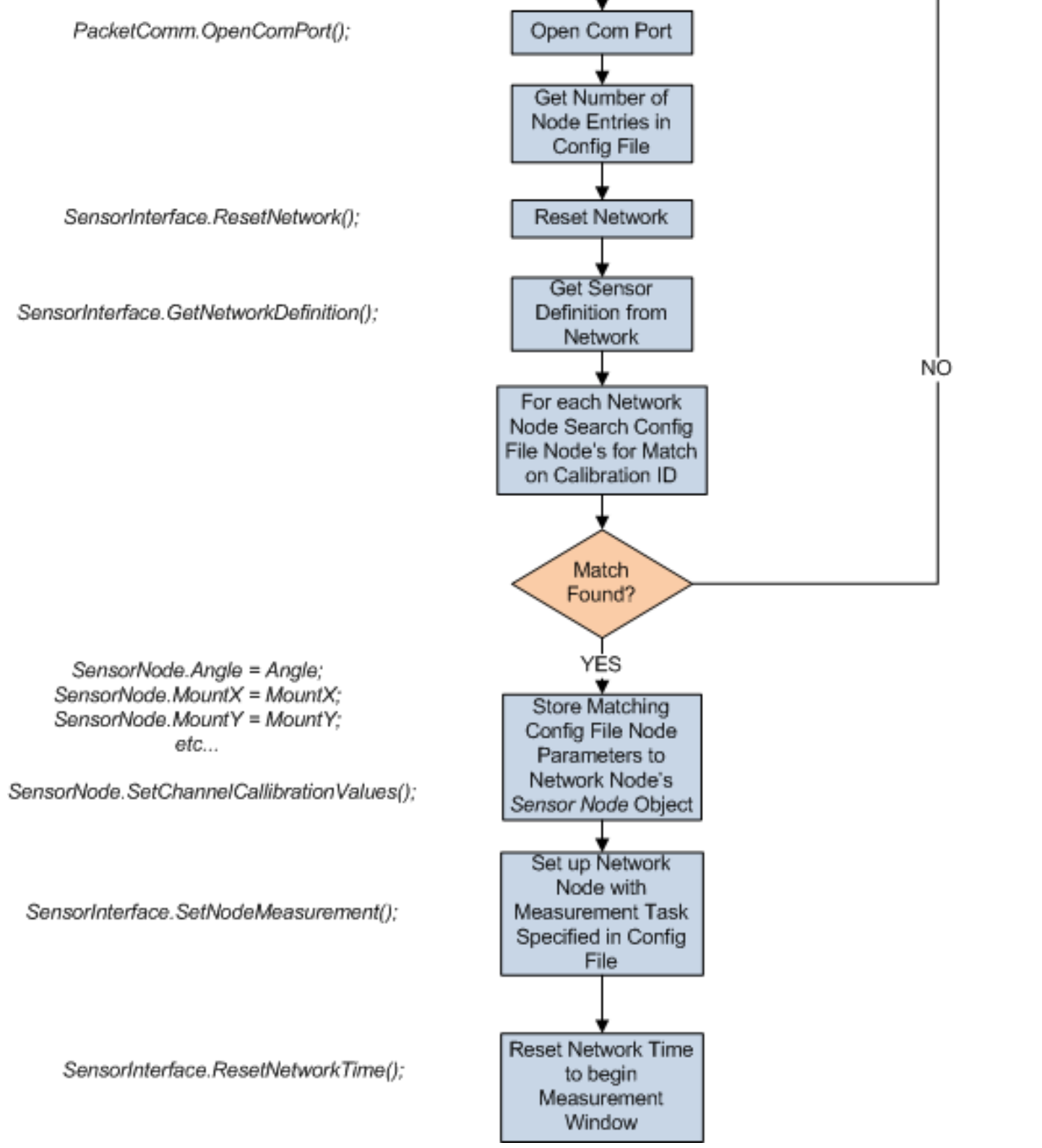

Figure 6.14 SensorInterface Constructor Process 
The constructor calls functions from the Sensor Interface, PacketCom and Sensor Node classes to discover the identity of the nodes that make up of the network, match them to entries in the configuration file and configure the network nodes with default measurement tasks defined in the configuration file. In Figure 6.14 the functions called by the Constructor are listed to the left of the flow chart and illustrate the interaction between the different classes.

\section{void ResetNetwork()}

This function instructs the Sensor Network interface board to reset the network. This causes every sensor node to be reset and the Sensor Network Interface board to query the network for a new network definition. Ideally an application should pause accessing the network for 2 seconds after calling this function to ensure the hardware has completed initialisation. Figure 6.15 illustrates the code for resetting the network. Two new Packet objects are instantiated, a request packet to send the reset network command and a response packet to locally store the packet sent from the network in response. The resquest packet's PacketID field is set to the ID value for a reset network command. Finally both packets are passed to the WritePacket function to be transmitted to the network. The majority of Sensor Interface functions follow this function structure.

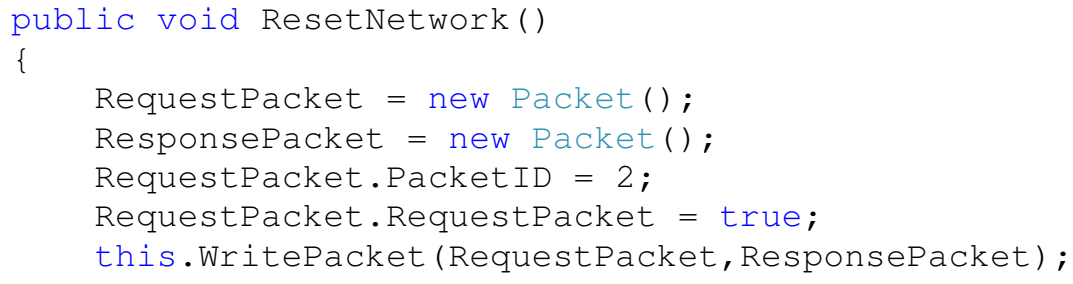

Figure 6.15 Code Example: ResetNetwork Function

\section{int SetAutoUpdate(int state)}

This function allows an application to instruct the Sensor Network Interface board to actively poll the sensor network for new sensor data. The polling rate is set to $50 \mathrm{~ms}$ in the Sensor Network Interface board's firmware. By setting the state parameter to 1 active polling will be enabled, by setting state to 0 active polling is disabled. 


\section{int SetGlobalMode(int mode)}

This function allows an application to set the measurement mode of all connected sensor nodes on the network. It is useful for issuing a Stop mode to force the network to stop taking measurements, as well as globally setting the Step measurement mode to ensure all sensors are ready to begin measurements when the next measurement window begins.

\section{int GetNetworkDefinition()}

This function requests the network definition from the Sensor Network Interface board. It uses the network definition to redefine the size of the array holding the sensor node objects to match the number of detected sensors on the network. It then populates each sensor node object with the network address and calibration ID it received in the network definition. Figure 6.16 illustrates the GetNetworkDefinition function. The GetNetworkDefiniton request packet is sent to the network and the response packet's data payload is parsed for the network definition. The number of network nodes is read from the response packet and is used to redefine the array of Sensor Node objects. These node objects are then populated with node addresses and calibration IDs from the response packet. The function returns the response packet's ErrorCode value to indicate if the command was succesfully executed by the network.

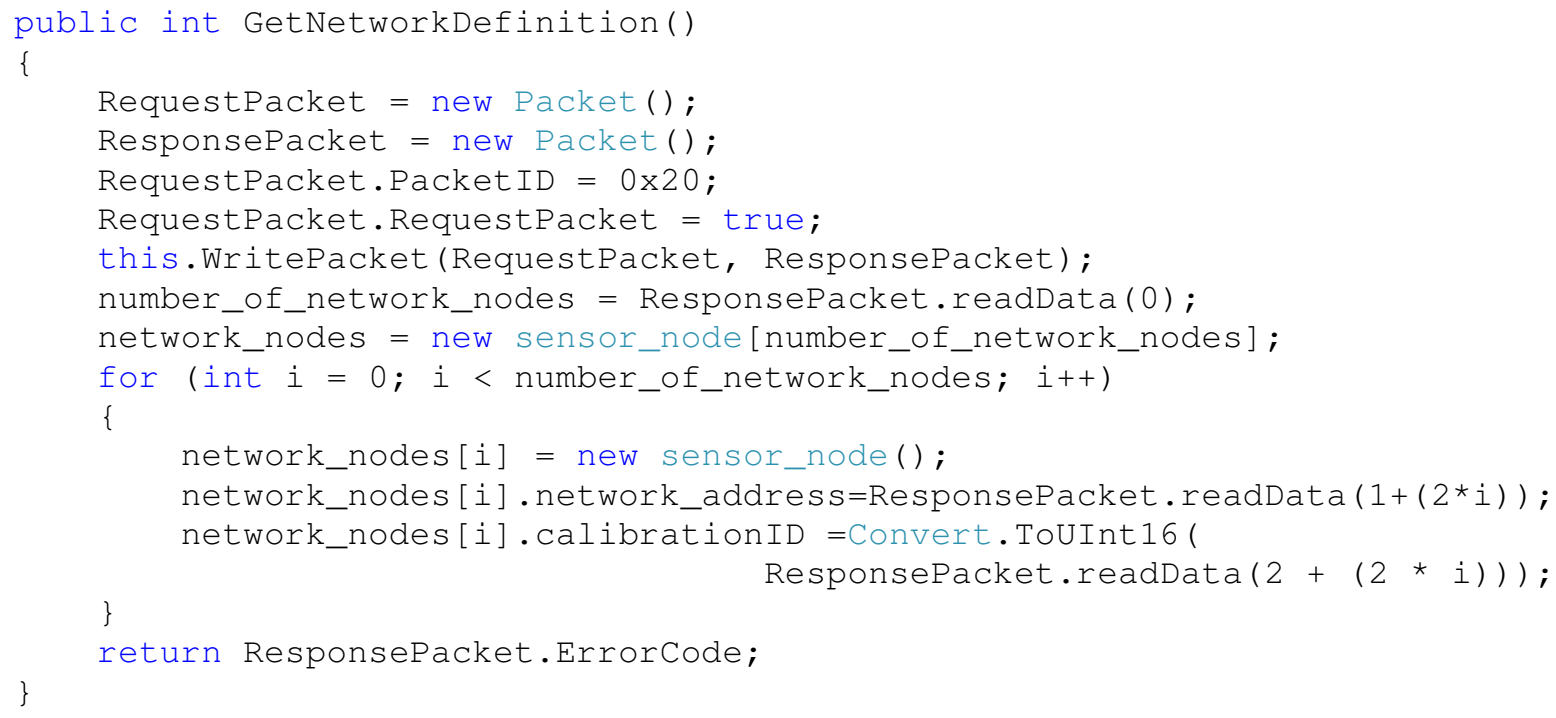

Figure 6.16 Code Example: GetNetworkDefinition Function 


\section{int GetNodeInfo(int node)}

This function requests sensor information from a specified sensor. It returns the calibration ID, number of sensor channels, the measurement mode, the start time and the step time of the sensor.

\section{int SetNodeMode(int node, int mode)}

This function allows an application to set the measurement mode for a specific sensor.

int SetNodeMeasurement(int node, int mode, uint startTime,uint stepTime, int channel )

This function allows an application to write a sensor measurement task to a specific sensor. Figure 6.17 illustrates the SetNodeMeasurement function. The request packet's PacketID field is set to the value for a Set Node Measurement command. The data payload for the request packet is populated with the measurement task information. The response packet's ErrorCode value is returned to indicate if the command was successfully executed by the network.

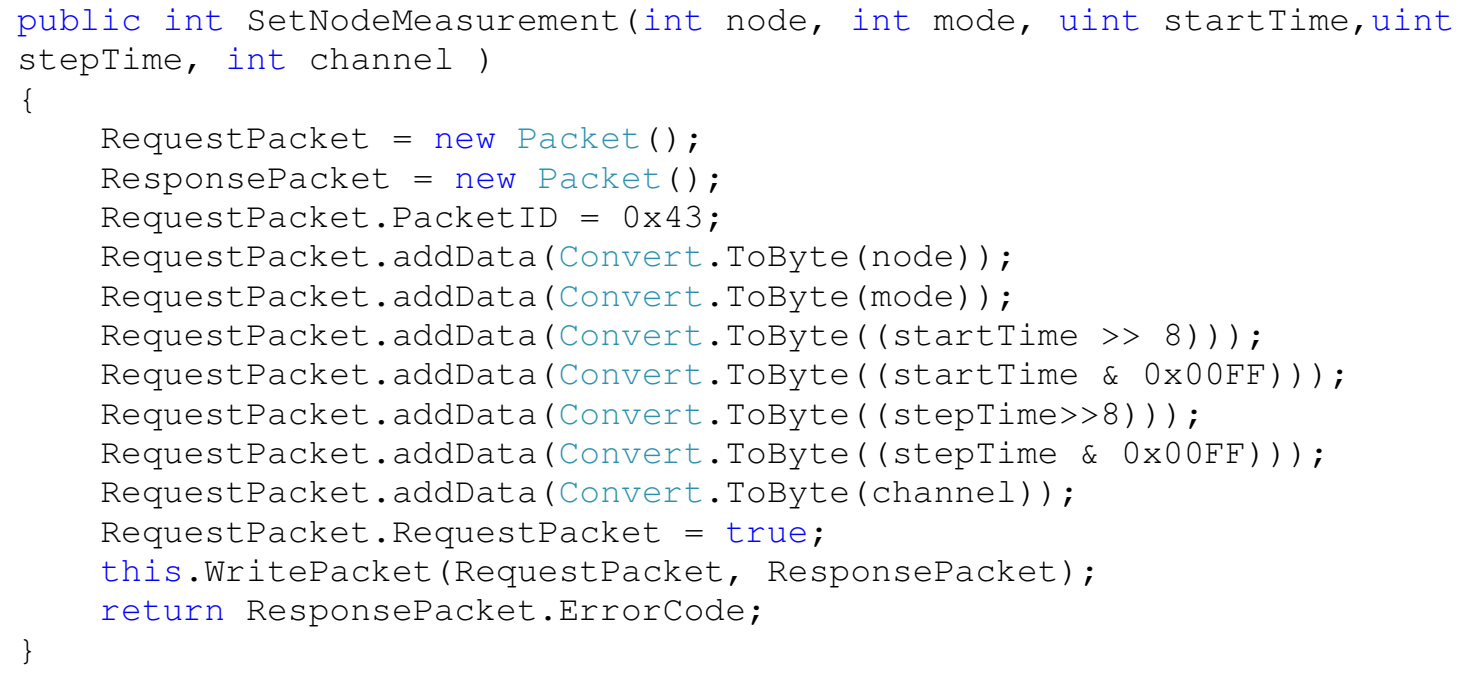

Figure 6.17 Code Example: SetNodeMeasurement Function

\section{int GetNodeData(int node, int channel)}

This function requests the latest sensor data on a single channel of a specified sensor. The sensor data returned is the latest data that was read by the sensor network interface board during its last update of the network. Note that if the active polling of the network was disabled then the sensor data may not be accurate. 


\section{int GetNodeDataFull(int node, int[] arr)}

This function requests the latest sensor data from all channels on a specified sensor. It returns the sensor data that was acquired by the sensor network interface board from the network the last time the network was polled. The data set is returned by storing it in the integer array passed as a function parameter. Note that if the active polling of the network was disabled then the sensor data may not be acurate.

\section{int GetNodeReading(int node, int channel)}

This function forces a specified sensor to take a reading on the nominated channel and return the result. It allows current sensor measurements to be obtained if active polling has been disabled on the Sensor network interface board.

\section{int GetNodeReadingFull(int node, int[] arr)}

This function forces a specified sensor to take a reading on all of its channels and return the results. It allows current sensor measurements to be obtained if active polling has been disabled on the Sensor network interface board. The data set is returned by storing it in the integer array passed as a function parameter.

\section{int ResetNetworkTime()}

This function instructs the Sensor Network Interface board to reset the network time across the network. It ensures that all sensors are synchronised to the universal network time and that measurement windows are aligned on every sensor.

\section{void kill_network_connection()}

This function allows an application to close the serial port connection to the sensor network interface board. It should be called when an application has finished with the sensor network. 


\subsection{Command Packet Interface}

A simple command interface application has been developed to test the functionality of the PacketCom and Packet classes. The application allows a user to create command interface packets and transmit them to the Sensor Network interface board. The application also displays any packets received from the Sensor Network interface board. The command interface application has been designed as a generic interface so that it can be used to test command interfaces implemented on other hardware devices, as well as the Sensor Network interface board. Figure 6.18 illustrates the graphical interface of the command packet interface application.

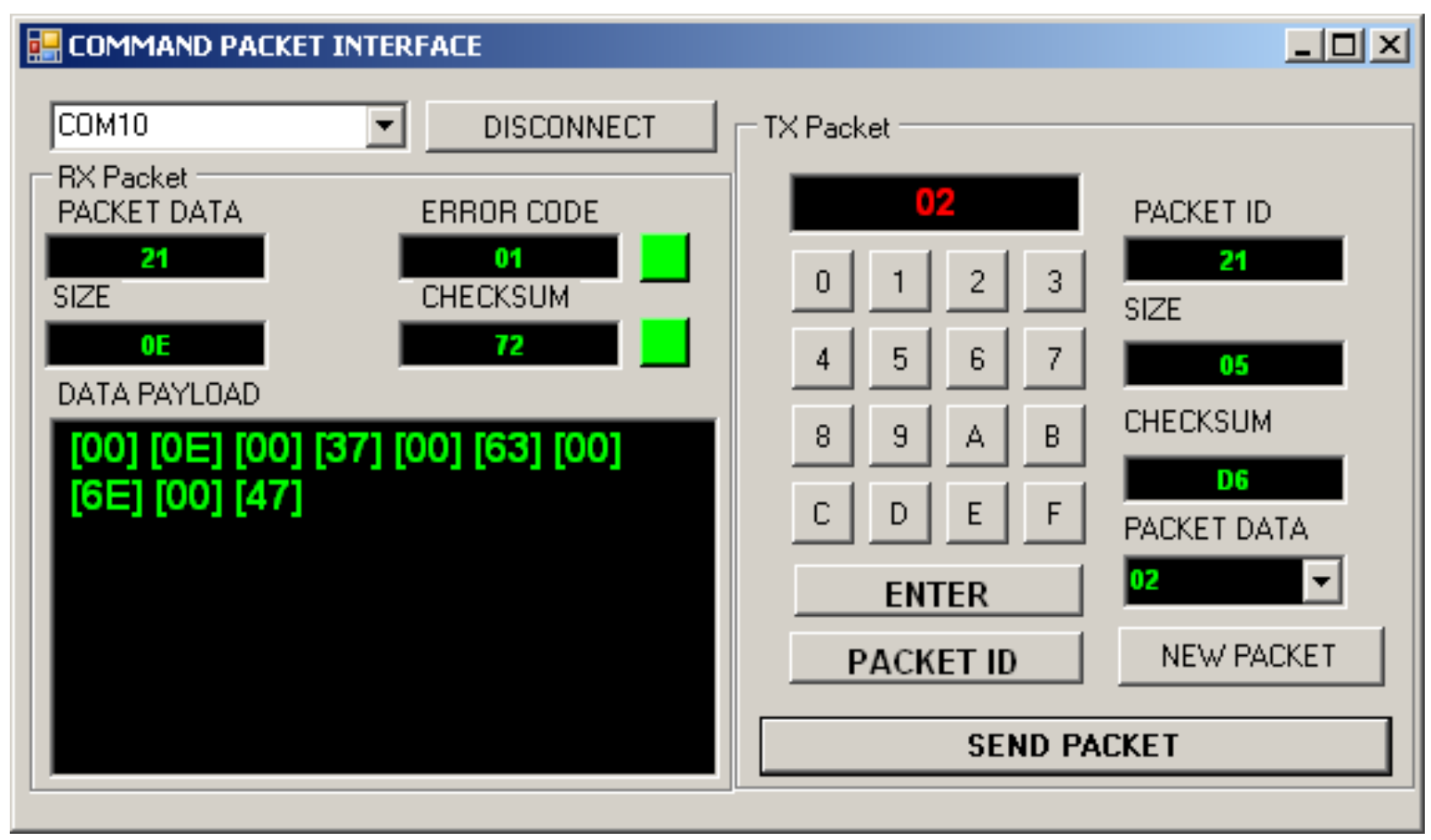

Figure 6.18 Command Packet Interface Application

A user can select a serial port from a drop-down list of serial ports available on the computer. Once connected, the user can create a command packet by using the controls in the TX Packet Panel. A user can specify the packet ID and enter hexadecimal values into the data payload of the packet. The packet size and checksum are calculated automatically and displayed.

The user can transmit the packet to the connected device by clicking the SEND PACKET button. Once a packet is sent the application will scan for a response packet on the serial port. When a response packet has been detected its contents are displayed in the RX Packet 
panel. Error Code and Checksum LEDs will light red to indicate an error has been detected in the response packet.

\subsection{Test Sensor Network Graphical Application}

A graphical user interface application provides a visual representation of sensor data received from the prototype sensor network. The application has been built on top of the sensor interface class. The prototype sensor network has been designed to probe an environment with a $360^{\circ}$ field of view in a single horizontal plane. The application displays the sensor data as a map, with each sensor data value plotted as a vector. The application uses the mount $X$, mount $Y$, mount $Z$ and angle fields of the configuration file to plot the position of each sensor and the horizontal and vertical components of the distance vector. An overview of the user interface application is illustrated in Figure 6.19.

Fixed distance reference rings spaced at $0.5 \mathrm{~m}$ increments are plotted on the map to provide a reference scale for the distance measurements. A slider bar allows a user to zoom in and out on the map. A Scan button at the bottom of the map allows a user to pause the distance map to examine a plot from a single set of sensor measurements. Controls on the right side of the application present the information for each sensor in the network. Using the Sensor Node control a user can select a sensor on the network to examine its information. The map will plot the currently selected sensor measurements as blue lines, allowing the user to identify the selected sensor on the map. A user can change the measurement mode and parameters of a sensor by setting the desired measurement parameters in the Sensor Node Information panel and send it to the sensor by clicking the Reconfigure Node button. The panel also displays the current distance calculations for the channels of the selected node.

A user can select the Disconnect from Network button and physically reconfigure the network by adding or removing sensors or changing the mounting position of sensors. Once the configuration file is updated to reflect the changes, the user can reconnect to the network without having to modify the user application. 


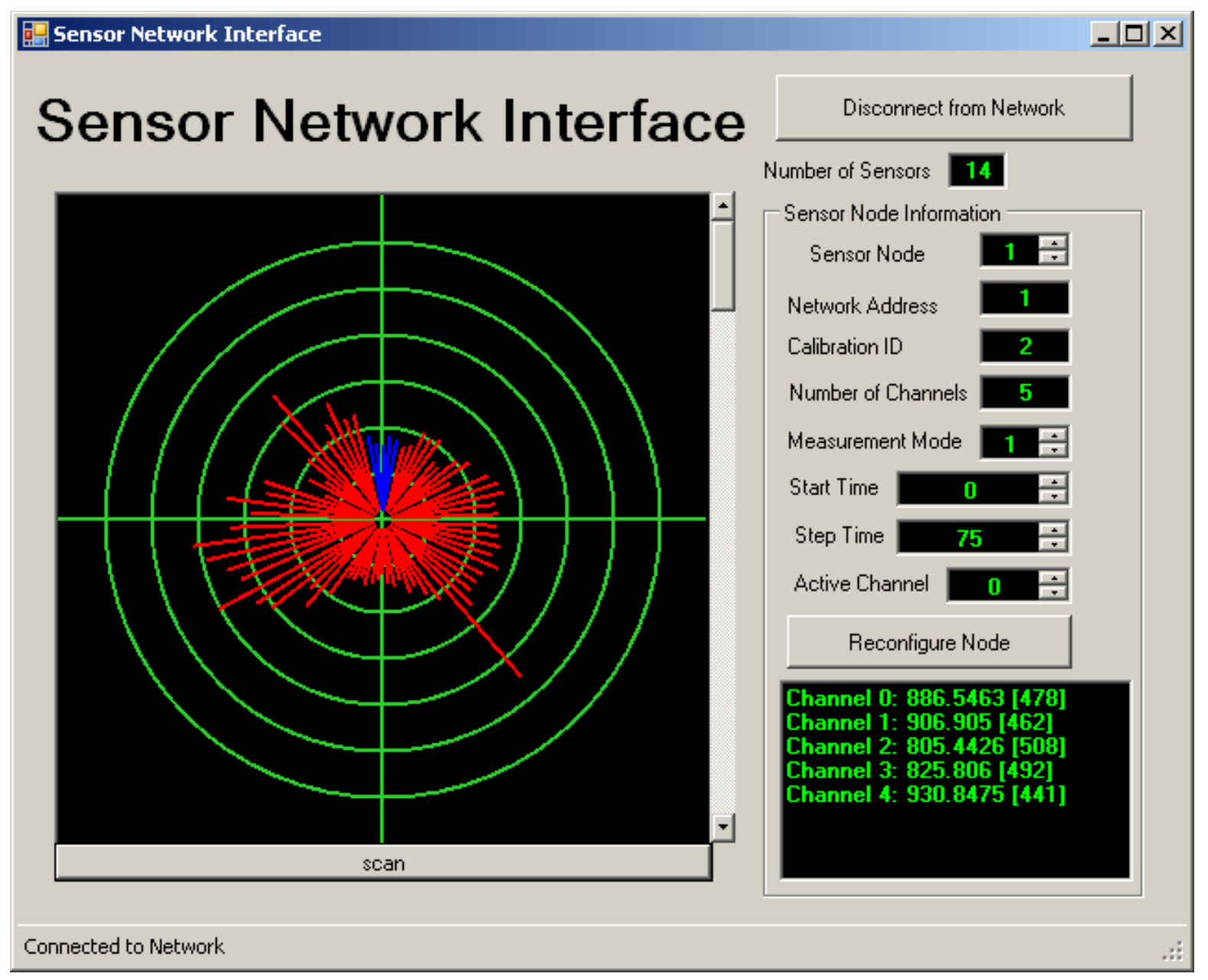

Figure 6.19 Sensor Network Interface Application

\subsection{Summary}

A C\# software library has been developed to interface a control computer with the sensor network. The library provides a suite of functions that allows applications to be developed to manipulate the network and process data from the sensors. The software library is built on top of a packet communication class that controls data transmission over the USB connection between the computer and the sensor network. A software application has been developed to test the packet communication class and test the command interface between the computer and network. A graphical user interface was developed to visually display sensor data from MARVIN's prototype network. The application was built on top of the Sensor Interface library to test the Network's command interface and the library's ability to be reconfigured through use of the configuration file. 


\section{Chapter 7 Hardware Methodology - Full- Field Image Ranger}

This chapter describes the hardware methodology adopted for the development of a mobile full-field image ranger system. The image ranger is a multi-board system that has been developed from a previously bench top based prototype system. The new system has been designed to be mounted on a mobile robotic platform and with the flexibility for future hardware upgrades to include an imaging sensor with higher resolution than the present prototype system.

\subsection{Initial Prototype Design}

Researchers at Victoria University of Wellington and the University of Waikato have developed a real time full-field image ranger system [11 - 13]. The prototype system employs a high frequency, amplitude modulated light source and image sensor. An Altera field programmable gate array (FPGA) development board has been used to develop the system, with additional peripheral hardware being designed to interface with the development kit. The complete system comprises a number of interconnected circuit boards. These boards are:

\section{Stratix III Development Kit}

This board is the primary control unit for the system. It controls all peripheral hardware and performs real time image processing.

\section{Illumination Board}

This board provides the active high frequency illumination of the scene to be imaged.

\section{PMD Daughter Board}

This board contains the imaging sensor from PMD technologies.

\section{PMD Main Board}

This board provides the interface between the FPGA development kit, the illumination board and PMD imaging sensor. The board is also responsible 
for the high frequency modulation of the PMD imaging sensor and analogue to digital conversion of the video output stream from the sensor.

\section{VGA/Ethernet Board}

This board provides two interfaces between the system and a control computer. It contains an Ethernet interface to allow processed and raw data images to be transferred from the system to a computer for storage. It also contains a VGA interface allowing the display, in real time, of both processed and raw data images.

Figure 7.1 illustrates the collection of electronic boards that form the image ranger system.

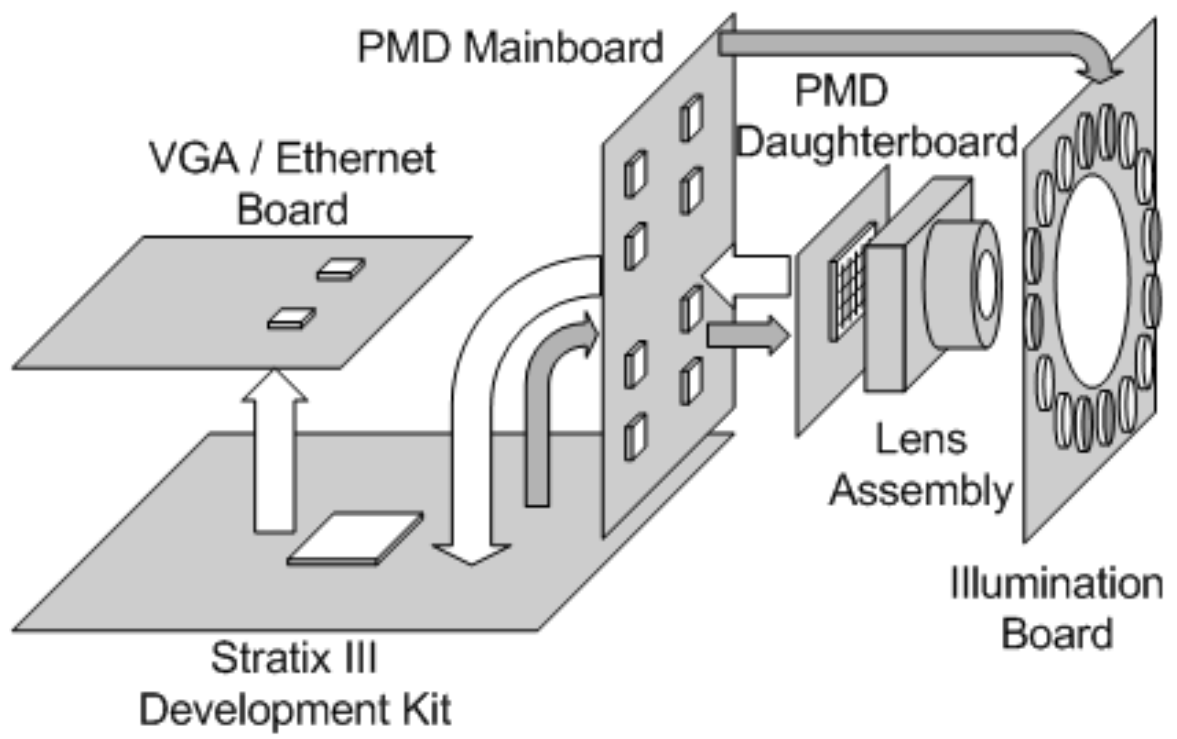

Figure 7.1 System Layout of Prototype Range Imaging System.

\subsubsection{FPGA Development Board}

The heart of the image ranger system is the Stratix III FPGA development board that hosts an Altera Stratix III EP3SL150F1152 FPGA. The FPGA development kit was originally selected due to the availability of reconfigurable phase lock loops that facilitate the phase stepping required for both the homodyne and heterodyne methods of distance ranging. The EP3SL150F1152 FPGA offers the following [39]: 
- 8 PLLs where each PLL can provide up to 10 phase synchronous output signals up to $700 \mathrm{MHz}$.

- 142,500 logic elements

- 5499 Kbits of memory

- 384 multiplier blocks

- 16 global clocks

- 744 user I/O pins

The development board provides various on-board memory blocks [40]. These include:

- 256 MByte DDR2 SDRAM DIMM

- 72 MBit QDRII SRAM

- 16 MByte DDR2 SDRAM

- 4 MByte SSRAM

- 64 MByte flash memory

The board has two high speed mezzanine card connectors to allow additional functionality through the development of custom boards.

\subsubsection{Illumination Board}

The illumination board has sixteen laser diode sources. The laser diodes can be switched to provide the modulated illumination required for the indirect time of flight method exploited by the system, as discussed in Section 2.2.4 Heterodyne Full Field Image Ranger System. The sixteen laser diodes are divided into two independent banks of eight diodes each. One bank comprises laser diodes with a wavelength of $658 \mathrm{~nm}$ and the other bank with lasers of wavelength $808 \mathrm{~nm}$. Lasers of two different wavelengths are used to investigate the potential advantages of IR versus visible light [3]. The illumination board has been designed with a circular cut-out to allow it to be mounted around an optical lens. The laser diodes are mounted in a circular layout around the cut-out to provide uniform illumination emanating from the imaging sensor.

The FPGA controls the modulation of the laser diodes via four differential pair output signals (two signals per bank). Each output signal controls the modulation of four laser 
diodes. All the diodes are driven in a constant current mode by eight $155 \mathrm{MHz}$ laser switch ICs. Each laser switch controls the current through two laser diodes in series. Low Voltage Differential Signal (LVDS) transceivers convert the FPGA modulation signal to the single ended CMOS signal required by the laser switch ICs.

The illumination board also features a Microchip Technology PIC16F684 microcontroller that supervises the driving of the constant current through the laser diodes. The main task of the MCU is to prevent damage to the laser diodes by providing a warm up period during which the diode drive current is slowly increased. The illumination board has on-board voltage regulation to provide separate $+8 \mathrm{~V}$ supplies to the two laser diode banks, a $+5 \mathrm{~V}$ supply for the MCU, and a $+3.3 \mathrm{~V}$ supply for the LVDS transceivers. These voltage regulators are powered from an external power supply.

\subsubsection{PMD Daughter Board}

The PMD daughter board houses a PMD Technologies PMD19k-2 imaging sensor used to capture images of the scene. This sensor operates from a $+5 \mathrm{~V}$ power supply provided from the PMD main board. The sensor provides a $160 \times 120$ array of pixels divided into four $40 \times 120$ pixel blocks that can be independently modulated [41]. The PMD daughter board contains only the sensor and passive components required to set voltage reference levels and to provide power supply filtering.

A lens assembly is mounted above the image sensor via a cage plate designed to hold a CMount lens. A $16 \mathrm{~mm}$ focal length lens with manual focus and iris, from Goyo Optical, is currently fitted on the prototype system. A dark red $(645 \mathrm{~nm})$ long pass filter attached to the lens assembly reduces the intensity of visible light incident on the sensor.

The PMD daughter board connects to the PMD main board via two 20 pin DIL $0.5 \mathrm{~mm}$ pitch connectors. 


\subsubsection{PMD Main Board}

The PMD Main board provides the electronics to interface the Stratix III development board to the illumination board and PMDTechnology imaging sensor. The FPGA I/O lines use LVDS signalling, while the imaging sensor uses single-ended CMOS signalling. The PMD main board provides the circuitry to convert between these two signal technologies.

The imaging sensor outputs two analogue signals (Video A and B) corresponding to the modulated intensity incident on a sensor pixel. The PMD main board converts the analogue video streams to a digital data stream through use of a 16-bit image signal processor ADC (AD9826 from Analog Devices [42]). This allows the FPGA to perform digital image processing on the image data in real time.

The imaging sensor has four independent modulation areas that are controlled in a push-pull configuration. The four modulation regions reduce the current requirements upon the driver. The PMD main board provides two high speed, high current drivers (EL7158 from Intersil [43]) that are needed to drive the high capacitance modulation inputs of the imaging sensor at the required modulation frequency.

The PMD main board also provides LVDS buffering/repeating to relay the modulation signal to the illumination board.

\subsubsection{Ethernet/VGA Board}

The Ethernet/VGA board provides an interface to allow image frames to be stored or displayed on peripheral equipment. The Ethernet interface provides a link to a control computer where image frames can be stored and displayed. The board also provides a VGA output to allow real time display of image frames on a VGA compatible monitor. Additional I/O lines are provided to a 14 pin header, providing $12 \mathrm{I} / \mathrm{O}$ lines, to allow debugging and future hardware boards to be added.

The Ethernet/VGA board connects to a TerasIC GPIO board via two 40-way IDC box headers. The TerasIC expansion board connects directly to the FPGA development kit via a high speed mezzanine connector. 


\subsection{Mobile Platform Design}

The mobile platform version of the full-field image ranger system packages the complete system into a small form factor that can be mounted on mobile robotic vehicles. The main modification of the mobile platform system is the design of an application specific FPGA board replacing the Stratix III FPGA development board. The main reason for this is that the development board has a large footprint $(210 \mathrm{~mm} \times 180 \mathrm{~mm})$ and peripheral hardware resources that are not required for the full-field image ranger application.

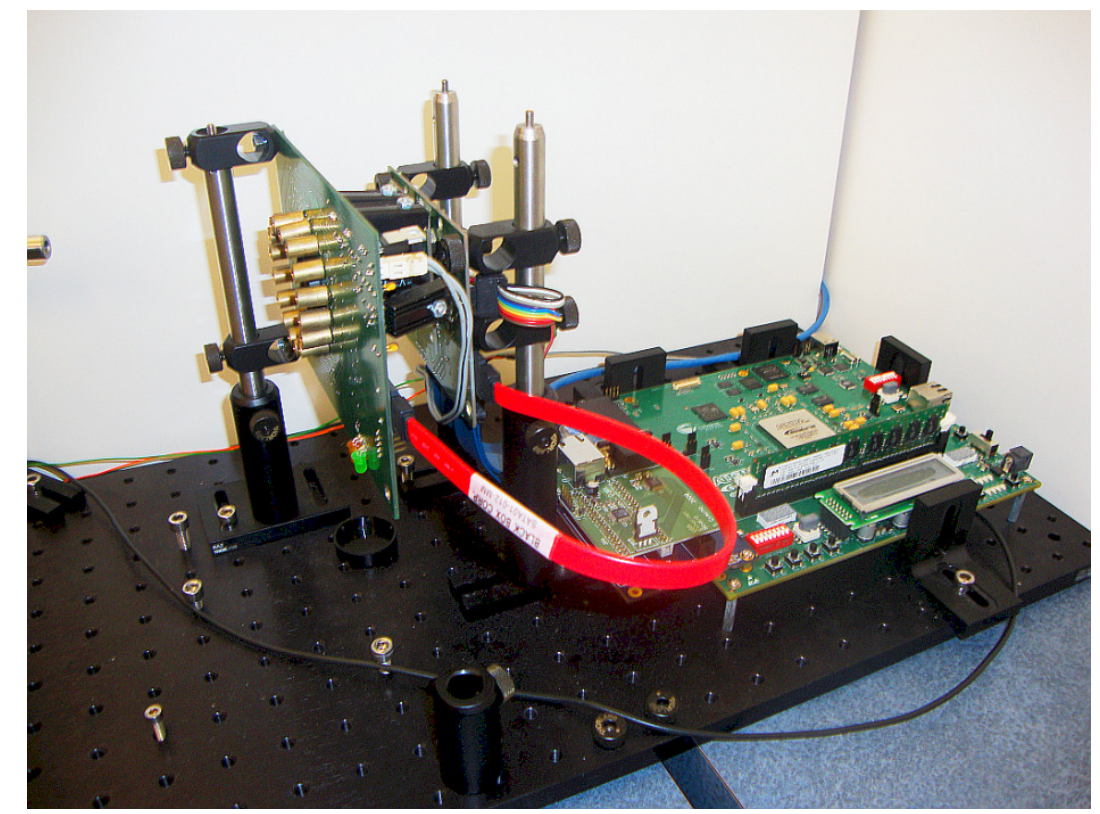

Figure 7.2 The Prototype Full-Field Image Ranger System.

The geometry of the prototype full-field image ranger system, pictured in Figure 7.2, required the design of several electronic boards. Each individual board has been responsible for implementing a sub-system.

These sub-systems are:

- Illumination

- Image Sensing

- Image Capture/Conditioning

- System supervision and control

- External Interface
(Illumination board)

(PMD Daughter board)

(PMD Main board)

(Stratix III Development board)

(Ethernet/VGA board) 
This system architecture has been adopted for several reasons;

- It has allowed the laser diodes, used for active illumination of the scene under measurement, to be positioned at the end of the optical lens and in a concentric layout around the lens.

- It has allowed the image sensor to be easily upgraded without radical revisions required to the entire system.

- It has allowed individual boards to be designed independently by various people.

- The division of sub-systems on to individual boards has provided flexibility in altering the configuration of the ranger, such as changing the optical hardware, and increased upgradability to the system.

This modular system architecture will be continued in the mobile platform system. The subsystems for the mobile platform system are:

- Laser Illumination

- Image Capture

- System Supervision and control

- External Interface

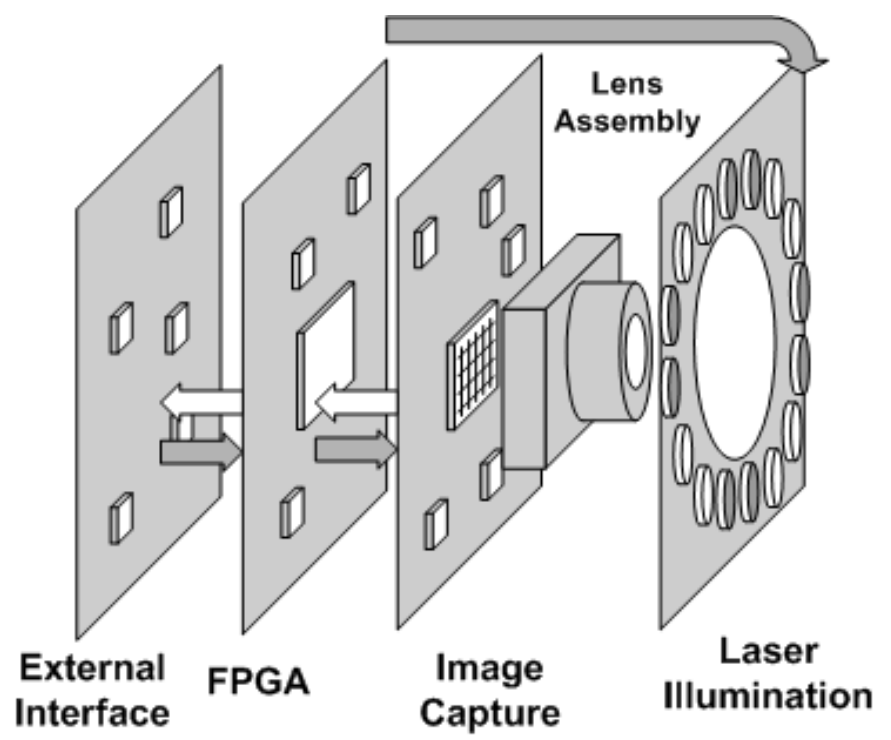

Figure 7.3 System Architecture of Full-field Imaging System

Each sub-system has been implemented on a separate circuit board to help reduce the overall system size by allowing the circuit boards to be stacked on top of each other. The stacked system also provides flexibility in optical setup by allowing the position of the illumination board to vary to accommodate differing optical lenses. The stacked system also provides 
upgradability by allowing the image capture board to be modified to house new image sensors without having to change the remaining circuit boards. The external interface, image capture and laser illumination sub-system circuit boards have the same board dimensions and mounting holes. The FPGA board has a slightly larger physical size due to the number of components it contains but has similar mounting holes to the rest of the circuit boards allowing them to be easily stacked providing a compact system size, as illustrated in Figure 7.3.

The major difference between the prototype and mobile platform systems is the interface between the FPGA and image capture boards. A suitable imaging sensor has not been available during the design process of the mobile platform system. The Image Capture board has been designed to interface to the imaging sensor used in the prototype system. It is predicted that the availability of higher resolution imaging sensors will become greater in the next couple of years. The mobile platform system has been designed to provide flexibility to change the imaging sensor. The main issue with providing this flexibility is that imaging sensors from different companies have varying interfaces and power requirements. The interface between the image capture board and the FPGA has been developed as a generic I/O interface. The image capture board handles all necessary signal conditioning, pin drivers and LVDS conversion to drive the imaging sensor.

An unregulated power bus connects through all circuit boards allowing on-board regulation on each circuit board; refer to Section 7.3.5.1 Unregulated Power Bus for more information.

\subsubsection{Circuit Board Dimensions}

The complete mobile platform system comprises multiple circuit boards stacked together. The dimensions of the external interface, image capture and laser illumination boards have been defined, primarily by the physical size of the optical components used in the system. In the stacked system, the optical lens assembly mounts to the image capture board and protrudes through a circular cut-out in the laser illumination board.

The circular cut-out in the laser illumination board must be a minimum of $35 \mathrm{~mm}$ in diameter to accommodate the optical lens, which has a diameter of $30 \mathrm{~mm}$. Immediately surrounding the circular cut-out are the laser diode modules. 


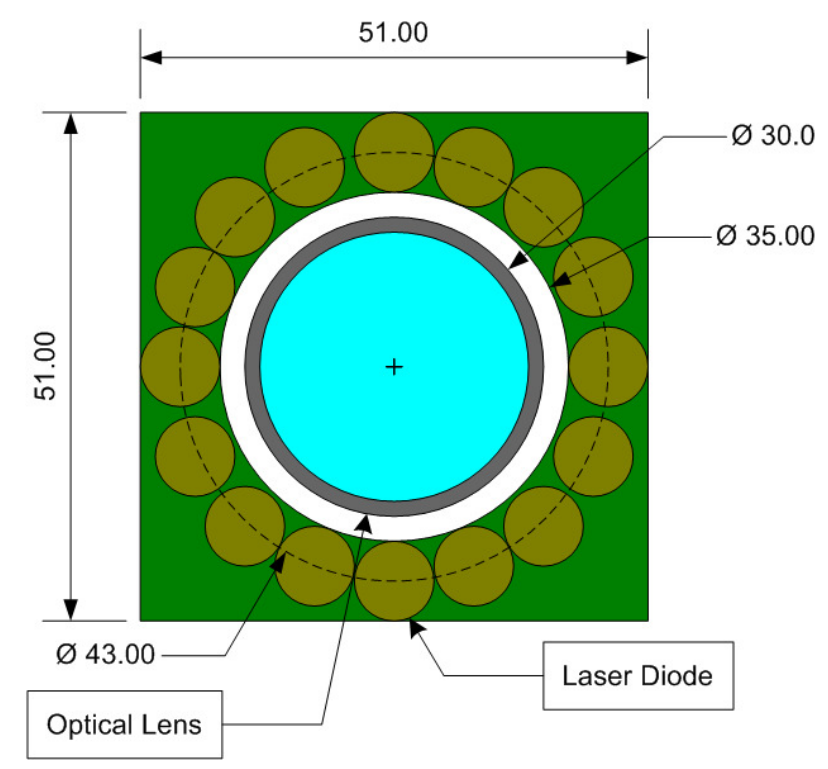

Figure 7.4 Minimum Size Limitations for Laser Illumination Printed Circuit Board

These modules have a diameter of $8 \mathrm{~mm}$. To physically accommodate the sixteen laser diode modules, with an $8 \mathrm{~mm}$ diameter each, around a $35 \mathrm{~mm}$ cut-out requires these laser modules to be centred on a circle with a minimum diameter of approximately $43 \mathrm{~mm}$. The physical size of the lens and laser diodes restrain the PCB to having a minimum width of $51 \mathrm{~mm}$. Figure 7.4 illustrates the physical layout of the laser diodes around the system lens.

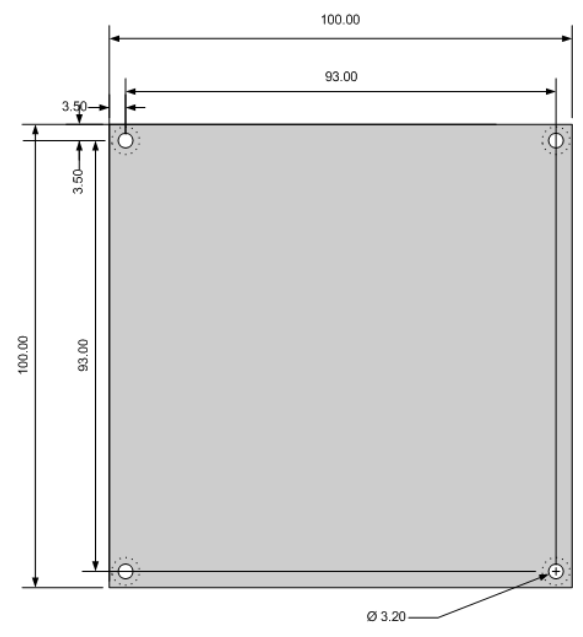

Figure 7.5 Printed Circuit Board Template for Mobile Platform Full-field Imaging System 
The laser illumination board also requires space for on-board voltage regulation and the laser diode driving circuitry. Conservatively the laser illumination PCB has been designed to be $100 \mathrm{~mm}$ square.

Each circuit board is separated from the next in the stack by four M3 standoffs. The mounting holes for the stand offs are positioned at each corner, $3.5 \mathrm{~mm}$ in from each side of the board. Figure 7.5 illustrates the PCB template used for the external interface, image capture and laser illumination boards.

\subsection{FPGA Board}

The FPGA board connects to the image capture board via one of its two generic I/O ports. Through this port connection the FPGA controls the image capture and illumination processes of the ranger system. The FPGA also connects to an external interface board via the other generic I/O port. The external interface board allows the FPGA to send captured images to peripheral devices such as a computer or VGA monitor. The FPGA board also performs image processing which requires significantly large amounts of fast memory.

\subsubsection{FPGA Selection}

The prototype system was designed around the Altera Stratix III FPGA family. After evaluation, through use of a Stratix III development kit, it has been decided to switch to the Altera Cyclone III family of FPGAs. The main motivating factor for the switch is the difference in cost. Stratix III FPGAs are an order of magnitude more expensive than the Cyclone III and offer little more in functionality required for the mobile platform system.

Due to the ability to migrate across different FPGA models in the Cyclone III family, the mobile platform system has been designed to operate using two different Cyclone III FPGAs [44]. These FPGAs are:

- EP3C40 (Packaged in F780 BGA)

- $\mathrm{EP} 3 \mathrm{C} 120$ (Packaged in F780 BGA) 
Table 7.1 outlines the main features of these FPGAs.

Table 7.1 Cyclone III FPGA Information

\begin{tabular}{c|c|c|c|c|}
\hline Device & $\begin{array}{c}\text { Logic } \\
\text { Elements }\end{array}$ & $\begin{array}{c}\text { On board } \\
\text { RAM } \\
\text { (bits) }\end{array}$ & PLLs & Maximum User I/O \\
& \multicolumn{5}{c|}{535} \\
\hline EP3C40 & 39,600 & $1,161,216$ & 4 & 531 \\
\hline EP3C120 & 119,088 & $3,981,312$ & 4 & \\
\hline
\end{tabular}

A $50 \mathrm{MHz}$ oscillator has been used as the system clock. The FPGA on-board phase lock loops are used to multiple this clock frequency to the desired clock frequencies needed for operation of the system.

\subsubsection{External Memory Selection}

The design of the full-field image ranger system utilises four discrete blocks of memory for processing and storage of images. The prototype system implements all four of these memory blocks on the FPGA's internal 5499 Kbit static random access memory [44]. The amount of internal memory on the FPGA is sufficient for storage and processing of image frames using the $160 \times 120(19 \mathrm{~K} 2)$ pixel PMD sensor but is insufficient for higher resolution sensors. The full-field image ranger system converts each sensor pixel into a 16-bit number. With a $19 \mathrm{~K} 2$ sensor the frame size is $38.4 \mathrm{kB}$. It is anticipated that the imaging sensor may achieve a resolution of 1 Mega-pixel (MP) in the next couple of years. The mobile platform system has been designed to handle image sensors of this resolution. At 16 bits per pixel a 1 Mega pixel frame will be approximately $2 \mathrm{MB}$ in size.

To provide the storage and processing capacity for a high resolution sensor the mobile platform system has external memory available to the FPGA. Five different types of memory, as illustrated in Figure 7.6, have been identified and implemented on the mobile platform system. These memory banks are:

1 Accumulator - this memory bank is required for the storage of images during image processing. The memory holds semi-processed and fully processed image data that determine phase measurements.

2 Output Buffer - this memory bank is required to store images that will be accessed by an NIOS processor, the VGA output and the Ethernet output. 
3 NIOS Program - this memory bank is required to store the firmware of the NIOS processor running on-board the FPGA.

4 NIOS Ethernet Frame Buffer - this memory bank is required to store image frames that will be transferred to a peripheral computer via the Ethernet interface. It is used to buffer images to handle the latency of the Ethernet connection.

5 Flash FPGA Configuration Memory - this memory bank is required to store the FPGA configuration that is loaded at power up.

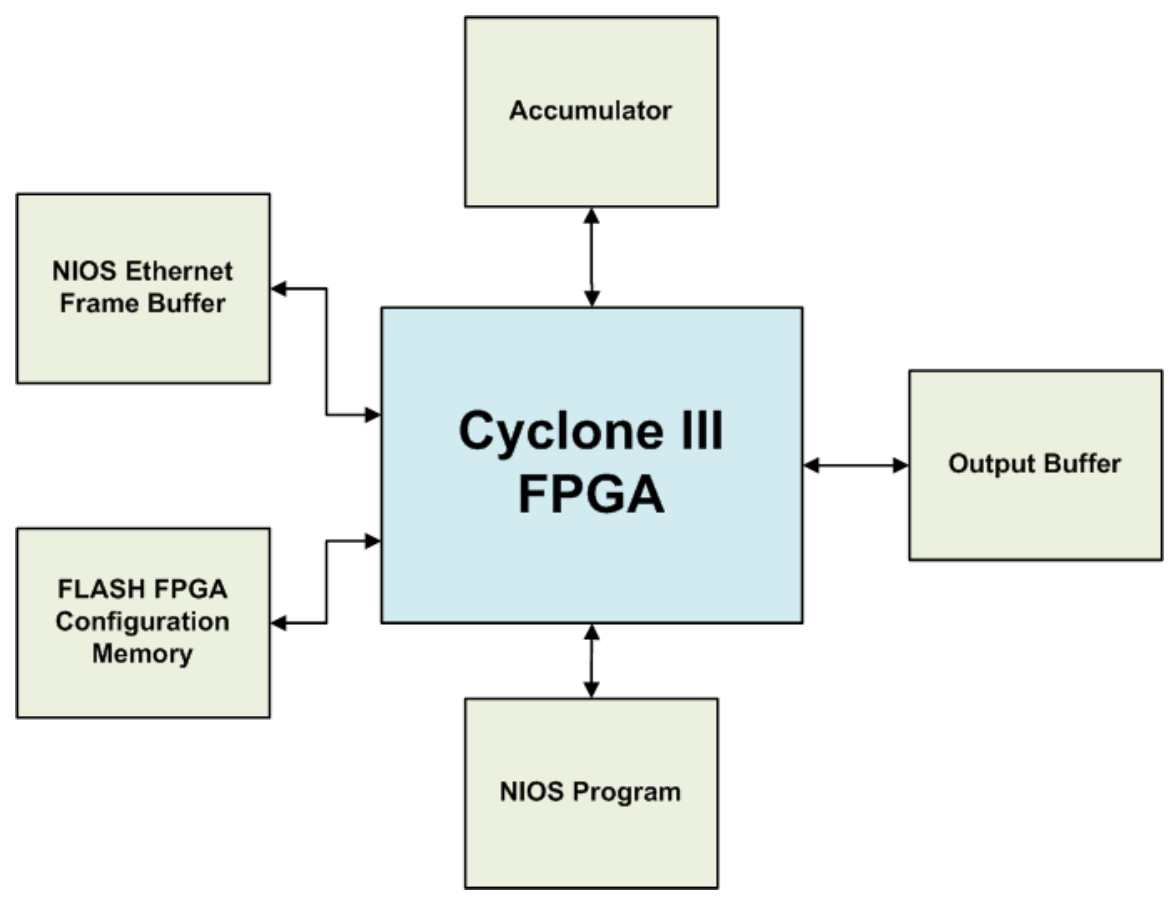

Figure 7.6 External Memory Banks of Mobile Platform System

The accumulator is used to store the accumulated real and imaginary terms of the phase for each pixel. These terms require 16 bits each per frame pixel, 32 bits combined per pixel. For a $1 \mathrm{MP}$ frame this would require slightly less than $4 \mathrm{MB}$ of memory per frame. Currently, the prototype system's image processing routine operates at a clock frequency of $10 \mathrm{MHz}$. At this frequency the accumulator must operate at a data rate of $320 \mathrm{MBit} / \mathrm{s}$ (32 bits/pixel $\times 10 \mathrm{MHz}$ ). The accumulator must read and write a pixel value each time it performs a mathematical operation. This increases the required data rate of the accumulator to at least $640 \mathrm{MBit} / \mathrm{s}$.

The output buffer is required to hold four frames: the A image frame, B image frame, phase frame and A-B frame (where A and B are the modulated pixel values). The output buffer is accessed by the NIOS processor, the VGA output operation, and the image processing 
operation. On the current prototype system all three accessing processes are clocked at $10 \mathrm{MHz}$. At this clock frequency the memory must be able to transfer data to each process at a speed of $320 \mathrm{Mbit} / \mathrm{s}$. Time multiplexing is utilised to handle the three accessing processes. This increases the data transfer speed to $960 \mathrm{Mbit} / \mathrm{s}$ (320 Mbit/s by each process).

The NIOS processor is a 32-bit processor running at $100 \mathrm{MHz}$. At this processing clock frequency the processor requires a memory bank with a data speed of $3.2 \mathrm{Gbit} / \mathrm{s}$ to access its firmware. The NIOS Program RAM requires $32 \mathrm{MB}$ of memory space.

The NIOS Ethernet Buffer RAM is used to store frames that will be transferred to a peripheral computer via the Ethernet connection. As the Ethernet connection has some finite latency it is anticipated that image frames will be produced faster than the Ethernet connection can transfer. It is necessary therefore to use a buffer to prevent the loss of frames. Ideally the memory would have as much RAM as possible to buffer the frames to be transferred. With a sensor resolution of $1 \mathrm{MP}, 1 \mathrm{~GB}$ of memory will be able to buffer 125 output frames (where an output frame contains four frames: A, B, Phase and A-B).

The Flash Configuration memory size has been chosen to be $64 \mathrm{MB}$ based on flash memory devices used on FPGA development boards.

Several types of random access memory have been investigated to be used as external memory on the mobile platform system.

\section{Static Random Access Memory (SRAM)}

SRAM is a type of memory that requires no regular refreshing of its memory contents. It uses a bi-stable latching circuit to store each bit of memory. Even though no refreshing of memory is required, SRAM is still a volatile memory scheme because data will be lost when the memory is not powered. The latching circuit of the memory requires six transistors. The number of transistors per memory bit limits the amount of memory that can be manufactured on a silicon die [45, 46, 47]. The largest, easily available and affordable SRAM device is $64 \mathrm{MBit}$ (8 MByte) running at a data access speed of $290 \mathrm{Mbit} / \mathrm{s}$. This device has a unit cost of approximately $\$ 100$. The device would provide enough memory for a 1 Mpixel frame but does not have the required data access speed for any of the memory banks. There is a tradeoff between memory size and cost with any memory device. SRAM memory has not been selected as a memory type due to its low data rate and high cost. 


\section{Double Data Rate Synchronous Dynamic Random Access Memory (DDR SDRAM)}

DDR SDRAM is a type of DRAM that operates in a manner allowing twice the data transfer rate compared with standard DRAM. Unlike SRAM, DRAM requires only one transistor per memory bit and therefore can be manufactured in much larger memory sizes on a similar sized silicon die. Each memory bit is stored on a single capacitor. As capacitors leak charge the information eventually fades unless refreshed periodically [48]. DRAM is used extensively in personal computers because of its high density of memory compared with cost. Where standard DRAM clocks in one data word on a single clock pulse, DDR SDRAM clocks two data words in on a single clock pulse. Data is latched in on both the rising and falling edges of a clock signal (known as double pumping the data bus). DDR2 SDRAM is an improvement on DDR SDRAM where the data rate is further increased by two. This is achieved by effectively doubling the width of the data bus inside the memory, allowing two blocks of memory to be transferred to the external data bus at a time. This is known as prefetching the data and requires a pre-fetch data queue to buffer the memory words at the external data bus before being transferred. The consequence of pre-fetching data is an increase in read latencies. Currently the largest DDR2 SDRAM device that is easily obtainable and affordable is 4 Gbit (512 MB) running at a data rate of $6.4 \mathrm{Gbit} / \mathrm{s}$. This device has a unit cost of approximately \$50. DDR2 SDRAM offers large memory sizes at very high data rates, but requires a more complex memory interface to refresh the data contents.

\section{Pseudostatic Random Access Memory (PSRAM)}

PSRAM is a type of dynamic RAM that emulates SRAM by having built-in refresh and address-control circuitry. It combines the high density of DRAM with the ease of use of SRAM [49]. Currently the largest PSRAM device that is easily obtainable and affordable is $128 \mathrm{Mbit}(16 \mathrm{MB})$ running at a data access speed of $2.1 \mathrm{Gbit} / \mathrm{s}$. This device has a unit price of approximately $\$ 10$ but is currently available only in quantities of 1000 .

\section{Quad Data Rate Static Random Access Memory QDR SRAM}

QDR SRAM is a type of SRAM that can transfer up to four words of data in each clock cycle [50]. Like DDR SDRAM, QDR SRAM transfers data on both the rising and falling edges of a clock signal. QDR also has dedicated read and write data buses effectively doubling the data bus width by eliminating the need to time multiplex read and write operations on a single data bus. QDR SRAM has been designed for high speed applications 
where data throughput is more important than cost or density. The largest, easily available and affordable QDR SRAM device is $72 \mathrm{MBit}$. This is equivalent to $8 \mathrm{MB}$ of memory as each byte stored in memory requires 9 bits of memory space, 8 data bits and an additional $9^{\text {th }}$ parity bit. The data rate of this memory device is $8.5 \mathrm{GBit} / \mathrm{s}$ but has a unit cost of approximately $\$ 200$.

\subsubsection{Selected Memory Devices}

The Cyclone III family of FPGAs support numerous high speed external memory interfaces which allow external memory to be connected to the FPGA with little customisation of hardware or software. Supported memory devices include DDR, DDR2 and SDR SDRAM.

DDR2 SDRAM was selected as the preferred type of memory due to its high density and data rates compared with cost. The cyclone family of FPGAs provide a DDR2 SDRAM memory interface to handle data transfer and refreshing of the memory.

The following memory devices have been selected for the mobile platform system's external memory banks:

Accumulator and Output Buffer - Micron MT47H64M8 DDR2 SDRAM. This device has a memory size of $512 \mathrm{Mbit}$ and operates at a clock frequency of $333 \mathrm{MHz}$. It has an 8-bit wide data bus allowing data transfer rates of $2.7 \mathrm{Gbit} / \mathrm{s}$ [51].

NIOS Program - Micron MT47H32M16 DDR2 SDRAM. This device has a memory size of 512 Mbit and operates at a clock frequency of $333 \mathrm{MHz}$. It has a 16-bit wide data bus allowing data transfer rates of $5.3 \mathrm{Gbit} / \mathrm{s}$ [51].

NIOS Ethernet Buffer - M47H512M8 DDR2 SDRAM. This device has a memory size of $4 \mathrm{Gbit}$ and operates at a frequency of $333 \mathrm{MHz}$. It has an 8-bit wide data bus allowing data transfer rates of $2.7 \mathrm{Gbit} / \mathrm{s}$. Two of these devices operating in parallel increase the memory density to 8 Gbit (1 Gbyte) and double the data transfer rate to $5.3 \mathrm{Gbit} / \mathrm{s}$ [52]. 
Table 7.2 External Memory Devices of Mobile Platform System

\begin{tabular}{|c|c|c|c|c|c|c|}
\hline Memory & Type & Size & Speed & I/O lines & Voltages & Current \\
\hline Accumulator & $\begin{array}{c}\text { DDR2 } \\
\text { SDRAM }\end{array}$ & $8 \mathrm{MB}$ & $333 \mathrm{MHz}$ & 34 & $1.8 \mathrm{~V}$ & $240 \mathrm{~mA}$ \\
\hline $\begin{array}{l}\text { Output } \\
\text { Buffer }\end{array}$ & $\begin{array}{c}\text { DDR2 } \\
\text { SDRAM }\end{array}$ & $8 \mathrm{MB}$ & $333 \mathrm{MHz}$ & 34 & $1.8 \mathrm{~V}$ & $240 \mathrm{~mA}$ \\
\hline $\begin{array}{c}\text { NIOS } \\
\text { Program }\end{array}$ & $\begin{array}{c}\text { DDR2 } \\
\text { SDRAM }\end{array}$ & $64 \mathrm{MB}$ & $333 \mathrm{MHz}$ & 43 & $1.8 \mathrm{~V}$ & $350 \mathrm{~mA}$ \\
\hline $\begin{array}{c}\text { NIOS } \\
\text { Ethernet } \\
\text { Buffer }\end{array}$ & $\begin{array}{c}\text { DDR2 } \\
\text { SDRAM }\end{array}$ & $1 \mathrm{~GB}$ & $333 \mathrm{MHz}$ & 48 & $1.8 \mathrm{~V}$ & $340 \mathrm{~mA}$ \\
\hline $\begin{array}{c}\text { FPGA } \\
\text { Configuration }\end{array}$ & FLASH & $64 \mathrm{MB}$ & $10 \mathrm{MHz}$ & 48 & $\begin{array}{l}3.3 \mathrm{~V}, \\
1.8 \mathrm{~V}\end{array}$ & $60 \mathrm{~mA}$ \\
\hline
\end{tabular}

Table 7.2 outlines the memory types, I/O lines required and power requirements for the memory devices on the FPGA board.

\subsubsection{FPGA Configuration Scheme}

During normal operation, FPGAs store their configuration data in their internal SRAM. Because SRAM is a volatile memory, the configuration data must be loaded into the SRAM each time the FPGA powers up. Typically a FLASH memory device is used to permanently store the configuration data. The configuration data can be loaded using an active, passive or JTAG configuration scheme [44].

Active configuration involves the FPGA generating the control and synchronisation signals between itself and the configuration device (typically FLASH memory).

Passive configuration requires an external intelligent host, such as a microcontroller, to control the configuration between the FPGA and the memory. Passive configuration allows the FPGA's functionality to be reconfigured even while the system is operating.

JTAG configuration requires the use of an external JTAG programmer to load the configuration data directly onto the FPGA. JTAG configuration typically operates where the configuration data is stored on a peripheral device external to the system, such as a personal computer.

Altera Cyclone III FPGAs can be configured in one of five possible schemes. These schemes are: 


\section{Passive Serial Configuration (PS)}

PS configuration (illustrated in Figure 7.7) can be performed by using an Altera download cable, an Altera configuration device or an intelligent host. During PS configuration, configuration data is transferred from a storage device to the FPGA on a serial data-in pin (DATA0). The configuration data is latched into the FPGA on the rising edge of a clock (DCLK). Configuration data is transferred one bit per clock cycle.

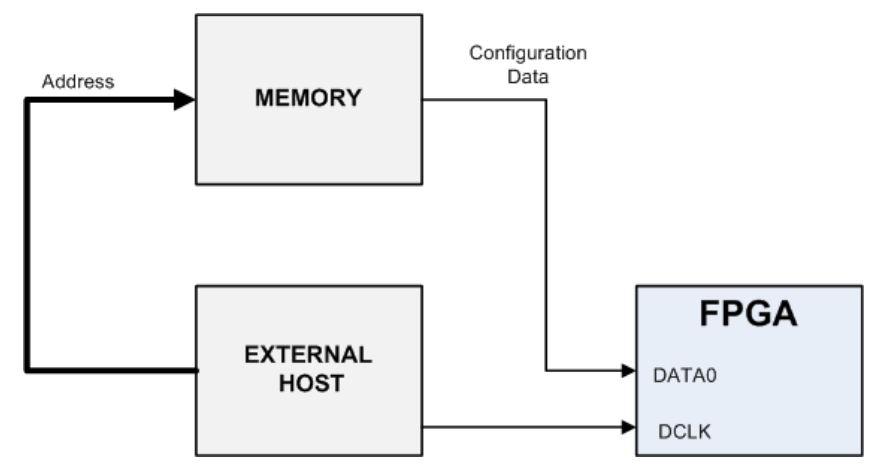

Figure 7.7 Passive Serial Configuration

\section{Active Serial Configuration (AS)}

AS configuration (illustrated in Figure 7.8) can be performed by using an Altera Serial Configuration device. During AS configuration, the FPGA device is the master and the configuration device is the slave. Configuration data is transferred to the FPGA on a serial data-in pin (DATA0). The configuration data is synchronised to a clock signal (DCLK). Configuration data is transferred one bit per clock cycle.

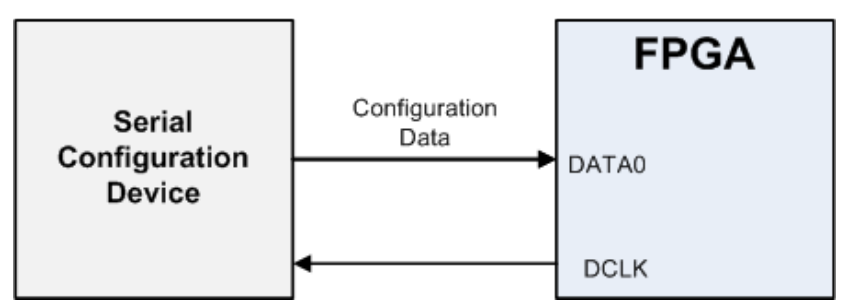

Figure 7.8 Active Serial Configuration

\section{Active Parallel Configuration (AP)}

During AP configuration (illustrated in Figure 7.9), the FPGA is configured using 16-bit parallel flash memory. These external non-volatile configuration 
devices are industry standard microprocessor flash memories. The FPGA is the master and the parallel flash memory is the slave. Configuration data is transferred to the FPGA on a 16-bit wide data bus (DATA15:0). This configuration data is synchronised to a clock input (DCLK). Data is transferred at a rate of 16 bits per clock cycle. The DCLK frequency is driven out of the FPGA during AP configuration at approximately $40 \mathrm{MHz}$.

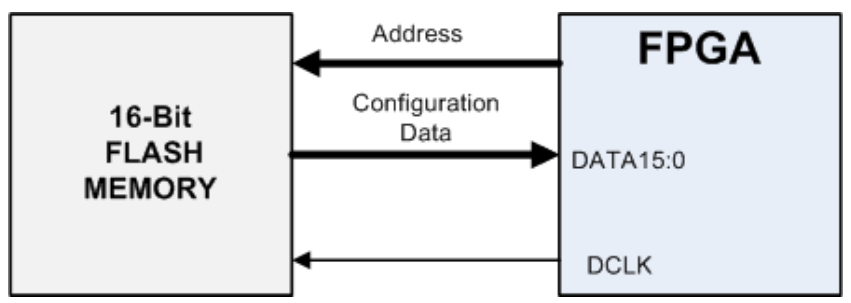

Figure 7.9 Active Parallel Configuration

\section{Fast Passive Parallel Configuration (FPP)}

FPP configuration (illustrated in Figure 7.10) can be performed by using an Altera enhanced configuration device, or an intelligent host. During FPP configuration, the configuration data is transferred from a storage device to the FPGA on a 8-bit wide data bus (DATA7:0). This configuration data is latched into the FPGA on the rising edge of DCLK. Configuration data is transferred one byte per clock cycle.

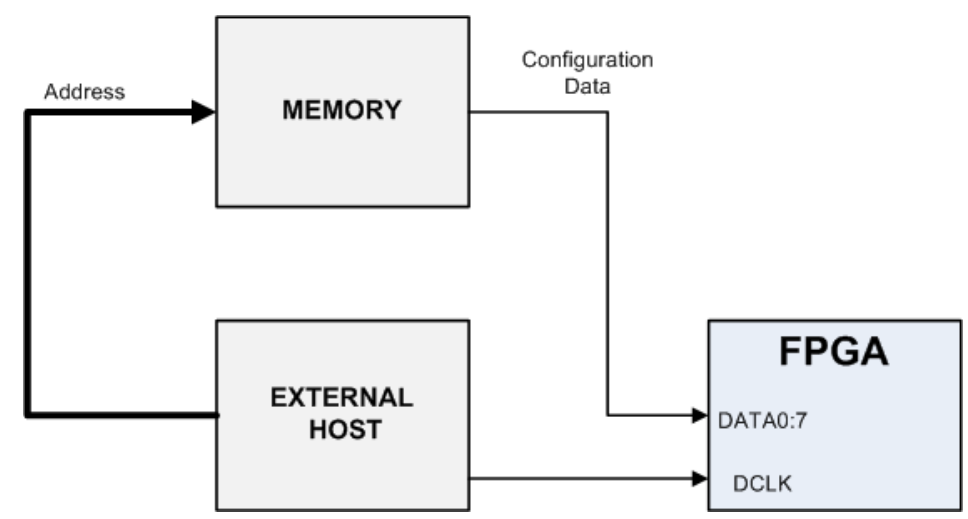

Figure 7.10 Fast Passive Parallel Configuration

\section{JTAG Configuration}

JTAG configuration (illustrated in Figure 7.11) uses the IEEE STD 1149.1 JTAG interface pins. JTAG configuration can be performed by using an Altera download cable or an intelligent host. 


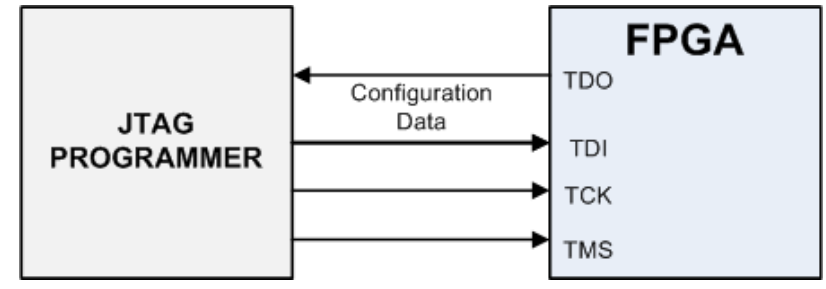

Figure 7.11 JTAG Configuration

PS, AS and FPP configuration schemes have not been adopted on the mobile platform system as they all require external configuration devices which would add the extra complexity of programming the configuration device as well as the storage device. External configuration devices would also add additional cost to the FPGA board. JTAG and AP configuration schemes have been adopted on the mobile platform system. The 16-bit parallel flash memory can be programmed with configuration data by configuring the FPGA, via JTAG, as a Parallel Flash Loader. This allows the FPGA to write new configuration data to the flash memory which will then be loaded into the FPGA on the next reset.

\subsubsection{General Purpose Interface}

The interface between the FPGA board and other boards has been designed as a generic interface to accommodate future upgrades to the system as a whole. The generic interface also allows the FPGA board to be used in other hardware systems and research projects.

The generic I/O interface has been modelled on the external interfaces available on the Stratix III FPGA development kit [40]. These external interfaces use a high speed mezzanine female 172 pin connector. Maintaining the same interface as adopted by the Stratix III development kit allows $3^{\text {rd }}$ party expansion boards to be used with the FPGA board.

The generic I/O interface has the following data and power lines:

- 8 dedicated LVDS pair transmit lines.

- 8 dedicated LVDS pair receive lines.

- 80 single ended transmit/receive lines.

- 4 JTAG programming/debugging lines.

- $20+3.3 \mathrm{~V}$ lines.

- $20+12 \mathrm{~V}$ lines. 
The FPGA board has two generic I/O interface ports. One port is mounted on the side of the board that mates with the image capture board. The other generic I/O port is mounted on the other side of the FPGA board and connects to the external interface board. FPGA differential drive and receive lines are not used on the generic interface as there are not enough migratable pairs between the EP3C40 and EP3C120 FPGAs. Instead LVDS receivers (SN65LVDS390 and SN65LVDS388 from Texas instruments [53, 54]) and transmitters (SN65LVDS391 and SN65LVDS389 from Texas instruments [55]), external to the FPGA, have been used to drive the LVDS lines of the generic interface. The I/O interface for the image capture board requires more than the dedicated eight LVDS transmit and receive lines; twenty transmit and receive pairs respectively. This has resulted in a modification to the generic interface between the FPGA and the image capture board to include extra LVDS pairs that are driven by additional LVDS transmitters and receivers.

\subsubsection{Power Supply}

\subsubsection{Unregulated Power Bus}

The full-field image ranger system requires a significant amount of power. The laser illumination sub-system alone requires approximately $40 \mathrm{~W}$ when running at full power. Due to the system's high power demand the majority of voltage regulators employed are switching regulators. Switching regulators have a higher efficiency than standard linear regulators which is particularly important for large current applications. Linear regulators, due to their low component count and size, have been employed for voltage rails that either supply low current circuits or for circuits that require a clean, spike free voltage supply.

The number of independent voltage rails required by each sub-system and the high current draw has been the prime factor in having an unregulated power bus throughout the system. The power bus allows voltage regulators to be implemented onboard the sub-system circuit board where the voltage rail is required. This simplifies the system to requiring only a single high current capacity rail bussed throughout the system, instead of several buses if voltage rails were shared across several circuit boards. The unregulated power bus allows power separation between sub-system circuit boards facilitating greater flexibility for upgrading and modifying.

All of Victoria's robotic vehicles run off a single unregulated power supply, typically a pair of lead acid batteries. Following the model of having a single power supply source on these 
vehicles, the use of an unregulated power bus has been adopted to allow the ranger system to be compatible with existing robotic vehicles.

\subsubsection{FPGA Power Supply}

The FPGA circuit board has eight independent voltage supply rails for powering the Cyclone III and peripheral components. All voltage rails are regulated onboard the FPGA board from the unregulated power supply that is bussed through the system. The unregulated power supply must be in the range of $14 \mathrm{~V}$ to $20 \mathrm{~V}$ to ensure correct operation of the regulators. Table 7.3 presents the FPGA board voltage rails and lists the devices they power.

Table 7.3 FPGA Board Voltage Rails

\begin{tabular}{|c|c|c|c|}
\hline Voltage Rail & Regulator & $\begin{array}{l}\text { Current } \\
\mathrm{O} / \mathrm{P}\end{array}$ & Description \\
\hline$+12 \mathrm{~V}$ & LT3481 & $2 \mathrm{~A}$ & $\begin{array}{l}\text { This switching voltage regulator powers the }+12 \\
\mathrm{~V} \text { supply on the generic } \mathrm{I} / \mathrm{O} \text { interfaces. It also } \\
\text { provides the input voltage for the }+5 \mathrm{~V} \text { regulator. }\end{array}$ \\
\hline$+5 \mathrm{~V}$ & LT1761 & $100 \mathrm{~mA}$ & $\begin{array}{l}\text { This linear voltage regulator powers the DDR } \\
\text { termination regulators that provide sinking and } \\
\text { sourcing for the active termination of control, } \\
\text { address and data lines to the DDR2 SDRAM } \\
\text { devices. }\end{array}$ \\
\hline$+3.3 \mathrm{~V}$ & LTM4601 & $12 \mathrm{~A}$ & $\begin{array}{l}\text { This switching voltage regulator supplies the }+3.3 \\
\mathrm{~V} \text { rail on the generic I/O interface. It also } \\
\text { provides the power supply for the LVDS receivers } \\
\text { and transmitters, and the FPGA I/O banks that } \\
\text { connect with the LVDS lines. This rail also } \\
\text { provides the input voltage for the }+2.5 \mathrm{~V},+1.8 \mathrm{~V} \text {, } \\
+1.2 \mathrm{~V} \text { and }+1.2 \mathrm{~V}_{\mathrm{PLL}} \text { voltage regulators. }\end{array}$ \\
\hline$+2.5 \mathrm{~V}$ & LTC3026 & $1.5 \mathrm{~A}$ & $\begin{array}{l}\text { This linear voltage regulator powers the FPGA } \\
\text { analogue voltage rail and the JTAG interface. }\end{array}$ \\
\hline$+1.8 \mathrm{~V}$ & LTC3418 & $8 \mathrm{~A}$ & $\begin{array}{l}\text { This switching voltage regulator powers all the } \\
\text { DDR2 SDRAM devices and the Flash memory. It } \\
\text { also powers the FPGA banks that interface to the } \\
\text { external memory. }\end{array}$ \\
\hline$+1.2 \mathrm{~V}$ & LTC3418 & $8 \mathrm{~A}$ & $\begin{array}{l}\text { This switching voltage regulator powers the } \\
\text { internal voltage of the FPGA. }\end{array}$ \\
\hline$+1.2 V_{\text {PLL }}$ & LTC3026 & $1.5 \mathrm{~A}$ & $\begin{array}{l}\text { This linear voltage regulator supplies the digital } \\
\text { voltage for the FPGA's phase lock loops. }\end{array}$ \\
\hline$+0.9 \mathrm{~V}$ & TPS51100 & $3 \mathrm{~A}$ & $\begin{array}{l}\text { This linear voltage regulator provides the } \\
\text { termination voltage required for active termination } \\
\text { of the control, address and data lines of the DDR2 } \\
\text { SDRAM devices. }\end{array}$ \\
\hline
\end{tabular}


It is important that the sequence of powering up the voltage rails on the FPGA board be controlled to prevent damage to the FPGA and ensure correct operation on power start-up. Figure 7.12 presents the power-up sequence for the voltage rails on the FPGA board. All regulators are disabled until their input voltage source has reached a stable level. This guarantees that the input voltages will be stable and glitch free, reducing the likelihood of voltage rail spikes cascading through the power supply. The oscillator that provides the system clock for the FPGA is not enabled until its voltage supply, $+1.8 \mathrm{~V}$, has reached a stable level.

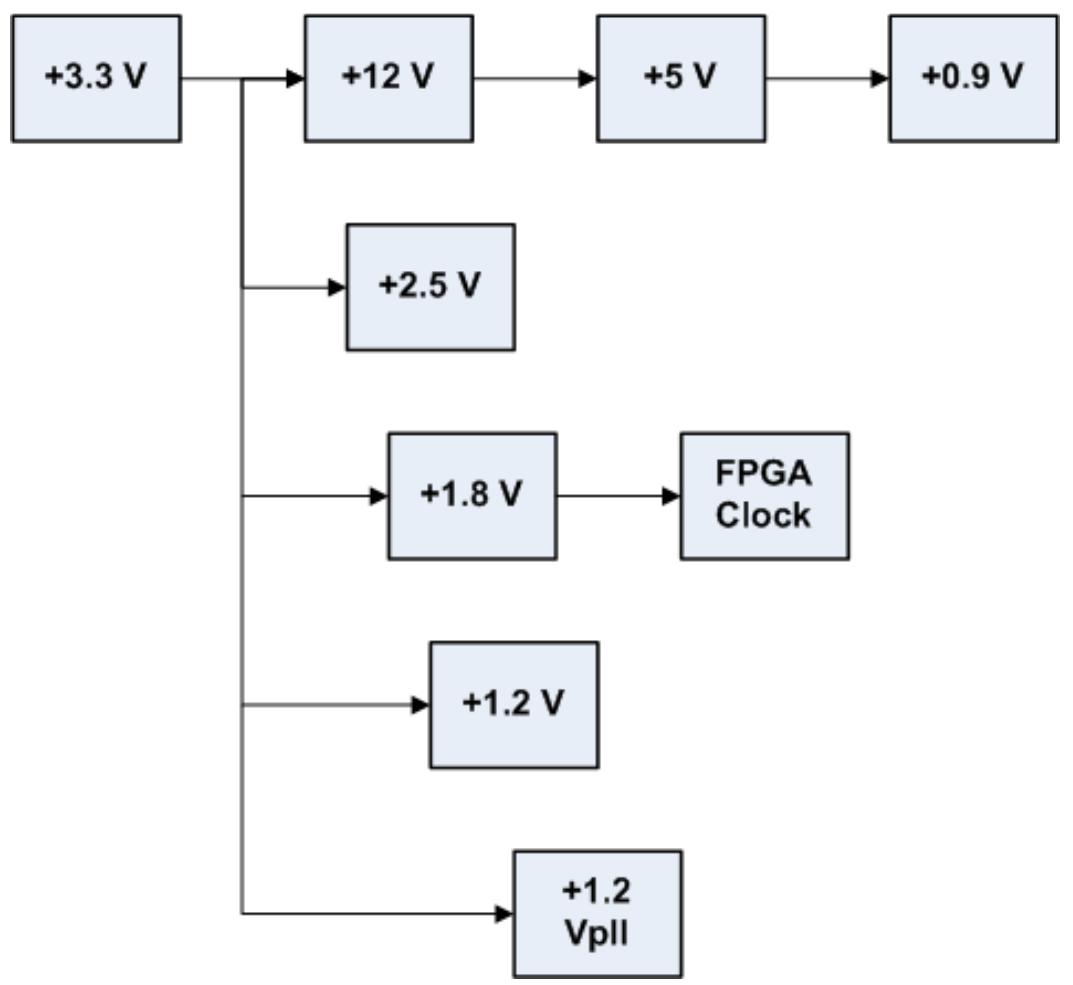

Figure 7.12 Voltage Supply Power Up Sequence for FPGA Board

Each of the design sub-circuits discussed in the previous section have been integrated into a single printed circuit board. Figures 7.13 and 7.14 illustrate the placement of the sub-circuits and primary interface headers of the FPGA PCB. A block diagram of the major sub-circuits of the FPGA board is presented in Figure 7.15. 


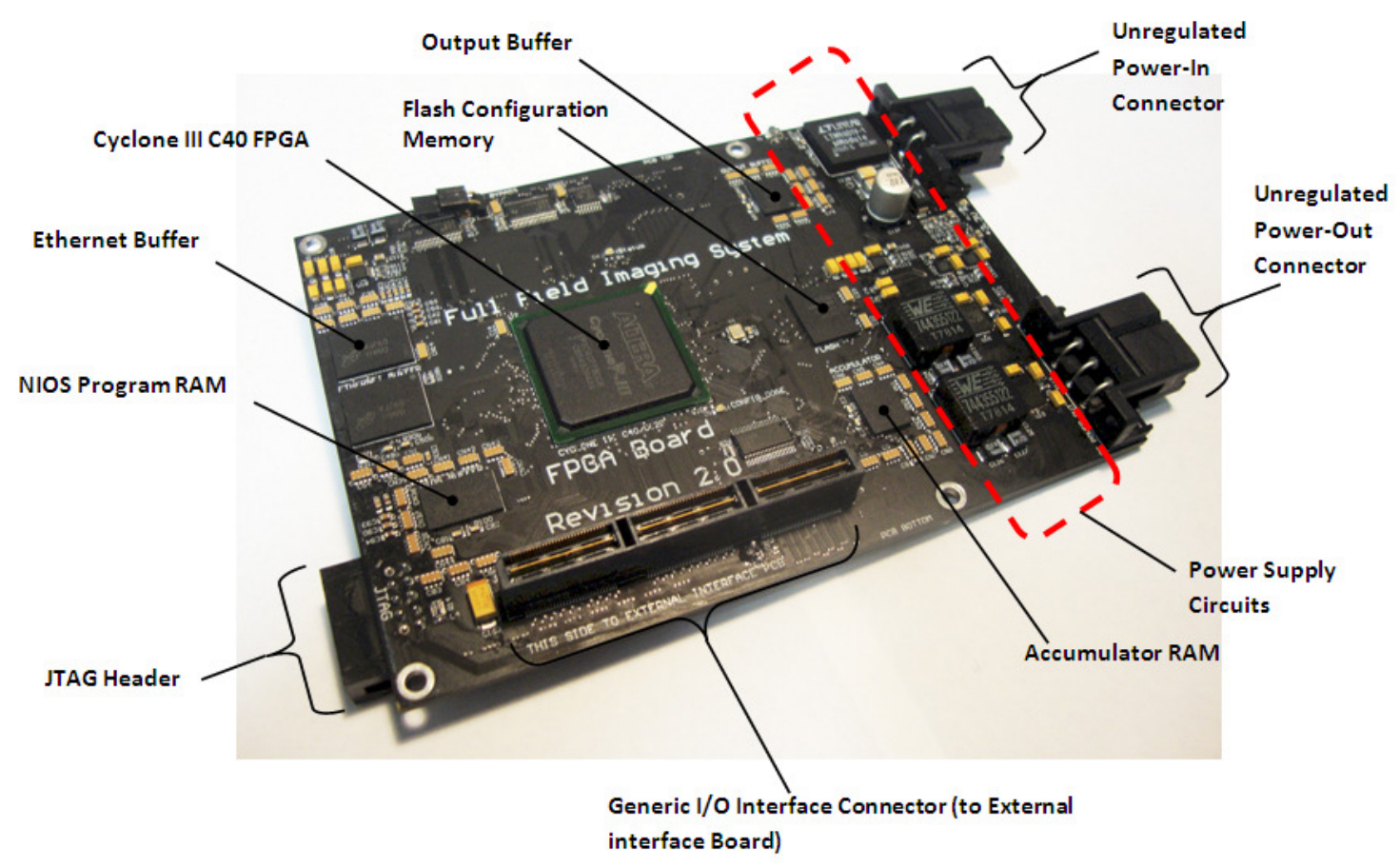

Figure 7.13 FPGA PCB layout - Top Side

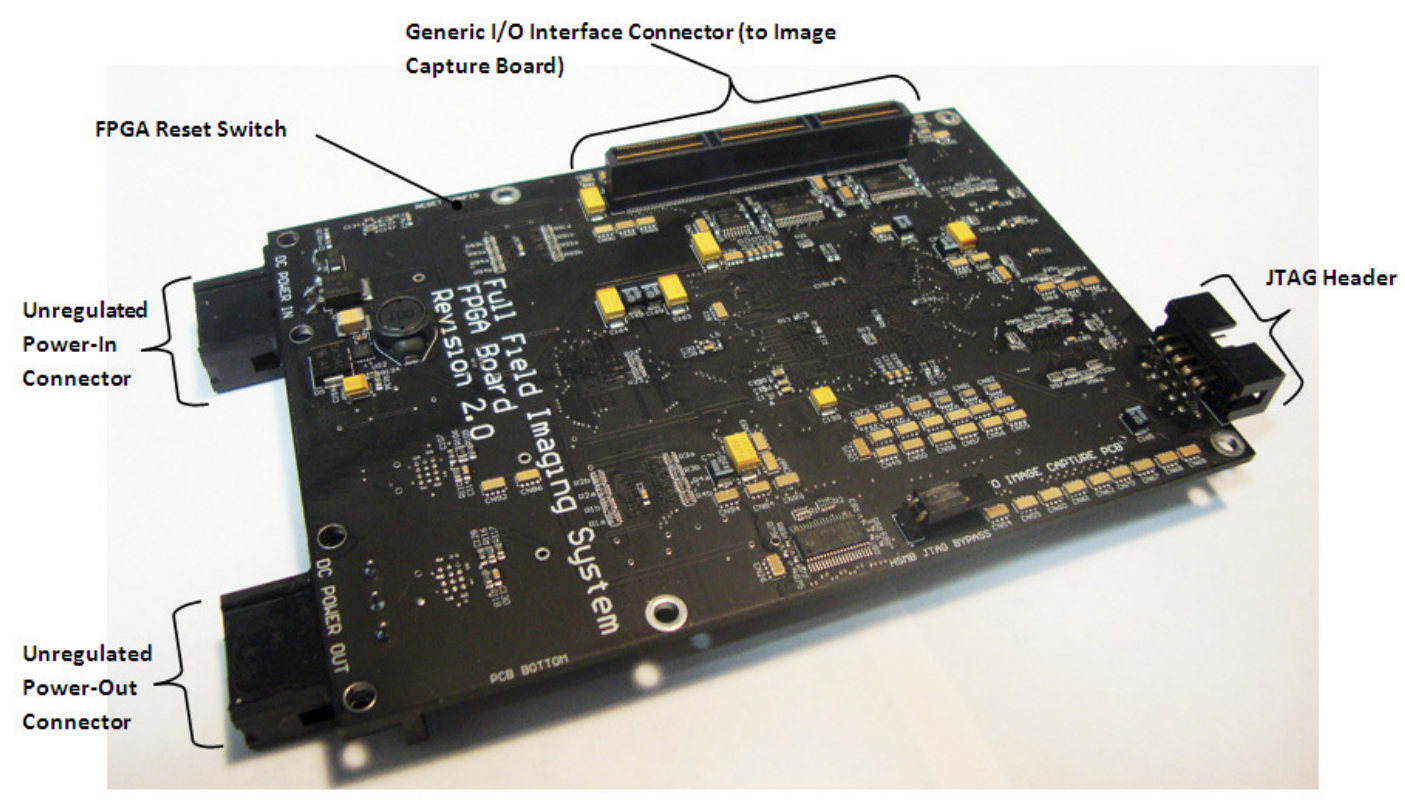

Figure 7.14 FPGA PCB Layout - Bottom Side 


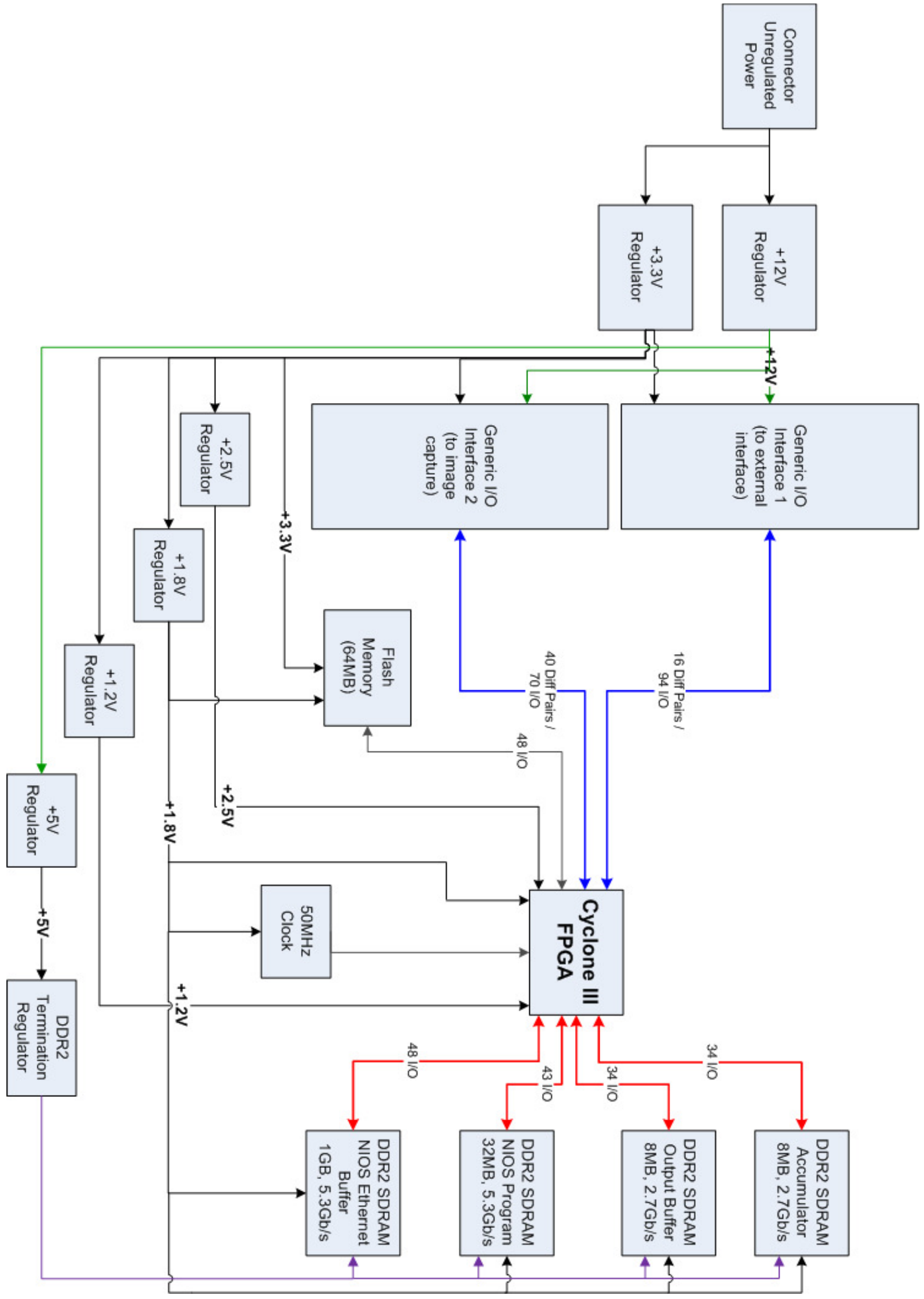

Figure 7.15 Mobile Platform Imaging Ranger - FPGA Block Diagram 


\section{Chapter 8 Hardware Methodology -Image Ranger Peripherals}

This chapter describes the hardware design for the peripheral modules of mobile full-field image ranger system. The peripheral modules interconnect with the FPGA board, described in Section 7.3 FPGA Board, to provide image capture, laser illumination and external interface functionality to the system.

\subsection{Image Capture Board}

The image capture board connects to the FPGA board via a generic I/O interface. The image capture board handles digitising of the captured images from the sensor and all signal modifications to drive the sensor. The board provides bus power and modulation control signals from the FPGA board to the Illumination board. Figure 8.1 illustrates the main components of the image capture board.

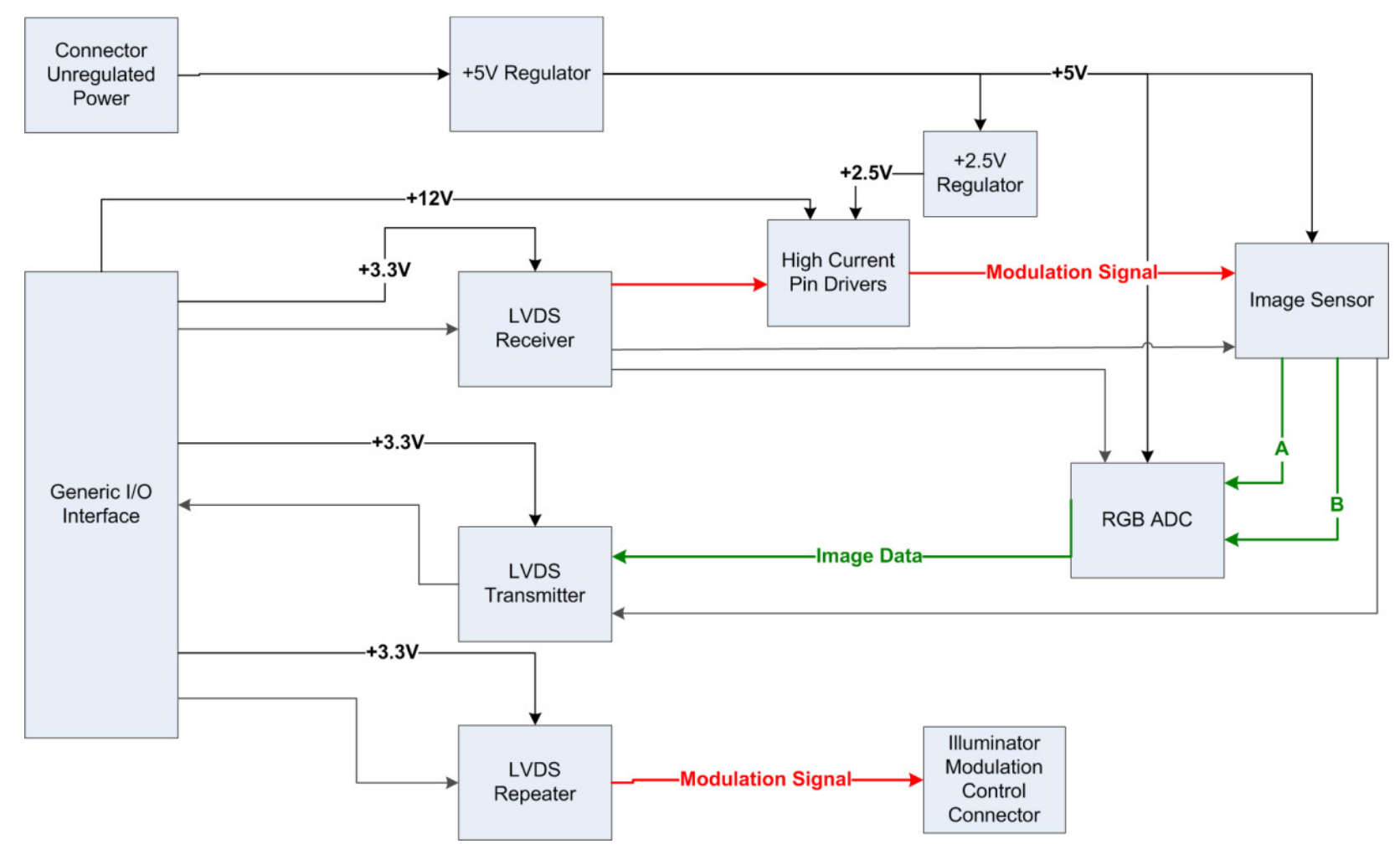

Figure 8.1 Image Capture Board Block Diagram 
The image capture board has been designed as a piggyback board to allow the PMD Daughter board from the prototype system to be integrated into the mobile platform system. As previously mentioned, this modification has been implemented due to the unavailability of imaging sensors at the time of the system design. The full-field image ranger system requires control over the modulation of the sensor and access to the raw pixel values. This requirement to have modulation control and raw pixel access eliminated several image sensors currently available on the market. Several manufacturers of suitable image sensors were contacted but none were able to provide sensors or documentation on sensors in production. An imaging sensor similar to the one used on the prototype system could not be sourced as it was no longer in production. As the image capture board is a sub-system of the complete mobile platform system it will be straight forward to design and 'drop-in' a new image capture board when an imaging sensor does become available.

The PMD Daughter board connects to the image capture board such that the imaging sensor is centred on the board. This ensures that the attached optical lens will be aligned with the circular cut-out on the laser illumination board.

\subsubsection{Imaging Sensor}

The PMD 19K-2 3D Video Sensor Array from PhotonICs has been used as the imaging sensor. As mentioned in section 7.1.3 PMD Daughter Board, this sensor features a $160 \times 120$ array of pixels grouped into four independently modulated blocks of $40 \times 120$ pixels. Figure 8.2 illustrates the PhotonICs sensor.

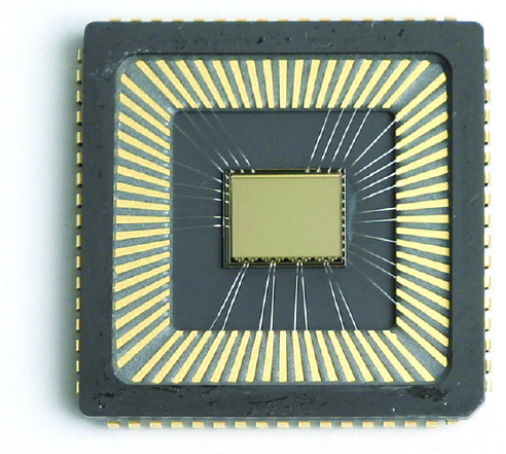

Figure 8.2 The PhotonICs PMD 19K-2 Sensor Array [41]

Each modulation block has two modulation inputs (ModA and ModB). The modulation blocks represent a capacitive load of $250 \mathrm{pF}$. At a modulation frequency of $10 \mathrm{MHz}$, the 
large capacitive load of the modulation blocks produces small input impedances requiring high current sources to drive them. Ultra high current pin drivers (EL7158 from Intersil) are used to drive the two modulation signals of each modulation block. This requires eight high current drivers for all four modulation blocks.

The high current pin drivers are powered from the $+12 \mathrm{~V}$ rail from the generic $\mathrm{I} / \mathrm{O}$ interface. From Figure 8.3 it can be seen that the high current pin drivers provide the best performance in the rise/fall times of approximately $12 \mathrm{~ns}$ at $+11 \mathrm{~V}$ with only a minimal difference at the available $+12 \mathrm{~V}$ supply from the $\mathrm{I} / \mathrm{O}$. These rise and fall times limit the maximum modulation frequency, without skew, to $41 \mathrm{MHz}$.

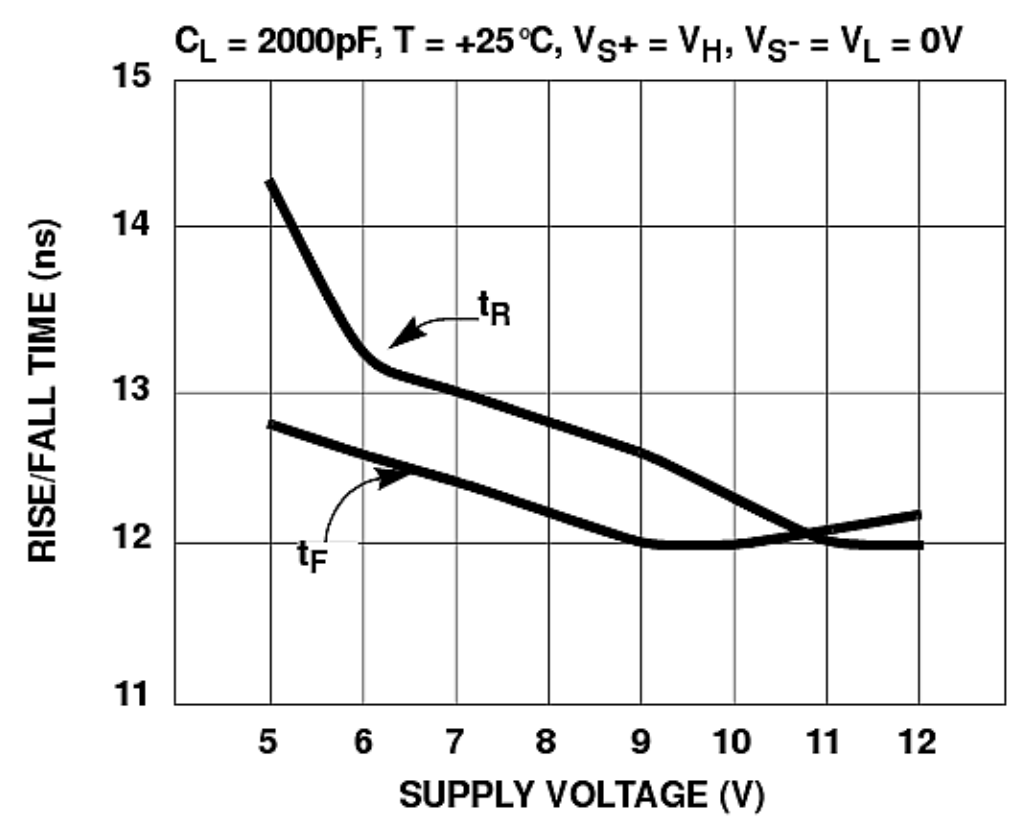

Figure 8.3 High Current Pin Driver Rise and Fall Times Vs Supply Voltage [43]

As can be seen from Figure 8.4, the PMD Image Sensor Modulation inputs require a $+2.5 \mathrm{~V}$ signal to achieve maximum contrast. 


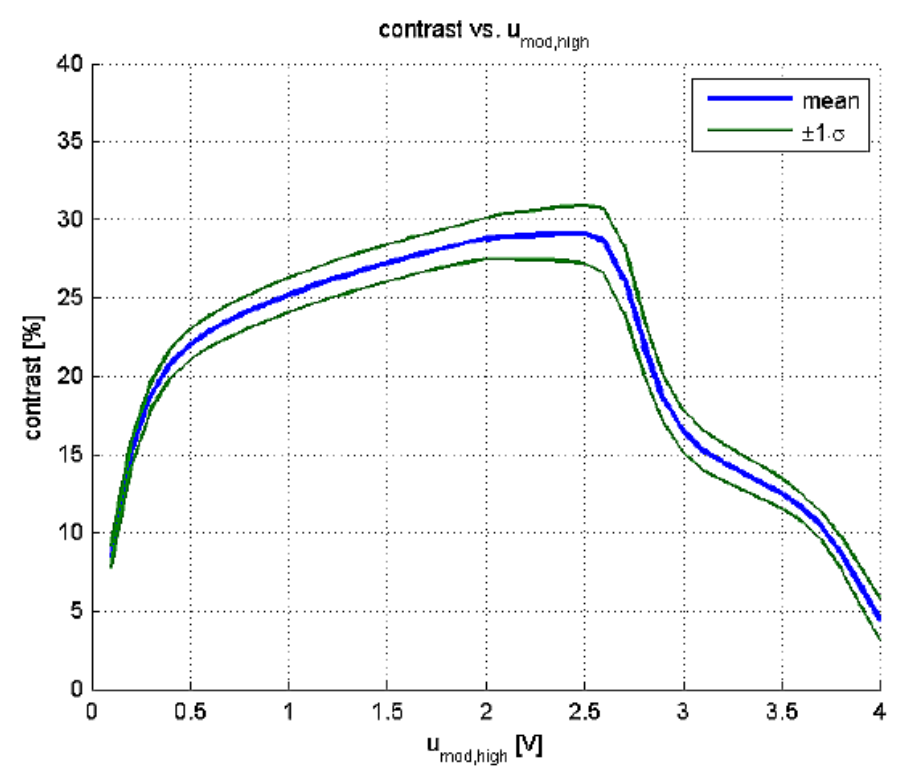

Figure 8.4 Image Contrast vs. Modulation Signal Voltage for PMD 19K2 Sensor [41]

At a modulation frequency of $41 \mathrm{MHz}$ the imaging sensor's modulation inputs have an impedance of approximately $15.5 \Omega$ each. At $+2.5 \mathrm{~V}$ the current draw per modulation input will be approximately $160 \mathrm{~mA}$. At a worst case of four modulation inputs sourcing current at one time, the total current draw will be $640 \mathrm{~mA}$ from the $+2.5 \mathrm{~V}$ supply. This is provided in the system by a TPS79625 linear regulator from Texas Instruments [56].

\subsubsection{Analogue to Digital Conversion}

The image sensor has two analogue video outputs, corresponding to the ModA and ModB modulation inputs for each block. A 16-bit Imaging Signal processor ADC (AD9826 from Analog Devices [42]) is provided to convert the two analogue video streams from the image sensor into digital frames to be processed by the FPGA. This ADC can sample the video streams at a maximum rate of 15 million samples a second. Each 16-bit data word is transferred to the FPGA in two sequential bytes on an 8-bit data bus via the generic $\mathrm{I} / \mathrm{O}$ interface.

\subsubsection{Low Voltage Differential Signals}

The generic I/O interface between the FPGA and image capture board utilises LVDS signals to improve the quality of signals carried across the interface. As such LVDS receivers and 
transmitters are required to convert the LVDS signals to the single-ended CMOS signals required by the image sensor, ADC and high current pin drivers. The same type of LVDS transmitters and receivers employed on the FPGA board are utilised on the image capture board (SN65LVDS388, SN65LVDS389 and SN65LVDS9637 from Texas instruments [54, $55,57])$.

The image capture board also busses the modulation and control signals for the illumination board. These signals are transmitted from the FPGA board as LVDS signals. The image capture board employs LVDS repeaters (SN65LVDT100DGK from Texas Instruments [58]) to boost the strength of the signals. The modulation and control signals are bussed from the image capture board to the illumination board via two Serial Advanced Technology Attachment (SATA) connections, one for the modulation signals and one for the control signals.

\subsubsection{Power Supply}

The voltage rails for the image capture board are derived from the unregulated power bus through on-board regulators and the generic I/O interface. Table 8.1 presents the image capture board voltage rails and lists the devices that are powered by the rails.

Table 8.1 Image Capture Board Voltage Rails

\begin{tabular}{|c|c|c|c|}
\hline Voltage Rail & Regulator & $\begin{array}{c}\text { Current } \\
\mathrm{O} / \mathrm{P}\end{array}$ & Description \\
\hline$+12 \mathrm{~V}$ & $\begin{array}{l}\text { Generic } \mathrm{I} / \mathrm{O} \\
\text { Interface }\end{array}$ & $2 \mathrm{~A}$ & $\begin{array}{l}\text { The }+12 \mathrm{~V} \text { rail from the generic } \mathrm{I} / \mathrm{O} \text { interface } \\
\text { powers the high current pin drivers. }\end{array}$ \\
\hline$+5 V$ & LT3481 & $2 \mathrm{~A}$ & $\begin{array}{l}\text { The switching voltage regulator powers the PMD } \\
19 \mathrm{~K} 2 \text { imaging sensor, the Imaging Signal } \\
\text { processor ADC and provides the input voltage for } \\
\text { the }+2.5 \mathrm{~V} \text { regulator. }\end{array}$ \\
\hline$+3.3 \mathrm{~V}$ & $\begin{array}{l}\text { Generic I/O } \\
\text { Interface }\end{array}$ & $3.3 \mathrm{~A}$ & $\begin{array}{l}\text { The }+3.3 \mathrm{~V} \text { rail from the generic } \mathrm{I} / \mathrm{O} \text { interface } \\
\text { powers the LVDS transmitters, receivers and } \\
\text { repeaters. }\end{array}$ \\
\hline$+2.5 \mathrm{~V}$ & TPS79625 & $1 \mathrm{~A}$ & $\begin{array}{l}\text { The voltage regulator provides the output voltage } \\
\text { for the high current pin drivers that feed the } \\
\text { imaging sensor modulation inputs. }\end{array}$ \\
\hline
\end{tabular}


Each of the design sub-circuits discussed in the previous section have been integrated into a single printed circuit board. Figures 8.5 and 8.6 illustrate the placement of the sub-circuits and primary interface headers of the image capture PCB.

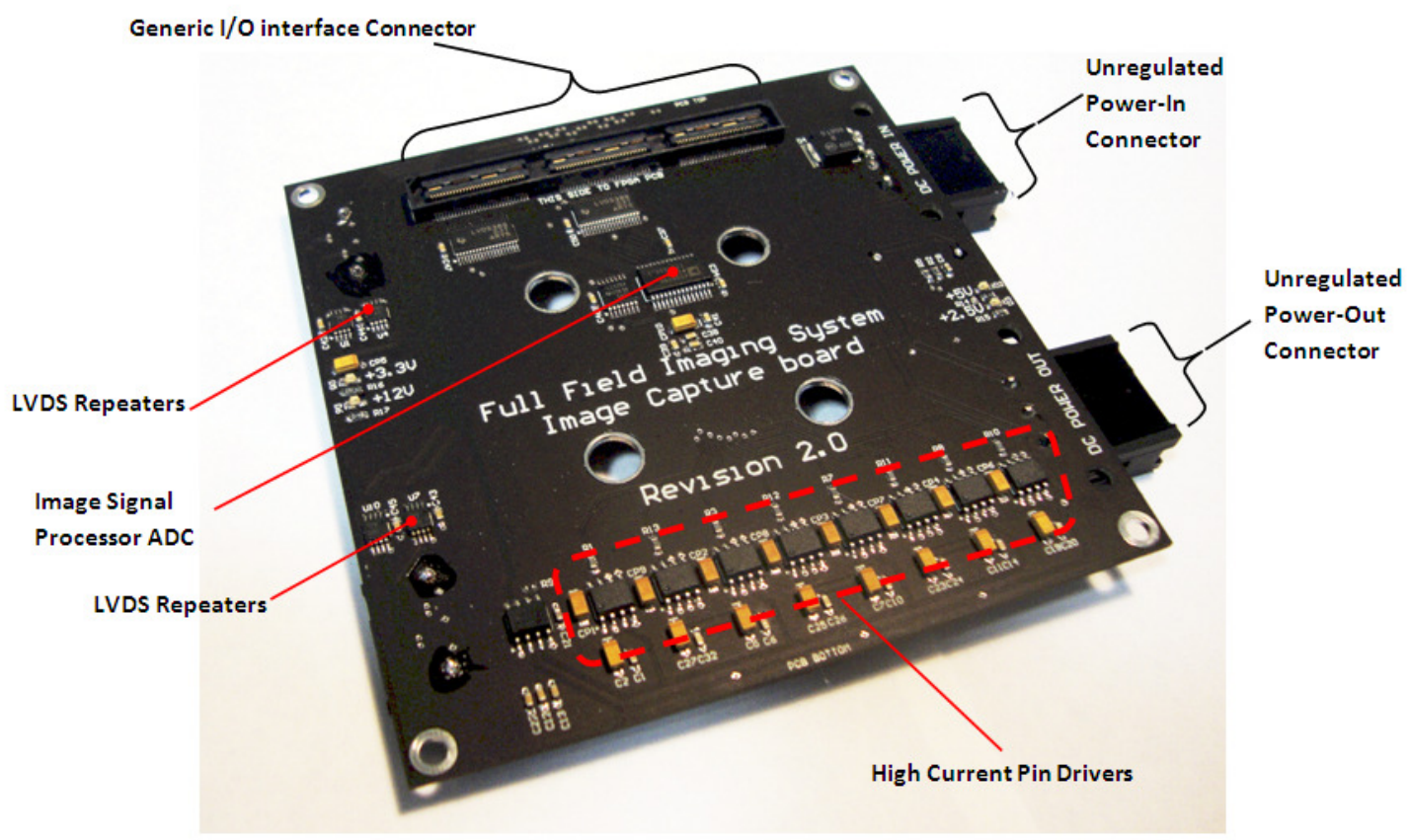

Figure 8.5 Image Capture PCB layout - Top Side

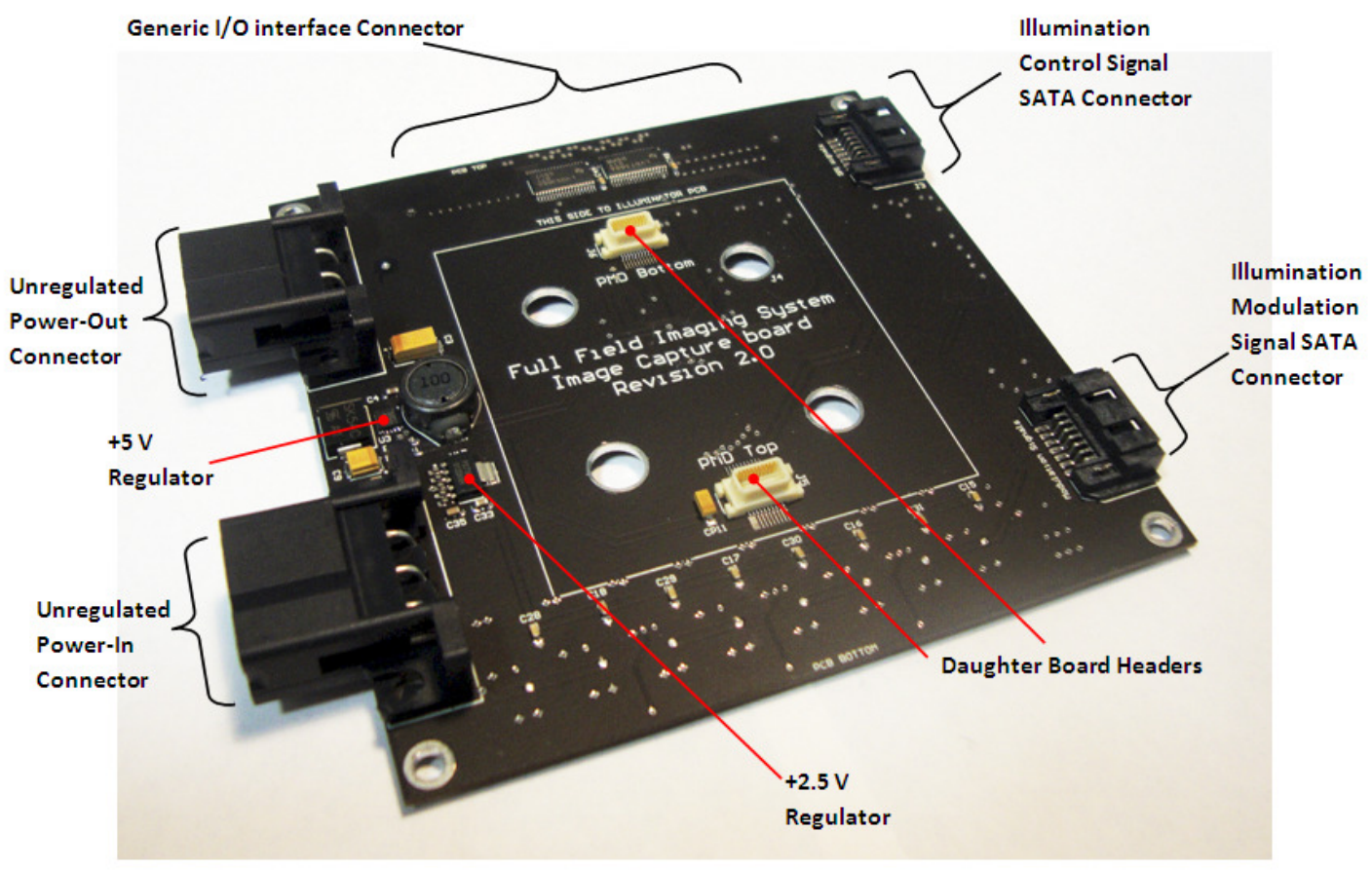

Figure 8.6 Image Capture PCB layout - Bottom Side 


\subsection{Laser Illumination Board}

The laser illumination board contains the modulated light source for the image ranger system. The board has sixteen laser diodes arranged into two independent banks of eight laser diodes each. The two independent banks allow the system to illuminate a scene with two different wavelengths of light at different modulation frequencies. Due to the unavailability of the $880 \mathrm{~nm}$ IR laser diodes from existing suppliers, the illumination board has both banks populated with $130 \mathrm{~mW}$ laser diodes of wavelength $660 \mathrm{~nm}$. Laser switch integrated circuits (ic-HK from icHaus [59]) are used to modulate the intensity of the laser diodes. The laser switch ICs can handle modulation frequencies of up to $155 \mathrm{MHz}$ and can switch the laser between two different intensities. Each laser diode requires its own laser switch IC to provide enough current to reach maximum light intensity. Figure 8.7 presents a block diagram of the main sub-systems of the laser illumination board.

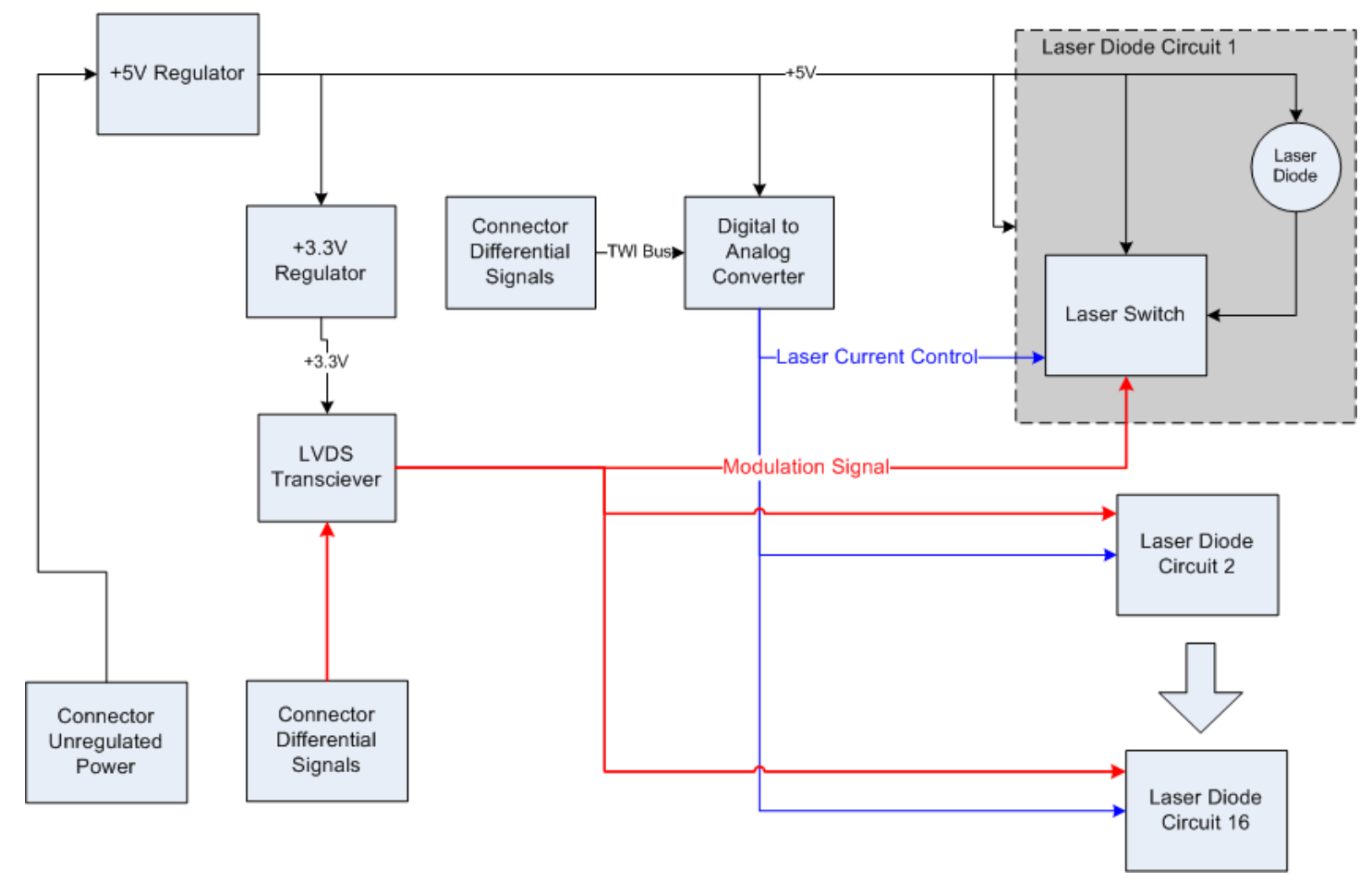

Figure 8.7 Laser Illumination Board Block Diagram

As mentioned in the section 7.2.1 Circuit Board Dimensions, the laser illumination circuit board requires a circular cut-out of diameter $35 \mathrm{~mm}$ to allow the optical lens to protrude through. This cut-out is positioned in the centre of the board and is surrounded by the sixteen laser diodes. The laser diodes from the two independent banks are interleaved in position around the cut-out to provide similar dispersion of laser light. 


\subsubsection{Laser Diode Current}

As mentioned in Section 7.1.2 Illumination Board, it is important that the current through the laser diode be tightly controlled to prevent damage. Sudden increases in the temperature of a laser diode can significantly reduce its life span. It is important at start-up that the diode current be gradually increased to allow stable heat dissipation to the laser diode heat sink. On the laser illumination board, the current through the laser diodes is set for each bank independently, from the output voltage of a digital to analogue convertor (AD5311 from Analog Devices [60]). The DAC output voltage is controlled by a two wire interface (TWI) that is driven by control signals, bussed through the Image Capture board, from the FPGA. This allows the FPGA to control the laser diode current at start-up to ensure a smooth increase in diode current. Figure 8.8 illustrates the flow of the laser diode current control signals from the FPGA to the laser switch.

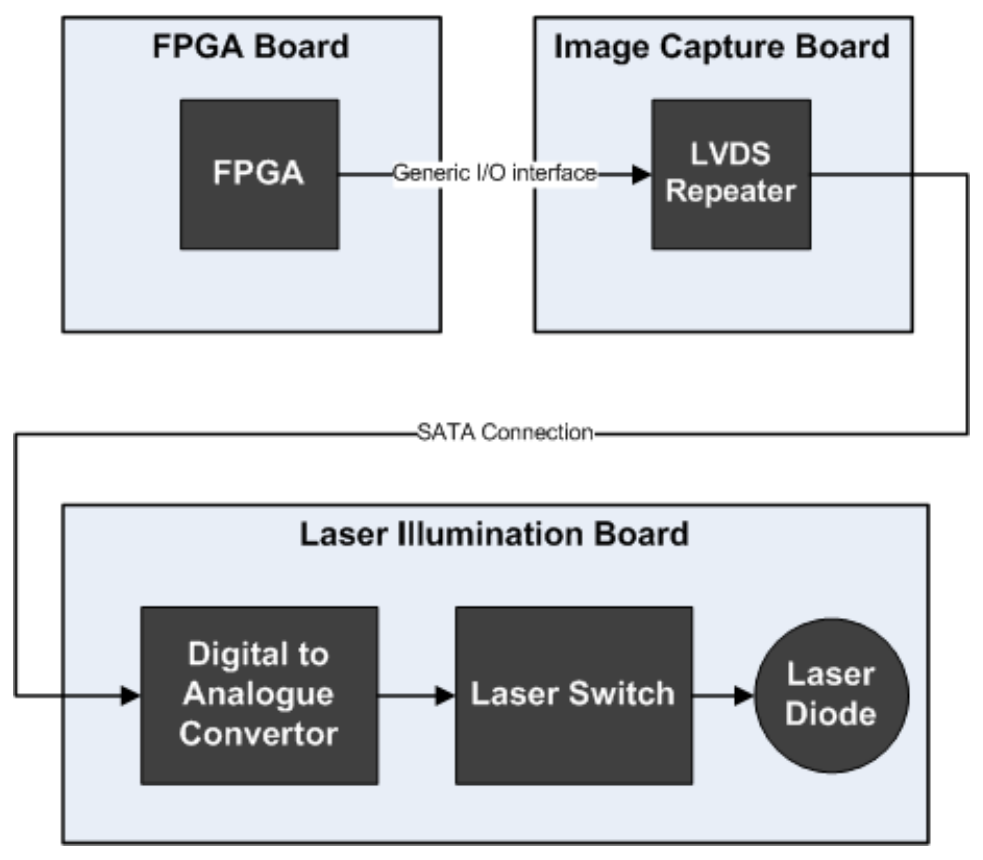

Figure 8.8 Laser Diode Current Control

A switch allows the user to select between running the laser diodes at maximum power or half power. The half power option operates the laser diodes at a safer optical intensity when output power is not a critical consideration for image ranging a scene.

A protection circuit has also been included to switch off the laser diodes if the control or modulation signals from FPGA board become disconnected from the laser illumination board. A simple low pass filter is employed to filter the modulation signal. The output of the 
filter is compared with a reference voltage. If the filter output is above the reference voltage the laser diodes are switched off. Figure 8.9 illustrates the protection circuit for the laser diode current.

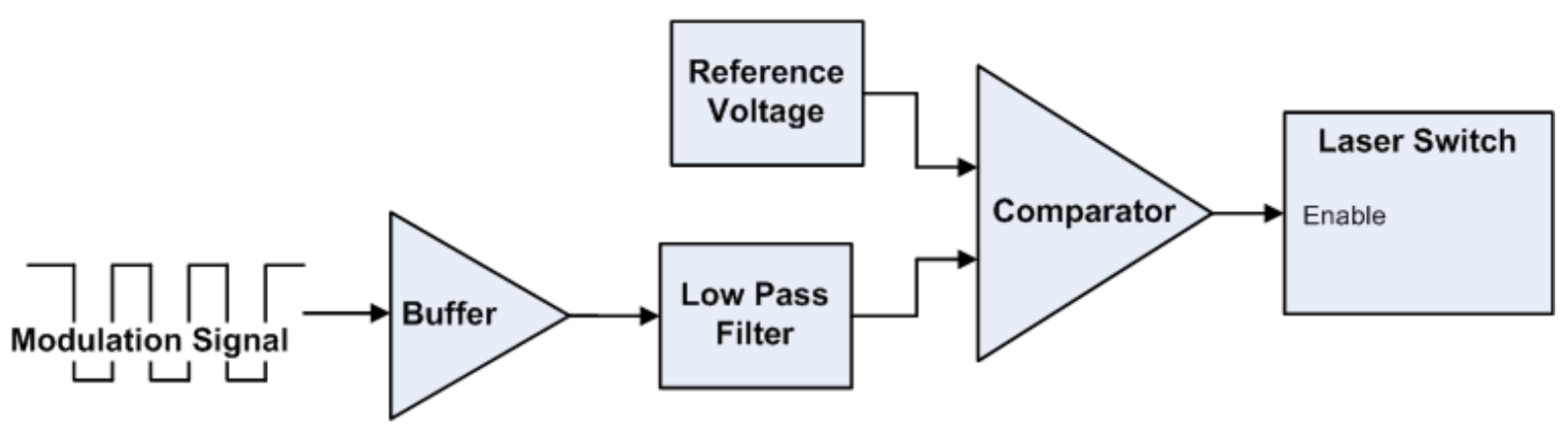

Figure 8.9 Protection Circuit for Laser Diode Current

\subsubsection{Power Supply}

The power requirements for the laser illumination board are significant. The worst case current draw for each laser diode circuit is $350 \mathrm{~mA}$. With an array of sixteen lasers the current draw on the $+5 \mathrm{~V}$ could be up to 5.6 A. For this reason the laser illumination board has an on-board high current regulator to provide the $+5 \mathrm{~V}$ supply for the laser circuit. The input voltage for the regulator is sourced directly from the unregulated power supply that is bussed through the system. Table 8.2 presents the voltage rails on the laser illumination board and lists the devices that are powered from the rails.

Table 8.2 Laser Illumination Board Voltage Rails

\begin{tabular}{c|c|c|l|}
$\begin{array}{c}\text { Voltage } \\
\text { Rail }\end{array}$ & Regulator & \multicolumn{1}{c|}{ Current } \\
O/P \\
$+\mathbf{+ 5 ~ V}$ & LTM4601 & 12 A & $\begin{array}{l}\text { This switching regulator provides the voltage } \\
\text { that powers the laser diodes, laser switches, } \\
\text { DAC and associated protection circuitry. It also } \\
\text { provides the input voltage for the +3.3 V V } \\
\text { regulator. }\end{array}$ \\
\hline$+3.3 \mathbf{~ V}$ & $\begin{array}{c}\text { ADP3300ART- } \\
3.3\end{array}$ & $50 \mathrm{~mA}$ & $\begin{array}{l}\text { This linear regulator provides the voltage for the } \\
\text { LVDS receivers that convert the LVDS } \\
\text { modulation and control signals from the FPGA } \\
\text { to single-ended signals. }\end{array}$ \\
\hline
\end{tabular}


Each of the design sub-circuits discussed in the previous section have been integrated into a single printed circuit board. Figures 8.10 and 8.11 illustrate the placement of the sub-circuits and primary interface headers of the laser illumination PCB.

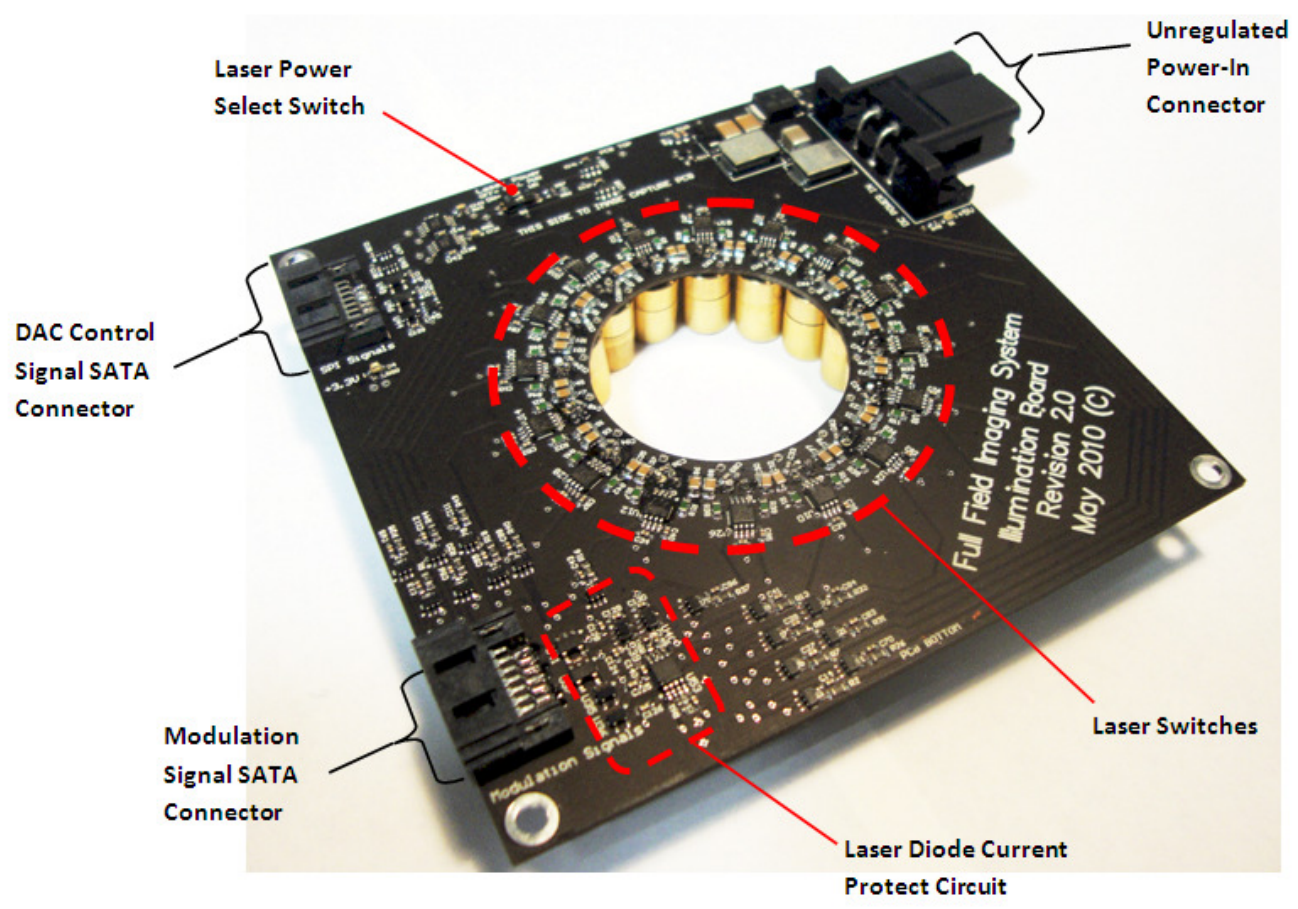

Figure 8.10 Laser Illumination PCB layout - Top Side

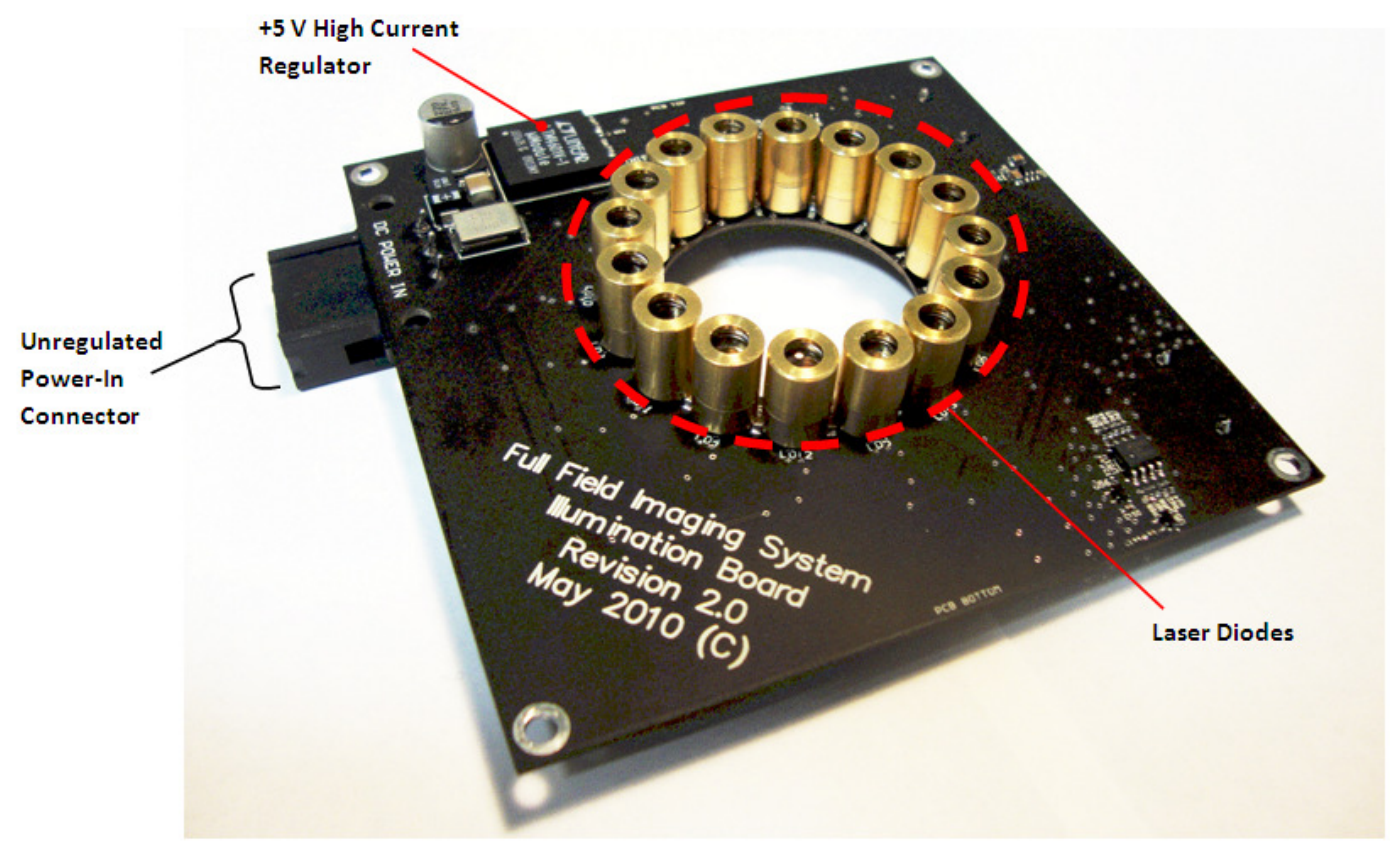

Figure 8.11 Laser Illumination PCB layout - Bottom Side 


\subsection{External Interface Board}

The external interface board provides all the necessary communication drivers to allow the system to connect to peripheral devices such as an external computer or VGA monitor. It also provides communication interfaces to allow configuration and management of the imaging system by an external control computer. The external interface board has an input connector for the unregulated power supply that powers the system. Figure 8.12 presents a block diagram of the main sub-systems of the external interface board.

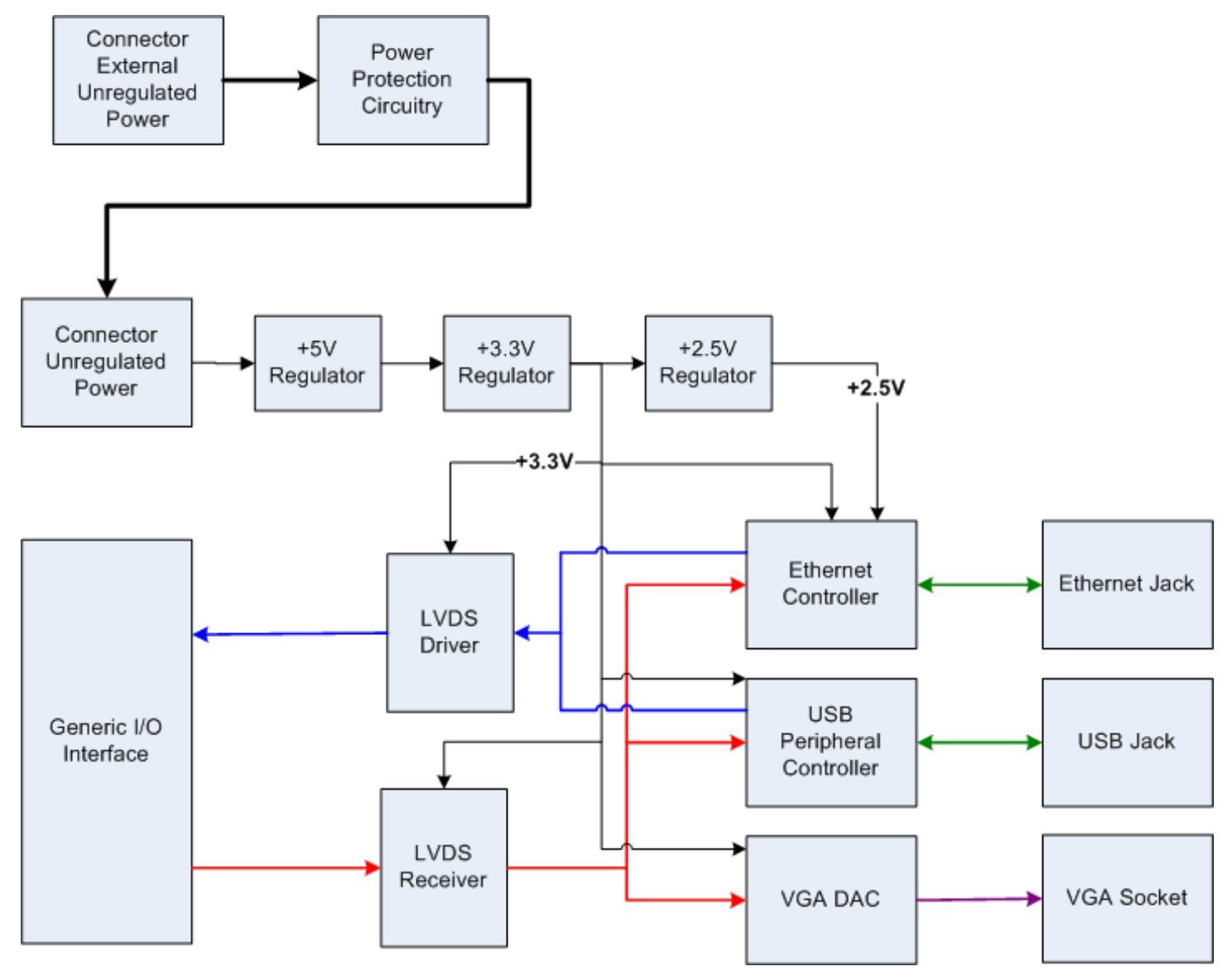

Figure 8.12 External Interface Board Block Diagram 


\subsubsection{Video Graphics Array (VGA) Interface}

The image ranger system can display image frames on an external standard $640 \times 480$ resolution VGA monitor over a VGA interface. This allows the user to examine four frames of raw and processed data simultaneously in real time.

VGA hardware produces a colour image through a raster scan method. Historically this has been by scanning an electron beam over the view screen in a sequence of horizontal lines, corresponding to a row of pixels in the image. Figure 8.13 illustrates the raster scan process for a $640 \times 480$ pixel image. The scan begins by sweeping the electron beam from pixel $(0,0)$ to pixel $(639,0)$.

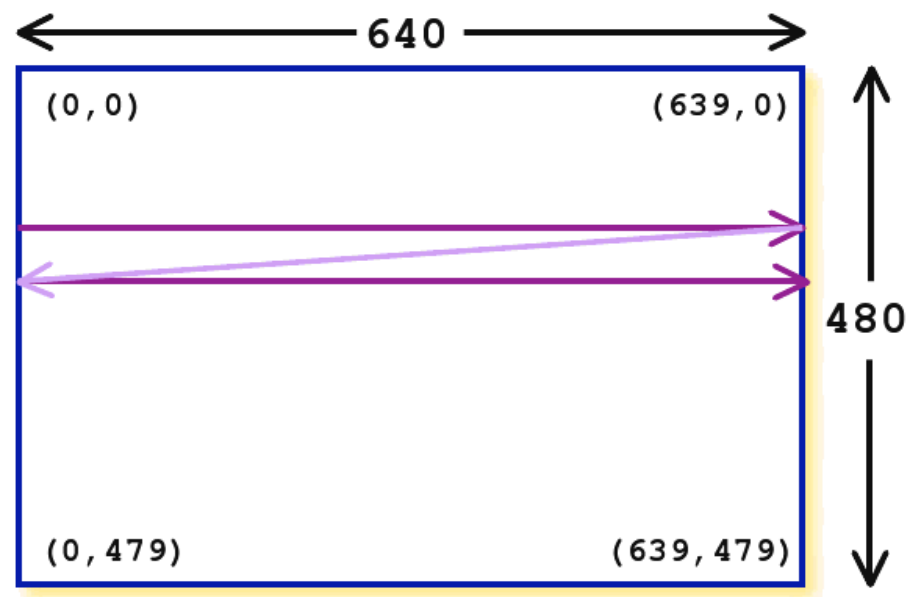

Figure 8.13 Raster Scan of 640x480 pixel image

The electron beam is repositioned to pixel $(0,1)$ and sweeps across to pixel $(639,1)$. The electron beam continues to sweep across the screen until it reaches pixel $(639,479)$ where it is reset to pixel $(0,0)$ to begin displaying a new frame.

Three analogue video streams corresponding to the red, blue and green image content of the frame are used to control the strength of the electron beam. The VGA hardware must be refreshed with new image data at least sixty times a second. A horizontal sync signal is used to instruct the VGA hardware to refresh another row of pixels. A vertical sync signal is used to instruct the VGA hardware to start displaying a new image. It is important that the RGB analogue video streams are set to zero during both a horizontal and vertical sync to ensure erroneous image data is not produced by the repositioning of the electron beam. Figure 8.14 
illustrates the relationship between the horizontal and vertical sync signals and the raster scan.

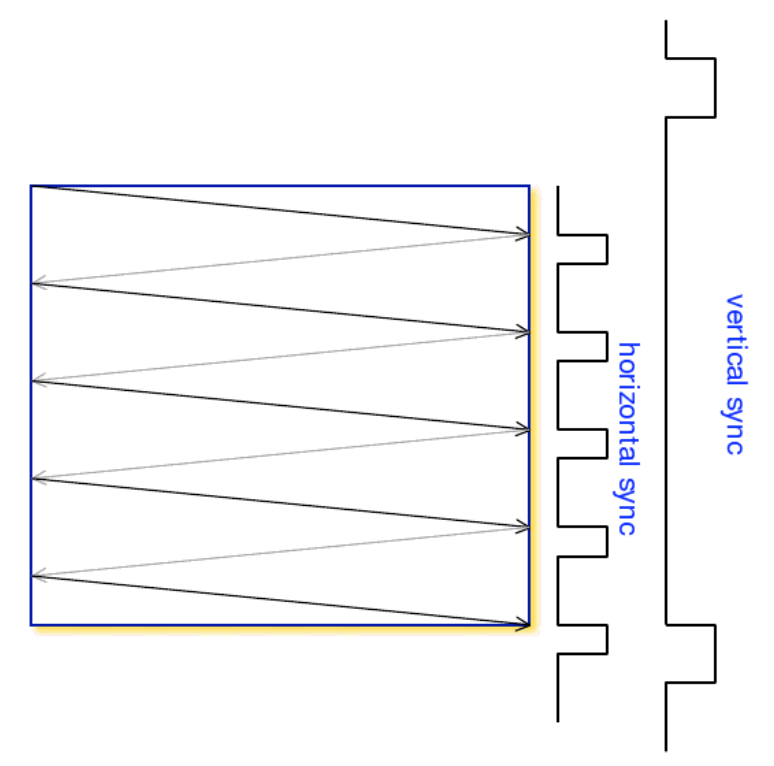

\section{Figure 8.14 Horizontal and Vertical Sync signals in VGA Display}

The VGA output is driven by a triple 10-bit high speed video DAC (ADV7123, from Analog Devices). This DAC has three separate 10-bit input ports that drive three complementary outputs to produce the analogue red, green and blue video streams to display images on a VGA monitor. The NIOS processor, running on the FPGA, is responsible for outputting the digital data streams to the DAC. The NIOS processor also controls the horizontal and vertical sync signals to ensure they are synchronised with the digital data input streams of the DAC. All the VGA data and control lines are sent over the generic I/O interface from the FPGA board to the external interface board. The external interface board uses a standard VGA jack to allow the connection of any standard VGA monitor.

\subsubsection{Ethernet Interface}

The external Interface board has an Ethernet interface to allow long term storage of processed and raw image frames on an external computer. The Ethernet interface allows a user to send commands to configure and manage the imaging system. A stand-alone Ethernet controller (DM9000A from Davicom [61]) provides both a MAC and PHY transceiver to meet the IEEE 802.3 specification for Ethernet connections. The DM9000A is interfaced to the FPGA via a 16-bit data bus over the generic I/O interface. The Ethernet controller chip is clocked from a $25 \mathrm{MHz}$ clock signal generated by the FPGA and sent, via LVDS signals, over the 
generic I/O interface. The NIOS processor is responsible for writing image frames to the Ethernet controller chip. A RJ-45 jack with integrated Ethernet transformer and status LEDs is used to reduce the component count on the board.

\subsubsection{USB Interface}

The external interface board provides a USB interface between the image ranger system and a control computer. The USB interface is provided to allow a user to send commands and data to the system from an external control computer.

An 8-bit microcontroller (ATMega32U4 from Atmel [62]) incorporating a USB controller is employed to provide the communication interface between the control computer and the image ranger system. The MCU provides full-speed and low-speed USB connections and allows the image ranger system to appear as a USB device to any USB host running on an external computer. The ATMega32U4 is an 8-bit AVR MCU with $32 \mathrm{~KB}$ of flash memory and $2.5 \mathrm{~KB}$ of SRAM. The MCU has a JTAG interface allowing programming and in-circuit emulation. A single supply rail of $+3.3 \mathrm{~V}$ is required for MCU operation. An $8 \mathrm{MHz}$ crystal provides the base clock frequency for the MCU. Due to the AVR's RISC architecture, an $8 \mathrm{MHz}$ clock allows up to 8 million instructions per second (MIPS). An onboard PLL provides a $48 \mathrm{MHz}$ clock signal allowing the MCU to support a data transfer rate of $12 \mathrm{Mbit} / \mathrm{s}$ required for full-speed USB connections. The MCU is interfaced to the FPGA via a 20 bit data bus over the generic $\mathrm{I} / \mathrm{O}$ interface.

The USB specification requires standard connectors for USB end devices, such as the ATMega32U4. Hence a Mini-B USB connector is used on the external interface board.

\subsubsection{Power Supply}

The external interface board provides the main power connector to connect an unregulated external power source (bench top PSU, battery etc) to the system. The power consumption of the image ranger sub-systems has been calculated based on the maximum ratings of the components utilised on each board. These calculated values are:

- FPGA Board - $23 \mathrm{~W}$.

- External interface Board - 3.72 W.

- Image Capture Board - 5.15 W.

- Laser Illumination Board - 47.7 W. 
The FPGA board power consumption is primarily set by the configuration of the FPGA, specifically the speed and the number of resources utilised. The power consumption stated here for the FPGA board is based on power consumption measurements on the Stratix III development board when programmed with the prototype ranger system configuration.

Based on the power consumption calculation the input power supply must be able to deliver $80 \mathrm{~W}$ of power. The power supply must be in the range of $14 \mathrm{~V}-20 \mathrm{~V}$ and, given a worst case input voltage of $14 \mathrm{~V}$, be capable of supplying $5.7 \mathrm{~A}$. The external interface board incorporates protection and filter circuits to provide a clean power supply to the unregulated power bus that runs through the entire system. The external interface board provides two power-out connectors, fused at $10 \mathrm{~A}$ each, to allow the unregulated power supply bus to be connected to the other boards in the system. A 40 A rated Schottky diode (48CTQ060SPBF, from Vishay) provides reverse polarity protection on the input voltage. An inductor-capacitor (LC) filter, rated at $23 \mathrm{~A}$, provides filtering on the input power supply to prevent high frequency noise.

All voltage supplies for the external interface board are provided from on-board regulators. The input voltage for the regulators is sourced directly from the unregulated power supply that is bussed through the system. Table 8.3 presents the voltage rails on the external interface board and lists the devices that are powered from the rails.

Table 8.3 External Interface Board Voltage Rails

\begin{tabular}{c|c|c|l|}
$\begin{array}{c}\text { Voltage } \\
\text { Rail }\end{array}$ & Regulator & \multicolumn{1}{c|}{ Description } \\
$\mathbf{+ 5 ~ V}$ & LT3481 & 2 A & $\begin{array}{l}\text { This switching regulator provides the voltage } \\
\text { required to power the voltage level shifting } \\
\text { circuit for the JTAG interface on the USB MCU. } \\
\text { It also provides the input voltage for the +3.3 V } \\
\text { and +2.5 V regulators. }\end{array}$ \\
\hline$+3.3 ~ V$ & ADP1715ARMZ- & 300 mA & $\begin{array}{l}\text { This linear regulator provides the voltage for the } \\
\text { LVDS transmitters and receivers for the generic } \\
\text { I/O interface. It also provides the power for the } \\
\text { video DAC, USB MCU and Ethernet Controller. }\end{array}$ \\
\hline$+\mathbf{2 . 5 ~ V}$ & TPS79625 & $1 \mathrm{~A}$ & $\begin{array}{l}\text { This linear regulator provides the +2.5 V V } \\
\text { required for correct termination of the transmit } \\
\text { and receive lines of the Ethernet interface. }\end{array}$ \\
\hline
\end{tabular}


Each of the design sub-circuits discussed in the previous section have been integrated into a single printed circuit board. Figures 8.15 and 8.16 illustrate the placement of the sub-circuits and primary interface headers of the external interface PCB.

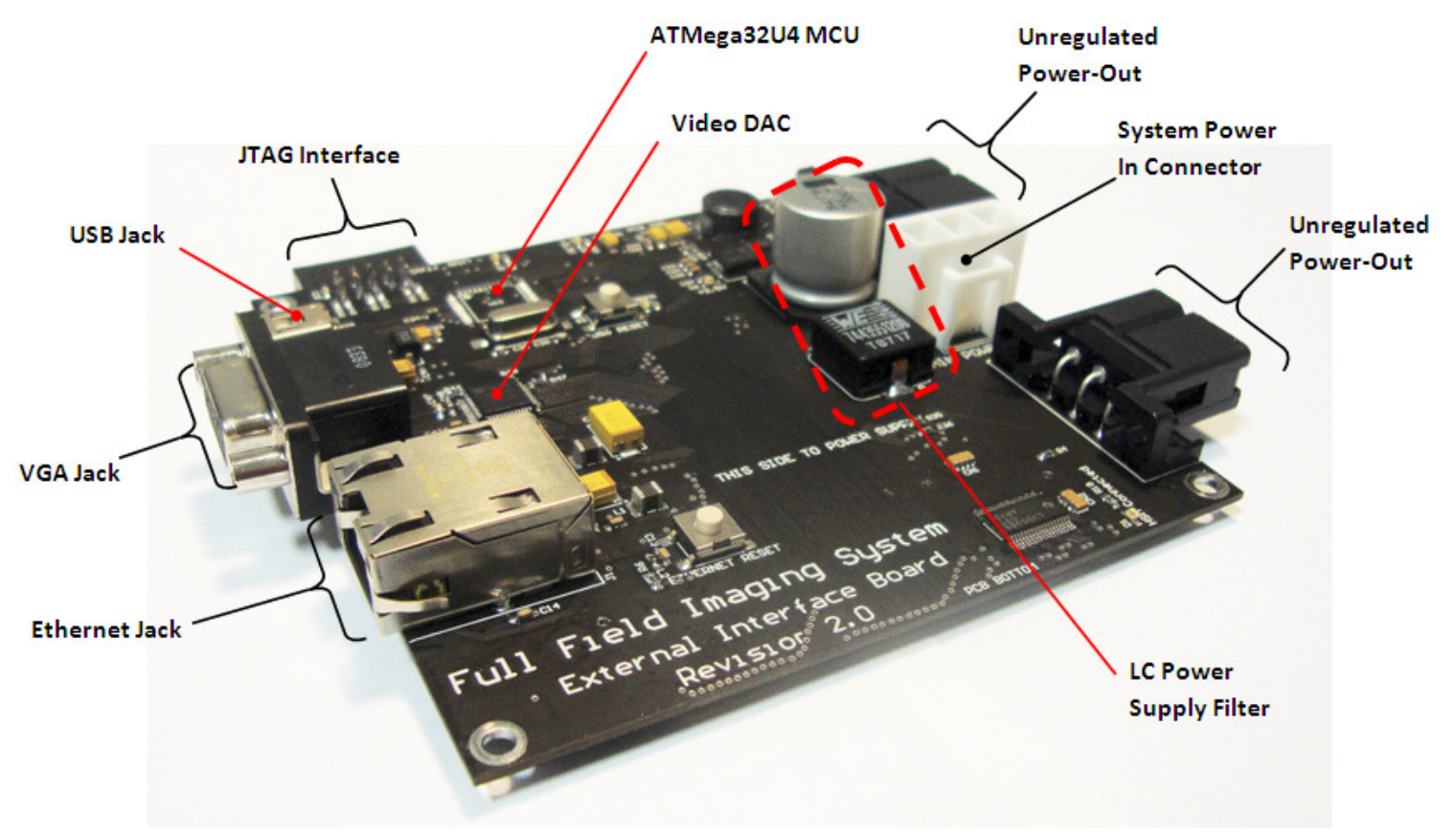

Figure 8.15 External Interface PCB layout - Top Side

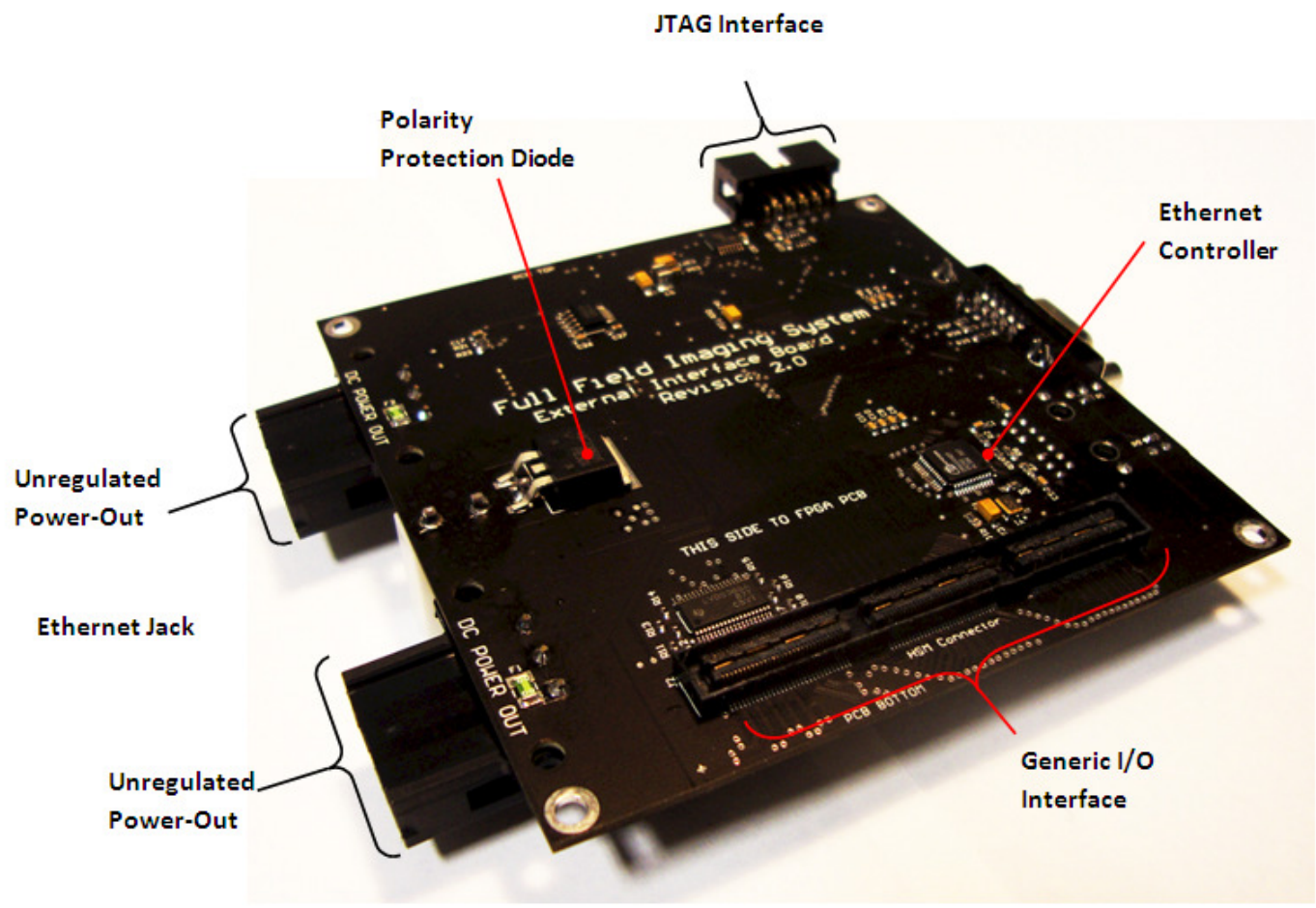

Figure 8.16 External Interface PCB layout - Bottom Side 


\subsection{System Summary}

A mobile image ranger system has been implemented with four circuit boards. Each circuit board controls an independent sub-system which allows the optical configuration of the system to be altered with no modifications required to the illumination and image sensor hardware. The distributed circuit board layout allows the boards to be stacked together to form a complete system with a reduced footprint size. An unregulated power bus provides input power to the onboard voltage regulators on each sub-system circuit board.

The primary supervisory sub-system board is implemented with a Cyclone III FPGA. The FPGA board provides real-time image processing and controls all other sub-systems. An Active Parallel configuration scheme has been implemented to store the FPGA configuration. Four banks of DDR2 SDRAM have been incorporated into the design to provide enough storage capacity to handle image frames up to 1 megapixel. A generic I/O interface has been designed to provide a communication interface between the FPGA board and the remaining sub-system boards.

An Image Capture board has been designed to control the operation and digitisation of an imaging array. Due to the lack of availability of an image array that offers access to the raw image data, the Image Capture board has been designed to piggy back the daughter board from the prototype ranger system.

The Laser Illumination board provides sixteen $660 \mathrm{~nm}, 130 \mathrm{~mW}$ laser diodes grouped into two banks of eight. Each bank can be independently modulated. Protection circuits have been designed to prevent the diodes from operating in continuous wave mode and to provide a controlled warm-up period to extend the life of the diodes.

The External Interface board provides an Ethernet interface to allow the transfer of image data to a peripheral computer for long term storage and post capture analysis. A USB interface has been added to provide a secondary interface to an external computer for future system upgrades. A VGA interface allows the system to display four image frames at once on a standard $640 \times 480$ VGA monitor. A power-in connector provides the input for the system power and protection circuitry has been implemented to provide filtering of the input power and current limits. 


\section{Chapter 9 Results - Sensor Network}

This chapter details the calibration and testing of the individual components developed for the sensor network. Results obtained from tests performed on the prototype sensor network developed for MARVIN are presented. The chapter is concluded with a summary of the project outcomes.

\subsection{Sensor Calibration}

\subsubsection{IR Ranging Modules}

The analogue voltage output from each IR ranging module is sampled by a microcontroller's 10-bit ADC on the infrared ranging node PCB. A basic calibration procedure was performed to obtain conversion functions for both the long-range and mid-range wide angle IR modules. During calibration a white cardboard surface was held perpendicular to the beam at various ranges. The calibration was performed under office fluorescent lighting during the day. A calibration data logger application, built on top of the Sensor Interface software library, was developed to collect 500 samples from each channel of the IR module at a set distance. The application uses an array to store and sort the sample set. The average, standard deviation, minimum value, maximum value, lower quartile value and upper quartile value of the sample set are calculated and logged to a text file as a comma delimited spreadsheet.

Figure 9.1 presents a code segment of the calibration application illustrating the collection of data and the calculation of the data set statistics. The data was stored in an array allowing the data to be sorted with the Array. Sort () function to find the minimum, maximum, upper and lower quartile values. The standard deviation was calculated using the discrete random variable method illustrated in equation 9.1 .

$$
\sigma=\sqrt{\left(\frac{1}{N} \sum_{i=1}^{N}\left(x_{i}-\mu\right)^{2}\right)}
$$

where $\sigma$ represents the standard deviation, $N$ represents the sample set total, $\mu$ represents the sample set average and $x_{i}$ represents the $i^{\text {th }}$ sample in the sample set. 


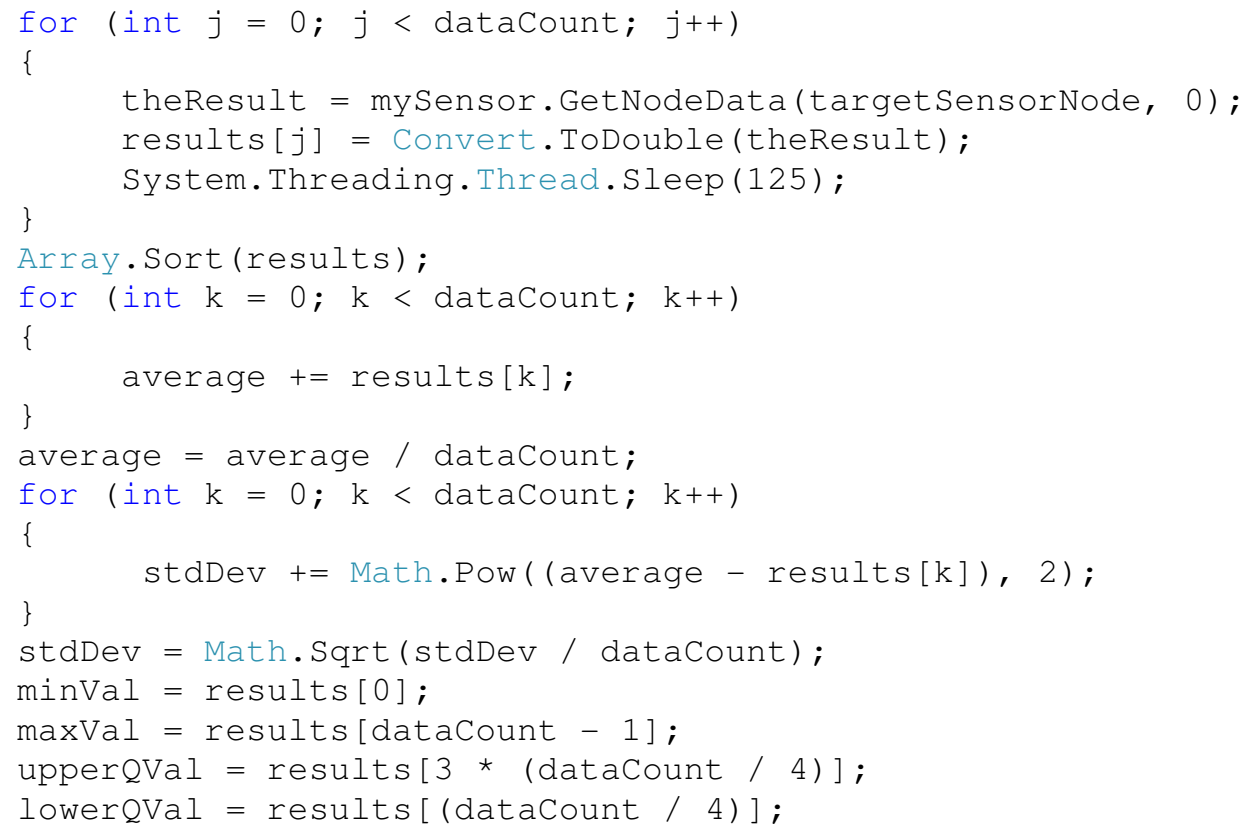

Figure 9.1 Code Segment of Sensor Calibration Application

The upper quartile, lower quartile, minimum and maximum values of a sample set from one of the five channels of a GP2Y3A003K0F medium-to-long range IR module are illustrated by box plots in Figure 9.2. The samples are logged at $0.1 \mathrm{~m}$ intervals.

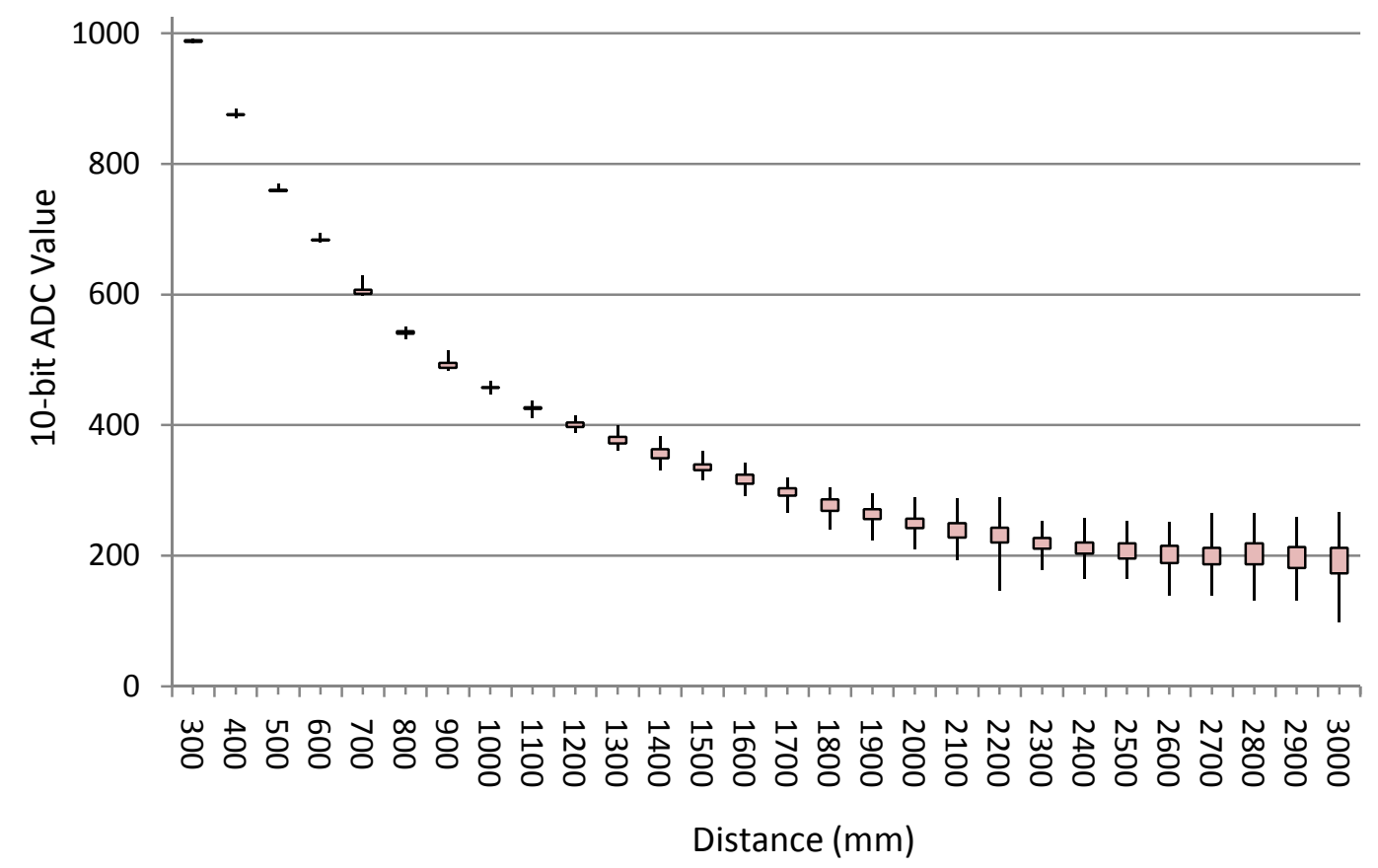

Figure 9.2 IR Module (GP2Y3A003K0F) Calibration Data

The accuracy of the placement of the object was confirmed with a laser ranger that has an accuracy of $\pm 1 \mathrm{~mm}$. 
The non-linear relationship between range and output voltage is a result of the IR module's internal signal processing circuit and position sensitive detector. The Sharp datasheet specifies a minimum operating distance of $0.4 \mathrm{~m}$ for the GP2Y3A003K0F sensor [17]. Below this distance the analogue output drops to zero hence data samples were taken for distances that fell within the specified operating range of the sensor. From the data set it can be seen that the variation in output voltage at a fixed distance increases with distance from the sensor. The standard deviation for ADC readings at $400 \mathrm{~mm}$ is 2.6 least significant bits (LSB) while at $3000 \mathrm{~mm}$ the standard deviation increases to $18.1 \mathrm{LSBs}$.

Figure 9.3 illustrates the relationship between range and output voltage for all five channels of a GP2Y3A003K0F sensor. At short distances the variation in ADC readings between the five channels tends to be greater, $30 \mathrm{LSBs}$ at $300 \mathrm{~mm}$ compared to $14 \mathrm{LSBs}$ at $2900 \mathrm{~mm}$. The standard deviations of the sample sets at shorter distances, typically $1-2.5 \mathrm{LSBs}$ at $300 \mathrm{~mm}$, compared with the ADC reading variations across the five channels, 30 LSBs at $300 \mathrm{~mm}$, warrant individual calibration of each channel in a sensor.

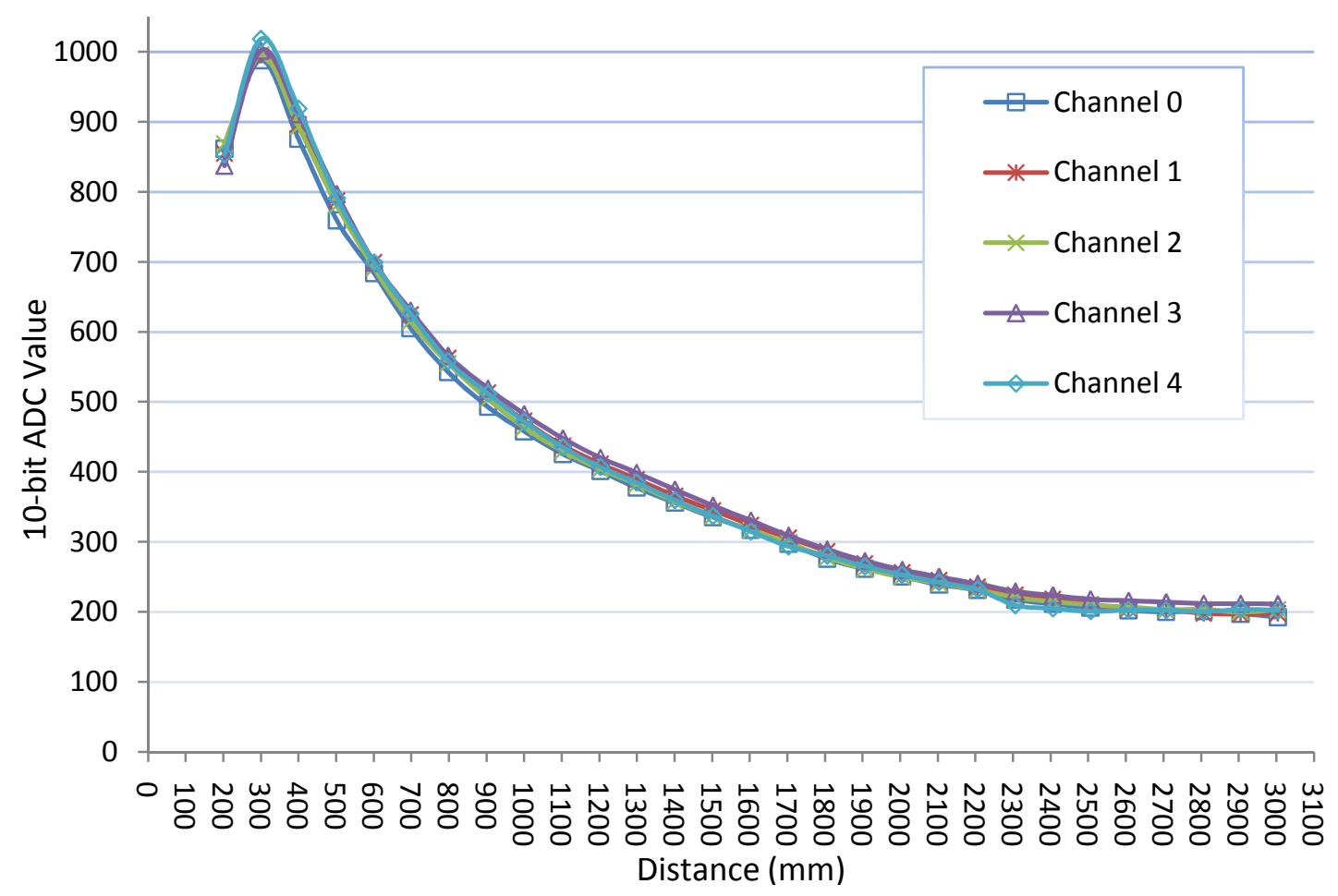

Figure 9.3 Average ADC Values vs Distance for Five channels of GP2Y3A003K0F Sensor

The variation in ADC reading was investigated for differing ambient light conditions. The calibration procedure was repeated in an office environment with the ambient fluorescent light off and on. The measurements were taken at night to provide a low ambient light environment when the fluorescent lights were off. Figure 9.4 presents the difference of the 
average of ADC readings for both data sets. It also presents the standard deviations of both sets. It can be seen that the ADC reading variation is significantly less than the standard deviations of both sets, indicating that the ambient lighting condition in an indoor office set had little effect on the IR sensor's output. Examination of the standard deviations for both data sets suggests an unusual fluctuation at $2200 \mathrm{~mm}$. Repetition of the measurements resulted in the same fluctuation at $2200 \mathrm{~mm}$. The cause of this fluctuation was not determined but is most likely due to an artefact of the PSD unit inside the sensor.

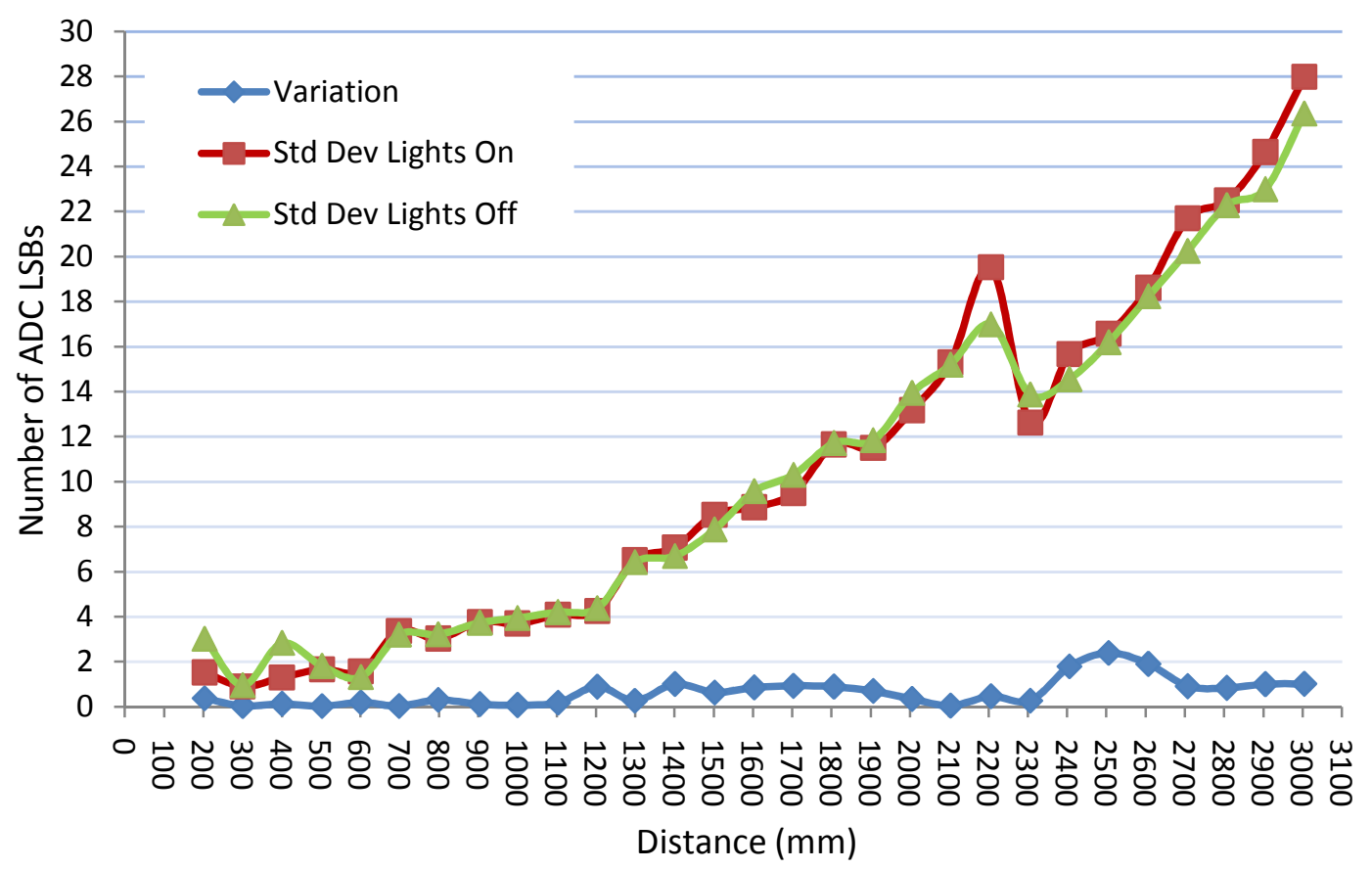

Figure 9.4 Variation in ADC Readings for Lights On and Off Compared with Standard Deviation.

The calibration procedure was repeated using a different IR PSD module to determine the variation in ADC readings between different GP2Y3A003K0F sensors. Figure 9.5 presents the relationship between output voltage and range for channel 0 of two different sensors. Error bars corresponding to the standard deviation for each sample set have been plotted to indicate the overlap of each sample set at a fixed distance. It can be seen that the output voltage relationship differs significantly between IR sensors. For short distances the difference greatly exceeds the standard deviations indicating that data sets are distinct groups and do not overlap. At long distances the difference tends to match the standard deviation indicating the data sets have an overlap. At large distances variation in the data set for PSD 1 is observable and is most likely due to output characteristics of the PSDs sensor. These results indicate that each PSD module requires individual calibration to guarantee accurate distance ranging. 


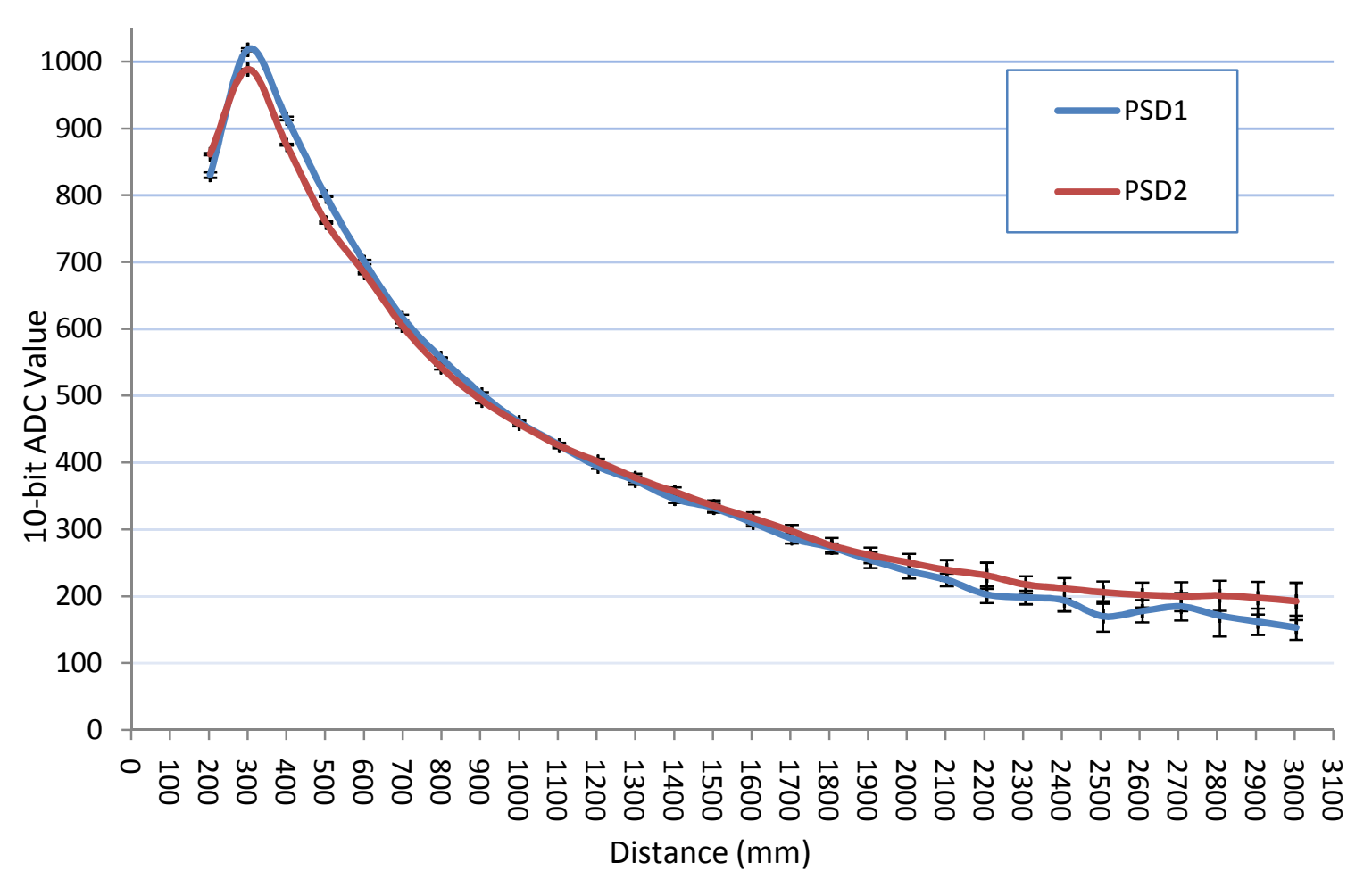

Figure 9.5 Variation in ADC Readings between different GP2Y3A003K0F Modules

The response of a sensor to range an object illuminated from a source with a significant amount of IR content was compared with its response to range object illuminated from light sources with low IR content. The calibration procedure was repeated for a GP2Y3A003K0F sensor for objects illuminated by sunlight and objects illuminated by fluorescent light. Figure 9.6 illustrates the ADC readings for both lighting conditions. It can be seen that there is a large difference between the ADC readings for the same sensor. This indicates the PSD sensor's output is dependent on the IR content of the background illumination source. At increasing distance the divergence between the two data set increases, this can be attributed to the decreasing signal to noise ratio due to the reduced signal strength from the output of the PSD sensor compared with the static noise level from the background IR. Application of these sensors outside would require the measurement of the IR content of the background illumination to compensate for the variance in output. It is recommended that these sensors not be used in environments where the background light contains significant IR content if high precision is required. An investigation into the long term drift of a sensor output for an object at a fixed distance to observe the influence of ambient IR light is recommended to further characterise the effects of background IR but due to time restraints has not be carried out in this project. 


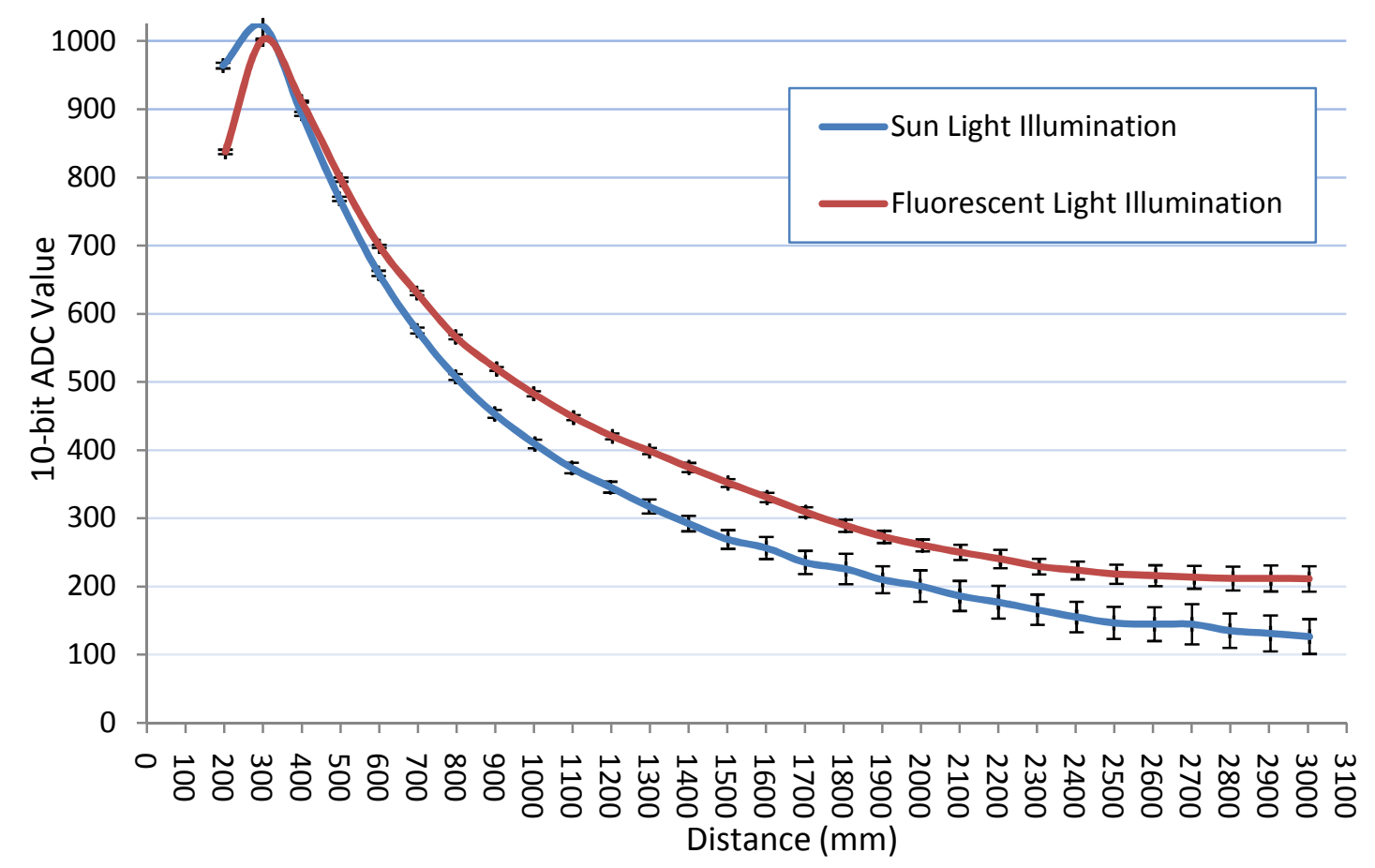

Figure 9.6 Variation in ADC Readings for a GP2Y3A003K0F Module Under Sunlight and Artificial Illumination.

Output voltages have also been sampled for the GP2Y3A002K0F short-to-medium range IR module. The box plot illustrated in Figure 9.7 presents the upper quartile, lower quartile, minimum and maximum values of a sample set from one of the five channels of the sensor.

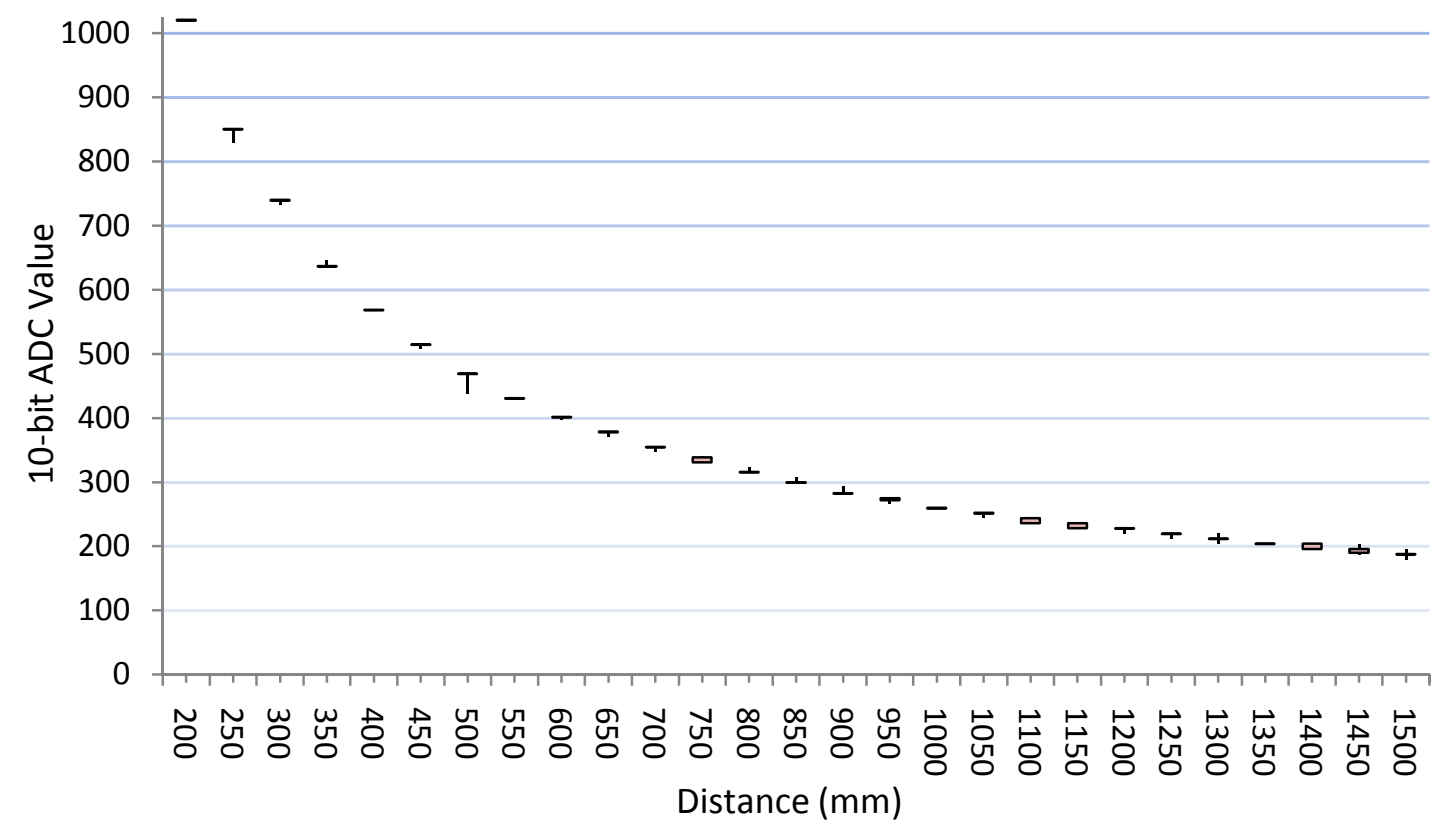

Figure 9.7 IR Module (GP2Y3A002K0F) Calibration Data 
The samples are logged at $0.05 \mathrm{~m}$ intervals. The accuracy of the placement of the object was confirmed with a laser ranger that has an accuracy of $\pm 1 \mathrm{~mm}$. Again a non-linear relationship between output voltage and range can be seen. The variation in the output voltage at a fixed difference is uniform at both short and long distances from the sensor. The standard deviation at $200 \mathrm{~mm}$ is $0.81 \mathrm{LSB}$ and at $1500 \mathrm{~mm}$ is $4.89 \mathrm{LSBs}$.

Figure 9.8 illustrates the relationship between range and output voltage for all five channels of a GP2Y3A002K0F sensor. The variation in ADC readings between the five channels, $26 \mathrm{LSBs}$ at $200 \mathrm{~mm}$ and $17 \mathrm{LSBs}$ at $1500 \mathrm{~mm}$, tends to be greater than the standard deviation of the sample sets, less than $1 \mathrm{LSB}$ at $200 \mathrm{~mm}$ and $4-6$ LSBs at $1500 \mathrm{~mm}$. This indicates that the five channels have significant variations in the output voltage to range relationship and individual calibration of each channel in a sensor should be undertaken.

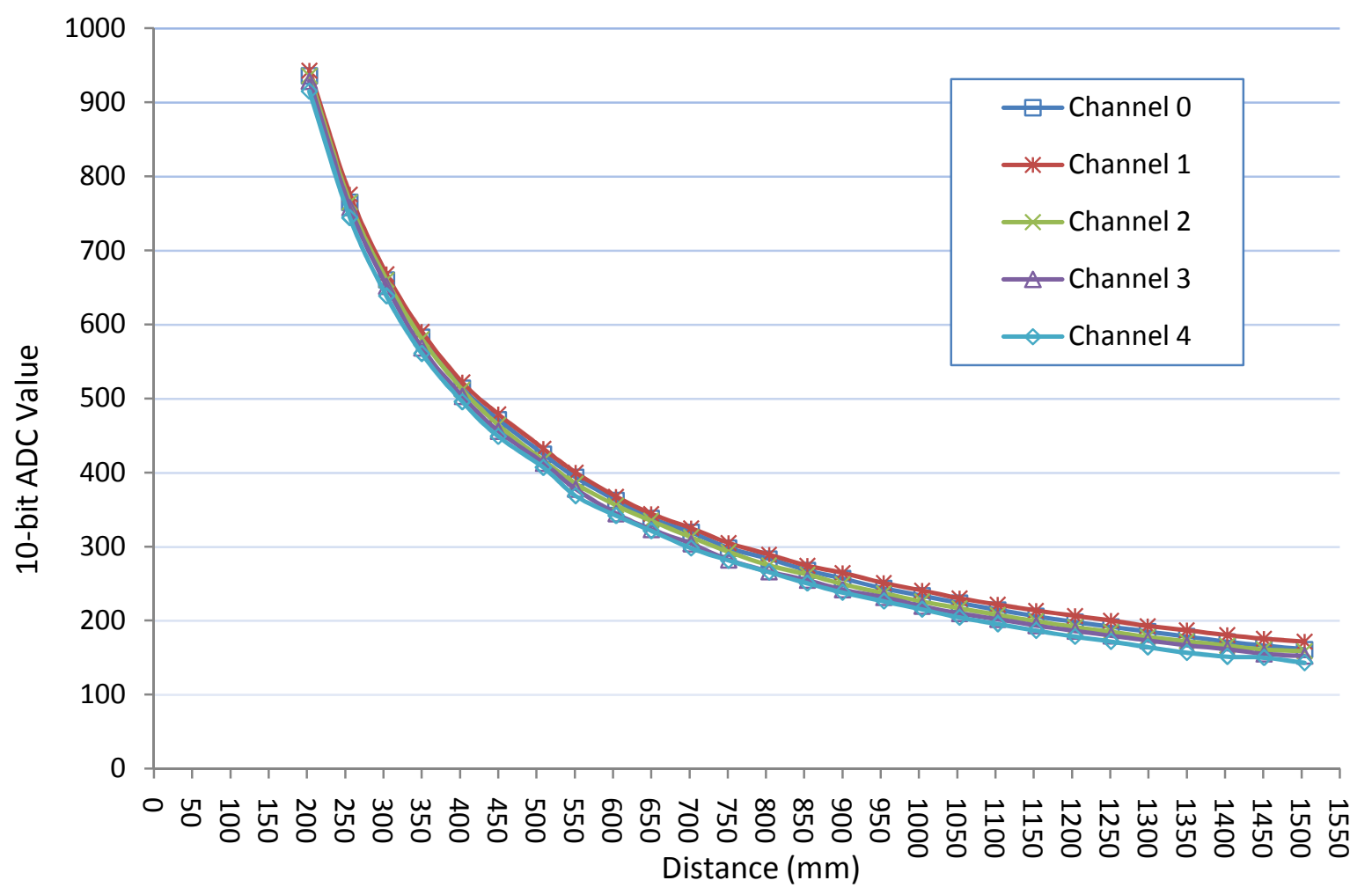

Figure 9.8 Average ADC Values vs Distance for Five channels of GP2Y3A002K0F Sensor

The variation in ADC reading was investigated for differing ambient light conditions for the short range sensor. The same procedure used for the GP2Y3A003K0F sensor was undertaken for the GP2Y3A002K0F sensor. Figure 9.9 presents the difference of the average of ADC readings for both data sets. It also presents the standard deviations of both sets. 
Similar to the GP2Y3A003K0F, the ADC reading variation is significantly less than the standard deviations of both sets indicating that the ambient lighting condition in an indoor office set also had little effect on this IR sensor's output.

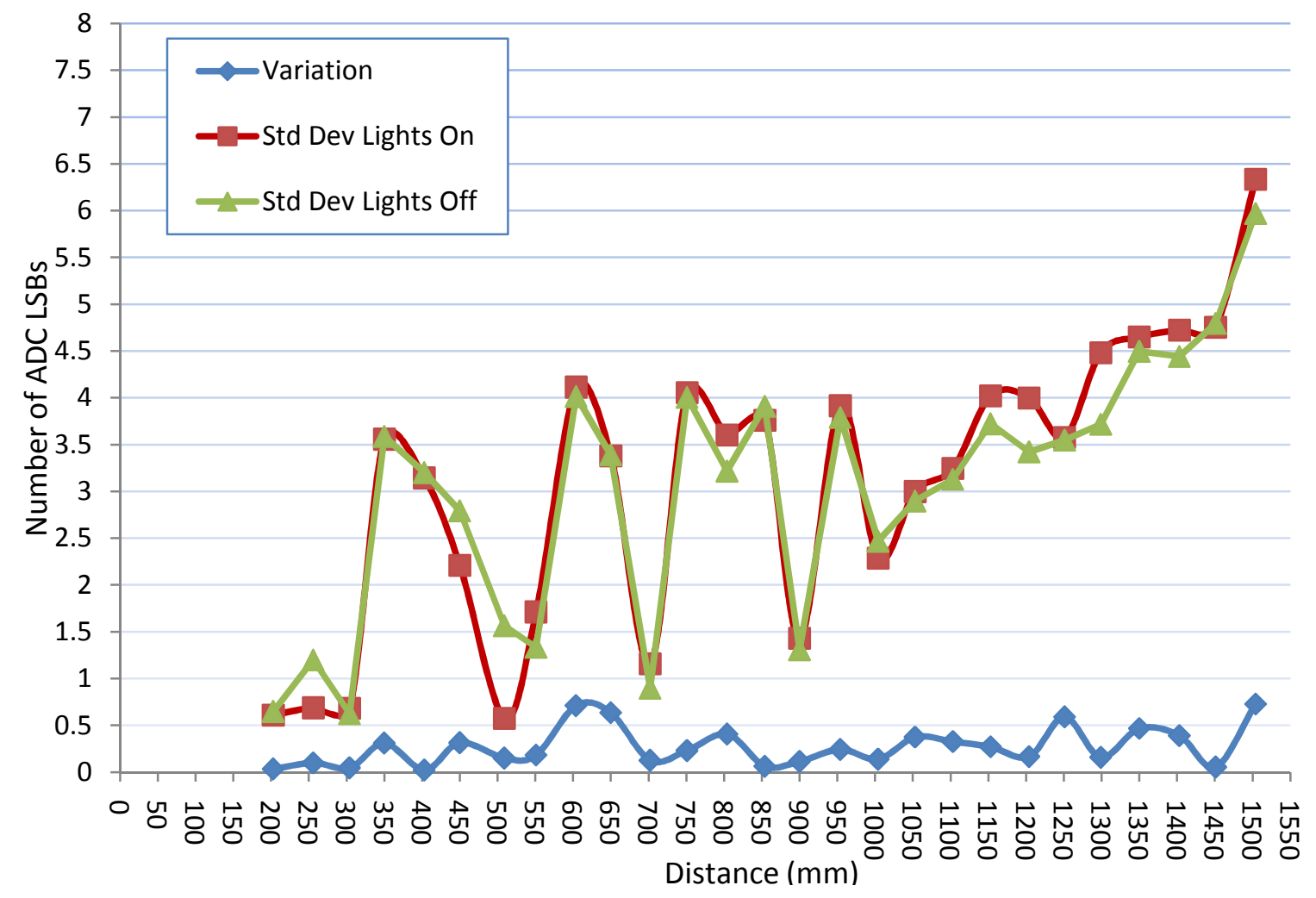

Figure 9.9 Variation in ADC Readings for Lights On and Off Compared with Standard Deviation.

The calibration procedure was repeated using a different IR PSD module to determine the variation in ADC readings between different GP2Y3A002K0F sensors. Figure 9.10 presents the relationship between output voltage and range for channel 0 of two different sensors. Error bars corresponding to the standard deviation for each sample set have been plotted to indicate the overlap of each sample set at a fixed distance. It can be seen that the output voltage relationship differs significantly between IR sensors. Across all distances the difference greatly exceeds the standard deviations indicating that data sets are distinct groups and do not overlap. This indicates that each PSD module requires individual calibration to guarantee accurate distance ranging similar to the results of the GP2Y3A003K0F sensor. 


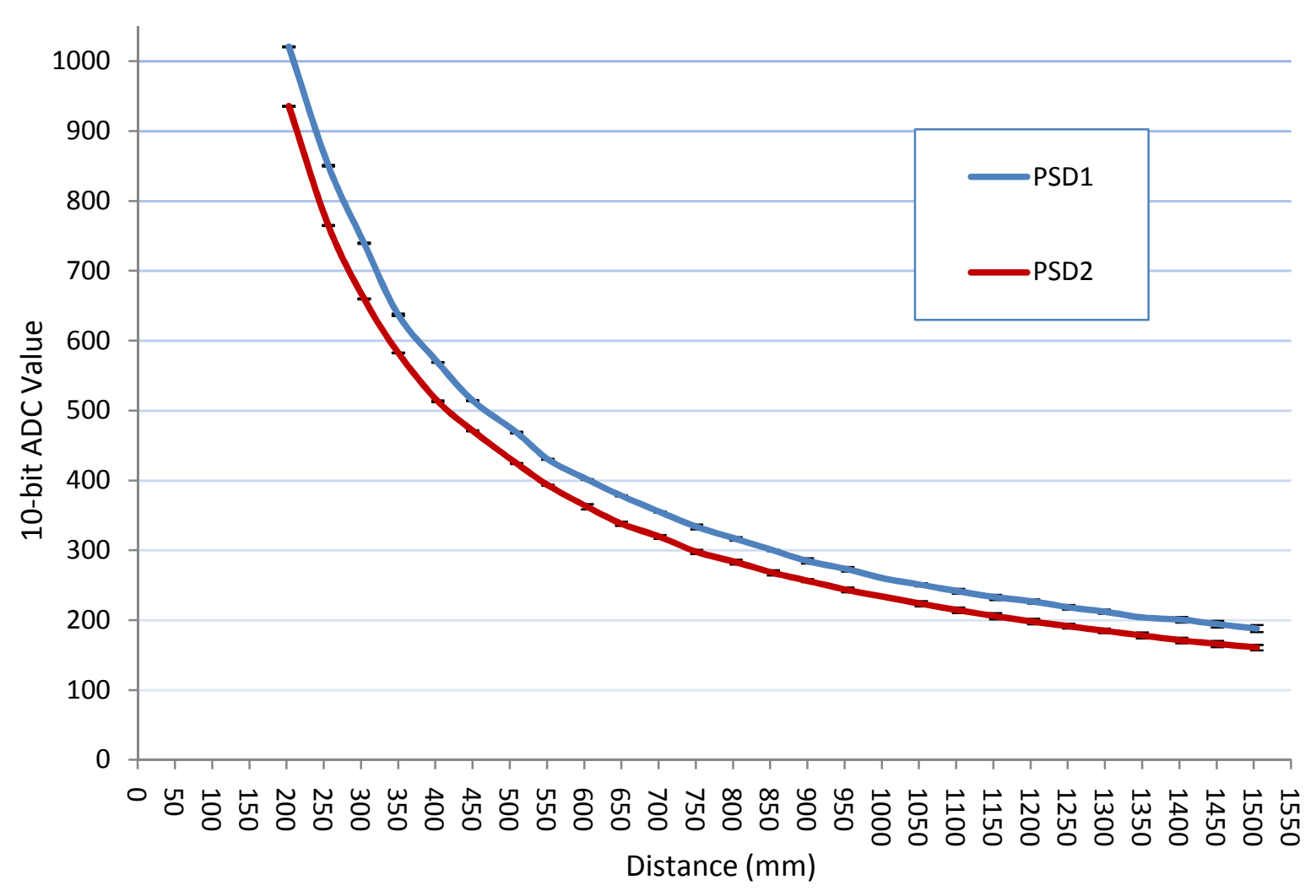

Figure 9.10 Variation in ADC Readings between different GP2Y3A002K0F Modules

Curves have been fitted to the average values of each sample set for both plots using linear regression. The calibration functions are given by Equation 9.2 and Equation 9.3 for the GP2Y3A003K0F medium-to-long range module and GP2Y3A002K0F short-to-medium range module, respectively.

$$
\begin{aligned}
& D=\left(1.90 \times 10^{6}\right) x^{-1.239}+24 \\
& D=\left(7.48 \times 10^{5}\right) x^{-1.186}+6
\end{aligned}
$$

Equation 9.2

Equation 9.3

where

$$
\begin{aligned}
& D=\text { distance }(\mathrm{mm}) \\
& x=10 \text {-bit ADC sample (with a reference voltage of } 1.25 \mathrm{~V} \text { ) }
\end{aligned}
$$

The sample spread in output voltage was investigated to determine the variation in range measurements at a fixed distance and hence the error in the range calculated. The range precision is quantified by comparing the calculated range based on the average from the ADC sample set with the calculated range given at one standard deviation $(\sigma)$ from the average in the same sample set. Figure 9.11 illustrates the error in range calculations for the 
GP2Y3A003K0F IR module under fluorescent lighting in an indoor setting. It can be seen that the instability of the output voltage causes the error in the range to rise dramatically at distances above $1200 \mathrm{~mm}$.

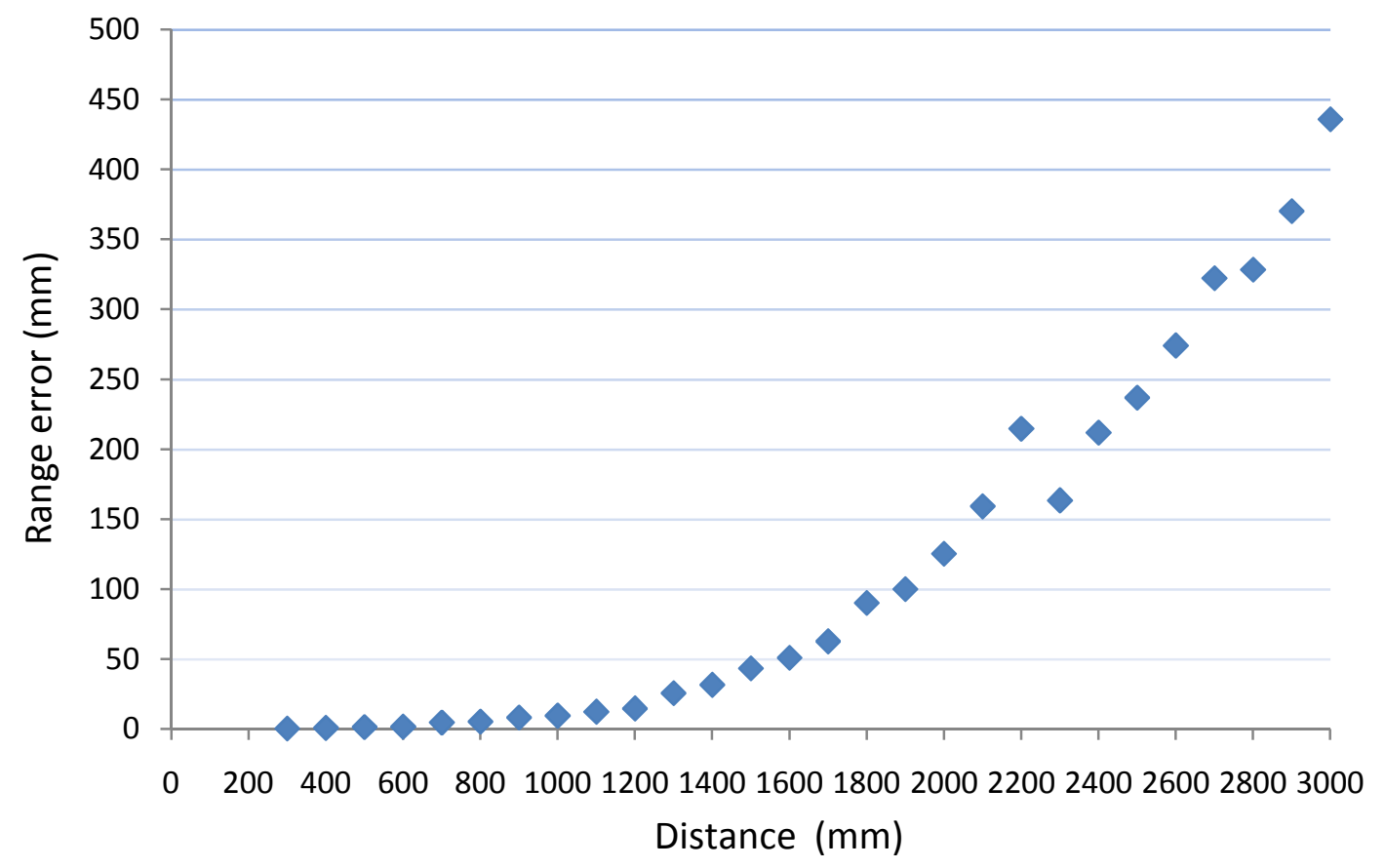

Figure 9.11 Error Analysis of GP2Y3A003K0F IR Module

The same analysis of sample spread in output voltage has been applied to the GP2Y3A002K0F IR module. Figure 9.12 illustrates the error in ranges for this IR module.

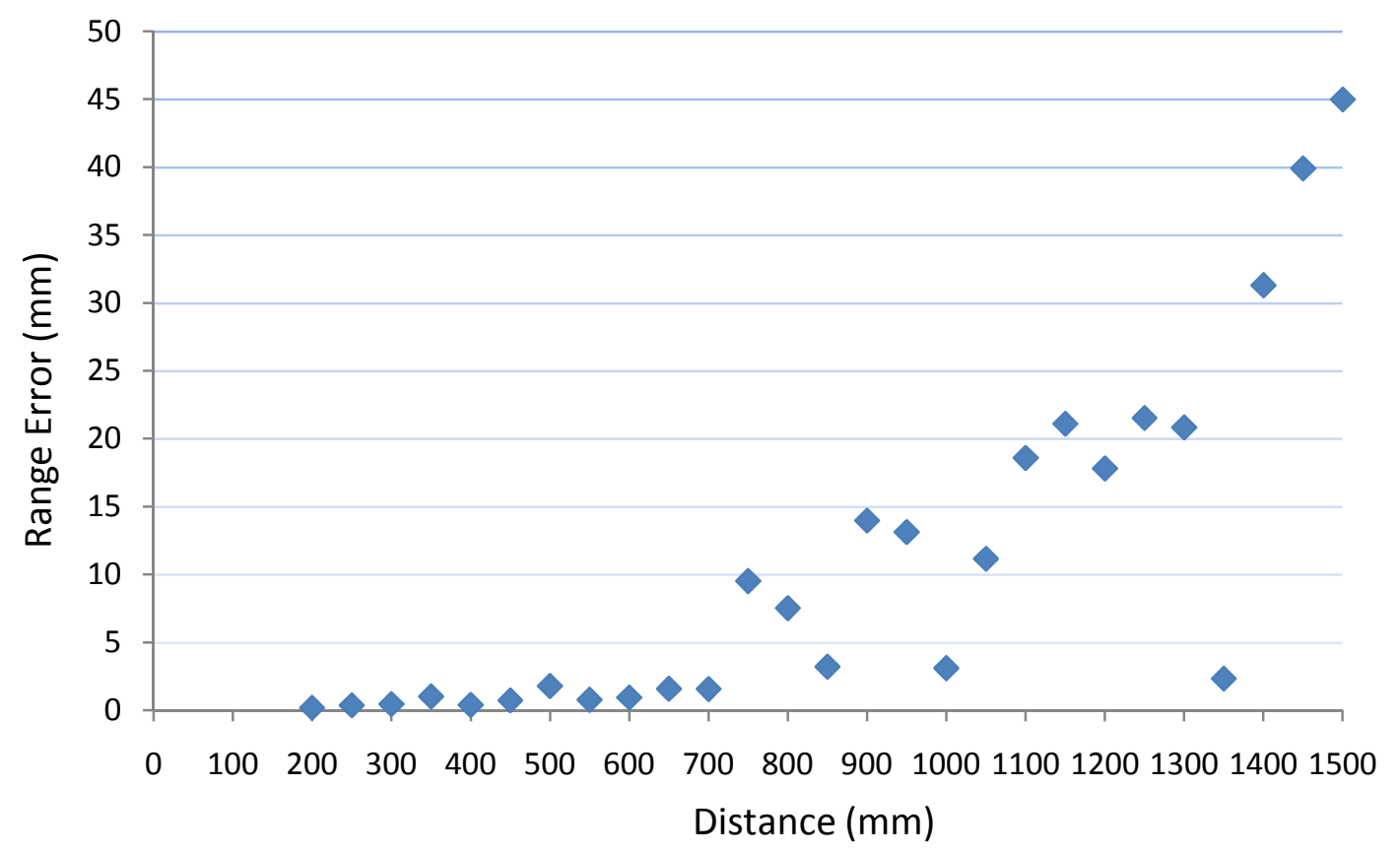

Figure 9.12 Error Analysis of GP2Y3A002K0F IR Module 
The precision in range measurements of the GP2Y3A002K0F module is an order of magnitude better than the GP2Y3A003K0F module. The range error in $99.7 \%$ of samples $(3 \sigma)$ for the GP2Y3A002K0F IR module is less than $5 \mathrm{~mm}$ up to a range of $700 \mathrm{~mm}$ and $135 \mathrm{~mm}$ at full range. These figures increase to approximately $14 \mathrm{~mm}$ and $1305 \mathrm{~mm}$ respectively for the GP2Y3A003K0F IR module.

The calibrated output of both sensors was investigated to determine the accuracy of the calibration. An object was placed at varying distances from the calibrated sensor and the calculated distance was compared with the distance measurements from a laser ranger finder with an accuracy of $\pm 1 \mathrm{~mm}$. Figure 9.13 plots the distance calculated by the sensor network with the actual distance for a calibrated GP2Y3A003K0F IR module. The slope of the linear trend line fitted to the data indicates the calibration has an accuracy of approximately $98 \%$.

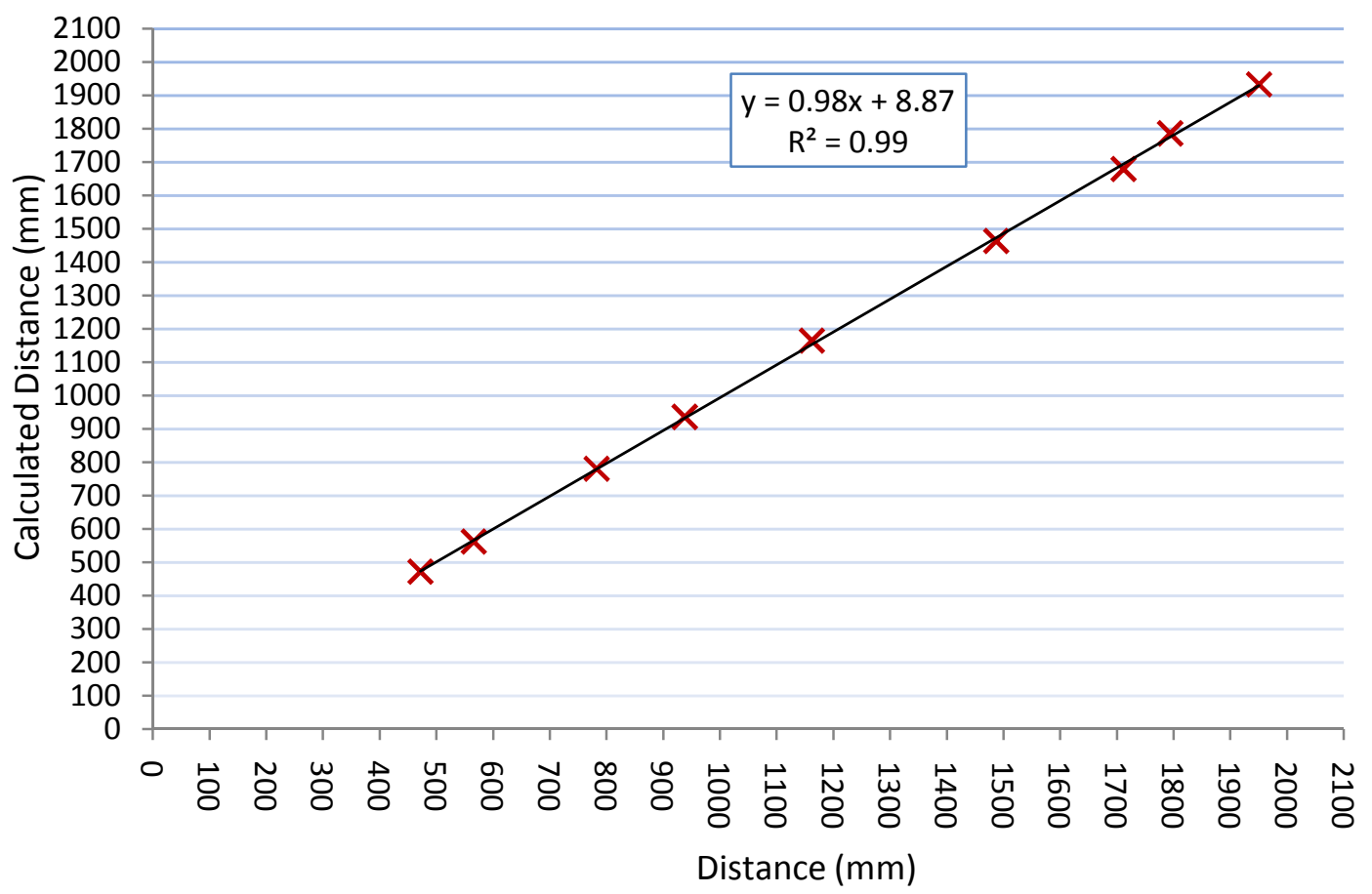

Figure 9.13 Calculated Distance vs Actual Distance for Calibrated GP2Y3A003K0F IR Module

Variation between the calculated range and the actual range as a percentage of the actual range is less than $2 \%$ between the distances of $400 \mathrm{~mm}$ to $2000 \mathrm{~mm}$.

Figure 9.14 plots the calculated distance against the actual distance for measurement undertaken on a calibrated GP2Y3A002K0F IR module. A linear trend line fitted to the data indicates the calibration varies from the actual distance by approximately $98 \%$ similar to the GP2Y3A003K0F IR module. 


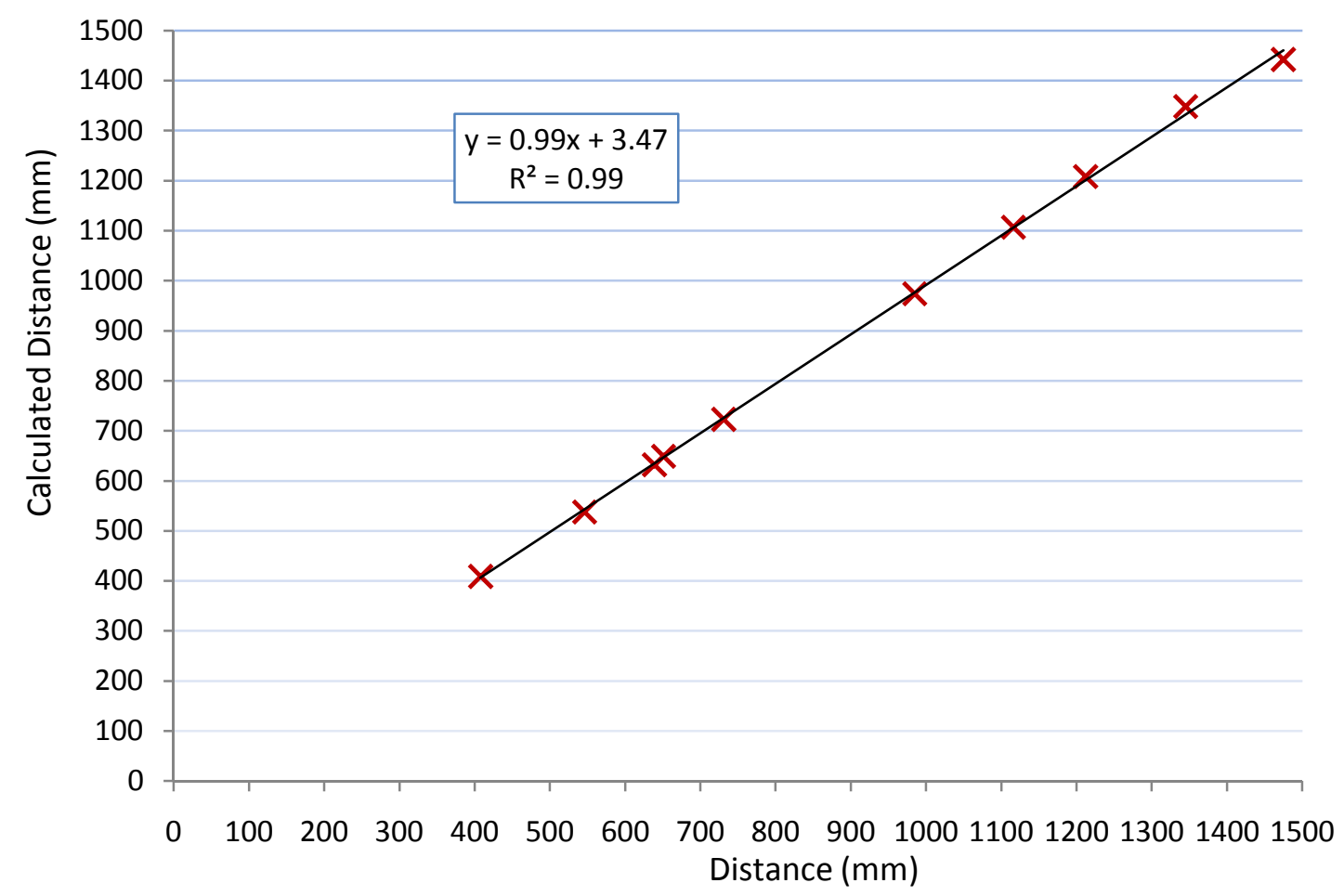

Figure 9.14 Calculated Distance vs Actual Distance for Calibrated GP2Y3A002K0F IR Module

From the measurements it can be seen that the percentage variation is less than $2.5 \%$ from the actual distance between the distances of $250 \mathrm{~mm}$ to $1500 \mathrm{~mm}$.

Calibration values have been calculated for all five channels on both the GP2Y3A003K0F and GP2Y3A002K0F IR modules. These calibration values have been used to determine ranges for all the IR modules in the prototype sensor network. Without calibration of each IR module, an error of $10 \%$ or greater can be expected. The calibration of every module is outside the scope of this thesis but is recommended for future work.

\subsubsection{Ultrasonic Ranging Modules}

The time between emitting an ultrasonic pulse and receiving its echo is measured by an MCU's 16-bit counter. A calibration procedure has been performed to determine the conversion function between the 16-bit timer count and distance. During the procedure the ultrasonic sensor was positioned at various ranges from a solid wall. The calibration data logger mentioned in section 9.1.1 IR Ranging Modules was used to collect and process the data. Sample sizes of 500 were taken at steps of $250 \mathrm{~mm} \pm 2 \mathrm{~mm}$ over a range of $0-10,000 \mathrm{~mm}$. In Figure 9.15 the upper quartile, lower quartile, minimum and maximum values of the sample set are illustrated by box plots (the boxes represent the spread of data 
between the lower and upper quartiles while the lines plot the data spread between the lower quartile and minimum as well as the upper quartile and maximum values).

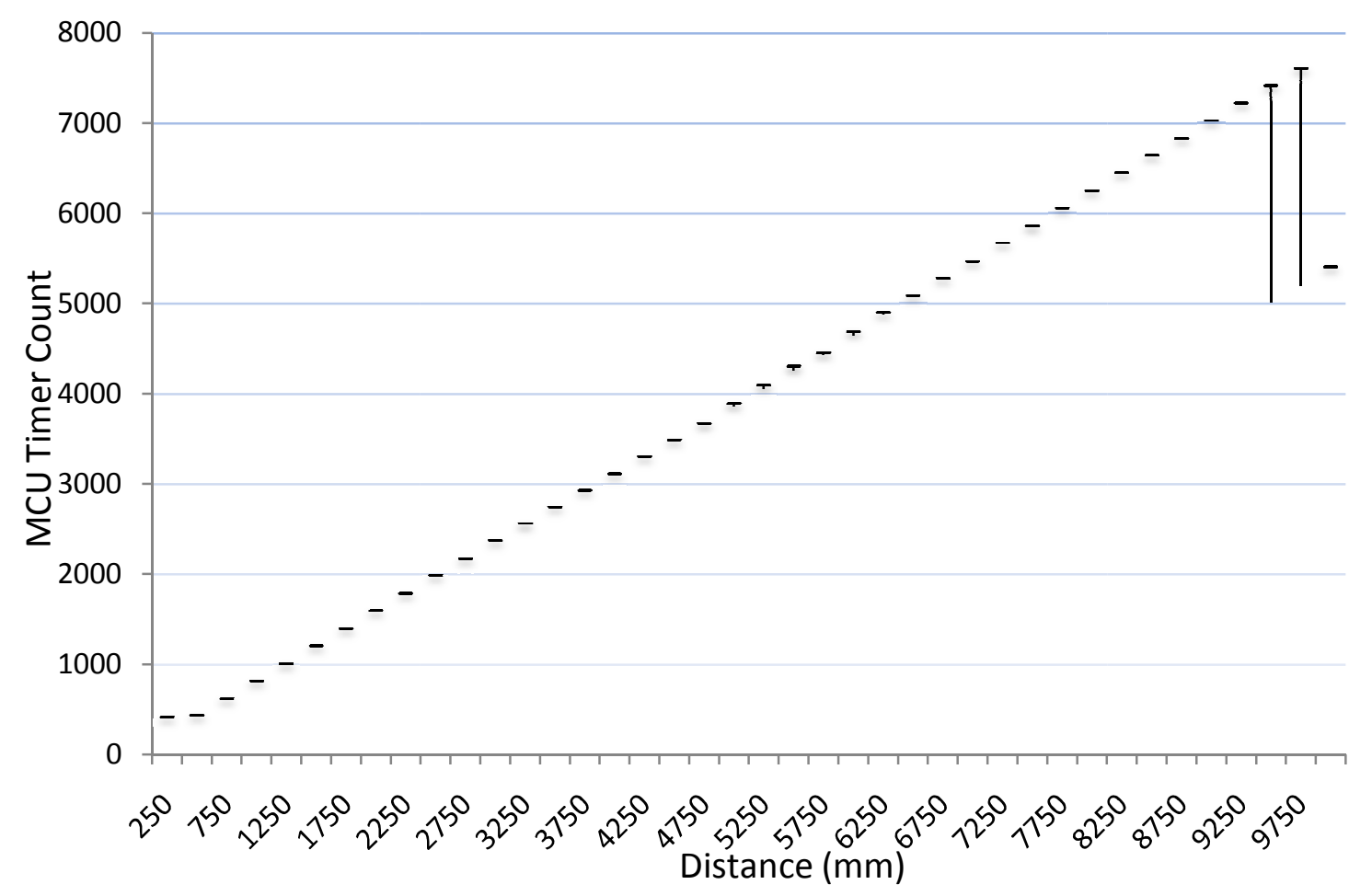

Figure 9.15 SensComp Ultrasonic Module Calibration Data

The linear relationship between range and timer count can be seen over a range from $500-9250 \mathrm{~mm}$. Below $500 \mathrm{~mm}$ the internal blanking circuit of the SensComp module prevents the accurate timing of the ultrasonic pulse because of the time required for ringing from the ultrasonic pulse generation to decay below the threshold of the reception circuit. Above $9250 \mathrm{~mm}$ the variation in the data set becomes increasingly large. This variation can be attributed to the amplitude of the reflected wave becoming comparable to the minimum detection level of the sensor's reception circuit. The increase in variation at distances above $9250 \mathrm{~mm}$ limits the ultrasonic sensor's useful range to between $500 \mathrm{~mm}$ and $9250 \mathrm{~mm}$.

A line has been fitted to the average values of the sample set using linear regression over the range of $500-9250 \mathrm{~mm}$. The calibration function for the sensor is given by Equation 9.4.

$$
D=1.29 x-39
$$

where

$D=$ distance $(\mathrm{mm})$

$x=16$-bit timer count (with an overflow time of $7.5 \mu \mathrm{s}$ ) 
Figure 9.16 presents the theoretical timer count values and the measured timer count values compared with distance for the ultrasonic sensor. The speed of sound was assumed to be $340.3 \mathrm{~m} / \mathrm{s}$ at $15.5^{\circ} \mathrm{C}$ for the calculation of the theoretical values. It can be seen that the measured values closely match the theoretical values calculated for the MCU timer with an overflow time of $7.5 \mu$ s corresponding to one tick of the ultrasonic pulse timer.

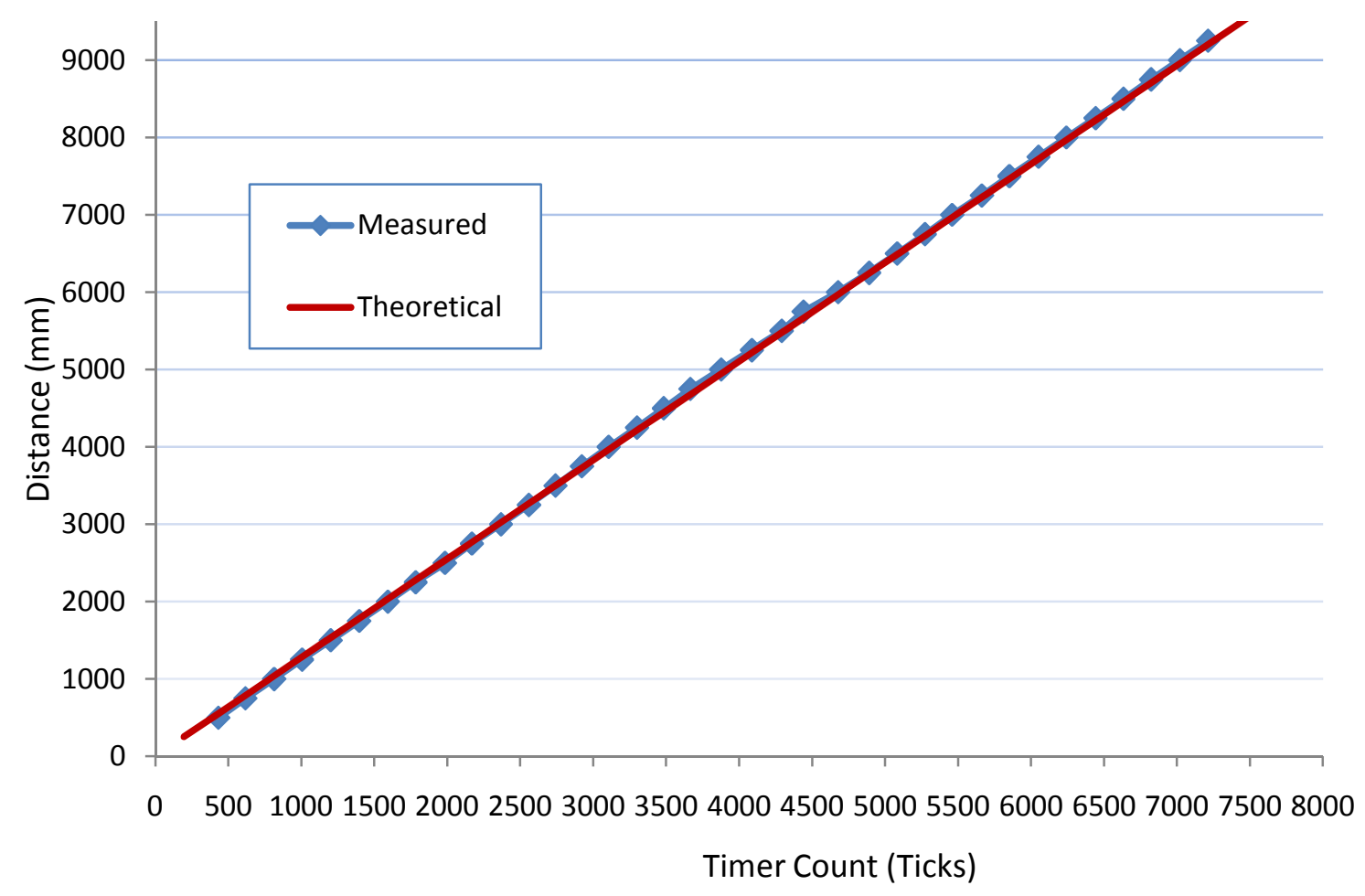

Figure 9.16 Theoretical and Measured Tick Count Vs Distance for Ultrasonic Sensor.

An analysis on the spread of the sample set has been performed to determine the variation in range measurements at a fixed distance and hence the error in the range calculated. From Figure 9.17, it can be observed that below $1500 \mathrm{~mm}$ the range error remains fairly constant at less than $1 \mathrm{~mm}$ while above this distance the error increases in a logarithmic manner. Above $6250 \mathrm{~mm}$ the range error stabilises to a value of approximately $10 \mathrm{~mm}$. It is unclear why a peak in the error occurs around $5500 \mathrm{~mm}$ and warrants further investigation that is beyond the scope of the project. 


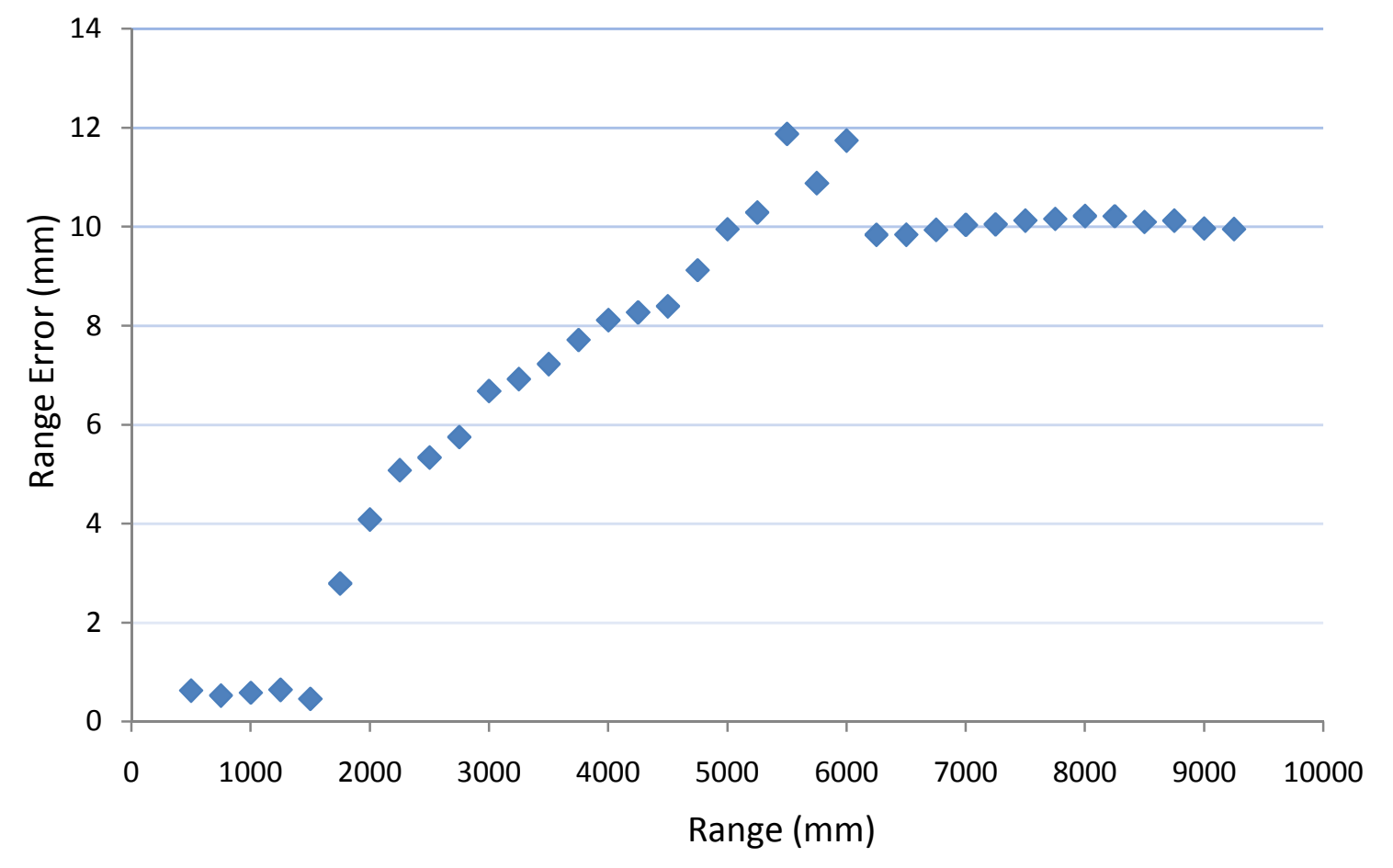

Figure 9.17 Error Analysis of SensComp Ultrasonic Module

The range error in $68 \%$ of samples $(\sigma)$ for the SensComp module is less than $1 \mathrm{~mm}$ up to a range of $1500 \mathrm{~mm}$ and approximately $10 \mathrm{~mm}$ at full range. This error provides a minimum range resolution of $0.1 \%$ at full range.

\subsection{Communications}

A number of tests have been performed to characterise the operation of both network and command interface communications. The command packet interface application, first described in Section 6.3 Command Packet Interface, has been used to issue commands to the network. Oscilloscope traces have been captured to determine the speed and correct operation of the MLP and HLP protocols. 


\subsubsection{Network Frame}

As described in section 5.1.3 Media Layer Protocol, the transfer of data in MLP packets occurs during a network frame. Following the request - response model, a network frame begins with the transmission of a Data type MLP packet by the network master and closes with the transmission of an acknowledge type MLP packet by the addressed sensor node. Network frames have a lifetime of $5 \mathrm{~ms}$. After this time the network master will close the network frame by issuing a Cancel Frame type MLP packet. Figure 9.18 illustrates oscilloscope traces on the network master's receive and transmit lines for a successful network frame. The yellow trace is the receive data line and the blue trace is the transmit data line.

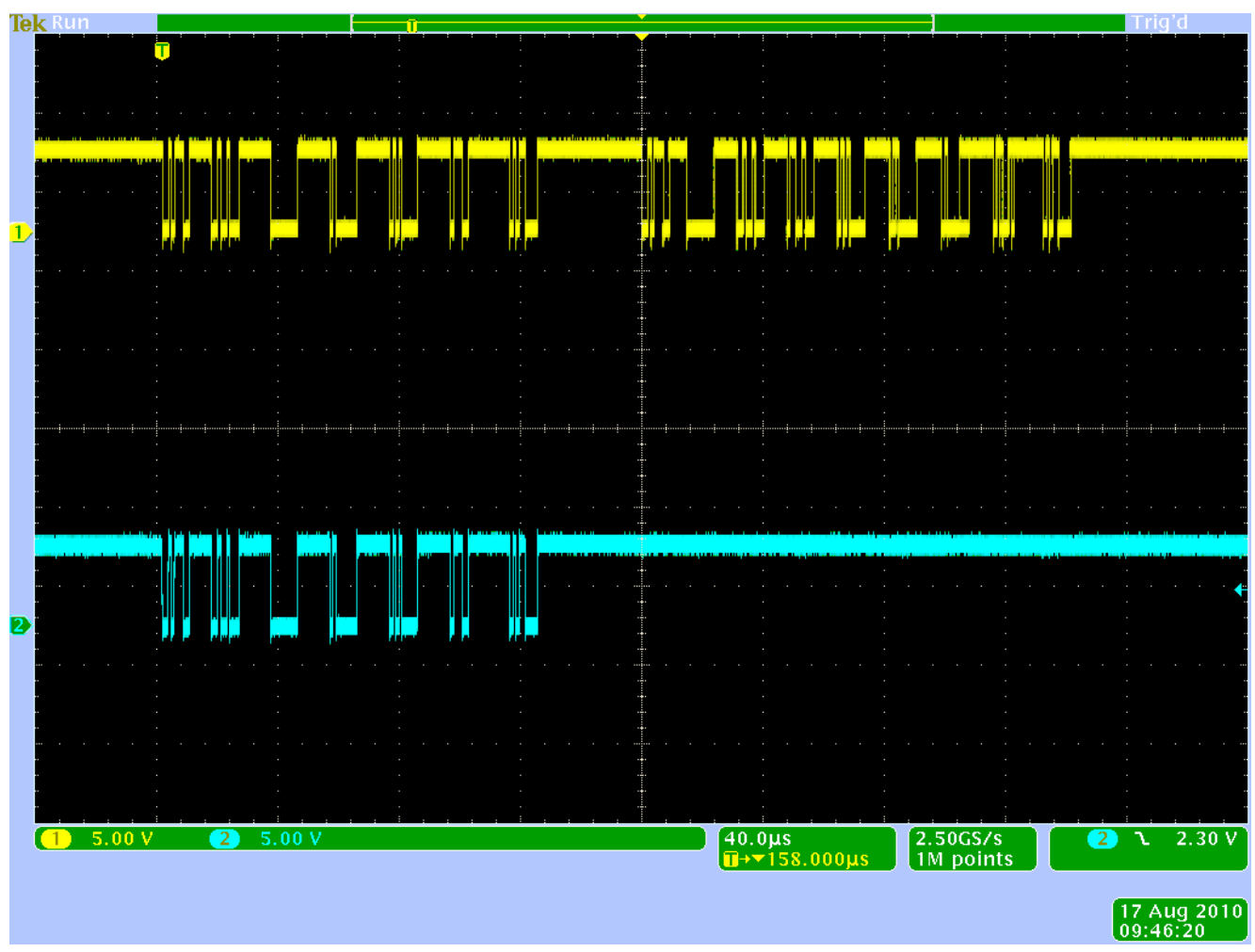

Figure 9.18 Sensor Network Frame

The data rate is set at $1 \mathrm{Mb} / \mathrm{s}$. As the network is half duplex, any data the network master transmits is echoed back on the receive line. The request packet is transmitted, and echoed back on the receive line, and the response packet is received approximately $32 \mu$ s later.

Figure 9.19 illustrates oscilloscope traces on the network master's receive and transmit line for a situation where a network frame expires and is closed by the master. It can be seen on 
the trace that the period between the start of the network frame and the issuing of the Cancel type MLP packet is $5 \mathrm{~ms}$.

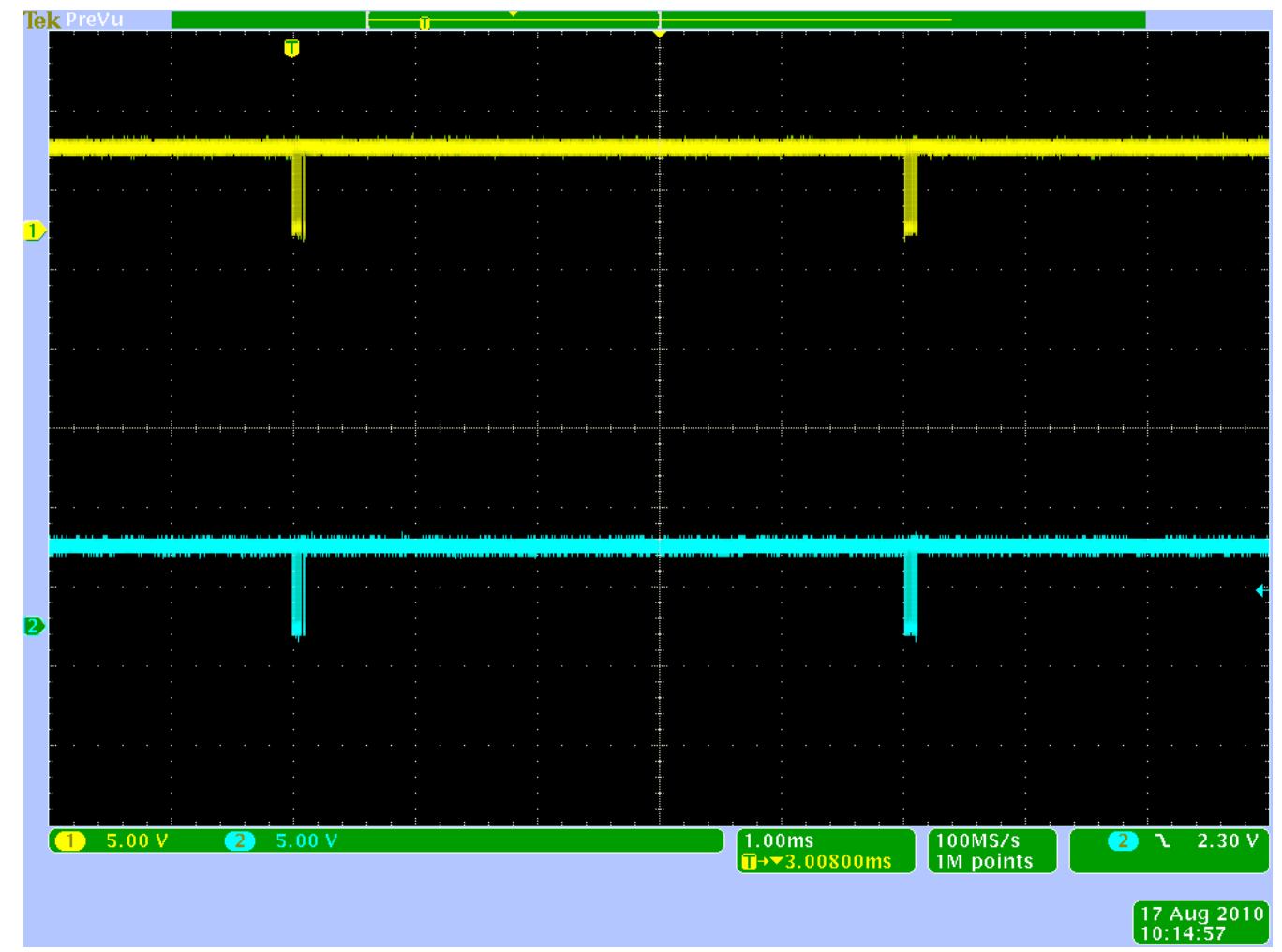

Figure 9.19 Example of a Cancelled Network Frame

\subsection{Sensor Control}

\subsubsection{PSD Timing}

As discussed in Section 4.1.1 Sharp IR PSD Ranging Modules the time between activating an IR sensor channel and sampling the output voltage affects the stability and accuracy of the output voltage. The IR module requires a minimum of $20.2 \mathrm{~ms}$ after activating an IR channel before the output voltage can be sampled $[17,18]$. The module also requires a minimum of $5 \mathrm{~ms}$ between activating a new IR channel to allow the internal signal processing circuit to reset. This effectively sets the minimum time required to sample an IR channel to $25.2 \mathrm{~ms}$. This limits the maximum sampling rate of a five channel PSD to approximately $8 \mathrm{~Hz}$. As mentioned in Section 5.4 IR PSD Sensor Control, the finite state machine controlling the sensor waits $25 \mathrm{~ms}$ after activating an IR channel and before beginning the ADC sampling process. 
The timing of the IR PSD Sensor has been examined to ensure the $25 \mathrm{~ms}$ minimum timing requirement is actually met. Figure 9.20 presents the oscilloscope traces of the IR PSD control lines during free-running measurements. The yellow trace represents the $\mathrm{V}_{\text {in }}$ control line used to reset the signal processing circuit. The blue, pink and green traces represent the LED activation control signal for Channel 1, 2 and 3 respectively. It can be seen that the $V_{\text {in }}$ control line is held low for $5 \mathrm{~ms}$, meeting the timing requirement to reset the signal processing circuit. The remaining traces are activated for approximately $28 \mathrm{~ms}$ each. This time comprises the $25 \mathrm{~ms}$ wait cycle of the FSM and the collection of 32 ADC samples for averaging.

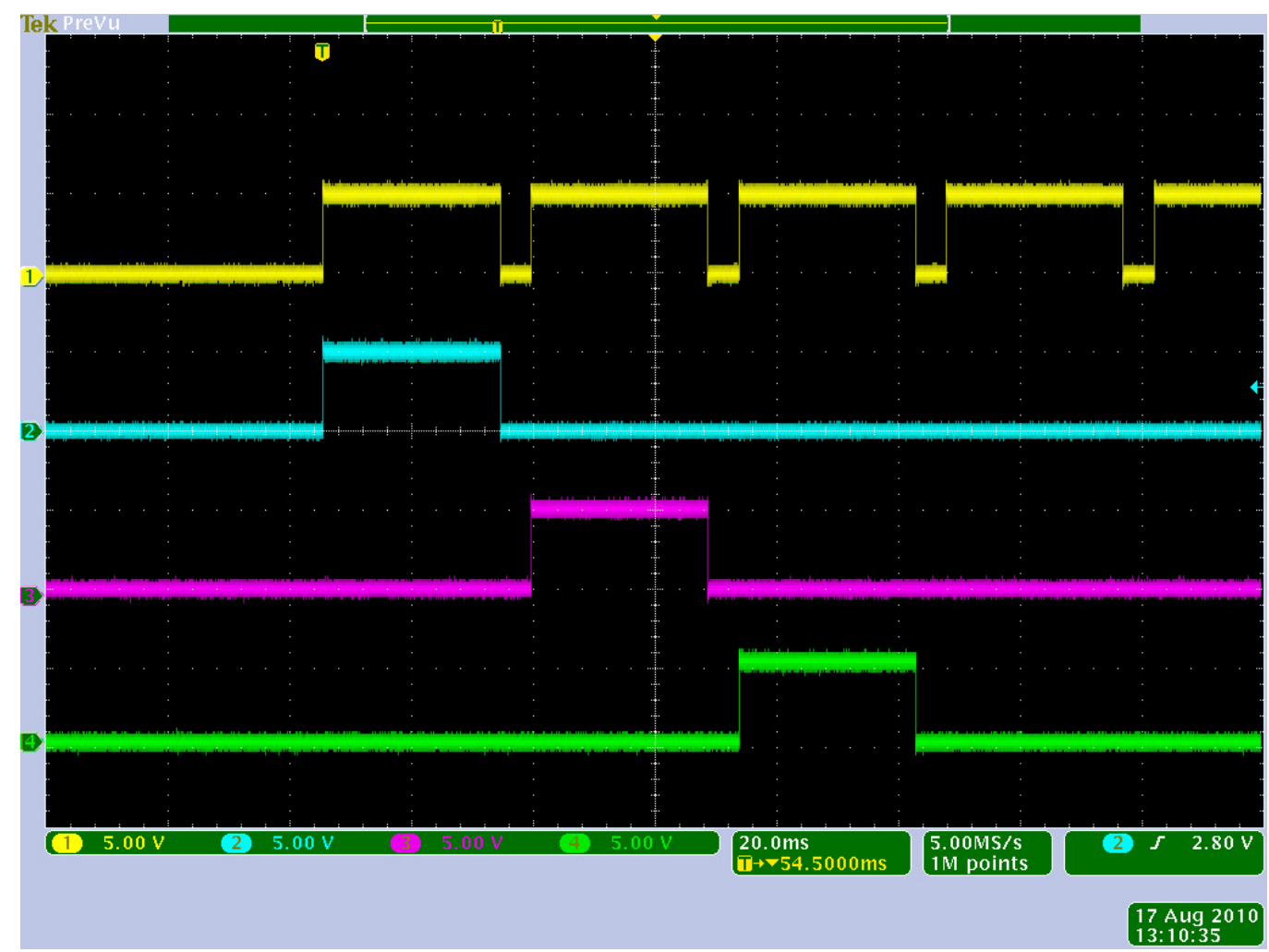

Figure 9.20 IR PSD Timing Oscilloscope Trace

The oscilloscope trace indicates that $33 \mathrm{~ms}$ are required to complete the sampling of one IR channel. To sample all five IR channels requires $165 \mathrm{~ms}$ and limits the maximum sampling rate of the IR sensor, in free running mode, to $6 \mathrm{~Hz}$.

In fast measurement mode only one IR channel is sampled. In this situation the sample rate is determined by the speed of the ADC. The MCU requires approximately $3 \mathrm{~ms}$ to sample the output voltage 32 times for averaging. At this speed the maximum sample rate increases to approximately $330 \mathrm{~Hz}$. 


\subsubsection{Ultrasonic timing}

The ultrasonic sensor determines distances by measuring the time of flight for an ultrasonic pulse. This dependence on timing means the maximum sample rate is a function of the maximum range to be measured. An ultrasonic pulse ranging an object at a distance of $10 \mathrm{~m}$ will require approximately $58 \mathrm{~ms}$ of flight time. This limits the maximum sample rate to approximately $17 \mathrm{~Hz}$. From Section 9.1.2 Ultrasonic Ranging Module, the effective range of the ultrasonic sensor is $9.25 \mathrm{~m}$. At this distance the time of flight of the ultrasonic pulse will be approximately $54 \mathrm{~ms}$, limiting the maximum sample rate to $18.5 \mathrm{~Hz}$.

\subsubsection{Measurement Window.}

As discussed in Section 5.1.2.1 Sensor Tasks, sensor nodes are given sensor tasks in order to allow the network to operate in a co-ordinated manner. The primary means of co-ordinating measurements across the network is achieved through the use of the Step measurement mode. This mode requires the sensor to take sensor measurements at regular fixed intervals over a $60 \mathrm{~s}$ measurement window. The finite state machines, discussed in Section 5.4 IR PSD Sensor Control and Section 5.5 Ultrasonic Sensor Control, implemented on the sensor nodes are responsible for taking measurements at the specified times in the measurement window. The timing of the measurements has been investigated on an oscilloscope to ensure the FSMs are initiating measurements at the correct time. Figure 9.21 presents the oscilloscope traces for step measurements undertaken on the ultrasonic sensor node. The ultrasonic sensor node was issued with a Step measurement task that had a start time in the measurement window of $25 \mathrm{~ms}$ and a step time of $35 \mathrm{~ms}$ between each measurement. The ultrasonic sensor node firmware produces a $15 \mathrm{~ms}$ pulse at the beginning of each measurement window. 


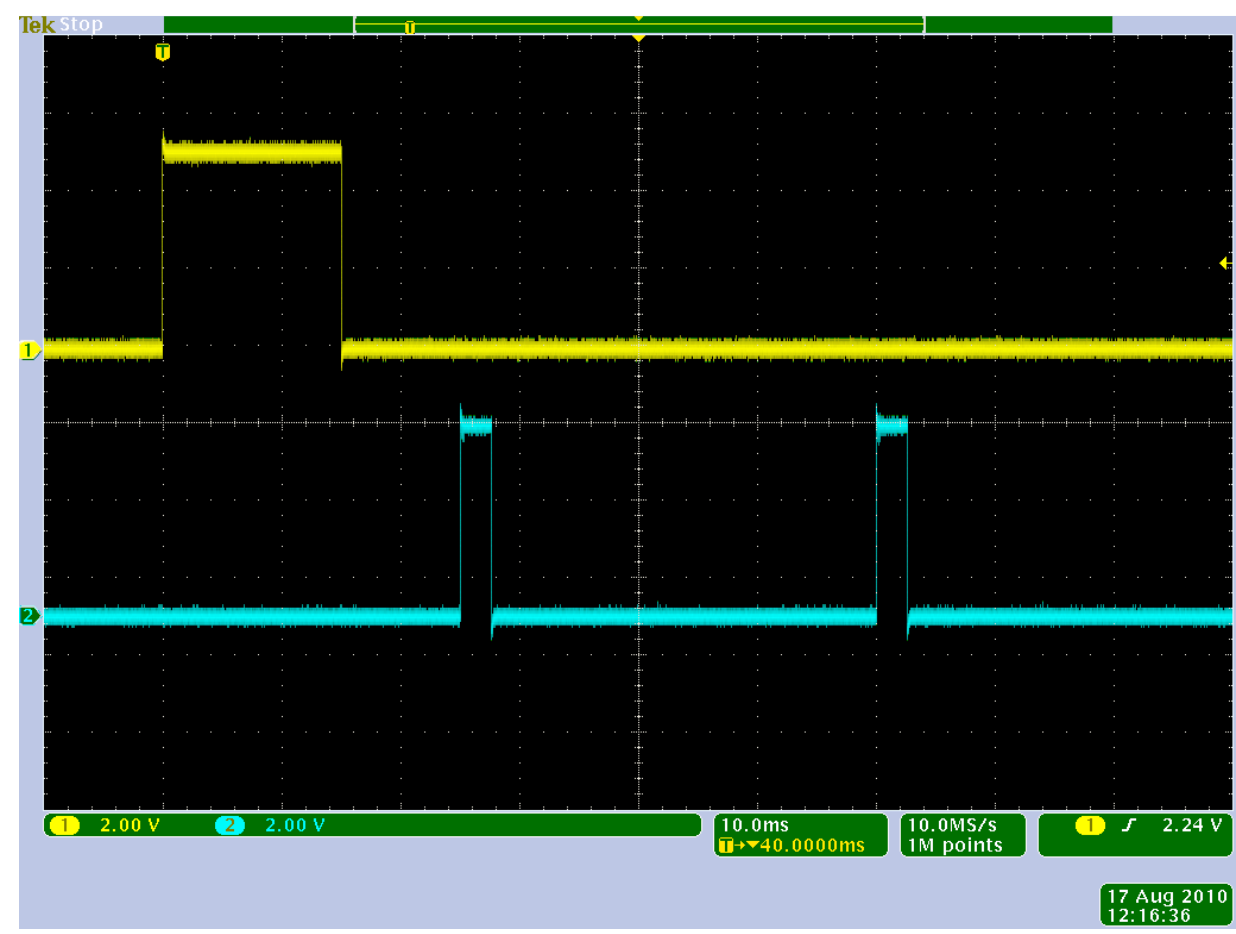

Figure 9.21 Example of Step Measurements on the Ultrasonic Sensor Node

In Figure 9.21 the yellow trace represents the $15 \mathrm{~ms}$ pulse indicating the start of a measurement window. The blue trace represents the Init line of the ultrasonic module that initiates a measurement. It can be seen that the first measurement occurs $25 \mathrm{~ms}$ after the beginning of the measurement window, and a subsequent measurement begins $35 \mathrm{~ms}$ after the first measurement as required.

\subsection{Prototype Sensor Network}

The prototype sensor network, first mentioned in Section 4.3 Prototype Sensor Network, has been tested to determine the accuracy of the calibration equations and its ability to map its surroundings.

\subsubsection{Sensor Accuracy}

An enclosed area measuring $1970 \mathrm{~mm} \times 1850 \mathrm{~mm}$ was set up to measure the ability of the prototype sensor network to measure and map its environment. As seen in Figure 9.22, the sensor network was positioned in the centre of the enclosure. All sensor nodes were issued with a free-running measurement task. 


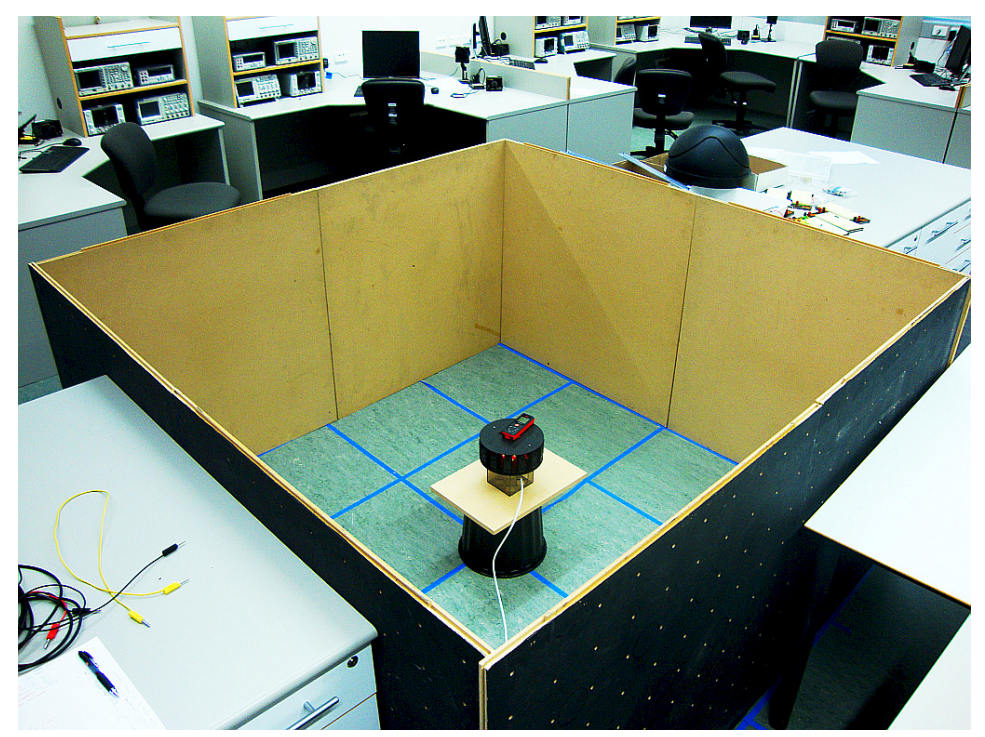

Figure 9.22 Prototype Sensor network Testing Enclosure.

The sensor network data was recorded by the sensor network interface application first discussed in Section 6.4 Test Sensor Network Graphical Application. Figure 9.23 presents a screen capture of the application program. The sensor network data was logged to a data file for further analysis. As time did not permit the calibration of each IR PSD sensor in the test network, the conversion functions, defined in Equations 9.2 and 9.3 were applied to all IR modules in the network.

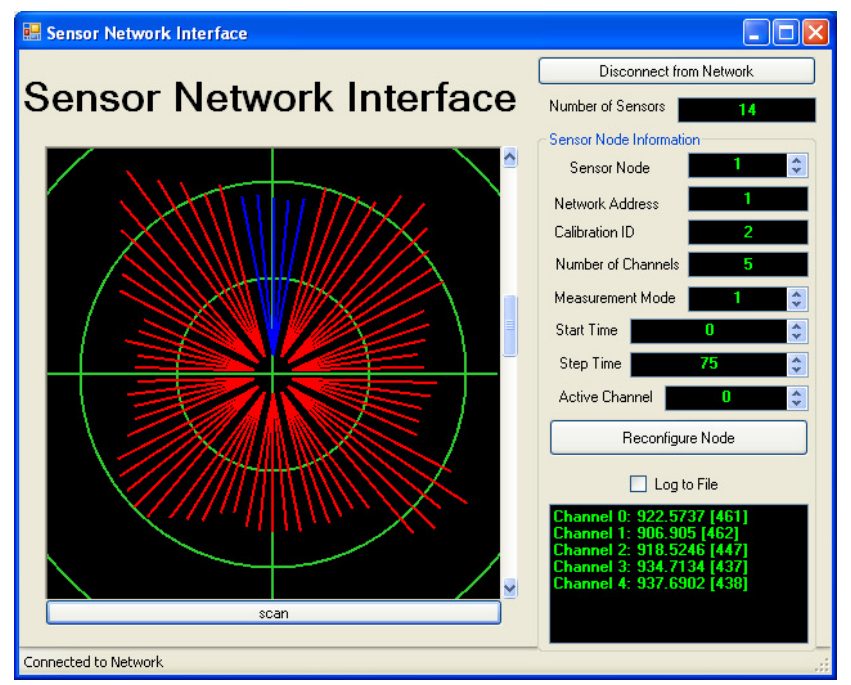

Figure 9.23 Screen Capture of Sensor Network Data for Test Enclosure.

The vector map in Figure 9.23 provides a qualitative view of the sensor network data collected inside the test enclosure. Analysis of the data was carried out in Microsoft Excel. Figure 9.24 presents a radar graph of the sensor data. The angular resolution between sensor channels of the network is $5^{\circ}$. 


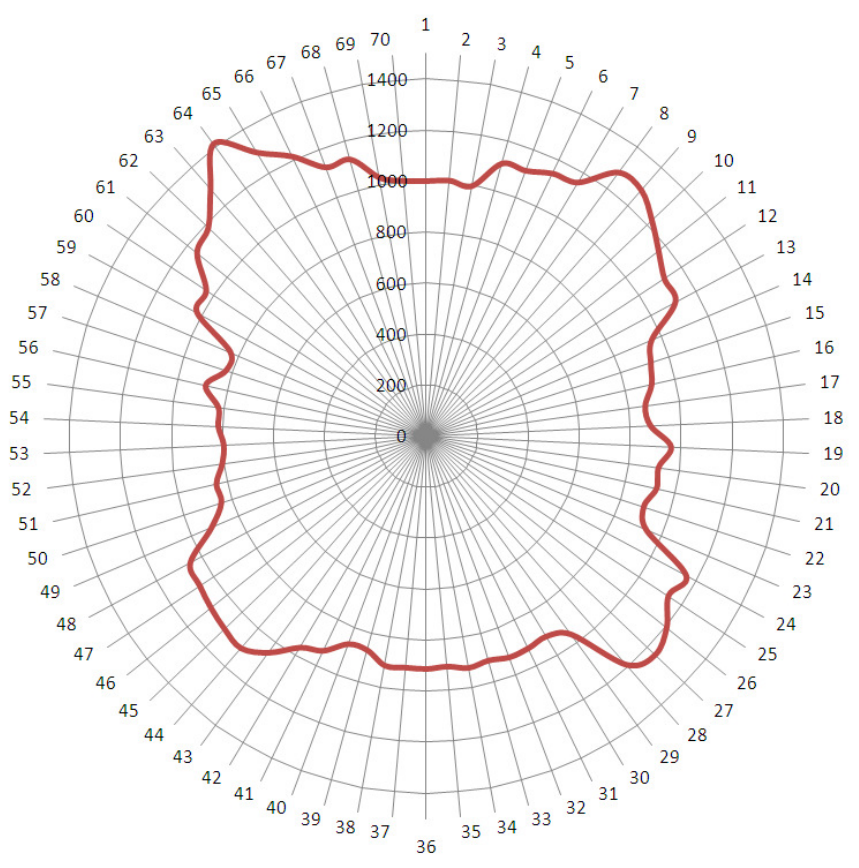

Figure 9.24 Radar Map for Sensor Network Data in Test Enclosure.

Averaging of the $\mathrm{X}$ and $\mathrm{Y}$ magnitudes of the sensor vectors indicated the measured size of the enclosure to be $1905 \mathrm{~mm} \times 1780 \mathrm{~mm}$. This represents an error of approximately $4 \%$ in the dimensions of the test enclosure, within the range error calculated at this range $(8 \%$ at $2000 \mathrm{~mm}$ ). The irregular shape of the mapped enclosure data is due to the blanket use of conversion functions across the network. It can be noted that the maximum deviation of the data from the actual enclosure occurs at the corners of the enclosure, this is most likely due to the increasing angle of incidence between the sensor IR beams and the enclosure walls, an investigation into the performance of the PSD sensors in determination the distance to objects at varying angles of incidence is recommended to provide further characterisation of the sensors but is beyond the scope of this project. A more accurate measurement of the enclosure can be obtained through the individual calibration of each IR module. As previously mentioned this calibration is recommended for further work.

Several objects of varying reflectivity were introduced into the test enclosure to examine the sensor network's ability to map and range a separate object from the boundaries of the test enclosure. Initially a black plastic container was introduced in front of the calibrated GP2Y3A002K0F IR module. Figure 9.25 illustrates the set-up for the test enclosure. A laser range finder, with an accuracy of $\pm 1 \mathrm{~mm}$, measured the container to be $497 \mathrm{~mm}$ in front of the sensor. 


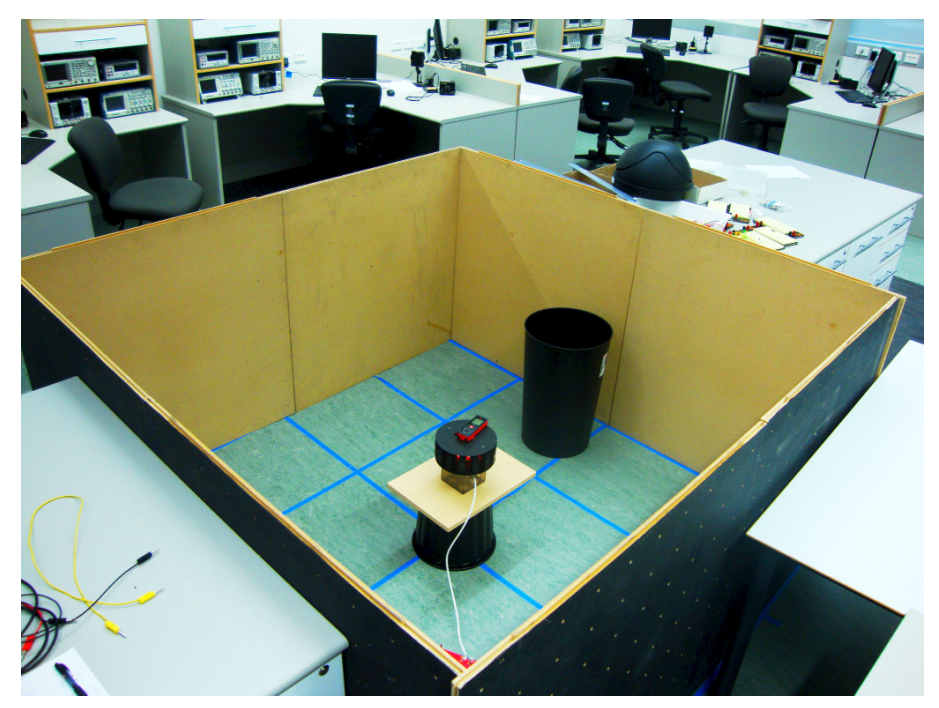

Figure 9.25 Test Enclosure Setup for Object Detection

Data was collected and logged by the Sensor Network Interface application. Analysis performed on the captured sensor data determined the average range to the container to be $480 \mathrm{~mm}$. This represents an error of $4 \%$ in the actual range to the object. This error is greater than the expected range error at this distance, $1.5 \%$ at $500 \mathrm{~mm}$, suggesting the precision of the sensor varies with the reflectivity of the object being ranged. Figure 9.26 presents a radar graph illustrating the mapped test enclosure with and without the container.

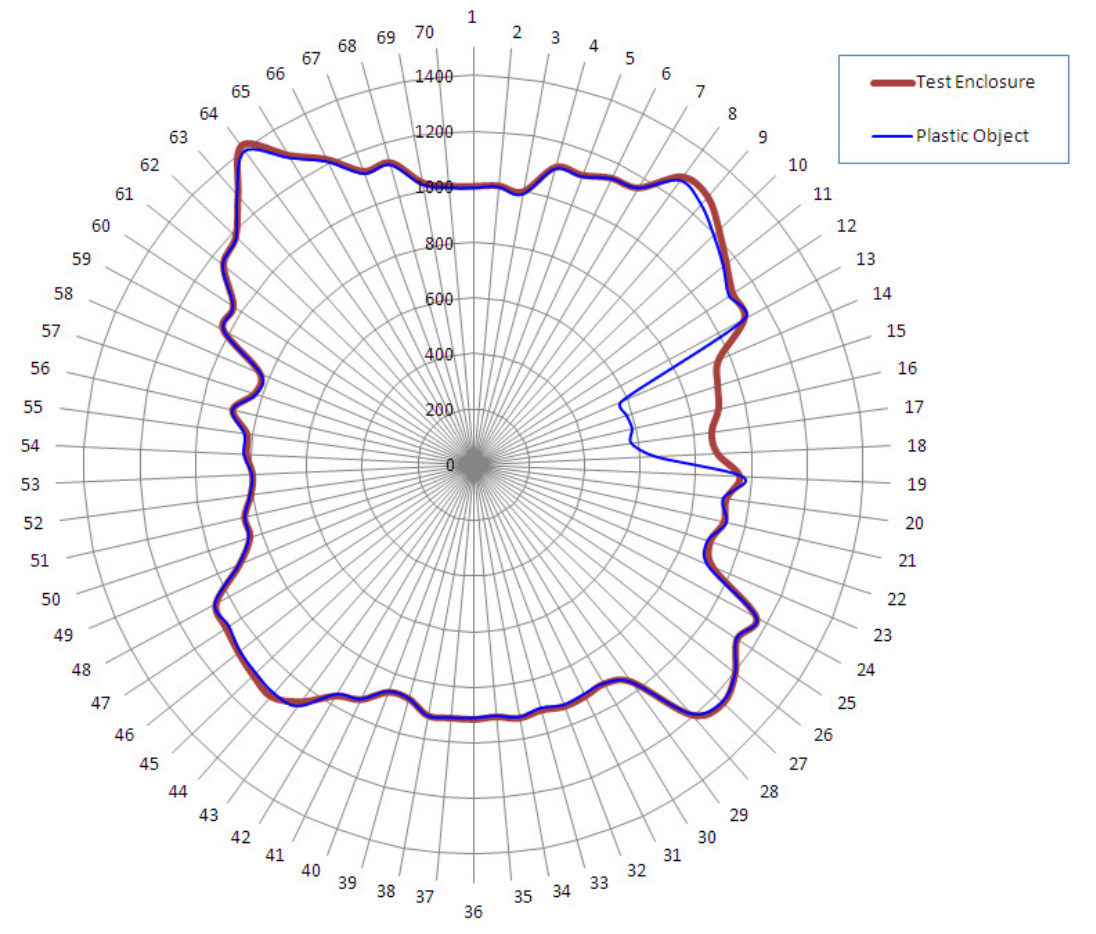

Figure 9.26 Radar Graph for Test Enclosure with Plastic Object at 497 mm 
Both a cardboard object and a metal object were introduced in front of the same calibrated GP2Y3A002K0F IR module to further examine the sensor's accuracy with objects of different reflectivity. Figure 9.27 illustrates the radar graph for the test enclosure with and without the cardboard object in front of the calibrated sensor. The laser range finder measured the cardboard object to be $500 \mathrm{~mm}$ in front of the calibrated sensor. Analysis performed on the sensor data determined the average range to the cardboard object to be $494 \mathrm{~mm}$. This represents an error of $1.5 \%$ in the actual range to the object in agreement with the calculated range error at this distance $(1.5 \%$ at $500 \mathrm{~mm})$.

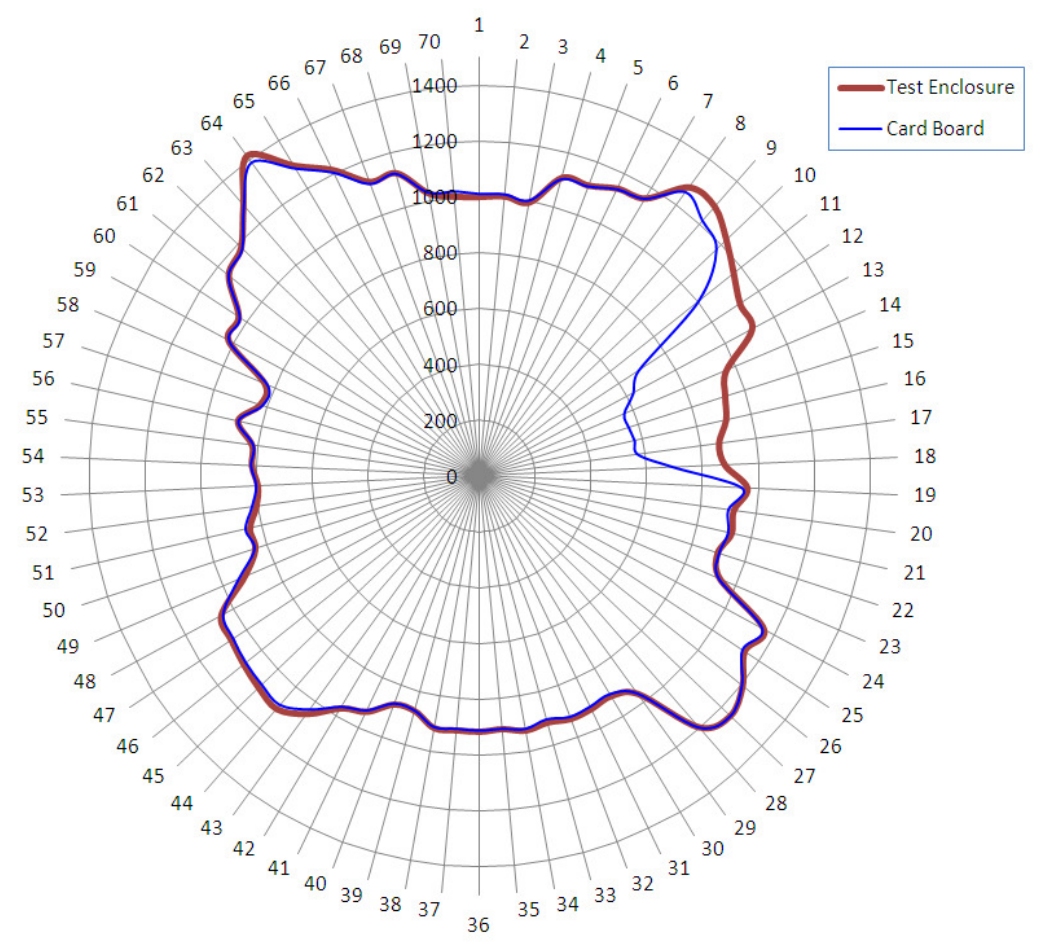

Figure 9.27 Radar Graph for Test Enclosure with Cardboard Object at $500 \mathbf{~ m m}$.

Figure 9.28 illustrates the radar graph for the test enclosure with and without the metal object. The laser range finder measured the metal object to be $498 \mathrm{~mm}$ in front of the calibrated sensor. Analysis performed on the sensor data determined the average range to the metal object to be $546 \mathrm{~mm}$. This represents an error of $9.6 \%$ in the actual range to the object. This variance in error between the metal object and the other objects exceeds the expected range error at $500 \mathrm{~mm}$ and indicates the sensor output varies with reflectivity. Both the plastic container and the cardboard object have low reflectivities relative to the brush aluminium metal object. 


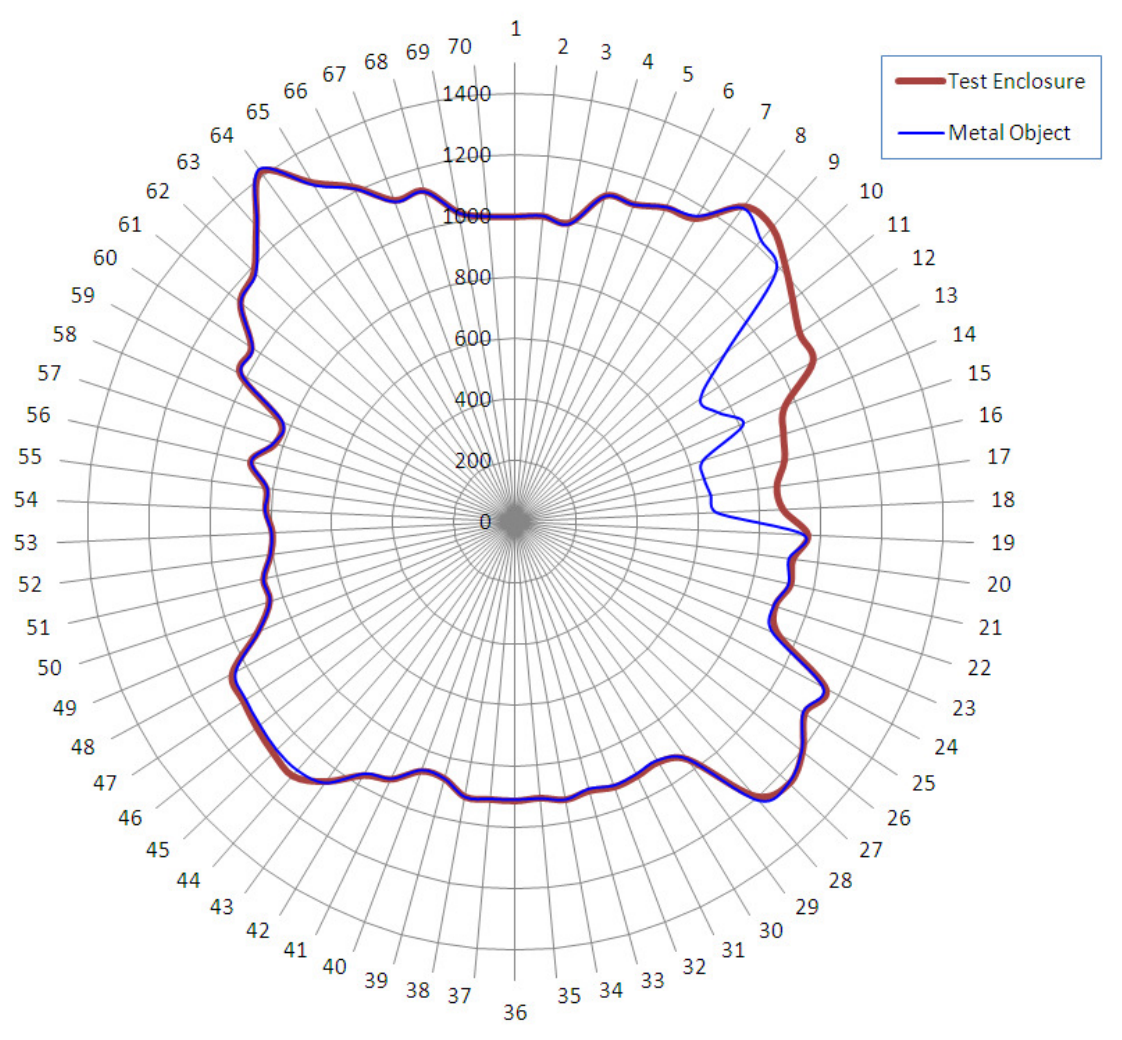

Figure 9.28 Radar Graph for Test Enclosure with Aluminium Object at 498 mm.

Tests on the prototype sensor network and calibration results indicate that the sensors of the network can determine distances to objects with low reflectivities within a range of $2000 \mathrm{~mm}$ to approximately $4 \%$ with the current conversion functions. The accuracy of ranging objects with higher relative reflectivities increases, approximately $10 \%$ for brushed aluminium. Distances greater than $2000 \mathrm{~mm}$ are prone to larger errors due to the variation in the output voltage measured in the GP2Y3A003K0F IR modules.

\subsection{Summary}

The network protocol developed for the sensor network works successfully to co-ordinate a collection of sensor nodes and report back sensor data. The firmware implementation of the network architecture, based on the OSI model, was successful in controlling network communication. The finite state machines implemented to control both the IR PSD sensors and ultrasonic sensors operate correctly to provide reliable sensor data.

Calibration of the GP2Y3A003K0F and GP2Y3A002K0F IR modules indicate a useful range of $200 \mathrm{~mm}-1500 \mathrm{~mm}$ and $400 \mathrm{~mm}-2000 \mathrm{~mm}$ respectively, where the average variation in sample readings was less than $50 \mathrm{~mm}$. Calibration of the ultrasonic sensor indicated a useful 
range of $500 \mathrm{~mm}-9250 \mathrm{~mm}$. Analysis of sensor output for different IR sensor and across channel of an IR sensor indicates variance significant enough to warrant individual calibration of every sensor channel in an IR sensor and individual calibration for each IR sensor.

Implementation of a software library to interface to the network hardware has provided the capability to rapidly develop user applications that can adapt flexibly to reconfigurations of a sensor network.

Implementation of a test sensor network for MARVIN resulted in a sensor network capable of ranging a $360^{\circ}$ field in a horizontal plane with a useful range of $2000 \mathrm{~mm}$ with a maximum range error of $4 \%$ for objects of low reflectivity (plastic and cardboard) and range errors greater than $10 \%$ for objects of relatively higher reflectivities (metals). 


\section{Chapter 10 Results - Full-Field Image Ranger}

This chapter outlines the methodology adopted to validate the correct operation of the hardware of the full-field image ranger system. The existing Stratix III based FPGA embedded software currently operating on the prototype system will be ported to run on the Cyclone III FPGA utilised on the new FPGA board. The porting of the software is a separate project that will be undertaken by another student and as such this section focuses on confirming the hardware functions to allow the software porting. The chapter is concluded with a summary of the results.

\subsection{FPGA Board}

The FPGA board represents the greatest change from the prototype system. The major change from the previous design is the implementation of a Cyclone III FPGA. The key areas tested on this board include the power supplies, the ability to configure the FPGA, the external memory interface and the generic I/O interface.

\subsubsection{FPGA Power Supply}

The FPGA board has eight separate power supplies. As discussed in Section 7.3.5 Power Supply, these power supplies are powered up in a controlled sequence. Figure 7.11 presents the power-up sequence of the power supplies. It is critical that the power supply sequence operates correctly and the voltage regulator outputs are within their specified operational range to ensure stable power is delivered to the FPGA and DDR2 SDRAM devices. The $+3.3 \mathrm{~V}$ regulator is the first power supply in the sequence to be powered. All remaining power supplies rely on a Power Good signal from the $+3.3 \mathrm{~V}$ regulator to either enable them, or an upstream regulator that provides the input voltage for them. This Power Good signal was initially hardwired to be de-asserted to allow the $+3.3 \mathrm{~V}$ regulator's operation to be tested independently of the other voltage regulators. The unregulated power supply to the FPGA board was derived from a current limited bench top power supply to provide the ability to limit the input current if a fault existed. A Fluke 175 digital multi-meter was used to measure the output voltage of the regulators. With power applied, the $+3.3 \mathrm{~V}$ regulator 
reached a stable voltage of $+3.34 \mathrm{~V}$, which is within the design specification tolerances of the LVDS technology of $\pm 10 \%[53,55]$.

The $+1.8 \mathrm{~V}$ and $+1.2 \mathrm{~V}$ derive their input power from the output voltage of the $+3.3 \mathrm{~V}$ regulator. The hardwiring of the Power Good signal was removed to allow these downstream regulators to be enabled. Upon applying input power the $+1.2 \mathrm{~V}$ reached a stable output voltage of $+1.22 \mathrm{~V}$. The $+1.8 \mathrm{~V}$ regulator produced an output voltage of $+3.3 \mathrm{~V}$. Upon closer examination it was observed that a short circuit existed between the $+1.8 \mathrm{~V}$ and $+3.3 \mathrm{~V}$ power rails. This short circuit was found to be the result of a design error in the Altium schematic part for the Cyclone III FPGA. The Cyclone III FPGA powers its I/O banks from the $+3.3 \mathrm{~V}$ and $+1.8 \mathrm{~V}$ supplies. It was discovered that in the Altium schematic part of the Cyclone III an I/O power supply pin for I/O bank 7 was located in the group of I/O power supply pins for I/O bank 6 . This resulted in the power supply pins for I/O bank 7 being connected to both the $+3.3 \mathrm{~V}$ and $+1.8 \mathrm{~V}$ rails causing a short circuit between these two rails. Figure 10.1 shows I/O bank 7 power pin A16 grouped with the power pins for I/O bank 6, and wrongly connected to $+3.3 \mathrm{~V}$. Figure 9.22 also shows that pad A16 of the FPGA was connected directly to the $+3.3 \mathrm{~V}$ internal plane of the FPGA PCB through a via under the FPGA. The short circuit was removed by drilling out the via between the pad and the power plane. This resulted in a disconnected power supply pin for I/O bank 7 and in reducing the current drive capacity of the I/O bank by $11 \%$.
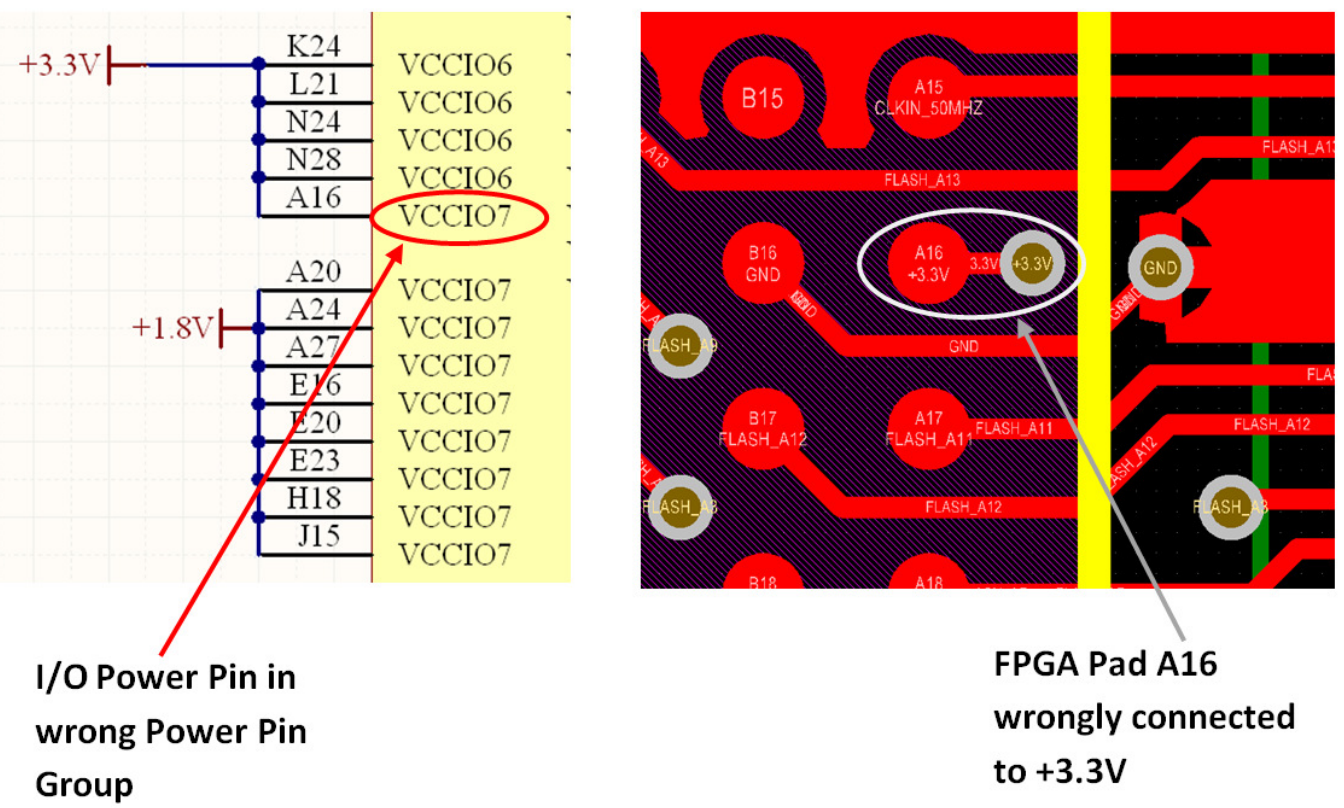

Figure 10.1 FPGA Board Power Supply Design Error 
Upon removal of the short circuit the $+1.8 \mathrm{~V}$ regulator reached a stable voltage of $+1.83 \mathrm{~V}$.

The $+12 \mathrm{~V}$ regulator requires a minimum input voltage of $+14 \mathrm{~V}$ which it derives directly from the unregulated power bus. It also requires the Power Good signal from the $+3.3 \mathrm{~V}$ regulator before it is enabled. Initial testing of the $+3.3 \mathrm{~V}$ regulator required the unregulated power bus to be only $+7 \mathrm{~V}$ which prevented the $+12 \mathrm{~V}$ regulator from operating even with the Power Good signal enabled. An input voltage of $+14 \mathrm{~V}$ was applied to the unregulated power bus to test the operation of the $+12 \mathrm{~V}$ regulator. The output of the regulator reached a stable voltage of $+11.95 \mathrm{~V}$.

The $+5 \mathrm{~V}$ regulator is powered from the output of the $+12 \mathrm{~V}$ regulator and requires a $+12 \mathrm{~V}$ Power Good signal from the $+12 \mathrm{~V}$ regulator to enable it. When powered up, after the $+12 \mathrm{~V}$ regulator reaches a stable voltage and asserts the $+12 \mathrm{~V}$ Power Good signal, the $+5 \mathrm{~V}$ regulator reaches a stable voltage of $+4.99 \mathrm{~V}$.

The $+2.5 \mathrm{~V}$ and $+1.2 \mathrm{~V}_{\mathrm{PLL}}$ regulators derive their input voltage from the $+3.3 \mathrm{~V}$ rail. They also require a boost voltage supply which is sourced from the $+5 \mathrm{~V}$ rail, and the Power Good signal from the $+3.3 \mathrm{~V}$ regulator, to enable them. With the $+3.3 \mathrm{~V}$ and $+5 \mathrm{~V}$ rails powered and stable the $+2.5 \mathrm{~V}$ and $+1.2 \mathrm{~V}_{\mathrm{PLL}}$ regulators reached stable output voltages of $+2.50 \mathrm{~V}$ and $+1.21 \mathrm{~V}$ respectively.

The two $+0.9 \mathrm{~V}$ regulators are powered directly from the output of the $+5 \mathrm{~V}$ regulator. With the $+5 \mathrm{~V}$ regulator powered and stable the two $+0.9 \mathrm{~V}$ regulators reached stable output voltages of $+0.92 \mathrm{~V}$.

\subsubsection{FPGA Configuration and Programming}

Examination of the active parallel configuration scheme revealed a significant design error that prevented its successful implementation. Active parallel configuration requires $47 \mathrm{I} / \mathrm{O}$ lines that are dispersed through four of the eight I/O banks of the FPGA (I/O banks 1, 6, 7 and 8) [44]. The Flash memory device selected as the configuration device is powered by $+1.8 \mathrm{~V}$ and requires $\mathrm{I} / \mathrm{O}$ voltages not to exceed $+2.3 \mathrm{~V}$. The I/O banks of the FPGA that contain the control, address and data lines to the Flash are required to be powered from a $+1.8 \mathrm{~V}$ supply to allow them to connect to the Flash device safely. The current FPGA design, as illustrated in Figure 10.2, has I/O banks 1 and 6 powered from $+3.3 \mathrm{~V}$ and banks 7 and 8 powered from $+1.8 \mathrm{~V}$. The split voltage supply to these I/O banks prevents the FPGA from 
communicating with the Flash device. I/O banks 1,2, 5 and 6 connect with the generic I/O interface and require a $+3.3 \mathrm{~V}$ power supply to interface with the LVDS drivers and receivers. I/O banks 3, 4, 7 and 8 interface with the four DD2 SDRAM memory banks and require a $+1.8 \mathrm{~V}$ supply.

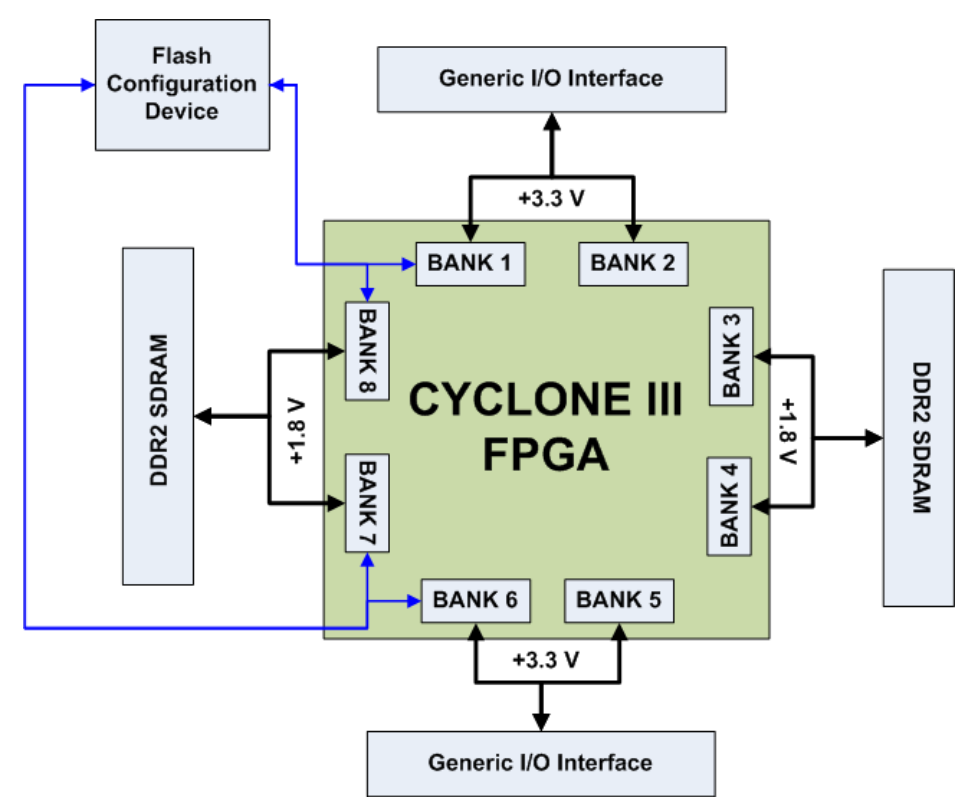

Figure 10.2 I/O Bank Allocation in Current FPGA Design

A complete redesign of the circuit board is required to allow active parallel configuration to be possible on this design as it would require changing the I/O banks which interface with the generic I/O interface or the DDR2 SDRAM. Figure 10.3 presents a suggested I/O bank allocation scheme that would allow active parallel configuration with the Flash device.

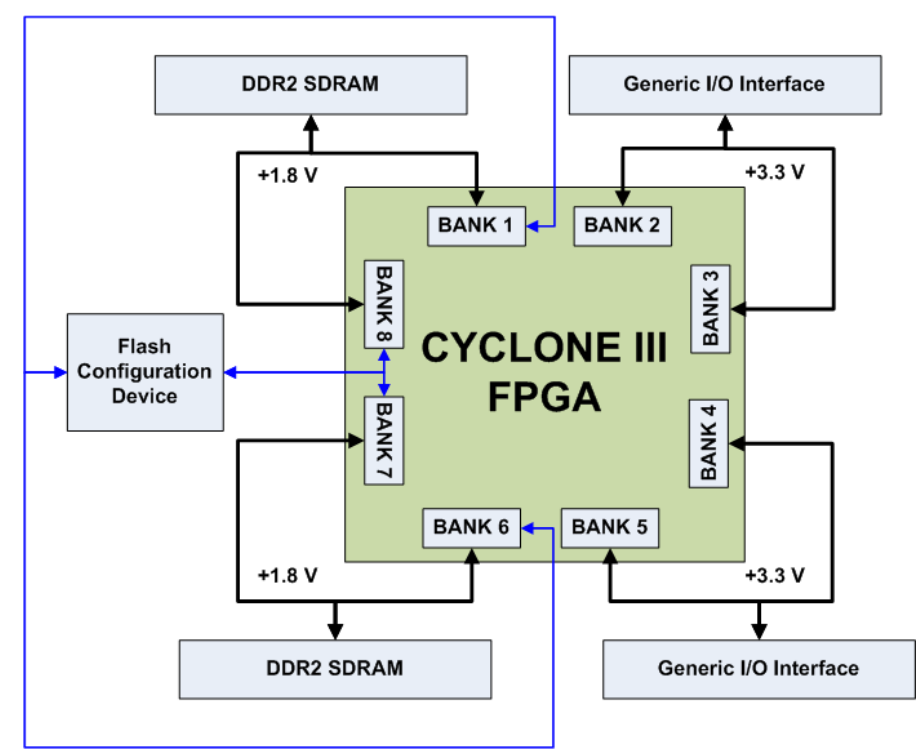

Figure 10.3 Suggested I/O Bank Allocation to Allow Active Parallel Configuration 
The Flash device was removed from the board to prevent erroneous operation from occurring due to Flash I/O lines being both $+3.3 \mathrm{~V}$ and $+1.8 \mathrm{~V}$. Consequently non-volatile storage of the configuration file is not possible on the design but the FPGA board can still be configured through the JTAG interface.

FPGA configuration is possible through the JTAG interface using an Altera USB Blaster programmer. This configuration scheme requires the FPGA configuration to be loaded from an external PC via the USB Blaster every time the FPGA is powered up. A simple 8-bit counter was implemented in VHSIC Hardware Description Language (VHDL) to test the configuration of the FPGA via the JTAG interface. Figure 10.4 presents a block diagram of the simple counter program. The external $50 \mathrm{MHz}$ clock drives a divide by 500 phase lock loop to generate a clock frequency of $100 \mathrm{kHz}$ to clock the 8-bit counter. Output bit 4 of the counter was connected to a test point on the FPGA board to validate an output frequency of $3125 \mathrm{~Hz}$.

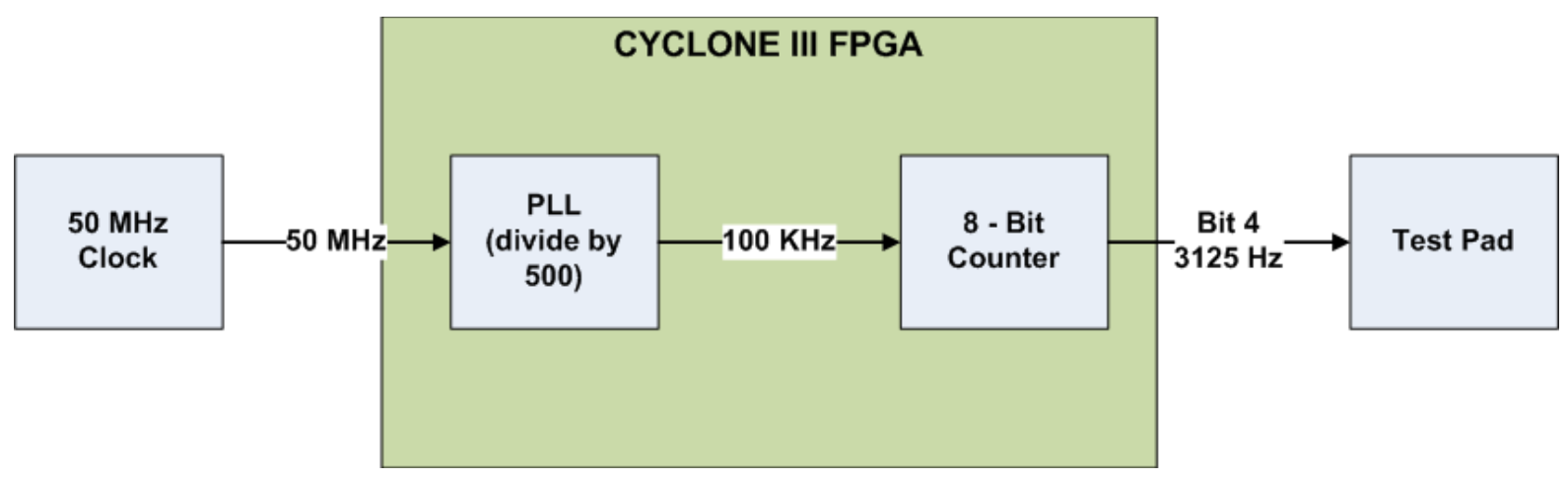

Figure 10.4 Block Diagram of Simple Counter Program for Testing FPGA Configuration

Initial testing of the JTAG interface revealed a design error with the connection between the JTAG header and the FPGA JTAG pads. In a JTAG chain the data output line (TDO) of one device is connected to the data input line (TDI) of the next device in the chain. The JTAG interface was designed with the JTAG header considered as a device in the JTAG chain and as such had the TDO pin connected to the TDI input of the FPGA, and the TDI pin connected to the TDO output of the FPGA. The JTAG header is not considered a device in the JTAG chain and as such its TDI and TDO pins should be connected to the FPGA's TDI and TDO input and output pads respectively. An adaptor cable was created that swapped the TDI and TDO lines between the USB Blaster programmer and the JTAG header on the FPGA board. With the modified cable the FPGA was able to be configured with the simple counter configuration via the USB Blaster programmer. Oscilloscope measurements of the test point 
connected to bit 4 of the simple counter validated that the expected frequency of $3125 \mathrm{~Hz}$ was outputted.

\subsubsection{FPGA External Memory}

Altera's DDR2 SDRAM Controller provides a simplified interface between the embedded FPGA code and the external DDR2 SDRAM devices. The controller handles the complex aspects of using DDR2 SDRAM such as initializing the memory device, managing the SDRAM banks and keeping the devices refreshed at appropriate levels. It also translates read and write requests from the local interface on the FPGA into the necessary SDRAM command signals. When compiling a DDR2 SDRAM controller an example driver is automatically generating to allow the memory interface to be tested. Through use of Altera's SignalTap II Logic Analyser the local interface between the example driver and the DDR2 SDRAM controller can be monitored in real time to confirm correct operation of the SDRAM. Figure 10.5 illustrates the set up of the DDR2 memory test. The example driver is a self-checking test generator for the DDR2 SDRAM controller. The driver writes data patterns to a range of column addresses within a range of row addresses in all memory banks and then reads back the data from the same locations and checks the data matches. A passnot-fail (pnf) output transitions low if any read data fails the comparison [63].

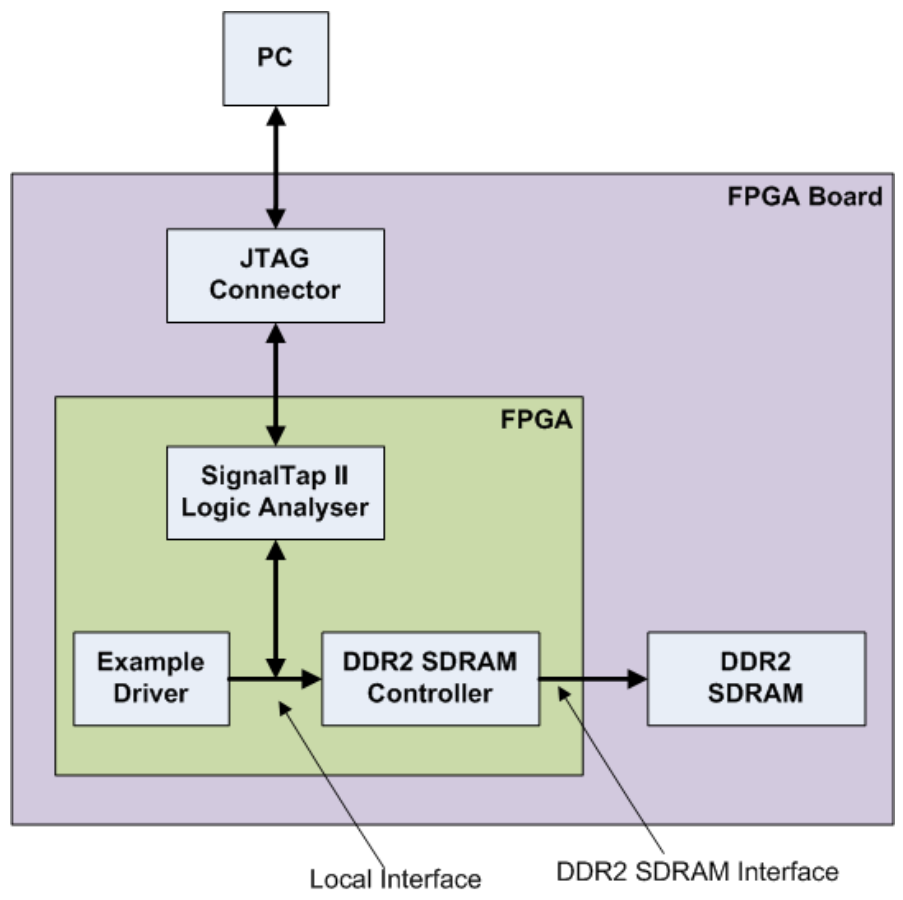

Figure 10.5 Block Diagram of DDR2 SDRAM Test Set up 
DDR2 SDRAM Controllers were compiled for the DDR2 memory devices on the FPGA board. The automatically generated example drivers were used to test the functionality of the DDR2 SDRAM. SignalTap Analysis indicated that the Accumulator and Output Buffer memory banks operated correctly. Figure 10.6 presents the SignalTap waveforms captured for the Output buffer test. In the highlighted sections it can be seen that the local_rdata value (representing the data read back from the SDRAM) matches the local_wdata value (representing the original data written to the SDRAM) indicating a successful write and read from the memory device.

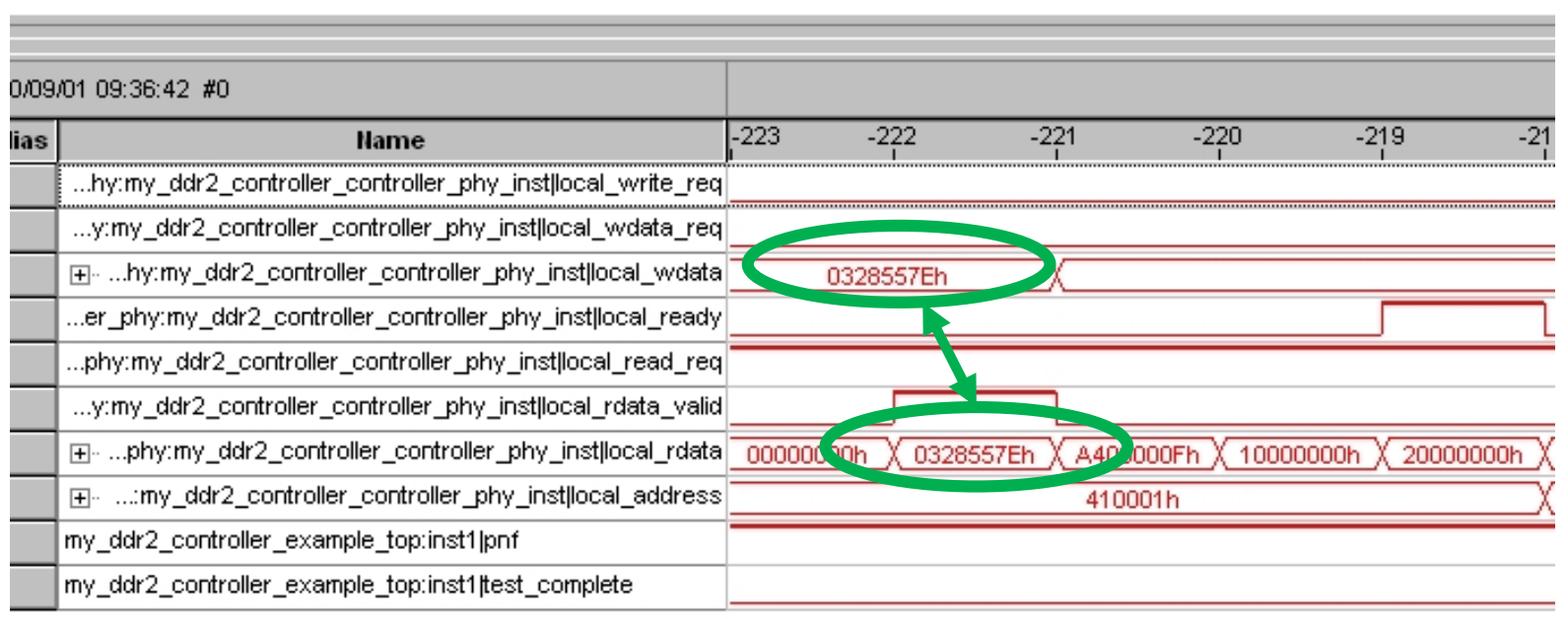

Figure 10.6 SignalTap II Captured Data for Output Buffer Memory Bank Test

Both the Accumulator and Output Buffer DDR2 SDRAM controllers were compiled to run at a memory clock frequency of $200 \mathrm{MHz}$, allowing a maximum data rate of $3.2 \mathrm{~Gb} / \mathrm{s}$.

The tests on the NIOS Program and Ethernet Buffer DDR2 SDRAMs could not be completed due to a design error. Both these memory banks operate with a 16 bit wide data bus. DDR2 SDRAM designs require a bi-directional Data Strobe (DQS) signal to accompany Data lines (DQ) from the memory device. The DQS line allows the FPGA to signal the SDRAM when data is ready on the data bus during a write operation. It also allows the SDRAM to signal the FPGA when data is ready to be read from the data bus during a read operation. In Altera FPGAs dedicated groups of pins are associated with DQ and DQS lines. This requires the placement of DQ lines in close proximity to a DQS line to minimise timing skew between them when the FPGA pin fitting is carried out during compilation of a configuration file. The memory interface of the Ethernet buffer and NIOS Program memory banks were designed as $\times 16$ DDR2 SDRAM interfaces. The 16 DQ lines from the SDRAM were assigned to the 
dedicated DQ pins for $\times 16$ interface mode as specified by Altera. During programming of the example driver for the NIOS Program buffer it was discovered that Altera define a $\times 16$ interface as requiring only one DQS line. For Cyclone III FPGAs, a DDR2 SDRAM with a 16-bit data bus must be designed as two separate 8-bit data buses with corresponding independent DQS lines. The Altera DDR2 SDRAM controller is instantiated as two sets of $\times 8$ DQ/DQS groups. The $\times 16$ interfaces between the FPGA and the Ethernet Buffer and NIOS Program memories are not compatible with the required two $\times 8$ interfaces instantiated by the controller. This incongruity means that several DQ lines from the DDR2 SDRAM are not connected to the required DQ lines of the FPGA and as such these memory banks cannot reliably store data. Due to the FPGA and DDR2 device packaging, the impedance controlled routing and the length tuning undertaken when designing the circuit board, it is not possible to modify these connections. A new design of the circuit board is required to enable the use of these external memory banks.

It is possible to connect to the two SDRAM banks by implementing a custom DDR2 SDRAM controller that would deskew the timing of the DQ lines to minimise timing skew between them and the DQS line. This would involve controlled fitting of the FPGA to ensure the internal FPGA routing of the DQ lines are of a similar length.

\subsubsection{FPGA Generic I/O Interface}

The input and output lines of the two generic I/O interfaces were tested to ensure communication is possible between the FPGA and the peripheral image capture and external interface boards. A simple 32-bit counter program was written in VHDL to provide modulating signals to drive the input and output lines of the generic interface. Oscilloscope measurements were employed to inspect the I/O lines of the image capture, and external interface boards to confirm the modulated signals were received across the interfaces. The single-ended low voltage transistor to transistor logic (LVTTL) lines and the LVDS driver lines on both interfaces correctly transmitted the modulated signals.

The interface's LVDS receivers were tested by looping back the output of the LVDS drivers to the receivers' inputs. The 32-bit counter was used to produce a modulating signal to the LVDS drivers. The output of the LVDS receivers was captured by the FPGA and routed to test points on the FPGA board for inspection with an oscilloscope. The LVDS receivers on 
both interfaces operated correctly and received the modulated signals sent to the LVDS drivers.

Inspection of the image capture board reveals a design error with the generic I/O interface. The LVDS lines for the Serial Data Read from the Imaging Signal Processor ADC were connected to interface lines reserved for JTAG TMS line. The image capture board was originally designed to be compatible with the interface adopted on the prototype full-field image ranging system which used the reserved TMS interface connector pin to carry this data line. The design of the generic $\mathrm{I} / \mathrm{O}$ interface on the mobile platform ranging system incorporates the JTAG interface lines to allow JTAG enabled devices on peripheral boards to be connected to the JTAG chain and programmed via the JTAG header on the FPGA board. Figure 10.7 illustrates the design error. The image capture interface schematic on the left has the LVDS lines connected to the JTAG TMS and SCL lines of the interface. The FPGA interface schematic on the right has the JTAG TMS interface pin connected to TMS and the SCL pin connected to ground. The image capture board was modified to connect the LVDS Serial Data Read lines to the RX0 differential interface pins.

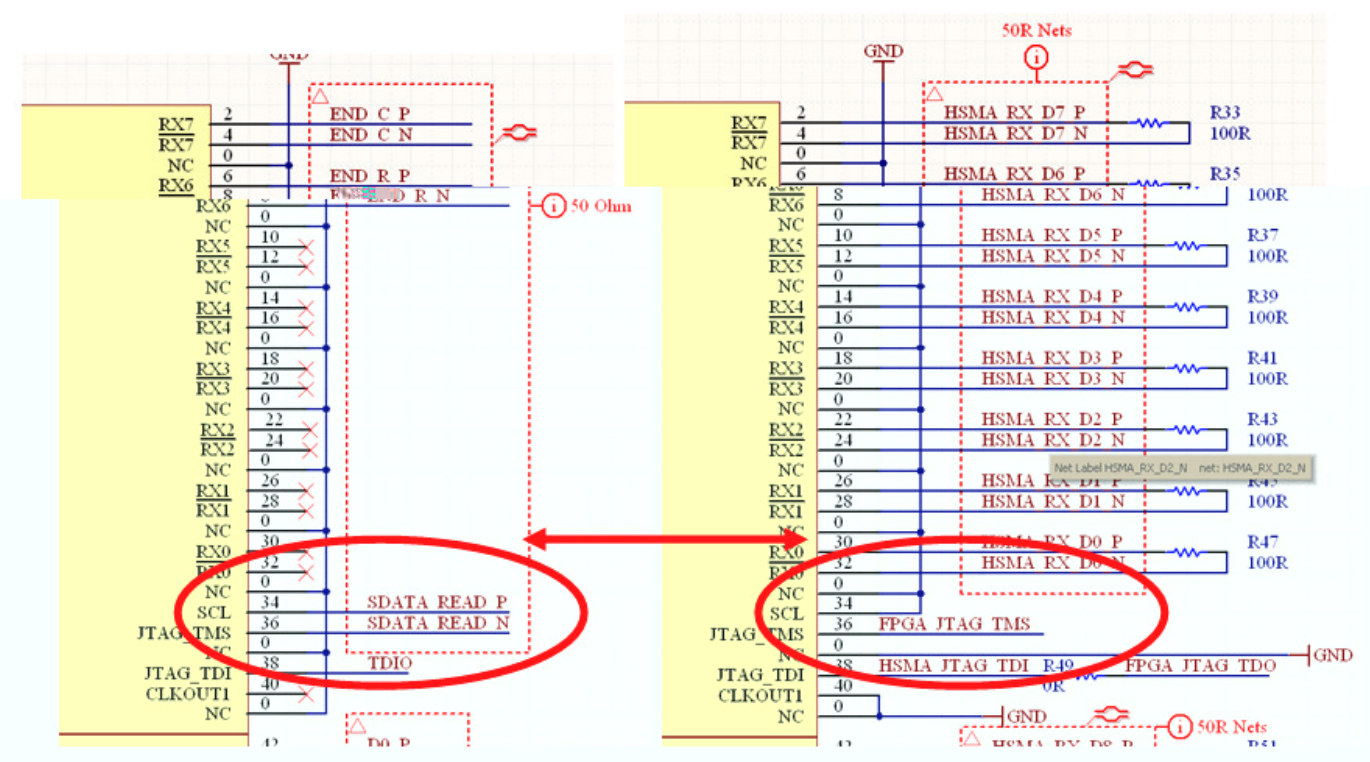

Figure 10.7 Generic I/O Interface Design Error on Image Capture Board 


\subsection{External Interface Board}

\subsubsection{Power Supply}

The External interface board has three separate voltage rails; $+5 \mathrm{~V},+3.3 \mathrm{~V}$ and $+2.5 \mathrm{~V}$. These voltages are generated from onboard regulators. The $+5 \mathrm{~V}$ regulator sources its input voltage from the system's unregulated power bus, and its $+5 \mathrm{~V}$ output voltage provides the input voltage for the $+3.3 \mathrm{~V}$ and $+2.5 \mathrm{~V}$ regulators. The $+5 \mathrm{~V}$ regulator generates a Power Good signal when its output voltage reaches a stable level. The Power Good signal drives the enable lines of the downstream regulators to ensure they are not activated until a stable $+5 \mathrm{~V}$ input source is available to them. To initially test the $+5 \mathrm{~V}$ regulator, the Power Good signal was hardwired to disable the $+3.3 \mathrm{~V}$ and $+2.5 \mathrm{~V}$ regulators. A current controlled bench top power supply, set at $+14 \mathrm{~V}$, was used as the power source for the system's power bus. The power supply's current limit was gradually increased until the supply's output voltage reached $+14 \mathrm{~V}$ and the $+5 \mathrm{~V}$ regulator's output voltage reached a stable level of $+5.00 \mathrm{~V}$.

The Power Good signal was restored to the $+3.3 \mathrm{~V}$ and $+2.5 \mathrm{~V}$ regulators to test their operation. Both the $+3.3 \mathrm{~V}$ and $+2.5 \mathrm{~V}$ regulators reached stable output voltages of $+3.30 \mathrm{~V}$ and $+2.53 \mathrm{~V}$ respectively.

\subsubsection{Generic I/O Interface}

The communication channels across the generic I/O interface between the FPGA and the External Interface boards were tested to ensure all I/O signals from the FPGA were received by the External Interface board. A simple 32 bit counter was implemented in VHDL to configure the FPGA to produce modulating signals on all the communication channels to the External Interface board. An oscilloscope was used to examine the External Interface's I/O line to confirm that the modulating signals generated by the FPGA were clearly received. All I/O lines on the External Interface board were confirmed to transmit clearly. 


\subsection{Image Capture Board}

\subsubsection{Power Supply}

The Image Capture board has four independent voltage rails. Two are generated by onboard regulators: $+2.5 \mathrm{~V}$ and $+5 \mathrm{~V}$. Two are provided by the generic $\mathrm{I} / \mathrm{O}$ interface: $+3.3 \mathrm{~V}$ and $+12 \mathrm{~V}$. The $+5 \mathrm{~V}$ on-board regulator derives its input voltage from the system's unregulated power bus and its $+5 \mathrm{~V}$ output drives the input voltage of the $+2.5 \mathrm{~V}$ regulator. A current controlled bench top power supply was used to provide a current limited power source to the system's unregulated power bus. The voltage of the bench top supply was set at $+14 \mathrm{~V}$, the minimum specified input voltage for the power bus, and the current limit gradually increased until the $+5 \mathrm{~V}$ regulator reached a stable output voltage. Voltage measurements indicate an output voltage of $+5.02 \mathrm{~V}$. The $+2.5 \mathrm{~V}$ regulator reached a stable output voltage of $+2.52 \mathrm{~V}$. Voltage measurement on the $+12 \mathrm{~V}$ and $+3.3 \mathrm{~V}$ rails from the generic I/O interface indicate voltages of $+11.42 \mathrm{~V}$ and $+3.34 \mathrm{~V}$ respectively.

\subsubsection{Generic I/O Interface}

Communication between the FPGA and Image Capture boards across the generic I/O interface was tested to ensure all I/O signal from the FPGA were received on the Image Capture board. A simple 32 bit counter was implemented in VHDL to configure the FPGA to provide a range of modulating output signals to be sent across the interface. An oscilloscope was used to examine the I/O lines on the Image Capture board to confirm that the modulating signals were received. All I/O lines from the FPGA to the Image Capture board were confirmed to transmit clearly.

\subsubsection{Signal Modulation}

The modulation pins for the image sensor are driven by high current pin drivers due to the capacitance of the pins. The drive signal for the sensor modulation is generated from the FPGA. This modulation signal is converted from a LVTTL single-ended signal to a LVDS signal to improve noise immunity across the generic $\mathrm{I} / \mathrm{O}$ interface to the Image Capture board. The LVDS signal is converted back to a LVTTL signal which is required to drive the high current pin driver chips. A simple PLL was implemented in VHDL to configure the FPGA to generate both a $10 \mathrm{MHz}$ and $40 \mathrm{MHz}$ signal on the modulation line. Figure 10.8 presents the waveforms captured on an oscilloscope at the input pin of a high current pin 
driver on the image capture board. The $10 \mathrm{MHz}$ modulation signal is clearly communicated between the FPGA and the pin drivers. The $40 \mathrm{MHz}$ modulation signal exhibits some distortion but is within tolerances of the pin driver's voltage specification for the input pins.
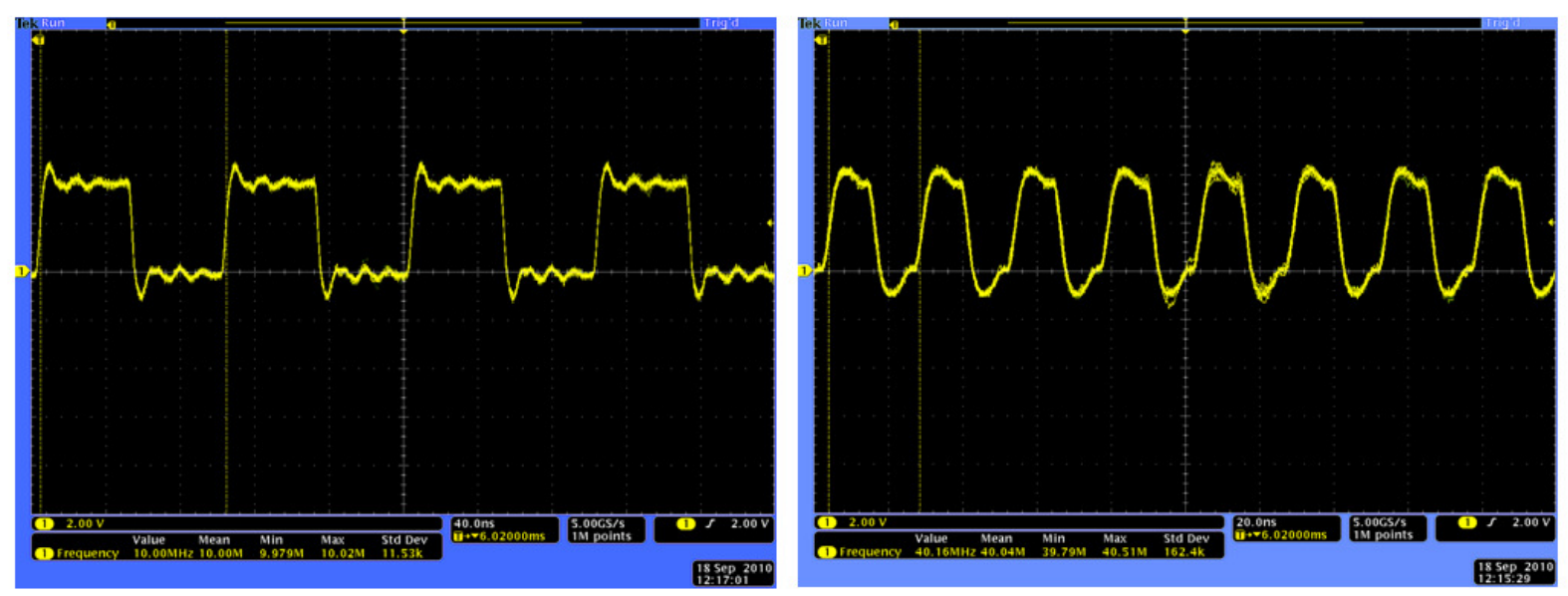

Figure 10.8 10 MHz and 40 MHz Modulation Signals Received at High Current Pin Driver Chip.

\subsubsection{Pin Driver Power Consumption}

The power consumption of the high current pin drivers is a function of the modulation frequency they are driven with. Even with the outputs floating, the internal capacitance of the pin drivers results in significant power consumption when driven with modulation frequencies of the order of $10 \mathrm{MHz}$. At $40 \mathrm{MHz}$ each pin driver consumes approximately $500 \mathrm{~mW}$ and generates significant amounts of heat. It was observed that the pin drivers exceeded their maximum ambient temperature after approximately three minutes when driven by a $40 \mathrm{MHz}$ modulation signal. Active cooling through use of a $12 \mathrm{~V}$ fan was employed to reduce the temperature of the pin driver at this modulation frequency. To investigate this heating, temperature profiles were recorded for a pin driver with passive cooling and active cooling using a type $\mathrm{K}$ thermocouple and a Fluke 566 IR Thermometer. Figure 10.9 presents the temperature profiles measured for a pin driver. It can be seen that the pin driver exceeds its specified operating temperature, $85^{\circ} \mathrm{C}$, after approximately $185 \mathrm{~s}$ when passively cooled. Active cooling significantly reduced the temperature of the pin driver to approximately $34^{\circ} \mathrm{C}$ after $120 \mathrm{~s}$. Temperature profiles for the pin drivers at $10 \mathrm{MHz}$ was also measured for both active and passive cooling schemes and are presented in Figure 10.10. With passive cooling the pin driver reaches a temperature of approximately $49^{\circ} \mathrm{C}$ after five minutes. Active cooling reduced the temperature of the drivers to $24^{\circ} \mathrm{C}$ after approximately $120 \mathrm{~s}$. 


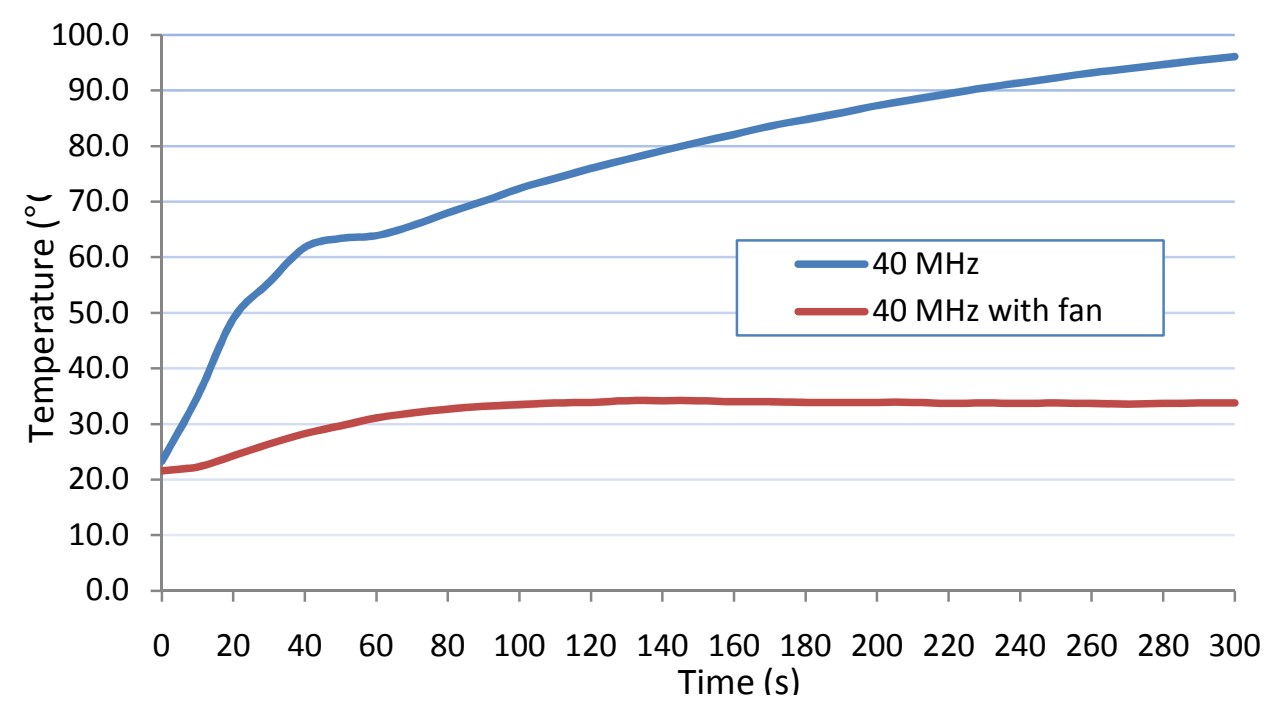

Figure 10.9 Temperature of High Current Pin Driver With and Without Active Cooling vs Time for 40 MHZ Modulation Signal.

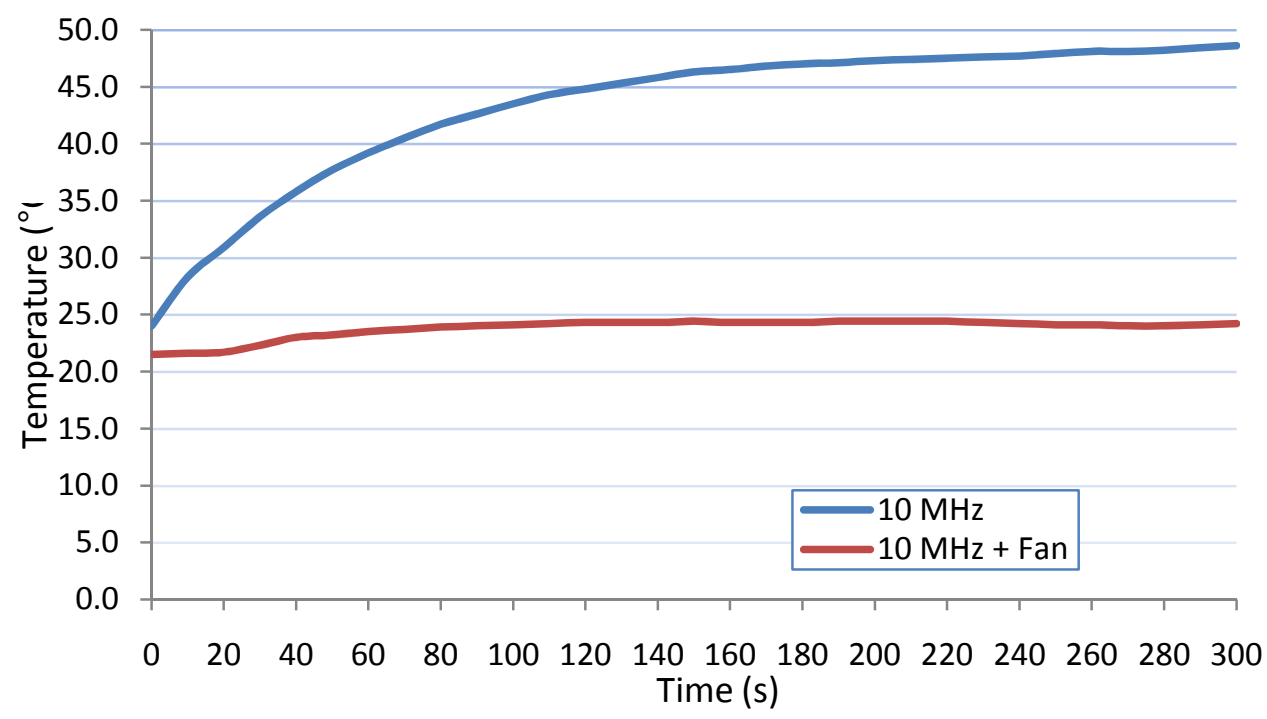

Figure 10.10 Temperature of High Current Pin Driver With and Without Active Cooling vs Time for 10 MHZ Modulation Signal.

The addition of a fan to actively cool the pin drivers significantly reduces their operating temperature and therefore extends the life time of the drivers. Ideally a passive cooling scheme should be employed as the addition of a fan can inject noise into a system and increase the power consumption of the system therefore reducing the operating time from a battery source. 


\subsection{Laser Illumination Board}

\subsubsection{Power Supply}

As discussed in Section 8.2.2 Power Supply, the Laser Illumination requires two power supplies. The laser diodes and laser switch devices require a $+5 \mathrm{~V}$ supply capable of delivering 5.6 A. A +3.3 V supply is required to power the LVDS receivers carrying the modulation signals for the laser switches and the two wire interface signals for the DAC controlling the diode current.

A current-controlled bench top power supply was used to power the unregulated power bus input from which the $+5 \mathrm{~V}$ regulator sources its input voltage. The bench top power supply output voltage was set at $+7 \mathrm{~V}$ and the current gradually increased until the $+5 \mathrm{~V}$ regulator reached a stable output voltage of $+4.98 \mathrm{~V}$. The $+3.3 \mathrm{~V}$ regulator, powered from the $+5 \mathrm{~V}$ rail, produced a stable output of $+3.27 \mathrm{~V}$.

\subsubsection{Laser Switching}

The laser diodes of the Laser Illumination board have been designed to be driven by a modulation signal from the FPGA. The FPGA generated modulation signal is converted from a LVTTL single-ended signal to a LVDS signal to improve noise immunity across the generic I/O interface to the image capture board. LVDS repeaters on the image capture board boost the signal strength of the modulation signal which is fed to the Laser Illumination board via a SATA connection. On the Laser illumination board, the LVDS signal is converted back to a LVTTL single-ended signal and finally to a TTL signal to drive the laser switch chip. Each laser bank has its own modulation signal to allow independent modulation. A simple PLL was implemented in VHDL to provide both a $10 \mathrm{MHz}$ and a $40 \mathrm{MHz}$ modulation signal source in the FPGA. Figure 10.11 presents the waveforms captured by an oscilloscope at a laser switch chip on the Laser Illumination board. Impedance driven routing and parallel termination schemes were employed to reduce transmission line effects on the modulation signals. Minor ringing effects can still be observed at $10 \mathrm{MHz}$ but are not significant enough to cause erroneous switching. The $40 \mathrm{MHz}$ signal exhibits distortion but is still within the specified voltage limits of the laser driver input pins to provide a reliable switching at this frequency. 

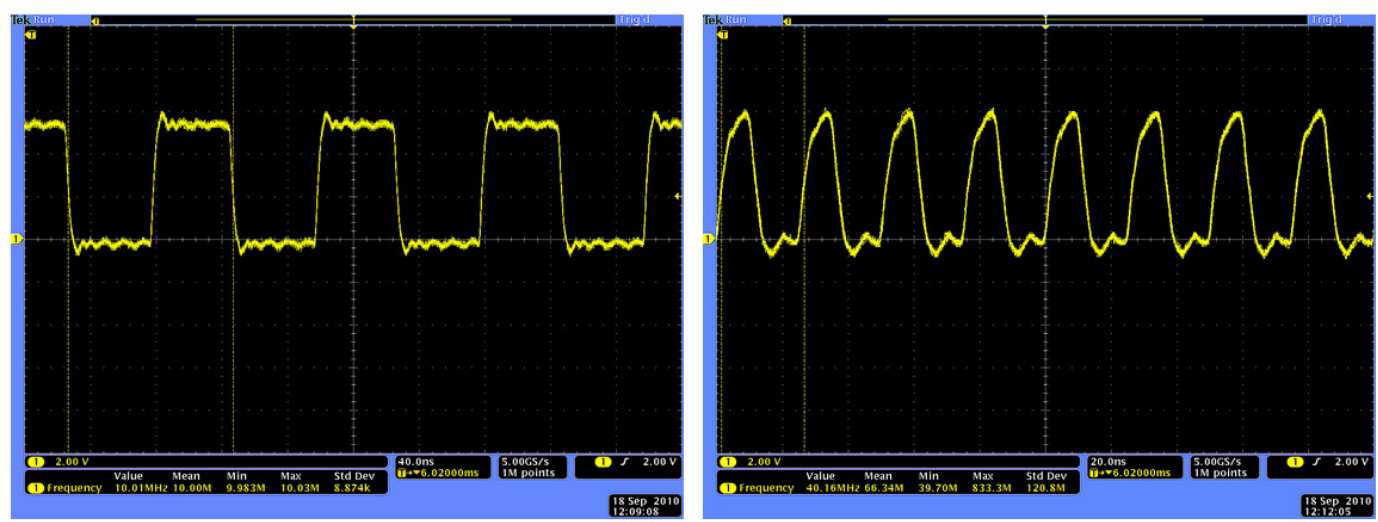

Figure 10.11 10 MHz and 40 MHz Modulation Signals received at Laser Switch.

Both bank modulation signals were inspected to confirm clear communication between the FPGA board and the Laser Illumination board. An external voltage source applied to the laser switch chip's current control pin confirmed visually that the laser diodes illuminate when driven by the modulation signals from the FPGA.

The output of the laser diodes was examined with a silicon diode to confirm that the lasers were switching at the modulation frequency driving the laser switches. A silicon diode detector, DET10A from Thorlabs, was positioned in front of the laser diodes and the output of the detector was captured on an oscilloscope. Figure 10.12 illustrates the silicon detector output when the laser diodes were switched with a $10 \mathrm{MHz}$ modulation signal. The output clearly indicates the laser diodes are switching at the desired modulation signal.

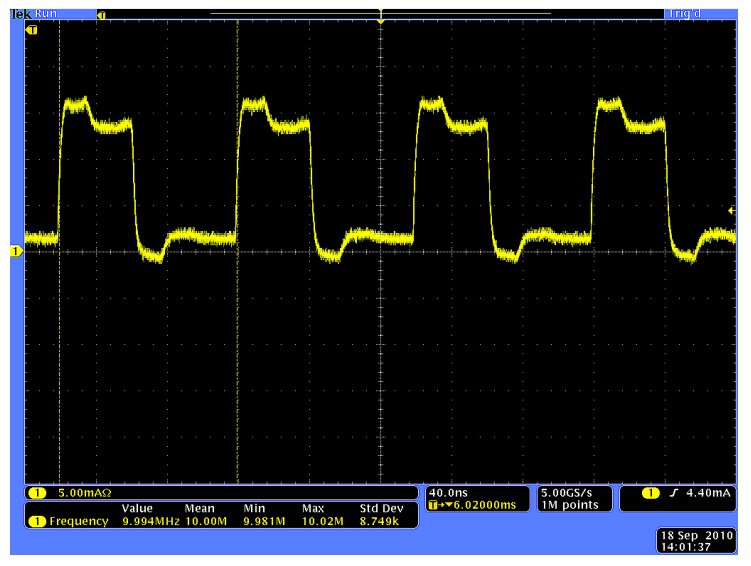

Figure 10.12 Silicon Detector signal for $10 \mathrm{MHz}$ modulation signal.

The laser diodes driven with both $20 \mathrm{MHz}$ and $40 \mathrm{MHz}$ modulation signals were also examined with the silicon detector. Figure 10.13 presents the waveforms captured by the oscilloscope for the two modulation frequencies. The $20 \mathrm{MHz}$ modulation signal drives the laser diodes with a clear $20 \mathrm{MHz}$ output. The $40 \mathrm{MHz}$ modulation signal suffers from 
distortion resulting in an asymmetrical duty cycle with the ON time approximately $25 \%$ of the OFF time. This results in a reduction in light intensity and indicates at higher modulation frequencies that the laser diode light output will reduce and could limit the maximum range that objects can be illuminated at. The modulation signal at $40 \mathrm{MHz}$ still produces a laser diode output which switches at $40 \mathrm{MHz}$.
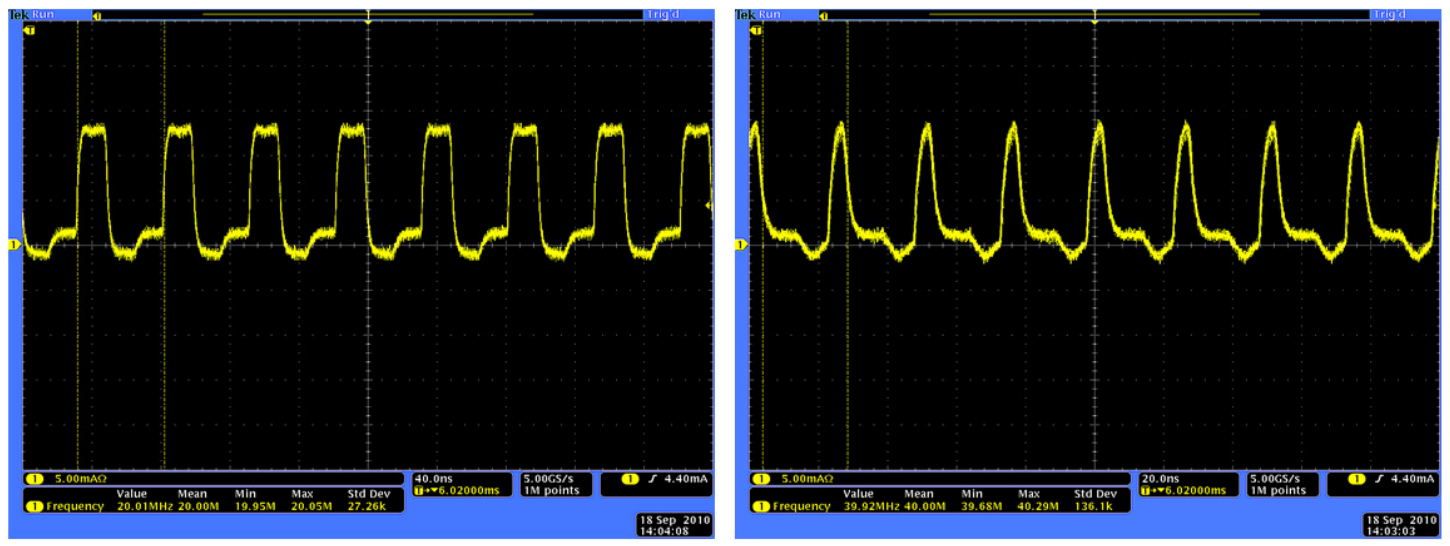

Figure 10.13 Silicon Detector signals for $20 \mathrm{MHz}$ and $40 \mathrm{MHz}$ Modulation Signals.

\subsubsection{Protection Circuit}

The Laser Illumination board has a protection circuit to prevent the laser diodes from being operated in a continuous wave mode. The laser diodes are operated at current levels permitted for pulse operation. Continuous wave operation would provide forward diode currents that exceed the maximum value specified for the laser diodes. The protection circuit filters the modulation signal for each laser bank and compares it with a reference voltage. If the filtered modulation signal is greater than the reference the drive signal to the laser switches is deasserted. A simple PLL was implemented in VHDL to configure the FPGA to output a low frequency modulation signal $(10 \mathrm{MHz})$ that would effectively operate the laser diodes in a continuous wave mode. The modulation and laser switch drive signals at the Laser Illumination board were inspected with an oscilloscope to determine the performance of the protection circuit. Figure 10.14 presents the waveforms captured by the oscilloscope. The yellow trace represents the modulation signal and the blue trace represents the laser switch drive signal. 


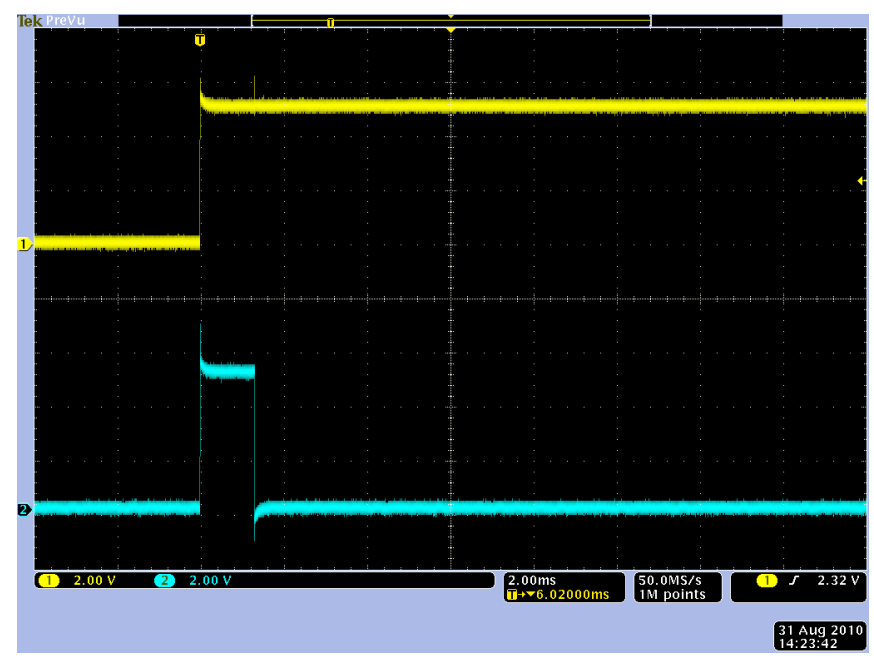

Figure 10.14 Laser Switch Enable Signal Response for DC Drive Signal

It can be seen that the laser switch's drive signal becomes low approximately $1.5 \mathrm{~ms}$ after being asserted. The protection circuit causes this low transition to occur due to the filtered modulation signal being greater than the voltage reference. The $1.5 \mathrm{~ms}$ delay indicates that the current reference voltage level activates the protection circuit for modulation signals less than approximately $700 \mathrm{~Hz}$.

\subsubsection{DAC Two Wire Interface}

The laser diode forwards current is set by a reference voltage input on the laser switch chip. This reference voltage is controlled by an AD5311 DAC with a two wire interface (TWI). The Serial Data and Serial Clock lines of the TWI of the DAC are driven by the FPGA allowing the FPGA to control the laser diode current and therefore the intensity of the laser light. As discussed in Section 8.2.1 Laser Diode Current, at power-up the laser diodes must be gradually warmed up to prevent damage. This warm-up period is achieved by the FPGA gradually increasing the reference voltage produced by the DAC. The FPGA drives the TWI signals as single-ended LVTTL signals which are subsequently converted to LVDS signals to provide greater noise immunity in transmitting them across the generic I/O interface to the Image Capture board. An LVDS repeater on the Image Capture board boosts the strength of the TWI signals prior to transmitting them to the Laser Illumination board, via a SATA cable. The TWI signals are subsequently converted back to single-ended LVTTL signals and finally to TTL signals capable of interfacing with the DAC. A simple 8-bit counter was implemented in VHDL to configure the FPGA to produce modulating signals on the FPGA's TWI outputs. An oscilloscope was used to examine the TWI signal lines of the DAC on the Laser Illumination board. The FPGA-generated modulated signals were clearly received at the 
DAC indicating no problem with the communication channel between the FPGA and the DAC.

\subsection{Summary}

The FPGA board is capable of configuration through use of the JTAG interface. Active Parallel Configuration was not possible due to a design error resulting in the I/O lines to the Flash memory device being driven by two different I/O voltages. JTAG configuration requires the FPGA to be configured via a PC when first powered up. Both generic I/O interfaces operate correctly allowing communication between the FPGA and peripheral boards. Two of the four DDR2 SDRAM memory banks operate providing the FPGA with two banks of $8 \mathrm{MB}$ with data transfer speeds of $3.2 \mathrm{~Gb} / \mathrm{s}$. The remaining two DDR2 SDRAM banks cannot be accessed with the existing DDR2 SDRAM controller IP from Altera due to the restriction on placement of DQ and DQS lines. Implementation of a custom DDR2 SDRAM controller could allow access to these memory banks.

The communication lines between the FPGA and the Image Capture board have been tested and verified to transmit data signals with modulation frequencies up to $40 \mathrm{MHz}$. The LVDS repeaters on the Image Capture board correctly transmit modulation signals between the FPGA and the Laser Illumination board.

The Laser Illumination board is capable of driving the two banks of eight laser diodes at modulation frequencies of $40 \mathrm{MHz}$. The diode current protection circuit prevents modulation signals less than $700 \mathrm{~Hz}$ from driving the laser diodes, preventing the diodes from operating in a continuous wave mode with forward currents that would damage the diodes. The TWI communication channel between the FPGA and the DAC on the Laser Illumination board has been verified to operate at signal frequencies of $10 \mathrm{MHz}$.

The communication channels between the FPGA and the External interface board have been verified to operate at signal frequencies of $10 \mathrm{MHz}$.

The complete full-field image ranging system is in a stable state to allow porting of the Stratix III FPGA configuration code implemented on the prototype ranging system to the Cyclone III FPGA configuration required. The FPGA's onboard SRAM and two banks of DDR2 SDRAM provide sufficient memory size for image frames from the currently available PMD 19K-2 Sensor Array that will be utilised in the system for the foreseeable future. 


\section{Chapter 11 Conclusions}

This chapter presents a review of the sensor network and full-field image ranger implemented in this project. Each extrospective system is summarised including project outcomes. Possible future work on both systems is then discussed.

\subsection{Project Review}

\subsubsection{Sensor Network}

An intelligent network of distance sensors has been developed to provide an integrated approach to interface such sensors to robotic platforms for Victoria University's fleet of robots.

The topology and architecture of the network has been defined to allow distributed networks of multiple sensor technologies to be controlled independently of a robotic platform's control computer. Such a system allows the installation of sensors to existing robotic platforms, the expansion of networks with extra sensors and the addition of new sensor technology.

The sensor network follows a star / bus hybrid topology with the central hub acting as the network master and communication interface to a control computer. Two sub-network interfaces on the central hub connect two bussed networks of distributed microcontrollers. Each sub-network interface is capable of supporting 31 devices allowing the network master to support networks of up to 62 devices. Each microcontroller interfaces to a sensor, managing its control and sampling procedure. The microcontroller is also responsible for managing communication from the network master.

A network protocol based on the Open Systems Interconnectivity model has been developed to manage network communication. Three basic layers define the protocol on network nodes: Physical, DataLink, and Network layers. A fourth Sensor Network layer is implemented on the network master providing the master control over the entire network. The network protocol follows a request - response relationship where sensor nodes communicate on the network only in response to a request from the network master.

The Physical layer manages the differential line RS-485 communication link between the master and the nodes which form a multi-drop network on each sub-network interface. 
The DataLink layer handles the routing and transfer of data between nodes across the network. It utilises a Media Layer Protocol that provides a managed access system to the RS485 half duplex network. The Media Layer Protocol controls data framing and packet transfer to control the flow of network traffic. The protocol provides basic error checking to validate data integrity upon reception at the destination node.

The Network layer operates as the host layer on sensor nodes. The host layer handles tasks and commands issued to a sensor node by the network master. The layer utilises the Host Layer Protocol to control how sensor nodes logically interact on the network. The Host Later Protocol defines what tasks and configuration settings may be allocated to sensors and how they are sent. The protocol defines a standard communication interface with a sensor irrespective of the type of sensor attached.

Finally the Sensor Network layer defines the services available to the master to manage the network. It manages node discovery at initialisation to determine a network definition defining what sensor nodes are connected and it maintains information on every attached node including copies of the last sensor data received from every attached node. It is also responsible for mapping the logical network addresses, utilised by itself and the control computer, to the physical addresses of the nodes on both sub-networks.

A command interface protocol over a USB interface has been developed to provide the control computer with functionality to query the master for sensor data and to issue instructions to the master on how to manage and configure the network. The command interface protocol follows a request - response relationship where the master node will communicate over the USB interface only in response to a request from the control computer. The command interface uses packets and packet framing to ensure delivery of data. Error checking is enabled to validate data integrity upon packet reception.

Two types of sensor nodes have been designed to interface with the network: an ultrasonic sensor node that is capable of measuring distances between $0.5-9.25 \mathrm{~m}$ and an infrared position sensitive detector node capable of measuring distances either between $0.2-1.5 \mathrm{~m}$ or $0.4-3 \mathrm{~m}$.

A test sensor network to mount on the robotic platform MARVIN has been designed and constructed. The test sensor network consists of fourteen position sensitive detectors: ten GP2Y3A003K0F and four GP2Y3A002K0F. The network provides a $360^{\circ}$ field of view in a 
single plane that is positioned $950 \mathrm{~mm}$ horizontally above the ground when mounted to MARVIN. The network provides an active sensing area $6000 \mathrm{~mm}$ in diameter with a dead zone of $1000 \mathrm{~mm}$ in diameter centred on the network mounting point. Within a $3800 \mathrm{~mm}$ diameter the sensor network can determine distances with a range error of less than $5 \%$.

A software library of functions has been developed to allow user applications to be written on the control computer to interface to, and control, the sensor network. A graphical user interface has been built on top of the software library to visually present and log sensor information from the test sensor network.

\subsubsection{Full-Field Image Ranger}

A full-field image ranger system capable of being mounting on mobile robotic platforms has been developed. The mobile ranging system is based on a prototype bench top ranging system developed by researchers at Victoria University of Wellington and the University of Waikato. The system can utilise both homodyne and heterodyne indirect time of flight (ToF) schemes to encode distance information into the phase of a modulated light source.

System supervision is handled by an Altera Cyclone III FPGA primarily to utilise its reconfigurable phase lock loops to facilitate high frequency signal generation required for the heterodyne and homodyne ranging schemes. The ranger has been designed as a modular system to facilitate future hardware upgrades to the image sensor array, allow various optical lenses to be utilised and changes made to the illumination source. The modular design also allows the FPGA hardware to be used for other research and teaching projects.

The system has been separated into four sub-systems:

1. System Supervision - responsible for modulation signal generation, image processing and communication with peripheral devices such as a PC or VGA monitor.

2. External interface - responsible for providing the hardware interfaces to connect to an external PC via Ethernet or USB and to display image frames on a VGA monitor.

3. Image Capture - responsible for interfacing the image sensor array to the FPGA.

4. Illumination - responsible for providing the active modulated illumination of a scene to be range imaged.

Each sub-system has been developed on a separate circuit board. The sub-system circuit boards are stacked together to provide a compact system with a footprint of 
$120 \times 200 \times 120 \mathrm{~mm}$, approximately $30 \%$ the size of the prototype system, as presented in Figure 11.1.

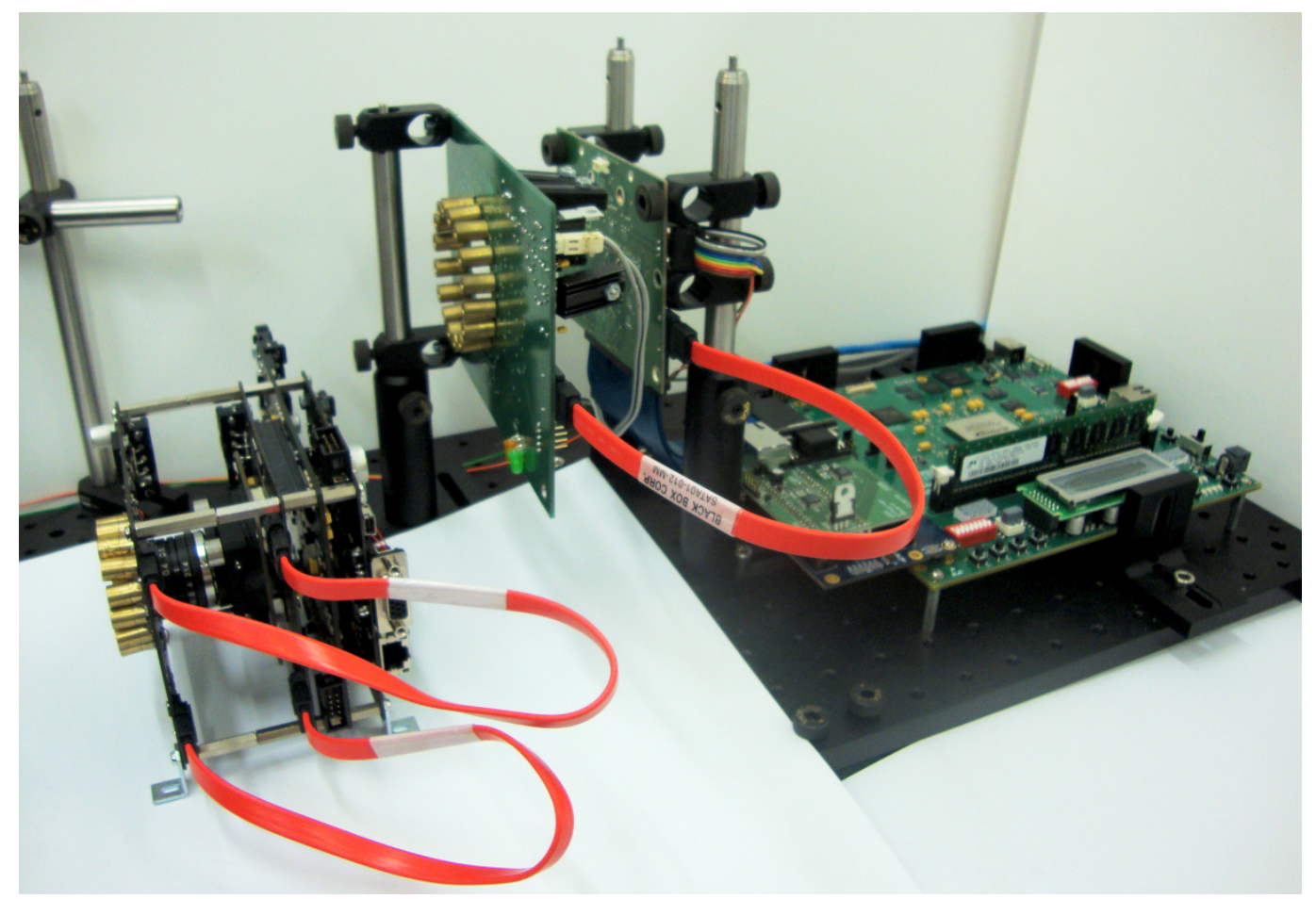

Figure 11.1 Size Comparison between the Mobile and Prototype Full-Field Image Ranger Systems

The system requires a single unregulated $80 \mathrm{~W}$ power supply providing a DC voltage between $14-20 \mathrm{~V}$ which is easily provided by the two flooded lead acid batteries provided on most of VUW's robotic vehicles. A system bus provides the unregulated power to each circuit board to supply the onboard voltage regulators. High current draw voltage rails have been powered from switching regulators to improve efficiency.

The FPGA supervisory board houses a Cyclone III EP3C40 FPGA, four external banks of DDR2 SDRAM and two generic I/O interfaces providing communication interfaces with the other sub-systems. FPGA configuration is possible via JTAG through the use of an Altera USB blaster programmer.

The External interface board provides an Ethernet interface to an external computer allowing the transfer of image frames from the FPGA to an external PC for long term storage and analysis. A USB interface is also provided for frame transfer and system configuration by an external PC. A VGA interface allows raw and processed image frames to be displayed in real time on a VGA monitor. The board also provides the input, power supply filtering and 
protection for the unregulated power bus. The board communicates with the FPGA via a generic I/O interface.

The Image Capture board provides an interface to connect the image sensor circuit board from the prototype system. The image sensor array is $160 \times 120$ pixels that are grouped into four independently modulated blocks of $40 \times 120$ pixels. The Image Capture board provides the digitisation of the analogue video streams from the image sensor. The board communicates with the FPGA via a generic I/O interface.

The Laser Illumination board provides sixteen $130 \mathrm{~mW} 660 \mathrm{~nm}$ laser diodes arranged into two independent banks of eight diodes each. Each bank can be modulated independently via signals from the FPGA. An onboard DAC, controlled by the FPGA, provides a slow ramp of the lasers' intensity to ensure an adequate warm up time occurs to prevent damage to the diodes. As the laser diodes are operated at their rated pulse currents, a protection circuit has been designed to prevent them from operating in continuous wave mode. The lasers can be selected to operate at full power or half power by a user set switch onboard the circuit board.

\subsection{Future Work}

\subsubsection{Cyclone III FPGA Code}

The development of a full-field image ranger capable of mounting onto a mobile robotic platform has focussed primarily on the redesign of the hardware setup of the prototype system to reduce the system footprint and provide flexibility in upgrading the image sensor as higher resolution sensor arrays become available. The design decision to move to the Altera Cyclone III FPGA family from the Stratix III family requires the FPGA code on the Stratix II based prototype system to be ported to run on the Cyclone III based mobile system. The selection of the Cyclone III EP3C40 FPGA has resulted in a reduction of built-in SRAM compared with the Stratix III EP3SL150 FPGA. This requires the Ethernet communication code to be rewritten to access an external DDR2 SDRAM memory bank to provide the necessary memory space to buffer image frames.

FPGA code must be written to provide a two-wire communication interface between the FPGA and the two DACs onboard the Laser Illumination board. Code must also be developed to allow the FPGA to control the laser diode's warm-up routine via this two-wire 
interface, as previously this was managed by a microcontroller onboard the laser illumination board.

\subsubsection{FPGA Configuration}

Due to a design fault, FPGA configuration is currently only possible via an Altera JTAG USB blaster cable. This requires the configuration file to be uploaded to the FPGA from an external computer each time the system is powered. A redesign of the FPGA board is desirable to correct the design fault and allow Active Parallel configuration from a Flash device. As mentioned in Section 9.5.1.2 FPGA Configuration and Programming, this would require a significant change to which I/O banks are allocated to the external DDR2 SDRAM memory banks and the generic I/O interfaces to ensure the I/O banks necessary for Active Parallel configuration are powered from a voltage compatible with the Flash device.

\subsubsection{DDR2 SDRAM}

Currently Altera's DDR2 SDRAM external interface mega-function allows only two of the four DDR2 SDRAM memory banks to be accessed on the FPGA board due to restrictions in FPGA pin allocation. Development of a custom DDR2 SDRAM memory interface would allow the other two memory banks to be accessed. The custom memory interface would require careful timing and use of delay lock loops for the DQ and DQS groups of the Ethernet and NIOS program memory banks to prevent skew between these lines.

A redesign of the FPGA board to allow Active Parallel configuration would also require the four DDR2 SDRAM memory banks to be interfaced to different I/O banks of the FPGA. The Ethernet Buffer and NIOS program memory banks could be redesigned at this stage to ensure their DQ and DQS lines are attached to FPGA pins specified by Altera to allow Altera's DDR2 SDRAM external memory interface mega-function to be used to access them. Both memory banks feature a 16-bit wide data bus which should be connected to the FPGA as two groups of eight DQ lines, with attention paid to ensure the correct DQS signal is associated with its appropriate DQ group.

\subsubsection{Thermal Management}

The high current pin drivers of the Image Capture board consume significant amounts of power when operated at high frequencies $(10-50 \mathrm{MHz})$. At $40 \mathrm{MHz}$ each driver consumes 
approximately $500 \mathrm{~mW}$ with no load attached. For reliable operation the drivers must be kept below $85^{\circ} \mathrm{C}$. Currently the temperature of the pin drivers, when driven at $40 \mathrm{MHz}$, exceeds this temperature after approximately three minutes. Placement of the pin drivers on the board prevents suitable passive cooling through the use of heat-sinks due to the close proximity of other circuit boards when stacked together. A fan has been incorporated to provide active cooling to maintain a temperature below $85^{\circ} \mathrm{C}$.

Redesign of the Image Capture board for a new image sensor should also factor in the thermal characteristics of these driver chips at high frequencies to provide a suitable cooling scheme. Passive cooling is more desirable than active cooling as it would reduce the overall power consumption of the system, extending the operating time of the system on a mobile robotic platform.

\subsection{Summary}

The two systems developed mark significant progress in the development of extrospective systems capable of being mounted on Victoria's fleet of robotic platforms. The outcome of the intelligent sensor network extends beyond the development of a medium range $360^{\circ}$ field of view distance sensing network for MARVIN; the development of the network architecture and communication protocols provides a model for future implementation of sensors on VUW's robotic platforms. The master node at the heart of the hybrid network topology provides the ability for sensor networks to be hardware independent of a control computer, facilitating computer upgrades with little or no modifications required to the network.

Development of the mobile ranging system has provided a compact, streamlined hardware platform for current research projects to further examine the capability and performance of heterodyne and homodyne time-of-flight schemes on mobile robotic platforms and now permit these ranging systems to be integrated into a robotic platform's hardware for real world evaluation.

One paper (McClymont, J., Carnegie, D.A., "A Hardware Design Philosophy for an Autonomous Robotic System", Electronics New Zealand Conference, 2008) has been published and presented at national conference and was awarded Best Novice Paper. Two papers (McClymont, J., Carnegie, D.A., “The Development of an Intelligent Sensor Network for Mobile Robotic Platforms", $6^{\text {th }}$ International Symposium on Electronic Design, Test and Applications, 2011) (McClymont, J., Carnegie, D.A., Jongenelen, A., Drayton, B., “The 
Development of a Full-Field Image Ranger System for Mobile Robotic Platforms", $6^{\text {th }}$ International Symposium on Electronic Design, Test and Applications, 2011) have been written and are awaiting acceptance at an international conference as a result of the work completed during this project.

Figures 11.2 and 11.3 present the completed extrospective systems implemented in this project.

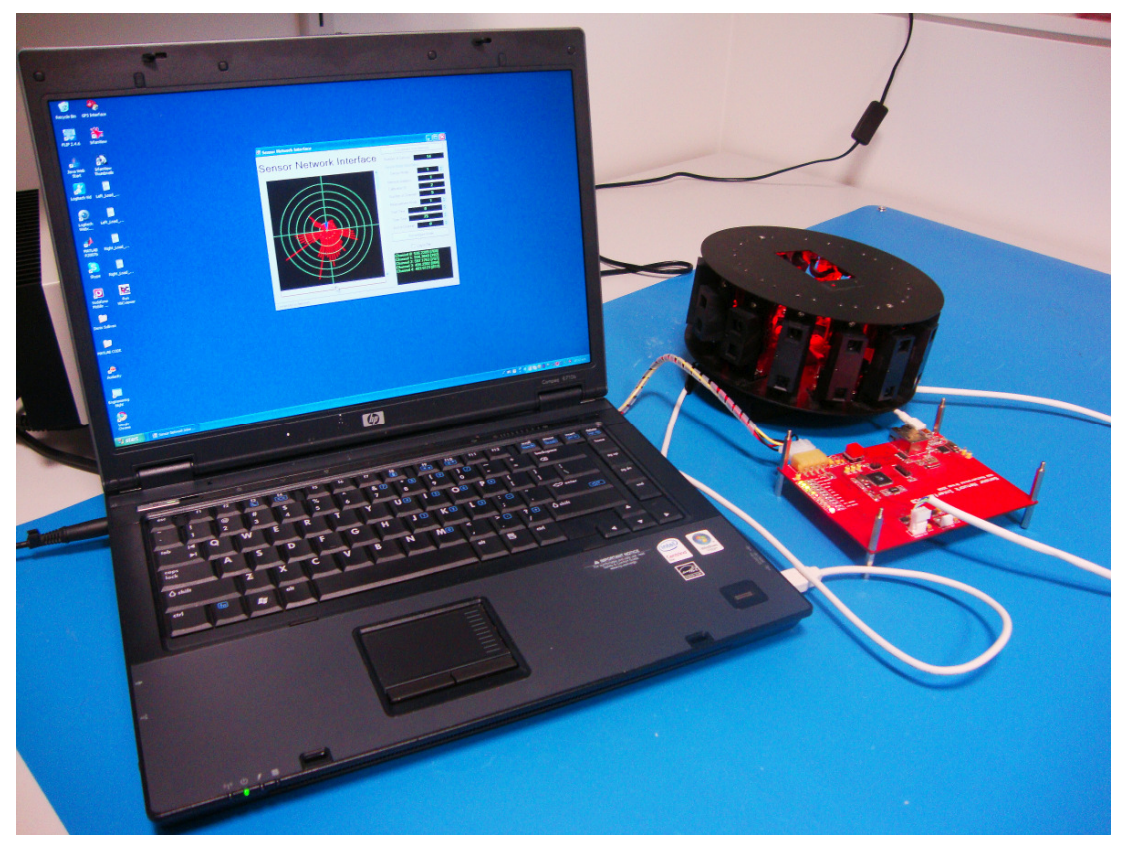

Figure 11.2 Completed Intelligent Sensor Network for Mobile Robotic Platforms

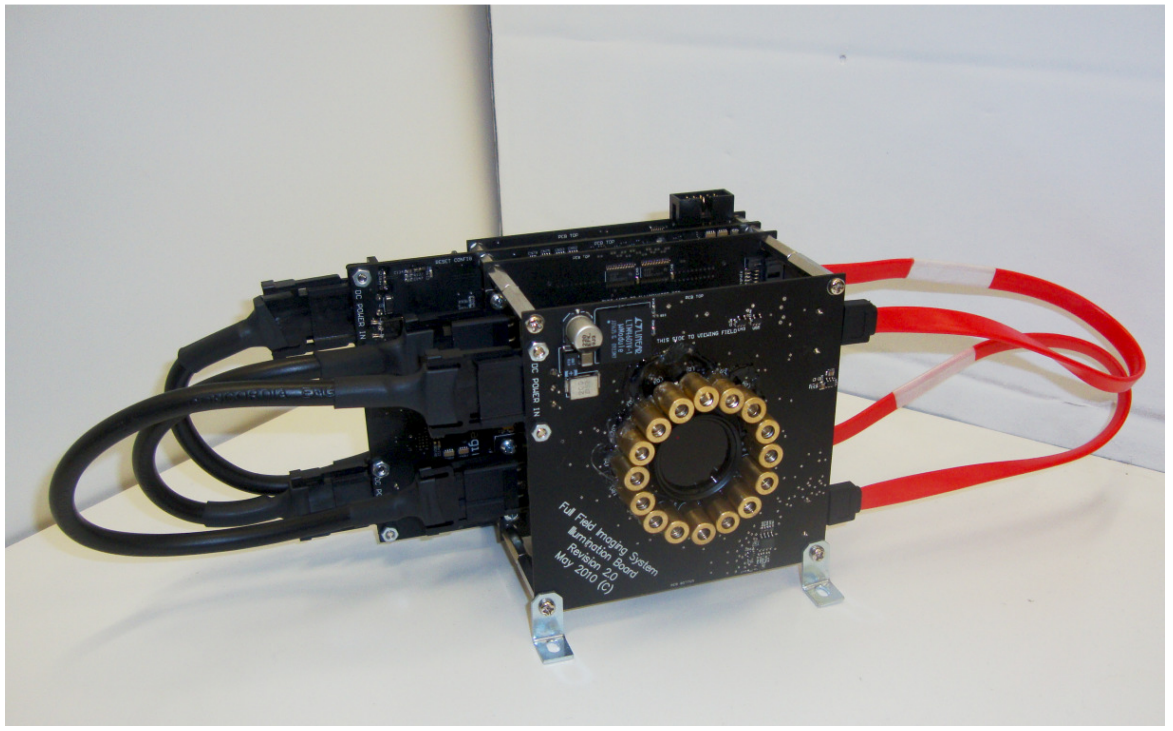

Figure 11.3 Completed Full-Field Image Ranger System for Mobile Robotic Platforms 


\section{Appendix A: Sensor Network Hardware}

\section{A.1 Sensor Network Interface MCU Schematic}
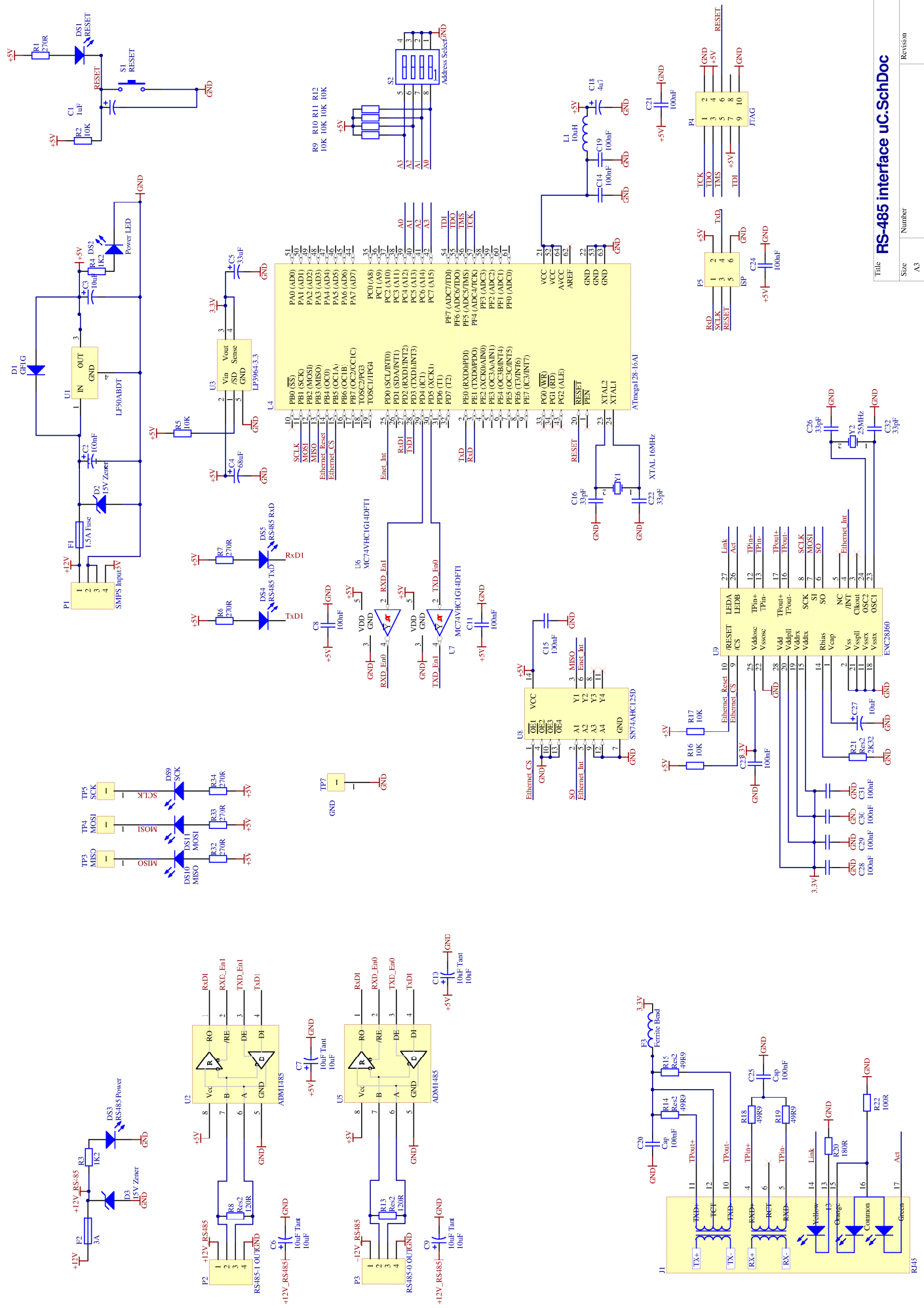


\section{A.2 Sensor Network Interface USB Schematic}
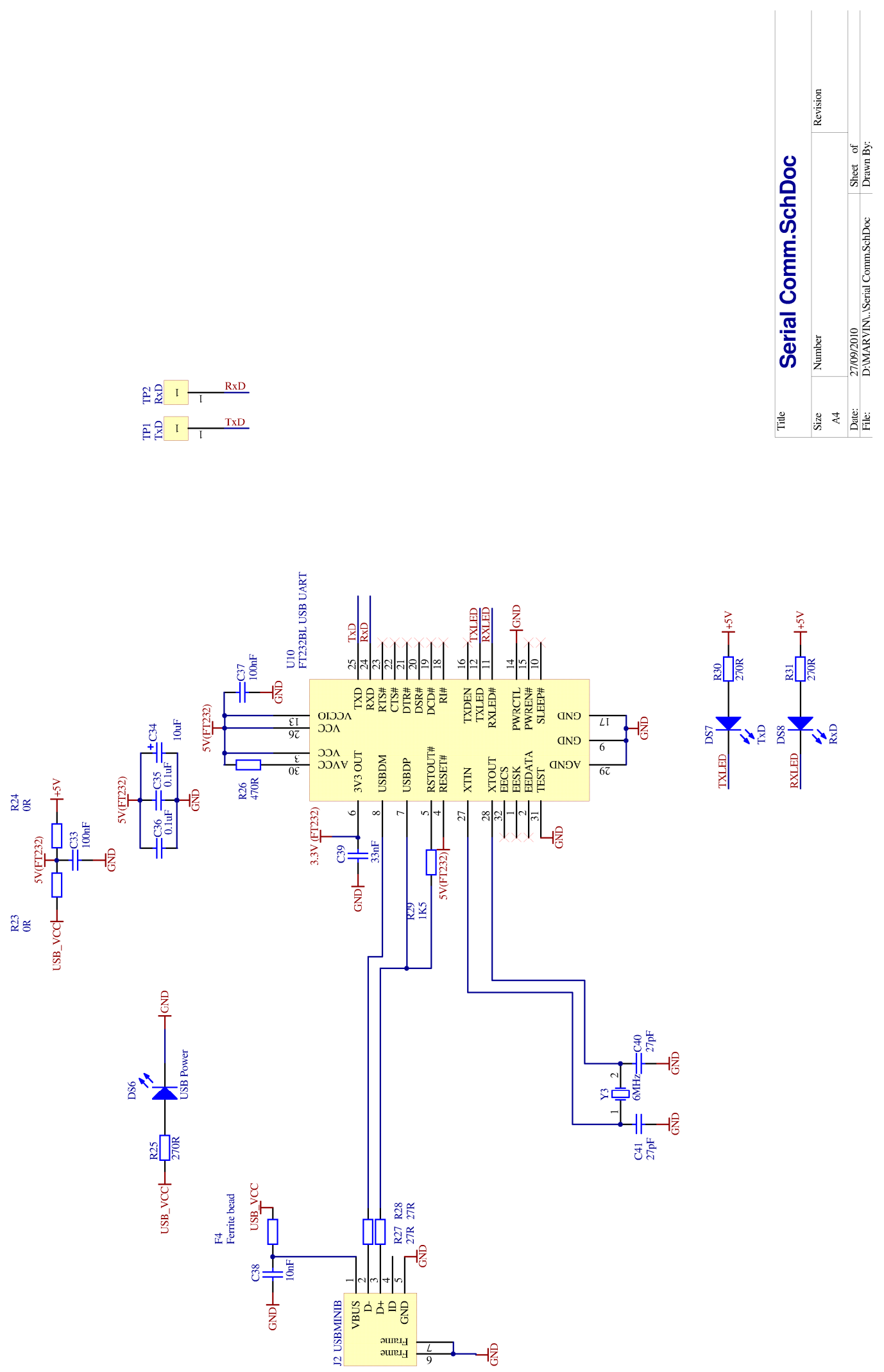


\section{A.3 Infirared Ranging Node Schematic}
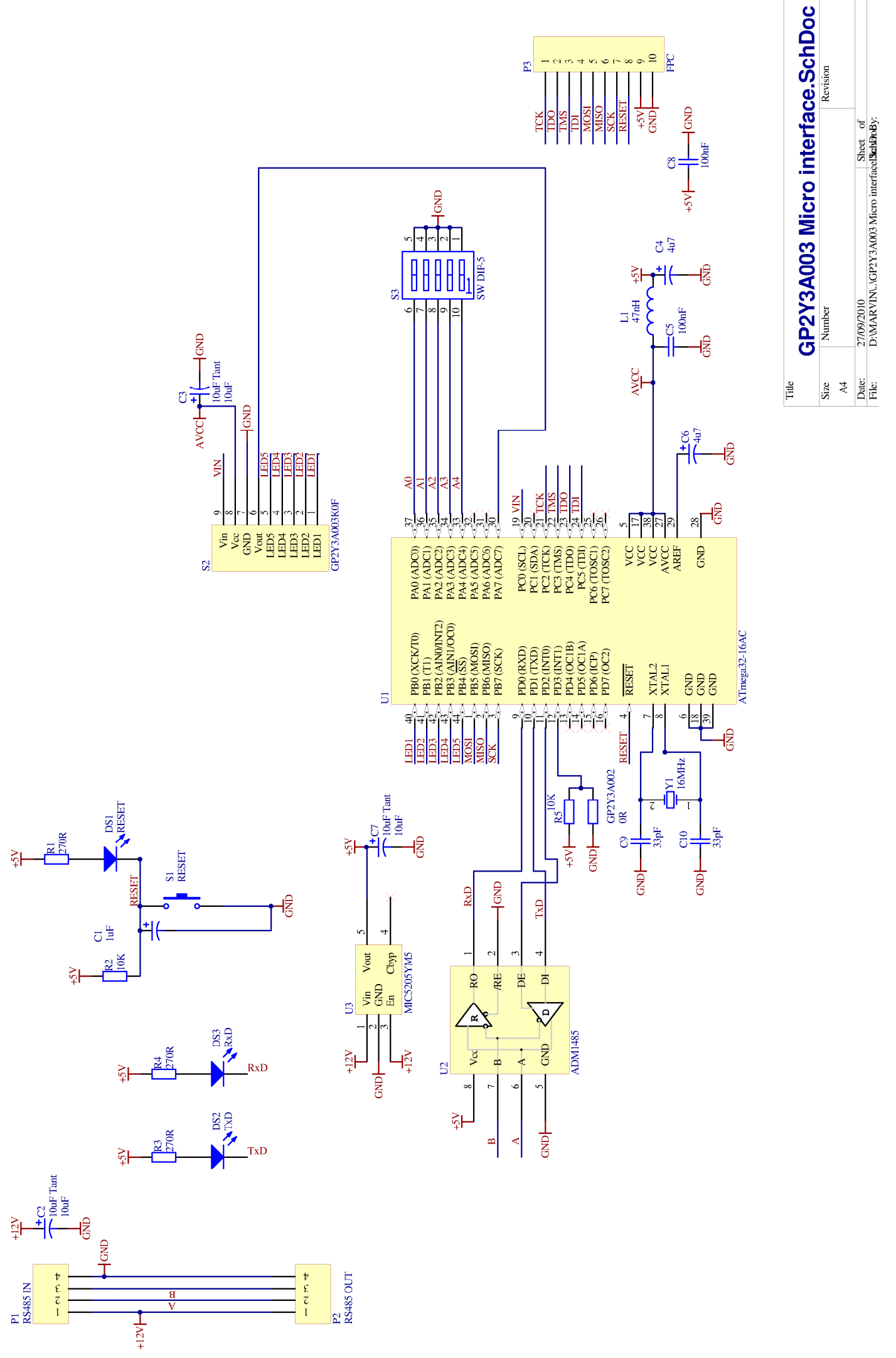


\section{A.4 Ultrasonic Ranging Node Schematic}
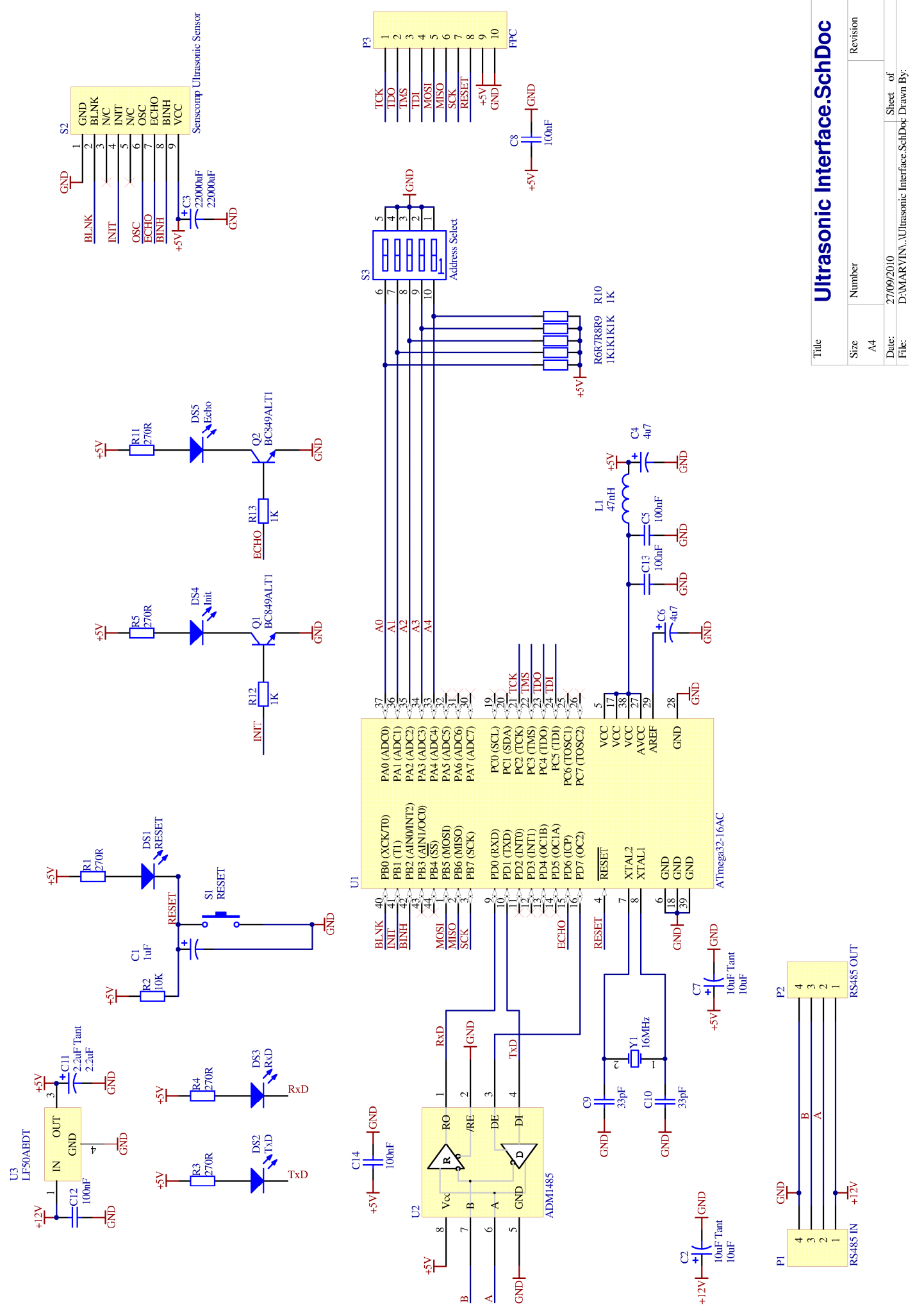

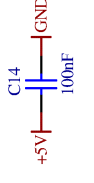




\title{
Appendix B: Full-Field Image Ranger
}

\author{
B.1 FPGA Board - Power 1 Schematic
}
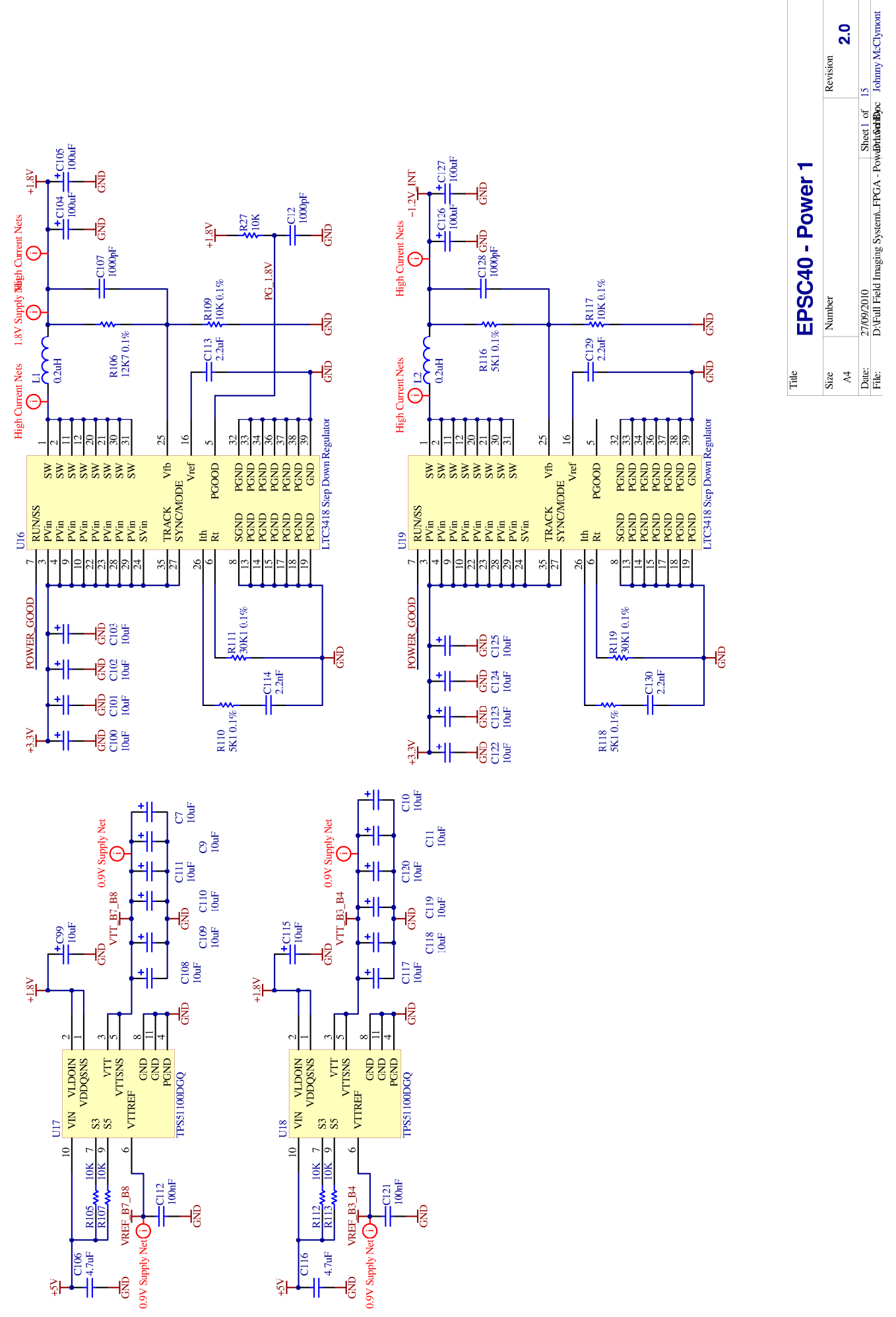


\section{B.2 FPGA Board - Power 2 Schematic}
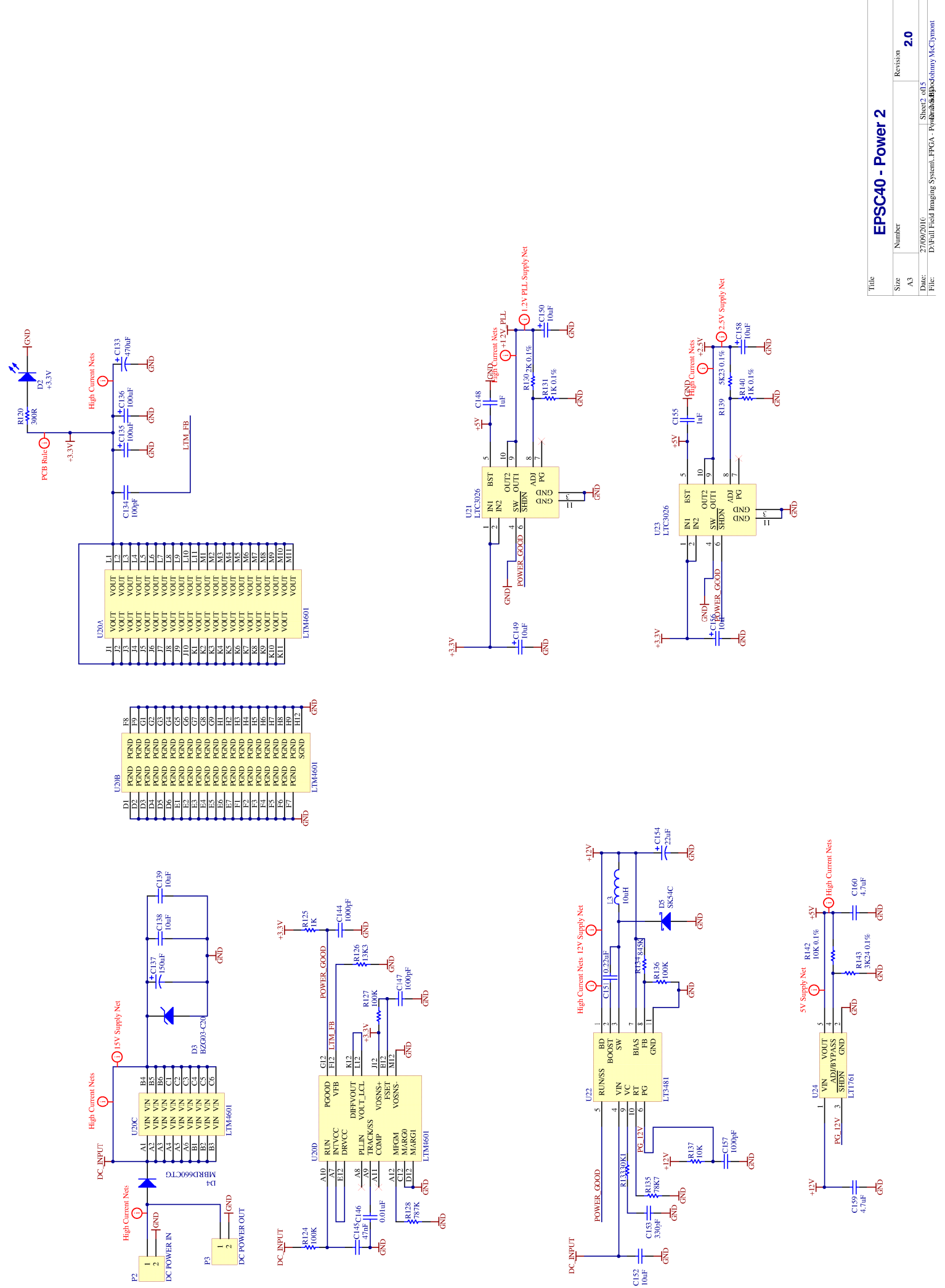


\section{B.3 FPGA Board - FPGA Power Bank Schematic}
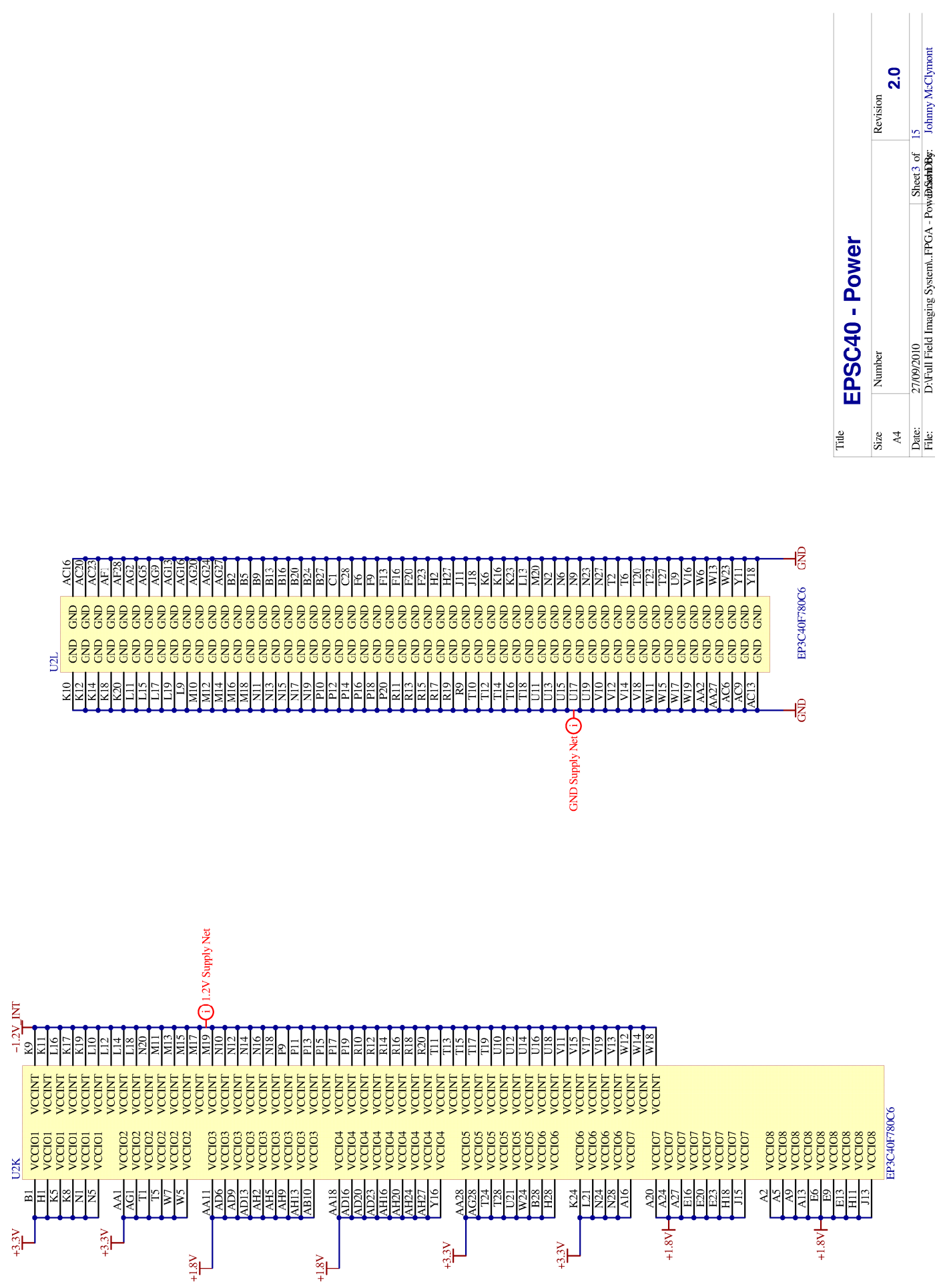


\section{B.4 FPGA Board - FPGA Configuration Schematic}
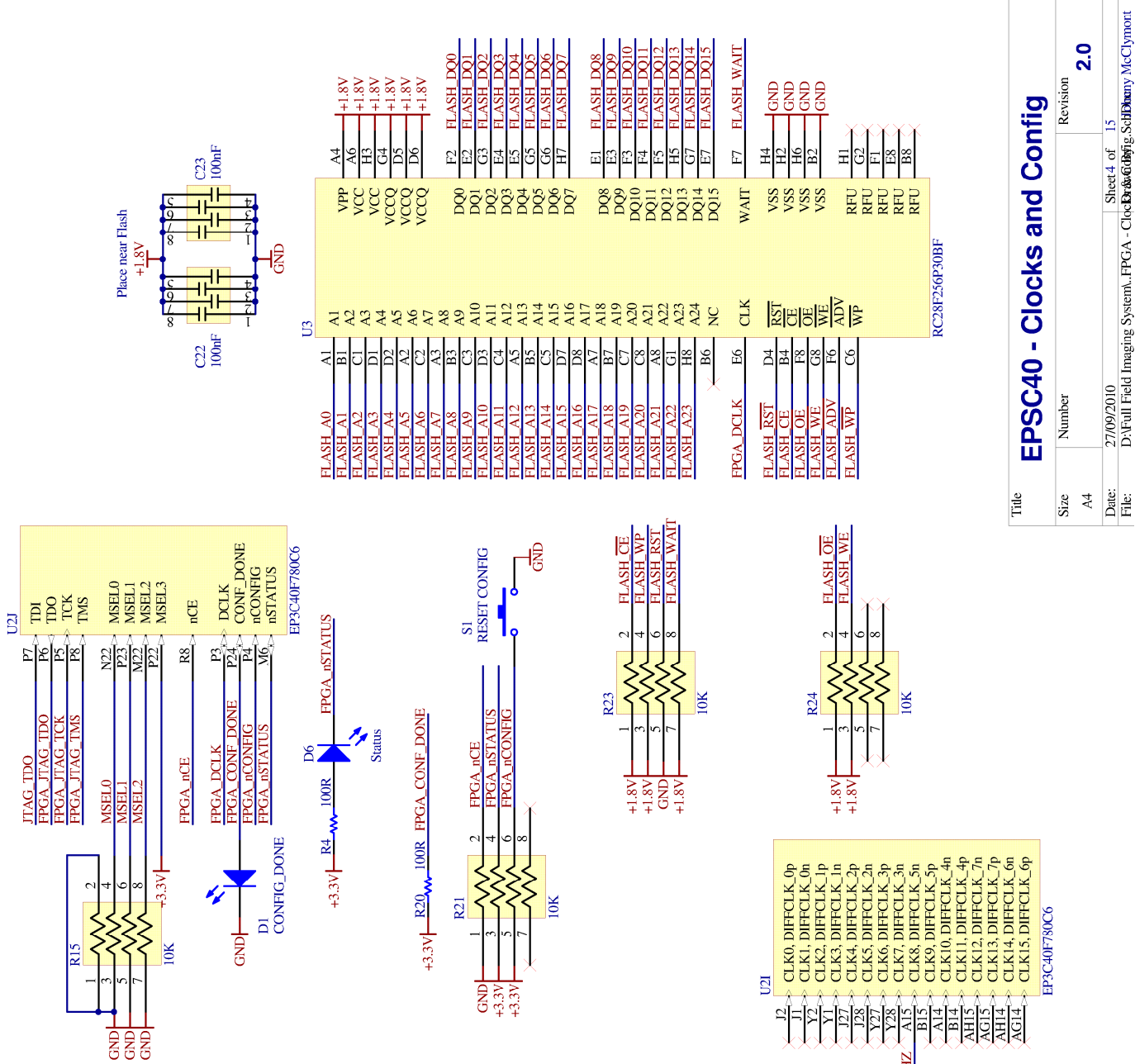

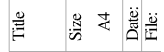
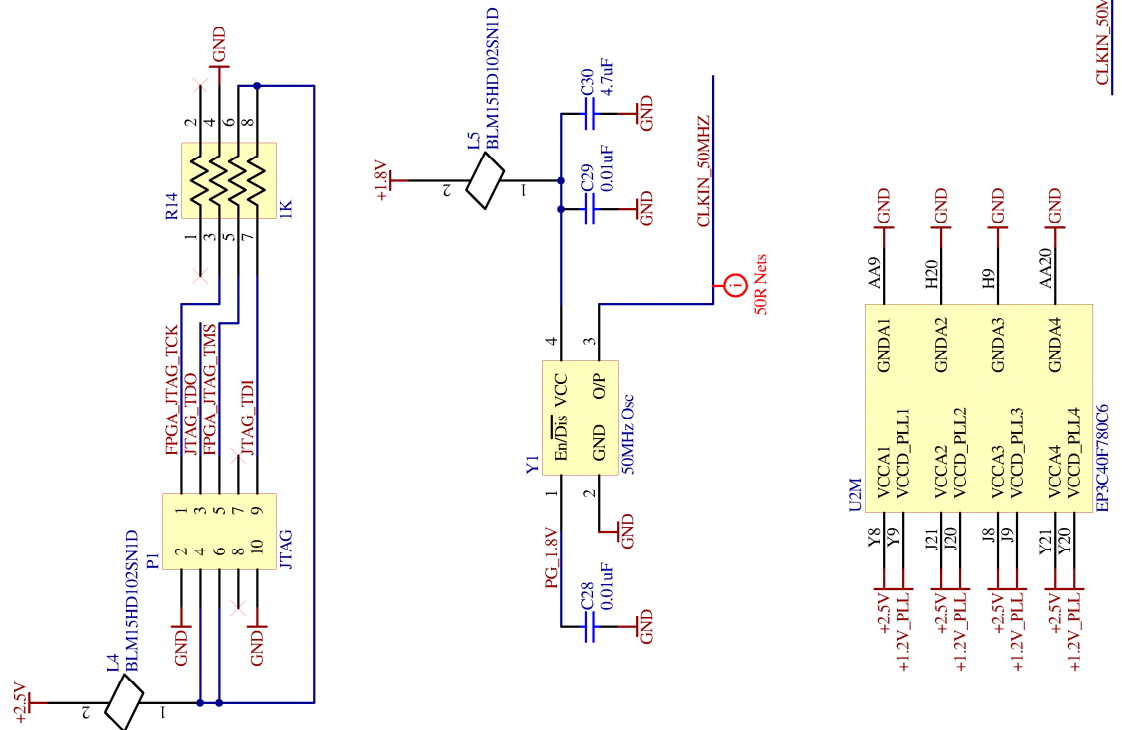


\section{B.5 FPGA Board - FPGA Bank 1 \& 2 Schematic}
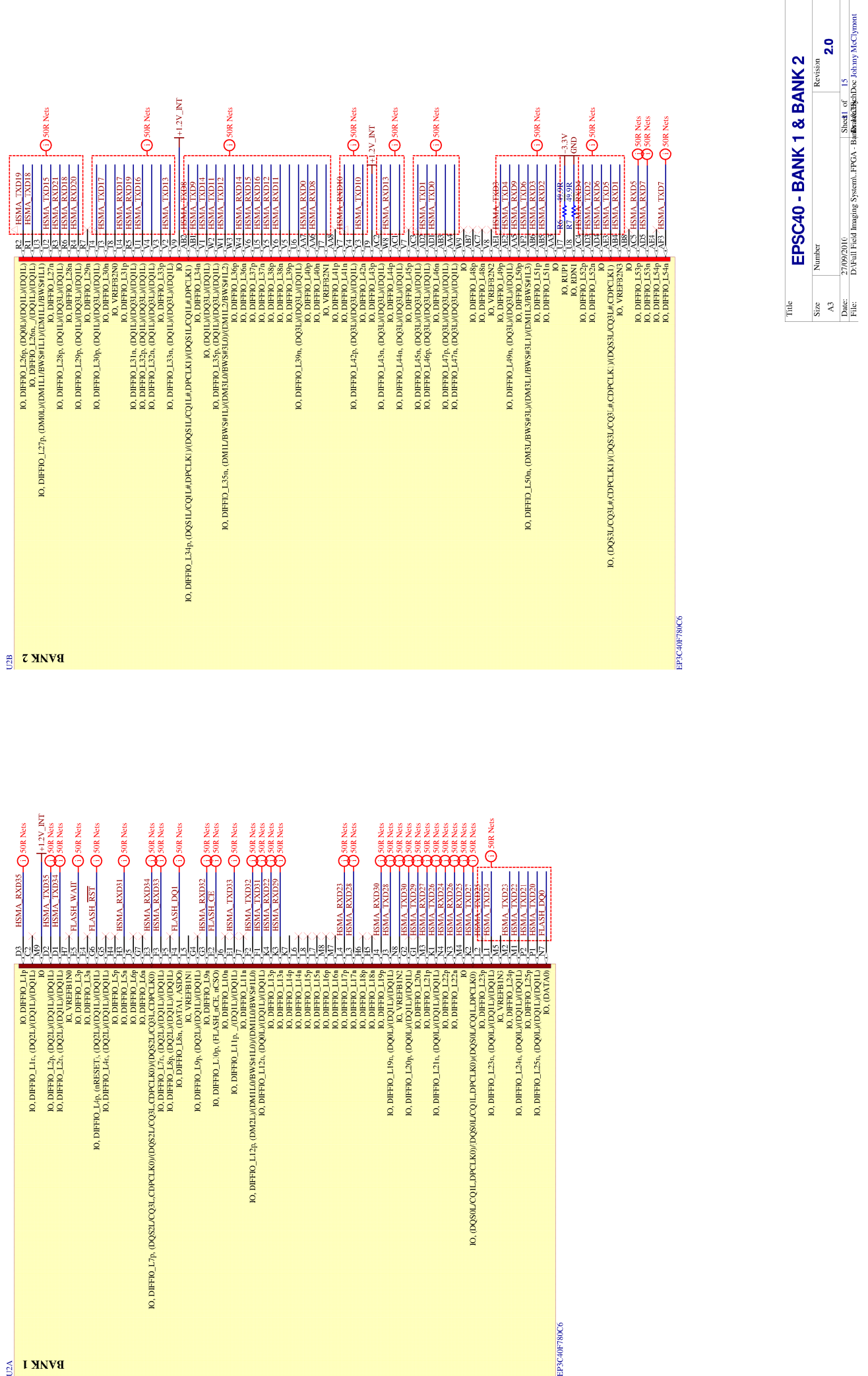


\section{B.6 FPGA Board - FPGA Bank 3 \& 4 Schematic}
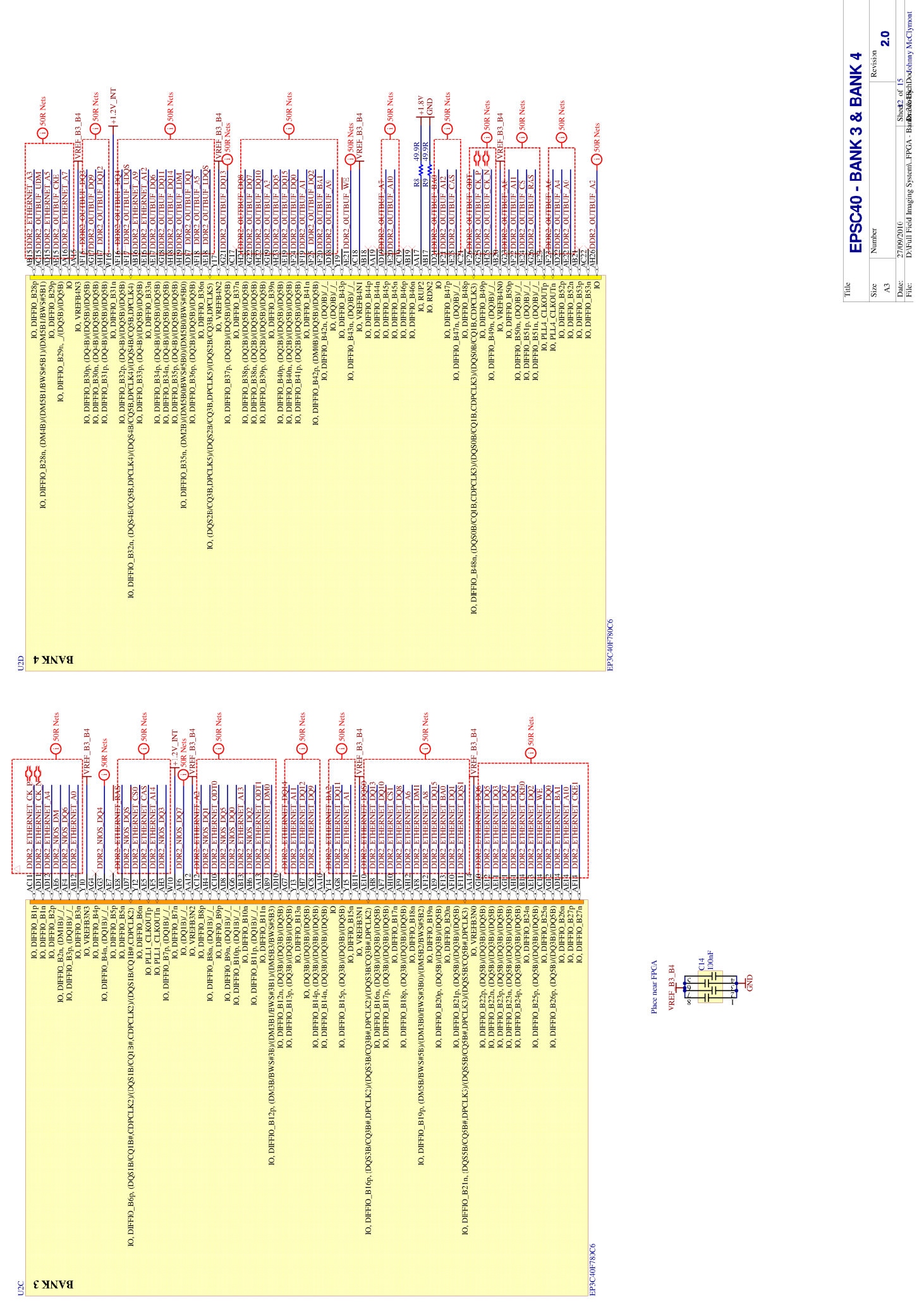


\section{B.7 FPGA Board - FPGA Bank 5 \& 6 Schematic}
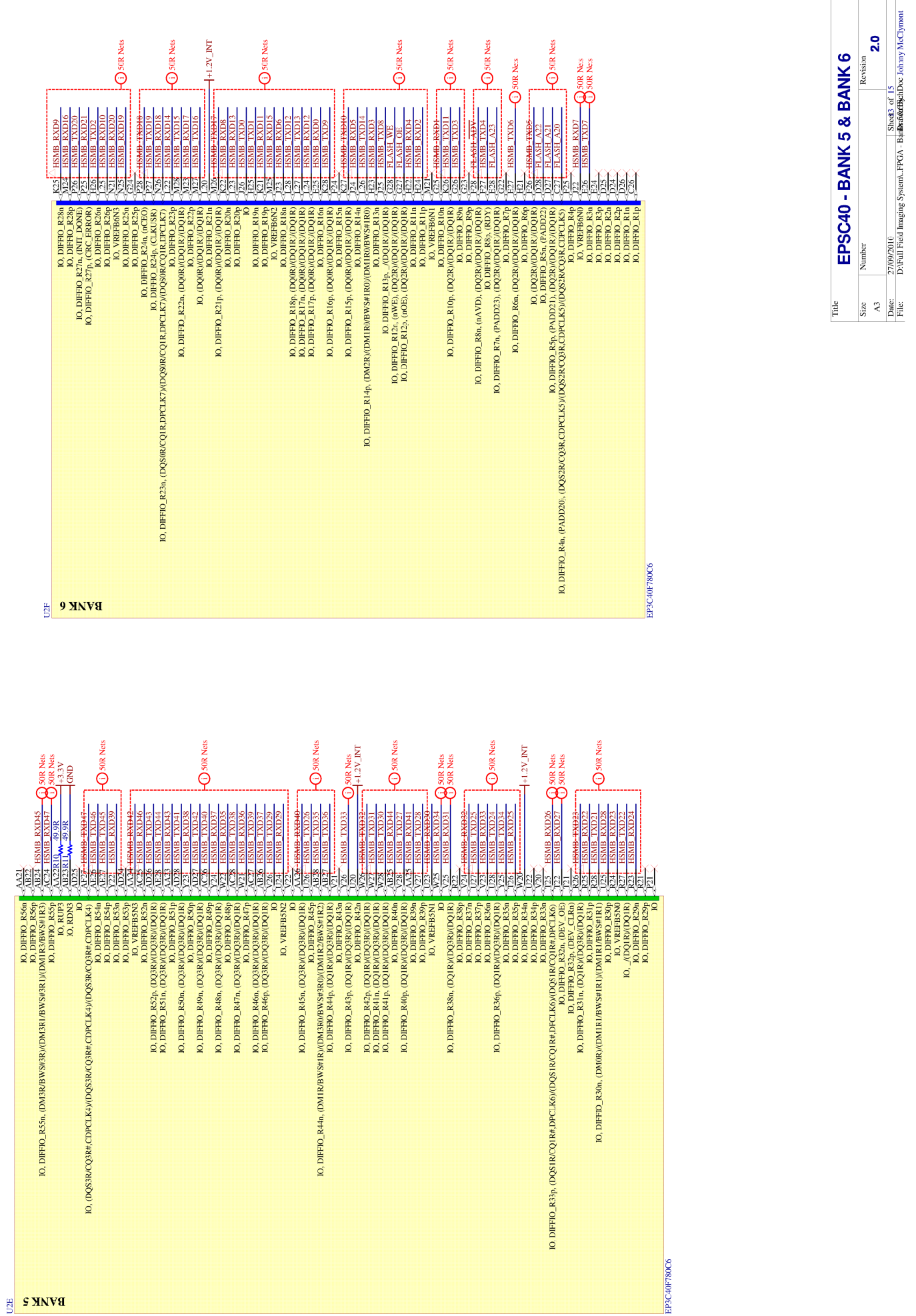


\section{B.8 FPGA Board - FPGA Bank 7 \& 8 Schematic}
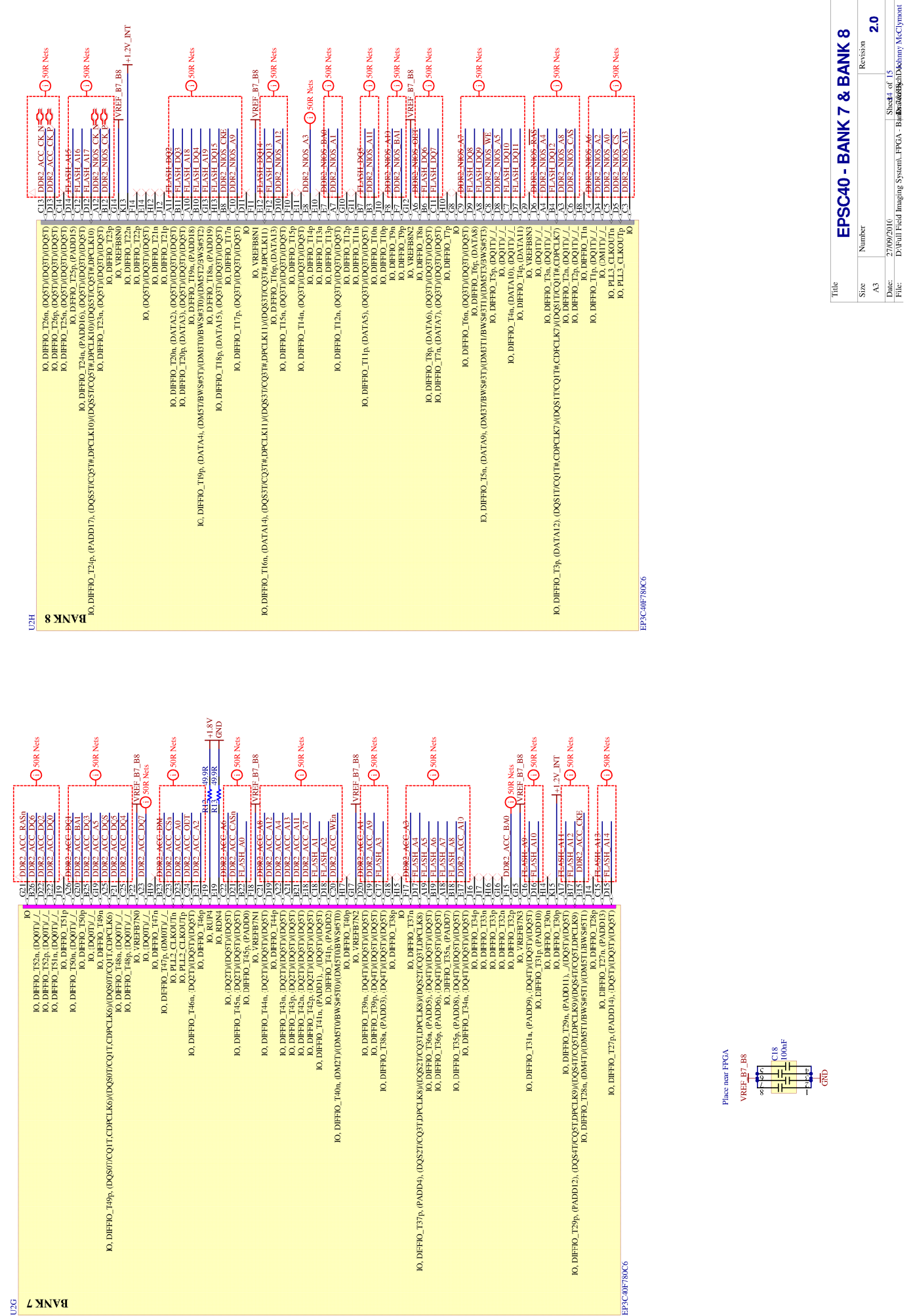


\section{B.9 FPGA Board - Accumulator SDRAM Schematic}
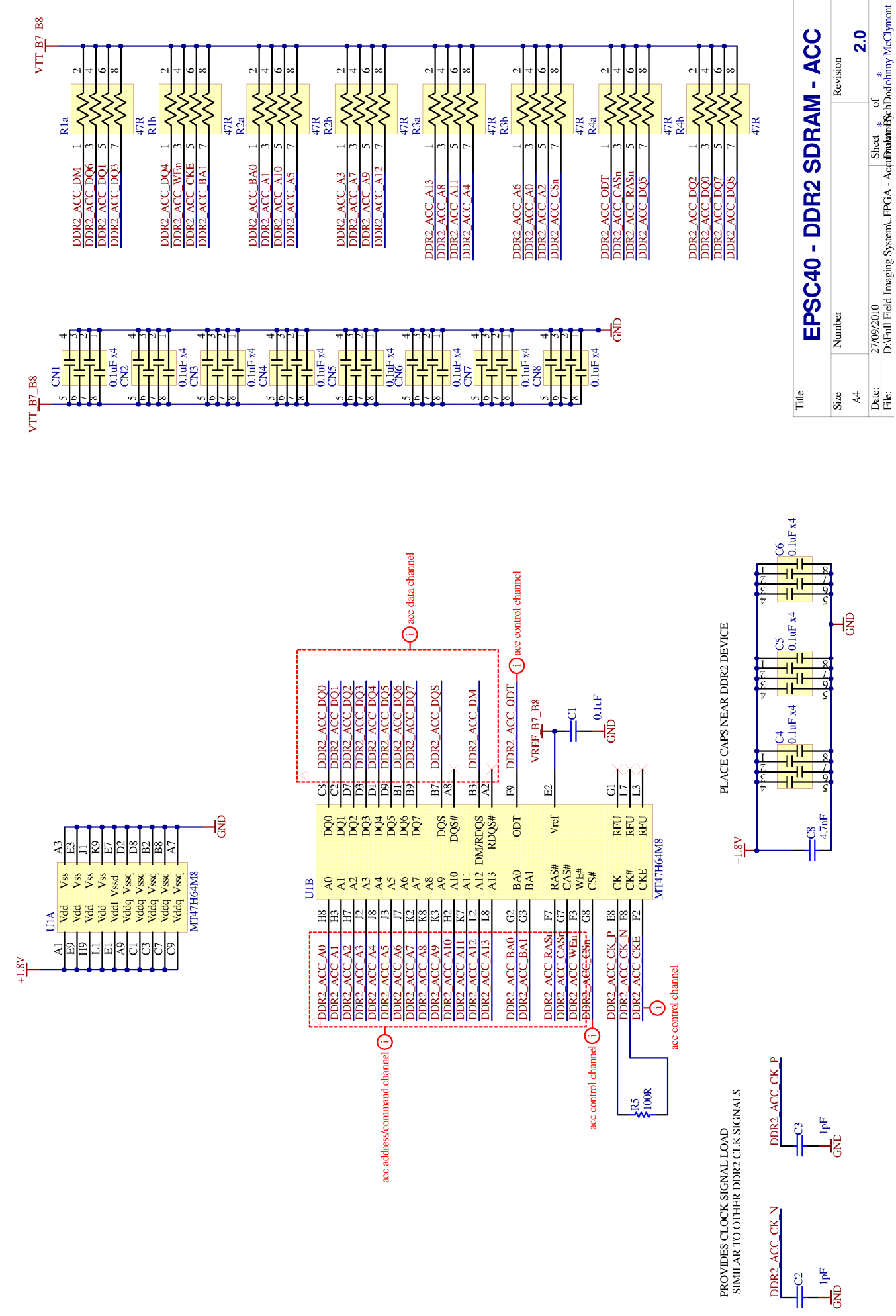


\section{B.10 FPGA Board - Output Buffer SDRAM Schematic}
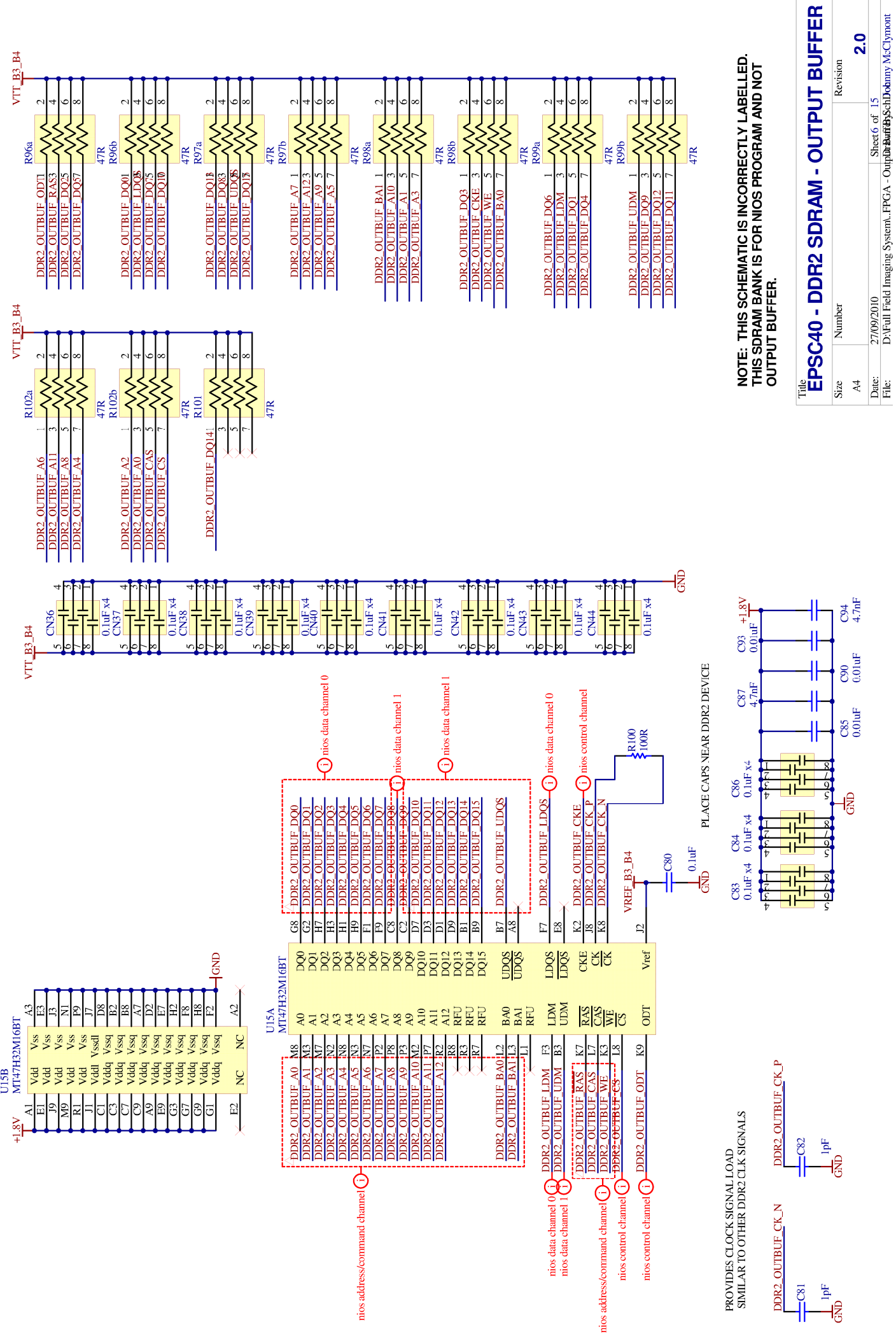


\section{B.11 FPGA Board - NIOS SDRAM Schematic}
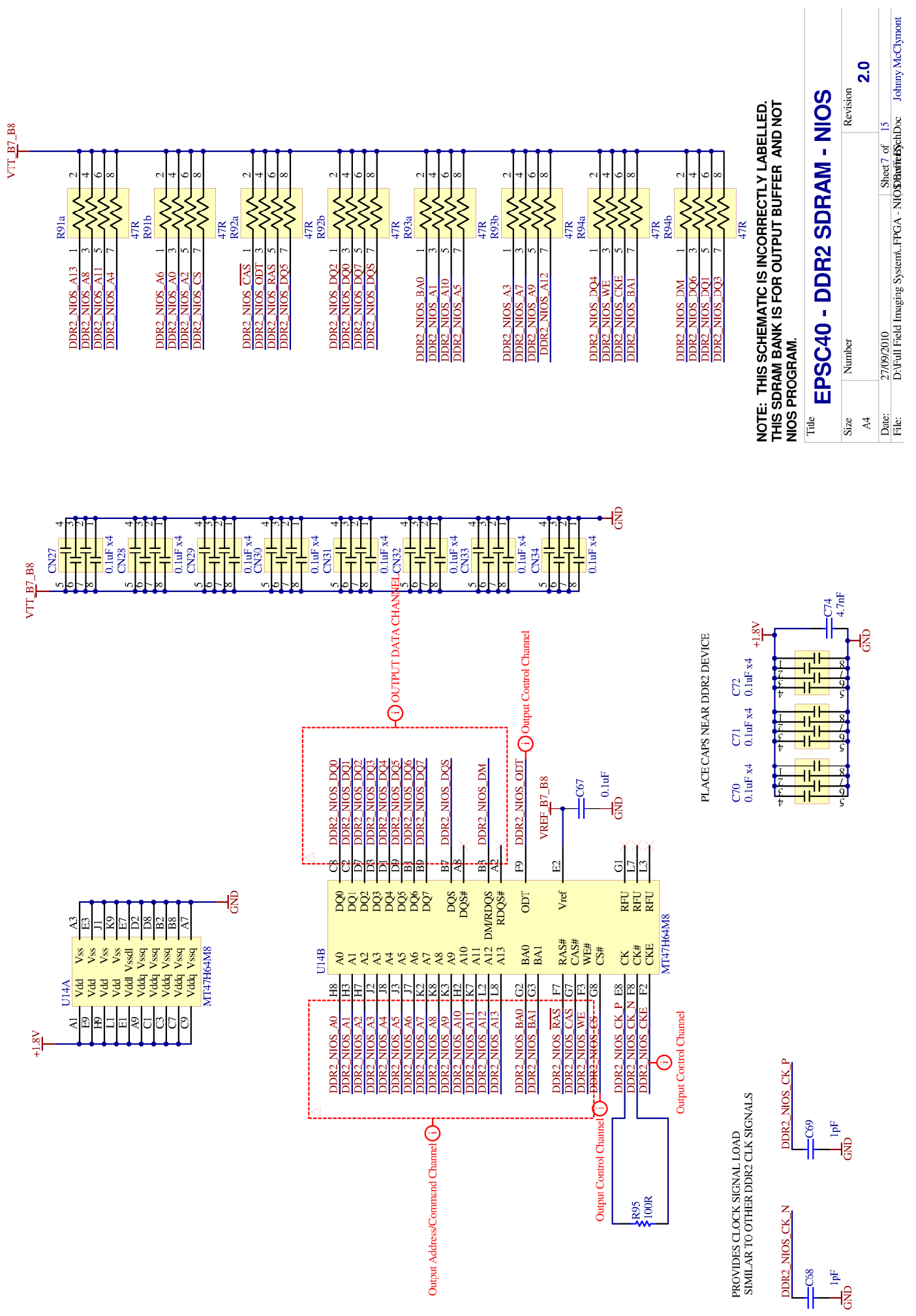


\section{B.12 FPGA Board - Ethernet Buffer SDRAM Schematic}
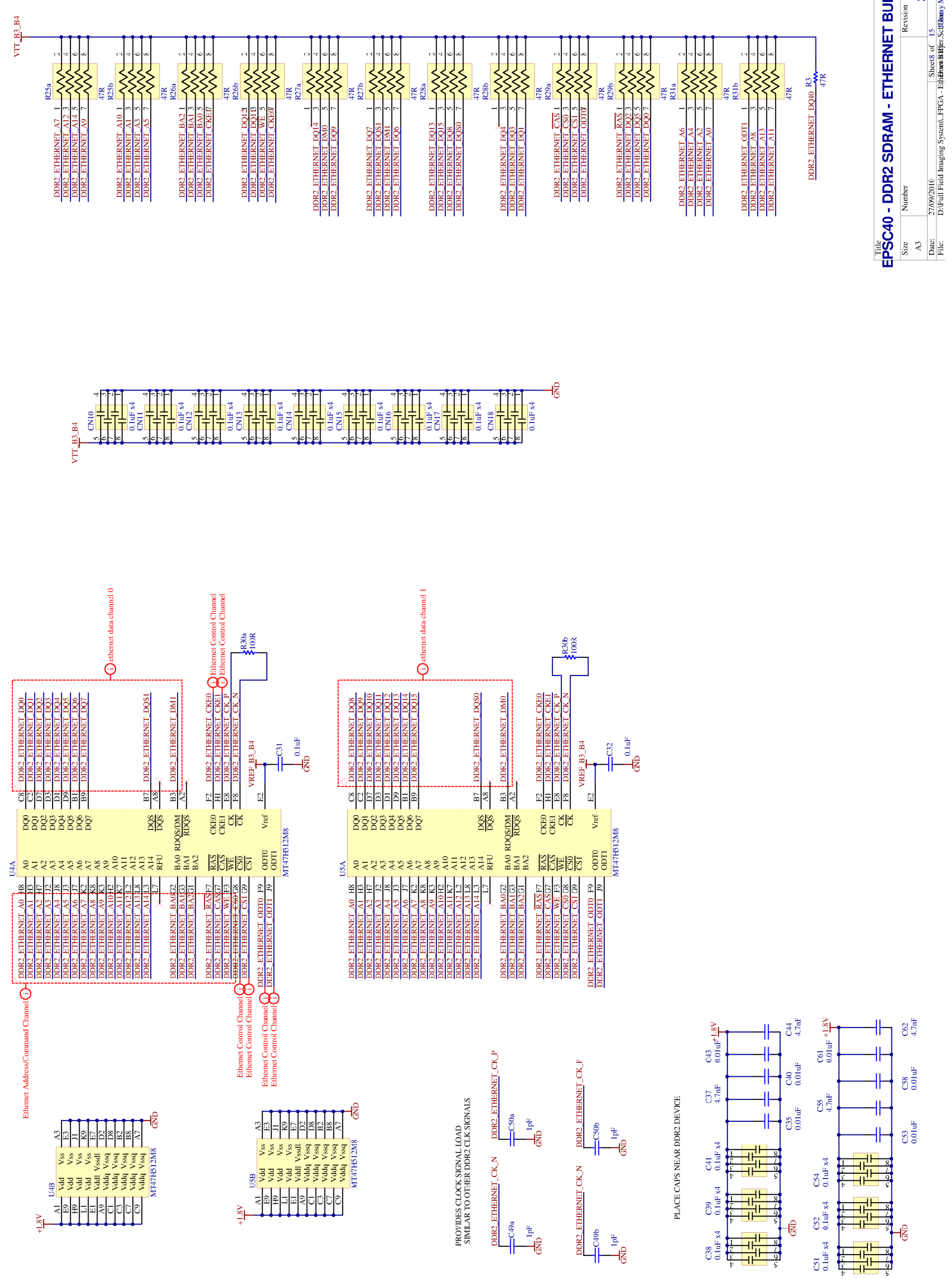


\section{B.13 FPGA Board - HSM-A Generic Interface Schematic}
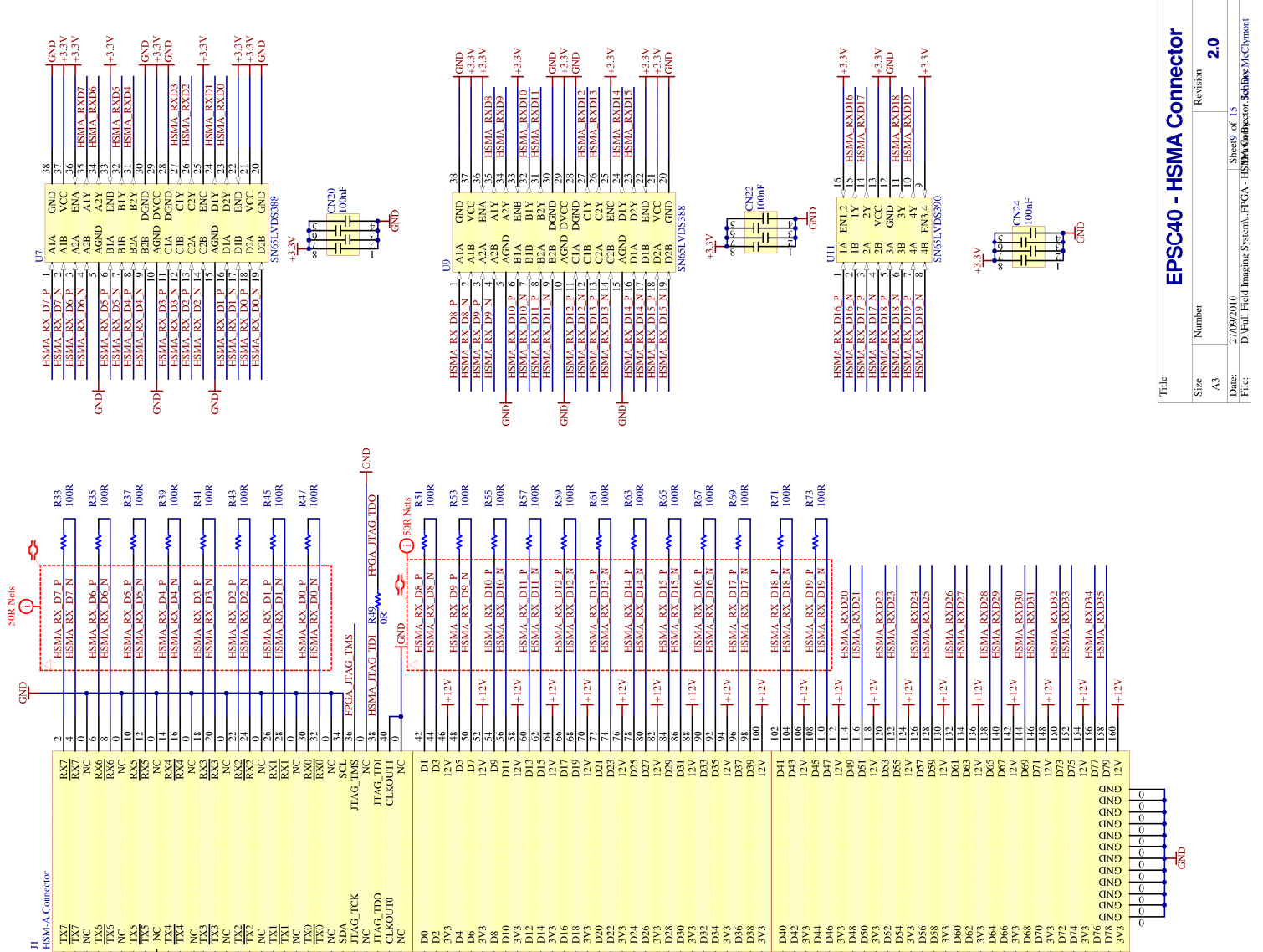

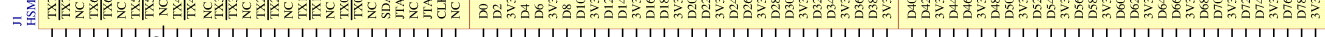
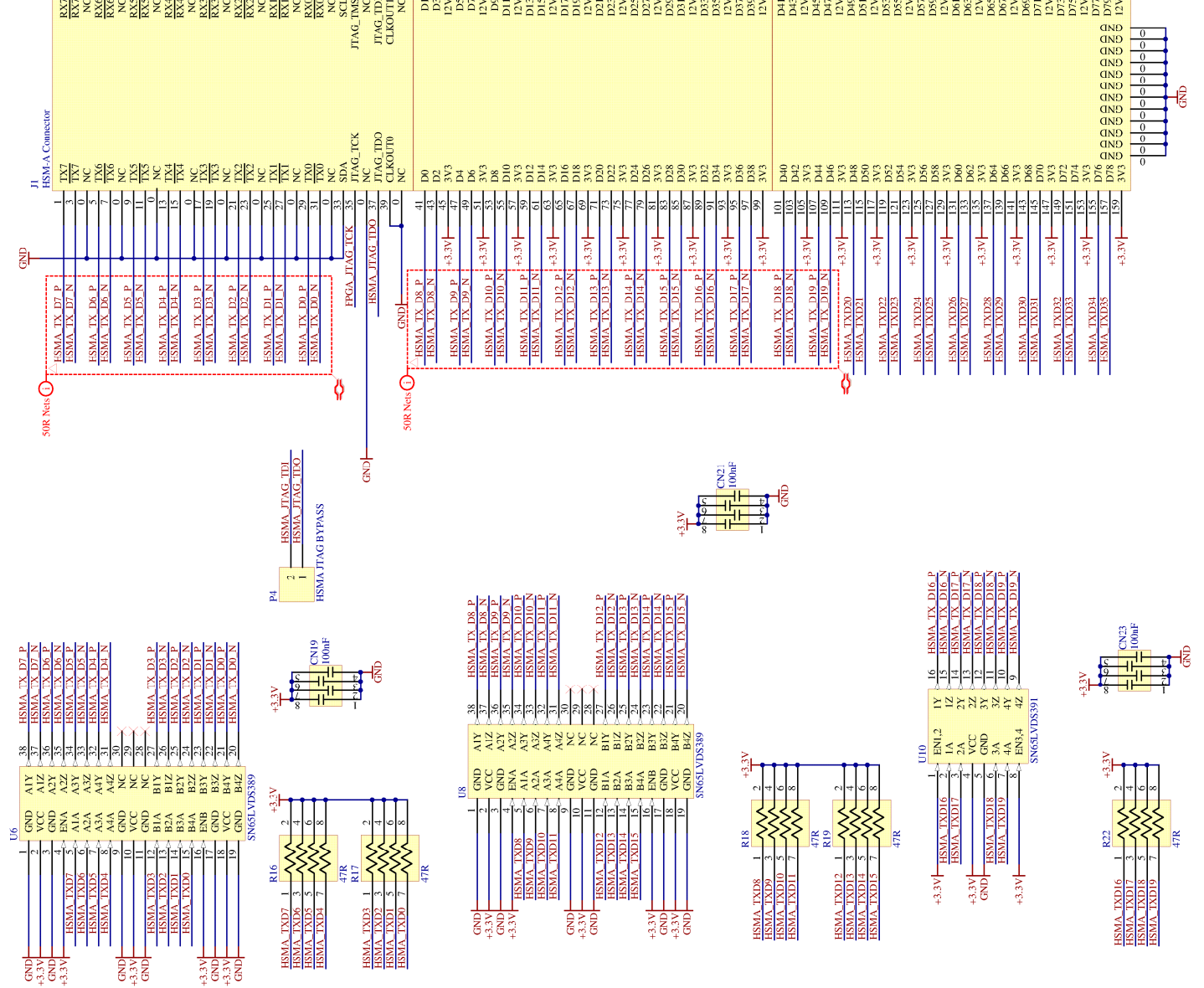


\section{B.14 FPGA Board - HSM-B Generic Interface Schematic}
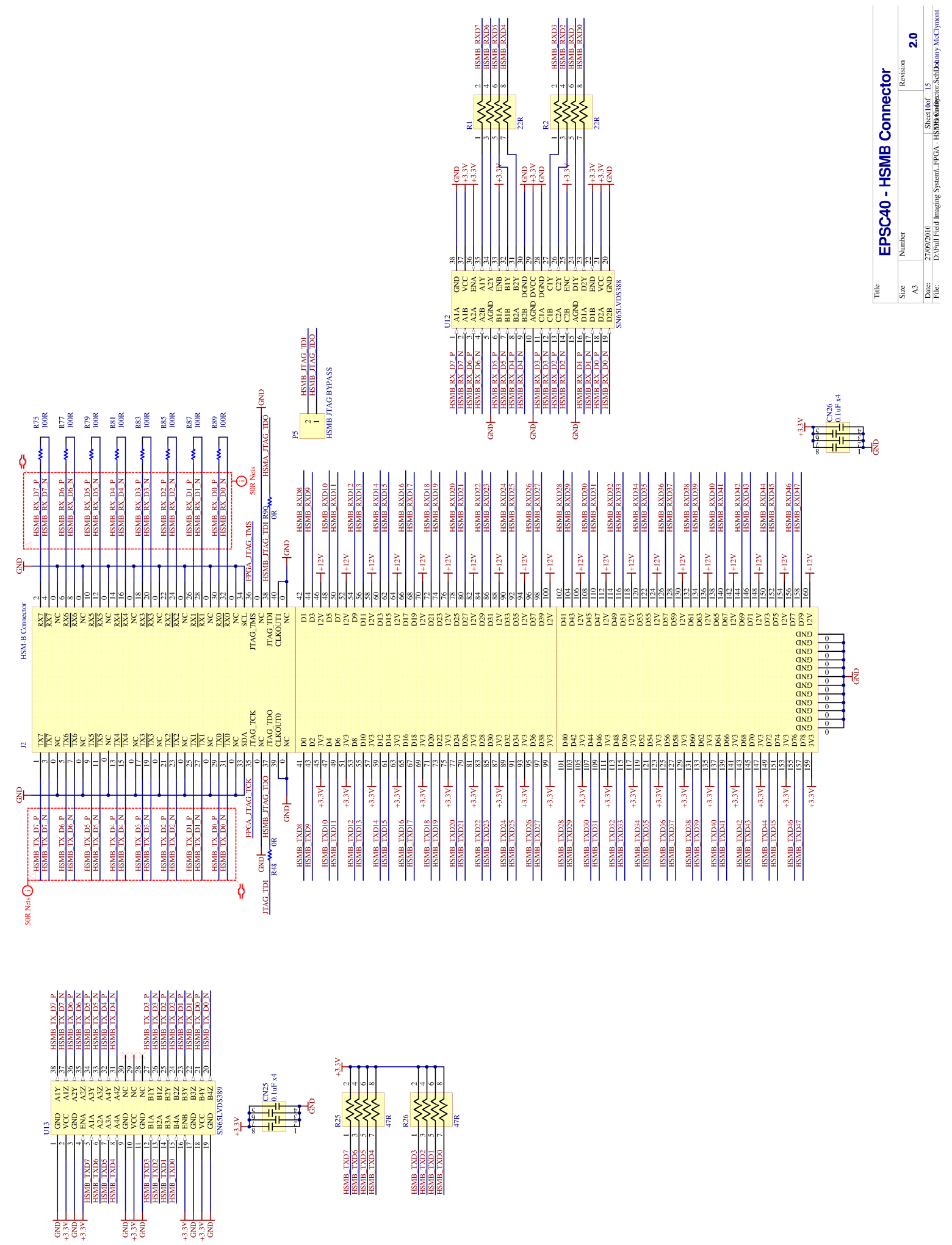


\section{B.15 FPGA Board - Power Decoupling Schematic}
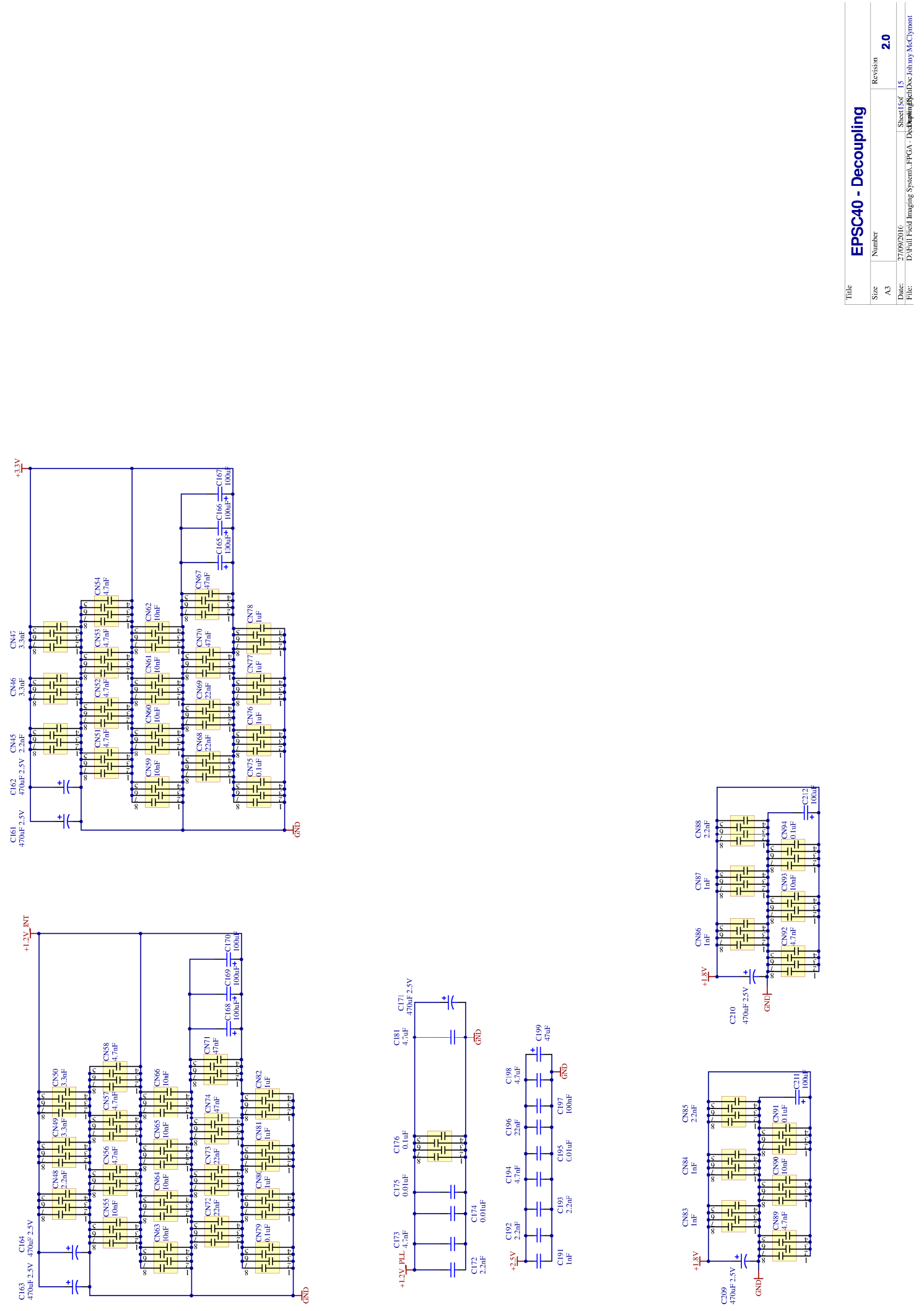


\section{B.16 External Interface - Power Schematic}
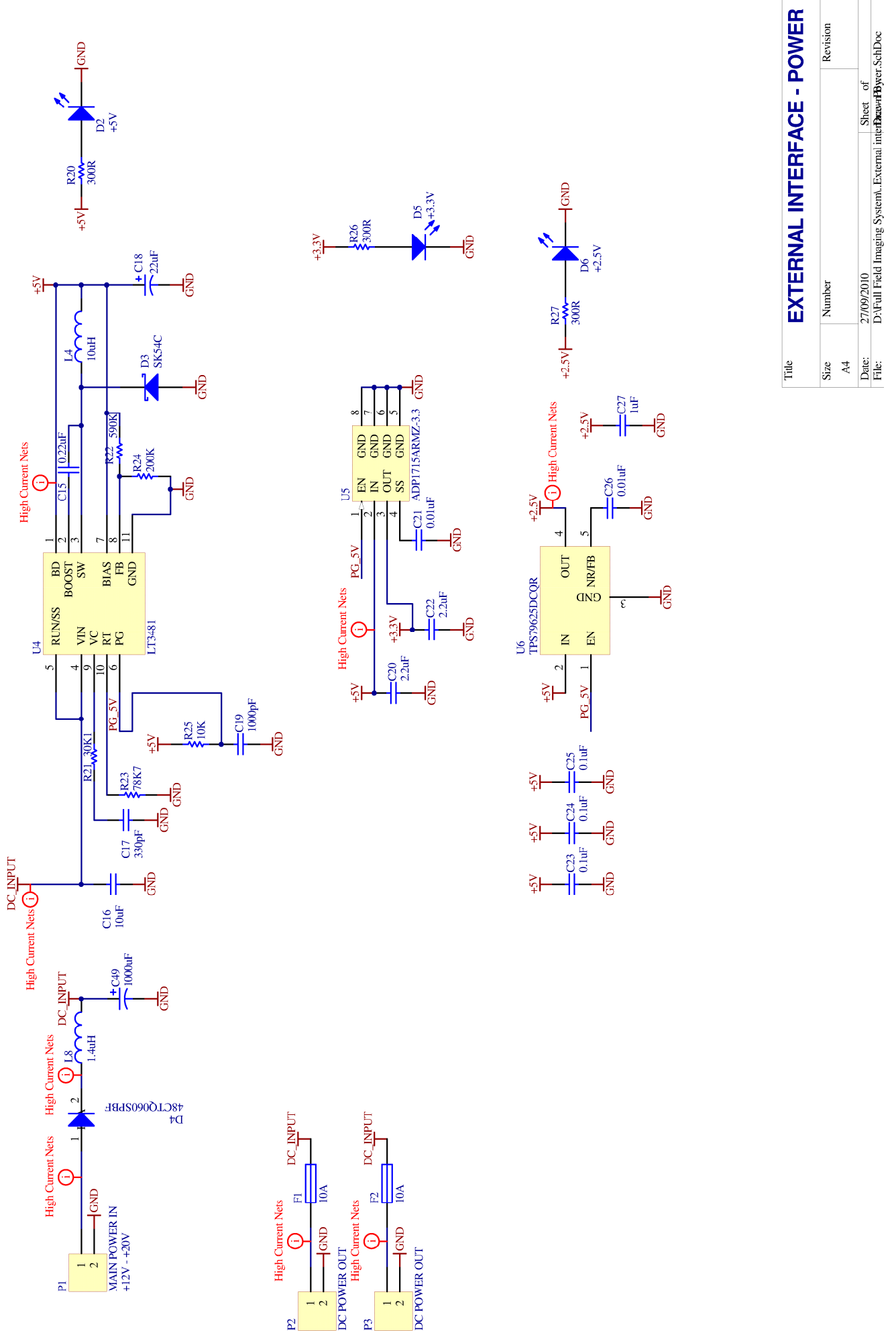


\section{B.17 External Interface - Ethernet Schematic}
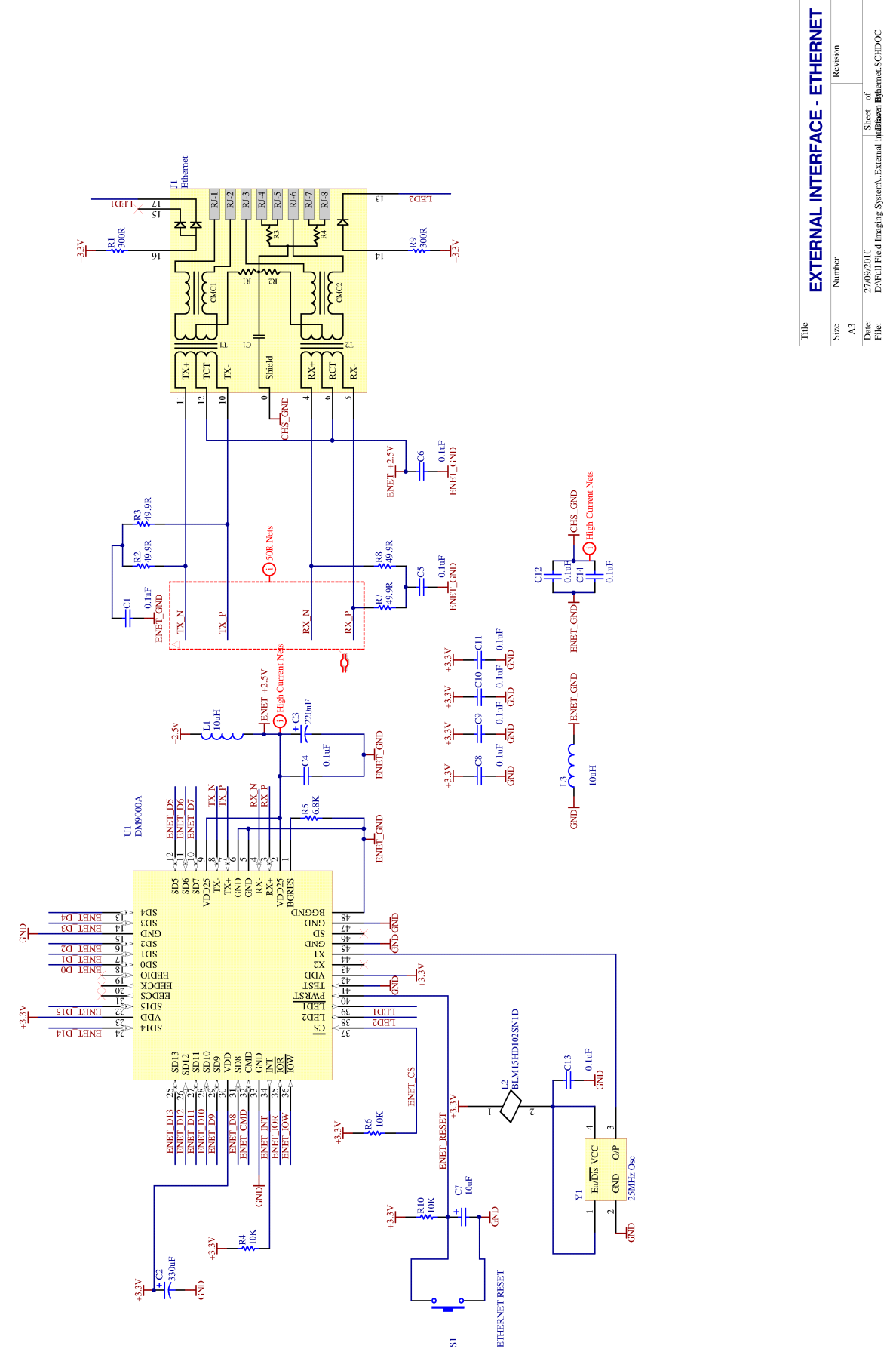


\section{B.18 External Interface - USB Schematic}
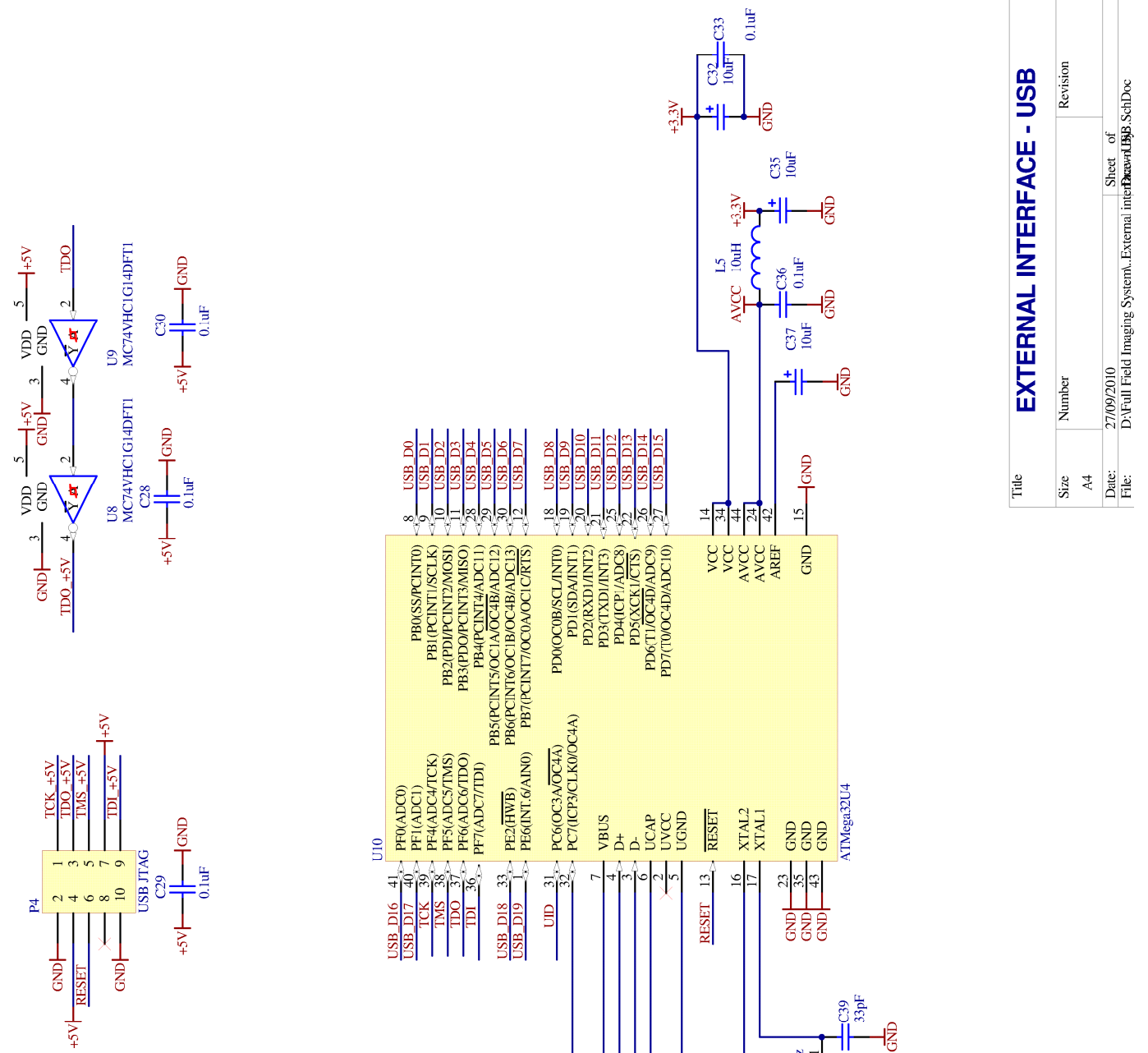

善を产产

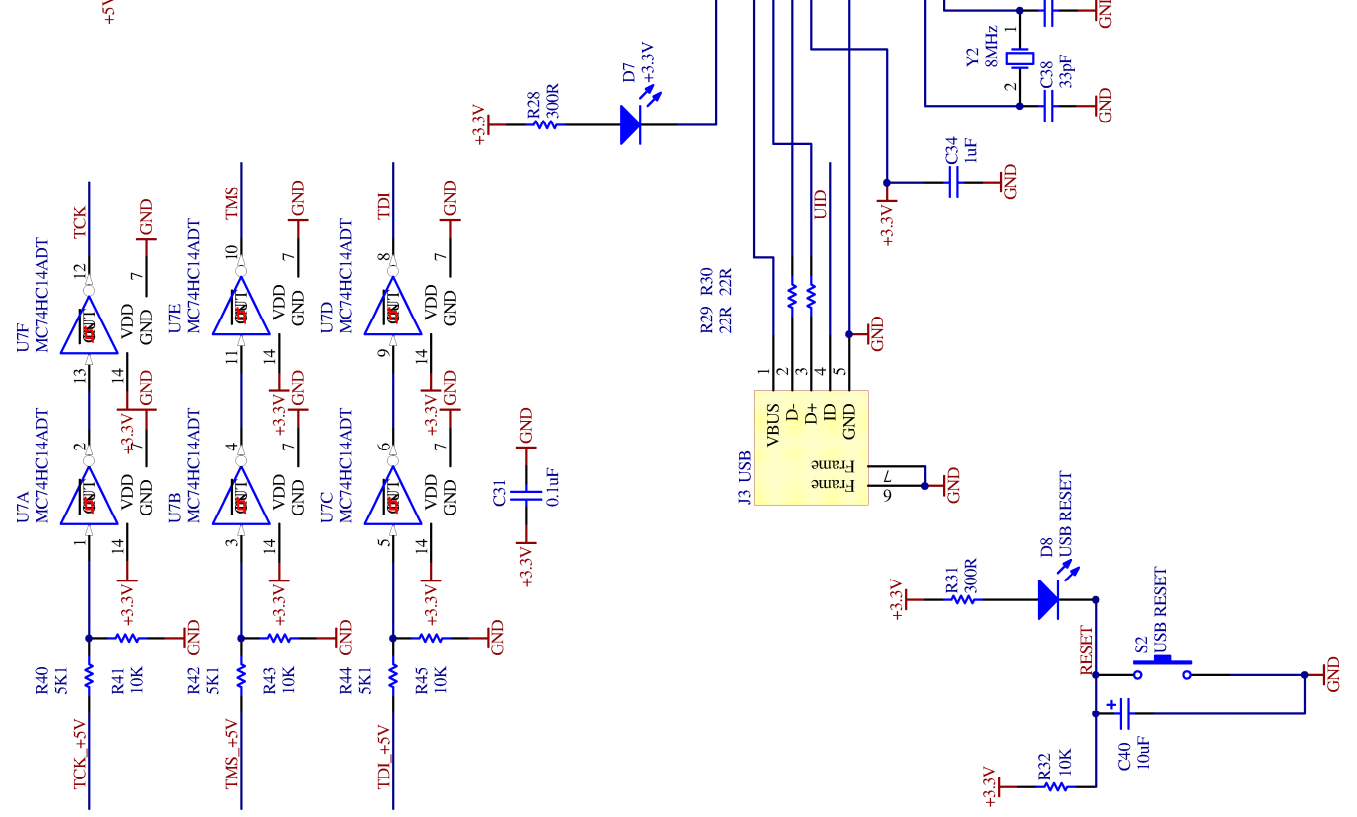




\section{B.19 External Interface - VGA Schematic}
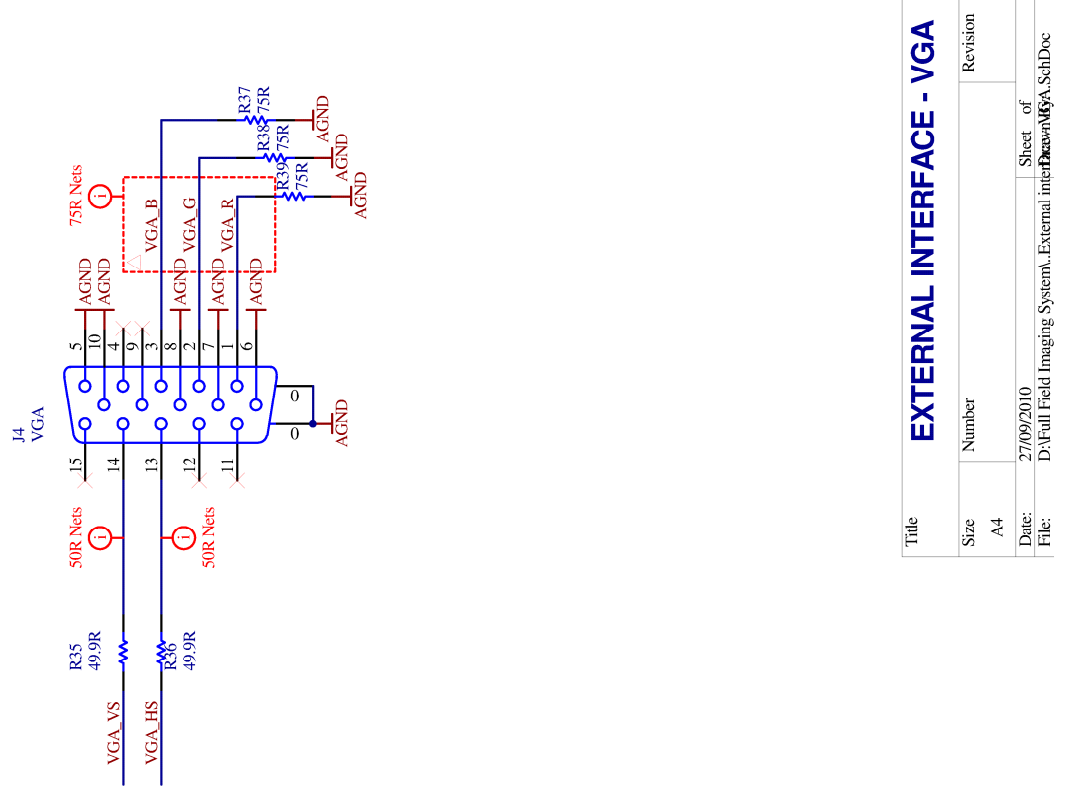

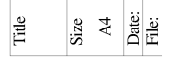
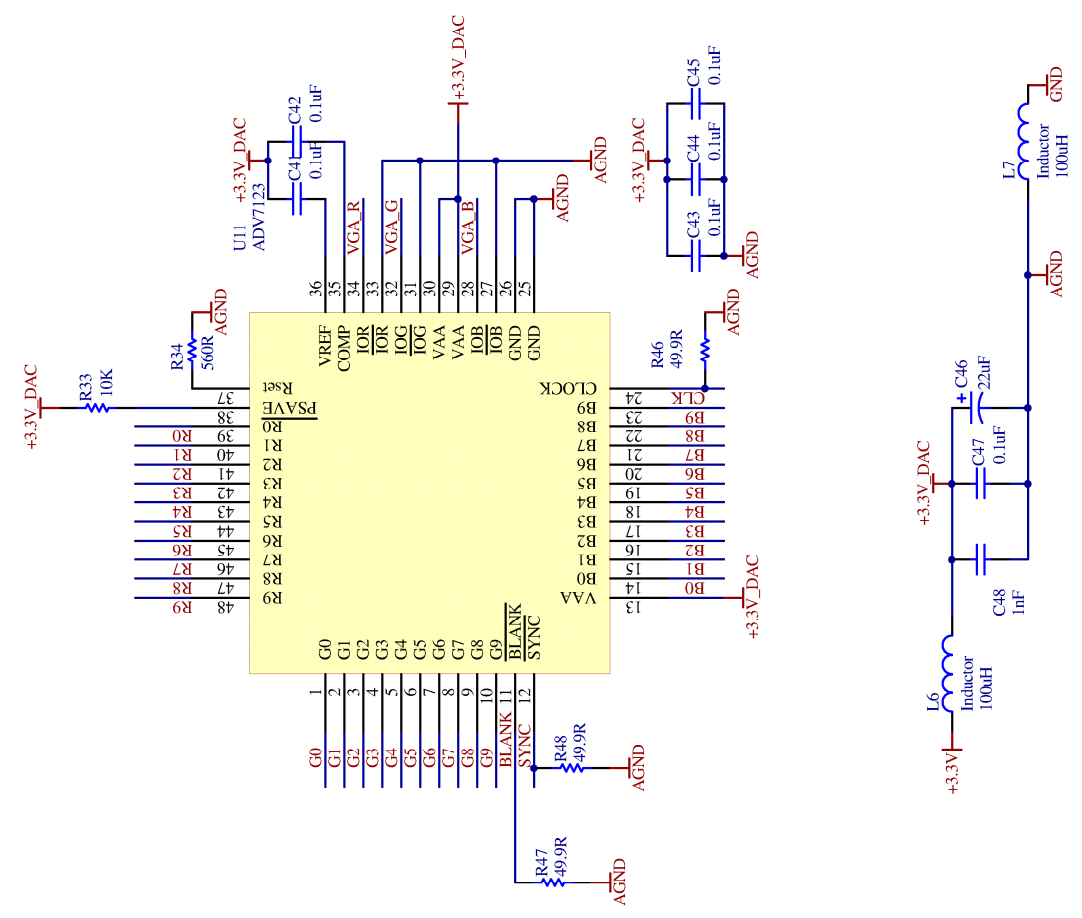


\section{B.20 External Interface - Generic Interface Schematic}
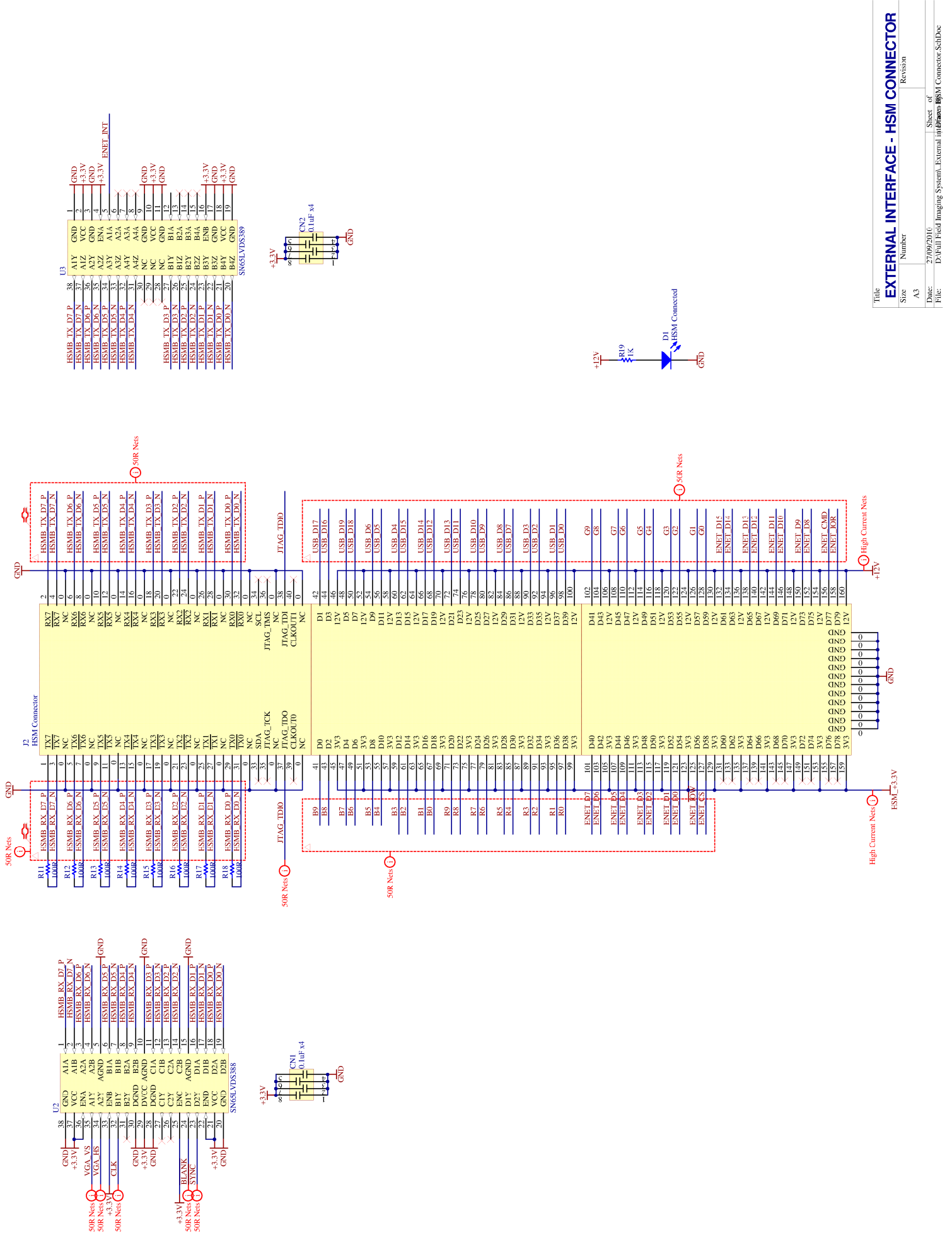


\section{B.21 Image Capture - LVDS Schematic}
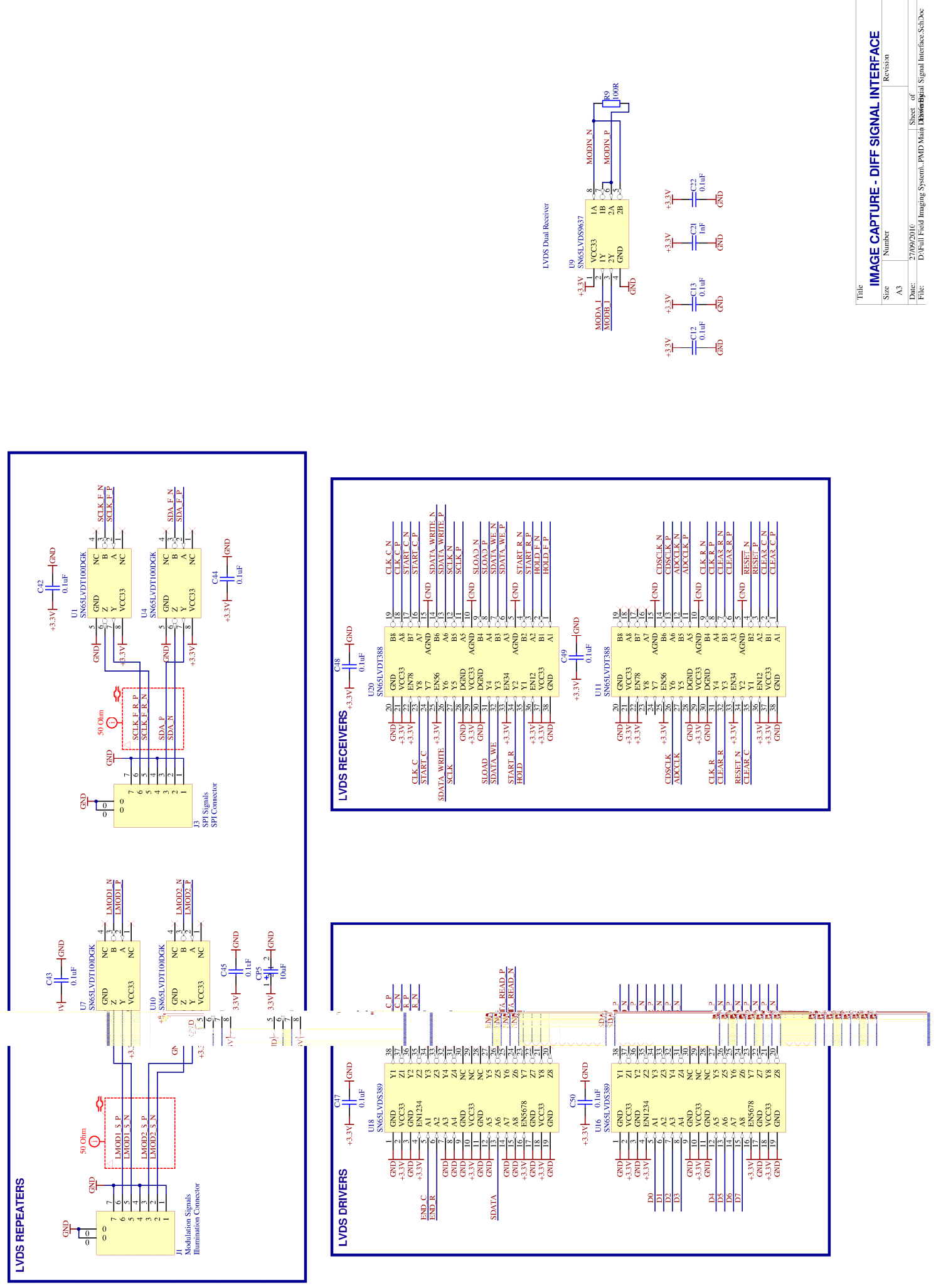


\section{B.22 Image Capture - Daughter Interface Schematic}
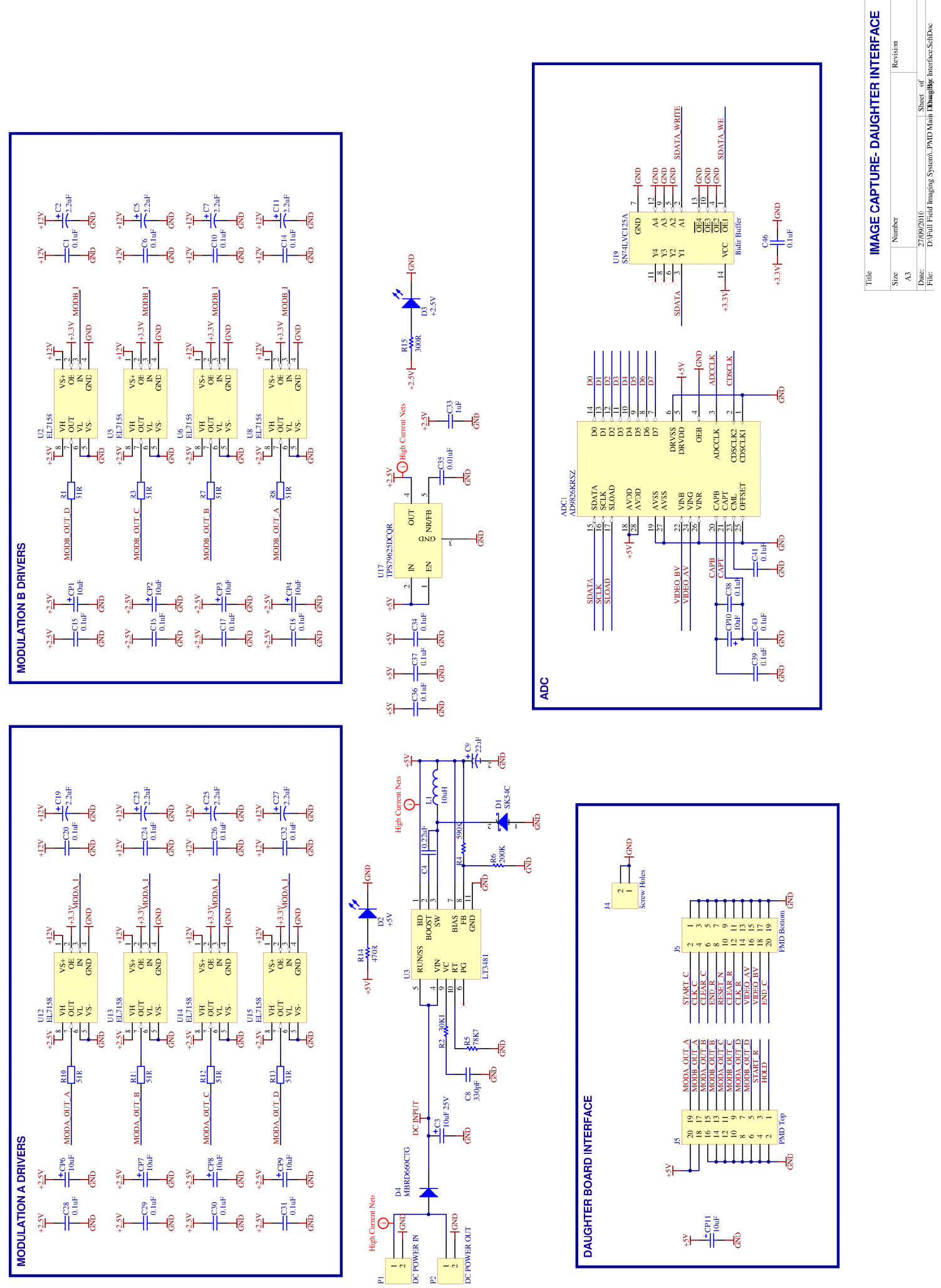


\section{B.23 Image Capture - Generic Interface Schematic}
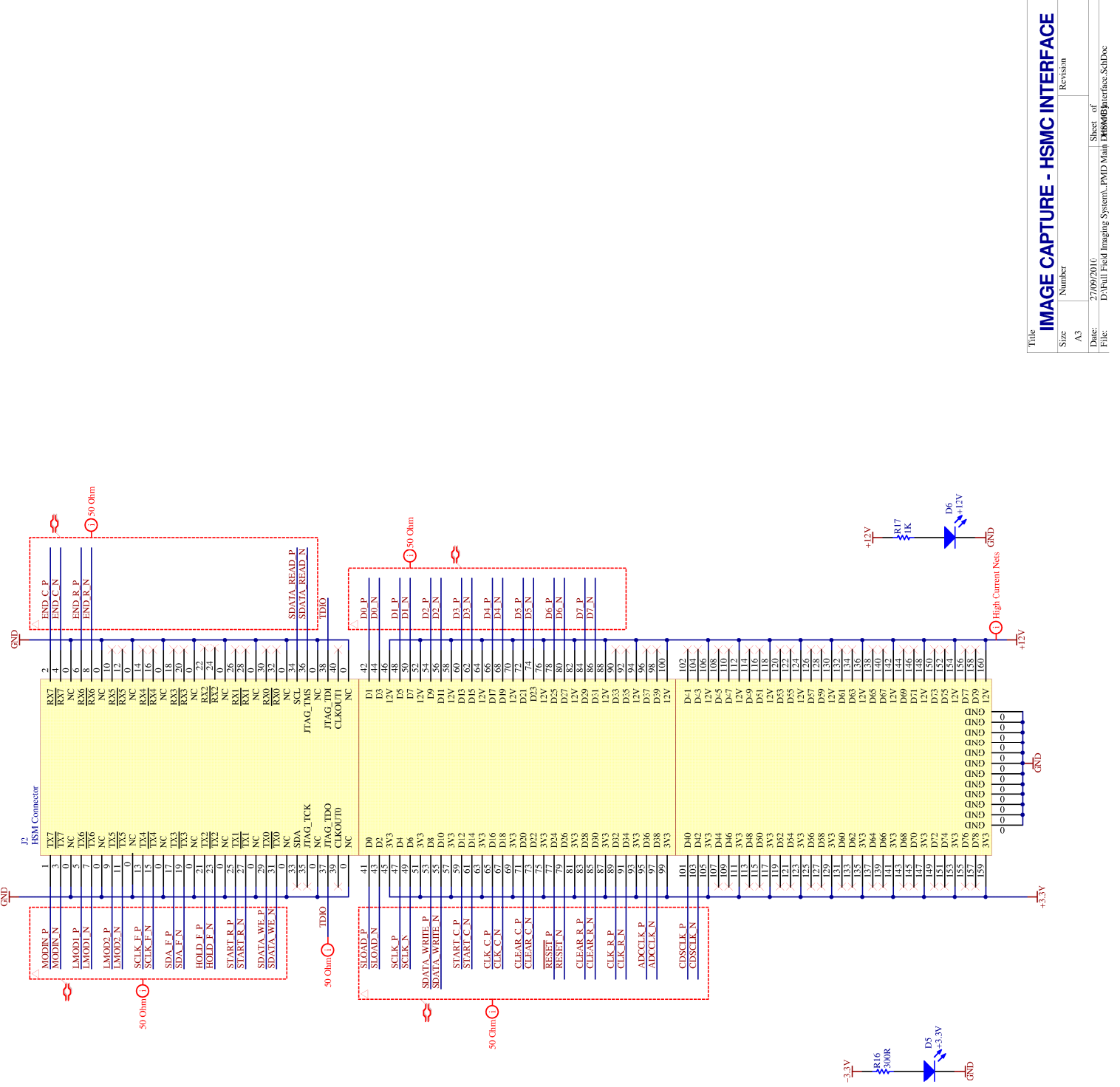


\section{B.24 Laser Illumination - Power Schematic}
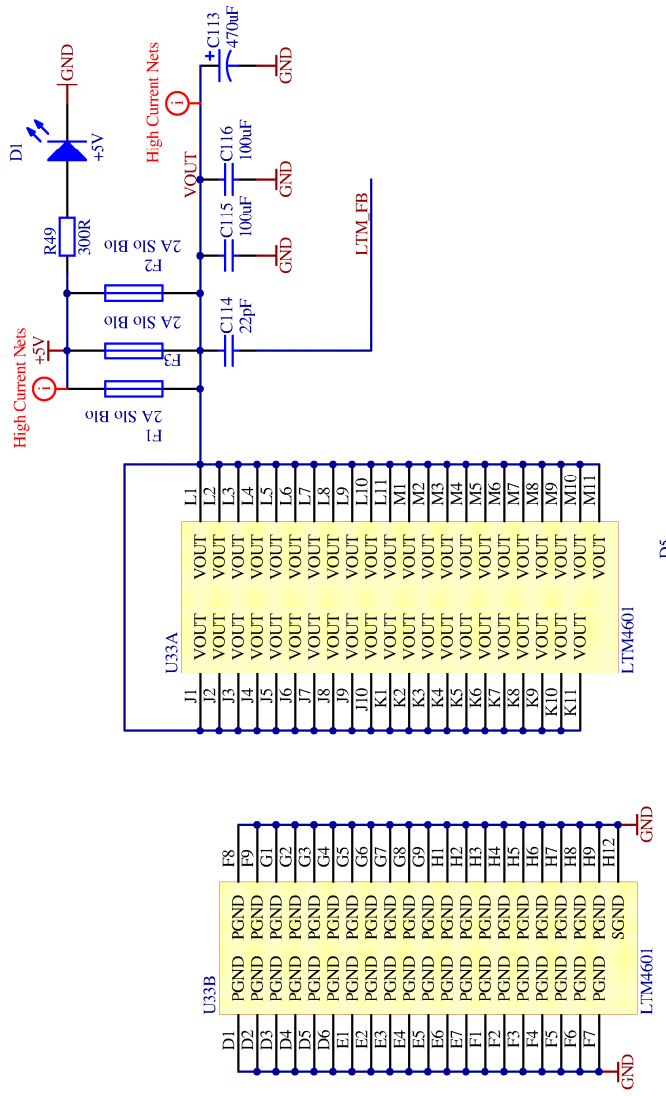
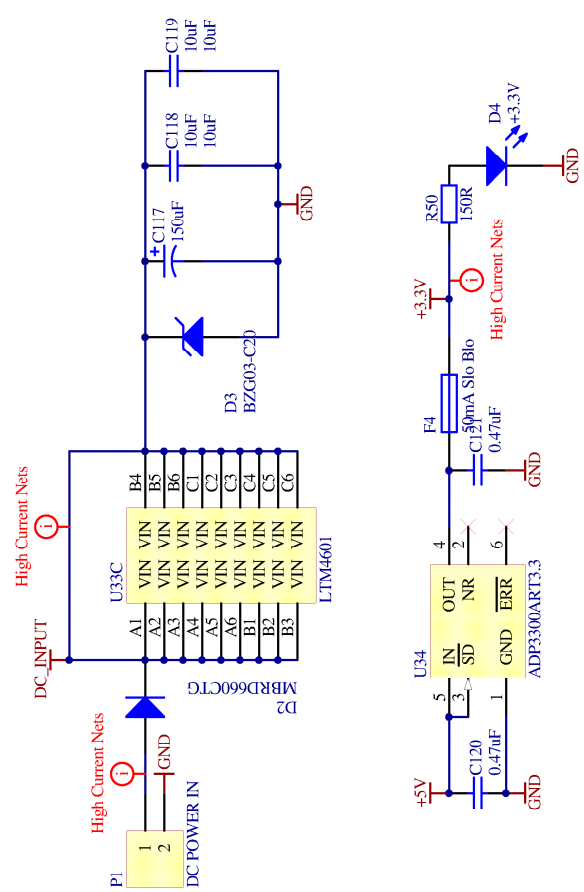

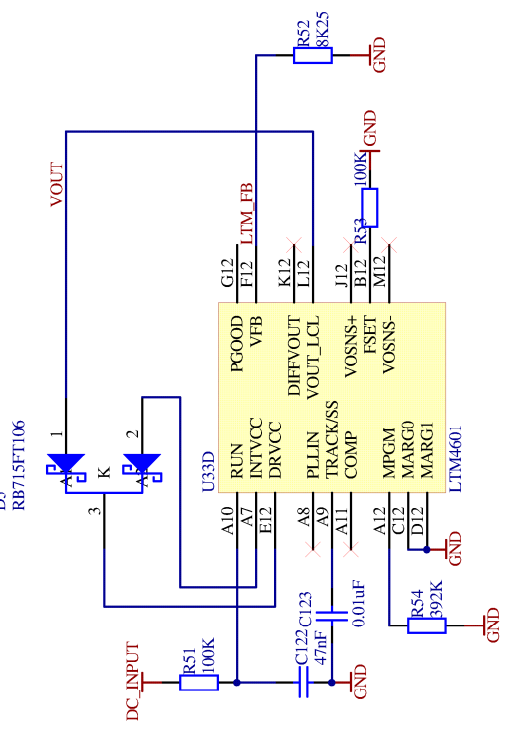

㟧

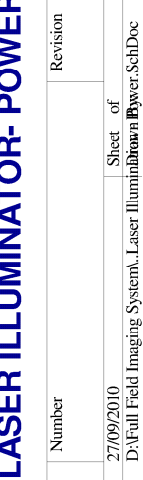

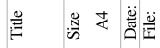




\section{B.25 Laser Illumination - Control Interface Schematic}

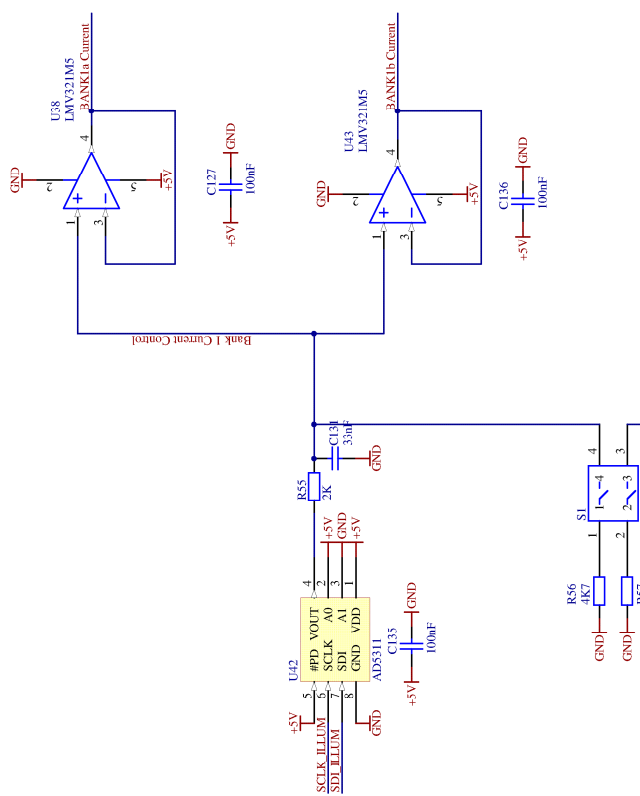

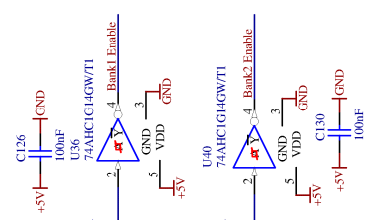

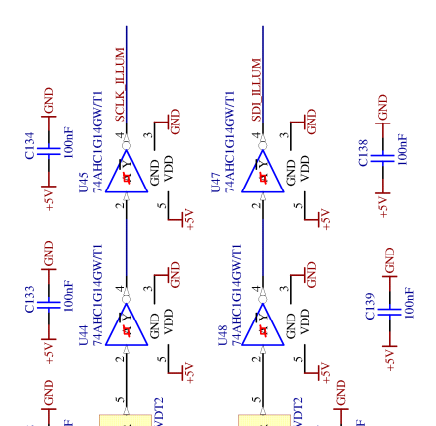

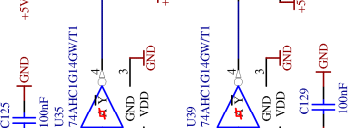

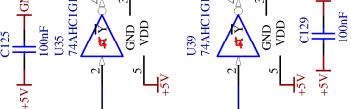

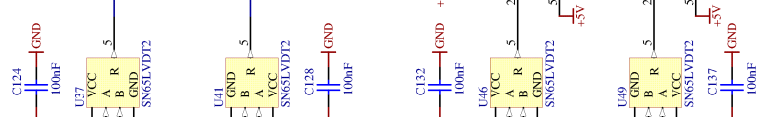
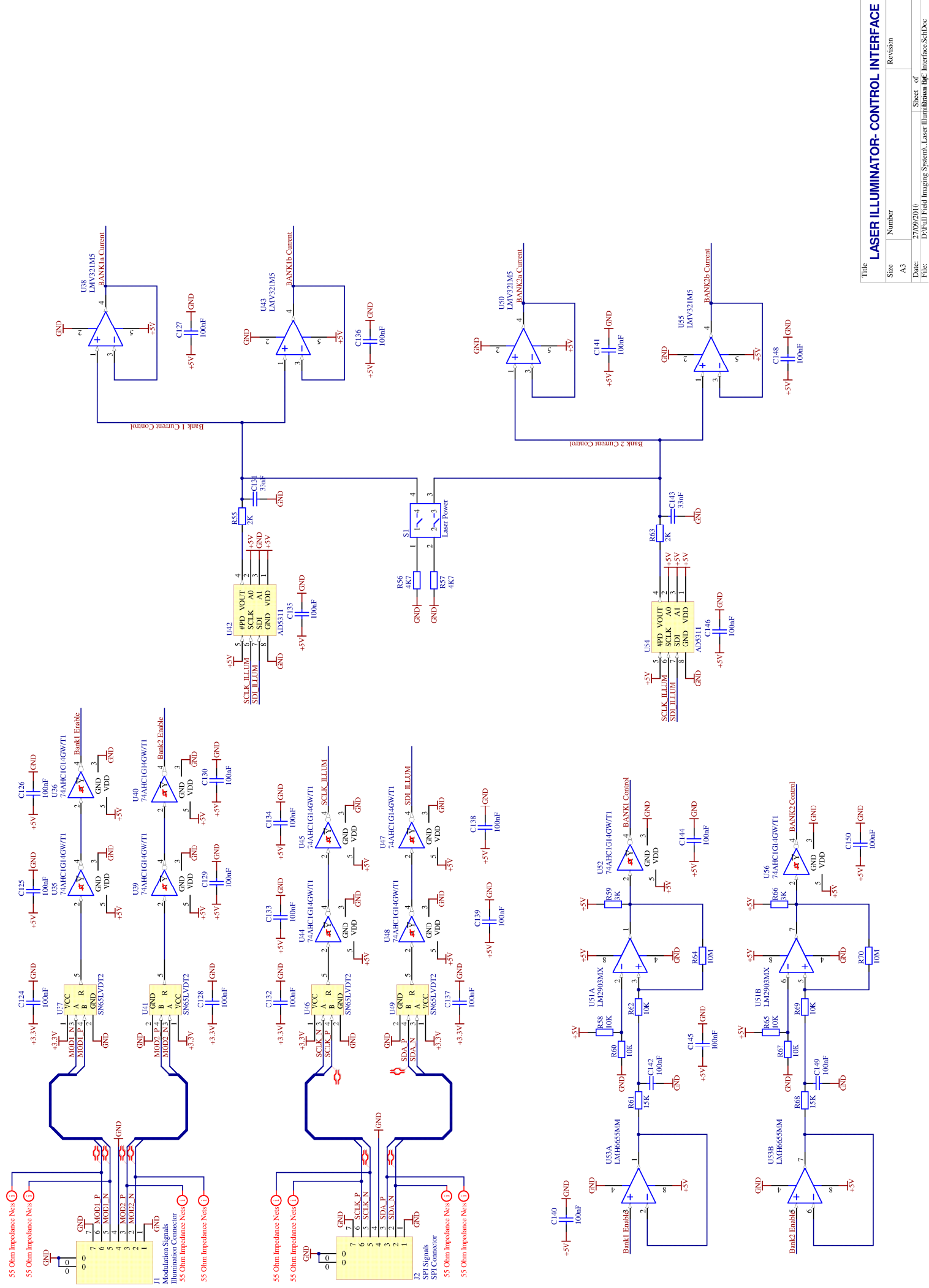


\section{B.26 Laser Illumination - Laser Bank 1 Schematic}
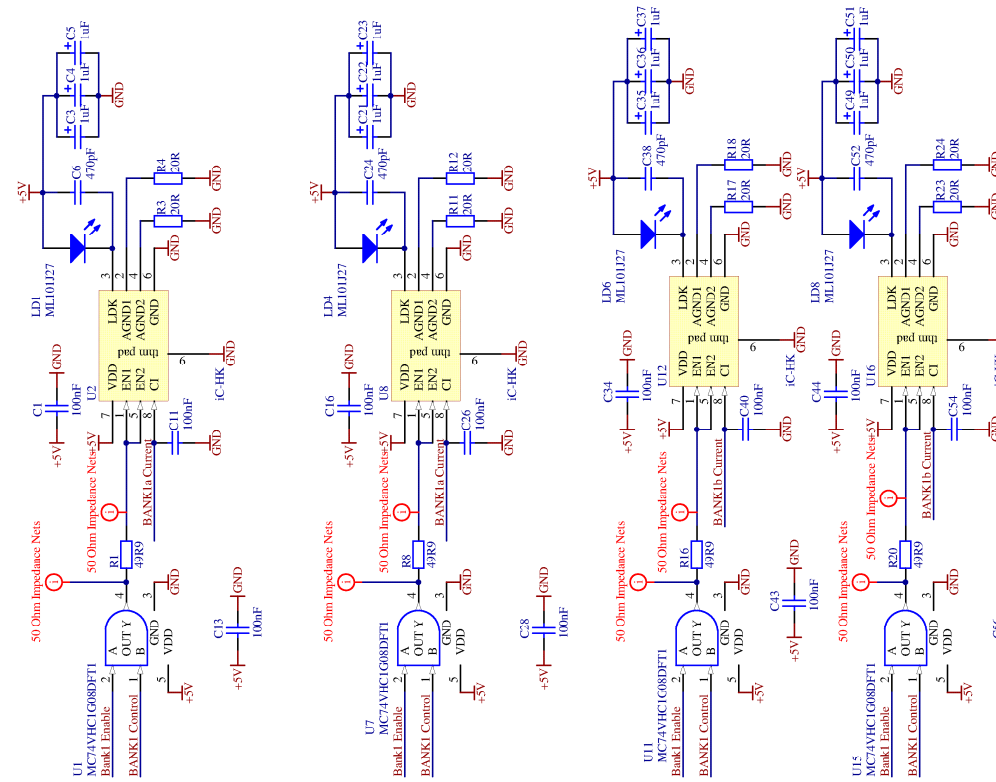

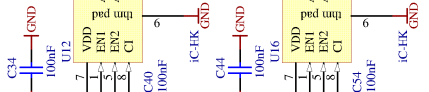
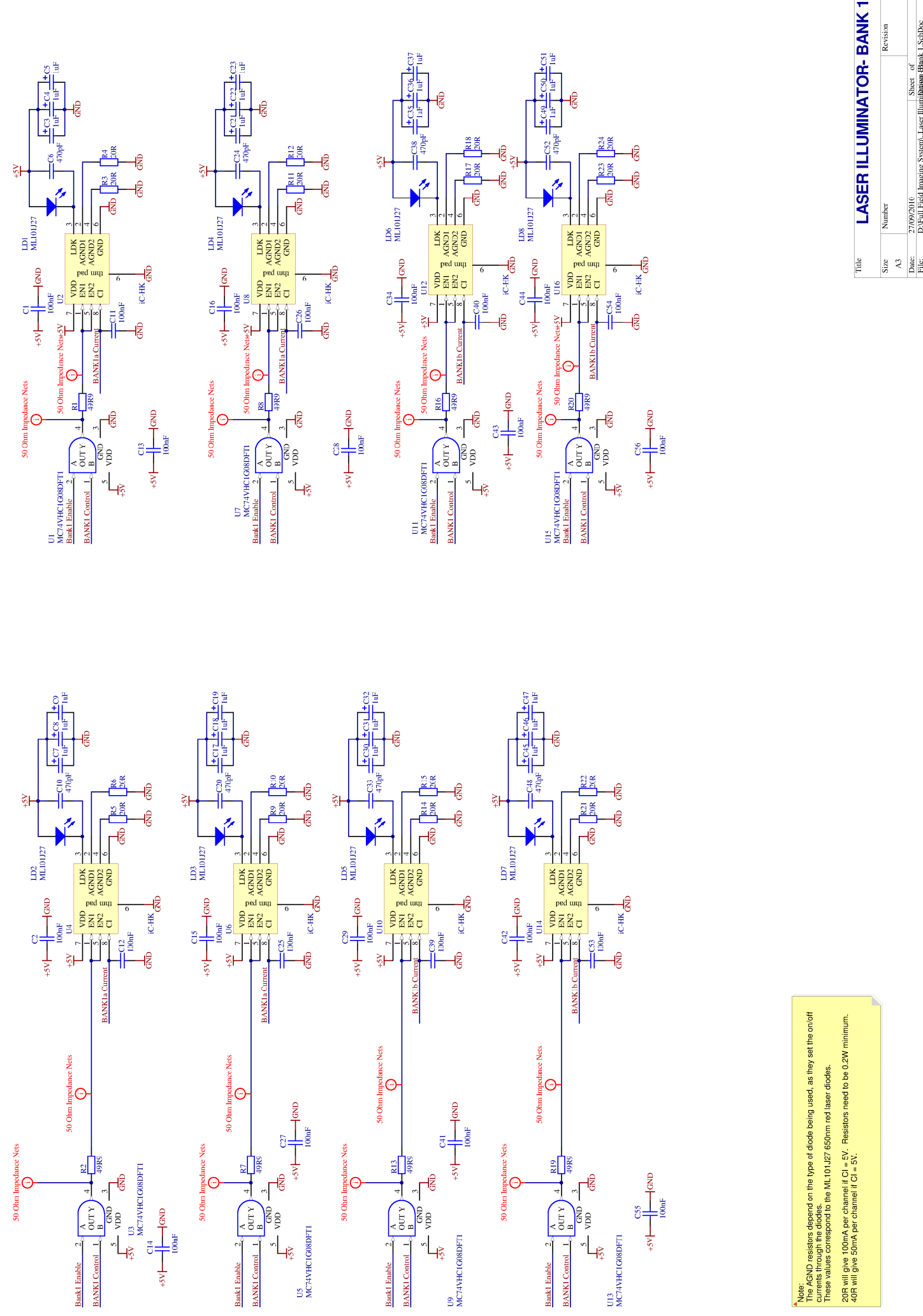


\section{B.27 Laser Illumination - Laser Bank 2 Schematic}
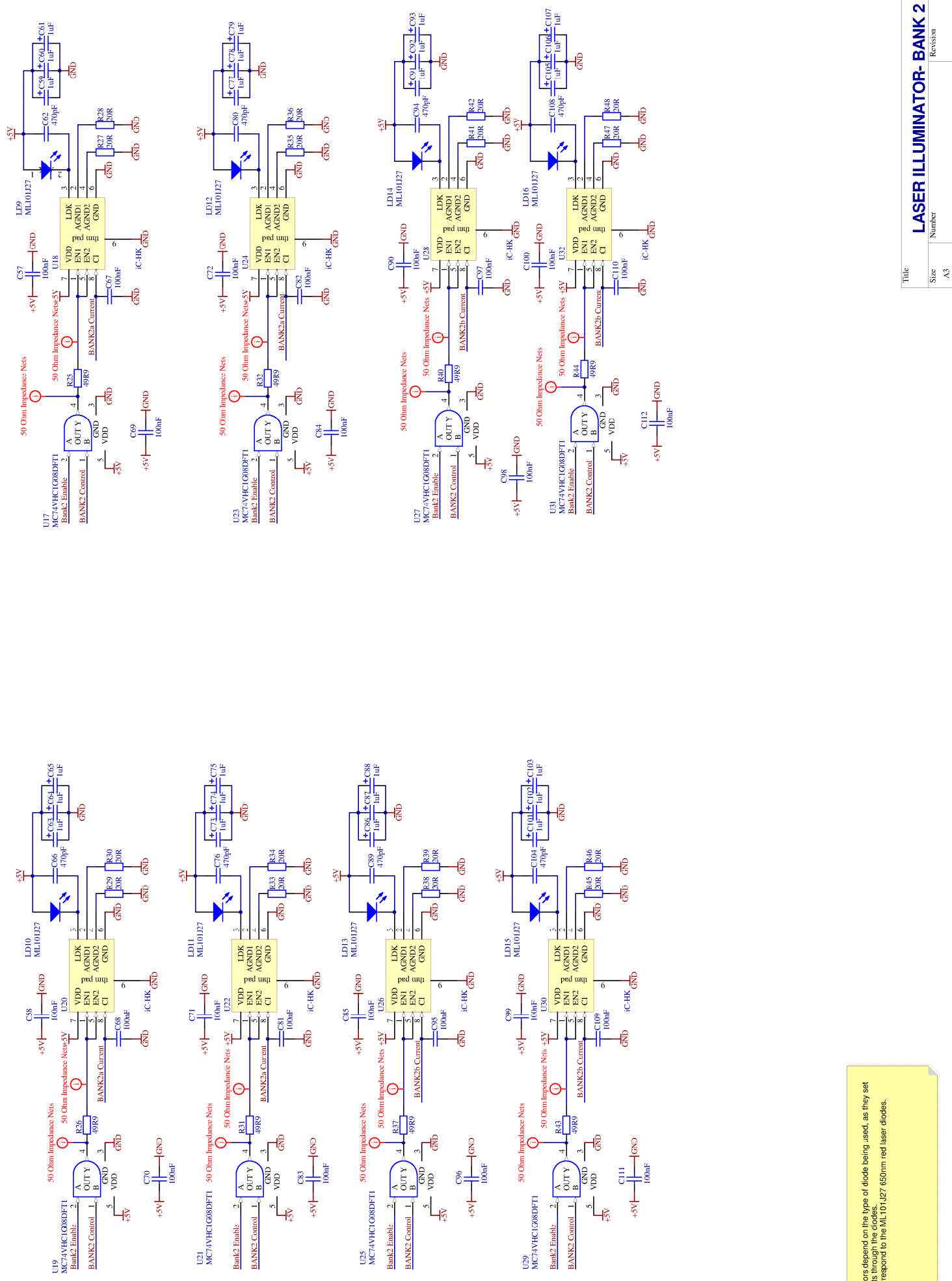


\section{Appendix C: CD Contents}

The attached CD contains the following:

- Soft copy of this thesis

- Photo Gallery

- Software Projects

\section{Altium:}

- Sensor Network Interface Module

- IR Ranging Node

- Ultrasonic Ranging Node

- Full-Field Image Ranger - FPGA Board

- Full-Field Image Ranger - Image Capture Board

- Full-Field Image Ranger - External Interface Board

- Full-Field Image Ranger - Laser Illumination Board

\section{GCC:}

- Sensor Network Interface Module Firmware

- IR Ranging Node Firmware

- Ultrasonic Ranging Node Firmware

\section{C\# Project:}

- Command Packet Interface

- Sensor Calibration

- Sensor Network Ranger

- Sensor Network Interface

- Datasheets. 


\section{Glossary}

AC Alternating Current

ADC Analogue to Digital Conversion

AMCW Amplitude modulated Continuous Wave

AP Active Parallel Configuration

AS Active Serial Configuration

ASCII American Standard Code for Information Interchange

CCD Charged Coupled Device

DAC Digital to Analogue Conversion

DAQ Data Acquisition

DDR Double Data Rate Synchronous Dynamic Random Access Memory

DIP Dual Inline Package

DLE Data Link Escape

DLL Data Link Layer

DQ Data

DQS Data Strobe

EBCDIC Extended Binary Coded Decimal Interchange Code

ESR Equivalent Series Resistance

ETX End of Text

FPGA Field Programmable Gate Array

FPP Fast Passive Parallel Configuration

FSM Finite State Machine

HDD Hard Disk Drive 


\begin{tabular}{|c|c|}
\hline HLP & Host Layer Protocol \\
\hline IDC & Insulation Displacement Connector \\
\hline IDE & Integrated Design Environment \\
\hline $\mathbf{I} / \mathbf{O}$ & Input / Output \\
\hline IR & Infrared \\
\hline ISP & In-System Programming \\
\hline ISR & Interrupt Service Routine \\
\hline JTAG & Joint Test Action Group \\
\hline LED & Light Emitting Diode \\
\hline LSB & Least Significant Bit \\
\hline LVDS & Low Voltage Differential Signal \\
\hline LVTTL & Low Voltage Transistor to Transistor Logic \\
\hline MAC & Media Access Controller \\
\hline MCU & Microcontroller Unit \\
\hline MIPS & Millions of Instructions Per Second \\
\hline MLP & Media Layer Protocol \\
\hline MP & Mega Pixel \\
\hline MTA & Mass Terminal Assembly \\
\hline OSI & Open Systems Interconnection \\
\hline PC & Personal Computer \\
\hline PCB & Printed Circuit Board \\
\hline PHY & Physical Layer Transceiver \\
\hline PLL & Phase Lock Loop \\
\hline PS & Passive Serial Configuration \\
\hline
\end{tabular}




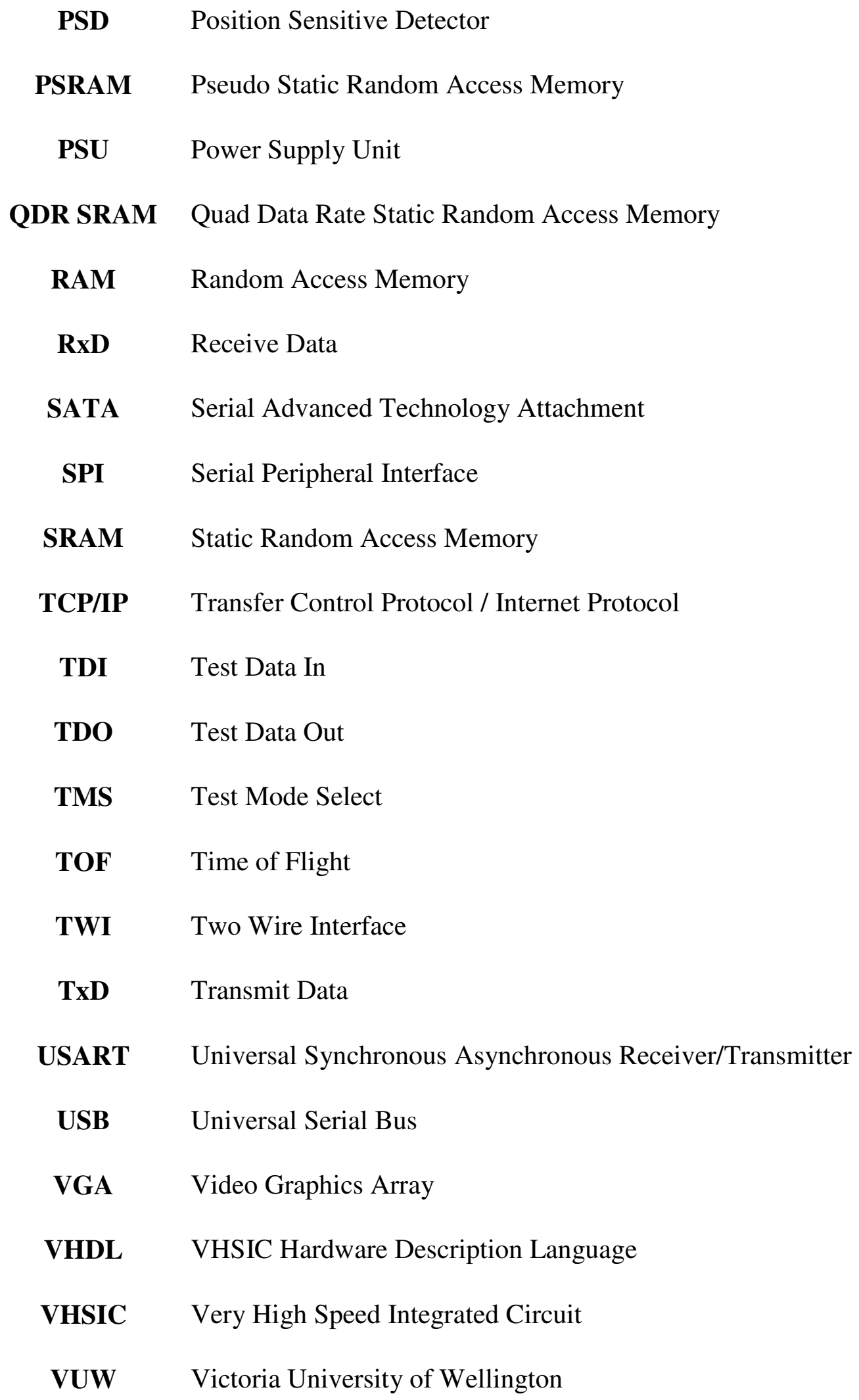

USART Universal Synchronous Asynchronous Receiver/Transmitter

USB Universal Serial Bus

VGA Video Graphics Array

VHDL VHSIC Hardware Description Language

VHSIC Very High Speed Integrated Circuit

VUW Victoria University of Wellington 


\section{References}

[1] http://www.pmdtec.com/products-services/pmdvisionr-cameras/pmdvisionr-camcube30/ last accessed 22/09/2010.

[2] http://www.mesa-imaging.ch/index.php last accessed 22/09/2010.

[3] A. P. P. Jongenelen, "Development of a Compact, Portable, Real-Time Range Imaging System", PhD Thesis, Victoria University of Wellington, 2010.

[4] Krishna, S.M., "Sensors For Robots” Electronics For You, pp. 124 -130i, Sep. 2007.

[5] Williamson D,A,. The Development of a "Mother" Agent for a Hierarchical MultiRobot Urban Search and Rescue System, MSc Thesis, Victoria University of Wellington, 2007.

[6] Halliday, D., Resnick, R., Walker, J., "Fundamentals of Physics", $5^{\text {th }}$ edition, John Wiley \& Sons, 1997.

[7] http://en.wikipedia.org/wiki/File:LaserPrinciple.png last accessed 13/07/2010.

[8] Maslin, G.D., "A Simple Ultrasonic Ranging System" in Proceedings of the 102nd Convention of the Audio Engineering Society, Cincinnati, USA, 12 May. 1983.

[9] Biber, C., Ellin, S., Shenk, E., Stempeck, J., "The Polaroid Ranging System" in Proceedings of the 67th Convention of the Audio Engineering Society, New York, USA, 31 Oct - 3 Nov. 1980.

[10] Cracknell, A. P., Hayes, Ladson, "Introduction to Remote Sensing" $2^{\text {nd }}$ edition, Taylor and Francis, 2007.

[11] Jongenelen, A.P.P, Payne, A.D., Carnegie, D.A., Dorrington, A.A., and Cree, M.J., "Development of a Real-Time Full Field Range Imaging System" Recent Advances in Sensing Technology, LNEE49, pp. 113 - 129, 2009.

[12] Jongenelen, A.P.P., Carnegie, D.A.,Dorrington, A.A., Payne, A.D., "Heterodyne Range Imaging in Real-time" in Proceedings of the $3^{\text {rd }}$ International Conference on Sensing Technology, Tainan, Taiwan, 30 Nov - 3 Dec. 2008.

[13] Jongenelen, A.P.P., Carnegie, D.A., Payne, A.D., Dorrington, A.A., "Development and Characterisation of an Easily Configurable Range Imaging System" in 
Proceedings of the 2009 Image and Vision Computing New Zealand Conference, Wellington, New Zealand, 23 - 25 Nov. 2009.

[14] Cawley, C.L., The Enhancement of a Multi-Terrain Mechatron for Autonomous Outdoor Applications, MSc Thesis, University of Waikato, 2006.

[15] Lee-Johnson, C.P., The Development of a Control System for an Autonomous Mobile Robot, MSc Thesis, University of Waikato, 2004.

[16] Payne, A.D., Carnegie, D.A., "Design and Construction of a Pair of Tricycle Based Robots to Investigate Cooperative Robotic Interaction", Proceedings of the $10^{\text {th }}$ Electronics New Zealand Conference, Hamilton, New Zealand, September 2003.

[17] "GP2Y3A003K0F Wide Angle Distance Measuring Sensor Unit", Sharp datasheet ID: E4-A01401EN, (December 2006).

[18] “GP2Y3A002K0F Wide Angle Distance Measuring Sensor Unit”, Sharp datasheet ID: E4-A01301EN, (December 2006).

[19] “Ultrasonic Ranging System", Polaroid Corporation Datasheet ID: P1834, (May 1992).

[20] Winch, S., "A laser range finding system with use of a webcam”, Post Graduate Diploma in Science Project Report, Victoria University of Wellington, 2009.

[21] Parziale, L., Britt, D.T, Davis, C., Forrester, J., Liu, W., Matthews, C., Rosselot, N. "TCP/IP Tutorial and Overview", $8^{\text {th }}$ edition, Redbooks, 2006.

[22] Piscatello, D., Lyman Chapin, A., "Open Systems Networking", $1^{\text {st }}$ edition, Addison Wesley, 1993.

[23] "IEEE Standard for Local and Metropolitian Area Networks: Overview and Architecture", Institute of Electrical and Electronics Engineers, Inc datasheet ID: IEEE Std 802-2001, (February 2002).

[24] “Universal Serial Bus Specification Revision 2.0”, USB Working Group. (April 2000).

[25] “Application Note 3947 Daisy Chaining SPI Devices", Maxim Integrated Products, AN-3947, (2006). 
[26] "Application Note: AA-00255 Introduction to $I^{2} C$ and SPI Protocols", ByteParadigm.com, AA-00255, (2010).

[27] "EIA Standard RS-232-C Interface Between Data Terminal Equipment and Data Communication Equipment Employing Serial Data Interchange", Electronics Industries Association, reprinted in Telebyte Technology Data Communication Library, Greenlawn NY, 1985.

[28] "EIA Standard RS-485 Electrical Characteristics of Generators and Receivers for Use in Balanced Multipoint Systems”, Electronic Industries Association, reprinted in Telebyte Technology "Data Communication Library" Greenlawn NY, 1985.

[29] “Application Note 763 Guidelines for Proper Wiring of an RS-485 (TIA/EIA-485-A) Network”, Maxim Integrated Products, AN-763, (2001).

[30] “ATmega128, ATmega128L 8-bit AVR Microcontroller with 128K Bytes In-System Programmable Flash", Atmel Corporation datasheet ID: 2467M-AVR-11/04, (2004).

[31] "ENC28J60 Stand-Alone Ethernet Controller with SPI Interface", Microchip Technologies Inc datasheet ID: DS39662C, (2008).

[32] “FT232BM USB UART IC”, Future Technology Devices International Ltd datasheet ID: FT_000206,(2010).

[33] "5 V Low Power EIA RS-485 Transceiver", Analog Devices Inc datasheet ID: ADM1485 Rev E, (2003).

[34] "LFxxAB Very Low Drop Voltage Regulators with Inhibit", STMicroelectronics datasheet ID: 2574 Rev 23, (2010).

[35] "LP3961/LP3964 800mA fast Ultra Low Dropout Linear Regulators", National Semiconductor Corporation datasheet ID: DS101129, (2006).

[36] “ATmega32, ATmega32L 8-bit AVR Microcontroller with 32K Bytes In-System Programmable Flash", Atmel Corporation datasheet ID: 2503-AVR-06/08, (2008).

[37] “MIC5205 150mA Low-noise LDO Regulator" Micrel Inc datasheet ID: M9999020806, (2006). 
[38] Gross, C., “Beginning C\# 2008, From Novice to Professional”, APRESS, November, 2007.

[39] “Stratix III Device Handbook, Volume 1”, Altera Corporation datasheet ID: SIII5V1$2.1,(2010)$.

[40] “Stratix III Development Kit, Reference Manual”, Altera Corporation datasheet ID: MNL-01030-1.2, (2008).

[41] "PHOTONICS PMD 19K-2 High Resolution 3D Video Sensor Array", PMD Technologies datasheet ID: PhotonICs PMD 19K2 Datasheet, (2008).

[42] “Complete 16-Bit Imaging Signal Processor AD9826”, Analog Devices Ltd datasheet ID: AD9826 Rev A, (2001).

[43] "EL7158 Ultra-High Current Pin Driver", Intersil Americas Inc datasheet ID: FN7349.2, (2007).

[44] “Cyclone III Device Handbook, Volume 1", Altera Corporation datasheet ID: CIII5V1-3.3, (2010).

[45] "NV SRAM Frequently Asked Questions”, Maxim Integrated Products Ltd Application Note: AN202, (2004).

[46] “Using Nonvolatile Static RAMs", Maxim Integrated Products Ltd Application Note ID: AN540, (2001).

[47] "Understanding Static SRAM Operation", International Business Machines Corporation Application Note, (1997).

[48] “General DDR SDRAM Functionality", Micron Technology Inc Technical Note ID: TN-46-05, (2001).

[49] "PSRAM 101: An Introduction to Micron CellularRAM and PSRAM", Micron Technology Inc Technical Note ID: TN-45-30, (2007).

[50] "Section II. Memory Standards Overview", Altera Corporation datasheet ID: EMI_INTRO_OVER-2.0,(2010). 
[51] “512Mb: x4, x8, x16 DDR2 SDRAM", Micron Technology Inc datasheet ID: 09005aef82f1e6e2, (2009).

[52] “4Gb: x4, x8 TwinDie DDR2 SDRAM", Micron Technology Inc datasheet ID: 09005aef8227ee4d, (2006).

[53] "SN65LVDS390 High-Speed Differential Line Receivers", Texas Instruments Incorporated datasheet ID: SLLS394G, (2004).

[54] “SN65LVDS388 High-Speed Differential Line Receivers", Texas Instruments Incorporated datasheet ID: SLLS448AG, (2001).

[55] "SN65LVDS389, SN65LVDS391 High-Speed Differential Line Drivers", Texas Instruments Incorporated datasheet ID: SLLS362D, (2001).

[56] "Ultralow-Noise, High PSRR, Fast, RF, 1A Low-Dropout Linear Regulators", Texas Instruments Incorporated datasheet ID: SLVS351L, (2010).

[57] "SN65LVDS9637 High-Speed Differential Line Receivers", Texas Instruments Incorporated datasheet ID: SLLS262Q, (2007).

[58] “SN65LVDT100 Differential Translator / Repeater”, Texas Instruments Incorporated datasheet ID: SLLS516C, (2004).

[59] “ic-HK, ic-HKB 155 MHz Laser Switch”, IC Haus datasheet ID: Rev F1, (2009).

[60] "2.5 V to $5.5 \mathrm{~V}, 120 \mu \mathrm{A}, 2-W i r e$ Interface, Voltage-Output 8-/10-/12-Bit DACs", Analog Devices Ltd datasheet ID: AD5301/AD5311/AD5321Rev B, (2007).

[61] "DM9000A Ethernet Controller with General Processor Interface", Davicom Semiconductor Inc datasheet ID: DM9000A-17-DS-F01, (2006).

[62] "ATmega16U4, ATmega32U4, 8-bit Microcontroller with 16/32K Bytes of ISP Flash and USB Controller", Atmel Corporation datasheet ID: 7766E-AVR-04/10, (2010).

[63] "Application Note 380: Test DDR SDRAM Interfaces on Hardware", Altera Corporation, AN-380-1.1, (2006) 